\title{
2015 American Thyroid Association Management Guidelines for Adult Patients with Thyroid Nodules and Differentiated Thyroid Cancer
}

\author{
The American Thyroid Association Guidelines Task Force \\ on Thyroid Nodules and Differentiated Thyroid Cancer
}

\author{
Bryan R. Haugen, ${ }^{1,{ }^{*}}$ Erik K. Alexander ${ }^{2}$ Keith C. Bible, ${ }^{3}$ Gerard M. Doherty, ${ }^{4}$ Susan J. Mandel,${ }^{5}$ \\ Yuri E. Nikiforov, ${ }^{6}$ Furio Pacini, ${ }^{7}$ Gregory W. Randolph, ${ }^{8}$ Anna M. Sawka, ${ }^{9}$ Martin Schlumberger, ${ }^{10}$ \\ Kathryn G. Schuff, ${ }^{11}$ Steven I. Sherman, ${ }^{12}$ Julie Ann Sosa, ${ }^{13}$ David L. Steward, ${ }^{14}$ \\ R. Michael Tuttle, ${ }^{15}$ and Leonard Wartofsky ${ }^{16}$
}

Background: Thyroid nodules are a common clinical problem, and differentiated thyroid cancer is becoming increasingly prevalent. Since the American Thyroid Association's (ATA's) guidelines for the management of these disorders were revised in 2009, significant scientific advances have occurred in the field. The aim of these guidelines is to inform clinicians, patients, researchers, and health policy makers on published evidence relating to the diagnosis and management of thyroid nodules and differentiated thyroid cancer.

Methods: The specific clinical questions addressed in these guidelines were based on prior versions of the guidelines, stakeholder input, and input of task force members. Task force panel members were educated on knowledge synthesis methods, including electronic database searching, review and selection of relevant citations, and critical appraisal of selected studies. Published English language articles on adults were eligible for inclusion. The American College of Physicians Guideline Grading System was used for critical appraisal of evidence and grading strength of recommendations for therapeutic interventions. We developed a similarly formatted system to appraise the quality of such studies and resultant recommendations. The guideline panel had complete editorial independence from the ATA. Competing interests of guideline task force members were regularly updated, managed, and communicated to the ATA and task force members.

Results: The revised guidelines for the management of thyroid nodules include recommendations regarding initial evaluation, clinical and ultrasound criteria for fine-needle aspiration biopsy, interpretation of fine-needle aspiration biopsy results, use of molecular markers, and management of benign thyroid nodules. Recommendations regarding the initial management of thyroid cancer include those relating to screening for thyroid cancer, staging and risk assessment, surgical management, radioiodine remnant ablation and therapy, and thyrotropin suppression therapy using levothyroxine. Recommendations related to long-term management

\footnotetext{
${ }^{1}$ University of Colorado School of Medicine, Aurora, Colorado.

${ }^{2}$ Brigham and Women's Hospital, Harvard Medical School, Boston, Massachusetts.

${ }^{3}$ The Mayo Clinic, Rochester, Minnesota.

${ }^{4}$ Boston Medical Center, Boston, Massachusetts.

${ }^{5}$ Perelman School of Medicine, University of Pennsylvania, Philadelphia, Pennsylvania.

${ }^{6}$ University of Pittsburgh Medical Center, Pittsburgh, Pennsylvania.

${ }^{7}$ The University of Siena, Siena, Italy.

${ }^{8}$ Massachusetts Eye and Ear Infirmary, Massachusetts General Hospital, Harvard Medical School, Boston, Massachusetts.

${ }^{9}$ University Health Network, University of Toronto, Toronto, Ontario, Canada.

${ }^{10}$ Institute Gustave Roussy and University Paris Sud, Villejuif, France.

${ }_{11}^{11}$ Oregon Health and Science University, Portland, Oregon.

${ }^{12}$ University of Texas M.D. Anderson Cancer Center, Houston, Texas.

${ }^{13}$ Duke University School of Medicine, Durham, North Carolina.

${ }^{14}$ University of Cincinnati Medical Center, Cincinnati, Ohio.

${ }^{15}$ Memorial Sloan Kettering Cancer Center, New York, New York.

${ }^{16}$ MedStar Washington Hospital Center, Washington, DC.

*Chair.

Authors are listed in alphabetical order and were appointed by ATA to independently formulate the content of this manuscript. None of the scientific or medical content of the manuscript was dictated by the ATA.
} 
of differentiated thyroid cancer include those related to surveillance for recurrent disease using imaging and serum thyroglobulin, thyroid hormone therapy, management of recurrent and metastatic disease, consideration for clinical trials and targeted therapy, as well as directions for future research.

Conclusions: We have developed evidence-based recommendations to inform clinical decision-making in the management of thyroid nodules and differentiated thyroid cancer. They represent, in our opinion, contemporary optimal care for patients with these disorders.

\section{INTRODUCTION}

$\mathbf{T}$ HYROID NODULES ARE A common clinical problem. Epidemiologic studies have shown the prevalence of palpable thyroid nodules to be approximately $5 \%$ in women and $1 \%$ in men living in iodine-sufficient parts of the world $(1,2)$. In contrast, high-resolution ultrasound (US) can detect thyroid nodules in $19 \%-68 \%$ of randomly selected individuals, with higher frequencies in women and the elderly $(3,4)$. The clinical importance of thyroid nodules rests with the need to exclude thyroid cancer, which occurs in $7 \%-15 \%$ of cases depending on age, sex, radiation exposure history, family history, and other factors $(5,6)$. Differentiated thyroid cancer (DTC), which includes papillary and follicular cancer, comprises the vast majority ( $>90 \%$ ) of all thyroid cancers (7). In the United States, approximately 63,000 new cases of thyroid cancer were predicted to be diagnosed in 2014 (8) compared with 37,200 in 2009 when the last ATA guidelines were published. The yearly incidence has nearly tripled from 4.9 per 100,000 in 1975 to 14.3 per 100,000 in 2009 (9). Almost the entire change has been attributed to an increase in the incidence of papillary thyroid cancer (PTC). Moreover, $25 \%$ of the new thyroid cancers diagnosed in 1988-1989 were $\leq 1 \mathrm{~cm}$ compared with $39 \%$ of the new thyroid cancer diagnoses in 2008-2009 (9). This tumor shift may be due to the increasing use of neck ultrasonography or other imaging and early diagnosis and treatment (10), trends that are changing the initial treatment and follow-up for many patients with thyroid cancer. A recent population-based study from Olmsted County reported the doubling of thyroid cancer incidence from 2000 to 2012 compared to the prior decade as entirely attributable to clinically occult cancers detected incidentally on imaging or pathology (11). By 2019, one study predicts that PTC will become the third most common cancer in women at a cost of \$19-21 billion in the United States (12). Optimization of longterm health outcomes and education about potential prognosis for individuals with thyroid neoplasms is critically important.

In 1996, the American Thyroid Association (ATA) published treatment guidelines for patients with thyroid nodules and DTC (13). Over the last 15-20 years, there have been many advances in the diagnosis and therapy of both thyroid nodules and DTC, but clinical controversy exists in many areas. A long history of insufficient peer-reviewed research funding for high-quality clinical trials in the field of thyroid neoplasia may be an important contributing factor to existing clinical uncertainties (12). Methodologic limitations or conflicting findings of older studies present a significant challenge to modern-day medical decision-making in many aspects of thyroid neoplasia. Although they are not a specific focus of these guidelines, we recognize that feasibility and cost considerations of various diagnostic and therapeutic options also present important clinical challenges in many clinical practice settings.

\section{AIM AND TARGET AUDIENCE}

Our objective in these guidelines is to inform clinicians, patients, researchers, and health policy makers about the best available evidence (and its limitations), relating to the diagnosis and treatment of adult patients with thyroid nodules and DTC. These guidelines should not be applied to children ( $<18-20$ years old); recent ATA guidelines for children with thyroid nodules and DTC were published in 2015 (14). This document is intended to inform clinical decision-making. A major goal of these guidelines is to minimize potential harm from overtreatment in a majority of patients at low risk for disease-specific mortality and morbidity, while appropriately treating and monitoring those patients at higher risk. These guidelines should not be interpreted as a replacement for clinical judgement and should be used to complement informed, shared patient-health care provider deliberation on complex issues. It is important to note that national clinical practice guidelines may not necessarily constitute a legal standard of care in all jurisdictions (15). If important differences in practice settings present barriers to meaningful implementation of the recommendations of these guidelines, interested physicians or groups (in or outside of the United States) may consider adapting the guidelines using established methods $(16,17)$ (ADAPTE Collaboration, 2009; www.g-i-n.net). The ADAPTE Collaboration is an international group of researchers, guideline developers, and guideline implementers who aim to promote the development and use of clinical practice guidelines through the adaption of existing guidelines. Because our primary focus was reviewing the quality of evidence related to health outcomes and diagnostic testing, we decided a priori not to focus on economic resource implications in these guidelines. As part of our review, we identified some knowledge gaps in the field, with associated future research priorities.

Other groups have previously developed clinical practice guidelines, including the American Association of Clinical Endocrinologists, Associazione Medici Endocrinologi, and the European Thyroid Association (18), the British Thyroid Association and The Royal College of Physicians (19), and the National Comprehensive Cancer Network (www.nccn .org). The European Thyroid Association has published consensus guidelines for postoperative US in the management of DTC (20). The Society for Nuclear Medicine and Molecular Imaging (21) and the European Association of Nuclear Medicine have also published guidelines for radioiodine (RAI) therapy of DTC (22). The Japanese Society of Thyroid Surgeons and the Japanese Association of Endocrine Surgeons 
Table 1. Interpretation of the American College of Physicians' Guideline Grading SYSTEM (FOR THERAPEUTIC INTERVENTIONS)

\begin{tabular}{|c|c|c|}
\hline Recommendation & Clarity of risk/benefit & Implications \\
\hline Strong recommendation & $\begin{array}{l}\text { Benefits clearly outweigh } \\
\text { harms and burdens, } \\
\text { or vice versa. }\end{array}$ & $\begin{array}{l}\text { Patients: Most would want course of action; a person should } \\
\text { request discussion if an intervention is not offered. } \\
\text { Clinicians: Most patients should receive the recommended } \\
\text { course of action. } \\
\text { Policymakers: The recommendation can be adopted as policy } \\
\text { in most circumstances. }\end{array}$ \\
\hline Weak recommendation & $\begin{array}{l}\text { Benefits closely balanced } \\
\text { with harms and burdens. }\end{array}$ & $\begin{array}{l}\text { Patients: Many would want course of action, but some may } \\
\text { not; the decision may depend on individual circumstances. } \\
\text { Clinicians: Different choices will be appropriate for different } \\
\text { patients; the management decision should be consistent } \\
\text { with patients' preferences and circumstances. } \\
\text { Policymakers: Policymaking will require careful consideration } \\
\text { and stakeholder input. }\end{array}$ \\
\hline No recommendation & $\begin{array}{l}\text { Balance of benefits and } \\
\text { risks cannot be determined. }\end{array}$ & Decisions based on evidence cannot be made. \\
\hline
\end{tabular}

have recently revised guidelines on treatment of patients with thyroid tumors (23). Given the existing controversies in the field, differences in critical appraisal approaches for existing evidence, and differences in clinical practice patterns across geographic regions and physician specialties, it should not be surprising that the organizational guidelines are not in complete agreement for all issues. Such differences highlight the importance of clarifying evidence uncertainties with future high quality clinical research.

\section{METHODS}

ATA Thyroid Nodules and Differentiated Thyroid Cancer guidelines were published in 2006 (24) and revised in 2009
(25). Because of the rapid growth of the literature on this topic, plans for revising the guidelines within approximately 4 years of publication were made at the inception of the project. A task force chair was appointed by the ATA President with approval of the Board. A task force of specialists with complementary expertise (endocrinology, surgery, nuclear medicine, radiology, pathology, oncology, molecular diagnostics, and epidemiology) was appointed. In order to have broad specialty and geographic representation, as well as fresh perspectives, one-third of the task force is replaced for each iteration of the guidelines, per ATA policy. Upon discussion among the panel members and the Chair with other Chairs of other ATA guideline committees, the American College of Physicians' (ACP) Grading System was adopted

Table 2. Recommendations (for Therapeutic Interventions) Based on Strength of Evidence

Recommendation

and evidence quality

Description of supporting evidence $\mathrm{a}^{\mathrm{a}}$

Interpretation

Strong recommendation

High-quality evidence

Moderate-quality evidence

Low-quality evidence

Weak recommendation

High-quality evidence

Moderate-quality evidence

Low-quality evidence

Insufficient
RCT without important limitations or overwhelming evidence from observational studies

RCT with important limitations or strong evidence from observational studies

Observational studies/case studies

RCT without important limitations or overwhelming evidence from observational studies

RCT with important limitations or strong evidence from observational studies

Observational studies/case studies

Evidence is conflicting, of poor quality, or lacking
Can apply to most patients in most circumstances without reservation

Can apply to most patients in most circumstances without reservation

May change when higher-quality evidence becomes available

Best action may differ based on circumstances or patients' values

Best action may differ based on circumstances or patients' values

Other alternatives may be equally reasonable

Insufficient evidence to recommend for or against

${ }^{\text {a }}$ This description of supporting evidence refers to therapy, therapeutic strategy, or prevention studies. The description of supporting evidence is different for diagnostic accuracy studies.

$\mathrm{RCT}$, randomized controlled trial. 
Table 3. Interpretation of the American Thyroid Association Guideline Grading System For Diagnostic Tests

\begin{tabular}{cc}
\hline $\begin{array}{c}\text { Accuracy of diagnostic } \\
\text { information versus risks } \\
\text { and burden of testing }\end{array}$ & Implications \\
\hline
\end{tabular}

$\begin{array}{ll}\text { Strong } & \text { Knowledge of the diagnostic } \\ \text { recommendation } & \begin{array}{l}\text { test result clearly outweighs } \\ \text { risks and burden of testing } \\ \text { or vice versa. }\end{array}\end{array}$

\section{Weak recommendation}

\section{No recommendation}

Balance of knowledge of the diagnostic test result cannot be determined.

Knowledge of the diagnostic test result is closely balanced with risks and burden of testing.
Patients: In the case of an accurate test for which benefits outweigh risks/burden, most would want the diagnostic to be offered (with appropriate counseling). A patient should request discussion of the test if it is not offered. In contrast, for a test in which risks and burden outweigh the benefits, most patients should not expect the test to be offered.

Clinicians: In the case of an accurate test for which benefits outweigh risks/burden, most patients should be offered the diagnostic test (and provided relevant counseling). Counseling about the test should include a discussion of the risks, benefits, and uncertainties related to testing (as applicable), as well as the implications of the test result. In contrast, for a test in which risks and burden outweigh the perceived benefits, most patients should not be offered the test, or if the test is discussed, the rationale against the test should, for the particular clinical situation, be explained.

Policymakers: In the case of an accurate test for which benefits outweigh risks/burden, availability of the diagnostic test should be adopted in health policy. In contrast, for a test in which risks and burden outweigh the perceived benefits, some restrictions on circumstances for test use may need to be considered.

Patients: Most would want to be informed about the diagnostic test, but some would not want to seriously consider undergoing the test; a decision may depend on the individual circumstances (e.g., risk of disease, comorbidities, or other), the practice environment, feasibility of optimal execution of the test, and consideration of other available options.

Clinicians: Different choices will be appropriate for different patients, and counseling about the test (if being considered) should include a discussion of the risks, benefits, and uncertainties related to testing (as applicable), as well as the implications of the test result. The decision to perform the test should include consideration of the patients' values, preferences, feasibility, and the specific circumstances. Counseling the patient on why the test may be helpful or not, in her/his specific circumstance, may be very valuable in the decision-making process.

Policymakers: Policymaking decisions on the availability of the test will require discussion and stakeholder involvement.

Decisions on the use of the test based on evidence from scientific studies cannot be made.

\footnotetext{
${ }^{a}$ Frequently in these guidelines, the accuracy of the diagnosis of thyroid cancer (relative to a histologic gold standard) was the diagnostic outcome unless otherwise specified. However, prognostic, disease staging, or risk stratification studies were also included in the grading scheme of diagnostic studies. For disease staging systems, the implication for use would be on the part of the clinician, in reporting results in the medical record and communicating them to the patient (at the applicable time point in disease or follow-up trajectory), as opposed to offering a specific choice of staging/risk stratification system to the patient.
}

for use in these guidelines, relating to critical appraisal and recommendations on therapeutic interventions (26) (Tables 1 and 2). An important component of these guidelines was judged to be critical appraisal of studies of diagnostic tests; however, the ACP Guideline Grading System is not designed for this purpose. We reviewed a number of appraisal systems for diagnostic tests, but some of the complexity and the timeconsuming nature of some systems limited their feasibility for implementation in our group (27-31). We drafted, revised, and piloted the use of a newly developed diagnostic test appraisal system that was acceptable to panel members. This system included consideration of the following 
Table 4. Recommendations (for Diagnostic Interventions) Based on Strength of Evidence

\begin{tabular}{|c|c|}
\hline $\begin{array}{l}\text { Recommendation and } \\
\text { evidence quality }\end{array}$ & Methodologic quality of supporting evidence \\
\hline \multicolumn{2}{|l|}{ Strong recommendation } \\
\hline High-quality evidence & $\begin{array}{l}\text { Evidence from one or more well-designed } \\
\text { nonrandomized diagnostic accuracy studies } \\
\text { (i.e., observational-cross-sectional or cohort) } \\
\text { or systematic reviews/meta-analyses of such } \\
\text { observational studies (with no concern about } \\
\text { internal validity or external generalizability } \\
\text { of the results) }\end{array}$ \\
\hline Moderate-quality evidence & $\begin{array}{l}\text { Evidence from nonrandomized diagnostic accuracy } \\
\text { studies (cross-sectional or cohort), with one or more } \\
\text { possible limitations causing minor concern about } \\
\text { internal validity or external generalizability of the } \\
\text { results }\end{array}$ \\
\hline Low-quality evidence & $\begin{array}{l}\text { Evidence from nonrandomized diagnostic accuracy } \\
\text { studies with one or more important limitations } \\
\text { causing serious concern about internal validity } \\
\text { or external generalizability of the results }\end{array}$ \\
\hline \multicolumn{2}{|l|}{ Weak recommendation } \\
\hline High-quality evidence & $\begin{array}{l}\text { Evidence from one or more well-designed nonrando- } \\
\text { mized diagnostic accuracy studies } \\
\text { (i.e., observational-cross-sectional or cohort) } \\
\text { or systematic reviews/meta-analyses of such ob- } \\
\text { servational studies (with no concern about internal } \\
\text { validity or external generalizability of the results) }\end{array}$ \\
\hline Moderate-quality evidence & $\begin{array}{l}\text { Evidence from nonrandomized diagnostic accuracy } \\
\text { studies (cross-sectional or cohort), with one or more } \\
\text { possible limitations causing minor concern about } \\
\text { internal validity or external generalizability of the } \\
\text { results }\end{array}$ \\
\hline Low-quality evidence & $\begin{array}{l}\text { Evidence from nonrandomized diagnostic accuracy } \\
\text { studies with one or more important limitations } \\
\text { causing serious concern about internal validity or } \\
\text { external generalizability of the results. }\end{array}$ \\
\hline Insufficient & $\begin{array}{l}\text { Evidence may be of such poor quality, conflicting, } \\
\text { lacking (i.e., studies not done), or not externally } \\
\text { generalizable to the target clinical population such } \\
\text { that the estimate of the true effect of the test is } \\
\text { uncertain and does not permit a reasonable } \\
\text { conclusion to be made. }\end{array}$ \\
\hline
\end{tabular}

Implies the test can be offered to most patients in most applicable circumstances without reservation.

Implies the test can be offered to most patients in most applicable circumstances without reservation.

Implies the test can be offered to most patients in most applicable circumstances, but the utilization of the test may change when higher-quality evidence becomes available.

The degree to which the diagnostic test is seriously considered may differ depending on circumstances or patients' or societal values.

The degree to which the diagnostic test is seriously considered may differ depending on individual patients'/ practice circumstances or patients' or societal values.

Alternative options may be equally reasonable.

Insufficient evidence exists to recommend for or against routinely offering the diagnostic test.

methodologic elements: consecutive recruitment of patients representative of clinical practice, use of an appropriate reference gold standard, directness of evidence (e.g., target population of interest, testing procedures representative of clinical practice, and relevant outcomes), precision of diagnostic accuracy measures (e.g., width of confidence intervals for estimates such as sensitivity, specificity), and consistency of results among studies using the same test (Tables 3 and 4). In the majority of circumstances (unless otherwise specified), the outcome of interest for the diagnostic test was the diagnosis of thyroid cancer (relative to a histologic gold standard). However, prognostic studies were also graded using the diagnostic study critical appraisal framework. In terms of strength of recommendation for use of diagnostic studies, we modeled our approach on the ACP system for therapeutic studies, as previously described, but the target outcome was the accuracy in establishing a definitive diagnosis, largely relating to the diagnosis of new or recurrent malignancy (unless otherwise specified). Diagnostic tests or risk stratification systems used for estimation of prognosis were also appraised using the diagnostic test grading system. An important limitation of our diagnostic test appraisal system is that it does not specifically examine the clinical utility of a test in improving long-term health outcomes by execution of the test as part of an intended therapeutic strategy (unless specifically noted). However, as much as possible, we tried to separate recommendations on the diagnostic accuracy of a test from therapeutic management based on the test result, with the latter grading being more rigorous and based on longer term outcomes (whenever possible). It is important to note that according to our diagnostic test grading system, a body of well-executed nonrandomized diagnostic accuracy 
studies could be considered high-quality evidence; yet, a therapeutic strategy incorporating the use of the diagnostic test would require one or more well-executed randomized controlled trials (RCTs) to be considered high-quality evidence. In developing and applying our diagnostic test critical appraisal system, we considered American societal values, relating to the importance of informing patients about potentially helpful tests developed for their clinical situation (with counseling on relevant limitations) and the role of patients in informed, shared decision-making relating to diagnostic and therapeutic strategies. Such input was based on thoughtful consideration of stakeholder input, including input from physician stakeholders who were committee members. Because this was a preliminary pilot utilization of this diagnostic test critical appraisal system by our group, we have labeled recommendations using this system in the manuscript (diagnostic test recommendation). Moreover, we anticipate that the future iterations of these guidelines will likely incorporate further refinements to the system, or even possible adoption of another system, if it is superior and feasible to execute by contributing physicians.

Prior to initiating the reviews, all task force members were provided written and verbal group advice on conducting electronic literature searches, critical appraisal of articles, and rationale for formulating strength of recommendations from a panel member with epidemiology and systematic review expertise (via e-mail documents, a teleconference meeting on February 21, 2012). For each question, a primary reviewer performed a literature search, appraised relevant literature, generated recommendations, accompanying text, and a relevant bibliography. This was then reviewed by the secondary reviewer, revised as needed, and presented for review by the entire panel. Feedback and suggestions for revisions from the Chair and panel members were obtained via e-mail, regularly scheduled teleconferences, and face-toface meetings held in conjunction with scientific meetings. Once the manuscript was drafted, all suggestions for revi- sions were regularly reviewed by all panel members in the form of a tracked changes draft manuscript and teleconferences. The draft document continued to be revised until no further suggestions for further revisions were requested by any panel members. Thus, general consensus on acceptability of recommendations and manuscript text was achieved, with the fundamental understanding that not all recommendations may be feasible in all practice settings.

Formal stakeholder input in development of these guidelines was sought from ATA membership in an online survey distributed in October 2011. Thyroid cancer survivor group leadership input was sought from three North American thyroid cancer groups via e-mail correspondence in January to March of 2012. We also reviewed any letters, editorials, or reviews of the 2009 iteration of the guidelines (25) that were collected by the current Chair of the committee. Prepublication verbal feedback on some of the key guideline recommendations was received at a formal Satellite Symposium held in conjunction with the Endocrine Society meeting in Chicago on June 19, 2014. The guideline manuscript was reviewed and approved by the ATA Board of Directors, then made available to the ATA membership for review and comments in September 2014. Substantive comments were received from 33 members representing endocrinology, surgery, pathology, and nuclear medicine. Feedback and suggestions were formally discussed by the panel, and revisions were made to the manuscript prior to journal submission. The organization of management guideline recommendations is shown in Table 5.

The medical opinions expressed here are those of the authors, and the committee had complete editorial independence from the ATA in writing the guidelines. No funding was received by individual committee members from the ATA or industry for work on these guidelines. Competing interests of all committee members were reviewed at inception of the group, yearly, and upon completion of the guidelines and are included with this document.

Table 5. Organization of the 2015 ATA Guidelines fOr Thyroid Nodules AND DiffERentiated Thyroid CANCER

\begin{tabular}{|c|c|c|c|}
\hline Page & Location key & Sections and subsections & Item $^{\mathrm{a}}$ \\
\hline 10 & [A1] & THYROID NODULE GUIDELINES & \\
\hline 10 & [A2] & $\begin{array}{l}\text { What is the role of thyroid cancer screening in people with } \\
\text { familial follicular cell-derived DTC? }\end{array}$ & $\mathrm{R} 1^{\mathrm{b}}$ \\
\hline 10 & [A3] & $\begin{array}{l}\text { What is the appropriate laboratory and imaging evaluation for } \\
\text { patients with clinically or incidentally discovered thyroid } \\
\text { nodules? }\end{array}$ & \\
\hline 10 & [A4] & Serum thyrotropin measurement & $\mathrm{R} 2$ \\
\hline 11 & [A5] & Serum thyroglobulin measurement & R3 \\
\hline 11 & [A6] & Serum calcitonin measurement & R4 \\
\hline 11 & [A7] & {$[18 \mathrm{~F}]$ Fluorodeoxyglucose positron emission tomography ${ }^{\mathrm{b}}$} & $\mathrm{R}^{\mathrm{b}}$ \\
\hline 12 & [A8] & Thyroid sonography & R6 \\
\hline 12 & [A9] & US for FNA decision-making & $\mathrm{R} 7$ \\
\hline 12 & {$[\mathrm{~A} 10]$} & $\begin{array}{l}\text { Recommendations for diagnostic FNA of a thyroid nodule based on } \\
\text { sonographic pattern }^{c}\end{array}$ & $\mathrm{R} 8^{\mathrm{c}} \mathrm{F} 1^{\mathrm{c}}, \mathrm{F} 2^{\mathrm{c}}, \mathrm{T} 6^{\mathrm{c}}$ \\
\hline 16 & [A11] & $\begin{array}{l}\text { What is the role of FNA, cytology interpretation, and molecular } \\
\text { testing in patients with thyroid nodules? }\end{array}$ & $\mathrm{R} 9^{\mathrm{c}}, \mathrm{F} 1^{\mathrm{c}}, \mathrm{T} 7^{\mathrm{c}}$ \\
\hline 17 & [A12] & Nondiagnostic cytology & $\mathrm{R} 10$ \\
\hline 17 & [A13] & Benign cytology & R11 \\
\hline 18 & [A14] & Malignant cytology & $\mathrm{R} 12$ \\
\hline 19 & [A15] & Indeterminate cytology (AUS/FLUS, FN, SUSP) ${ }^{\mathrm{c}}$ & \\
\hline
\end{tabular}


Table 5. (Continued)

\begin{tabular}{|c|c|c|c|}
\hline Page & Location key & Sections and subsections & Item $^{\mathrm{a}}$ \\
\hline 19 & [A16] & $\begin{array}{l}\text { What are the principles of the molecular testing of FNA } \\
\text { samples? }\end{array}$ & R13-14 \\
\hline 21 & [A17] & AUS/FLUS cytology ${ }^{\mathrm{c}}$ & $\mathrm{R} 15^{\mathrm{c}}$ \\
\hline 22 & [A18] & Follicular neoplasm/suspicious for follicular neoplasm cytology ${ }^{c}$ & $\mathrm{R} 16^{\mathrm{c}}$ \\
\hline 23 & [A19] & Suspicious for malignancy cytology ${ }^{\mathrm{c}}$ & $\mathrm{R} 17^{\mathrm{c}}$ \\
\hline 23 & [A20] & $\begin{array}{l}\text { What is the utility of }{ }^{18} \text { FDG -PET scanning to predict malignant } \\
\text { or benign disease when FNA cytology is indeterminate (AUS/ } \\
\text { FLUS, FN, SUSP)? }\end{array}$ & $\mathrm{R} 18^{\mathrm{b}}$ \\
\hline 23 & [A21] & $\begin{array}{l}\text { What is the appropriate operation for cytologically indeterminate } \\
\text { thyroid nodules? }^{\mathrm{c}}\end{array}$ & $\mathrm{R} 19-20^{\mathrm{c}}$ \\
\hline 25 & [A22] & $\begin{array}{l}\text { How should multinodular thyroid glands (i.e., two or more } \\
\text { clinically relevant nodules) be evaluated for malignancy? }\end{array}$ & R21-22 \\
\hline 25 & [A23] & $\begin{array}{l}\text { What are the best methods for long-term follow-up of patients } \\
\text { with thyroid nodules? }\end{array}$ & \\
\hline 25 & [A24] & $\begin{array}{l}\text { Recommendations for initial follow-up of nodules with benign FNA } \\
\text { cytology }\end{array}$ & $\mathrm{R} 23 \mathrm{~A}-\mathrm{C}^{\mathrm{c}}$ \\
\hline 25 & [A25] & $\begin{array}{l}\text { Recommendation for follow-up of nodules with two benign FNA } \\
\text { cytology results }\end{array}$ & $\mathrm{R} 23 \mathrm{D}^{\mathrm{b}}$ \\
\hline 26 & [A26] & Follow-up for nodules that do not meet FNA criteria ${ }^{\mathrm{b}}$ & $\mathrm{R} 24^{\mathrm{b}}$ \\
\hline 27 & [A27] & $\begin{array}{l}\text { What is the role of medical or surgical therapy for benign thyroid } \\
\text { nodules? }\end{array}$ & R25-29 \\
\hline 27 & [A28] & How should thyroid nodules in pregnant women be managed? & \\
\hline 27 & [A29] & FNA for thyroid nodules discovered during pregnancy & $\mathrm{R} 30$ \\
\hline 28 & [A30] & $\begin{array}{l}\text { Approaches to pregnant patients with malignant or indeterminate } \\
\text { cytology }\end{array}$ & R31 \\
\hline 28 & [B1] & $\begin{array}{l}\text { DIFFERENTIATED THYROID CANCER: INITIAL } \\
\text { MANAGEMENT GUIDELINES }\end{array}$ & \\
\hline 29 & [B2] & Goals of initial therapy of DTC & \\
\hline 29 & [B3] & $\begin{array}{l}\text { What is the role of preoperative staging with diagnostic imaging } \\
\text { and laboratory tests? }\end{array}$ & \\
\hline 29 & [B4] & Neck imaging-ultrasound & R32 F3, T6, T8 \\
\hline 30 & [B5] & Neck imaging-CT/MRI/PET ${ }^{\mathrm{c}}$ & $\mathrm{R} 33^{\mathrm{c}}$ \\
\hline 31 & [B6] & Measurement of serum $\mathrm{Tg}$ and anti-Tg antibodies & R34 \\
\hline 31 & [B7] & $\begin{array}{l}\text { Operative approach for a biopsy diagnostic for follicular cell-derived } \\
\text { malignancy }\end{array}$ & $\mathrm{R} 35^{\mathrm{c}}$ \\
\hline 33 & [B8] & Lymph node dissection & R36-37, F3 \\
\hline 35 & [B9] & Completion thyroidectomy & $\mathrm{R} 38$ \\
\hline 35 & [B10] & $\begin{array}{l}\text { What is the appropriate perioperative approach to voice and } \\
\text { parathyroid issues? }\end{array}$ & \\
\hline 35 & [B11] & Preoperative care communication ${ }^{b}$ & $\mathrm{R} 39^{\mathrm{b}}$ \\
\hline 35 & [B12] & Preoperative voice assessment ${ }^{\mathrm{b}}$ & $\mathrm{R} 40-41^{\mathrm{b}}, \mathrm{T} 9^{\mathrm{b}}$ \\
\hline 36 & [B13] & Intraoperative voice and parathyroid management ${ }^{\mathrm{b}}$ & $\mathrm{R} 42-43^{\mathrm{b}}$ \\
\hline 37 & [B14] & Postoperative care ${ }^{\mathrm{b}}$ & $\mathrm{R} 44-45^{\mathrm{b}}$ \\
\hline 37 & {$[\mathrm{~B} 15]$} & $\begin{array}{l}\text { What are the basic principles of histopathologic evaluation of } \\
\text { thyroidectomy samples? }\end{array}$ & $\mathrm{R} 46^{\mathrm{b}}$ \\
\hline 40 & [B16] & $\begin{array}{l}\text { What is the role of postoperative staging systems and risk } \\
\text { stratification in the management of DTC? }\end{array}$ & \\
\hline 40 & [B17] & Postoperative staging & $\mathrm{R} 47$ \\
\hline 40 & [B18] & AJCC/UICC TNM staging & T10 \\
\hline 41 & [B19] & $\begin{array}{l}\text { What initial stratification system should be used to estimate } \\
\text { the risk of persistent/recurrent disease? }\end{array}$ & $\mathrm{R} 48^{\mathrm{c}}, \mathrm{T} 11^{\mathrm{b}}, \mathrm{T} 12^{\mathrm{c}}$ \\
\hline 43 & [B20] & $\begin{array}{l}\text { Potential impact of specific clinico-pathologic features on the risk } \\
\text { estimates in PTC }\end{array}$ & \\
\hline 44 & [B21] & $\begin{array}{l}\text { Potential impact of } B R A F^{V 600 E} \text { and other mutations on risk of } \\
\text { estimates in PTC }\end{array}$ & \\
\hline 45 & [B22] & Potential impact of postoperative serum Tg on risk estimates ${ }^{\mathrm{b}}$ & \\
\hline 46 & [B23] & $\begin{array}{l}\text { Proposed modifications to the } 2009 \text { ATA initial risk stratification } \\
\text { system }^{\mathrm{b}}\end{array}$ & $\mathrm{T} 12^{\mathrm{c}}$ \\
\hline 46 & [B24] & Risk of recurrence as a continuum of risk ${ }^{b}$ & $\mathrm{~F} 4^{\mathrm{b}}$ \\
\hline 46 & [B25] & How should initial risk estimates be modified over time? ${ }^{\text {b }}$ & R49 \\
\hline 47 & [B26] & Proposed terminology to classify response to therapy and clinical & \\
\hline
\end{tabular}


TABle 5. (CONTINUED)

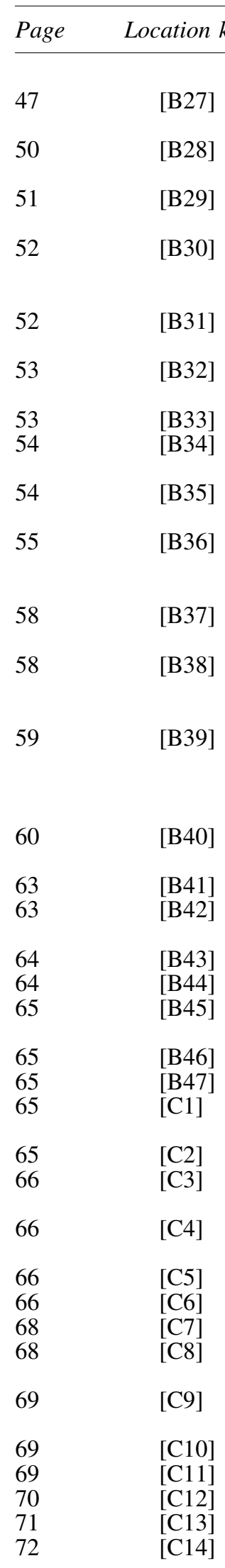

Sections and subsections

Item $^{\mathrm{a}}$

implications $^{\mathrm{b}}$

Excellent response: no clinical, biochemical, or structural evidence of $\quad \mathrm{T}^{\mathrm{b}}{ }^{\mathrm{b}}$ disease after initial therapy (remission, NED) ${ }^{\mathrm{b}}$

Biochemical incomplete response: abnormal Tg values in the absence $\quad \mathrm{T}^{\mathrm{b}} 3^{\mathrm{b}}$ of localizable disease ${ }^{\mathrm{b}}$

Structural incomplete response: persistent or newly identified loco- $\quad \mathrm{T}^{\mathrm{b}}{ }^{\mathrm{b}}$ regional or distant metastases ${ }^{\mathrm{b}}$

Indeterminate response: biochemical or structural findings that cannot be classified as either benign or malignant (acceptable response) $)^{\mathrm{b}}$

Using risk stratification to guide disease surveillance and therapeutic management decisions ${ }^{\mathrm{b}}$

Should postoperative disease status be considered in decisionmaking for RAI therapy for patients with DTC?

Utility of postoperative serum $\mathrm{Tg}$ in clinical decision-making

Potential role of postoperative US in conjunction with postoperative serum $\mathrm{Tg}$ in clinical decision-making

Role of postoperative radioisotope diagnostic scanning in clinical decision-making

What is the role of RAI (including remnant ablation, adjuvant therapy, or therapy persistent disease) after thyroidectomy in the primary management of differentiated thyroid cancer?

What is the role of molecular marker status in therapeutic RAI decision-making? ${ }^{\mathrm{b}}$

How long does thyroid hormone need to be withdrawn in preparation for RAI remnant ablation/treatment or diagnostic scanning?

Can rhTSH (Thyrogen) be used as an alternative to thyroxine withdrawal for remnant ablation or adjuvant therapy in patients who have undergone near-total or total thyroidectomy?

What activity of ${ }^{131}$ I should be used for remnant ablation or adjuvant therapy ${ }^{\mathrm{c}}$

Is a low-iodine diet necessary before remnant ablation?

Should a posttherapy scan be performed following remnant ablation or adjuvant therapy?

Early management of DTC after initial therapy

What is the appropriate degree of initial TSH suppression?

Is there a role for adjunctive external beam radiation or chemotherapy?

External beam radiation

Systemic adjuvant therapy DTC: LONG-TERM MANAGEMENT AND ADVANCED CANCER MANAGEMENT GUIDELINES

What are the appropriate features of long-term management?

What are the criteria for absence of persistent tumor (excellent response)?

What are the appropriate methods for following patients after initial therapy?

What is the role of serum Tg measurement in the follow-up of DTC?

Serum $\mathrm{Tg}$ measurement and clinical utility

Anti-Tg antibodies

What is the role of serum $\mathrm{Tg}$ measurement in patients who have not undergone RAI remnant ablation?

What is the role of US and other imaging techniques (RAI SPECT/CT, CT, MRI, PET-CT) during follow-up?

Cervical ultrasonography

Diagnostic whole-body RAI scans

${ }^{18}$ FDG-PET scanning

$\mathrm{CT}$ and $\mathrm{MRI}^{\mathrm{b}}$

Using ongoing risk stratification (response to therapy) to guide disease long-term surveillance and therapeutic management decisions $^{b}$

R51

T14

$\mathrm{R} 52^{\mathrm{b}}$

R53

R54

R55-56

R57

R58

R59

R60

R61

R62-63 ${ }^{\mathrm{c}}$

R64

R65

R66-67

R68

R69 ${ }^{\mathrm{b}}$ 
TABle 5. (CONTINUED)

\begin{tabular}{|c|c|c|c|}
\hline Page & Location key & Sections and subsections & Item $^{2}$ \\
\hline 72 & {$[\mathrm{C} 15]$} & $\begin{array}{l}\text { What is the role of TSH suppression during thyroid hormone } \\
\text { therapy in the long-term follow-up of DTC? }\end{array}$ & $\begin{array}{l}\mathrm{R} 70^{\mathrm{c}} \\
\mathrm{T} 15^{\mathrm{b}}\end{array}$ \\
\hline 74 & {$[\mathrm{C} 16]$} & $\begin{array}{l}\text { What is the most appropriate management of DTC patients with } \\
\text { metastatic disease? }\end{array}$ & \\
\hline 74 & {$[\mathrm{C} 17]$} & $\begin{array}{l}\text { What is the optimal directed approach to patients with suspected } \\
\text { structural neck recurrence? }\end{array}$ & R71 \\
\hline 74 & [C18] & Nodal size threshold & \\
\hline 75 & [C19] & Extent of nodal surgery & \\
\hline 75 & [C20] & Ethanol injection ${ }^{b}$ & \\
\hline 75 & {$[\mathrm{C} 21]$} & Radiofrequency or laser ablation ${ }^{\mathrm{b}}$ & \\
\hline 75 & [C22] & Other therapeutic options ${ }^{\mathrm{b}}$ & \\
\hline 76 & [C23] & What is the surgical management of aerodigestive invasion? & R72 \\
\hline 76 & {$[\mathrm{C} 24]$} & $\begin{array}{l}\text { How should RAI therapy be considered for loco-regional or } \\
\text { distant metastatic disease? }\end{array}$ & \\
\hline 76 & [C25] & Administered activity of ${ }^{131} \mathrm{I}$ for loco-regional or metastatic disease ${ }^{c}$ & $\mathrm{R} 73^{\mathrm{c}}$ \\
\hline 77 & [C26] & $\begin{array}{l}\text { Use of rhTSH (Thyrogen) to prepare patients for }{ }^{131} \mathrm{I} \text { therapy for } \\
\text { loco-regional or metastatic disease }\end{array}$ & R74-75 \\
\hline 77 & [C27] & Use of lithium in ${ }^{131}$ I therapy & R76 \\
\hline 77 & [C28] & $\begin{array}{l}\text { How should distant metastatic disease to various organs be } \\
\text { treated? }\end{array}$ & \\
\hline 78 & [C29] & Treatment of pulmonary metastases & R77-78 \\
\hline 78 & [C30] & RAI treatment of bone metastases & R79 \\
\hline 79 & [C31] & $\begin{array}{l}\text { When should empiric RAI therapy be considered for Tg-positive, } \\
\text { RAI diagnostic scan-negative patients? }\end{array}$ & R80-82 \\
\hline 79 & [C32] & What is the management of complications of RAI therapy? & R83-85 \\
\hline 80 & [C33] & $\begin{array}{l}\text { How should patients who have received RAI therapy be } \\
\text { monitored for risk of secondary malignancies? }\end{array}$ & R86 \\
\hline 80 & [C34] & What other testing should patients receiving RAI therapy undergo? & R87 \\
\hline 80 & {$[\mathrm{C} 35]$} & $\begin{array}{l}\text { How should patients be counseled about RAI therapy and } \\
\text { pregnancy, breastfeeding, and gonadal function? }\end{array}$ & R88-90 \\
\hline 81 & [C36] & How is RAI-refractory DTC classified? ${ }^{\mathrm{b}}$ & $\mathrm{R} 91^{\mathrm{b}}$ \\
\hline 82 & [C37] & $\begin{array}{l}\text { Which patients with metastatic thyroid cancer can be followed } \\
\text { without additional therapy? }\end{array}$ & $\mathrm{R} 92^{\mathrm{b}}$ \\
\hline 82 & [C38] & What is the role for directed therapy in advanced thyroid cancer? ${ }^{c}$ & $\mathrm{R} 93^{\mathrm{c}}$ \\
\hline 84 & [C39] & Treatment of brain metastases & R94 \\
\hline 84 & {$[\mathrm{C} 40]$} & Who should be considered for clinical trials? ${ }^{\mathrm{b}}$ & $\mathrm{R} 95^{\mathrm{b}}$ \\
\hline 84 & [C41] & $\begin{array}{l}\text { What is the role of systemic therapy (kinase inhibitors, other } \\
\text { selective therapies, conventional chemotherapy, } \\
\text { bisphosphonates) in treating metastatic DTC }{ }^{\text {c }}\end{array}$ & \\
\hline 85 & [C42] & Kinase inhibitors ${ }^{\mathrm{b}}$ & $\mathrm{R} 96^{\mathrm{b}}, \mathrm{T} 16^{\mathrm{b}}$ \\
\hline 87 & [C43] & Patients for whom first-line kinase inhibitor therapy fails $\mathrm{s}^{\mathrm{b}}$ & $\mathrm{R} 97^{\mathrm{b}}$ \\
\hline 87 & [C44] & Management of toxicities from kinase inhibitor therapy ${ }^{\mathrm{b}}$ & $\mathrm{R} 98^{\mathrm{b}}, \mathrm{T} 17^{\mathrm{b}}$ \\
\hline 87 & [C45] & Other novel agents $\mathrm{b}^{\mathrm{b}}$ & R99 \\
\hline 87 & [C46] & Cytotoxic chemotherapy & R100 \\
\hline 88 & [C47] & Bone-directed agents ${ }^{\mathrm{c}}$ & $\mathrm{R} 101^{\mathrm{c}}$ \\
\hline 89 & [D1] & DIRECTIONS FOR FUTURE RESEARCH & \\
\hline 89 & [D2] & $\begin{array}{l}\text { Optimizing molecular markers for diagnosis, prognosis, and } \\
\text { therapeutic targets }\end{array}$ & \\
\hline 89 & [D3] & Active surveillance of DTC primary tumors & \\
\hline 90 & [D4] & Improved risk stratification & \\
\hline 90 & [D5] & $\begin{array}{l}\text { Improving our understanding of the risks and benefits of DTC } \\
\text { treatments and optimal implementation/utilization }\end{array}$ & \\
\hline 90 & [D6] & Issues with measurement of $\mathrm{Tg}$ and anti-Tg antibodies & \\
\hline 90 & [D7] & Management of metastatic cervical adenopathy detected on US & \\
\hline 91 & [D8] & Novel therapies for systemic RAI-refractory disease & \\
\hline 91 & [D9] & Survivorship care & \\
\hline
\end{tabular}

${ }^{\mathrm{a}} \mathrm{F}$, figure; R, recommendation; T, table.

${ }^{\mathrm{b}}$ New section/recommendation.

'Substantially changed recommendation compared with 2009.

ATA, American Thyroid Association; AUS/FLUS, atypia of undetermined significance/follicular lesion of undetermined significance; CT, computed tomography; DTC, differentiated thyroid cancer; FN, follicular neoplasm; FNA, fine-needle aspiration; ${ }^{18} \mathrm{FDG}-\mathrm{PET}$, $\left[{ }^{18}\right.$ F]fluorodeoxyglucose positron emission tomography; MRI, magnetic resonance imaging; NED, no evidence of disease; PET, positron emission tomography; RAI, radioactive iodine (radioiodine); rhTSH, recombinant human thyrotropin; SPECT/CT, single photon emission computed tomography-computed tomography; SUSP, suspicious for malignancy; Tg, thyroglobulin; TSH, thyrotropin; US, ultrasound. 


\section{[A1] THYROID NODULE GUIDELINES}

A thyroid nodule is a discrete lesion within the thyroid gland that is radiologically distinct from the surrounding thyroid parenchyma. Some palpable lesions may not correspond to distinct radiologic abnormalities (32). Such abnormalities do not meet the strict definition for thyroid nodules. Nonpalpable nodules detected on US or other anatomic imaging studies are termed incidentally discovered nodules or "incidentalomas." Nonpalpable nodules have the same risk of malignancy as do sonographically confirmed palpable nodules of the same size (33). Generally, only nodules $>1 \mathrm{~cm}$ should be evaluated, since they have a greater potential to be clinically significant cancers. Occasionally, there may be nodules $<1 \mathrm{~cm}$ that require further evaluation because of clinical symptoms or associated lymphadenopathy. In very rare cases, some nodules $<1 \mathrm{~cm}$ lack these sonographic and clinical warning signs yet may nonetheless cause future morbidity and mortality. This remains highly unlikely, and given the unfavorable cost/benefit considerations, attempts to diagnose and treat all such small thyroid cancers in an effort to prevent exceedingly rare outcomes is deemed to cause more harm than good. In general, the guiding clinical strategy acknowledges that most thyroid nodules are low risk, and many thyroid cancers pose minimal risk to human health and can be effectively treated.

\section{[A2] What is the role of thyroid cancer screening in people with familial follicular cell-derived DTC?}

\section{- RECOMMENDATION 1}

Screening people with familial follicular cell-derived DTC may lead to an earlier diagnosis of thyroid cancer, but the panel cannot recommend for or against US screening since there is no evidence that this would lead to reduced morbidity or mortality.

\section{(No recommendation, Insufficient evidence)}

Screening programs for patients at risk of oncological disease are usually advocated based on the following evidence: (a) a clear demonstration that the patient is indeed at risk; (b) demonstration that screening allows the detection of the disease at an earlier stage; (c) early diagnosis has an impact on subsequent outcome, both recurrence and survival.

Family members of patients with nonmedullary DTC may be considered at risk based on epidemiological evidence showing that $5 \%-10 \%$ of DTCs have a familial occurrence. However, in most of the pedigrees only two members are affected. There is controversy on whether two family members are sufficient to define a real familial disease rather than a fortuitous association. The probability estimates reported by Charkes (34) suggests that when only two first-degree family members are affected, the probability that the disease is sporadic is $62 \%$. This probability decreases when the number of affected family members is three or more. In contrast, the study by Capezzone et al. (35), which was statistically adjusted to minimize risk of "insufficient follow-up bias," demonstrates that even when only two family members are affected, the disease displays the features of "genetic anticipation" (occurrence of the disease at an earlier age and with more aggressive presentation in the subsequent generation compared with the first generation), which is considered good evidence for a distinct clinical entity possibly representing true familial disease. Appearance of the disease at an earlier age has also been found by Moses et al. (36). More advanced disease at presentation and slightly worse outcomes have been reported in familial cases by Capezzone et al. (35). More frequent multicentricity has been reported by Ito et al. (37), but disease-free and overall survival were similar to sporadic cases. In the study by Park et al. (38), familial follicular cell-derived DTC patients with parent-offspring relationship were found to have a higher recurrence rate compared with sporadic cases and the second generation had even higher rates compared with the first generation. Mazeh et al. (39) found that familial DTC patients had more aggressive disease compared with sporadic cases regardless of the number of family members affected. In contrast, Robenshtok et al. (40) found that staging at diagnosis and outcomes were not different in familial DTC patients compared with sporadic DTC patients. Syndromes associated with DTC (e.g., PTEN [phosphatase and tensin homolog] hamartoma tumor syndrome [Cowden's disease], familial adenomatous polyposis [FAP], Carney complex, multiple endocrine neoplasia [MEN] 2, Werner syndrome/progeria) in a first-degree relative, warrant screening based on various components of that syndrome (41).

It is not possible to speculate on the impact of screening in preventing or reducing recurrence and deaths, since no interventional screening programs have ever been reported in at-risk family members. Patients with familial DTC should have a careful history and directed neck examination as a part of routine health maintenance. One should also consider thyroid cancer syndromes as noted above (41).

\section{[A3] What is the appropriate laboratory and imaging evaluation for patients with clinically or incidentally discovered thyroid nodules?}

\section{[A4] Serum thyrotropin measurement}

\section{- RECOMMENDATION 2}

(A) Serum thyrotropin (TSH) should be measured during the initial evaluation of a patient with a thyroid nodule.

\section{(Strong recommendation, Moderate-quality evidence)}

(B) If the serum TSH is subnormal, a radionuclide (preferably ${ }^{123} \mathrm{I}$ ) thyroid scan should be performed. (Strong recommendation, Moderate-quality evidence)

(C) If the serum TSH is normal or elevated, a radionuclide scan should not be performed as the initial imaging evaluation.

\section{(Strong recommendation, Moderate-quality evidence)}

With the discovery of a thyroid nodule, a complete history and physical examination focusing on the thyroid gland and adjacent cervical lymph nodes should be performed. Pertinent historical factors predicting malignancy include a history of childhood head and neck radiation therapy, total body radiation for bone marrow transplantation (42), exposure to ionizing radiation from fallout in childhood or adolescence (43), familial thyroid carcinoma, or thyroid cancer syndrome (e.g., PTEN hamartoma tumor syndrome [Cowden's disease], FAP, Carney complex, Werner syndrome/progeria, or MEN 2, a risk for medullary thyroid cancer [MTC]) in a firstdegree relative, rapid nodule growth, and/or hoarseness. 
Pertinent physical findings suggesting possible malignancy include vocal cord paralysis, cervical lymphadenopathy, and fixation of the nodule to surrounding tissue.

With the discovery of a thyroid nodule $>1 \mathrm{~cm}$ in any diameter, a serum TSH level should be obtained. If the serum TSH is subnormal, a radionuclide thyroid scan should be obtained to document whether the nodule is hyperfunctioning ("hot," i.e., tracer uptake is greater than the surrounding normal thyroid), isofunctioning ("warm," i.e., tracer uptake is equal to the surrounding thyroid), or nonfunctioning ("cold," i.e., has uptake less than the surrounding thyroid tissue) (44). Since hyperfunctioning nodules rarely harbor malignancy, if one is found that corresponds to the nodule in question, no cytologic evaluation is necessary. If overt or subclinical hyperthyroidism is present, additional evaluation is required. A higher serum TSH level, even within the upper part of the reference range, is associated with increased risk of malignancy in a thyroid nodule, as well as more advanced stage thyroid cancer $(45,46)$.

\section{[A5] Serum thyroglobulin measurement}

\section{- RECOMMENDATION 3}

Routine measurement of serum thyroglobulin ( $\mathrm{Tg}$ ) for initial evaluation of thyroid nodules is not recommended.

\section{(Strong recommendation, Moderate-quality evidence)}

Serum $\mathrm{Tg}$ levels can be elevated in most thyroid diseases and are an insensitive and nonspecific test for thyroid cancer (47-49).

\section{[A6] Serum calcitonin measurement}

\section{- RECOMMENDATION 4}

The panel cannot recommend either for or against routine measurement of serum calcitonin in patients with thyroid nodules.

\section{(No recommendation, Insufficient evidence)}

The utility of serum calcitonin has been evaluated in a series of prospective, nonrandomized studies (50-54). These data suggest that the use of routine serum calcitonin for screening may detect C-cell hyperplasia and MTC at an earlier stage, and overall survival consequently may be improved. However, most studies relied on pentagastrin stimulation testing to increase specificity. This drug is not available in the United States, Canada, and some other countries, and there remain unresolved issues of sensitivity, specificity, assay performance, cut-offs using calcium stimulation (55), and cost effectiveness. Two retrospective studies have shown improved survival in patients diagnosed with MTC after routine calcitonin testing compared with historical controls $(53,56)$, but they were unable to show a decreased number of MTC-related deaths. A cost-effectiveness analysis suggested that calcitonin screening would be cost effective in the United States (57). However, prevalence estimates of MTC in this analysis included patients with C-cell hyperplasia and microMTC, which have uncertain clinical significance. Based on the retrospective nature of the survival data, unresolved issues of assay performance, lack of availability of pentagastrin in North America, and potential biases in the cost-effective analysis, the task force cannot recommend for or against the routine measurement of serum calcitonin as a screening test in patients with thyroid nodules, although there was not uniform agreement on this recommendation. There was, however, agreement that serum calcitonin may be considered in the subgroup of patients in whom an elevated calcitonin may change the diagnostic or surgical approach (i.e., patients considered for less than total thyroidectomy, patients with suspicious cytology not consistent with PTC). If the unstimulated serum calcitonin determination has been obtained and the level is greater than $50-100 \mathrm{pg} / \mathrm{mL}$, a diagnosis of MTC is common (58).

There is emerging evidence that a calcitonin measurement from a thyroid nodule fine-needle aspiration (FNA) washout may be helpful in the preoperative evaluation of patients with a modestly elevated basal serum calcitonin $(20-100 \mathrm{pg} / \mathrm{mL})(59)$.

\section{[A7] $]^{18}$ F]Fluorodeoxyglucose positron emission tomography scan}

\section{- RECOMMENDATION 5}

(A) Focal $\left[{ }^{18} \mathrm{~F}\right]$ fluorodeoxyglucose positron emission tomography $\left({ }^{18}\right.$ FDG-PET) uptake within a sonographically confirmed thyroid nodule conveys an increased risk of thyroid cancer, and FNA is recommended for those nodules $\geq 1 \mathrm{~cm}$.

(Strong recommendation, Moderate-quality evidence)

B) Diffuse ${ }^{18}$ FDG-PET uptake, in conjunction with sonographic and clinical evidence of chronic lymphocytic thyroiditis, does not require further imaging or FNA.

(Strong recommendation, Moderate-quality evidence)

${ }^{18}$ FDG-PET is increasingly performed during the evaluation of patients with both malignant and nonmalignant illness. While ${ }^{18}$ FDG-PET imaging is not recommended for the evaluation of patients with newly detected thyroid nodules or thyroidal illness, the incidental detection of abnormal thyroid uptake may nonetheless be encountered. Importantly, incidental ${ }^{18}$ FDG-PET uptake in the thyroid gland can be either focal or diffuse. Focal ${ }^{18}$ FDG-PET uptake in the thyroid is incidentally detected in $1 \%-2 \%$ of patients, while an additional $2 \%$ of patients demonstrate diffuse thyroid uptake (60-62).

Focal thyroid uptake most often corresponds to a clinically relevant thyroid nodule, and US examination is thus recommended to define thyroid anatomy. Importantly, focal ${ }^{18}$ FDG-PET uptake increases malignancy risk in an affected nodule, and therefore clinical evaluation and FNA of nodules $\geq 1 \mathrm{~cm}$ is recommended. ${ }^{18}$ FDG-PET positive thyroid nodules $<1 \mathrm{~cm}$ that do not meet FNA criteria (see Recommendation 8) can be monitored similarly to thyroid nodules with high-risk sonographic patterns that do not meet FNA criteria (see Recommendation 24A). A recent meta-analysis confirmed that approximately one in three $(\sim 35 \%){ }^{18}$ FDG-PET positive thyroid nodules proved to be cancerous (60), with higher mean maximum standardized uptake value in malignant compared to benign nodules ( 6.9 vs. $4.8, p<0.001)$. In contrast, diffuse thyroid uptake most often represents benign disease corresponding to inflammatory uptake in the setting 
of Hashimoto's disease or other diffuse thyroidal illness. However, if detected, diffuse ${ }^{18}$ FDG-PET uptake in the thyroid should also prompt sonographic examination to ensure there is no evidence of clinically relevant nodularity. Most patients with diffuse ${ }^{18}$ FDG-PET uptake demonstrate diffuse heterogeneity on sonographic examination, and no further intervention or FNA is required. It is appropriate to evaluate thyroid function in these patients.

\section{[A8] Thyroid sonography}

\section{- RECOMMENDATION 6}

Thyroid sonography with survey of the cervical lymph nodes should be performed in all patients with known or suspected thyroid nodules.

\section{(Strong recommendation, High-quality evidence)}

Diagnostic thyroid/neck US should be performed in all patients with a suspected thyroid nodule, nodular goiter, or radiographic abnormality suggesting a thyroid nodule incidentally detected on another imaging study (e.g., computed tomography [CT] or magnetic resonance imaging [MRI] or thyroidal uptake on ${ }^{18}$ FDG-PET scan) (www.aium.org/ resources/guidelines/thyroid.pdf). Thyroid US can answer the following questions: Is there truly a nodule that corresponds to an identified abnormality? How large is the nodule? What is the nodule's pattern of US imaging characteristics? Is suspicious cervical lymphadenopathy present? Is the nodule greater than $50 \%$ cystic? Is the nodule located posteriorly in the thyroid gland? These last two features might decrease the accuracy of FNA biopsy performed with palpation $(63,64)$.

Ultrasound should evaluate the following: thyroid parenchyma (homogeneous or heterogeneous) and gland size; size, location, zand sonographic characteristics of any nodule(s); the presence or absence of any suspicious cervical lymph nodes in the central or lateral compartments. The US report should convey nodule size (in three dimensions) and location (e.g., right upper lobe) and a description of the nodule's sonographic features including composition (solid, cystic proportion, or spongiform), echogenicity, margins, presence and type of calcifications, and shape if taller than wide, and vascularity. The pattern of sonographic features associated with a nodule confers a risk of malignancy, and combined with nodule size, guides FNA decision-making $(65,66)$ (see Recommendation 8).

In the subset of patients with low serum TSH levels who have undergone radionuclide thyroid scintigraphy suggesting nodularity, US should also be performed to evaluate both the presence of nodules concordant with the hyperfunctioning areas on the scan, which do not require FNA, as well as other nonfunctioning nodules that meet sonographic criteria for FNA (67).

\section{[A9] US for FNA decision-making}

\section{- RECOMMENDATION 7}

FNA is the procedure of choice in the evaluation of thyroid nodules, when clinically indicated.

\section{(Strong recommendation, High-quality evidence)}

FNA is the most accurate and cost-effective method for evaluating thyroid nodules. Retrospective studies have reported lower rates of both nondiagnostic and false-negative cytology from FNA procedures performed using US guidance compared to palpation $(68,69)$. Therefore, for nodules with a higher likelihood of either a nondiagnostic cytology (>25\%-50\% cystic component) (64) or sampling error (difficult to palpate or posteriorly located nodules), US-guided FNA is preferred. If the diagnostic US confirms the presence of a predominantly solid nodule corresponding to what is palpated, the FNA may be performed using palpation or US guidance.

[A10] Recommendations for diagnostic FNA of a thyroid nodule based on sonographic pattern

Figure 1 provides an algorithm for evaluation and management of patients with thyroid nodules based on sonographic pattern and FNA cytology, which is discussed in subsequent sections.

\section{- RECOMMENDATION 8}

I. Thyroid nodule diagnostic FNA is recommended for (Fig. 2, Table 6):

(A) Nodules $\geq 1 \mathrm{~cm}$ in greatest dimension with high suspicion sonographic pattern.

(Strong recommendation, Moderate-quality evidence)

(B) Nodules $\geq 1 \mathrm{~cm}$ in greatest dimension with intermediate suspicion sonographic pattern.

\section{(Strong recommendation, Low-quality evidence)}

(C) Nodules $\geq 1.5 \mathrm{~cm}$ in greatest dimension with low suspicion sonographic pattern.

\section{(Weak recommendation, Low-quality evidence)}

II. Thyroid nodule diagnostic FNA may be considered for (Fig. 2, Table 6):

(D) Nodules $\geq 2 \mathrm{~cm}$ in greatest dimension with very low suspicion sonographic pattern (e.g., spongiform). Observation without FNA is also a reasonable option.

\section{(Weak recommendation, Moderate-quality evidence)}

III. Thyroid nodule diagnostic FNA is not required for (Fig. 2, Table 6):

(E) Nodules that do not meet the above criteria.

(Strong recommendation, Moderate-quality evidence)

(F) Nodules that are purely cystic.

(Strong recommendation, Moderate-quality evidence)

Thyroid US has been widely used to stratify the risk of malignancy in thyroid nodules, and aid decision-making about whether FNA is indicated. Studies consistently report that several US gray scale features in multivariate analyses are associated with thyroid cancer, the majority of which are PTC. These include the presence of microcalcifications, nodule hypoechogenicity compared with the surrounding thyroid or strap muscles, irregular margins (defined as either infiltrative, microlobulated, or spiculated), and a shape taller than wide measured on a transverse view. Features with the highest specificities (median $>90 \%$ ) for thyroid cancer are microcalcifications, irregular margins, and tall shape, 


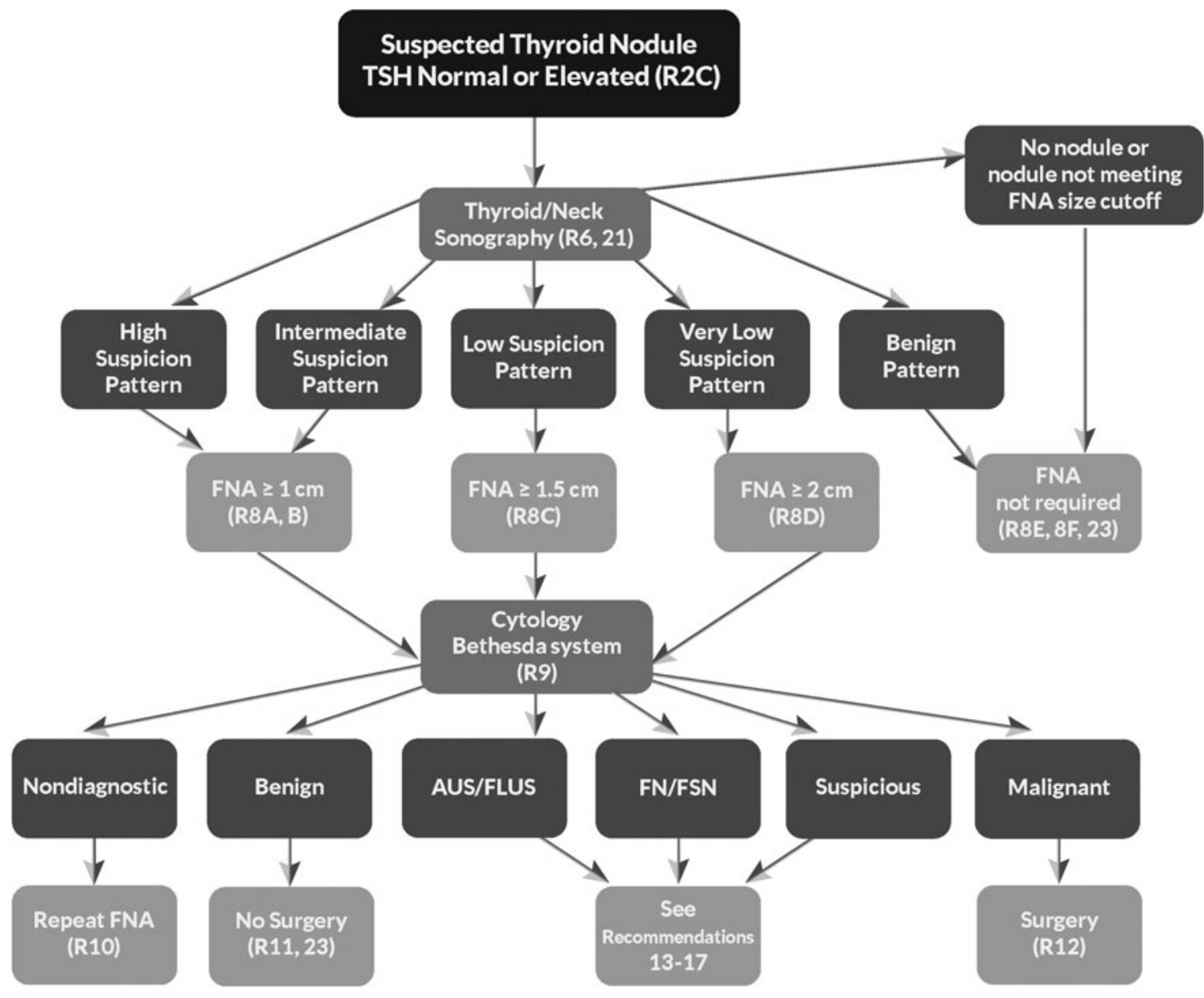

FIG. 1. Algorithm for evaluation and management of patients with thyroid nodules based on US pattern and FNA cytology. $\mathrm{R}$, recommendation in text.

although the sensitivities are significantly lower for any single feature (70-77). It is important to note that poorly defined margins, meaning the sonographic interface between the nodule and the surrounding thyroid parenchyma is difficult to delineate, are not equivalent to irregular margins. An irregular margin indicates the demarcation between nodule and parenchyma is clearly visible but demonstrates an irregular, infiltrative or spiculated course. Up to $55 \%$ of benign nodules are hypoechoic compared to thyroid parenchyma, making nodule hypoechogenicity less specific. In addition, subcentimeter benign nodules are more likely to be hypoechoic than larger nodules (71). Multivariable analyses confirm that the probability of cancer is higher for nodules with either microlobulated margins or microcalcifications than for hypoechoic solid nodules lacking these features (70). Macrocalcifications within a nodule, if combined with microcalcifications, confer the same malignancy risk as microcalcifications alone $(70,74)$. However, the presence of this type of intranodular macrocalcification alone is not consistently associated with thyroid cancer (78). On the other hand, a nodule that has interrupted peripheral calcifications, in association with a soft tissue rim outside the calcification, is highly likely to be malignant, and the associated pathology may demonstrate tumor invasion in the area of disrupted calcification $(79,80)$.

In a recent study where $98 \%$ of the cancers were PTC, intranodular vascularity did not have independent predictive value for malignancy in multivariate logistic regression model including gray-scale features (72). Two other studies and a meta-analysis with higher proportions of follicular thyroid cancer (FTC) (10\%-22\%) have shown that intranodular vascularity was correlated with malignancy $(66,74,81)$. FTC exhibits other differences in sonographic features compared to PTC. These tumors are more likely to be iso- to hyperechoic, noncalcified, round (width greater than anterioposterior dimension) nodules with regular smooth margins (82). Similarly, the follicular variant of papillary cancer (FVPTC) is also more likely than conventional PTC to have this same appearance as FTC (79). Distant metastases are rarely observed arising from follicular cancers $<2 \mathrm{~cm}$ in diameter, which therefore justifies a higher size cutoff for hyperechoic nodules (83).

The vast majority $(82 \%-91 \%)$ of thyroid cancers are solid $(70,73,75,77,84)$. Of 360 consecutively surgically removed thyroid cancers at the Mayo clinic, $88 \%$ were solid or minimally cystic $(<5 \%), 9 \%$ were $<50 \%$ cystic, and only $3 \%$ were more than $50 \%$ cystic (85). Therefore, FNA decision-making for partially cystic thyroid nodules must be tempered by their lower malignant risk. In addition, evidence linking sonographic features with malignancy in this subgroup of nodules is less robust, originating from univariate rather than multivariate analyses. However, an eccentric rather than concentric position of the solid component along the cyst wall, an acute rather than obtuse angle interface of the 


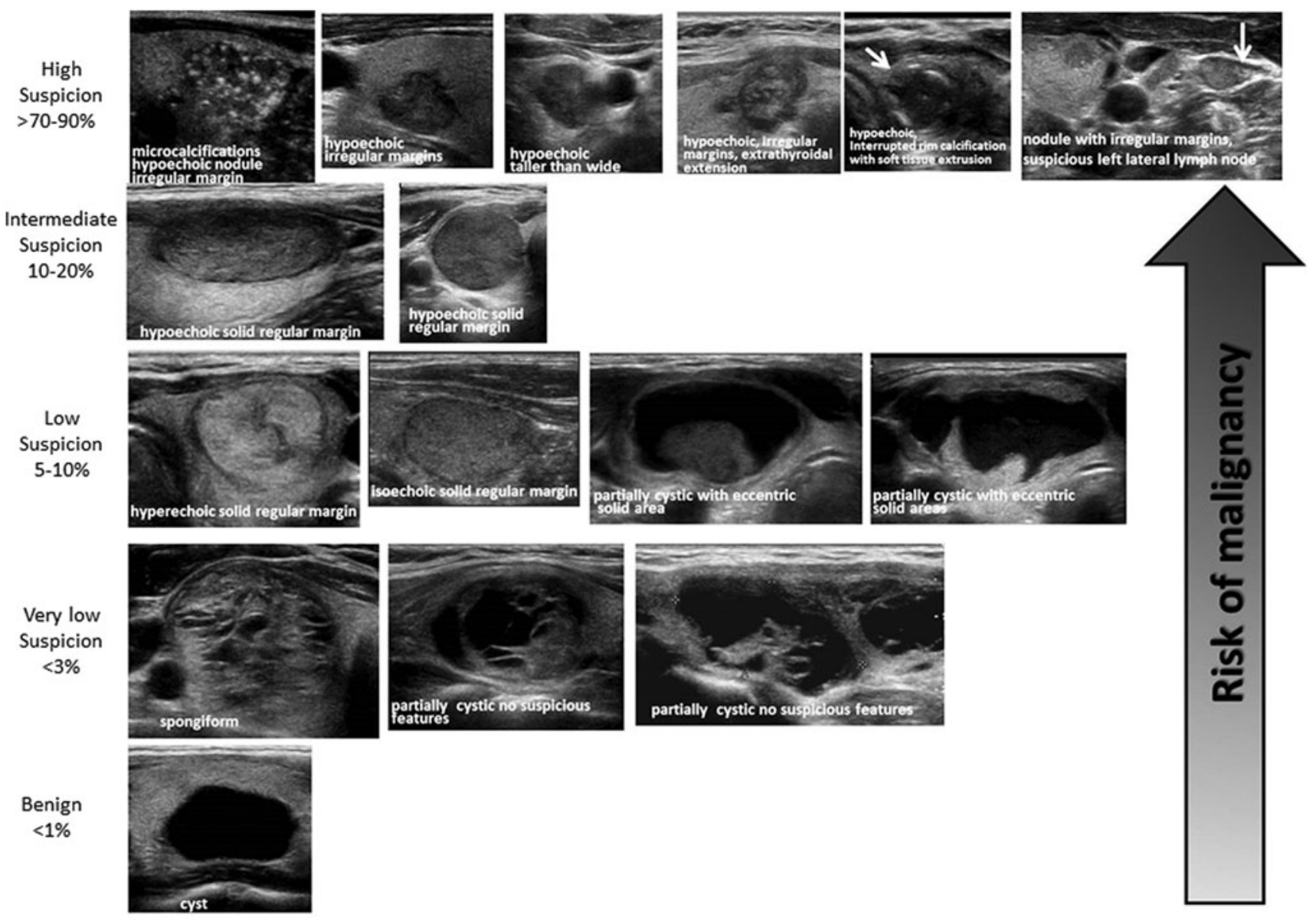

FIG. 2. ATA nodule sonographic patterns and risk of malignancy.

Table 6. Sonographic Patterns, Estimated Risk of Malignancy, and Fine-Needle Aspiration GUIDANCE FOR THYROID NODULES

\begin{tabular}{|c|c|c|c|}
\hline Sonographic pattern & US features & $\begin{array}{l}\text { Estimated risk } \\
\text { of malignancy, \% }\end{array}$ & $\begin{array}{c}\text { FNA size cutoff } \\
\text { (largest dimension) }\end{array}$ \\
\hline High suspicion & $\begin{array}{l}\text { Solid hypoechoic nodule or solid hypoechoic } \\
\text { component of a partially cystic nodule } \\
\text { with one or more of the following features: } \\
\text { irregular margins (infiltrative, microlobu- } \\
\text { lated), microcalcifications, taller than wide } \\
\text { shape, rim calcifications with small extru- } \\
\text { sive soft tissue component, evidence } \\
\text { of ETE }\end{array}$ & $>70-90^{\mathrm{a}}$ & Recommend FNA at $\geq 1 \mathrm{~cm}$ \\
\hline Intermediate suspicion & $\begin{array}{l}\text { Hypoechoic solid nodule with smooth mar- } \\
\text { gins without microcalcifications, ETE, } \\
\text { or taller than wide shape }\end{array}$ & $10-20$ & Recommend FNA at $\geq 1 \mathrm{~cm}$ \\
\hline Low suspicion & $\begin{array}{l}\text { Isoechoic or hyperechoic solid nodule, or } \\
\text { partially cystic nodule with eccentric solid } \\
\text { areas, without microcalcification, irregular } \\
\text { margin or ETE, or taller than wide shape. }\end{array}$ & $5-10$ & Recommend FNA at $\geq 1.5 \mathrm{~cm}$ \\
\hline Very low suspicion & $\begin{array}{l}\text { Spongiform or partially cystic nodules with- } \\
\text { out any of the sonographic features de- } \\
\text { scribed in low, intermediate, or high } \\
\text { suspicion patterns }\end{array}$ & $<3$ & $\begin{array}{l}\text { Consider FNA at } \geq 2 \mathrm{~cm} \\
\text { Observation without FNA } \\
\text { is also a reasonable option }\end{array}$ \\
\hline Benign & Purely cystic nodules (no solid component) & $<1$ & No biopsy ${ }^{\mathrm{b}}$ \\
\hline
\end{tabular}

US-guided FNA is recommended for cervical lymph nodes that are sonographically suspicious for thyroid cancer (see Table 7).

${ }^{a}$ The estimate is derived from high volume centers, the overall risk of malignancy may be lower given the interobserver variability in sonography.

${ }^{\mathrm{b}}$ Aspiration of the cyst may be considered for symptomatic or cosmetic drainage.

ETE, extrathyroidal extension. 
solid component and cyst, and the presence of microcalcifications consistently confer a higher risk of malignancy (85-87). Other findings such as lobulated margins or increased vascularity of the solid portion are risk factors that are not as robust $(86,87)$. However, a spongiform appearance of mixed cystic solid nodules is strongly correlated with benignity $(66,70,71,88)$. A spongiform appearance is defined as the aggregation of multiple microcystic components in more than $50 \%$ of the volume of the nodule (71). Spongiform and other mixed cystic solid nodules may exhibit bright reflectors on US imaging, caused by colloid crystals or posterior acoustic enhancement of the back wall of a microcystic area. These may be confused with microcalcifications by less proficient sonographers, and a recent meta-analysis confirmed that operator experience is correlated with accurate evaluation of internal calcifications (66). Therefore, because of potential for misclassification, FNA may still be considered for nodules interpreted as spongiform, but with a higher size cutoff. Lastly, pure cysts, although rare ( $<2 \%$ of thyroid lesions), are highly likely to be benign $(66,89,90)$.

Given the nuances in sonographic appearances of different thyroid cancer histologies, as well as the challenges posed by partially cystic nodules, some authors have suggested risk stratification based upon a constellation of sonographic features (89-91). In the absence of sonographically suspicious cervical lymph nodes, features associated with the highest risk for thyroid cancer can be used to triage smaller nodules for fine-needle biopsy, whereas nodules with sonographic appearance suggesting lower risk might be considered for fine-needle biopsy at a larger size as determined by maximal diameter (Figs. 1 and 2, Table 6). The sonographic appearance for the vast majority of thyroid nodules can be generally classified in the following categories of US patterns, which combine several individual sonographic characteristics. Since the interobserver variability in reporting individual characteristics is moderate even within controlled studies (72), the use of patterns exhibiting correlated sonographic features is more robust. Two recent studies have reported substantial interobserver correlation for identification for nodule sonographic patterns (multirater kappa statistics $>0.6)(92,93)$.

High suspicion [malignancy risk $>70 \%-90 \%(89,90,94)]$. High suspicion of malignancy is warranted with a solid hypoechoic nodule or a solid hypoechoic component in a partially cystic nodule with one or more of the following features: irregular margins (specifically defined as infiltrative, microlobulated, or spiculated), microcalcifications, taller than wide shape, disrupted rim calcifications with small extrusive hypoechoic soft tissue component, or evidence of extrathyroidal extension (Fig. 2, Table 6). A nodule demonstrating this US pattern is highly likely to be a PTC. Nodules with the high suspicion pattern and measuring $\geq 1 \mathrm{~cm}$ should undergo diagnostic fine-needle biopsy to refute or confirm malignancy. However, in the absence of evidence of extrathyroidal extension, metastatic cervical lymph nodes, or distant metastases, micropapillary thyroid cancers $(<1 \mathrm{~cm})$ often have an indolent course, but this may depend upon patient age (95). Although no distant metastases or deaths occurred in a recent observational series of 1235 Japanese patients with biopsy-proven PTC, tumor growth and new appearance of lymph node metastases occurred more frequently in patients younger than 40 years of age compared with those over age 60 (5.9\% vs. $2.2 \%$ for size increase; $5.3 \%$ vs. $0.4 \%$ for new nodal metastases, $p<0.05$ ). Thus although a sonographically suspicious subcentimeter thyroid nodule without evidence of extrathyroidal extension or sonographically suspicious lymph nodes may be observed with close sonographic follow-up of the nodule and cervical lymph nodes, rather than pursuing immediate FNA, patient age and preference may modify decision-making (95).

Intermediate suspicion [malignancy risk 10\%-20\% $(89,90,94)]$. Intermediate suspicion of malignancy is attached to a hypoechoic solid nodule with a smooth regular margin, but without microcalcifications, extrathyroidal extension, or taller than wide shape (Fig. 2, Table 6). This appearance has the highest sensitivity $(60 \%-80 \%)$ for PTC, but a lower specificity than the preceding high suspicion pattern, and fine-needle biopsy should be considered for these nodules $\geq 1 \mathrm{~cm}$ to refute malignancy.

Low suspicion [malignancy risk 5\%-10\% $(89,90,94)]$. Isoechoic or hyperechoic solid nodule, or partially cystic nodule with eccentric uniformly solid areas without microcalcifications, irregular margin or extrathyroidal extension, or taller than wide shape prompts low suspicion for malignancy (Fig. 2, Table 6). Only about 15\%-20\% of thyroid cancers are iso- or hyperechoic on US, and these are generally the follicular variant of PTC or FTCs (71). Fewer than $20 \%$ of these nodules are partially cystic. Therefore, these appearances are associated with a lower probability of malignancy and observation may be warranted until the size is $\geq 1.5 \mathrm{~cm}$.

Very low suspicion $[\leq 3 \%(66,89,90,94)]$. Spongiform or partially cystic nodules without any of the sonographic features described in the low, intermediate, or high suspicion patterns have a low risk of malignancy $(<3 \%)$. If FNA is performed, the nodule should be at least $2 \mathrm{~cm}$. Observation without FNA may also be considered for nodules $\geq 2 \mathrm{~cm}$ (Fig. 2, Table 6).

Benign $[\leq 1 \%(89,90,94)]$. Purely cystic nodules are very unlikely to be malignant, and fine-needle biopsy is not indicated for diagnostic purposes (Fig. 2, Table 6). Aspiration with or without ethanol ablation may be considered as a therapeutic intervention if a cyst is large and symptomatic; cytology should be performed if aspiration is done.

Sonographic evaluation of the anterior cervical lymph node compartments (central and lateral) should be performed whenever thyroid nodules are detected. If US detects cervical lymph nodes that are sonographically suspicious for thyroid cancer (Table 7), FNA of the suspicious lymph node should be performed for cytology and washout for Tg measurement if indicated. In addition, this scenario also warrants USguided FNA of a subcentimeter nodule that is likely to represent the primary tumor based upon sonographic features.

Although there are several known clinical risk factors for thyroid cancer in patients with thyroid nodules including immobility with swallowing, pain, cough, voice change, growth, lymphadenopathy, and a history of childhood radiation therapy (either therapeutic, such as cranial radiation in childhood leukemia, or for benign conditions, such as enlarged thymus or tonsils) or familial thyroid cancer (96), these have not been incrementally included in multivariate analyses of gray-scale sonographic features and thyroid 
Table 7. Ultrasound Features of Lymph Nodes Predictive of Malignant Involvement ${ }^{\mathrm{a}}$

\begin{tabular}{lcc}
\hline Sign & $\begin{array}{c}\text { Reported } \\
\text { sensitivity, } \%\end{array}$ & $\begin{array}{c}\text { Reported } \\
\text { specificity, } \%\end{array}$ \\
\hline Microcalcifications & $5-69$ & $93-100$ \\
Cystic aspect & $10-34$ & $91-100$ \\
Peripheral vascularity & $40-86$ & $57-93$ \\
Hyperechogenicity & $30-87$ & $43-95$ \\
Round shape & 37 & 70 \\
\hline
\end{tabular}

${ }^{a}$ Adapted with permission from the European Thyroid Association guidelines for cervical ultrasound (20).

cancer risk. However, given the higher pretest likelihood of thyroid cancer associated with these clinical risk factors, FNA can be considered at lower size cutoffs for all of the sonographic appearances described above.

Ultrasound elastography (USE) has similarly been investigated for its ability to modify thyroid cancer risk assessment among clinically relevant thyroid nodules. Elastography is a measurement of tissue stiffness. Performance requires an US machine, as well as an elastography computational module that most often must be purchased separately. An initial prospective study of 92 selected, nonrandomized patients suggested positive and negative predictive values (NPVs) near $100 \%$ (97). However, more recently, larger trials have reported substantially different results. Moon and colleagues retrospectively studied 703 thyroid nodules in comparison to grayscale US (78). Performance of USE was inferior to that of gray-scale US assessment. The largest prospective study of 706 patients with 912 thyroid nodules was recently published by Azizi et al. (98). In this study, the positive predictive value (PPV) of USE was only 36\%, comparable to that of microcalcifications. The NPV of USE was $97 \%$ in a population with cancer prevalence of $9 \%$. Thus, while USE holds promise as a means by which to noninvasively assess cancer risk, its performance is highly variable and operator dependent. Perhaps most importantly, USE can only be effectively applied to solid nodules, thus excluding its utility for cystic or partially cystic nodules. Furthermore, to be amenable to direct pressure and determination of tissue strain, the index nodule must not overlap with other nodules in the anterioposterior plane. Obese patients, those with multinodular goiters and coalescent nodules, or patients in whom the nodule is posterior or inferior are not candidates for USE. Thus, at present, USE cannot be widely applied to all thyroid nodules in a similar fashion to gray-scale or Doppler US examination. The committee therefore believes USE (when available) may prove to be a helpful tool for preoperative risk assessment in those patients in whom accurate assessment can be performed. However, the committee cannot presently recommend its universal use or widespread adoption. Importantly, the ability to perform (or not perform) USE should not modify the recommendation for traditional gray-scale sonographic evaluation.

Finally, while most thyroid nodules meeting the preceding sonographic patterns and sizes should undergo FNA, we acknowledge that a conservative approach of active surveillance management may be appropriate as an alternative to FNA in selected patients. These may include patients with very low-risk tumors (e.g., no clinical or radiographic evidence of invasion or metastases), patients at high surgical risk, or those with a relatively short life span expectancy in whom the benefits of intervention may be unrealized.

\section{[A11] What is the role of FNA, cytology interpretation, and molecular testing in patients with thyroid nodules?}

\section{- RECOMMENDATION 9}

Thyroid nodule FNA cytology should be reported using diagnostic groups outlined in the Bethesda System for Reporting Thyroid Cytopathology.

\section{(Strong recommendation, Moderate-quality evidence)}

To address a significant variability in the reporting of cytological findings in thyroid FNA samples, the 2007 National Cancer Institute Thyroid Fine-Needle Aspiration State of the Science Conference provided consensus recommendations known as the Bethesda System for Reporting Thyroid Cytopathology $(99,100)$. The Bethesda system recognizes six diagnostic categories and provides an estimation of cancer risk within each category based upon literature review and expert

Table 8. The Bethesda System for Reporting Thyroid Cytopathology: Diagnostic Categories AND RisK OF MALIGNANCY ${ }^{a}$

\begin{tabular}{|c|c|c|}
\hline Diagnostic category & $\begin{array}{l}\text { Estimated/predicted risk } \\
\text { of malignancy by the } \\
\text { Bethesda system, } \%^{\mathrm{a}}\end{array}$ & $\begin{array}{c}\text { Actual risk of malignancy } \\
\text { in nodules surgically excised, } \\
\% \text { median }(\text { range })^{\mathrm{b}}\end{array}$ \\
\hline Nondiagnostic or unsatisfactory & $1-4$ & $20(9-32)$ \\
\hline Benign & $0-3$ & $2.5(1-10)$ \\
\hline $\begin{array}{l}\text { Atypia of undetermined significance } \\
\text { or follicular lesion of undetermined } \\
\text { significance }\end{array}$ & $5-15$ & $14(6-48)$ \\
\hline $\begin{array}{l}\text { Follicular neoplasm or suspicious for } \\
\text { a follicular neoplasm }\end{array}$ & $15-30$ & $25(14-34)$ \\
\hline Suspicious for malignancy & $60-75$ & $70(53-97)$ \\
\hline Malignant & 97-99 & $99(94-100)$ \\
\hline
\end{tabular}

\footnotetext{
${ }^{a}$ As reported in The Bethesda System by Cibas and Ali (1076).

${ }^{\mathrm{b}}$ Based on the meta-analysis of eight studies reported by Bongiovanni et al. (103). The risk was calculated based on the portion of nodules in each diagnostic category that underwent surgical excision and likely is not representative of the entire population, particularly of nondiagnostic and benign diagnostic categories.
} 
opinion (Fig. 1, Table 8). These categories are (i) nondiagnostic/ unsatisfactory; (ii) benign; (iii) atypia of undetermined significance/follicular lesion of undetermined significance (AUS/ FLUS); (iv) follicular neoplasm/suspicious for follicular neoplasm (FN/SFN), a category that also encompasses the diagnosis of Hürthle cell neoplasm/suspicious for Hürthle cell neoplasm; (v) suspicious for malignancy (SUSP), and (vi) malignant. Recent studies that applied the criteria and terminology of the Bethesda System to a large series of patients have shown a relatively good concordance in reporting FNA cytology, with $89 \%-95 \%$ of samples being satisfactory for interpretation and $55 \%-74 \%$ reported as definitively benign and $2 \%-5 \%$ as definitively malignant (101-104). The remaining samples are cytologically indeterminate, including AUS/FLUS in $2 \%-18 \%$ of nodules, FN in $2 \%-25 \%$, and SUSP in $1 \%-6 \%$. In these studies, the probability of malignancy for each Bethesda category demonstrated significant variability, but was overall compatible with the range predicted by the Bethesda System, with the exception of the AUS/FLUS diagnosis, for which the risk of malignant outcome in some studies was significantly higher than predicted (Table 8$)(103,105)$. Recently, a blinded prospective evaluation of inter-observer concordance using Bethesda classification was performed. These data confirm an inherent limitation to the reproducibility of interpreting any cytology specimen (106). Specimens diagnosed as AUS/ FLUS and SUSP were associated with the highest discordance rates. Some studies suggest that the AUS/FLUS category should be further subdivided into AUS with cytologic atypia (higher risk for malignancy) and FLUS with architectural atypia (lower risk for malignancy), but this has not yet been widely adopted (107). Nonetheless, classification using the Bethesda system has proven highly beneficial, allowing practitioners to speak with the same terminology and better convey malignant risk. The risk of malignancy in each of the six diagnostic categories should be independently defined at each cytology center or institution to guide clinicians on risk estimates and help choose appropriate molecular testing for patients with indeterminate cytology.

\section{[A12] Nondiagnostic cytology}

\section{- RECOMMENDATION 10}

(A) For a nodule with an initial nondiagnostic cytology result, FNA should be repeated with US guidance and, if available, on-site cytologic evaluation

\section{(Strong recommendation, Moderate-quality evidence)}

(B) Repeatedly nondiagnostic nodules without a high suspicion sonographic pattern require close observation or surgical excision for histopathologic diagnosis

\section{(Weak recommendation, Low-quality evidence)}

(C) Surgery should be considered for histopathologic diagnosis if the cytologically nondiagnostic nodule has a high suspicion sonographic pattern, growth of the nodule (>20\% in two dimensions) is detected during US surveillance, or clinical risk factors for malignancy are present

\section{(Weak recommendation, Low-quality evidence)}

Nondiagnostic or unsatisfactory FNA biopsies are those that fail to meet the established quantitative or qualitative criteria for cytologic adequacy (i.e., the presence of at least six groups of well-visualized follicular cells, each group containing at least 10 well-preserved epithelial cells, preferably on a single slide) $(99,108)$. Although an FNA specimen found to have abundant colloid and few epithelial cells may be considered nondiagnostic by the above criteria, this is also likely a benign biopsy. After an initial nondiagnostic cytology result, repeat FNA with US guidance and, if available, on-site cytologic evaluation, will substantially increase the rate of specimen adequacy (109-113). It has been suggested that repeat FNA should be performed no sooner than 3 months after the initial FNA to prevent false-positive interpretation due to biopsy-induced reactive/reparative changes (114). Two recent studies have questioned the necessity for a 3-month waiting period after the first FNA because they did not find a correlation between the diagnostic yield/accuracy of repeated FNA and the waiting time between the procedures $(115,116)$. A 3-month waiting period after a nondiagnostic biopsy is likely not necessary. If clinical and US features are suspicious for malignancy, a shorter waiting period may be appropriate. Repeat FNA with US guidance will yield a diagnostic cytology specimen in $60 \%-80 \%$ of nodules, particularly when the cystic component is $<50 \%(64,112,117)$. Nodules with larger cystic portion have a higher chance to yield nondiagnostic samples on the initial and repeated FNA.

Most nodules with a nondiagnostic cytology interpretation are benign. In large series of patients classified based on the Bethesda System, nondiagnostic samples constituted 2\%-16\% of all FNA samples, of which 7\%-26\% were eventually resected (101-103). The frequency of malignancy among all initially nondiagnostic samples was $2 \%-4 \%$ and among those nondiagnostic samples that were eventually resected was $9 \%-32 \%$.

Sonographic features are also useful for identifying which nodules with repeat nondiagnostic FNA cytology results are more likely to be malignant. Of 104 nodules with two nondiagnostic cytology results, thyroid cancer was found in $25 \%$ of those with microcalcifications, irregular margins, a taller than wide shape, or hypoechogenicity, but in only $4 \%$ lacking these features (118).

In some studies, the use of thyroid core-needle biopsy (119) and molecular testing for $B R A F(120,121)$ or a panel of mutations (122) helped to facilitate appropriate management of these patients, although the full clinical impact of these approaches for nodules with nondiagnostic cytology remains unknown. Some studies have found that core biopsy offers a higher adequacy rate, but may be less sensitive for the detection of papillary cancer $(123,124)$. Mutational testing may be informative in samples considered inadequate by qualitative criteria (i.e., due to poor preparation or poor staining of cells) but is unlikely to be contributory in samples with insufficient quantity of cells.

\section{[A13] Benign cytology}

\section{- RECOMMENDATION 11}

If the nodule is benign on cytology, further immediate diagnostic studies or treatment are not required

\section{(Strong recommendation, High-quality evidence)}

Accurate FNA cytology diagnosis depends upon a number of factors including the skill of the operator, FNA technique, specimen preparation, and cytology interpretation. 
Ultrasound-guided FNA with real-time visualization of needle placement in the target nodule decreases the false-negative rate of a benign cytology diagnosis $(68,69,126,128)$. Although prospective studies are lacking, malignancy rates of only $1 \%-2 \%$ have been reported in large retrospective series that analyzed the utility of systematic repeat FNA in nodules with prior benign cytology results (129-133). A pooled analysis of 12 studies by Tee et al. (134) showed that of 4055 patients with benign cytology who underwent surgery, the rate of malignancy was $3.2 \%$.

Studies have also attempted to correlate nodule size with accuracy of FNA cytology. Several surgical series have reported higher malignancy rates in nodules $>3-4 \mathrm{~cm}$, but these suffer from both selection bias (only a subset of patients underwent preoperative FNA) and potential sampling error (FNA performed by palpation) $(135,136)$. A recent study evaluated the accuracy of FNA and US features in patients with thyroid nodules $\geq 4 \mathrm{~cm}$ (137). This was a single-center study in which the practice is to offer thyroidectomy or lobectomy to all patients with nodules $\geq 4 \mathrm{~cm}$. The investigators identified thyroid cancer in $22 \%$ of 382 nodules. A subset of thyroid nodules underwent preoperative FNA, and of the 125 cytologically benign nodules, $10.4 \%$ were malignant on final histopathology. The investigators further showed that individual US characteristics were not predictive for malignancy, although they did not look at sonographic patterns. Two other recent reports of consecutive US-guided FNA evaluations in over 1400 nodules $>3 \mathrm{~cm}$ with initial benign cytology followed for a mean of 3 years confirmed a lower false-negative rate of $<1.5 \%(138,139)$. Interestingly, in both these studies $66 \%$ of the missed cancers were found in nodules with high suspicion sonographic pattern, despite initial benign cytology. A recent retrospective study analyzed the long-term follow-up of 2010 cytologically benign nodules from 1369 patients. Over a mean follow-up of 8.5 years 18 falsenegative malignancies were detected. However, no deaths attributable to thyroid cancer were identified in this cohort. These data confirm that an initially benign FNA confers negligible mortality risk during long-term follow-up despite a low but real risk of false negatives in this cytologic category (140). Based on the evidence, it is still unclear if patients with thyroid nodules $\geq 4 \mathrm{~cm}$ and benign cytology carry a higher risk of malignancy and should be managed differently than those with smaller nodules.

Follow-up for patients with benign cytology is discussed in section [A24] and Recommendation 23.

\section{[A14] Malignant cytology}

\section{- RECOMMENDATION 12}

If a cytology result is diagnostic for primary thyroid malignancy, surgery is generally recommended.

(Strong recommendation, Moderate-quality evidence)

A cytology diagnostic for a primary thyroid malignancy will almost always lead to thyroid surgery. However, an active surveillance management approach can be considered as an alternative to immediate surgery in

(A) patients with very low risk tumors (e.g., papillary microcarcinomas without clinically evident metastases or local invasion, and no convincing cytologic evidence of aggressive disease),
(B) patients at high surgical risk because of comorbid conditions,

(C) patients expected to have a relatively short remaining life span (e.g., serious cardiopulmonary disease, other malignancies, very advanced age), or

(D) patients with concurrent medical or surgical issues that need to be addressed prior to thyroid surgery.

Following thyroid surgery for papillary thyroid microcarcinoma (PTMC), defined as a tumor $1 \mathrm{~cm}$ or less in size, disease-specific mortality rates have been reported to be $<1 \%$, loco-regional recurrence rates are $2 \%-6 \%$, and distant recurrence rates are $1 \%-2 \%(141,142)$. It is quite likely that these excellent outcomes are more related to the indolent nature of the disease rather than to the effectiveness of treatment, since two prospective clinical studies of active surveillance from Japan reported similar clinical outcomes in 1465 patients with biopsy-proven PTMC that were not surgically removed and were followed for up to 15 years (average $5-6$ years, range $1-17$ years) $(95,143)$. In the study by Ito et al. (95), observation was offered to 1235 patients with PTMC that did not have (i) location adjacent to the trachea or on the dorsal surface of the lobe close to the recurrent laryngeal nerve, (ii) FNA findings suggestive of high-grade malignancy; (iii) presence of regional lymph node metastases; or (iv) signs of progression during follow-up. Of those, most patients showed stable tumor size on average follow-up of 60 months (range 18-227 months), whereas 5\% showed tumor enlargement ( $>3 \mathrm{~mm}$ ) by US on 5-year follow-up, and $8 \%$ on 10-year follow-up. Furthermore, $1.7 \%$ and $3.8 \%$ of patients at 5-year and 10-year follow-up showed evidence for lymph node metastases. Of 1235 patients, 191 underwent surgical treatment after observation, including those with tumor enlargement and new lymph node metastases. These patients have been followed an average of 75 months (range 1-246 months) after the surgical intervention. Only one of the patients treated with surgery after observation developed tumor recurrence. In the study by Sugitani et al. (143), 230 patients with asymptomatic PTMC were followed for 5 years on average. Of those patients, tumor size enlargement was observed in $7 \%$, and $1 \%$ developed apparent lymph node metastasis. Seven percent of patients underwent surgery after 1-12 years of follow-up, and no recurrences were identified in those on limited follow-up, suggesting that the delayed surgery did not affect the outcome. A more recent study by Ito and colleagues followed 1235 patients with PTMC under active surveillance for an average of 60 months (95). Only 43 patients $(3.5 \%)$ had clinical progression of disease by their stated criteria (tumor growing to $>12 \mathrm{~mm}$ or appearance of new lymph node metastases). Interestingly, the younger patients ( $<40$ years old) had an $8.9 \%$ rate of clinical progression, while those 40-60 years old had a $3.5 \%$ rate of progression and those $>60$ years old had the lowest rate of clinical progression $(1.6 \%)$.

Despite the evidence that cautious observation is a safe and effective alternative to immediate surgical resection, very few PTMC patients outside of those two centers in Japan are given the option of an active surveillance approach. This is in part due to reports in the literature of a small percentage of patients with PTMC presenting with clinically significant regional or distant metastases $(141,142,144)$. Unfortunately, no clinical features (145-151) can reliably differentiate the relatively small number 
of PTMC patients destined to develop clinically significant progression from the larger population of people that harbor indolent PTMCs that will not cause significant disease.

Similarly, well-known thyroid cancer oncogenes, such as $B R A F$, when taken in isolation, are not able to specifically identify the microcarcinomas that will progress and spread outside of the thyroid. The prevalence of BRAF V600E mutations in PTMC with lymph node metastases and tumor recurrence is higher than PTMC without LN metastases or recurrence, and in some studies the presence of a $B R A F \mathrm{mu}-$ tation was associated with lymph node metastasis from PTMC on multivariate analysis $(150,152,153)$. These studies showed that although the presence of a $B R A F^{V 600 E}$ mutation identifies $65 \%-77 \%$ of patients with PTMC that develop lymph node metastases, the BRAF status taken in isolation has a low PPV for detecting PTMC with extrathyroidal spread and therefore has a limited role for guiding patient management. However, recent data suggest that specific molecular profiles, such as the coexistence of $B R A F$ with other oncogenic mutations (such as PIK3CA, AKT1), TERT promoter, or TP53 mutations may serve as more specific markers of less favorable outcome of PTC. Therefore, it is likely that finding of one of these genetic profiles in a small tumor would suggest that it represents an early stage of a clinically relevant PTC (154-157). Future studies are expected to establish the impact of molecular profiling involving multiple mutations or other genetic alterations on clinical management of patients with PTMC.

\section{[A15] Indeterminate cytology (AUS/FLUS, FN, SUSP)*}

\section{[A16] What are the principles of the molecular testing of FNA samples?}

Molecular markers may be classified according to intended use; that is, diagnostic (classification of a disease state), prognostic, or predictive purposes (providing information on the estimated probability of therapeutic benefit or harm of a specific therapy) (158). Furthermore, companion use of predictive molecular markers involves the identification of patient subgroups in which a therapeutic intervention is proven to be either beneficial or harmful, with intended implications for appropriate clinical stratification of therapies (158). Validation studies of molecular marker tests may include examination of (a) analytic validity (including test accuracy and reproducibility in ascertaining the molecular event), (b) clinical validity (the performance of the test in distinguishing different groups of patients, based on biology or expected disease outcome, including measures of sensitivity and specificity or predictive values), and (c) clinical utility (examination of the test's ability to improve outcomes, with direct clinical decision-making implications) (158). Furthermore, an NCCN Tumor Marker Task Force has indicated that the clinical utility of a molecular test should be founded in strong evidence proving that use of the marker "improves patient outcomes sufficiently to justify its incorporation into routine clinical practice." ${ }^{\dagger}$

*The final draft for the sections (A15-A19) and recommendations (13-17) were revised and approved by a subgroup of seven members of the task force with no perceived conflicts or competing interests in this area.

${ }^{\dagger}$ From the NCCN Biomarkers Compendium (www.ncen.org/ professionals/biomarkers/default.aspx).
The principal proposed use of molecular markers in indeterminate thyroid FNA specimens is diagnostic (ruling out or in the presence of thyroid malignancy), with the implication of a companion use to inform decision-making on primary surgical treatment (i.e., the decision to perform surgery and if so, the extent of surgery). However, the focus of this section is restricted to the clinical validity of molecular testing of indeterminate FNA specimens. It is important to note that longterm outcome data on companion use of molecular marker status to guide therapeutic decision-making is currently lacking, and therefore we do not know if implementation of molecular marker use in routine clinical practice would result in a significant overall benefit in health outcomes in patients with thyroid nodules. Surgical decision-making on indeterminate FNA specimens is reviewed in another section of these guidelines, with some reference to molecular marker testing (if performed). As summarized in a Disease State Commentary from the AACE Thyroid Scientific Committee and a consensus statement from the ATA Surgical Affairs Committee, use of molecular marker testing on indeterminate FNA specimens should not be intended to replace other sources of information or clinical judgment $(159,160)$. The pretest probability of malignancy (based on clinical risk factors, cytology, US findings), feasibility considerations, and patient preferences are some additional factors that need to be considered in decision-making related to molecular marker testing of FNA specimens. Because this is a rapidly evolving field of investigation, it will be important to perform interval evaluations of the published evidence to ensure that recommendations remain contemporary.

A number of molecular approaches have been studied in the clinical setting of indeterminate FNA cytologic interpretation (161). One could surmise that an ideal "rule-in" test would have a PPV for histopathologically proven malignancy similar to a malignant cytologic diagnosis (98.6\%), and an ideal "rule-out" test would have a NPV similar to a benign cytologic diagnosis (96.3\%) (predictive value estimates based on a recent meta-analysis of performance of the Bethesda system) (103), and these would hold true with a reasonable degree of precision and reproducibility.

\section{- RECOMMENDATION 13}

If molecular testing is being considered, patients should be counseled regarding the potential benefits and limitations of testing and about the possible uncertainties in the therapeutic and long-term clinical implications of results.

\section{(Strong recommendation, Low-quality evidence)}

The largest studies of preoperative molecular markers in patients with indeterminate FNA cytology have respectively evaluated a seven-gene panel of genetic mutations and rearrangements (BRAF, RAS, RET/PTC, PAX8/PPAR $\gamma)$ (162), a gene expression classifier (167 GEC; mRNA expression of 167 genes) (163), and galectin-3 immunohistochemistry (cell blocks) (164). These respective studies have been subject to various degrees of blinding of outcome assessment (162-164).

Mutational testing has been proposed for use as a rule-in test because of relatively high reported specificity $(86 \%-100 \%)$ 
and PPV (84\%-100\%) (105,122,162,165-168). Although $B R A F^{V 600 E}$ single mutation testing has been estimated to have a specificity of approximately 99\% (pooled data from 1117 nodules with histopathologic confirmation from multiple studies), the sensitivity has been deemed to be too low to reliably rule out the presence of malignancy (169). Therefore, mutational panels have been expanded to include multiple mutations/translocations including $B R A F, N R A S, H R A S$, and $K R A S$ point mutations, as well as RET/PTC1 and RET/PTC3, with or without $P A X 8 / P P A R \gamma$ rearrangements $(105,122,162$, 165-168) and other gene rearrangements (170). In indeterminate cytology thyroid nodules, the sensitivity of the sevengene mutational panel testing is variable, with reports ranging from $44 \%$ to $100 \%(162,165,167)$. The reported variability in sensitivity of mutational analysis with the seven-gene panel in indeterminate nodules suggests that traditional limited mutation panels may not reliably rule out malignancy with a negative test in this population. Next-generation sequencing of an expanded panel of point mutations, single base insertions/deletions (indels), and gene rearrangements has been reported to have a sensitivity of $90 \%$ for FN/SFN FNA cytology specimens from a single-center study (170). A limitation was that the pathologists evaluating the gold standard surgical pathology specimens were aware of the results of earlier generation molecular tests previously conducted on the FNAs (170). It is not known to what extent differences in techniques used to perform mutational testing by various groups $(162,167,170)$ may affect test performance, and direct, head-to-head comparisons of these tests within the same population are lacking. The currently available seven-gene mutational panels have been proposed to be most useful when surgery is favored. However, this is based on the assumption that the surgical approach would be altered with a positive test, and long-term outcome data proving the overall benefit of this therapeutic strategy are needed. It is important to acknowledge that algorithms employing seven-gene mutational testing as a means to inform decisionmaking on extent of primary thyroid surgery (i.e., lobectomy or total thyroidectomy) (162) were developed at a time when the ATA guidelines favored total thyroidectomy for most PTCs $>1 \mathrm{~cm}$ in diameter (25). However, this does not reflect recommendations in these guidelines (see Recommendation 35 on surgical management of malignant cytology nodules). Furthermore, long-term outcome data from a strategy of using molecular markers in indeterminate FNA specimens to stratify surgical approach are currently lacking.

Use of a 167 GEC has been proposed as a rule-out test due to relatively high sensitivity $(92 \%)$ and NPV $(93 \%)$, as reported in a prospective multicenter study (163). The relatively low specificity of the 167 GEC test (mean values $48 \%-53 \%$ in indeterminate nodules subject to histopathologic confirmation) suggests that the test cannot definitively rule-in malignancy in indeterminate nodules. In a retrospective analysis of 167 GEC results from five institutions, Alexander et al. (171) reported that the prevalence of 167 GEC benign readings by institution varied up to $29 \%$, which was not statistically significant. The distribution of recruitment from each of the five study sites was highly variable (total $n=339$ nodules), with two sites contributing only 30 and 37 patients, and the other three sites accounting for the majority of the study population. The prevalence of malignancy confirmed by histopathology in 167 GEC "suspicious" nodules ranged from $33 \%$ to $80 \%$ in the 48 nodules in the AUS/FLUS group across institutions (no $95 \%$ confidence interval $[95 \% \mathrm{CI}]$ reported, $p=0.11$ ), and from $33 \%$ to $67 \%$ in the 65 nodules in the FN cytology group (no 95\% CI reported, $p=0.87$ ) (171). For the 174 patients with 167 GEC "benign" readings, four patients were advised to undergo surgery (2\%), and $41 \%$ (71 patients) of this group had short documented follow-up for a mean of 8.5 months (median 8 months, range 1-24 months); ultimately, $6 \%$ of patients in this group (11/174) had surgery, with one histopathologically confirmed malignancy (171). The reproducibility of 167 GEC NPV measures in different populations of patients with indeterminate cytology thyroid nodules has recently been questioned in three smaller, independent, unblinded studies (172-174). None of these studies reported any degree of blinding. Furthermore, 95\% CI of predictive estimates in indeterminate cytology nodules were not reported in two single-center studies $(172,173)$, and one multi-center study was reported to be a retrospective analysis (174), making it difficult to interpret the findings. However, such data highlight the need for additional independent research examining the reproducibility of diagnostic efficacy of the 167 GEC in more institutions, and the importance of reporting precision estimates for diagnostic accuracy measures. Furthermore, as in the case of other molecular-based diagnostic tests in the field, long-term outcome data from clinical utility studies are needed to inform potential future clinical practice implications of the 167 GEC.

Immunohistochemical stains such as galectin-3 and HBME-1 have been examined in multiple studies of histologically confirmed thyroid FNA samples with indeterminate cytology, with reports of relatively high rates of specificity, but low sensitivity, for cancer detection $(164,175,176)$. Two of these studies were reported to be prospective $(164,175)$, but only one of the studies reported any degree of blinding (blind central histopathologic review) (164). Immunohistochemical stains require the availability of a cell block to perform the staining. Additional diagnostic molecular marker strategies are also under development. Specifically, mRNA markers (177-179), as well as miRNA markers (180-185), have shown initial diagnostic utility in FNA samples with indeterminate cytological diagnoses, but they have not been thoroughly validated. A recent study combining seven-gene mutational testing with expression of a set of 10 miRNA genes on preoperative FNA sampling from 109 patients with AUS/ FLUS or FN cytology, showed $89 \%$ sensitivity, $85 \%$ specificity, with a $73 \%$ PPV and $94 \%$ NPV on this group with a $32 \%$ prevalence of malignancy (186). Finally, peripheral blood TSH receptor mRNA assay has been reported to have a 90\% PPV and 39\% NPV in FNA-based assessment of thyroid nodules with atypical or suspicious cytology in a single-center, prospective validation study (187).

In summary, there is currently no single optimal molecular test that can definitively rule in or rule out malignancy in all cases of indeterminate cytology, and long-term outcome data proving clinical utility are needed.

\section{- RECOMMENDATION 14}

If intended for clinical use, molecular testing should be performed in Clinical Laboratory Improvement Amendments/ College of American Pathologists (CLIA/CAP)-certified molecular laboratories, or the international equivalent, 
because reported quality assurance practices may be superior compared to other settings.

\section{(Strong recommendation, Low-quality evidence)}

Many molecular marker tests are available in hospital-based molecular pathology laboratories and in reference laboratories. Importantly, all molecular marker tests intended for clinical use should be performed only in CLIA/CAP-certified molecular laboratories after appropriate analytical and clinical validation of all assays in each laboratory (158). In a survey of American molecular genetic testing laboratory directors, laboratory process quality assurance score (for multiple relevant domains) was associated with the presence of CLIA certification (188). In a large, international survey of medical genetic testing laboratory directors, accreditation of the laboratory was associated with a higher quality assurance index score (189).

\section{[A17] AUS/FLUS cytology}

\section{- RECOMMENDATION 15}

(A) For nodules with AUS/FLUS cytology, after consideration of worrisome clinical and sonographic features, investigations such as repeat FNA or molecular testing may be used to supplement malignancy risk assessment in lieu of proceeding directly with a strategy of either surveillance or diagnostic surgery. Informed patient preference and feasibility should be considered in clinical decision-making.

(Weak recommendation, Moderate-quality evidence)

(B) If repeat FNA cytology, molecular testing, or both are not performed or inconclusive, either surveillance or diagnostic surgical excision may be performed for an AUS/ FLUS thyroid nodule, depending on clinical risk factors, sonographic pattern, and patient preference.

\section{(Strong recommendation, Low-quality evidence)}

Based on the Bethesda System, this diagnostic category is reserved for specimens that contain cells with architectural and/or nuclear atypia that is more pronounced than expected for benign changes but not sufficient to be placed in one of the higher risk diagnostic categories $(99,190)$. Although this diagnostic category has been recommended for limited use and has an expected frequency in the range of $7 \%$, recent reports based on the Bethesda System have found this cytologic diagnosis to be used in $1 \%-27 \%$ of all thyroid FNA samples $(105,191)$. In studies that utilized the criteria established by the Bethesda System, the risk of cancer for patients with AUS/FLUS nodules who underwent surgery was $6 \%-48 \%$, with a mean risk of $16 \%$ (191).

A second opinion review of the cytopathology slides by a high-volume cytopathologist may be considered for patients with AUS/FLUS cytology. There is some evidence that this approach may reclassify many of these patients into the benign and nondiagnostic categories $(106,192)$. Furthermore, the overall diagnostic accuracy may be improved. Unfortunately, there is a relatively high intra-observer variability in this difficult diagnostic category (106).

A repeat FNA yields a more definitive cytologic diagnosis in many cases, whereas $10 \%-30 \%$ of nodules are repeatedly AUS/FLUS (104,193-195). The rate of malignancy on surgical follow-up has been shown to be similar for patients with a single AUS/FLUS diagnosis (37/90, 41\%), two successive AUS/FLUS diagnoses $(22 / 51,43 \%)$, and patients with a benign cytologic interpretation following the initial AUS/FLUS diagnosis $(2 / 7,29 \%)$ (196). Use of thyroid core-needle biopsy was reported by some to be more informative than repeated FNA for sampling nodules that were AUS/FLUS on initial FNA (119), and it is reasonably well-tolerated (197).

The refinement of risk stratification of nodules with AUS/ FLUS cytology using molecular testing has been examined in multiple studies. The interpretation of molecular testing is complex, however, and its utility is strongly influenced by the prevalence of cancer in the tested population of nodules. Only two molecular tests have been separately evaluated and validated for the individual AUS/FLUS, FN, and SUSP categories. Mutational testing for $B R A F$ in AUS/FLUS samples has high specificity for cancer, but low sensitivity $(198,199)$. Testing for a panel of mutations (BRAF, NRAS, HRAS, KRAS, RET/PTC1, RET/PTC3, PAX8/PPAR $\gamma$ ) offers a significantly higher sensitivity of $63 \%-80 \%(162,165)$. In the largest prospective study to date of nodules with AUS/FLUS cytology (653 consecutive nodules, of which 247 had surgical follow-up) from a single institution, detection of any of these mutations using RT-PCR with fluorescent melting curve analysis was reported to convey an $88 \%$ risk of cancer among nodules with surgical follow-up; $63 \%$ of cancers on final histopathology were identified with a positive mutation preoperatively ( 22 of 35 ), and $94 \%$ of nodules that were negative on mutation analysis had a benign final histopathology (209 of 222) (162). Positive testing for BRAF, RET/PTC or PAX8/ $P P A R \gamma$ was specific for a malignant outcome in $100 \%$ of cases, whereas RAS mutations had an $84 \%$ risk of cancer and a $16 \%$ chance of benign follicular adenoma. A recent study utilizing RT-PCR with liquid bead array flow cytometry with the sevengene mutation panel reported on 11 AUS/FLUS cytology nodules that had malignant histopathologic confirmation, of which seven had a negative molecular test and four had a positive test (167). There were also 11 AUS/FLUS cytology nodules, which were benign on histopathologic evaluation; 9 of 11 had a negative molecular test result and 2 of 11 had a positive result. Interpretation of results from the AUS/FLUS subgroup is limited by the small reported sample size (167).

Molecular testing using the 167 GEC has been studied for its diagnostic use in nodules with AUS/FLUS cytology. A multi-institutional study of 129 FNA samples with AUS/ FLUS cytology and surgical follow-up reported a $90 \%$ sensitivity [95\% CI 74\%-98\%] and 95\% NPV [95\% CI 85\%$99 \%]$, but only a $53 \%$ specificity [95\% CI $43 \%-63 \%$ ] and $38 \%$ PPV [95\% CI 27\%-50\%] for cancer (163). Although the specificity of the 167 GEC was low (53\% [95\% CI 43\%$63 \%]$ ), the negative test result was reported to decrease the risk estimate of malignancy in AUS/FLUS nodules in this study from $24 \%$ to $5 \%$. This observation has led to a clinical extrapolation suggesting that nodules that have a negative 167 GEC test results may be followed without surgery (163). In a recent single-center retrospective study including 68 cases of AUS/FLUS nodules, 16 AUS/FLUS cases were reported to have a 167 GEC suspicious result, and the PPV was $61 \%$ (11 of 18) for those with surgical pathology confirmation (200). There were insufficient data to confirm the NPV of the 167 GEC test for AUS/FLUS cytology, since the vast majority of patients with a benign 167 GEC test did not undergo surgery, and no long-term follow-up of such cases was reported 
(200). In three other recent studies, there were insufficient data for analysis in the AUS/FLUS subgroup to draw any meaningful conclusion on 167 GEC test performance in that subgroup (172-174). In addition, published follow-up for the 167 GEC is currently limited to a mean of 8.5 months in a subgroup of 71 patients (171), hence long-term outcome data are needed to ensure durability of benign 167 GEC findings with correlation to clinical and histologic outcomes.

Several recent studies (201-205) have examined the feasibility of using sonographic features to estimate risk of malignancy in nodules with AUS/FLUS cytology. The PPV of suspicious sonographic features has been estimated to range from $60 \%$ to $100 \%$ depending on the pretest probability of malignancy of AUS/FLUS cytology and the specific sonographic criteria selected in respective studies. A limitation of all of these studies is that a gold-standard surgical excisional diagnosis was not required for confirmation of malignancy and long-term follow-up data were generally lacking. The prevalence of suspicious sonographic features among studies of AUS/ FLUS cytology nodules ranged from $18 \%$ to $50 \%$, assuming that one or more suspicious features were deemed to be sufficient to be categorized as a sonographically suspicious nodule. The findings of these studies must be interpreted in the context of each study's overall risk of malignancy for this cytology classification because of its effect on the PPVs obtained by subsequent application of sonographic features to cytologically AUS/FLUS nodules. From the four Korean studies (overall malignancy rate $40 \%-55 \%$ ), the reported cancer risk in AUS/ FLUS nodules with the high suspicion sonographic pattern is 90\%-100\% (201-204), and the presence of even one suspicious US feature (irregular margins, taller than wide shape, marked hypoechogenicity or microcalcifications) increases the cancer risk to $60 \%-90 \%$. However, when the reported cancer rate in AUS/FLUS nodules is lower [e.g., 23\% in a study from Brazil (205)], the high suspicion sonographic pattern still raises the risk of malignancy, but the PPV is lower at $70 \%$. Nonetheless, the incidence of cancer in AUS/FLUS nodules with either the high suspicion pattern US or just one suspicious US feature is significantly higher than that generally accepted for this cytology category. In a secondary analysis of a retrospective single-center study of indeterminate FNA specimens subject to 167 GEC testing, Lastra et al. studied whether reexamining US characteristics could assist in distinguishing malignancy in indeterminate cytology nodules with a 167 GEC suspicious result (200). The presence of "nodular calcifications" or hyper- versus hypo-echogenicity did not alter the prediction of malignancy. It is unclear whether this study was adequately powered because the analysis was limited to a subgroup of 48 cases for analysis of microcalcifications and 20 cases for analysis of echogenicity (200). Further research is needed to examine the impact of considering clinical and sonographic features on the potential utility and interpretation of molecular testing of FNA specimens.

\section{[A18] Follicular neoplasm/suspicious for follicular neo- plasm cytology}

\section{- RECOMMENDATION 16}

(A) Diagnostic surgical excision is the long-established standard of care for the management of FN/SFN cytology nodules. However, after consideration of clinical and sonographic features, molecular testing may be used to supplement malignancy risk assessment data in lieu of proceeding directly with surgery. Informed patient preference and feasibility should be considered in clinical decision-making.

\section{(Weak recommendation, Moderate-quality evidence)}

(B) If molecular testing is either not performed or inconclusive, surgical excision may be considered for removal and definitive diagnosis of an FN/SFN thyroid nodule.

\section{(Strong recommendation, Low-quality evidence)}

This diagnostic category of the Bethesda System is used for cellular aspirates (i) comprised of follicular cells arranged in an altered architectural pattern characterized by cell crowding and/or microfollicle formation and lacking nuclear features of papillary carcinoma or (ii) comprised almost exclusively of oncocytic (Hürthle) cells $(99,206,207)$. This is an intermediate risk category in the Bethesda System, with a $15 \%-30 \%$ estimated risk of malignancy. Studies that applied the Bethesda System reported the use of this diagnostic category in $1 \%-25 \%$ (mean, 10\%) of all thyroid FNA samples, with the risk of cancer on surgery found to range from $14 \%$ to $33 \%$ (mean, 26\%) (191).

The refinement of risk stratification of nodules with FN/ SFN/Hürthle cell neoplasm cytology has been examined using ancillary molecular testing. Testing for a seven-gene panel of mutations (including $B R A F, R A S, R E T / P T C$, and $/ P P A R \gamma$ ) in nodules with follicular or Hürthle cell neoplasm or SFN has been reported to be associated with a sensitivity of 57\%-75\%, specificity of $97 \%-100 \%$, PPV of $87 \%-100 \%$, and NPV of $79 \%-86 \%(162,165)$. Many of these benign tumors are follicular adenomas driven by oncogenic $R A S$ mutation with uncertain malignant potential (208). Nodules lacking all of these mutations still have a substantial cancer risk, which is due to the presence of a subset of tumors that lack any of the mutations tested by this seven-gene panel (162). Expansion of the current panels to include additional mutations and gene rearrangements with this next-generation sequencing assay was associated with a sensitivity of $90 \%$ [95\% CI $80 \%-99 \%$ ], specificity of $93 \%$ [95\% CI 88\%-98\%], PPV of $83 \%$ [95\% CI 72\%-95\%], and NPV of $96 \%$ [95\% CI $92 \%-100 \%$ ] in a recent single-center study of 143 consecutive FN/SFN FNA specimens with known surgical outcomes. In this study (170), retrospective $(n=91)$ and prospective data $(n=52)$ were combined. A limitation was that the pathologists reviewing the surgical specimens were aware of earlier generation molecular marker seven-gene panel test results, although they were blinded to results of the nextgeneration mutation panel $(162,170)$. Given the overlap of some of the markers detected in earlier and later generation assays, there is a potential for bias (170), and the results need to be replicated in other studies.

Molecular testing using the GEC was reported to have a 94\% NPV [95\% CI 79\%-99\%], and a 37\% PPV [95\% CI $23 \%-52 \%$ ] in the FN/SFN/Hürthle cell neoplasm Bethesda subgroup (163). A recent unblinded study from the Mayo Clinic utilizing a prospective patient registry reported the following diagnostic accuracy estimates in a subgroup of 31 indeterminate nodules from the same Bethesda subgroup that were subject to histopathologic confirmation: sensitivity $80 \%$ (four of five nodules), specificity 12\% (3 of 26 nodules), PPV 
$15 \%$ ( 4 of 27), and NPV $75 \%$ (three of four) (173). In a singlecenter retrospective study including 64 nodules subjected to 167 GEC testing and a cytology read as FN/FN with oncocytic features, the PPV for a suspicious GEC result was 37\% (11 of 30 ), although the PPV was significantly higher in the FN group $(53 \%)$ compared with the FN with oncocytic features group (15\%) (200). There were insufficient numbers of patients with benign 167 GEC results who underwent surgery to draw conclusions on NPV; moreover, no long-term follow-up data were reported (200). The relatively small number of indeterminate nodules is an important limitation. Furthermore, precision estimates (95\% CIs) for the diagnostic accuracy measures were not reported but could be assumed to be relatively wide given the small sample size $(173,200)$.

\section{[A19] Suspicious for malignancy cytology}

\section{- RECOMMENDATION 17}

(A) If the cytology is reported as suspicious for papillary carcinoma (SUSP), surgical management should be similar to that of malignant cytology, depending on clinical risk factors, sonographic features, patient preference, and possibly results of mutational testing (if performed).

(Strong recommendation, Low-quality evidence)

(B) After consideration of clinical and sonographic features, mutational testing for $B R A F$ or the seven-gene mutation marker panel (BRAF, RAS, RET/PTC, PAX8/ $P P A R \gamma)$ may be considered in nodules with SUSP cytology if such data would be expected to alter surgical decisionmaking.

(Weak recommendation, Moderate-quality evidence)

This diagnostic category of the Bethesda System is reserved for aspirates with cytologic features that raise a strong suspicion for malignancy (mainly for PTC) but are not sufficient for a conclusive diagnosis $(99,209)$. This is the highest risk category of indeterminate cytology in the Bethesda System, with an estimated cancer risk of $60 \%-75 \%$ (209). Studies that utilize the Bethesda System have reported this cytologic diagnosis in 1\%-6\% of thyroid FNAs and found malignancy after surgery in $53 \%-87 \%$ (mean, $75 \%$ ) of these nodules (191). Due to the high risk of cancer, the diagnosis of suspicious for papillary carcinoma is an indication for surgery.

Mutational testing has been proposed to refine risk prior to surgery, assuming that surgical management would change based on a positive test result. $B R A F$ mutations have been reported to confer close to $100 \%$ probability of malignancy (162,198,210,211). Testing for BRAF mutations in nodules suspicious for malignancy has been reported to have a sensitivity of 36\% (10 of 28) and specificity of 100\% (24 of 24) in a single-center retrospective study (162). In another single center retrospective study in which FNA slides deemed to be suspicious for PTC were tested after surgery, the sensitivity of $B R A F$ testing for PTC was $45.5 \%$ (15 of 33), and specificity was $85.7 \%$ (12 of 14) (212). Testing for a seven-gene panel of mutations (including $B R A F, R A S, R E T / P T C$, with or without $P A X 8 / P P A R \gamma)$ in nodules with cytology suspicious for malignancy is associated with a sensitivity of $50 \%-68 \%$, specificity of $86 \%-96 \%$, PPV of $80 \%-95 \%$, and NPV of $72 \%-75 \%$ in respective single-center studies $(162,165,168)$. Molecular testing using the 167 GEC has a PPV that is similar to cytology alone (76\%) and a NPV of $85 \%$ (163), and it is therefore not indicated in patients with this cytologic diagnosis.

\section{[A20] What is the utility of ${ }^{18}$ FDG-PET scanning to predict malignant or benign disease when FNA cytology is indeterminate (AUS/FLUS, FN, SUSP)?}

\section{- RECOMMENDATION 18}

${ }^{18}$ FDG-PET imaging is not routinely recommended for the evaluation of thyroid nodules with indeterminate cytology.

(Weak recommendation, Moderate-quality evidence)

Eight studies have been performed and are the subject of two meta-analyses (213-222). While early data suggested a high NPV for ${ }^{18}$ FDG-PET in this setting, most studies failed to use the Bethesda System for Reporting Thyroid Cytopathology and included numerous small nodules $<1 \mathrm{~cm}$ in diameter (221). A recent meta-analysis included seven studies, of which five were prospective (222). The cancer prevalence was $26 \%$ inclusive of all combined data, confirming a typical study cohort. Sensitivity and specificity of ${ }^{18}$ FDG-PET were $89 \%$ and $55 \%$, respectively, resulting in a $41 \%$ PPV and $93 \%$ NPV, which is similar to the performance of the 167 GEC. Vriens et al. (223) performed a costeffectiveness analysis using ${ }^{18}$ FDG-PET performance data from their own meta-analysis and 2012 reimbursement rates of the Dutch system. They showed that ${ }^{18}$ FDG-PET was more cost effective than surgery, the 167 GEC, or mutational testing. A recent prospective analysis of 56 nodules with indeterminate FNA cytology used both ${ }^{18}$ FDG-PET and thyroid US to further evaluate the nodules (220). In a multivariate analysis, the authors demonstrated no additional diagnostic benefit or improved risk assessment when adding ${ }^{18}$ FDG-PET to that already obtained from thyroid US, bringing into question the incremental benefit of PET imaging in patients with cytologically indeterminate thyroid nodules.

\section{[A21] What is the appropriate operation for cytologically indeterminate thyroid nodules?}

\section{- RECOMMENDATION 19}

When surgery is considered for patients with a solitary, cytologically indeterminate nodule, thyroid lobectomy is the recommended initial surgical approach. This approach may be modified based on clinical or sonographic characteristics, patient preference, and/or molecular testing when performed (see Recommendations 13-16).

(Strong recommendation, Moderate-quality evidence)

\section{- RECOMMENDATION 20}

(A) Because of increased risk for malignancy, total thyroidectomy may be preferred in patients with indeterminate nodules that are cytologically suspicious for malignancy, positive for known mutations specific for carcinoma, sonographically suspicious, or large $(>4 \mathrm{~cm})$, or in patients with familial thyroid carcinoma or history of radiation exposure, if completion thyroidectomy would be 
recommended based on the indeterminate nodule being malignant following lobectomy.

\section{(Strong recommendation, Moderate-quality evidence)}

(B) Patients with indeterminate nodules who have bilateral nodular disease, those with significant medical comorbidities, or those who prefer to undergo bilateral thyroidectomy to avoid the possibility of requiring a future surgery on the contralateral lobe, may undergo total or near-total thyroidectomy, assuming completion thyroidectomy would be recommended if the indeterminate nodule proved malignant following lobectomy.

\section{(Weak recommendation, Low-quality evidence)}

The primary goal of thyroid surgery for a thyroid nodule that is cytologically indeterminate (AUS/FLUS or FN or SUSP) is to establish a histological diagnosis and definitive removal, while reducing the risks associated with remedial surgery in the previously operated field if the nodule proves to be malignant. Surgical options to address the nodule should be limited to lobectomy (hemithyroidectomy) with or without isthmusectomy, near-total thyroidectomy (removal of all grossly visible thyroid tissue, leaving only a small amount $[<1 \mathrm{~g}]$ of tissue adjacent to the recurrent laryngeal nerve near the ligament of Berry), or total thyroidectomy (removal of all grossly visible thyroid tissue). Removal of the nodule alone, partial lobectomy, and subtotal thyroidectomy, leaving $>1 \mathrm{~g}$ of tissue with the posterior capsule on the uninvolved side, are inappropriate operations for possible thyroid cancer (224).

Decisions regarding the extent of surgery for indeterminate thyroid nodules are influenced by several factors (225), including the estimated presurgical likelihood of malignancy based upon clinical risk factors ( $>4 \mathrm{~cm}$, family history, and/or history of radiation) (226-229), sonographic pattern (Table 6, Fig. 2) (202-203), cytologic category (Table 8), and ancillary test findings (see molecular testing section [A15-19]). These risk factors, as well as patient preference, presence of contralateral nodularity or coexistent hyperthyroidism, and medical comorbidities, impact decisions regarding thyroid lobectomy with the possible need for subsequent completion thyroidectomy versus total thyroidectomy up front.

Nodules that are cytologically classified as AUS/FLUS or FN and benign using the 167 GEC, or AUS/FLUS and negative using the seven-gene mutation panel have an estimated $5 \%-6 \%$ risk of malignancy $(162,163)$. Nodules that are cytologically classified as $\mathrm{FN}$ and negative using the seven-gene mutation panel have an estimated $14 \%$ risk of malignancy, which is slightly lower than the risk based upon the Bethesda classification alone (162).

Nodules that are cytologically classified as AUS/FLUS or $\mathrm{FN}$ and as suspicious using the 167 GEC have an estimated $37 \%-44 \%$ risk of malignancy, which is slightly higher than the risk based upon the Bethesda classification alone (Table 8) $(163,171)$.

Nodules that are cytologically classified as SUSP cytology and benign using the 167 GEC or negative using the mutation seven-gene panel, also have an estimated $15 \%-28 \%$ risk of malignancy.

In contrast, nodules that are cytologically classified as AUS/FLUS or FN and that are positive for known RAS mutations associated with thyroid carcinoma have an estimated
$84 \%$ risk of malignancy and should be considered in a similar risk category to cytologically suspicious for malignancy (Table 8) (103,230). Nodules that are cytologically classified as AUS/FLUS or FN or SUSP and that are positive for known $B R A F^{V 600 E}, R E T / P T C$, or PAX8/PPAR $\gamma$ mutations have an estimated risk of malignancy of $>95 \%$ and should be considered in a similar category to cytologically diagnosed thyroid carcinoma $(198,210,211)$.

Sonographic pattern of nodules with AUS/FLUS cytology may aid in risk stratification and management. In one study, sonographically benign or seemingly very low risk nodules with AUS/FLUS cytology were noted to be malignant in only $8 \%$ of cases, compared to $58 \%$ when sonographic suspicious was low or intermediate, and $100 \%$ when sonographic suspicion of malignancy was high (203). Another study supported this finding with sonographic findings highly suspicious for malignancy (taller than wide shape, irregular borders, and/or marked hypoechogenicity) having $>90 \%$ specificity and PPV for malignancy (202).

The risks of total thyroidectomy are significantly greater than that for thyroid lobectomy, with a recent meta-analysis suggesting a pooled relative risk (RR) significantly greater for all complications, including recurrent laryngeal nerve injury (transient $\mathrm{RR}=1.7$, permanent $\mathrm{RR}=1.9$ ), hypocalcemia (transient $\mathrm{RR}=10.7$, permanent $\mathrm{RR}=3.2$ ), and hemorrhage/ hematoma $(R R=2.6)(231)$. Further, total thyroidectomy is associated with the rare but potential risk of bilateral recurrent laryngeal nerve injury necessitating tracheostomy. Surgeon experience likely influences the risks of thyroidectomy, with higher volume surgeons having lower complication rates (232,233).

Total thyroidectomy necessitates thyroid hormone replacement, while lobectomy is associated with an incidence of postoperative biochemical hypothyroidism estimated at $22 \%$, with clinical or overt hypothyroidism estimated at $4 \%$ (234). A significantly increased risk of hypothyroidism following lobectomy has been reported in the presence of autoimmune thyroid disease (e.g., as reflected by the presence of thyroid antibodies) or high normal/elevated TSH $(231,234)$. Hypothyroidism is not an indication for thyroidectomy, and its use as justification for total thyroidectomy over lobectomy should be weighed against the higher risks associated with total thyroidectomy. In contrast, coexistent hyperthyroidism may be an indication for total thyroidectomy depending upon the etiology.

Thyroid lobectomy (hemithyroidectomy) provides definitive histological diagnosis and complete tumor removal for cytologically indeterminate nodules with a lower risk of complications compared to total thyroidectomy and may be sufficient for smaller, solitary intrathyroidal nodules that ultimately prove malignant. As the likelihood of malignancy increases, the potential need for a second operation also increases, if the cytologically indeterminate nodule ultimately proves malignant and if completion thyroidectomy would be recommended. Intraoperative evaluation, with or without frozen section, can occasionally confirm malignancy at the time of lobectomy allowing for conversion to total thyroidectomy if indicated. Frozen section is most helpful if the histopathologic diagnosis is classic PTC, whereas its impact is low in follicular variant of PTC and FTC. The individual patient must weigh the relative advantages and disadvantages of thyroid lobectomy with possible total thyroidectomy or 
subsequent completion thyroidectomy versus initial total thyroidectomy.

\section{[A22] How should multinodular thyroid glands (i.e., two or more clinically relevant nodules) be evaluated for malignancy?}

\section{- RECOMMENDATION 21}

(A) Patients with multiple thyroid nodules $\geq 1 \mathrm{~cm}$ should be evaluated in the same fashion as patients with a solitary nodule $\geq 1 \mathrm{~cm}$, excepting that each nodule that is $>1 \mathrm{~cm}$ carries an independent risk of malignancy and therefore multiple nodules may require FNA.

(Strong recommendation, Moderate-quality evidence)

(B) When multiple nodules $\geq 1 \mathrm{~cm}$ are present, FNA should be performed preferentially based upon nodule sonographic pattern and respective size cutoff (Table 6, Fig. 2).

\section{(Strong recommendation, Moderate-quality evidence)}

(C) If none of the nodules has a high or moderate suspicion sonographic pattern, and multiple sonographically similar very low or low suspicion pattern nodules coalesce with no intervening normal parenchyma, the likelihood of malignancy is low and it is reasonable to aspirate the largest nodules $(\geq 2 \mathrm{~cm})$ or continue surveillance without FNA.

(Weak recommendation, Low-quality evidence)

\section{- RECOMMENDATION 22}

A low or low-normal serum TSH concentration in patients with multiple nodules may suggest that some nodule(s) may be autonomous. In such cases, a radionuclide (preferably ${ }^{123} \mathrm{I}$ ) thyroid scan should be considered and directly compared to the US images to determine functionality of each nodule $\geq 1 \mathrm{~cm}$. FNA should then be considered only for those isofunctioning or nonfunctioning nodules, among which those with high suspicion sonographic pattern should be aspirated preferentially.

\section{(Weak recommendation, Low-quality evidence)}

Patients with multiple thyroid nodules have the same risk of malignancy as those with solitary nodules $(32,74)$. However, when evaluating the risk of cancer per individual nodule, one large study found that a solitary nodule had a higher likelihood of malignancy than did a nonsolitary nodule $(p<0.01)$, although in agreement with the other studies the risk of malignancy per patient was the same and independent of the number of nodules (77). A recent systematic review and meta-analysis confirmed the slightly higher risk of malignancy in a solitary nodule compared with an individual nodule in a MNG. However, this appeared to hold true mostly outside of the United States and in iodine-deficient populations (235). A diagnostic US should be performed to evaluate the sonographic risk pattern of each nodule, but if only the "dominant" or largest nodule is aspirated, the thyroid cancer may be missed (74). Therefore, multiple thyroid nodules $\geq 1 \mathrm{~cm}$ may require aspiration, based on sonographic pattern (Recommendation 8, Table 6, Fig. 2) to fully exclude clinically relevant thyroid cancer. Radionuclide scanning may also be considered in patients with multiple thyroid nodules with the goal of identifying and aspirating appropriate hypofunctioning nodules. Such imaging may prove especially useful when the serum TSH is below or in the low-normal range. Similarly, sonographic risk assessment of each nodule can assist in identifying those nodules with the highest likelihood of cancer (see section [A10]).

\section{[A23] What are the best methods for long-term follow-up of patients with thyroid nodules?}

[A24] Recommendations for initial follow-up of nodules with benign FNA cytology

\section{- RECOMMENDATION 23}

Given the low false-negative rate of US-guided FNA cytology and the higher yield of missed malignancies based upon nodule sonographic pattern rather than growth, the follow-up of thyroid nodules with benign cytology diagnoses should be determined by risk stratification based upon US pattern.

(A) Nodules with high suspicion US pattern: repeat US and US-guided FNA within 12 months.

(Strong recommendation, Moderate-quality evidence)

(B) Nodules with low to intermediate suspicion US pattern: repeat US at 12-24 months. If sonographic evidence of growth (20\% increase in at least two nodule dimensions with a minimal increase of $2 \mathrm{~mm}$ or more than a $50 \%$ change in volume) or development of new suspicious sonographic features, the FNA could be repeated or observation continued with repeat US, with repeat FNA in case of continued growth.

\section{(Weak recommendation, Low-quality evidence)}

(C) Nodules with very low suspicion US pattern (including spongiform nodules): the utility of surveillance US and assessment of nodule growth as an indicator for repeat FNA to detect a missed malignancy is limited. If US is repeated, it should be done at $\geq 24$ months.

\section{(Weak recommendation, Low-quality evidence)}

[A25] Recommendation for follow-up of nodules with two benign FNA cytology results

(D) If a nodule has undergone repeat US-guided FNA with a second benign cytology result, US surveillance for this nodule for continued risk of malignancy is no longer indicated.

(Strong recommendation, Moderate-quality evidence)

Given that there is a low but discrete false-negative rate for nodules with benign FNA cytology results, is there an optimal way to identify these missed malignancies? Although the risk of malignancy after two benign cytology results is virtually zero $(129-133,236)$, routine rebiopsy is not a viable or cost-effective option because of the low false-negative rate of an US-guided FNA benign cytology result. Prior guidelines have recommended repeat FNA for nodules that grow during serial sonographic observation. However, nodule growth can be variably defined. Because of interobserver variation, Brauer et al. (237) reported a 50\% increase in nodule volume as the minimally significant reproducibly recorded change in 
nodule size, which is equivalent to a $20 \%$ increase in two of the three nodule dimensions. If a $50 \%$ volume increase cutoff is applied, only $4 \%-10 \%$ of nodules were reported to be larger at a mean of 18 months $(133,238)$. However, using cutoffs of a $15 \%$ volume increase based upon internally assessed interobserver coefficients of variation, published series report that $32 \%-50 \%$ of nodules increase in size over a 4-5 year period $(239,240)$. Because of the stringent methodology of these studies, adoption of a $15 \%$ volume increase as statistically significant is not practically applicable.

A recent 5-year prospective multicenter study evaluated outcomes of 1597 nodules from 992 patients with either cytologically or sonographically benign nodules (241). Nodules $1 \mathrm{~cm}$ or larger underwent US-guided FNA and subcentimeter nodules were defined as sonographically benign based upon imaging characteristics equivalent to the ATA low or very low suspicion US patterns. All nodules were followed by annual US exams. The false-negative rate of a benign cytology diagnosis was $1.1 \%$. Of the four missed cancers, on baseline US imaging three were hypoechoic and solid and one was isoechoic with microcalcifications; none was spongiform or mixed cystic solid and noncalcified (ATA very low suspicion pattern). During sonographic surveillance, repeat FNA was prompted by either growth (two nodules) or development of a new suspicious sonographic feature (two nodules). In addition, the shortest time interval to detect change and repeat the FNA was 2 years. Another critical observation from this study was that only one cancer was detected in 5 years among the 852 subcentimeter nodules classified as sonographicially benign at baseline. This cancer was identified on the 5-year follow-up US, when its composition changed from mixed cystic/solid to hypoechoic solid with irregular margins prompting FNA (241). Currently, there are no follow-up studies of nodule growth that extend observation beyond 5 years to help inform decision-making about long-term surveillance. Additional research would be valuable because indefinite follow-up of nodules with benign cytology is costly and may be unnecessary.

Recent investigations of repeat US-guided FNA in nodules with initial benign cytology show higher detection rates for missed malignancy for those nodules with a high suspicion sonographic pattern rather than size increase $(236,242)$. Kwak et al. (236) reported a significantly higher malignancy rate of $20.4 \%$ in nodules with benign cytology that exhibited either marked hypoechogenicity, irregular borders, microcalcifications, or a taller than wide shape versus a $1.4 \%$ risk in those that exhibited a $15 \%$ volume increase but lacked these US features. Importantly, the low risk of malignancy did not differ between US negative nodules that grew and those that demonstrated no interval size change ( $1.4 \%$ vs. $0.5 \%, p=0.18)$. Similarly, Rosario et al. (242) detected cancer in $17.4 \%$ of nodules with benign cytologic diagnoses and suspicious US features versus $1.3 \%$ of those without suspicious characteristic that grew, using criteria of a $50 \%$ volume increase. These studies indicate that the use of suspicious US characteristics rather than nodule growth should be the indication for repeat FNA despite an initial benign cytology diagnosis. Repeat US and FNA should be repeated within 12 months as guided by clinical judgement. Given the low false-negative rate of US-guided FNA cytology and the higher yield of missed malignancies based upon nodule sonographic pattern rather than growth, the follow-up of thyroid nodules with benign cytology diagnoses should be determined by risk stratification based upon US pattern as defined in Recommendation 8. If follow-up US for surveillance is performed and the nodule size is stable, the utility of subsequent US imaging for detection of potential malignancy by nodule growth assessment is very low and if performed, the time interval for any additional US exam should be at least as long that between the initial benign FNA cytology result and first follow-up. However, even if a repeat US is not indicated based on a benign cytology, US pattern, or stability in nodule size, larger nodules may require monitoring for growth that could result in symptoms and thus prompt surgical intervention despite benign cytology.

One recent study evaluated the long-term consequences of a false-negative benign cytology (140). A total of 1369 patients with 2010 cytologically benign thyroid nodules were followed for a mean of 8.5 years. Eighteen false-negative cases were identified, although only a subset of patients underwent repeat FNA or thyroid surgery. Thirty deaths were documented in the entire cohort over this time period and none were attributable to thyroid cancer. These data support that an initial benign cytology conveys an overall excellent prognosis and a conservative follow-up strategy is reasonable.

\section{[A26] Follow-up for nodules that do not meet FNA criteria}

\section{- RECOMMENDATION 24}

Nodules may be detected on US that do not meet criteria for FNA at initial imaging (Recommendation 8). The strategy for sonographic follow-up of these nodules should be based upon the nodule's sonographic pattern.

(A) Nodules with high suspicion US pattern: repeat US in 6-12 months.

\section{(Weak recommendation, Low-quality evidence)}

(B) Nodules with low to intermediate suspicion US pattern: consider repeat US at 12-24 months.

\section{(Weak recommendation, Low-quality evidence)}

(C) Nodules $>1 \mathrm{~cm}$ with very low suspicion US pattern (including spongiform nodules) and pure cyst: the utility and time interval of surveillance US for risk of malignancy is not known. If US is repeated, it should be at $\geq 24$ months.

\section{(No recommendation, Insufficient evidence)}

(D) Nodules $\leq 1 \mathrm{~cm}$ with very low suspicion US pattern (including spongiform nodules) and pure cysts do not require routine sonographic follow-up.

\section{(Weak recommendation, Low-quality evidence)}

Ultrasound studies demonstrate that up to $50 \%$ of adults have thyroid nodules. The vast majority of these are subcentimeter, and FNA evaluation is generally not indicated. In addition, based upon both sonographic pattern and size cutoffs (Recommendation 8), many nodules $>1 \mathrm{~cm}$ may also be followed without FNA. Although no prospective studies address the optimal cost-effective surveillance strategy for these nodules that have not undergone FNA, a recent study by Durante et al. (241) confirms that subcentimeter thyroid nodules corresponding to the ATA very low suspicion risk pattern are highly 
unlikely to change during 5-year sonographic follow-up, and the risk of malignancy is exceedingly low. The findings from studies correlating sonographic features and malignancy risk in aspirated nodules can be extrapolated to inform a follow-up strategy for this group of nodules that do not meet FNA criteria at the time of their initial detection. For example, the interval for follow-up sonography for a nodule that is hypoechoic and taller than wide should be shorter than that for an isoechoic solid nodule with smooth borders.

US-guided FNA should be performed based upon followup US imaging if the nodule subsequently meets criteria based upon Recommendation 8 .

\section{[A27] What is the role of medical or surgical therapy for benign thyroid nodules?}

\section{- RECOMMENDATION 25}

Routine TSH suppression therapy for benign thyroid nodules in iodine sufficient populations is not recommended. Though modest responses to therapy can be detected, the potential harm outweighs benefit for most patients.

(Strong recommendation, High-quality evidence)

\section{- RECOMMENDATION 26}

Individual patients with benign, solid, or mostly solid nodules should have adequate iodine intake. If inadequate dietary intake is found or suspected, a daily supplement (containing $150 \mu \mathrm{g}$ iodine) is recommended.

(Strong recommendation, Moderate-quality evidence)

\section{- RECOMMENDATION 27}

(A) Surgery may be considered for growing nodules that are benign after repeat FNA if they are large $(>4 \mathrm{~cm})$, causing compressive or structural symptoms, or based upon clinical concern.

\section{(Weak recommendation, Low-quality evidence)}

(B) Patients with growing nodules that are benign after FNA should be regularly monitored. Most asymptomatic nodules demonstrating modest growth should be followed without intervention.

\section{(Strong recommendation, Low-quality evidence)}

\section{- RECOMMENDATION 28}

Recurrent cystic thyroid nodules with benign cytology should be considered for surgical removal or percutaneous ethanol injection (PEI) based on compressive symptoms and cosmetic concerns. Asymptomatic cystic nodules may be followed conservatively.

\section{(Weak recommendation, Low-quality evidence)}

\section{- RECOMMENDATION 29}

There are no data to guide recommendations on the use of thyroid hormone therapy in patients with growing nodules that are benign on cytology.

\section{(No recommendation, Insufficient evidence)}

Evidence from multiple prospective, RCTs, and from three meta-analyses suggest that thyroid hormone supplementation in doses that suppress the serum TSH to subnormal levels may result in a decrease in nodule size and may prevent the appearance of new nodules in regions of the world with borderline low iodine intake (239,243-245). However, the effect is modest, with most studies suggesting an average $5 \%-15 \%$ reduction in nodule volume when treated with suppressive levothyroxine ( $\left.\mathrm{LT}_{4}\right)$ therapy for 6-18 months. Two high-quality meta-analyses confirm that six to eight patients will require suppressive $\mathrm{LT}_{4}$ therapy to achieve one successful treatment response (246,247). The extent of TSH suppression achieved in high-quality studies is variable, though the majority suppressed TSH to $<0.2 \mathrm{mIU} / \mathrm{L}$, with many to $<0.1$ $\mathrm{mIU} / \mathrm{L}$. Hyperthyroidism to this degree has been significantly associated with an increased risk of cardiac arrhythmias and osteoporosis, as well as adverse symptomatology. Together, these data confirm that $\mathrm{LT}_{4}$ suppressive therapy demonstrates modest (though usually clinically insignificant) efficacy in nodule volume reduction, but increases the risk of adverse consequences related to iatrogenic thyrotoxicosis. One large prospective, randomized trial demonstrated that sufficient dietary iodine intake $(150 \mu \mathrm{g}$ daily) also reduced nodule size slightly more than placebo (248). The consumption of adequate dietary iodine is recommended for all adults and is without harm when not excessive. Data supporting $\mathrm{LT}_{4}$ therapy in non-TSHsuppressive doses for prevention of thyroid nodule growth are incomplete. One recent cohort analysis suggested nonsuppressive doses of $\mathrm{LT}_{4}$ therapy conferred protection from nodule growth over time (249). However, the nonblinded, nonrandomized nature of the trial precludes broad translation of the data, and the efficacy of nonsuppressive $\mathrm{LT}_{4}$ remains unproven.

Cystic nodules that are cytologically benign can be monitored for recurrence (fluid reaccumulation), which can be seen in $60 \%-90 \%$ of patients $(250,251)$. For those patients with subsequent recurrent symptomatic cystic fluid accumulation, surgical removal, generally by hemithyroidectomy, or PEI are both reasonable strategies. Four controlled studies demonstrated a 75\%$85 \%$ success rate after PEI compared with a $7 \%-38 \%$ success rate in controls treated by simple cyst evacuation or saline injection. Success was achieved after an average of two PEI treatments. Complications included mild to moderate local pain, flushing, dizziness, and dysphonia (250-253). Surgery may be considered for growing solid nodules that are benign on repeat cytology if they are large $(>4 \mathrm{~cm})$, causing compressive or structural symptoms, or based upon clinical concern $(254,255)$.

\section{[A28] How should thyroid nodules in pregnant women be managed?}

[A29] FNA for thyroid nodules discovered during pregnancy

\section{- RECOMMENDATION 30}

(A) FNA of clinically relevant thyroid nodules (refer to section [A10]) should be performed in euthyroid and hypothyroid pregnant women.

(Strong recommendation, Moderate-quality evidence)

(B) For women with suppressed serum TSH levels that persist beyond 16 weeks gestation, FNA may be deferred until after pregnancy and cessation of lactation. At that 
time, a radionuclide scan can be performed to evaluate nodule function if the serum TSH remains suppressed.

\section{(Strong recommendation, Moderate-quality evidence)}

It is uncertain if thyroid nodules discovered in pregnant women are more likely to be malignant than those found in nonpregnant women, since there are no population-based studies to address this question. Pregnancy does not appear to modify microscopic cellular appearance, thus standard diagnostic criteria should be applied for cytologic evaluation (256). Serial evaluation of nodules throughout pregnancy has demonstrated that thyroid nodules will enlarge slightly throughout gestation, though this does not imply malignant transformation (257). The recommended evaluation of a clinically relevant nodule in a pregnant patient is thus the same as for a nonpregnant patient, with the exception that a radionuclide scan is contraindicated. In addition, for patients with nodules diagnosed as DTC by FNA during pregnancy, delaying surgery until after delivery does not affect outcome (258). Surgery performed during pregnancy is associated with greater risk of complications, longer hospital stays, and higher costs (259).

\section{[A30] Approaches to pregnant patients with malignant} or indeterminate cytology

\section{- RECOMMENDATION 31}

PTC discovered by cytology in early pregnancy should be monitored sonographically. If it grows substantially (as defined in section [A24]) before 24-26 weeks gestation, or if US reveals cervical lymph nodes that are suspicious for metastatic disease, surgery should be considered during pregnancy. However, if the disease remains stable by midgestation, or if it is diagnosed in the second half of pregnancy, surgery may be deferred until after delivery.

\section{(Weak recommendation, Low-quality evidence)}

If FNA cytology is consistent with PTC, surgery is generally recommended. However, the decision to perform such surgery either during pregnancy or after delivery must be individualized. If surgery is not performed, the utility of thyroid hormone therapy targeted to lower serum TSH levels to improve the prognosis of thyroid cancer diagnosed during gestation is not known. Because higher serum TSH levels may be correlated with a more advanced stage of cancer at surgery (260), if the patient's serum TSH is $>2 \mathrm{mU} / \mathrm{L}$, it may be reasonable to initiate thyroid hormone therapy to maintain the TSH between 0.3 to $2.0 \mathrm{mU} / \mathrm{L}$ for the remainder of gestation.

Most data confirm that the prognosis of women with welldifferentiated thyroid cancer identified but not treated during pregnancy is similar to that of nonpregnant patients. Because of this, surgery in most pregnant patients is deferred until postpartum $(258,261)$, and no further testing is required. However, some studies differ from these findings. Two Italian cohort studies have investigated women diagnosed with DTC in relation to the timing of pregnancy. Messuti et al. (262) noted a statistically higher rate of persistence/recurrence when DTC was diagnosed during pregnancy or within 2 years postpartum. However, the stimulated $\mathrm{Tg}$ was found to be $>10 \mathrm{ng} / \mathrm{mL}$ during ${ }^{131} \mathrm{I}$ ablation in many cases, suggesting the extent of thyroidectomy and/or tumor resection may have been limited in this cohort and therefore contributed to biochemical persistence of disease. Vannucchi et al. (263) followed a small cohort of 10 patients with DTC during pregnancy or within 1 year post partum, again noting a large rate of persistent disease $(60 \%)$ compared to nonpregnant controls $(4.2 \%-13.1 \%)$. Similarly, the majority of cases with persistent disease were attributable to biochemical elevations in $\mathrm{Tg}$ or anti-Tg antibodies, again raising the question of whether the extent of initial resection was limited in comparison to nonpregnant controls. Given the likelihood that biochemical persistence could be attributable to an increased size of remnant tissue or incomplete surgical resection in both studies, these data should not refute previous, larger analyses showing no increased recurrence rates when DTC is diagnosed during pregnancy.

Theoretically, molecular marker analysis could be helpful in the evaluation of DTC or clinically relevant, cytologically indeterminate thyroid nodules detected during pregnancy. However, the application of molecular testing in pregnant women with indeterminate cytology remains uncertain. There are no published data validating the performance of any molecular marker in this population. Therefore, the committee cannot recommend for or against their use in pregnant women. However, it is theoretically possible that changes in a nodule's RNA expression may occur during gestation altering performance of the 167 GEC while the seven-gene mutational panel (BRAF, RAS, PAX8/PPAR $\gamma$, $R E T / P T C$ ) would be more likely to demonstrate similar performance to that of a nonpregnant population.

When surgery is advised during pregnancy, it is most often because of high-risk clinical or sonographic findings, nodule growth, or change over short duration follow-up or it is based upon physician judgement. To minimize the risk of miscarriage, surgery during pregnancy should be done in the second trimester before 24 weeks gestation (264). However, PTC discovered during pregnancy does not behave more aggressively than that diagnosed in a similar-aged group of nonpregnant women $(258,265)$. A retrospective study of pregnant women with DTC found no difference in either recurrence or survival rates between women operated during or after pregnancy (258). Further, retrospective data suggest that treatment delays of $<1$ year from the time of thyroid cancer discovery do not adversely affect patient outcome (266). A separate study reported a higher rate of complications in pregnant women undergoing thyroid surgery compared with nonpregnant women (267). If FNA cytology is indeterminate, monitoring may be considered with further evaluation may be delayed until after delivery. Some experts recommend thyroid hormone suppression therapy for pregnant women with FNA suspicious for or diagnostic of PTC, if surgery is deferred until the postpartum period (259).

\section{[B1] DIFFERENTIATED THYROID CANCER: INITIAL MANAGEMENT GUIDELINES}

Differentiated thyroid cancer, arising from thyroid follicular epithelial cells, accounts for the vast majority of thyroid cancers. Of the differentiated cancers, papillary cancer comprises about $85 \%$ of cases compared to about $12 \%$ that have follicular histology, including conventional and oncocytic (Hürthle cell) carcinomas, and $<3 \%$ that are poorly 
differentiated tumors (268). In general, stage for stage, the prognoses of PTC and follicular cancer are similar $(266,269)$.

\section{[B2] Goals of initial therapy of DTC}

The basic goals of initial therapy for patients with DTC are to improve overall and disease-specific survival, reduce the risk of persistent/recurrent disease and associated morbidity, and permit accurate disease staging and risk stratification, while minimizing treatment-related morbidity and unnecessary therapy. The specific goals of initial therapy are to

1. Remove the primary tumor, disease that has extended beyond the thyroid capsule, and clinically significant lymph node metastases. Completeness of surgical resection is an important determinant of outcome, while residual metastatic lymph nodes represent the most common site of disease persistence/recurrence (270-272).

2. Minimize the risk of disease recurrence and metastatic spread. Adequate surgery is the most important treatment variable influencing prognosis, while RAI treatment, TSH suppression, and other treatments each play adjunctive roles in at least some patients (273-275).

3. Facilitate postoperative treatment with RAI, where appropriate. For patients undergoing RAI remnant ablation, or RAI treatment of presumed (adjuvant therapy) or known (therapy) residual or metastatic disease, removal of all normal thyroid tissue is an important element of initial surgery (276).

4. Permit accurate staging and risk stratification of the disease. Because disease staging and risk stratification should be used to guide initial prognostication, disease management, and follow-up strategies, accurate postoperative risk assessment is a crucial element in the management of patients with DTC $(277,278)$.

5. Permit accurate long-term surveillance for disease recurrence.

6. Minimize treatment-related morbidity. The extent of surgery and the experience of the surgeon both play important roles in determining the risk of surgical complications $(232,233,279,280)$.

\section{[B3] What is the role of preoperative staging with diagnostic imaging and laboratory tests?}

[B4] Neck imaging-ultrasound

\section{- RECOMMENDATION 32}

(A) Preoperative neck US for cervical (central and especially lateral neck compartments) lymph nodes is recommended for all patients undergoing thyroidectomy for malignant or suspicious for malignancy cytologic or molecular findings.

\section{(Strong recommendation, Moderate-quality evidence)}

(B) US-guided FNA of sonographically suspicious lymph nodes $\geq 8-10 \mathrm{~mm}$ in the smallest diameter should be performed to confirm malignancy if this would change management.

\section{(Strong recommendation, Moderate-quality evidence)}

(C) The addition of FNA-Tg washout in the evaluation of suspicious cervical lymph nodes is appropriate in select patients, but interpretation may be difficult in patients with an intact thyroid gland.

\section{(Weak recommendation, Low-quality evidence)}

Differentiated thyroid carcinoma (particularly papillary carcinoma) involves cervical lymph node metastases in $20 \%-50 \%$ of patients in most series using standard pathologic techniques $(84,145,281-283)$, and it may be present even when the primary tumor is small and intrathyroidal (284). The frequency of micrometastases $(<2 \mathrm{~mm})$ may approach $90 \%$, depending on the sensitivity of the detection method $(285,286)$. However, the clinical implications of micrometastases are likely less significant compared to macrometastases. Preoperative US identifies suspicious cervical adenopathy in $20 \%-31 \%$ of cases, potentially altering the surgical approach $(287,288)$ in as many as $20 \%$ of patients (289-291). However, preoperative US identifies only half of the lymph nodes found at surgery, due to the presence of the overlying thyroid gland (292).

Sonographic features suggestive of abnormal metastatic lymph nodes include enlargement, loss of the fatty hilum, a rounded rather than oval shape, hyperechogenicity, cystic change, calcifications, and peripheral vascularity (Table 7). No single sonographic feature is adequately sensitive for detection of lymph nodes with metastatic thyroid cancer. One study correlated the sonographic features acquired 4 days preoperatively directly with the histology of 56 cervical lymph nodes identified in 19 patients. Some of the most specific criteria were short axis $>5 \mathrm{~mm}(96 \%)$, presence of cystic areas (100\%), presence of hyperechogenic punctuations representing either colloid or microcalcifications $(100 \%)$, and peripheral vascularity $(82 \%)$. Of these, the only one with sufficient sensitivity was peripheral vascularity $(86 \%)$. The others had sensitivities of $<60 \%$ and would not be adequate to use as a single criterion for identification of malignant involvement (292). As shown by earlier studies $(293,294)$, the ultrasonographic feature with the highest sensitivity is absence of a hilum (100\%), but this has a low specificity of $29 \%$. Microcalcifications have the highest specificity; any lymph nodes with microcalcifications should be considered abnormal (292) (Table 7).

The location of the lymph nodes may also be useful for decision-making. Malignant lymph nodes are much more likely to occur in levels III, IV, and VI than in level II (Fig. 3) $(292,294)$, although this may not be true for PTC tumors arising in the upper pole of the thyroid, which have a higher propensity to demonstrate skip metastases to levels III and II (295). Figure 3 illustrates the delineation of cervical lymph node levels I through VI.

Confirmation of malignancy in lymph nodes with a suspicious sonographic appearance is achieved by US-guided FNA aspiration for cytology and/or measurement of Tg in the needle washout. A Tg concentration $<1 \mathrm{ng} / \mathrm{mL}$ is reassuring, and the probability of $\mathrm{N} 1$ disease increases with higher $\mathrm{Tg}$ levels (296). This FNA measurement of Tg is likely valid even in patients with circulating anti-Tg autoantibodies (297,298), although one study challenges the validity of this measurement in patients with anti-Tg autoantibodies (299). Tg washout may be helpful, particularly in cases in which the lymph nodes are cystic, cytologic evaluation of the lymph node is inadequate, or the cytologic and sonographic evaluations are divergent (i.e., normal cytologic biopsy of 


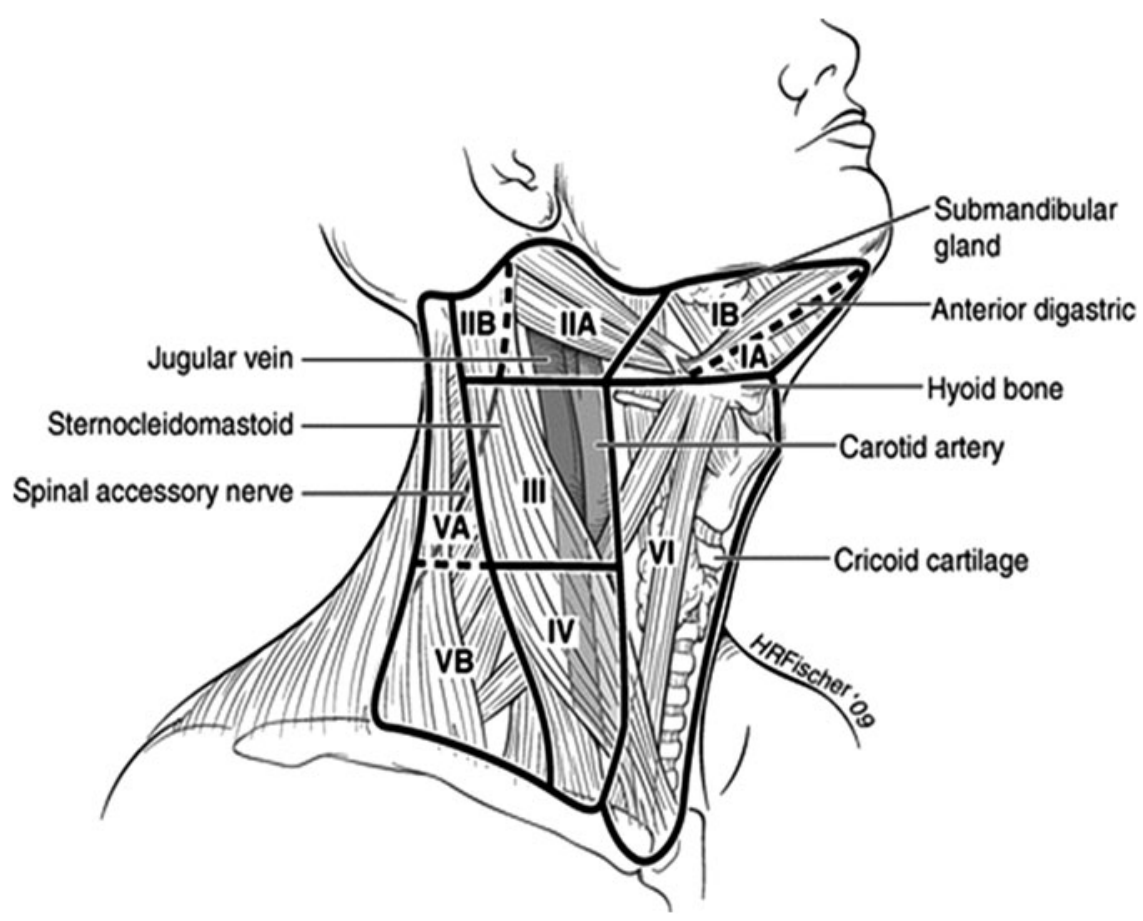

FIG. 3. Lymph node compartments separated into levels and sublevels. Level VI contains the thyroid gland, and the adjacent nodes bordered superiorly by the hyoid bone, inferiorly by the innominate (brachiocephalic) artery, and laterally on each side by the carotid sheaths. The level II, III, and IV nodes are arrayed along the jugular veins on each side, bordered anteromedially by level VI and laterally by the posterior border of the sternocleidomastoid muscle. The level III nodes are bounded superiorly by the level of the hyoid bone and inferiorly by the cricoid cartilage; levels II and IV are above and below level III, respectively. The level I node compartment includes the submental and submandibular nodes, above the hyoid bone, and anterior to the posterior edge of the submandibular gland. Finally, the level V nodes are in the posterior triangle, lateral to the lateral edge of the sternocleidomastoid muscle. Levels I, II, and V can be further subdivided as noted in the figure. The inferior extent of level VI is defined as the suprasternal notch. Many authors also include the pretracheal and paratracheal superior mediastinal lymph nodes above the level of the innominate artery (sometimes referred to as level VII) in central neck dissection (341).

a large lymph node with microcalcifications) (300). In a retrospective study of 241 lymph nodes in 220 patients who underwent US-guided FNA with $\mathrm{Tg}$ in FNA (FNA-Tg) washout fluid measurements for suspicious lymph nodes, additional FNA-Tg helped to diagnose a metastatic lymph node with one or two suspicious US features but did not offer incremental benefit for those lymph nodes with highly suspicious US features in which FNA alone was sufficient for diagnosis. Two recent systematic reviews showed that falsepositive Tg washout may occur, particularly in lymph nodes in the central compartment when the thyroid gland is still present $(301,302)$. The review by Pak et al. (302) suggests that an FNA-Tg cutoff of $32 \mathrm{ng} / \mathrm{mL}$ has the best sensitivity and specificity in patients with an intact thyroid gland. Others have suggested interpreting the FNA-Tg in context of the serum Tg and TSH in these patients $(303,304)$. There is no standardization of FNA-Tg procedures or assays to date, which makes this additional diagnostic tool sometimes difficult to interpret (305). Future standardization including matrix type (phosphate-buffered saline, $\mathrm{Tg}$-free serum, etc.) and volume of diluent matrix would help with interpretation of a $\mathrm{Tg}$ washout.

Accurate staging is important in determining the prognosis and tailoring treatment for patients with DTC. However, unlike many tumor types, the presence of metastatic disease does not obviate the need for surgical excision of the primary tumor in DTC (306). Because metastatic disease may respond to RAI therapy, removal of the thyroid as well as the primary tumor and accessible loco-regional disease remains an important component of initial treatment even in most patients with metastatic disease.

\section{[B5] Neck imaging-CT/MRI/PET}

\section{- RECOMMENDATION 33}

(A) Preoperative use of cross-sectional imaging studies (CT, MRI) with intravenous (IV) contrast is recommended as an adjunct to US for patients with clinical suspicion for advanced disease, including invasive primary tumor, or clinically apparent multiple or bulky lymph node involvement.

\section{(Strong recommendation, Low-quality evidence)}

(B) Routine preoperative ${ }^{18}$ FDG-PET scanning is not recommended.

\section{(Strong recommendation, Low-quality evidence)}

Since US evaluation is operator dependent and cannot always adequately image deep anatomic structures and those acoustically shadowed by bone or air, alternative imaging procedures may be preferable or useful as an adjunct in 
some clinical settings. Patients displaying bulky or widely distributed nodal disease on initial US examination may present with involvement of nodal regions beyond typical cervical regions, some of which maybe difficult to visualize on routine preoperative US, including the mediastinum, infraclavicular, retropharyngeal, and parapharyngeal regions. In a study of 37 consecutive patients who had preoperative CT and US and subsequently underwent total thyroidectomy and neck dissection, the sensitivity of CT was better than US for the evaluation central and lateral compartment lymph nodes examined together $(77 \%$ vs $62 \%, p=0.002)$, but there were no differences between the two imaging modalities when the central and lateral compartments were examined separately (307). In a series of 299 consecutively registered patients with pathologically proven PTC who underwent preoperative $\mathrm{CT}$ and US, US was more accurate than $\mathrm{CT}$ in predicting extrathyroidal tumor extension and multifocal bilobar disease $(p<0.05)$. The accuracy of staging was better overall with US $(p<0.01)$, and US had greater sensitivity than CT at predicting lateral compartment metastases $(p=0.041)(308)$. However, another study showed that combined preoperative mapping with US and CT was superior to US alone in the preoperative detection of nodal disease, especially in the central neck (309). The sensitivities of MRI and PET for the detection of cervical lymph node metastases are relatively low (30\%-40\%) (310). PET can also detect inflammatory lymph nodes, which reduces the specificity of this test in many patients with DTC. MRI can be used in the detection of cervical nodal metastasis. MRI is affected by respiratory artifacts and may be more difficult to interpret than CT scanning by surgeons in the operating room for low-volume nodal disease (311).

Invasive DTC has been reported to occur in $10 \%-15 \%$ of patients at the time of diagnosis (312). For this group of patients, cross-sectional imaging can also be a useful supplement for preoperative planning to accurately delineate the extent of laryngeal, tracheal, esophageal, or vascular involvement $(309,313)$. Endoscopy of the trachea and or esophagus, with or without ultrasonography, at the beginning of the initial operation looking for evidence of intraluminal extension can also be helpful in cases of suspected areodigestive tract invasion.

Locally invasive primary tumors may be associated with characteristic signs and symptoms including progressive dysphagia, respiratory compromise, hemoptysis, rapid tumor enlargement, significant voice change or the finding of vocal cord paralysis, and mass fixation to the airway or neck structures. Certain sonographic features of the primary tumor, including extrathyroidal extension especially with posterior capsular extension and extension into the mediastinum, may also prompt axial imaging (307). Chest CT is useful in defining the inferior border of disease and in determining the extent to which mediastinal structures are involved in cases with significant caudal spread. CT findings may influence management by indicating the need for sternotomy and/or tracheal or laryngeal resection/reconstruction, which would likely require assembling additional resources and personnel in preparation for surgery. Neck CT with contrast can therefore be useful in delineating the extent of laryngeal, tracheal, and/or esophageal involvement in tumors displaying aggressive local invasion, as well as delineating bulky nodal disease, which may harbor significant extranodal extension that involves muscle and/or blood vessels. Preoperative knowledge of these features of the primary tumor or metastases could significantly influence the surgical plan (314). ${ }^{18}$ FDG-PET scanning may be sensitive in some patients for neck or mediastinal involvement and may reveal distant metastases as well.

When cross-sectional imaging is performed, use of IV contrast is an important adjunct because it helps to delineate the anatomic relationship between the primary tumor or metastatic disease and these other structures. Iodine is generally cleared within 4-8 weeks in most patients, so concern about iodine burden from IV contrast causing a clinically significant delay in subsequent whole-body scans (WBSs) or RAI treatment after the imaging followed by surgery is generally unfounded (315). The benefit gained from improved anatomic imaging generally outweighs any potential risk of a several week delay in RAI imaging or therapy. When there is concern, a urinary iodine to creatinine ratio can be measured.

\section{[B6] Measurement of serum $\mathrm{Tg}$ and anti-Tg antibodies}

\section{- RECOMMENDATION 34}

Routine preoperative measurement of serum $\mathrm{Tg}$ or anti-Tg antibodies is not recommended.

\section{(Weak recommendation, Low-quality evidence)}

Data from a systematic review and meta-analysis suggested that high preoperative concentrations of serum $\mathrm{Tg}$ may predict a higher sensitivity for postoperative surveillance with serum $\mathrm{Tg}$ (316). Preoperative anti-Tg antibodies do not appear to be an independent preoperative predictor of stage in patients with DTC, but the evidence is limited. In a cross-sectional analysis of 1770 patients with perioperative anti-Tg antibodies status data in the National Thyroid Cancer Treatment Cooperative Study (a large thyroid cancer registry that included 11 North American centers and enrolled patients between 1987 and 2011), serum anti-Tg antibody status was not significantly associated with stage of disease on multivariate analysis, or with disease-free or overall survival on univariate or multivariate analyses (317). Evidence that preoperative measurement of serum $\mathrm{Tg}$ impacts patient management or outcomes is not yet available.

[B7] Operative approach for a biopsy diagnostic for follicular cell-derived malignancy

\section{- RECOMMENDATION 35}

(A) For patients with thyroid cancer $>4 \mathrm{~cm}$, or with gross extrathyroidal extension (clinical T4), or clinically apparent metastatic disease to nodes (clinical N1) or distant sites (clinical M1), the initial surgical procedure should include a near-total or total thyroidectomy and gross removal of all primary tumor unless there are contraindications to this procedure.

\section{(Strong recommendation, Moderate-quality evidence)}

(B) For patients with thyroid cancer $>1 \mathrm{~cm}$ and $<4 \mathrm{~cm}$ without extrathyroidal extension, and without clinical evidence of any lymph node metastases (cNO), the initial surgical procedure can be either a bilateral procedure (neartotal or total thyroidectomy) or a unilateral procedure 
(lobectomy). Thyroid lobectomy alone may be sufficient initial treatment for low-risk papillary and follicular carcinomas; however, the treatment team may choose total thyroidectomy to enable RAI therapy or to enhance followup based upon disease features and/or patient preferences.

\section{(Strong recommendation, Moderate-quality evidence)}

(C) If surgery is chosen for patients with thyroid cancer $<1 \mathrm{~cm}$ without extrathyroidal extension and $\mathrm{cN} 0$, the initial surgical procedure should be a thyroid lobectomy unless there are clear indications to remove the contralateral lobe. Thyroid lobectomy alone is sufficient treatment for small, unifocal, intrathyroidal carcinomas in the absence of prior head and neck radiation, familial thyroid carcinoma, or clinically detectable cervical nodal metastases.

\section{(Strong recommendation, Moderate-quality evidence)}

Surgery for thyroid cancer is an important element of a multifaceted treatment approach. The operation must be compatible with the overall treatment strategy and follow-up plan recommended by the managing team. Consideration should be given to referring patients with high-risk features (clinical N1 disease, concern for recurrent laryngeal nerve [RLN] involvement, or grossly invasive disease) to experienced surgeons, as both completeness of surgery and experience of the surgeon can have a significant impact on clinical outcomes and complication rates $(232,233,279,280)$. Previous guidelines have endorsed total thyroidectomy as the primary initial surgical treatment option for nearly all DTCs $>1 \mathrm{~cm}$ with or without evidence of loco-regional or distant metastases (25). This was based on retrospective data suggesting that a bilateral surgical procedure would improve survival (318), decrease recurrence rates (319-321), allow for routine use of RAI remnant ablation, and facilitate detection of recurrent/persistent disease during follow-up. However, recent data have demonstrated that in properly selected patients, clinical outcomes are very similar following unilateral or bilateral thyroid surgery (322-326). Furthermore, since the requirement for routine use of RAI ablation was one of the major reasons given in support of total thyroidectomy in low to intermediate risk patients, our current more selective approach to RAI ablation in these patients requires a critical reassessment of this indication. In some patients, the presence of the remaining lobe of the gland may obviate the lifelong need for exogenous thyroid hormone therapy. Finally, as our follow-up management paradigm has moved away from diagnostic whole body RAI scanning and toward a greater reliance on neck ultrasonography and serial serum $\mathrm{Tg}$ measurements (even in patients that did not receive RAI remnant ablation), we must also question whether total thyroidectomy and RAI remnant ablation is required to facilitate follow-up in low to intermediate risk patients.

In an analysis of 52,173 PTC patients diagnosed between 1985 and 1998 from the National Cancer Data Base (43,227 receiving total thyroidectomy, 8946 undergoing lobectomy), Bilimoria et al. (318) demonstrated a slightly higher 10-year relative overall survival for total thyroidectomy as opposed to thyroid lobectomy $(98.4 \%$ vs. $97.1 \%$, respectively, $p<0.05$ ) and a slightly lower 10 -year recurrence rate $(7.7 \%$ vs. $9.8 \%$, respectively, $p<0.05)$. When analyzed by size of the primary tumor, statistically significant differences in survival and recurrence were seen for all sizes $>1 \mathrm{~cm}$ based on the extent of initial surgery. However, data on extrathyroidal extension, completeness of resection, and other comorbid conditions, which could have had a major impact on survival and recurrence risk, were not available. Therefore, it is unclear how often lobectomy was done based on proper selection of low to intermediate risk patients versus how often lobectomy was done in high-risk patients because of comorbid conditions, inability to obtain a complete resection, or status of the contralateral RLN. This is an important distinction because thyroid lobectomy patients were found to have extrathyroidal extension in $7 \%$ of cases (325), underwent external beam radiation therapy (EBRT) in 1\%-2\% (324), and RAI therapy in $12 \%-18 \%(318,325)$, and high-risk features were present in $8 \%$ (325). Given the small magnitude of differences reported for survival and recurrence between the total thyroidectomy and the lobectomy patients, it is quite possible that the slightly poorer outcomes seen in the lobectomy group could have been influenced by lobectomy patients with concurrent high risk features. Adam et al. (327) performed an updated analysis of 61,775 patients in the National Cancer Database who underwent thyroid surgery between 1998 and 2006. The researchers demonstrated that the overall survival advantage seen for patients with 1-4 cm PTC who underwent thyroidectomy in the study by Bilimoria et al. (318) disappeared when further adjustment was made for additional variables related to complexity and severity of illness. This lack of overall survival advantage was also seen when the group was subdivided into patients with $1-2 \mathrm{~cm}$ and $2-4 \mathrm{~cm}$ PTC.

Previously, Haigh et al. (325) had analyzed 5432 PTC patients from the SEER database (4612 receiving total thyroidectomy and 820 undergoing lobectomy) and found no difference in 10-year overall survival between total thyroidectomy and thyroid lobectomy when risk stratified by the AMES classification system. Interestingly, patients selected for thyroid lobectomy included $7 \%$ with extrathyroidal extension, $1 \%$ with distant metastases, and $5 \%$ with primary tumors $>5 \mathrm{~cm}$, and $8 \%$ were classified as having high risk based on AMES.

More recently, two additional studies have analyzed the SEER database, and both have failed to demonstrate a significant difference in survival when comparing total thyroidectomy with thyroid lobectomy $(323,324)$. Barney et al. (323) included 23,605 DTC patients diagnosed between 1983 and 2002 (12,598 with total thyroidectomy, 3266 with lobectomy) and found no difference in 10-year overall survival (90.4\% for total thyroidectomy vs. $90.8 \%$ for lobectomy) or 10 -year cause-specific survival $(96.8 \%$ for total thyroidectomy vs. $98.6 \%$ for lobectomy). Furthermore, in a multivariate analysis that included age, T, N, M, sex, year of diagnosis, extent of surgery, and RAI use, no difference in overall survival or cause specific survival was seen with respect to the extent of initial surgery. Mendelsohn et al. (324) analyzed 22,724 PTC patients diagnosed between 1998 and 2001 (16,760 with total thyroidectomy, 5964 with lobectomy) and found no differences in overall survival or diseasespecific survival in a comparison of total thyroidectomy with lobectomy. Interestingly, of the patients that had lobectomy, $1.6 \%$ received external beam radiation therapy, $16 \%$ had extrathryoidal extension, $9 \%$ of tumors were $>4 \mathrm{~cm}$, and $20 \%$ received RAI ablation (once again indicating that lobectomy was done in some high-risk patients). 
Consistent with the SEER data analyses, two single-center studies also confirmed that lobectomy is associated with excellent survival in properly selected patients $(322,326)$. After a median follow-up of 8 years, only one diseasespecific death was seen in a cohort of 889 PTC patients with T1-T2 tumors treated with either total thyroidectomy $(n=528)$ or lobectomy $(n=361)$ (326). Furthermore, Matsuzu et al. (322) reported a cause-specific survival rate of $98 \%$ after a median of 17 years of follow-up in properly select PTC patients treated with lobectomy and ipsilateral neck dissection.

Given the propensity for PTC to be multifocal (often involving both lobes), it is not surprising that some studies have demonstrated a lower risk of loco-regional disease recurrence following total thyroidectomy as compared to thyroid lobectomy (319-321). However, with proper patient selection, loco-regional recurrence rates of less than $1 \%-4 \%$ and completion thyroidectomy rates of $<10 \%$ can be achieved following thyroid lobectomy $(326,328)$. Furthermore, the few recurrences that develop during long-term follow-up are readily detected and appropriately treated with no impact on survival $(322,326,328)$.

Therefore, we conclude that in properly selected low- to intermediate-risk patients (patients with unifocal tumors $<4 \mathrm{~cm}$, and no evidence of extrathyroidal extension or lymph node metastases by examination or imaging), the extent of initial thyroid surgery probably has little impact on disease-specific survival. While recurrence rates can be quite low in these patients, it is likely that the lowest rates of recurrence during long-term follow-up would be associated with a total thyroidectomy. But since salvage therapy is quite effective in the few patients that recur after thyroid lobectomy, a conservative management approach to completion surgery, accepting a slightly higher risk of locoregional recurrence, is a reasonable management strategy. Finally, a more selective use of RAI coupled with a greater reliance on neck US and serial serum Tg measurements for detection of recurrent disease is likely to significantly decrease the mandate for total thyroidectomies in low- and intermediate-risk patients done solely to facilitate RAI remnant ablation and follow-up.

Near-total or total thyroidectomy is necessary if the overall strategy is to include RAI therapy postoperatively, and thus is recommended if the primary thyroid carcinoma is $>4 \mathrm{~cm}$, if there is gross extrathyroidal extension, or if regional or distant metastases are clinically present. For tumors that are between 1 and $4 \mathrm{~cm}$ in size, either a bilateral thyroidectomy (total or near-total) or a unilateral procedure (thyroid lobectomy) may be suitable as treatment plan. Older age ( $>45$ years), contralateral thyroid nodules, a personal history of radiation therapy to the head and neck, and familial DTC may be criteria for recommending a bilateral procedure because of plans for RAI therapy or to facilitate follow-up strategies or address suspicions of bilateral disease $(270,278,322,326)$.

The relationship between surgeon volume and patient outcomes has been studied extensively over the last 20 years. Institutional studies examining outcomes following thyroidectomy by high-volume surgeons have been published demonstrating overall safety. In one of the first studies examining the relationship between surgeon volume and thyroidectomy outcomes at a state level, Sosa et al. (232) found a strong association between higher surgeon volume and favorable patient outcomes, especially with regard to RLN injury and wound complications. This was especially pronounced for patients undergoing total thyroidectomy for thyroid cancer. Others have made similar observations $(233,329,330)$. In a recent study of patients undergoing thyroidectomy in the Health Care Utilization Project Nationwide Inpatient Sample (HCUP-NIS), surgeons were divided into categories of low $(<10$ cases/year; encompassing 6072 surgeons), intermediate (10100 cases/year; 11,544 surgeons), and high volume ( $>100$ cases/ year; 4009 surgeons) (331). Over $80 \%$ of thyroid resections were performed by low- and intermediate-volume surgeons. On average, high-volume surgeons had the lowest complication rates for patients who underwent total thyroidectomy for cancer at $7.5 \%$; intermediate-volume surgeons had a rate of $13.4 \%$, and low-volume surgeons, $18.9 \%(p<0.001)$.

From robust population-level data such as these, it can be concluded that referral of patients to high-volume thyroid surgeons is associated, on average, with superior outcomes. However, such referral is not always possible, given the relative scarcity of high-volume surgeons and their geographic distribution. In addition, there are some data suggesting that other factors, such as surgeon age, should be considered (332). Therefore, conclusions at a population level cannot always be applied to individual surgeons and patient circumstances. It may, however, be reasonable to consider sending patients with more extensive disease and concern for grossly invasive disease to a high-volume surgeon experienced in the management of advanced thyroid cancer.

It is worth noting that even high-volume surgeons have a higher overall postoperative complication rate when performing total thyroidectomy compared with lobectomy (333). Using the HCUP-NIS, these authors found that highvolume thyroid surgeons had a complication rate of $7.6 \%$ following thyroid lobectomy but a rate of $14.5 \%$ following total thyroidectomy. For low-volume surgeons, the complication rates were $11.8 \%$ and $24.1 \%$, respectively. Therefore, patients should carefully weigh the relative benefits and risks of total thyroidectomy versus thyroid lobectomy, even when surgery is performed by high-volume surgeons.

\section{[B8] Lymph node dissection}

\section{- RECOMMENDATION 36}

(A) Therapeutic central-compartment (level VI) neck dissection for patients with clinically involved central nodes should accompany total thyroidectomy to provide clearance of disease from the central neck.

\section{(Strong recommendation, Moderate-quality evidence)}

(B) Prophylactic central-compartment neck dissection (ipsilateral or bilateral) should be considered in patients with papillary thyroid carcinoma with clinically uninvolved central neck lymph nodes (cN0) who have advanced primary tumors (T3 or T4) or clinically involved lateral neck nodes (cN1b), or if the information will be used to plan further steps in therapy.

\section{(Weak recommendation, Low-quality evidence)}

(C) Thyroidectomy without prophylactic central neck dissection is appropriate for small (T1 or T2), noninvasive, 
clinically node-negative PTC ( $\mathrm{cN} 0)$ and for most follicular cancers.

(Strong recommendation, Moderate-quality evidence)

\section{- RECOMMENDATION 37}

Therapeutic lateral neck compartmental lymph node dissection should be performed for patients with biopsy-proven metastatic lateral cervical lymphadenopathy.

\section{(Strong recommendation, Moderate-quality evidence)}

Regional lymph node metastases are present at the time of diagnosis in a majority of patients with papillary carcinomas and a lesser proportion of patients with follicular carcinomas $(290,334,335)$. Although PTC lymph node metastases are reported by some to have no clinically important effect on outcome in low risk patients, a study of the SEER database found, among 9904 patients with PTC, that lymph node metastases, age $>45$ years, distant metastasis, and large tumor size significantly predicted poor overall survival outcome in a multivariate analysis (336). All-cause survival at 14 years was $82 \%$ for PTC without lymph node metastases and $79 \%$ with nodal metastases $(p<0.05)$. Another SEER registry study concluded that cervical lymph node metastases conferred an independent risk of decreased survival, but only in patients with follicular cancer and patients with papillary cancer over age 45 years (337). However, characteristics of the lymph node metastases can further discriminate the risk of recurrence to the patient, especially in patients with clinically evident metastasis, multiple metastases, larger metastases, and/or extracapsular nodal extension $(338,339)$, compared with those with more limited microscopic nodal disease (335). A recent comprehensive analysis of the National Cancer Data Base and SEER, however, showed a small but significantly increased risk of death for patients younger than 45 years with lymph node metastases compared with younger patients without involved lymph nodes, and that having incrementally more metastatic lymph nodes up to six involved nodes confers additional mortality risk in this age group (340). This study underlines the importance of rigorous preoperative screening for nodal metastases and potentially raises questions about current thyroid cancer staging systems. Common to all of these studies is the conclusion that the effect of the presence or absence of lymph node metastases on overall survival, if present, is small.

The cervical node sites are well-defined (341), and the most common site of nodal metastases is in the central neck, which is cervical level VI (Fig. 3). A recent consensus conference statement describes the relevant anatomy of the central neck compartment, delineates the nodal subgroups within the central compartment commonly involved with thyroid cancer, and defines the terminology relevant to central compartment neck dissection (342). In many patients, lymph node metastases in this area do not appear abnormal on preoperative imaging $(289,334,343-345)$ or by inspection at the time of surgery (335), defining a $\mathrm{cN} 0$ group.

The role of therapeutic lymph node dissection for treatment of thyroid cancer nodal metastases is well accepted for $\mathrm{cN} 1$ disease $(336,346-348)$. However, the value of routine prophylactic level VI (central) neck dissection for cNO disease remains unclear. Central compartment dissection (therapeutic or prophylactic) can be achieved with low morbidity by experienced thyroid surgeons (349-351). The value for an individual patient depends upon the utility of the staging information to the treatment team in specific patient circumstances $(351,352)$. Based on limited and imperfect data, prophylactic dissection has been suggested to improve disease-specific survival (353), local recurrence $(345,354)$, and post-treatment $\mathrm{Tg}$ levels $(345,355)$. It has also been used to inform the use of adjuvant RAI $(344,347,350,356)$ and improve the accuracy of the estimates of risk of recurrence (356-358). However, in several studies, prophylactic dissection has shown no improvement in long-term patient outcome, while increasing the likelihood of temporary morbidity, including hypocalcemia, although prophylactic dissection may decrease the need for repeated RAI treatments $(334,346,347,349,359-364)$.

The removal of cNO level VI lymph nodes detects a substantial number of patients with pN1 disease; however, the direct effect of this on long-term outcome is small at best $(365,366)$. The use of staging information for the planning of adjuvant therapy depends upon whether this information will affect the team-based decision-making for the individual patient. For these reasons, groups may elect to include prophylactic dissection for patients with some prognostic features associated with an increased risk of metastasis and recurrence (older or very young age, larger tumor size, multifocal disease, extrathyroidal extension, known lateral node metastases) to contribute to decision-making and disease control $(345,351,355)$. Alternatively, some groups may apply prophylactic level VI dissection to patients with better prognostic features if the patient is to have a bilateral thyroidectomy, and if the nodal staging information will be used to inform the decision regarding use of adjuvant therapy $(344,350,356)$. Finally, for some groups it appears reasonable to use a selective approach that applies level VI lymph node dissection at the time of initial operation only to patients with clinically evident disease based on preoperative physical exam, preoperative radiographic evaluation, or intraoperative demonstration of detectable disease (cN1) $(335,359,367)$.

The information from prophylactic central neck dissection must be used cautiously for staging information. Since microscopic nodal positivity occurs frequently, prophylactic dissection often converts patients from clinical N0 to pathologic N1a, upstaging many patients over age 45 from American Joint Committee on Cancer (AJCC) stage I to stage III $(334,344-347)$. However, microscopic nodal positivity does not carry the recurrence risk of macroscopic clinically detectable disease (335). Thus microscopic nodal upstaging may lead to excess RAI utilization and patient follow-up. Alternatively, the demonstration of uninvolved lymph nodes by prophylactic dissection may decrease the use of RAI for some groups $(344,350,356)$. These effects may account for some of the existing extreme variability in utilization of RAI for thyroid cancer (368).

Studies of the $B R A F^{V 600 E}$ mutation have suggested an association between presence of the mutation and the risk of nodal disease (369-371), although results across all patients with papillary thyroid carcinoma are mixed (372-375). However, the presence of a $B R A F^{V 600 E}$ mutation has a limited PPV for recurrence and therefore, $B R A F^{V 600 E}$ mutation status in the primary tumor should not impact the decision for prophylactic central neck dissection (376). 
The preceding recommendations should be interpreted in light of available surgical expertise. For patients with small, noninvasive, cN0 tumors, the balance of risk and benefit may favor thyroid lobectomy and close intraoperative inspection of the central compartment, with the plan adjusted to total thyroidectomy with compartmental dissection only in the presence of involved lymph nodes.

Lymph nodes in the lateral neck (compartments II-V, Fig. 3), level VII (anterior mediastinum), and rarely in level I may also be involved by thyroid cancer $(282,335,377,378)$. For patients in whom nodal disease is evident clinically on preoperative US and nodal FNA cytology or Tg washout measurement or at the time of surgery, surgical resection by compartmental node dissection may reduce the risk of recurrence and possibly mortality (379-381).

\section{[B9] Completion thyroidectomy}

\section{- RECOMMENDATION 38}

(A) Completion thyroidectomy should be offered to patients for whom a bilateral thyroidectomy would have been recommended had the diagnosis been available before the initial surgery. Therapeutic central neck lymph node dissection should be included if the lymph nodes are clinically involved. Thyroid lobectomy alone may be sufficient treatment for low-risk papillary and follicular carcinomas.

\section{(Strong recommendation, Moderate-quality evidence)}

(B) RAI ablation in lieu of completion thyroidectomy is not recommended routinely; however, it may be used to ablate the remnant lobe in selected cases.

\section{(Weak recommendation, Low-quality evidence)}

Completion thyroidectomy may be necessary when the diagnosis of malignancy is made following lobectomy for an indeterminate or nondiagnostic biopsy. In addition, some patients with malignancy may require completion thyroidectomy to provide complete resection of multicentric disease and to allow for efficient RAI therapy. However, since intrathyroidal PTC or low-risk FTC can be managed with either lobectomy or total thyroidectomy (see Recommendation 35B), a completion thyroidectomy is not always required. The surgical risks of two-stage thyroidectomy (lobectomy followed by completion thyroidectomy) are similar to those of a near-total or total thyroidectomy (382-384). The marginal utility of prophylactic lymph node dissection for $\mathrm{cN} 0$ disease argues against its application in re-operations.

Ablation of the remaining lobe with RAI has been used as an alternative to completion thyroidectomy $(385,386)$. There are limited data regarding the long-term outcomes of this approach. The data suggest similar clinical outcomes with a slightly higher proportion of patients with persistent detectable $\mathrm{Tg}$. This approach may be helpful in patients for whom completion thyroidectomy carries some increased risk and for whom a delay in the length of time required to achieve destruction of the normal thyroid, which follows RAI (as opposed to surgical resection), is acceptable. In one unblinded, multicenter, randomized controlled equivalence trial comparing dose activities in achieving successful ablation of a remaining lobe in patients with $\mathrm{T} 1 \mathrm{~b}$ or $\mathrm{T} 2$ primary tumors, who had surgical contraindications or declined completion thyroidectomy, the remnant ablation success rate was significantly higher using $100 \mathrm{mCi}(75 \%$ success rate; $1 \mathrm{mCi}=37 \mathrm{MBq})$, compared with $30 \mathrm{mCi}(54 \%)$, although mild to moderate short-term neck pain was more frequently reported in the high-dose group (66\%) compared with the low-dose group (51\%) (387). Prednisone treatment for neck pain was used more frequently in the high-dose group (36\% of patients) than in the low-dose group.

\section{[B10] What is the appropriate perioperative approach to voice and parathyroid issues?}

\section{[B11] Preoperative care communication}

\section{- RECOMMENDATION 39}

Prior to surgery, the surgeon should communicate with the patient regarding surgical risks, including nerve and parathyroid injury, through the informed consent process and communicate with associated physicians, including anesthesia personnel, regarding important findings elicited during the preoperative workup.

\section{(Strong recommendation, Moderate-quality evidence)}

The preoperative consent process should include explicit discussion of the potential for temporary or permanent nerve injury (and its clinical sequelae, including voice change, swallowing disability, risk of aspiration, and tracheostomy) as well as hypoparathyroidism, bleeding, scarring, disease recurrence, need for additional postoperative treatment, and need for thyroid hormone and surveillance thyroid function tests. The conversation should be informed by the operating surgeon's own rates of complications. Results of the preoperative evaluation regarding extent of disease, risk stratification, and integrity of the airway should include results from imaging, cytology, and physical examination (388-392).

\section{[B12] Preoperative voice assessment}

\section{- RECOMMENDATION 40}

All patients undergoing thyroid surgery should have preoperative voice assessment as part of their preoperative physical examination. This should include the patient's description of vocal changes, as well as the physician's assessment of voice.

(Strong recommendation, Moderate-quality evidence)

\section{- RECOMMENDATION 41}

Preoperative laryngeal exam should be performed in all patients with

(A) Preoperative voice abnormalities

(Strong recommendation, Moderate-quality evidence)

(B) History of cervical or upper chest surgery, which places the RLN or vagus nerve at risk

(Strong recommendation, Moderate-quality evidence)

(C) Known thyroid cancer with posterior extrathyroidal extension or extensive central nodal metastases

(Strong recommendation, Low-quality evidence) 
Voice alteration is an important complication of thyroid surgery affecting patients' quality of life (with regard to voice, swallowing, and airway domains), and it can have medico-legal and cost implications (393-401).

Preoperative assessment provides a necessary baseline reference from which to establish perioperative expectations (402). Also, preoperative voice assessment may lead one to identify preoperative vocal cord paralysis or paresis, which provides presumptive evidence of invasive thyroid malignancy and is important in planning the extent of surgery and in perioperative airway management (403-405). Contralateral nerve injury at surgery in such patients could cause bilateral cord paralysis with airway implications.

Preoperative voice assessment should include the patient's historical subjective response to questions regarding voice abnormalities or changes, as well as the physician's objective assessment of voice, and should be documented in the medical record (Table 9) (406). Voice and laryngeal function may be further assessed through laryngoscopy, and the application of validated quality of life and auditory perceptual assessment voice instruments (402). It is important to appreciate that vocal cord paralysis, especially when chronic, may not be associated with significant vocal symptoms due to a variety of mechanisms, including contralateral vocal cord compensation. Voice assessment alone may not identify such individuals (402).

Incidence rates for preoperative vocal cord paresis or paralysis for patients with benign thyroid disease at preoperative laryngoscopy range from $0 \%$ to $3.5 \%$ and up to $8 \%$ in patients with thyroid cancer (407-411). Finding vocal cord paralysis on preoperative examination strongly suggests the presence of locally invasive disease. Approximately 10\%-15\% of thyroid cancers present with extrathyroidal extension, with the most common structures involved including strap muscle (53\%), the RLN (47\%), trachea (30\%), esophagus (21\%), and larynx (12\%) $(405,412-414)$.

Undiagnosed preoperative laryngeal nerve dysfunction conveys greater risk during total thyroidectomy of postoperative bilateral nerve paralysis, respiratory distress, and need for tracheostomy. Also, preoperative identification of vocal cord paralysis is important because surgical algorithms in the management of the invaded nerve incorporate nerve functional status (415).

A laryngeal exam should be performed if the voice is abnormal during preoperative evaluation. In addition, a patient

Table 9. Preoperative Factors Which May Be Associated with Laryngeal Nerve Dysfunction

\begin{tabular}{lc}
\hline Factor & \multicolumn{1}{c}{ Symptoms/signs } \\
\hline History & $\begin{array}{l}\text { Voice abnormality, dysphagia, } \\
\text { airway symptoms, hemoptysis, } \\
\text { pain, rapid progression, prior } \\
\text { operation in neck or upper chest } \\
\text { Extensive, firm mass fixed to the } \\
\text { larynx or trachea } \\
\text { Mass extending to/beyond periphery } \\
\text { of thyroid lobe posteriorly and/or } \\
\text { tracheoesophageal infiltration, or } \\
\text { bulky cervical adenopathy along } \\
\text { the course of the RLN or vagus } \\
\text { nerve }\end{array}$ \\
\hline
\end{tabular}

should have a laryngeal exam even if the voice is normal if he or she has a history of neck surgery that placed at risk either the RLN (such as past thyroid or parathyroid surgery) or the vagus nerve (such as carotid endarterectomy, cervical esophagectomy, and anterior approach to the cervical spine) or a history of prior external beam radiation to the neck. Correlation between vocal symptoms and actual vocal cord function is poor given the potential for variation in paralytic cord position, degree of partial nerve function, and contralateral cord function/ compensation; therefore, vocal symptoms may be absent in patients with vocal cord paralysis. Vocal cord paralysis may be present in $1.5 \%$ to $30 \%$ of such postsurgical patients; it can be asymptomatic in up to one-third (403,416-422).

A laryngeal exam is recommended in patients with the preoperative diagnosis of thyroid cancer if there is evidence for gross extrathyroidal extension of cancer posteriorly or extensive nodal involvement, even if the voice is normal. The laryngeal exam should be performed in the previously noted high-risk settings, but it can be performed in other patients based on the surgeon's judgment.

\section{[B13] Intraoperative voice and parathyroid management}

\section{- RECOMMENDATION 42}

(A) Visual identification of the RLN during dissection is required in all cases. Steps should also be taken to preserve the external branch of the superior laryngeal nerve (EBSLN) during dissection of the superior pole of the thyroid gland.

\section{(Strong recommendation, Moderate-quality evidence)}

(B) Intraoperative neural stimulation (with or without monitoring) may be considered to facilitate nerve identification and confirm neural function.

\section{(Weak recommendation, Low-quality evidence)}

\section{- RECOMMENDATION 43}

The parathyroid glands and their blood supply should be preserved during thyroid surgery.

\section{(Strong recommendation, Moderate-quality evidence)}

RLN injury rates are lower when the nerve is routinely visualized in comparison with surgeries in which the nerve is simply avoided $(402,416,423)$. If the EBSLN can be visualized and preserved, that is ideal. If the EBSLN cannot be visually identified, steps should be taken to avoid the nerve; this can be done by staying close to the thyroid capsule at the superior pole and by skeletonizing the superior vascular pedicle. Intraoperative nerve monitoring can be used to facilitate this dissection (419). Studies with or without intraoperative nerve monitoring demonstrate similar patient outcomes with regard to nerve injury rates (420), but studies likely have been underpowered to detect statistically significant differences $(413,424)$. A recent systematic metaanalysis of 20 randomized and nonrandomized prospective and retrospective studies suggested no statistically significant benefit of intraoperative neuromonitoring compared to visualization alone during thyroidectomy for the outcomes of overall, transient, or permanent RLN palsy when analyzed per nerve at risk or per patient (425). However, secondary subgroup analyses of high-risk patients (including those 
with thyroid cancer) suggested statistically significant heterogeneity (variability) in treatment effect for overall and transient RLN injury, when analyzed per nerve at risk. Several studies show that intraoperative nerve monitoring is more commonly utilized by higher volume surgeons to facilitate nerve management, and several studies show improved rates of nerve paralysis with the use of neural monitoring in reoperative and complex thyroid surgery $(401,426-430)$. Neural stimulation at the completion of lobectomy can be used as a test to determine the safety of contralateral surgery with avoidance of bilateral vocal cord paralysis, and it has been associated with a reduction of bilateral paralysis when loss of signal occurs on the first side $(428,431-433)$. Given the complexity of monitoring systems, training and observation of existing monitoring standards are important to provide optimal benefit $(424,434)$.

Typically, parathyroid gland preservation is optimized by gland identification via meticulous dissection $(435,436)$. If the parathyroid(s) cannot be located, the surgeon should attempt to dissect on the thyroid capsule and ligate the inferior thyroid artery very close to the thyroid, since the majority of parathyroid glands receive their blood supply from this vessel. There are exceptions to this rule; for example, superior glands in particular may receive blood supply from the superior thyroid artery. If the parathyroid glands are inadvertently or unavoidably removed (e.g., they are intrathyroidal, or require removal during a central lymph node dissection) or devascularized, confirmation of cancer-free parathyroid tissue should be performed, and then the glands can be autotransplanted into the strap or sternocleidomastoid muscles. It is important to inspect the thyroidectomy and/or central lymphadenectomy specimen when removed and before sending it to pathology to look for parathyroid glands that can be rescued.

\section{[B14] Postoperative care}

\section{- RECOMMENDATION 44}

Patients should have their voice assessed in the postoperative period. Formal laryngeal exam should be performed if the voice is abnormal

(Strong recommendation, Moderate-quality evidence)

\section{- RECOMMENDATION 45}

Important intraoperative findings and details of postoperative care should be communicated by the surgeon to the patient and other physicians who are important in the patient's postoperative care.

\section{(Strong recommendation, Low-quality evidence)}

Voice assessment should occur after surgery and should be based on the patient's subjective report and physician's objective assessment of voice in the office (409). Typically this assessment can be performed at 2 weeks to 2 months after surgery. Early detection of vocal cord motion abnormalities after thyroidectomy is important for facilitating prompt intervention (typically through early injection vocal cord medialization), which is associated with better long-term outcome, including a lower rate of formal open thyroplasty repair (437-439). Many options exist for the management of RLN paralysis, including voice therapy, vocal cord injection techniques, and open vocal cord medialization. Rates of vocal cord paralysis after thyroid surgery can only be assessed by laryngeal exam postoperatively.

Communication of intraoperative findings and postoperative care from the surgeon to other members of the patient's thyroid cancer care team is critical to subsequent therapy and monitoring approaches. Important elements of communication include (i) surgical anatomic findings, including RLN and parathyroid status (including nerve monitoring loss of signal information if monitoring is employed); (ii) surgical disease findings, including evidence for extrathyroidal spread, completeness of tumor resection, presence and distribution of nodal disease; and (iii) postoperative status, including voice/laryngeal exam, laboratory data regarding calcium/parathyroid hormone levels and need for calcium and/or vitamin D supplementation, and the surgical pathology report (440). The surgeon should remain engaged in the patient's pursuant care to facilitate appropriate communication and may remain engaged subsequent to endocrinologic consultation depending on regional practice patterns.

\section{[B15] What are the basic principles of histopathologic evaluation of thyroidectomy samples?}

\section{- RECOMMENDATION 46}

(A) In addition to the basic tumor features required for AJCC/UICC thyroid cancer staging including status of resection margins, pathology reports should contain additional information helpful for risk assessment, such as the presence of vascular invasion and the number of invaded vessels, number of lymph nodes examined and involved with tumor, size of the largest metastatic focus to the lymph node, and presence or absence of extranodal extension of the metastatic tumor.

(Strong recommendation, Moderate-quality evidence)

(B) Histopathologic variants of thyroid carcinoma associated with more unfavorable outcomes (e.g., tall cell, columnar cell, and hobnail variants of PTC; widely invasive FTC; poorly differentiated carcinoma) or more favorable outcomes (e.g., encapsulated follicular variant of PTC without invasion, minimally invasive FTC) should be identified during histopathologic examination and reported.

\section{(Strong recommendation, Low-quality evidence)}

(C) Histopathologic variants associated with familial syndromes (cribriform-morular variant of papillary carcinoma often associated with FAP, follicular or papillary carcinoma associated with PTEN-hamartoma tumor syndrome) should be identified during histopathologic examination and reported.

\section{(Weak recommendation, Low-quality evidence)}

Pathologic examination of thyroid samples establishes the diagnosis and provides important information for risk stratification of cancer and postsurgical patient management. Histopathologically, papillary carcinoma is a welldifferentiated malignant tumor of thyroid follicular cells that demonstrates characteristic microscopic nuclear features. Although a papillary growth pattern is frequently seen, it is not required for the diagnosis. Follicular carcinoma is a 
well-differentiated malignant tumor of thyroid follicular cells that shows transcapsular and/or vascular invasion and lacks the diagnostic nuclear features of papillary carcinoma. Oncocytic (Hürthle cell) follicular carcinoma shows the follicular growth pattern but is composed of cells with abundant granular eosinophilic cytoplasm, which has this appearance because of accumulation of innumerable mitochondria. This tumor is currently designated by the World Health Organization as a histopathologic variant of follicular carcinoma (441). However, oncocytic follicular carcinoma tumors have some differences in biological behavior as compared to the conventional type follicular carcinoma, such as the ability to metastasize to lymph nodes and a possibly higher rate of recurrence and tumor-related mortality $(269,442,443)$. Moreover, a growing body of genetic evidence suggests that oncocytic tumors develop via unique molecular mechanisms and therefore represent a distinct type of welldifferentiated thyroid cancer (444).

Traditionally, follicular carcinomas have been subdivided into minimally invasive (encapsulated) and widely invasive. In this classification scheme, minimally invasive carcinomas are fully encapsulated tumors with microscopically identifiable foci of capsular or vascular invasion, whereas widely invasive carcinomas are tumors with extensive vascular and/or extrathyroidal, invasion. More recent approaches consider encapsulated tumors with only microscopic capsular invasion as minimally invasive, whereas angioinvasive tumors are placed into a separate category (445-447). Such an approach is preferable because it distinguishes encapsulated tumors with capsular invasion and no vascular invasion, which are highly indolent tumors with a mortality $<5 \%$, from angioinvasive follicular carcinomas, which have a mortality ranging from $5 \%$ to $30 \%$, depending on the number of invaded blood vessels (448).

In addition to establishing a diagnosis for each nodule in a thyroidectomy or lobectomy specimen, the pathology report must provide characteristics required for AJCC/UICC TNM staging, such as tumor size and presence of extrathyroidal extension and lymph node metastasis. Extrathyroidal extension is defined as tumor extension into the adjacent tissues. It is subdivided into minimal, which is invasion into immediate perithyroidal soft tissues or sternothyroid muscle typically detected only microscopically (T3 tumors), and extensive, which is tumor invasion into subcutaneous soft tissues, larynx, trachea, esophagus, or RLN (T4a tumors). The status of the resection (inked) margins should be reported as "involved" or "uninvolved" with tumor, since positive margins are generally associated with intermediate or high risk for recurrence.

The size of the metastatic focus in a lymph node (335) and tumor extension beyond the capsule of a lymph node $(338,449,450)$ affect cancer risk. Therefore, the pathology report should indicate the size of the largest metastatic focus to the lymph node and the presence or absence of extranodal tumor extension, as well as the number of examined and involved lymph nodes.

Additionally, the presence of vascular (blood vessel) invasion is an unfavorable prognostic factor (451-453) and should be evaluated and reported. Vascular invasion is diagnosed as direct tumor extension into the blood vessel lumen or a tumor aggregate present within the vessel lumen, typically attached to the wall and covered by a layer of endothelial cells. More rigid criteria for vascular invasion proposed by some authors also require the presence of a fibrin thrombus attached to the intravascular tumor cells (453). The invaded blood vessels should not be located within the tumor nodule parenchyma, but rather in the tumor capsule or outside of it. Invasion of multiple (four or more) blood vessels appears to entail poorer outcomes, particularly in follicular carcinomas (454-456). Therefore, the number of invaded blood vessels (less than four or more) should be stated in the pathology report.

More than 10 microscopic variants of papillary carcinoma have been documented (457). Some of them are associated with more aggressive or conversely more indolent tumor behavior and can contribute to risk stratification. The variants with more unfavorable outcomes are the tall cell, columnar cell, and hobnail variants. The tall cell variant is characterized by predominance $(>50 \%)$ of tall columnar tumor cells whose height is at least three times their width. These tumors present at an older age and more advanced stage than classic papillary carcinoma (458-461) and demonstrate a higher recurrence rate and decreased diseasespecific survival $(458-460,462,463)$. Some studies found a higher rate of lymph node metastasis and poorer survival in patients with tall cell variant as compared to classic papillary carcinoma even in tumors without extrathyroidal extension, and this was independent of patient age and tumor size and stage $(464,465)$. The $B R A F^{V 600 E}$ mutation is found in $\sim 80 \%$ of these tumors $(156,466)$.

The columnar cell variant of papillary carcinoma is characterized by predominance of columnar cells with pronounced nuclear stratification $(467,468)$. These tumors have a higher risk of distant metastases and tumor-related mortality, the latter seen mostly in patients with an advanced disease stage at presentation (467-470). The $B R A F^{V 600 E}$ mutation is found in one-third of these tumors (467).

Papillary carcinoma with prominent hobnail features is a rare, recently described variant characterized by the predominance of cells with a hobnail appearance with apically placed nuclei and bulging of the apical cell surface $(471,472)$. The $B R A F^{V 6 O O E}$ mutation is frequently found in these tumors $(471,473)$. This variant of papillary carcinoma appears to be associated with frequent distant metastases (typically to lung) and increased risk of tumor-related death (471).

Other variants of papillary carcinoma, such as the solid variant and diffuse sclerosing variant, may be associated with a less favorable outcome, although the data remain conflicting. The solid variant tumors appear to be more frequently associated with distant metastases that are present in about $15 \%$ of cases, and with a slightly higher mortality rate, which was $10 \%-12 \%$ in two studies with 10 and 19 years mean follow-up (474,475). However, among children and adolescents with post-Chernobyl papillary carcinomas, which frequently were of the solid variant, the mortality was very low $(<1 \%)$ during the first 10 years of follow-up $(476,477)$. Importantly, the solid variant of papillary carcinoma should be distinguished from poorly differentiated thyroid carcinoma, with which it shares the insular, solid, and trabecular growth patterns. The distinction is based primarily on the preservation of nuclear features and lack of necrosis and high mitotic activity in the solid variant, as outlined by the Turin diagnostic criteria for poorly differentiated thyroid carcinoma (478). It is important to make the distinction because poorly differentiated thyroid carcinoma has a much poorer prognosis, with the 5-year survival of $72 \%$ and 10 -year survival of 
$46 \%$ in a series of 152 patients diagnosed using the Turin criteria (479).

The prognostic implication of the diffuse sclerosing variant of papillary cancer remains controversial. This variant is characterized by diffuse involvement of the thyroid gland and a higher rate of local and distant metastases at presentation, and it has lower disease-free survival than classic papillary carcinoma (480-482). The frequency of distant metastases, predominantly affecting the lung, varies between reported series and is $10 \%-15 \%$ based on almost 100 published cases summarized by Lam and Lo in 2006 (483) and more recent reports. Nevertheless, the overall mortality appears to be low, with a disease-specific survival of approximately $93 \%$ at 10 years of follow-up. The diffuse sclerosing variant tends to be found in younger patients in whom response to treatment is high.

The encapsulated follicular variant of papillary carcinoma is, in contrast, associated with a low risk of recurrence, particularly in the absence of capsular or vascular invasion. This variant is characterized by a follicular growth pattern with no papillae formation and total tumor encapsulation, and the diagnosis rests on the finding of characteristic nuclear features of papillary carcinoma. Although the encapsulated follicular variant of PTC shares the follicular growth pattern with the infiltrative, nonencapsulated follicular variant of PTC, these tumors differ in their molecular profiles and biological properties. The encapsulated follicular variant tumors frequently have $R A S$ mutations, whereas nonencapsulated follicular variants frequently harbor $B R A F^{V 600 E} \mathrm{mu}-$ tations, similar to classic papillary carcinomas $(484,485)$. Most of the encapsulated follicular variant papillary carcinomas show no invasive growth, whereas in about one-third of cases tumor capsule invasion, vascular invasion, or both are found $(486,487)$. Whereas in the past the encapsulated follicular variant was relatively rare, at the present time half to two-thirds of all follicular variant papillary carcinomas belong to this subtype (488). The behavior of these tumors is usually quite indolent. A summary of six studies that reported 107 cases of encapsulated follicular variant revealed $25 \%$ with lymph node metastases and $1 \%$ with distant metastases (489). Among these 107 patients, one died of disease and two were alive with disease, whereas the rest $(97 \%)$ of the patients were alive and well with various follow-up periods. In a study of 61 cases of encapsulated follicular variant, lymph node metastases were observed in 5\%, and there was no distant metastasis (486). With median follow-up of 11 years, one patient developed tumor recurrence, and this tumor had invasion. No adverse events were found in any of the encapsulated and noninvasive tumors, including 31 patients treated with lobectomy only. Similarly, no evidence of recurrence was found in 61 out of 62 encapsulated or well-circumscribed follicular variant of PTC in another series of patients with a median follow-up of 9.2 years, and the only case that developed recurrence had a positive resection margin after initial surgery (490). In another study of a cohort of thyroid tumors followed on average for 12 years, none of 66 patients with encapsulated follicular variant of papillary carcinoma died of disease (487). Despite a low probability, some patients with encapsulated follicular variants may present with distant metastases, particularly to the bones, or develop metastasis on follow-up $(491,492)$. Tumors prone to metastatic behavior often have a thick capsule and significant intratumoral fibrosis, and virtually all of them reveal vascular invasion or invasion of the tumor capsule. Therefore, pathologic evaluation of these tumors should include microscopic examination of the entire tumor capsule to rule out invasion, as well as careful evaluation of the tumor to rule out the presence of poorly differentiated carcinoma areas or other unfavorable diagnostic features such as tumor necrosis or high ( $\geq 3$ per 10 high-power fields) mitotic activity (493). In the absence of these features, a completely excised noninvasive encapsulated follicular variant of papillary carcinoma is expected to have a very low risk of recurrence or extrathyroidal spread, even in patients treated by lobectomy.

Similarly, excellent clinical outcomes are seen in FTCs that manifest only capsular invasion without vascular invasion (494-496). When vascular invasion is present, the tumor should no longer be designated as minimally invasive. However, some studies (456,494,497-500), although not all $(496,501)$, suggest that only those follicular carcinomas that have a greater extent of vascular invasion (more than four foci of vascular invasion, or extracapsular vascular invasion) are associated with poorer outcomes.

Some histopathologic variants of thyroid carcinomas are important to recognize because of their association with familial tumor syndromes $(41,502)$. The cribriform-morular variant of papillary carcinoma is frequently seen in patients with FAP due to a germline mutation in the adenomatous polyposis coli $(A P C)$ gene $(503,504)$. It is characterized by a prominent cribriform architecture and formation of whorls or morules composed of spindle cells. The presence of aberrant $\beta$-catenin immunoreactivity provides a strong evidence for this tumor variant (505-507). Approximately $40 \%$ of patients with this variant of papillary carcinoma are found to have FAP, whereas the rest have no evidence of the inherited disease $(505,508)$. Although no microscopic tumor features can distinguish between familial and sporadic disease, tumor multifocality is more common in the setting of the familial disease $(505,508)$. Since many patients with the cribriformmorular variant have FAP, and thyroid cancer can precede clinically detectable colonic abnormalities in $\sim 40 \%$ of patients (508), this diagnosis should raise the possibility of the familial disease and prompt consideration for colonic examination and genetic counseling.

Follicular carcinoma may develop as a manifestation of the PTEN hamartoma tumor syndrome, which is caused by a germline mutation in the PTEN gene (509-511). The histopathologic appearance of thyroid glands in these patients is very characteristic and should allow pathologists to suspect this syndrome $(510,511)$. The glands typically have numerous sharply delineated, frequently encapsulated thyroid nodules that microscopically are well-delineated and cellular and have variable growth patterns (510-513). Individuals affected by this syndrome also have a high risk for benign and malignant tumors of the breast and endometrium, colon hamartomas, and others, and in light of the characteristic appearance of the thyroid gland in these patients, genetic counseling should be recommended.

Well-differentiated papillary and follicular cancers should be histologically distinguished from poorly differentiated carcinoma. Poorly differentiated carcinoma is an aggressive thyroid tumor characterized by a partial loss of the features of thyroid differentiation that occupies morphologically and behaviorally an intermediate position between welldifferentiated papillary and follicular carcinomas and fully 
dedifferentiated anaplastic carcinoma. Another term used in the past for this tumor was "insular carcinoma." Diagnostic criteria for poorly differentiated carcinoma are based on the consensus Turin proposal and include the following three features: (i) solid/trabecular/insular microscopic growth pattern, (ii) lack of well-developed nuclear features of papillary carcinoma, and (iii) convoluted nuclei (evidence for partial loss of differentiation in papillary cancer), tumor necrosis, or three or more mitoses per 10 high-power fields (514). Poorly differentiated carcinomas have significantly worse outcome as compared to well-differentiated PTC and FTC, with a 10-year survival of $\sim 50 \%$ (514-516). Patient age over 45 years, larger tumor size, presence of necrosis, and high mitotic activity are additional factors that may influence a more unfavorable outcome in patients with poorly differentiated thyroid cancer $(514,517)$. It is not clear if the proportion of poorly differentiated carcinoma areas within the cancer nodule directly correlates with prognosis. Several studies have reported similarly decreased survival in patients with poorly differentiated carcinoma constituting more than $50 \%$ of the tumor and in those in whom it was observed as a minor component $(518,519)$. Tumors with insular, solid, or trabecular architecture, but lacking other diagnostic features of poorly differentiated carcinoma, do not demonstrate such an aggressive behavior and therefore should not be considered as poorly differentiated. On the other hand, some studies suggest that the presence of a high mitotic rate ( $\geq 5$ mitoses/10 high-power fields or Ki-67 labeling index $\geq 4 \%$ ) or tumor necrosis predicts a less favorable outcome irrespective of the presence of the solid/trabecular/insular growth pattern $(520,521)$.

\section{[B16] What is the role of postoperative staging systems and risk stratification in the management of DTC?}

\section{[B17] Postoperative staging}

\section{- RECOMMENDATION 47}

AJCC/UICC staging is recommended for all patients with DTC, based on its utility in predicting disease mortality, and its requirement for cancer registries.

\section{(Strong recommendation, Moderate-quality evidence)}

Postoperative staging for thyroid cancer, as for other cancer types, is used (i) to provide prognostic information, which is of value when considering disease surveillance and therapeutic strategies, and (ii) to enable risk-stratified description of patients for communication among health care professionals, tracking by cancer registries, and research purposes.

Accurate initial staging requires a detailed understanding of all pertinent risk stratification data, whether they were obtained as part of preoperative testing, during the operation(s), or as part of postoperative follow-up. It is also important to emphasize that in many cases the written pathology report of the surgical specimen does not convey critical risk factors such as preoperative vocal cord paralysis, extent of gross extrathyroidal invasion, completeness of resection, or remaining gross residual disease. Without these critical pieces of information, it is likely that initial risk stratification will be inaccurate and potentially misleading. Details and suggestions for effectively communicating specific risk factors between health care providers are outlined in a recent publication of the Surgical Affairs Committee of the ATA (440).

It is important to emphasize that the identification of a clinico-pathologic or molecular predictor of recurrence or mortality does not necessarily imply that more aggressive therapies (such as more extensive surgery, RAI therapy, aggressive thyroid hormone therapy with TSH suppression, targeted therapies) will have a significant impact on clinical outcomes. Similarly, the absence of a risk factor does not mean that more aggressive therapies are not indicated. Intervention studies are required to determine which at-riskpatients may benefit from additional therapies or a more conservative management approach. Until appropriate treatment intervention studies are completed, the risk stratification information associated with a clinico-pathologic risk factor or with a molecular profiling can be used as a prognostic factor to guide follow-up management decisions such as the type and frequency of imaging and biochemical testing.

\section{[B18] AJCC/UICC TNM staging}

Over the years, multiple staging systems have been developed to predict the risk of mortality in patients with DTC (522). Each of the systems uses some combination of age at diagnosis, size of the primary tumor, specific tumor histology, and extrathyroidal spread of the tumor (direct extension of the tumor outside the thyroid gland, loco-regional metastases, and/or distant metastases) to stratify patients into one of several categories with differing risks of death from thyroid cancer. Recently, a nomogram was developed and validated using the SEER data base, which provides a mathematical approach to integrating these important clinical predictive features into a specific mortality risk estimate for an individual patient (523). Using an approach similar to the MACIS system from the Mayo Clinic (270), a quantitative approach based on histology, age, lymph node metastases, tumor size, and extrathyroidal extension utilizing TNM staging has recently been proposed and validated $(524,525)$

While none of the staging systems has been shown to be clearly superior to the other systems, several studies have demonstrated that the AJCC/UICC TNM system (Table 10) and the MACIS system consistently provide the highest proportion of variance explained (PVE, a statistical measure of how well a staging system can predict the outcome of interest) when applied to a broad range of patient cohorts (277,501,526-530), and they have been validated in retrospective studies as well as prospectively in clinical practice.

Unfortunately, none of the staging systems designed to predict mortality from thyroid cancer can account for more than a small proportion (5\%-30\%, corresponding to PVE values of $0.05-0.30$ ) of the uncertainty associated with eventual death from thyroid cancer $(277,501,526-530)$. This relative inability to accurately predict the risk of death from thyroid cancer for an individual patient may be related to the failure of current staging systems to adequately integrate the risk associated with other potentially important clinicopathologic features such as the specific histology (welldifferentiated thyroid cancer versus poorly differentiated thyroid cancer), molecular profile, size and location of distant metastases (pulmonary metastases versus bone metastases versus brain metastases), functional status of the metastases (RAI avid versus ${ }^{18}$ FDG-PET avid), and effectiveness of initial therapy (completeness of resection, effectiveness of 
TABLE 10. AJCC 7TH Edition/TNM ClassifiCATION System for Differentiated Thyroid Carcinoma

\begin{tabular}{|c|c|}
\hline & Definition \\
\hline T0 & No evidence of primary tumor \\
\hline T1a & Tumor $\leq 1 \mathrm{~cm}$, without extrathyroidal extension \\
\hline T1b & $\begin{array}{l}\text { Tumor }>1 \mathrm{~cm} \text { but } \leq 2 \mathrm{~cm} \text { in greatest dimension, } \\
\text { without extrathyroidal extension }\end{array}$ \\
\hline $\mathrm{T} 2$ & $\begin{array}{l}\text { Tumor }>2 \mathrm{~cm} \text { but } \leq 4 \mathrm{~cm} \text { in greatest dimension, } \\
\text { without extrathyroidal extension. }\end{array}$ \\
\hline $\mathrm{T} 3$ & $\begin{array}{l}\text { Tumor }>4 \mathrm{~cm} \text { in greatest dimension limited to } \\
\text { the thyroid } \\
\text { or } \\
\text { Any size tumor with minimal extrathyroidal } \\
\text { extension (e.g., extension into sternothyroid } \\
\text { muscle or perithyroidal soft tissues). }\end{array}$ \\
\hline $\mathrm{T} 4 \mathrm{a}$ & $\begin{array}{l}\text { Tumor of any size extending beyond the thyroid } \\
\text { capsule to invade subcutaneous soft tissues, } \\
\text { larynx, trachea, esophagus, or recurrent } \\
\text { laryngeal nerve. }\end{array}$ \\
\hline $\mathrm{T} 4 \mathrm{~b}$ & $\begin{array}{l}\text { Tumor of any size invading prevertebral fascia } \\
\text { or encasing carotid artery or mediastinal vessels }\end{array}$ \\
\hline No & No metastatic nodes \\
\hline N1a & $\begin{array}{l}\text { Metastases to level VI (pretracheal, paratracheal, } \\
\text { and prelaryngeal/Delphian lymph nodes). }\end{array}$ \\
\hline N1b & $\begin{array}{l}\text { Metastases to unilateral, bilateral, or contralateral } \\
\text { cervical (levels I, II III, IV, or V) or } \\
\text { retropharyngeal or superior mediastinal } \\
\text { lymph nodes (level VII) }\end{array}$ \\
\hline M0 & No distant metastases \\
\hline M1 & Distant metastases \\
\hline
\end{tabular}

\begin{tabular}{|c|c|c|c|}
\hline \multicolumn{4}{|c|}{ Patient age $<45$ years old at diagnosis } \\
\hline I & Any T & Any N & M0 \\
\hline II & Any $\mathrm{T}$ & Any N & M1 \\
\hline
\end{tabular}

Patient age $\geq 45$ years old at diagnosis

\begin{tabular}{llll}
\hline I & T1a & N0 & M0 \\
II & T1b & N0 & M0 \\
III & T2 & N0 & M0 \\
& T1a & N1a & M0 \\
& T1b & N1a & M0 \\
& T2 & N1a & M0 \\
& T3 & N0 & M0 \\
IVa & T3 & N1a & M0 \\
& T1a & N1b & M0 \\
& T1b & N1b & M0 \\
& T2 & N1b & M0 \\
& T3 & N1b & M0 \\
& T4a & N0 & M0 \\
IVb & T4a & N1a & M0 \\
IVc & T4a & N1b & M0 \\
\hline
\end{tabular}

Used with the permission of the American Joint Committee on Cancer (AJCC), Chicago, Illinois. The original source for this material is the AJCC Cancer Staging Manual, Seventh Edition (1077) published by Springer Science and Business Media LLC (http://www.springer.com).
RAI, external beam radiation therapy or other systemic therapies). Furthermore, recent studies have questioned the use of the age of 45 years as a cutoff to upstage patients using the AJCC/UICC TNM system (340,531-533).

Even though the various staging systems designed to predict mortality from thyroid cancer were developed and validated using cohorts that were either exclusively or predominantly PTC patients, several small studies have demonstrated that MACIS and AJCC/UICC TNM staging systems were also predictive in patients with FTC $(501,534,535)$.

Currently, none of the mortality risk systems incorporate molecular testing results. This may need to be re-evaluated as studies emerge using molecular testing including $B R A F^{V 600 E}$, $T E R T$, and TP53 or combinations of markers. For example, in one study that analyzed more than 400 DTCs, the presence of a TERT mutation was found to be an independent predictor of mortality (hazard ratio [HR] 10.35 [95\% CI 2.01-53.24]) for all differentiated cancers and for papillary carcinomas (154). These potential prognostic markers are promising, but require further study.

\section{[B19] What initial stratification system should be used to estimate the risk of persistent/recurrent disease?}

\section{- RECOMMENDATION 48}

(A) The 2009 ATA Initial Risk Stratification System is recommended for DTC patients treated with thyroidectomy, based on its utility in predicting risk of disease recurrence and/or persistence.

(Strong recommendation, Moderate-quality evidence)

(B) Additional prognostic variables (such as the extent of lymph node involvement, mutational status, and/or the degree of vascular invasion in FTC), not included in the 2009 ATA Initial Risk Stratification system may be used to further refine risk stratification for DTC as described in the following text (and in Fig. 4) in the Modified Initial Risk Stratification system. However, the incremental benefit of adding these specific prognostic variables to the $2009 \mathrm{In}$ itial Risk Stratification system has not been established.

\section{(Weak recommendation, Low-quality evidence)}

(C) While not routinely recommended for initial postoperative risk stratification in DTC, the mutational status of $B R A F$, and potentially other mutations such as $T E R T$, have the potential to refine risk estimates when interpreted in the context of other clinico-pathologic risk factors.

(Weak recommendation, Moderate-quality evidence)

Because the AJCC/TNM risk of mortality staging system does not adequately predict the risk of recurrence in DTC (536-539), the 2009 version of the ATA thyroid cancer guidelines proposed a three-tiered clinico-pathologic risk stratification system that classified patients as having low, intermediate, or high risk of recurrence (25). Low-risk patients were defined as having intrathyroidal DTC with no evidence of extrathyroidal extension, vascular invasion, or metastases. Intermediate-risk patients demonstrated either microscopic extrathyroidal extension, cervical lymph node metastases, RAI-avid disease in the neck outside the thyroid 


\section{Risk of Structural Disease Recurrence \\ (In patients without structurally identifiable disease after initial therapy)}
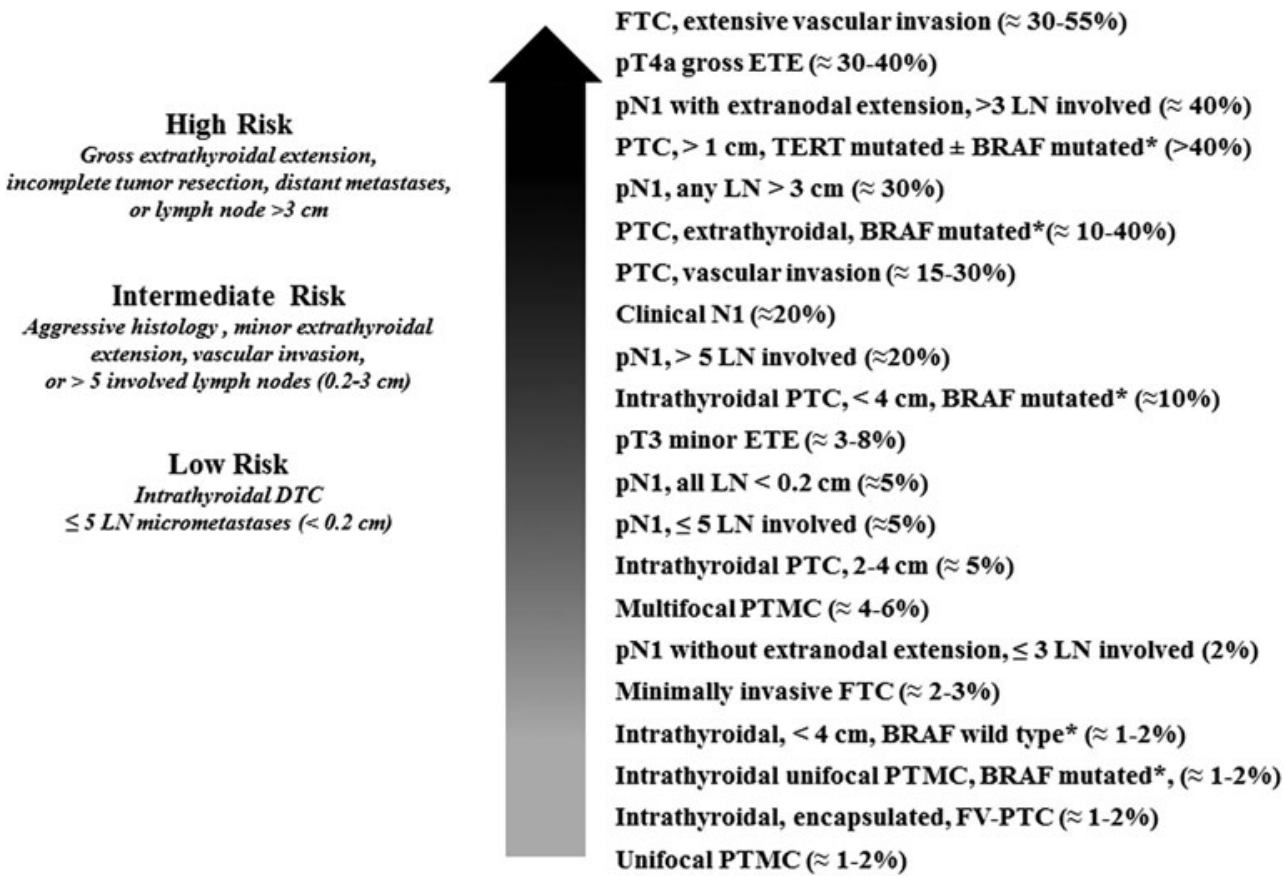

FIG. 4. Risk of structural disease recurrence in patients without structurally identifiable disease after initial therapy. The risk of structural disease recurrence associated with selected clinico-pathological features are shown as a continuum of risk with percentages (ranges, approximate values) presented to reflect our best estimates based on the published literature reviewed in the text. In the left hand column, the three-tiered risk system proposed as the Modified Initial Risk Stratification System is also presented to demonstrate how the continuum of risk estimates informed our modifications of the 2009 ATA Initial Risk System (see Recommendation 48). *While analysis of BRAF and/or TERT status is not routinely recommended for initial risk stratification, we have included these findings to assist clinicians in proper risk stratification in cases where this information is available. FTC, follicular thyroid cancer; FV, follicular variant; LN, lymph node; PTMC, papillary thyroid microcarcinoma; PTC, papillary thyroid cancer.

bed, vascular invasion, or aggressive tumor histology. Highrisk patients had gross extrathyroidal extension, incomplete tumor resection, distant metastases, or inappropriate postoperative serum $\mathrm{Tg}$ values (Table 11).

The 2009 ATA risk stratification system was somewhat different than staging systems proposed by a European Consensus conference (540) and the Latin American Thyroid Society (LATS) (541) which classify patients as either being at very low risk (unifocal, intrathyroidal T1aNOM0), low risk (T1b N0M0, T2N0M0, or multifocal T1N0M0,), or high risk (any T3 or T4, any N1, or any M1). The European and LATS very low risk and low risk categories would be classified as ATA low risk, while the ETA high risk category would be subdivided between ATA intermediate risk (minor extrathyroidal extension, N1 disease) and ATA high risk (gross extrathyroidal extension, M1, incomplete tumor resection).

Subsequent studies have retrospectively validated the 2009 ATA risk of recurrence staging system through analysis of independent datasets originating from three respective continents (Table 12). These studies have reported the estimates of patients who subsequently had no evidence of disease (NED) in each ATA Risk Category after total thyroidectomy and RAI remnant ablation: (a) low risk, 78\%-91\% NED, (b) intermediate risk, 52\%-64\% NED, and (c) high risk, 31\%$32 \%$ NED $(538,539,542,543)$. In these datasets, NED was defined as a stimulated $\mathrm{Tg}<1 \mathrm{ng} / \mathrm{mL}$ with no other radiological or clinical evidence of disease. Prospectively collected validation data for the ATA initial risk stratification system are needed.

Three additional studies, in which the ATA risk classification system was retrospectively evaluated, have also suggested that the ATA risk of recurrence model may be applied in low- and intermediate-risk patients in the absence of RAI remnant ablation $(328,544,545)$. Over a median follow-up period that ranged from 5 to 10 years, structural disease recurrence was identified in less than $1 \%-2 \%$ of ATA low-risk patients and $8 \%$ of ATA intermediate-risk patients who underwent thyroid surgery without RAI ablation as the initial therapy $(328,544,545)$.

The type of persistent disease also varies according to ATA initial risk stratification, with $70 \%-80 \%$ of the persistent disease in ATA low-risk patients manifested by abnormal serum Tg levels (suppressed or stimulated $\mathrm{Tg}>1 \mathrm{ng} / \mathrm{mL}$ ) without structurally identifiable disease, while only $29 \%-$ $51 \%$ of the ATA intermediate-risk patients and $19 \%-21 \%$ of ATA high-risk patients are classified as having persistent disease only on the basis of biochemical abnormalities $(538,539,543)$. With increasing ATA risk level, the RR of having structural persistent/recurrent disease increases. Thus, the ATA low-risk patients appear to have the highest RR of 
Table 11. ATA 2009 Risk Stratification System with Proposed Modifications

\begin{tabular}{|c|c|}
\hline ATA low risk & $\begin{array}{l}\text { Papillary thyroid cancer (with all of the following): } \\
\text { - No local or distant metastases; } \\
\text { - All macroscopic tumor has been resected } \\
\text { - No tumor invasion of loco-regional tissues or structures } \\
\text { - The tumor does not have aggressive histology (e.g., tall cell, hobnail variant, } \\
\text { columnar cell carcinoma) } \\
\text { - If }{ }^{131} \text { I is given, there are no RAI-avid metastatic foci outside the thyroid bed on } \\
\text { the first posttreatment whole-body RAI scan } \\
\text { - No vascular invasion } \\
\text { - Clinical N0 or } \leq 5 \text { pathologic N1 micrometastases }(<0.2 \mathrm{~cm} \text { in largest dimension) } \\
\text { Intrathyroidal, encapsulated follicular variant of papillary thyroid cancera } \\
\text { Intrathyroidal, well differentiated follicular thyroid cancer with capsular invasion and } \\
\text { no or minimal }\left(<4 \text { foci) vascular invasion }{ }^{\mathrm{a}}\right. \\
\text { Intrathyroidal, papillary microcarcinoma, unifocal or multifocal, including } B R A F^{V 600 E} \\
\text { mutated (if known) }\end{array}$ \\
\hline $\begin{array}{l}\text { ATA intermediate } \\
\text { risk }\end{array}$ & $\begin{array}{l}\text { Microscopic invasion of tumor into the perithyroidal soft tissues } \\
\text { RAI-avid metastatic foci in the neck on the first posttreatment whole-body RAI scan } \\
\text { Aggressive histology (e.g., tall cell, hobnail variant, columnar cell carcinoma) } \\
\text { Papillary thyroid cancer with vascular invasion } \\
\text { Clinical N1 or }>5 \text { pathologic N1 with all involved lymph nodes }<3 \mathrm{~cm} \text { in largest dimension } \\
\text { Multifocal papillary microcarcinoma with ETE and } B R A F^{V 600 E} \text { mutated (if known) }^{\mathrm{a}}\end{array}$ \\
\hline ATA high risk & $\begin{array}{l}\text { Macroscopic invasion of tumor into the perithyroidal soft tissues (gross ETE) } \\
\text { Incomplete tumor resection } \\
\text { Distant metastases } \\
\text { Postoperative serum thyroglobulin suggestive of distant metastases } \\
\text { Pathologic N1 with any metastatic lymph node } \geq 3 \mathrm{~cm} \text { in largest dimension } \\
\text { Follicular thyroid cancer with extensive vascular invasion }\left(>4 \text { foci of vascular invasion) }{ }^{\mathrm{a}}\right.\end{array}$ \\
\hline
\end{tabular}

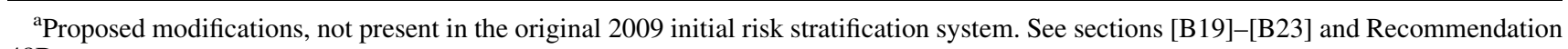
48B.

isolated thyroglobulinemia, which may be of less clinical significance than structural disease persistence or recurrence.

Similar to what was seen with the staging systems designed to predict risk of mortality from thyroid cancer (see section [B18] above), the PVE by the ATA risk of recurrence system was suboptimal, ranging from $19 \%$ to $34 \%(538,542)$. More recently, a novel mathematical clinico-pathologic staging system from the University of Yonsei yielded similar results with a PVE of $11.9 \%$ in predicting disease recurrence (546).
[B20] Potential impact of specific clinico-pathologic features on the risk estimates in PTC

As originally conceived, the ATA initial risk stratification system was a three-tiered system in which clinico-pathologic features available at the time of initial treatment were used to classify DTC patients as having either low, intermediate, or high risk of either recurrence or persistent disease. However, as with any categorical staging system, the risk of recurrence within the individual risk categories (low, intermediate, and

Table 12. American Thyroid Association Risk Stratification System: Clinical Outcomes Following Total Thyroidectomy and Radioiodine Remnant Ablation or Adjuvant Therapy

\begin{tabular}{|c|c|c|c|c|}
\hline ATA risk & Study & $N E D, \%$ & $\begin{array}{c}\text { Biochemical } \\
\text { incomplete, } \%\end{array}$ & $\begin{array}{c}\text { Structural } \\
\text { incomplete, \% }\end{array}$ \\
\hline Low & $\begin{array}{l}\text { Tuttle et al. }(538) \\
\text { Castagna et al. }(542) \\
\text { Vaisman et al. }(539) \\
\text { Pitoia et al. }(543)\end{array}$ & $\begin{array}{l}86 \\
91 \\
88 \\
78\end{array}$ & $\begin{array}{c}11 \\
\mathrm{ND}^{\mathrm{a}} \\
10 \\
15\end{array}$ & $\begin{array}{c}3 \\
\mathrm{ND}^{\mathrm{a}} \\
2 \\
7\end{array}$ \\
\hline Intermediate $^{\mathrm{a}}$ & $\begin{array}{l}\text { Tuttle et al. }(538) \\
\text { Vaisman et al. }(539) \\
\text { Pitoia et al. }(543)\end{array}$ & $\begin{array}{l}57 \\
63 \\
52\end{array}$ & $\begin{array}{l}22 \\
16 \\
14\end{array}$ & $\begin{array}{l}21 \\
21 \\
34\end{array}$ \\
\hline High & $\begin{array}{l}\text { Tuttle et al. }(538) \\
\text { Vaisman et al. }(539) \\
\text { Pitoia } \text { et al. }(543)\end{array}$ & $\begin{array}{l}14 \\
16 \\
31\end{array}$ & $\begin{array}{l}14 \\
12 \\
13\end{array}$ & $\begin{array}{l}72 \\
72 \\
56\end{array}$ \\
\hline
\end{tabular}

"Because the ATA intermediate- and high-risk groups were merged into a single "high-risk" group in the series by Castagna et al. (542), risk of persistent/recurrent disease for these subgroups is not presented.

${ }^{b}$ Proportion of patients with a biochemical incomplete response. Definition: suppressed $\mathrm{Tg}>1 \mathrm{ng} / \mathrm{mL}, \mathrm{TSH}-\mathrm{stimulated} \mathrm{Tg}>10 \mathrm{ng} / \mathrm{mL}$, or rising anti-Tg antibody levels in the absence of structural disease.

'Proportion of patients with persistent/recurrent disease that is structural. Definition: structural disease that is either biopsy-proven or highly suspicious for disease with or without abnormal serum $\mathrm{Tg}$.

ND, not determined. 
high) can vary depending on the specific clinical features of individual patients (Fig. 4). In addition, the three-tiered system did not specifically address the risk of recurrence associated with specific DTC histologies, multifocality, genotype, extent of vascular invasion, or extent of metastatic lymph node involvement.

For example, while intrathyroidal PTCs of all sizes are included in the ATA low-risk category, the risk of structural disease recurrence can vary from $1 \%-2 \%$ in unifocal papillary microcarcinomas, to $4 \%-6 \%$ in multifocal papillary microcarcinomas $(141,147)$, to $5 \%-6 \%$ in $2-4 \mathrm{~cm}$ intrathyroidal PTC (547), and 8\%-10\% in intrathyroidal PTC $>4 \mathrm{~cm}$ (547). Similarly, while all DTC patients with locoregional lymph node metastases were classified as having intermediate risk in the 2009 ATA risk stratification system, the risk of structural disease recurrence can vary from $4 \%$ in patients with fewer than five metastatic lymph nodes, to $5 \%$ if all involved lymph nodes are $<0.2 \mathrm{~cm}$, to $19 \%$ if more than five lymph nodes are involved, to $21 \%$ if more than 10 lymph nodes are involved, to $22 \%$ if macroscopic lymph node metastases are clinically evident (clinical N1 disease), and 27\%$32 \%$ if any metastatic lymph node is $>3 \mathrm{~cm}(335,548)$. These risk estimates apply to both N1a and N1b disease because there are insufficient data to determine risk based on location within the neck independent of size, number of involved nodes, and extranodal extension. It is very likely that these risk estimates could vary depending on the types and extent of nodal dissections done in individual patients. As defined by the ATA surgical affairs committee task force on thyroid cancer nodal surgery, $\leq 5 \mathrm{pN} 1$ micrometastases $(<0.2 \mathrm{~cm}$ in largest dimension) are classified as lower risk N1 disease ( $<5 \%$ risk of recurrence) (335). Clinical N1 disease, more than five metastatic lymph nodes, or any metastatic lymph node $>3 \mathrm{~cm}$ in largest dimension are classified as higher risk N1 disease ( $>20 \%$ risk of recurrence) (335). Identification of extranodal extension of the tumor through the metastatic lymph node capsule has also been associated with an increased risk of recurrent/persistent disease $(338,450)$. It is difficult to estimate the risk associated with extension of the tumor through the capsule of involved lymph nodes because this histologic finding is tightly linked with both the number of involved lymph nodes $(338,450)$ and invasion of the tumor through the thyroid capsule $(450,549)$.

It is also important to differentiate the clinical significance of minor extrathyroidal extension (ATA intermediate risk) from gross extrathyroidal invasion of surrounding structures (ATA high risk). The risk of recurrence associated with minor extrathyroidal extension (pT3 disease manifest by minimal extrathyroidal extension) ranges from $3 \%$ to $9 \%$ (550-554), while the risk of recurrence in patients with gross extrathyroidal extension (pT4a disease involving the subcutaneous soft tissues, larynx, trachea, esophagus, or RLN) ranges from $23 \%$ to $40 \%(537,550,552-555)$.

The encapsulated follicular variant of papillary thyroid carcinoma also appears to be associated with a low 10-year risk of recurrence $(486,490,556)$. Only two recurrences were reported in the 152 patients (1.3\% risk of recurrence) described in these three reports. No recurrences were described in the 42 patients that had encapsulated FVPTC without capsular or lymphovascular invasion in the series by Liu et al. (486). However, the study by Baloch et al. (556) included a single patient with encapsulated FVPTC that developed bone metastasis despite not having lymphovascular invasion or lymph node metastases identified at the time of diagnosis. Therefore, intrathyroidal FVPTC is best classified as a lowrisk tumor that is unlikely to recur or metastasize.

Well-differentiated FTCs demonstrating only capsular invasion (without vascular invasion) usually have an excellent prognosis with recurrence rates of $0 \%-7 \%$ and can be classified as low-risk tumors (494-496). Encapsulated, minimally invasive FTC with only minor vascular invasion (small number of foci confined to intracapsular vessels) also appears to have a low recurrence rate of approximately $0 \%-5 \%(456,500)$. Furthermore, some studies $(456,494,497-500)$, but not all $(496,501)$, suggest a greater extent of vascular invasion (more than four foci of vascular invasion, or extracapsular vascular invasion) is associated with poorer outcomes even in encapsulated FTCs. However, FTC is considered ATA high risk if extensive vascular invasion is present because the risk of the development of newly identified distant metastases is as high as $30 \%-55 \%(455,494,497,500)$.

In PTC, most (451-453,558-561), but not all (562) studies demonstrate that vascular invasion is associated with worse clinical outcomes. Recurrence rates were significantly higher if vascular invasion was present in the studies by Gardner et al. (16\%-20\% with vascular invasion, 3\%-6\% without), Nishida et al. (28\% with vascular invasion, $15 \%$ without), and Falvo et al. (30\% with vascular invasion, $5 \%$ without), but not in the studies by Furlan et al. (562) or Akslen et al. (559). Vascular invasion in PTC was also associated with higher rates of distant metastases $(453,561)$ and disease-specific mortality $(559,560)$.

[B21] Potential impact of BRAF ${ }^{\mathrm{V} 600 \mathrm{E}}$ and other mutations on risk estimates in PTC

In a pooled univariate analysis of 1849 PTC patients, the presence of a $B R A F^{V 600 E}$ mutation was associated with increased disease-specific mortality, although this was not significantly associated with mortality in a multivariate analysis (370). However, a significant interaction between $B R A F^{V 600 E}$ mutation and several conventional clinicopathological risk factors was seen, such that the risk of mortality was higher in patients with $B R A F$ mutation compared to those with wild-type $B R A F$ in the setting of lymph node metastases $(11.1 \%$ vs. $2.6 \%, p<0.001)$, distant metastases $(51.5 \%$ vs. $18.2 \%, p<0.001)$, AJCC stage IV disease $(31.4 \%$ vs. $13 \%$, p 0.004$)$, and age $\geq 45$ years at diagnosis $(8 \%$ vs. $1.9 \%, p<0.001)$. In a systematic review and metaanalysis of 14 publications that included 2470 PTC patients from nine different countries, the $B R A F^{V 600 E}$ mutation was associated with a significantly higher risk of recurrence than $B R A F$ wild-type tumors $(24.9 \%$ vs. $12.6 \%, p<0.00001[95 \%$ CI 1.61-2.32]) (563). In the studies included in this metaanalysis, the risk of recurrence in $B R A F^{V 600 E}$-positive tumors ranged from $11 \%$ to $40 \%$ (median $26.5 \%$ ), while the risk of recurrence in $B R A F$ wild-type tumors ranged from $2 \%$ to $36 \%$ (median $9.5 \%$ ). Because the $B R A F^{V 600 E}$ mutation is tightly linked with the presence of aggressive histologic phenotypes, lymph node metastases, and extrathyroidal extension, it is difficult to determine the proportion of risk that is attributable to the $B R A F$ mutation versus that attributable to the other clinico-pathologic features. Some studies $(564,565)$, but not all (566-568), have demonstrated that the $B R A F^{V 600 E}$ mutation is an independent predictor of risk of 
recurrence in multivariate analysis. Furthermore, a recent publication demonstrated a small, but statistically significant improvement in risk stratification if $B R A F$ status was used in conjunction with the 2009 ATA initial risk stratification system (569). In a meta-analysis of 2167 patients, the presence of a $B R A F^{V 600 E}$ mutation had a sensitivity of $65 \%$ in identifying those tumors that subsequently recurred, but had a PPV of only $25 \%$ in predicting the risk of recurrence (563). Unlike previous studies that were often small and primarily single-institution reports, a large multicenter pooled-data evaluation of 2099 patients (1615 women, 484 men, median age 45 years) demonstrated that the $B R A F^{V 600 E}$ mutation was significantly associated with the risk of recurrence in both classical PTC (20.7\% vs. $12.4 \%$, HR 1.46 [95\% CI 1.08-1.99] after adjustment for multiple clinico-pathological features), and FVPTC (21.3\% vs. 7.0\%, HR 3.20 [95\% CI 1.46-7.02] after adjustment for multiple clinico-pathological features). Based on these data, it appears that the $B R A F$ status in isolation is not sufficient to substantially contribute to risk stratification in most patients. However, an incremental improvement in risk stratification can be achieved if the BRAF mutational status is considered in the context of other standard clinico-pathological risk factors. Since the clinical implications of this incremental improvement in risk stratification are not clear, we are not routinely recommending $B R A F$ mutational evaluation for initial postoperative risk stratification in DTC.

The potential role of $B R A F$ status in isolation as an aid to risk stratification in patients clinico-pathologically classified as having ATA low risk is currently being evaluated. In a cohort of low-risk patients with intrathyroidal PTC ( $<4 \mathrm{~cm}, \mathrm{~N} 0, \mathrm{M} 0 ; 33 \%$ with $B R A F$ mutation), the overall risk of having structural disease recurrence over 5 years of follow up was $3 \%$ (565). However, $B R A F^{V 600 E}$-mutated tumors had a recurrence rate of $8 \%$ (8 of 106) compared with only $1 \%$ (2 of 213 ) in $B R A F$ wild-type tumors ( $p=0.003$, Fisher's exact). Furthermore, in multivariate analysis, the only clinico-pathological significant predictor of persistent disease after 5 years of follow-up was the presence of a $B R A F^{V 600 E}$ mutation. If these findings are verified in additional studies, it is possible that $B R A F$ testing could be used to help further risk stratify patients with intrathyroidal PTC as having very low risk of recurrence ( $B R A F$ wild-type) or intermediate risk of recurrence (BRAF $F^{V 600 E}$ mutation).

The impact of $B R A F$ status on the risk of recurrence in the very low-risk patients (intrathyroidal unifocal papillary microcarcinomas $<1 \mathrm{~cm}$ ) appears to be small. Even though $B R A F$ mutation is present in $30 \%-67 \%$ of papillary microcarcinomas $(152,153,156,566,570-576)$, the overall clinical recurrence rate is quite low, ranging from $1 \%$ to $6 \%(274,566)$. In a series of 99 papillary microcarcinoma patients with an overall recurrence rate of $7 \%$, no recurrences were detected in the patients with a $B R A F^{V 600 E}$ mutation and intrathyroidal, unifocal tumors. Conversely, $B R A F^{V 600 E}$-mutated multifocal PTMC with extrathyroidal extension demonstrated a $20 \%$ recurrence rate (150). Therefore, in the absence of data demonstrating that the $B R A F^{V 600 E}$ mutation is associated with increased structural recurrence in very low-risk tumors, we have classified intrathyroidal papillary microcarcinomas (T1a, N0, M0) harboring $B R A F^{V 600 E}$ mutations with no other worrisome features (such as extrathyroidal extension, aggressive histology, vas- cular invasion, or lymph node metastases) as ATA low-risk tumors. The few patients (about $10 \%$ of PTMC patients) that demonstrate multifocal PTMC with extrathyroidal invasion and a $B R A F^{V 600 E}$ mutation would be considered as having ATA intermediate risk for recurrence. Based on these data, there appears to be little role for $B R A F$ mutational testing as an aid to risk stratification in PTMC tumors that do not demonstrate other worrisome clinico-pathologic features.

More recent data suggest that aggressive behavior of a given thyroid carcinoma, including high probability of tumor recurrence, is likely when it harbors more than one known oncogenic mutation, and specifically a $B R A F$ mutation cooccurring with a TERT promoter, PIK3CA, TP53, or AKT1 mutation (155-157,577). Such a combination of several mutations is seen in a much smaller fraction of PTC as compared with a $40 \%-45 \%$ incidence of $B R A F$ mutations and is expected to serve as a more specific marker of unfavorable outcomes of PTC.

Two other molecular markers that appear to confer an increased risk of tumor recurrence and tumor-related mortality are TP53 and TERT mutations. TP53 mutations have been known to occur mostly in poorly differentiated and anaplastic thyroid cancers. However, more recent broad mutational analyses identified TP53 mutations in 2 of $57(3.5 \%)$ well-differentiated PTC and 4 of $36(11 \%)$ of well-differentiated FTC (578). Both PTCs in this series that were positive for TP53 mutations also showed mutations in $B R A F$ (or BRAF and PIK3CA) and developed lung metastases. All four TP53-positive FTC (with no other coexisting mutations) were oncocytic, and three out of four of those were widely invasive FTC.

Finally, recent studies identified TERT promoter mutations as a likely predictor of more unfavorable outcomes for patients with thyroid cancer. TERT mutations were found in $7 \%-22 \%$ of PTC and $14 \%-17 \%$ of FTC, but with a significantly higher prevalence in dedifferentiated thyroid cancers $(154,579-581)$. In some $(154,579)$, but not all (580) reports, TERT mutations were found more often in PTC carrying a $B R A F$ mutation. In the largest reported series (332 PTC and 70 FTC followed on average for 8 years), a TERT mutation was an independent predictor of diseasefree survival (odds ratio [OR] 4.68 [95\% CI 1.54-14.27]) and mortality (HR 10.35 [95\% CI 2.01-53.24]) for welldifferentiated thyroid cancer (154). Furthermore, the combination of a TERT mutation and a BRAF mutation within the same tumor was associated with a high risk of structural disease recurrence (155). These results, although pending confirmation in other studies, suggest that these molecular markers, alone or in combination, may be helpful for risk stratification of thyroid cancer and provide significantly more accurate risk assessment than $B R A F$ mutational status taken in isolation.

\section{[B22] Potential impact of postoperative serum Tg on risk} estimates

Several studies have demonstrated the clinical utility of a serum $\mathrm{Tg}$ measurement (either $\mathrm{TSH}$ stimulated or nonstimulated) obtained a few weeks after total thyroidectomy (postoperative $\mathrm{Tg}$ ) and before RAI remnant ablation as a tool to aid in initial risk stratification and adjuvant therapy decision-making (See Recommendations 50B and 50C). Please see section [C6] for discussion of Tg measurements. 
[B23] Proposed modifications to the 2009 ATA initial risk stratification system

While the 2009 risk stratification system has proven to be a valuable tool for initial risk stratification in PTC, modifications are required to better incorporate our new understanding regarding the risks associated with the extent of lymph node involvement, mutational status, and specific FTC histologies (Table 11). While the modified 2009 risk stratification system continues to classify intrathyroidal PTC without vascular invasion as low risk, the category was expanded to include patients with small-volume lymph node metastases (clinical N0 or $\leq 5$ pathologic N1 micrometastases, $<0.2 \mathrm{~cm}$ in largest dimension), intrathyroidal encapsulated follicular variant of PTC, intrathyroidal well-differentiated follicular cancer with capsular or minor vascular invasion ( $<4$ vessels involved), and intrathyroidal papillary microcarcinomas that are either $B R A F$ wild-type or $B R A F$ mutated. Similarly, the modified 2009 intermediate-risk category continues to include patients with microscopic invasion of the tumor into perithyroidal soft tissues, vascular invasion, uptake outside the thyroid bed at the time of remnant ablation, and aggressive histologies, but it has been modified to include only a subset of patients with lymph node metastases (clinical N1 or $>5$ pathologic N1 with all involved lymph nodes $<3 \mathrm{~cm}$ in largest dimension and multifocal papillary microcarcinoma with extrathyroidal extension and $B R A F$ mutated (if known). Finally, the highrisk category continues to include patients with macroscopic extrathyroidal extension, incomplete tumor resection, distant metastases, and postoperative serum Tg suggestive of distant metastases, but it has been expanded to include patients with large-volume lymph node involvement (any metastatic lymph node $\geq 3 \mathrm{~cm}$ in largest dimension), and FTC with extensive vascular invasion ( $>4$ foci of vascular invasion or extracapsular vascular invasion). While these modifications are based on our current literature review of the impact of the individual risk factors, future studies will be required to validate this proposed modification to determine if the additional modifying factors provide significant incremental improvement in risk stratification.

\section{[B24] Risk of recurrence as a continuum of risk}

While the ATA initial risk stratification system provides a meaningful and valuable tool for predicting risk of recurrence when used as a three-tiered categorical staging system, additional insights can be gained if one appreciates that the risk of structural disease recurrence is a continuum of risk that ranges from $<1 \%$ in very low-risk patients to $>50 \%$ in high-risk patients (see Fig. 4). Therefore, individualized management recommendations should be based not only on the categorical risk of recurrence estimate, but also on a more individualized estimate of risk in which the ATA lowand intermediate-risk categories are further characterized on the basis of respective clinical or pathologic features, such as the size of the primary tumor, number and size of locoregional lymph node metastases (as well as extranodal extension), specific histologic variant, vascular invasion, extent of extrathyroidal extension, or other potentially important factors.

While the risk estimates presented in this section may be useful in guiding initial treatment selections (extent of initial surgery, need for RAI ablation), it is important to recognize that these risk estimates likely reflect not only the tumor biology, but also the impact of initial therapy, which varies from thyroid lobectomy to total thyroidectomy with varying extents of lymph node dissection, either with or without RAI ablation, across the various studies. Additional studies are needed to better define the risk of recurrence in most of these clinical scenarios in patients randomized to receive one of several initial treatment options (lobectomy versus total thyroidectomy, extent of lymph node dissection, with or without RAI ablation).

\section{[B25] How should initial risk estimates be modified over time?}

\section{- RECOMMENDATION 49}

Initial recurrence risk estimates should be continually modified during follow-up, because the risk of recurrence and disease-specific mortality can change over time as a function of the clinical course of the disease and the response to therapy.

\section{(Strong recommendation, Low-quality evidence)}

While initial staging systems provide important insights into an individual patient's risk of recurrence and diseasespecific mortality, they provide static, single-point estimates of risk based only on data available at the time of initial therapy. None of the currently available initial staging systems are capable of using new data obtained during the course of follow-up to modify the initial risk estimate. For example, an ATA low-risk patient that demonstrates a rising serum Tg associated with cervical lymphadenopathy highly suspicious for recurrent disease at some point during follow-up would still be classified as ATA low risk despite the presence of clinical data demonstrating a high risk of recurrence. Conversely, an ATA high-risk patient that remains NED over 30 years of appropriate follow-up would still be classified as ATA high risk despite having a risk of recurrence that is significantly lower than would have been predicted at the time of initial therapy.

Therefore, while the initial staging systems can be informative in guiding therapeutic and early diagnostic follow-up strategy decisions, a risk stratification system that incorporates individual response to therapy into a real-time, dynamic risk stratification scheme is needed to provide an individualized approach to ongoing management (582,583). One approach that has been proposed is to use the risk estimates from the initial staging systems to guide initial management recommendations and then to incorporate a response-totherapy assessment during follow-up to modify these initial risk estimates in an ongoing, dynamic process (584).

Multiple studies have now shown that many patients initially classified as intermediate or high risk of recurrence using initial staging systems can be reclassified as having a subsequent low risk of recurrence based on having an excellent response to initial therapy $(538,539,542,586-601)$. Furthermore, the PVE values associated with staging systems that incorporated response-to-therapy variables into revised risk estimates were significantly higher $(62 \%-84 \%)$ than those seen with initial staging systems $(<30 \%)$ $(538,542)$. These data indicate that long-term outcomes can be more reliably predicted using systems that adjust to new data over time. 
[B26] Proposed terminology to classify response to therapy and clinical implications

All clinical, biochemical, imaging (structural and functional), and cytopathologic findings obtained during followup should be used to redefine the clinical status of the patient and to assess their individual response to therapy. Ideally, the global consideration of the composite findings of such an assessment would allow for the disease status to be classified into one of several discrete response to therapy risk strata as proposed by Tuttle et al. $(538,582)$. Potential challenges in applying this specific system in routine clinical practice include lack of validation in specific subgroups of patients (such as those who had less than total thyroidectomy or those not treated with RAI), the lack of published prospective data utilizing this system in clinical care, and some inconsistency with other authors in classifying the significance of varying levels of detectable Tg levels or imaging findings.

The response to therapy restaging system was also not designed or specifically tested by developers to guide specific therapeutic decisions on primary therapy (such as use of adjuvant treatment) because it has been designed for use after primary therapy is completed. Prospective studies of the value of this system for guiding extent of primary treatment, including adjuvant treatment decisions, are needed. However, given that there is emerging evidence that such a reclassification system has potential to be of great importance in ongoing clinical care of DTC patients after primary treatment, the details are described herein.

It is acknowledged that appropriate clinical application of such a system is highly dependent on the availability of high-quality biochemical testing and structural, and functional imaging with appropriate interpretation (e.g., standardized radiologic reporting). Therefore, applicability may be limited in settings where these procedures are not available or feasible.

The concept and initial validation of the four response-totherapy categories presented here were described by Tuttle et al. (538) and modified in Vaisman et al. (328). As originally conceived, these clinical outcomes described the best response to initial therapy during the first 2 years of follow-up $(538,582)$, but they are now being used to describe the clinical status at any point during follow-up.

- Excellent response: no clinical, biochemical, or structural evidence of disease

- Biochemical incomplete response: abnormal $\mathrm{Tg}$ or rising anti-Tg antibody levels in the absence of localizable disease. Please see section [C6] for discussion of Tg measurements.

- Structural incomplete response: persistent or newly identified loco-regional or distant metastases

- Indeterminate response: nonspecific biochemical or structural findings that cannot be confidently classified as either benign or malignant. This includes patients with stable or declining anti-Tg antibody levels without definitive structural evidence of disease.

The majority of published studies examining response to therapy in DTC have been performed in populations of patients whose primary treatment consisted of total thyroidectomy and RAI ablation. While the following sections will provide the details of multiple studies examining response to therapy, a simplified overview of the clinical implications of the response-to-therapy reclassification system in patients treated with total thyroidectomy and RAI ablation, based on the research from Tuttle et al. $(538,539)$ and supported by other studies, is described in Table 13. Specific suggestions with regard to the application of response-to-therapy assessments in patients treated with total thyroidectomy without RAI ablation or with thyroid lobectomy have recently been published by Momesso et al. (602).

[B27] Excellent response: no clinical, biochemical, or structural evidence of disease after initial therapy (remission, NED)

These patients have no clinical, biochemical, or structural evidence of disease identified on risk-appropriate follow-up studies (Table 13). If a total thyroidectomy and RAI ablation were done, an excellent response was usually defined as a $\mathrm{TSH}$-stimulated $\mathrm{Tg}$ of $<1 \mathrm{ng} / \mathrm{mL}$ in the absence of structural or functional evidence of disease (and in the absence of anti-Tg antibodies) (538,539,542,586-601). An excellent response to initial therapy is achieved in $86 \%-91 \%$ of ATA low-risk patients, $57 \%-63 \%$ of ATA intermediate-risk patients, and $14 \%-16 \%$ of ATA high-risk patients $(538,539,542)$.

In 20 retrospective studies, the risk of recurrence over 5-10 years of follow-up ranged from $1 \%$ to $4 \%$ (median $1.8 \%$ ) in patients who had an excellent response to therapy by $6-18$ months after total thyroidectomy and RAI remnant ablation $(538,539,542,586-601,603)$.

The potential impact of this reclassification is the most dramatic in the two-thirds of ATA intermediate-risk patients who achieve an excellent response and therefore have their risk of having recurrent/persistent disease decreased from $36 \%-43 \%$ (predicted by initial ATA risk stratification) to $1 \%-$ $2 \%$ (predicted by response-to-therapy reclassification) $(538,539)$. Because of the very low risk of structural disease recurrence in ATA low risk patients (1\%-3\%), reclassification based on an excellent response to therapy has less practical implications than in the intermediate- and high-risk patients.

While many of the studies reviewed primarily low-risk DTC patients $(586,588,595-598,601)$, the same low risk of recurrence following achievement of excellent response to therapy was seen in other studies that had substantial numbers of intermediate-risk patients $(538,539,542,587,591,592,604)$. Furthermore, most studies also demonstrate that the few high-risk patients that achieve an excellent response to therapy also have subsequent recurrence rates in the $1 \%-$ $2 \%$ range $(542,593,594,600)$. The one exception is the $14 \%$ risk of recurrence seen in the few ATA high-risk patients that achieved remission in a series from Memorial SloanKettering Cancer Center (538), which is likely due to the known referral bias of unusual and very high-risk cases. Nonetheless, high-risk patients that achieve an excellent response to therapy may require somewhat more intense follow-up than ATA low- and intermediate-risk patients demonstrating an excellent response to therapy. It is important to note that patients at intermediate to high risk of recurrence may require additional structural or functional imaging to rule out disease that may not be detected by US and $\mathrm{Tg}$ measurements prior to being classified as having an excellent response (602). The details for choice of follow-up tests are found in another section of these guidelines [C4C13], (Figs. 5-8). 
Table 13. Clinical Implications of Response to Therapy Reclassification in Patients With Differentiated Thyroid Cancer Treated with Total Thyroidectomy and Radioiodine Remnant Ablation

\begin{tabular}{|c|c|c|c|}
\hline Category & Definitions $^{\mathrm{a}}$ & Clinical outcomes & Management implications \\
\hline $\begin{array}{l}\text { Excellent } \\
\text { response }\end{array}$ & $\begin{array}{l}\text { Negative imaging } \\
\text { and either } \\
\text { Suppressed } \mathrm{Tg}<0.2 \mathrm{ng} / \mathrm{mL}^{\mathrm{b}} \\
\text { or } \\
\text { TSH-stimulated } \mathrm{Tg}<1 \mathrm{ng} / \mathrm{mL}^{\mathrm{b}}\end{array}$ & $\begin{array}{l}1 \%-4 \% \text { recurrence } \\
<1 \% \text { disease specific death }\end{array}$ & $\begin{array}{l}\text { An excellent response to therapy } \\
\text { should lead to an early } \\
\text { decrease in the intensity and } \\
\text { frequency of follow up and } \\
\text { the degree of TSH suppression }\end{array}$ \\
\hline $\begin{array}{l}\text { Biochemical } \\
\text { incomplete } \\
\text { response }\end{array}$ & $\begin{array}{l}\text { Negative imaging } \\
\text { and } \\
\text { Suppressed } \mathrm{Tg} \geq 1 \mathrm{ng} / \mathrm{mL}^{\mathrm{b}} \\
\text { or } \\
\text { Stimulated } \mathrm{Tg} \geq 10 \mathrm{ng} / \mathrm{mL}^{\mathrm{b}} \\
\text { or } \\
\text { Rising anti-Tg antibody levels }\end{array}$ & $\begin{array}{l}\text { At least } 30 \% \text { spontaneously }^{\mathrm{d}} \\
\text { evolve to NED } \\
20 \% \text { achieve NED after } \\
\text { additional therapy }{ }^{\mathrm{a}} \\
20 \% \text { develop structural disease }^{\mathrm{a}} \\
<1 \% \text { disease specific death }\end{array}$ & $\begin{array}{l}\text { If associated with stable or } \\
\text { declining serum Tg values, a } \\
\text { biochemical incomplete } \\
\text { response should lead to } \\
\text { continued observation with } \\
\text { ongoing TSH suppression in } \\
\text { most patients. Rising Tg or } \\
\text { anti-Tg antibody values should } \\
\text { prompt additional investigations } \\
\text { and potentially additional } \\
\text { therapies. }\end{array}$ \\
\hline $\begin{array}{l}\text { Structural } \\
\text { incomplete } \\
\text { response }\end{array}$ & $\begin{array}{l}\text { Structural or functional } \\
\text { evidence of disease } \\
\text { With any Tg level } \\
\text { With or without anti-Tg } \\
\text { antibodies }\end{array}$ & $\begin{array}{l}50 \%-85 \% \text { continue to have } \\
\text { persistent disease despite } \\
\text { additional therapy } \\
\text { Disease specific death rates as } \\
\text { high as } 11 \% \text { with loco-regional } \\
\text { metastases and } 50 \% \text { with } \\
\text { structural distant metastases }\end{array}$ & $\begin{array}{l}\text { A structural incomplete response } \\
\text { may lead to additional treatments } \\
\text { or ongoing observation depending } \\
\text { on multiple clinico-pathologic } \\
\text { factors including the size, } \\
\text { location, rate of growth, RAI } \\
\text { avidity, }{ }^{18} \text { FDG avidity, and } \\
\text { specific pathology of the } \\
\text { structural lesions. }\end{array}$ \\
\hline $\begin{array}{l}\text { Indeterminate } \\
\text { response }\end{array}$ & $\begin{array}{l}\text { Nonspecific findings on } \\
\text { imaging studies } \\
\text { Faint uptake in thyroid bed } \\
\text { on RAI scanning } \\
\text { Nonstimulated Tg detectable, } \\
\text { but }<1 \mathrm{ng} / \mathrm{mL} \\
\text { Stimulated Tg detectable, } \\
\text { but }<10 \mathrm{ng} / \mathrm{mL} \\
\text { or } \\
\text { Anti-Tg antibodies stable or } \\
\text { declining in the absence } \\
\text { of structural or functional } \\
\text { disease }\end{array}$ & $\begin{array}{l}15 \%-20 \% \text { will have structural } \\
\text { disease identified during } \\
\text { follow-up } \\
\text { In the remainder, the nonspecific } \\
\text { changes are either stable, } \\
\text { or resolve } \\
<1 \% \text { disease specific death }\end{array}$ & $\begin{array}{l}\text { An indeterminate response should } \\
\text { lead to continued observation } \\
\text { with appropriate serial imaging } \\
\text { of the nonspecific lesions and } \\
\text { serum Tg monitoring. } \\
\text { Nonspecific findings that } \\
\text { become suspicious over time } \\
\text { can be further evaluated with } \\
\text { additional imaging or biopsy. }\end{array}$ \\
\hline
\end{tabular}

NED denotes a patient as having no evidence of disease at final follow-up.

${ }^{\mathrm{a}}$ References $(538,539)$

${ }^{\mathrm{b}}$ In the absence of anti-Tg antibodies.

${ }^{c}$ References $(538,539,542,586-593,595-601,1078)$.

${ }^{\mathrm{d}}$ References $(598,599,617-621)$.

${ }^{\mathrm{e}}$ References $(328,607,626,627,898)$.

While most studies have assessed response to therapy using TSH-stimulated Tg values obtained 6-18 months after initial therapy $(538,539,542,586,588-590,593-595,597-600)$, at least 15 other studies have evaluated the response to surgical therapy using a stimulated Tg obtained at the time of RAI remnant ablation (605). Patients demonstrating an excellent response to therapy at this very early time point have a very low risk of disease recurrence (605).

Four additional studies used nonstimulated sensitive $\mathrm{Tg}$ assays to define an excellent response to therapy at various time points after initial therapy with total thyroidectomy and RAI remnant ablation $(587,595,601,606)$. A recurrence rate of $1.5 \%$ was seen in a cohort of 589 DTC patients who had a nonstimulated $\mathrm{Tg}<0.27 \mathrm{ng} / \mathrm{mL}$ at 3 months after initial therapy (595). Malandrino et al. (587) reported recurrence rates of $0 \%$ in low-risk patients, $1 \%$ in intermediate-risk patients, and $2.7 \%$ in high-risk patients who demonstrated nonstimulated $\mathrm{Tg}$ values $<0.15 \mathrm{ng} / \mathrm{mL}$ at $9-18$ months after initial therapy. Smallridge et al. (606) described a $4.3 \%$ recurrence rate in 163 low- to intermediate-risk DTC patients with nonstimulated Tg $<0.1 \mathrm{ng} / \mathrm{mL}$, measured a median of 1.8 years after initial surgery. Finally, Giovanella et al. (601) reported a $1.6 \%$ recurrence rate over 5-6 years of follow-up in 185 low-risk patients who had a nonstimulated $\mathrm{Tg}$ of $<0.2 \mathrm{ng} / \mathrm{mL}$ and normal postoperative neck US 6 months after remnant ablation.

Further studies are needed to refine the precise $\mathrm{Tg}$ value cutoff used to define what should be an excellent response to therapy in patients treated with total thyroidectomy with or without RAI remnant ablation. In addition to determining whether TSH-stimulated $\mathrm{Tg}$ values are clinically helpful in 
Initial Therapy

Total Thyroidectomy (R35)

valuation of Post-Operative Disease Status

Routine use of post-op serum thyroglobulin (R50B, R50C)

Post-op diagnostic RAI scanning (R50D) and/or ultrasound may be considered [B34]<smiles>[CH]1[CH]C1</smiles>

RAI Remnant Ablation Not Routinely Recommended (R51A, Table 14)

If done, $30 \mathrm{mCi}$ is generally favored over higher administered activities (R55)

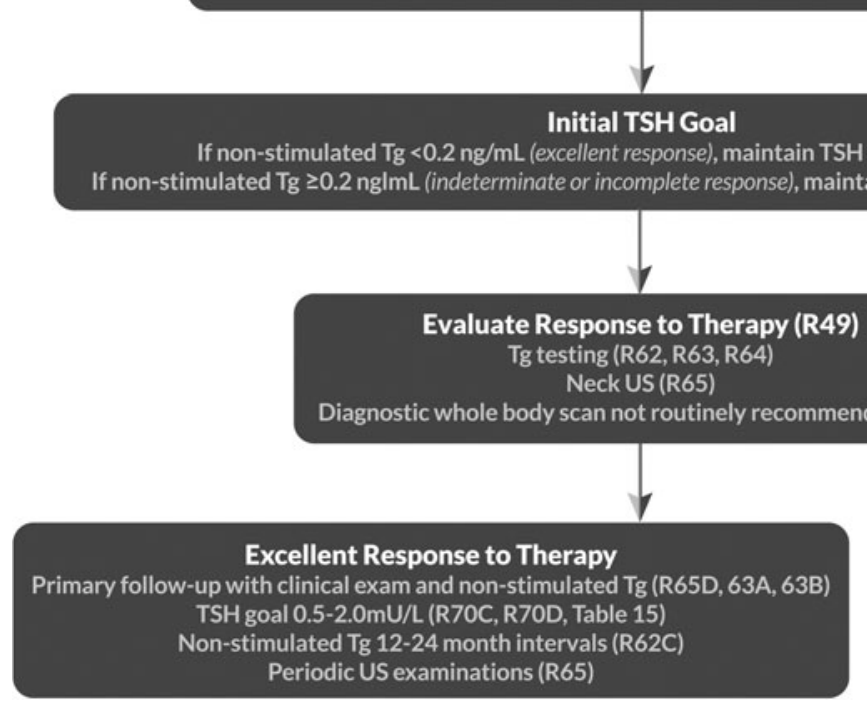

Biochemical Incomplete, Structural Incomplete,

or Indeterminate Response See text for guidance

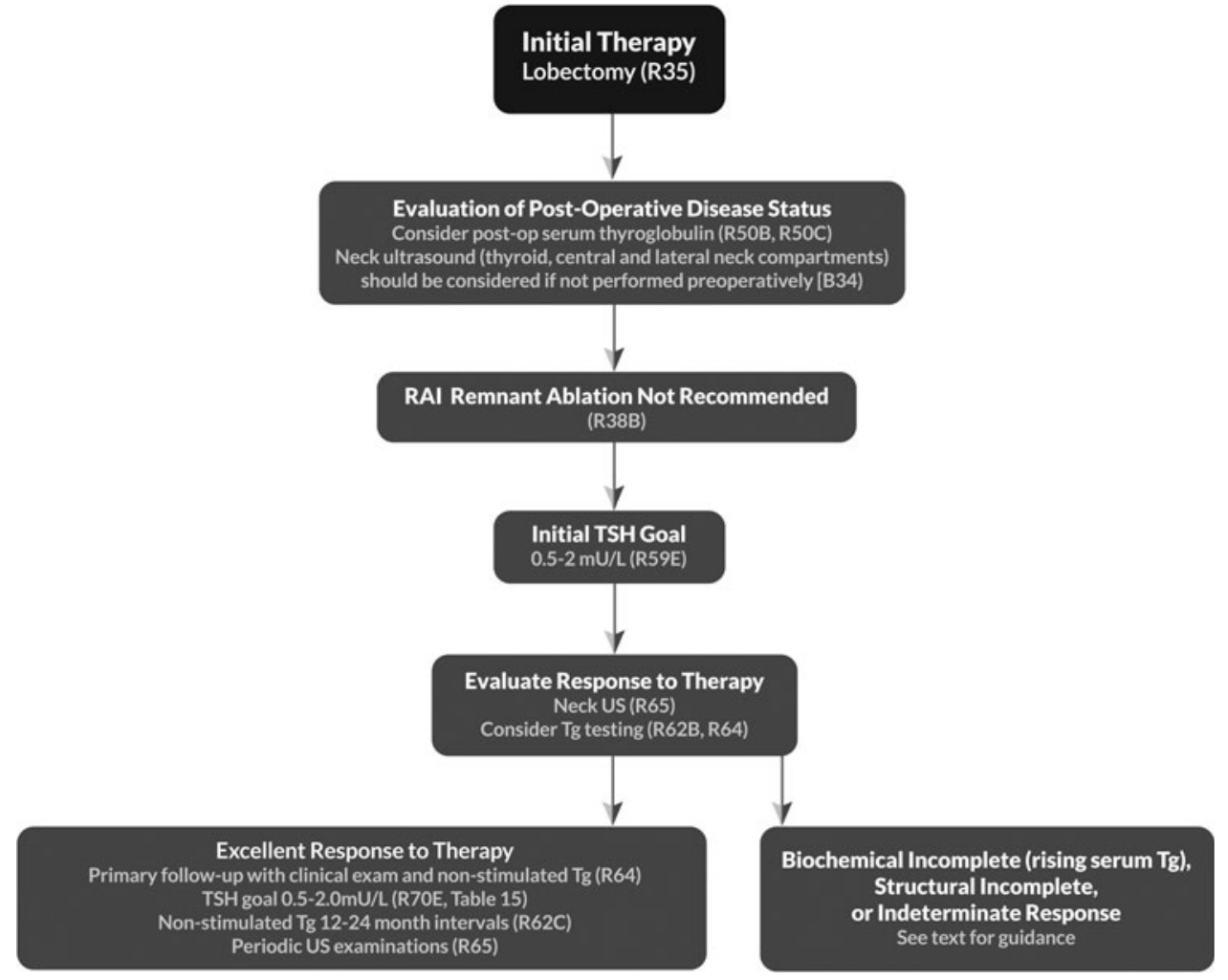

FIG. 5. Clinical decisionmaking and management recommendations in ATA low-risk DTC patients that have undergone total thyroidectomy. $\mathrm{R}$, recommendation in text.

FIG. 6. Clinical decisionmaking and management recommendations in ATA low risk DTC patients that have undergone less than total thyroidectomy (lobectomy or lobectomy with isthmusectomy). $\mathrm{R}$, recommendation in text. 
FIG. 7. Clinical decision-making and management recommendations in ATA intermediate risk DTC patients that have undergone total thyroidectomy. $\mathrm{R}$, recommendation in text.

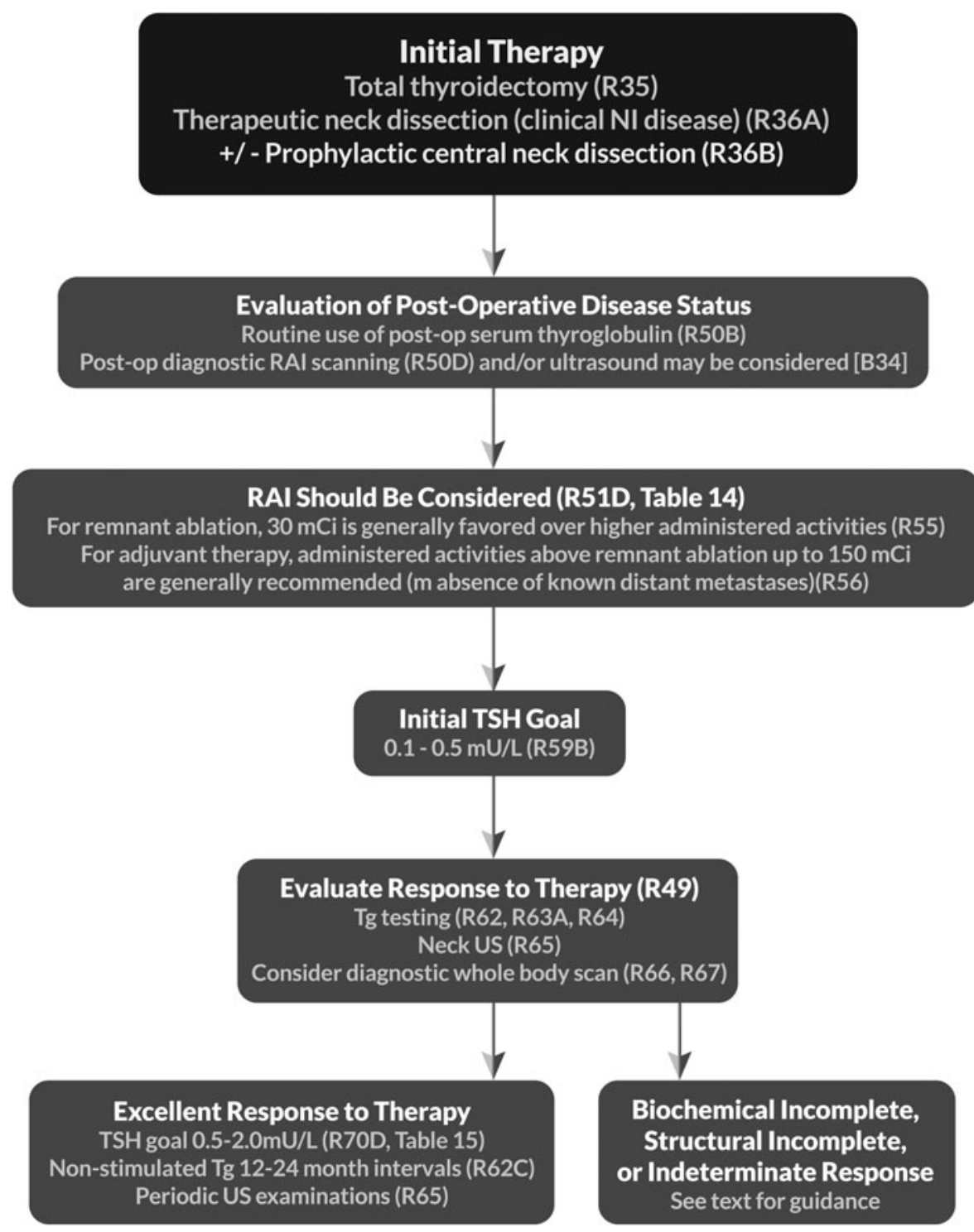

Total thyroidectomy (R35)

erapeutic neck dissection (clinical NI disease) (R36A)

+/ - Prophylactic central neck dissection (R36B)
Routine use of post-op serum thyroglobulin (R50B)

Post-op diagnostic RAl scanning (R50D) and/or ultrasound may be considered [B34]

Initial TSH Goal

$0.1-0.5 \mathrm{mU} / \mathrm{L}$ (R59B)
Excellent Response to Therapy

TSH goal 0.5-2.0mU/L (R70D, Table 15)

Periodic US examinations (R65)
Biochemical Incomplete,

Structural Incomplete,

See text for guidance patients at very low risk for recurrence, $\mathrm{Tg}$ cut points need to be defined for patients whose primary treatment consisted of thyroid lobectomy or total thyroidectomy without RAI ablation.

In summary, once a patient achieves an excellent response to therapy, the initial risk of recurrence estimate should be modified and the patient reclassified as having a subsequent very low risk of recurrence. This reclassification into a very low risk of recurrence status can occur as early as several weeks (or several months) after initial therapy and is applicable to all DTC patients initially stratified as ATA low or intermediate risk of recurrence and to the few ATA high-risk patients that achieve an excellent response to therapy. Appropriate reclassification into the excellent response category with its very low risk of recurrence should lead to reevaluation of intensity of diagnostic surveillance procedures and treatment, as discussed in other sections of these clinical practice guidelines [C4-C13] (Figs. 5-8).

[B28] Biochemical incomplete response: abnormal $\mathrm{Tg}$ values in the absence of localizable disease

These patients have persistently abnormal suppressed and/ or stimulated $\mathrm{Tg}$ values or rising anti-Tg antibodies without structural evidence of disease that can be detected using riskappropriate structural and functional imaging (Table 13). Please see section [C6] for discussion of Tg measurements. Previous studies have used nonstimulated Tg values of $>1 \mathrm{ng} / \mathrm{mL}$ or TSH-stimulated Tg values of $>10 \mathrm{ng} / \mathrm{mL}$ to define a biochemical incomplete response to therapy in patients treated with total thyroidectomy and RAI ablation $(538,539,542)$. These studies used Tg assays with variable functional sensitivities, so this definition may change over time, especially for the nonstimulated $\mathrm{Tg}$ values.

A biochemical incomplete response is not an uncommon outcome and is seen in 11\%-19\% of ATA low-risk patients, $21 \%-22 \%$ of ATA intermediate-risk patients, and 16\%-18\% of ATA high-risk patients $(538,539)$.

Clinical outcomes in these patients are usually very good, with as many as $56 \%-68 \%$ being classified as having NED at final follow-up, while $19 \%-27 \%$ continue to have persistently abnormal Tg values without structural correlate, and only $8 \%-17 \%$ developing structurally identifiable disease over 5-10 years follow-up $(538,539,607)$. No deaths have been reported in patients with a biochemical incomplete response to therapy followed for up to 10 years $(539,607)$. 


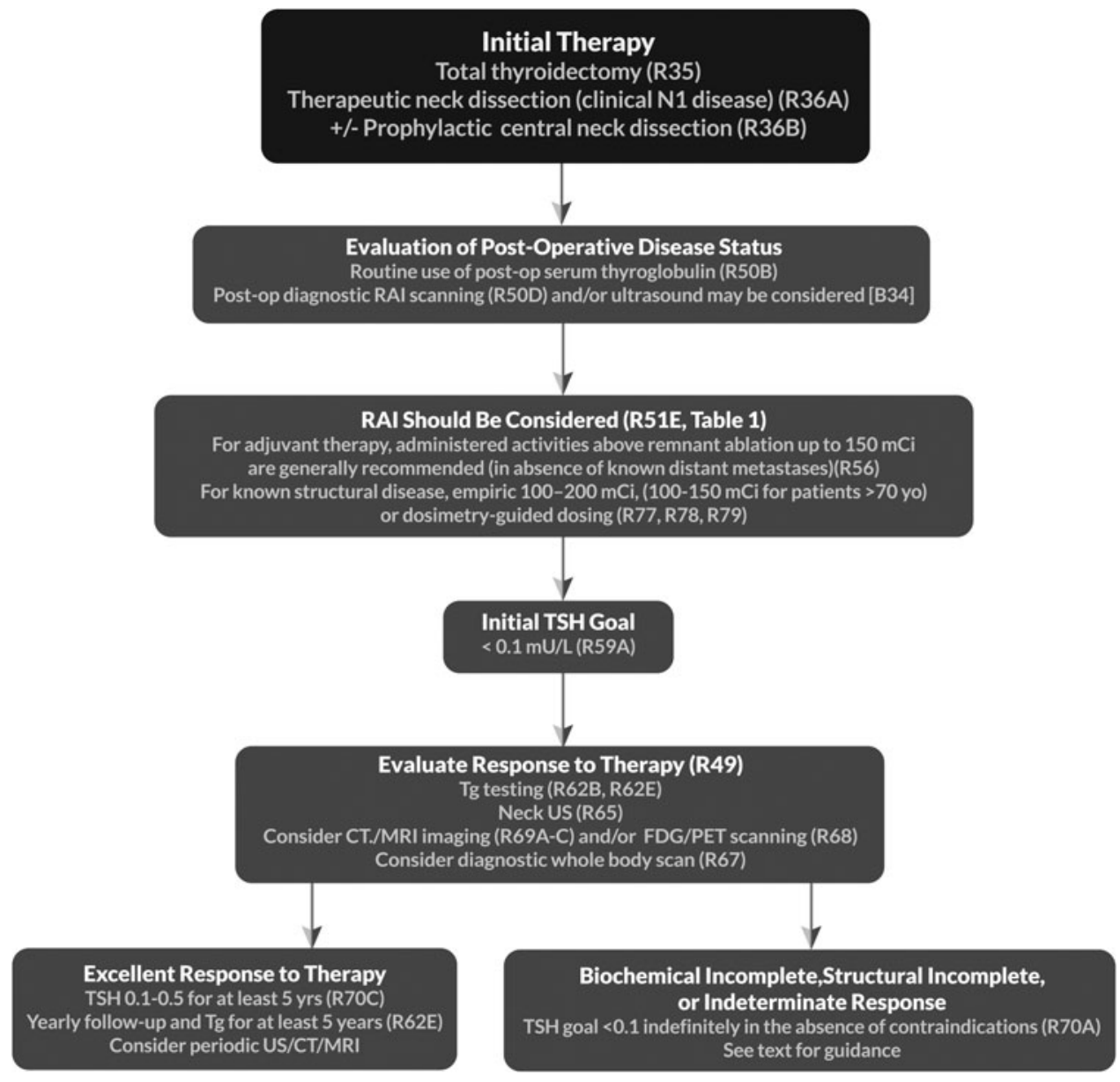

FIG. 8. Clinical decisionmaking and management recommendations in ATA high risk DTC patients that have undergone total thyroidectomy and have no gross residual disease remaining in the neck. $\mathrm{R}$, recommendation in text.
Anti-Tg antibody levels measured over time in the same assay can provide clinically useful information (608). Rising anti-Tg antibody titers (or new appearance of anti-Tg antibodies) are associated with an increased risk of disease recurrence (609-614). Conversely, patients rendered free of disease with initial therapy will usually demonstrate a decline in anti-Tg antibody titers over several years $(611,615,616)$.

Vaisman et al. (607) further demonstrated that the transition to NED status occurred without any additional RAI or surgical therapy (beyond $\mathrm{LT}_{4}$ suppressive therapy) in $34 \%$ of patients classified as having a biochemical incomplete response to initial therapy. These observations are consistent with several previous studies that have demonstrated that abnormal serum $\mathrm{Tg}$ values can gradually decline over time without additional RAI or surgical therapy, in the absence of structurally identifiable disease $(598,599,617,617-621)$.

A small percentage of patients with a biochemical incomplete response to therapy will demonstrate progressive increases in the nonstimulated $\mathrm{Tg}$ values over time. In patients treated with total thyroidectomy and RAI remnant ablation, clinically significant increases in unstimulated serum $\mathrm{Tg}$ values over time as described by $\mathrm{Tg}$ doubling times ( $<1$ year, 1-3 years, or $>3$ years) (622) or rate of rise in unstimulated $\mathrm{Tg}$ of $\geq 0.3 \mathrm{ng} / \mathrm{mL} /$ year over time (623), identify patients at increased risk of developing structurally identifiable loco-regional or distant metastases.

Just as with the excellent response to therapy category, additional studies are needed to more precisely define what
Tg levels define an incomplete biochemical response to therapy. The specific cut point that defines an "abnormal Tg", is dependent on the corresponding TSH value, the amount of residual normal thyroid tissue after thyroidectomy, whether or not RAI ablation was performed, and the duration of time since ablation, because Tg values often decline for months to years after ablation. To define a biochemical incomplete response, previous studies have used nonstimulated Tg values of $>5 \mathrm{ng} / \mathrm{mL}$ at 6 months (624), nonstimulated $\mathrm{Tg}$ values $>1 \mathrm{ng} / \mathrm{mL}$ more than 12 months after ablation $(538,539,542)$, or TSH-stimulated $\mathrm{Tg}$ values $>10 \mathrm{ng} / \mathrm{mL}$ more than 1 year after ablation $(538,539)$. The precise $\mathrm{Tg}$ value for defining a biochemical incomplete response to therapy in patients treated with lobectomy or total thyroidectomy without ablation has not been adequately defined. In addition, some studies also classified patients with persistent or rising anti-Tg antibodies in the absence of structurally identifiable disease as having a biochemical incomplete response to therapy $(538,539,625)$.

In summary, a biochemical incomplete response is seen in approximately $15 \%-20 \%$ of DTC patients. Fortunately, many of these patients are eventually reclassified as having NED at final follow-up, often without any additional RAI or surgical treatments.

[B29] Structural incomplete response: persistent or newly identified loco-regional or distant metastases

These patients have structural or functional (RAI scan, ${ }^{18}$ FDG-PET) evidence of loco-regional or distant metastases 
$(538,539,607)$. This category includes both patients with biopsy-proven disease and also patients in whom structural or functional disease is identified, which is highly likely to be metastatic disease based on the clinical scenario (Table 13). A structural incomplete response to initial therapy is seen in $2 \%-6 \%$ of ATA low-risk patients, $19 \%-28 \%$ of ATA intermediate-risk patients, and $67 \%-75 \%$ of ATA high-risk patients $(538,539)$.

Despite additional treatments, the majority of patients classified as having a structural incomplete response will have persistent structural and/or biochemical evidence of persistent disease at final follow-up $(539,607)$. Depending on the definition used to describe patients as free from disease, higher rates of remission $(29 \%-51 \%)$ have been described following surgical intervention for patients with persistent/ recurrent loco-regional disease (626-628). While no deaths were reported over a follow-up period that extended to 15 years in patients with biochemical incomplete response to therapy, death from disease was seen in $11 \%$ of patients with a loco-regional incomplete response and in $57 \%$ of patients with structurally identifiable distant metastases $(539,607)$.

In summary, a structural incomplete response to initial therapy identifies a cohort of DTC patients that may not be cured with additional therapies and consequently demonstrate the highest risk of disease-specific mortality of any of the response-to-therapy categories. Persistent/recurrent locoregional structural disease may have a higher likelihood of responding to additional treatments and has significantly lower disease-specific mortality rates than persistent/recurrent distant metastases.

[B30] Indeterminate response: biochemical or structural findings that cannot be classified as either benign or malignant (acceptable response)

Patients with an indeterminate response have biochemical, structural, or functional findings that cannot be confidently classified as either excellent response or persistent disease $(328,538,539,606,629)$ (Table 13). Rather than forcing these patients into either the excellent or incomplete response-totherapy categories, some investigators have recommended a separate category for these patients so that they can be continued to be carefully observed, with selected patients identified for further evaluation with testing designed to establish the presence or absence of disease $(538,539)$.

For example, this category includes patients with subcentimeter avascular thyroid bed nodules or atypical cervical lymph nodes that have not been biopsied, faint uptake in the thyroid bed with undetectable stimulated $\mathrm{Tg}$ on follow-up imaging, or nonspecific abnormalities on functional or crosssectional imaging. Also included in this category are patients with nonstimulated $\mathrm{Tg}$ values that are detectable but $<1 \mathrm{ng} /$ $\mathrm{mL}$, TSH-stimulated Tg values between 1 and $10 \mathrm{ng} / \mathrm{mL}$, and stable or declining $\mathrm{Tg}$ antibodies in the same assay over time in the absence of structural disease $(538,539)$.

This issue was exemplified in a recent study evaluating the prognostic value of a highly sensitive Tg assay in which the response to therapy could not be definitively established in 16 patients that had small indeterminate pulmonary micronodules without other evidence for persistent disease (606). A similar situation arises when trying to determine the response to therapy in the $34 \%$ of patients that demonstrated nonspecific subcentimeter thyroid bed nodules after total thyroidectomy (629). Similarly, it is often difficult to be certain whether or not very low-level detectable $\mathrm{Tg}$ values represent persistent disease or simply remnant normal thyroid cells remaining after initial therapy.

An indeterminate response to initial therapy is seen in $12 \%-$ $29 \%$ of ATA low-risk patients, $8 \%-23 \%$ of ATA intermediaterisk patients, and $0 \%-4 \%$ of ATA high-risk patients $(538,539)$. The clinical outcomes in patients with an indeterminate response to therapy are intermediate between patients with an excellent response and those with incomplete responses. Two series have demonstrated that only $13 \%-20 \%$ of patients with an indeterminate response to therapy are reclassified as persistent/recurrent disease over approximately 10 years of followup. In the remaining $80 \%-90 \%$ of patients, the nonspecific findings either remain stable or resolve with observation alone.

In summary, the majority of patients with an indeterminate response to therapy remain disease-free during prolonged follow-up. However, up to $20 \%$ of these patients will eventually have biochemical, functional, or structural evidence of disease progression and may require additional therapies.

[B31] Using risk stratification to guide disease surveillance and therapeutic management decisions

Risk stratification is the cornerstone of individualized thyroid cancer management. Initial risk estimates are useful to guide the wide variety of clinical management decisions that need to be made around the time of initial diagnosis and treatment. As described in this document, initial management decisions are largely made by balancing the estimates of the risk of recurrence and the risk of disease-specific mortality with the potential benefits and risks of proposed therapies. However, in clinical practice many other risk estimates can also significantly influence surveillance and therapeutic decision-making, including the risk of failing initial therapy, the risk of having non-RAI-avid disease, the risk of disease recurring without making appreciable amounts of serum $\mathrm{Tg}$, the risk of adjuvant RAI therapy, the risk of additional thyroid surgery, the risk of additional lymph node surgery, the risk of external beam radiation therapy, and the risk of systemic therapy. Individual management recommendations require that the risks and benefits of potential surveillance and therapeutic management decisions be carefully evaluated in the context of the specific clinicopathologic features of each patient.

Nonetheless, initial risk estimates can be used to guide recommendations with regard to the extent of thyroid surgery, the need for and extent of cervical lymph node dissection, the need for and the dose of administered activities of RAI, the need for and degree of TSH suppression, the need for and details of external beam radiation therapy, the need for and types of systemic therapy, the need for and types of studies required for initial staging, and the intensity and type of followup studies required for evaluating response to therapy in the early years following initial therapy. This approach tailors the aggressiveness of intervention and follow-up to the specific risks associated with the tumor in an individual patient.

In summary, this risk-adapted management approach utilizes initial risk estimates to guide early surveillance and therapeutic management decisions. These initial management plans are then modified over time as additional data accumulate and allow for restratification based on individual response to therapy. This system tailors the extent and 
intensity of therapy and follow-up studies to real-time risk estimates that evolve over time for individual patients.

\section{[B32] Should postoperative disease status be considered in decision-making for RAI therapy for patients with DTC?}

\section{- RECOMMENDATION 50}

(A) Postoperative disease status (i.e., the presence or absence of persistent disease) should be considered in deciding whether additional treatment (e.g., RAI, surgery, or other treatment) may be needed.

\section{(Strong recommendation, Low-quality evidence)}

(B) Postoperative serum $\mathrm{Tg}$ (on thyroid hormone therapy or after TSH stimulation) can help in assessing the persistence of disease or thyroid remnant and predicting potential future disease recurrence. The Tg should reach its nadir by 3-4 weeks postoperatively in most patients.

\section{(Strong recommendation, Moderate-quality evidence)}

(C) The optimal cutoff value for postoperative serum $\mathrm{Tg}$ or state in which it is measured (on thyroid hormone therapy or after TSH stimulation) to guide decision-making regarding RAI administration is not known.

\section{(No recommendation, Insufficient evidence)}

(D) Postoperative diagnostic RAI WBSs may be useful when the extent of the thyroid remnant or residual disease cannot be accurately ascertained from the surgical report or neck ultrasonography, and when the results may alter the decision to treat or the activity of RAI that is to be administered. Identification and localization of uptake foci may be enhanced by concomitant single photon emission computed tomography-computed tomography (SPECT/ CT). When performed, pretherapy diagnostic scans should utilize ${ }^{123} \mathrm{I}(1.5-3 \mathrm{mCi})$ or a low activity of ${ }^{131} \mathrm{I}(1-3 \mathrm{mCi})$, with the therapeutic activity optimally administered within 72 hours of the diagnostic activity.

\section{(Weak recommendation, Low-quality evidence)}

Postoperative disease status is a relevant consideration in postoperative treatment decision-making after initial consideration of clinic-pathologic stage. Evaluation of postoperative disease status may be performed by a number of means including serum Tg, neck ultrasonography, and iodine radioisotope scanning. There are currently no RCTs comparing any particular postoperative diagnostic strategy with the intention of modulating decision-making on RAI remnant ablation or RAI treatment for DTC.

[B33] Utility of postoperative serum Tg in clinical decisionmaking

Serum Tg measurements (with anti-Tg antibodies), with or without neck US, are frequently performed as part of the early postoperative evaluation. Please see section [C6] for discussion of $\mathrm{Tg}$ measurements. The predictive value of the postoperative $\mathrm{Tg}$ value will be significantly influenced by a wide variety of factors including the amount of residual thyroid cancer and/or normal thyroid tissue, the TSH level at the time of $\mathrm{Tg}$ measurement, the functional sensitivity of the Tg assay, the Tg cutoff used for analysis, the individual risk of having RAI-avid loco-regional or distant metastasis, the time elapsed since total thyroidectomy, and/or the sensitivity of the posttherapy scanning technique (SPECT/CT vs. planar imaging).

Multiple studies have confirmed an increase risk of recurrence following total thyroidectomy and RAI remnant ablation in patients that had a postoperative TSH-stimulated $\mathrm{Tg}>1-2 \mathrm{ng} / \mathrm{mL}$ at the time of ablation $(596,605,630-637)$. In multivariate analysis, the postoperative $\mathrm{Tg}$ is often found to be an independent predictor of persistent or recurrent disease $(596,630,631,636,637)$. Furthermore, the risk of having recurrent or persistent disease increases as the postoperative $\mathrm{Tg}$ rises $(634,636)$. Using receiver operator curve analyses, thyroid hormone withdrawal postoperative $\mathrm{Tg}$ values between 20 and $30 \mathrm{ng} / \mathrm{mL}$ achieve the optimal balance of sensitivity and specificity for predicting recurrent or persistent disease (638-640). Furthermore, high postoperative stimulated $\mathrm{Tg}$ values $(>10-30 \mathrm{ng} / \mathrm{mL})$ are also associated with poorer survival $(636,639,641)$. Conversely, postoperative stimulated Tg values less than $1-2 \mathrm{ng} / \mathrm{mL}$ are strong predictors of remission $(634,636)$. Even in ATA low- and intermediate-risk patients that did not receive RAI remnant ablation, a nonstimulated postoperative $\mathrm{Tg}<1 \mathrm{ng} / \mathrm{mL}$ was associated with excellent clinical outcomes and recurrence rates $<1 \%$ (642). The median follow-up in this study was 62 months (2-116 months). Therefore, a postoperative serum $\mathrm{Tg}$ can provide valuable information with regard to the likelihood of achieving remission or having persistent or recurrent disease in response to an initial therapy.

A postoperative $\mathrm{Tg}<10 \mathrm{ng} / \mathrm{mL}$ may not distinguish between nodal disease and thyroid remnant, when evaluated using concurrent RAI scans with SPECT/CT (643). In one of the prospective studies mentioned previously, a postoperative $\mathrm{Tg}$ threshold of $>5 \mathrm{ng} / \mathrm{mL}$ was suggested as an indication for RAI treatment (633). However, in a recent retrospective review of consecutive low-risk patients treated with total thyroidectomy without RAI, an unstimulated $\mathrm{Tg}$ of $\geq 2 \mathrm{ng} / \mathrm{mL}$ with a concomitant median TSH level of $0.48 \mathrm{mIU} / \mathrm{L}$ was reported to detect all patients with disease recurrence (76 patients followed for a median of 2.5 years) (644). Thus, there is some uncertainty as to what degree of postoperative stimulated or unstimulated thyroglobulinemia (with or without neck US interpretation) may be appropriate to prompt RAI treatment. Moreover, detection of unexplained inappropriate thyroglobulinemia may prompt consideration of further investigation for its cause (e.g., imaging studies).

The postoperative $\mathrm{Tg}$ can also be used to predict the likelihood of identifying RAI-avid metastatic thyroid cancer outside the thyroid bed on the posttherapy scan at the time of remnant ablation. No uptake outside the thyroid bed was identified in 63 low-risk patients with a nonstimulated postoperative $\mathrm{Tg}$ of $<0.4 \mathrm{ng} / \mathrm{mL}$ (630) or in 132 low-risk patients with a thyroid hormone withdrawal $\mathrm{Tg}$ of $<1 \mathrm{ng} / \mathrm{mL}$ (645). However, RAI-avid metastatic foci outside the thyroid bed were detected in $12 \%$ of intermediate-risk patients with a suppressed $\mathrm{Tg}$ of $<0.6 \mathrm{ng} / \mathrm{mL}$ (646), $5.6 \%$ of intermediate/ high risk patients with a suppressed Tg of $<1.0 \mathrm{ng} / \mathrm{mL}$ (647), and $6.3 \%$ of intermediate/high-risk patients with a thyroid hormone withdrawal stimulated $\mathrm{Tg}$ of $<2 \mathrm{ng} / \mathrm{mL}$ (648). The likelihood of finding RAI-avid metastatic disease on the posttherapy scan is substantially lower $(2.8 \%)$ if the postoperative 
$\mathrm{Tg}$ is undetectable in three different $\mathrm{Tg}$ assays than if it is undetectable only in a single assay (30\%) (649). Conversely, the likelihood of identifying either loco-regional or distant metastases on the posttherapy scan increases as either the suppressed or stimulated $\mathrm{Tg}$ values rise above $5-10 \mathrm{ng} / \mathrm{mL}(631,646,647,650)$. Therefore, neither a stimulated or suppressed postoperative $\mathrm{Tg}$ of $<1 \mathrm{ng} / \mathrm{mL}$ can completely eliminate the possibility that a posttherapy RAI scan will identify metastatic foci outside the thyroid bed. However, postoperative Tg values greater than $5-10 \mathrm{ng} / \mathrm{mL}$ increase the likelihood of identifying RAI-avid metastatic disease on the posttherapy scan.

The postoperative serum $\mathrm{Tg}$ value can also be used to predict the likelihood of successful remnant ablation. Postoperative thyroid hormone withdrawal stimulated $\mathrm{Tg}$ values $>5-6 \mathrm{ng} / \mathrm{mL}$ were associated with higher rates of failed ablation after administered activities of both $30 \mathrm{mCi}(651)$ and $100 \mathrm{mCi}$ (652). A TSH-stimulated $\mathrm{Tg}>6 \mathrm{ng} / \mathrm{mL}$ was associated with a 5-fold greater risk of failing ablation after an activity of $30 \mathrm{mCi}$ administered after preparation with thyroid hormone withdrawal (651).

It does appear that a postoperative $\mathrm{Tg}$ value (either TSHstimulated or nonstimulated) is an important prognostic factor that can be used to guide clinical management. Given a disappearance half-life of 1-3 days (653-658), the postoperative $\mathrm{Tg}$ should reach its nadir by $3-4$ weeks postoperatively in nearly all patients. In low-risk patients, a suppressed or stimulated $\mathrm{Tg}<1 \mathrm{ng} / \mathrm{mL}$ is very reassuring and further confirms classification of the patients as being at low risk. In intermediate-risk patients, postoperative Tg values $<1 \mathrm{ng} / \mathrm{mL}$ are reassuring, but do not completely rule out the presence of small-volume RAI-avid metastatic disease. However, even without RAI ablation, many intermediate risk patients have excellent clinical outcomes. Therefore, it is not clear that additional therapy is required in these intermediate-risk patients with postoperative $\mathrm{Tg}$ values $<1 \mathrm{ng} / \mathrm{mL}$ even though small-volume RAI-avid disease may still be present after thyroidectomy.

On the other hand, postoperative Tg values (stimulated or nonstimulated) greater than $10-30 \mathrm{ng} / \mathrm{mL}$ increase the likelihood of having persistent or recurrent disease, failing initial RAI ablation, having distant metastases, and dying of thyroid cancer. Therefore, postoperative $\mathrm{Tg}$ values $>10 \mathrm{ng} / \mathrm{mL}$ will likely lead to additional evaluations and possibly even additional therapies.

With regard to decision-making on the need for RAI remnant ablation, it appears that the postoperative serum $\mathrm{Tg}$ value will be more helpful in identifying patients that may benefit from RAI ablation rather than in identifying patients that do not require ablation. For example, a postoperative $\mathrm{Tg}$ value $>5-10 \mathrm{ng} / \mathrm{mL}$ may lead to selection of RAI ablation in an ATA low-risk patient or ATA intermediate-risk patient that otherwise would not have required RAI ablation (selective use) in order to improve initial staging and facilitate followup. Conversely, in high-risk patients, a postoperative Tg value $<1 \mathrm{ng} / \mathrm{mL}$ does not rule out RAI-avid disease and therefore is unlikely to alter the decision to proceed with RAI ablation.

[B34] Potential role of postoperative US in conjunction with postoperative serum $\mathrm{Tg}$ in clinical decision-making

In a prospective study of 218 DTC patients, Lee et al. (659) reported that a stimulated $\mathrm{Tg}<2 \mathrm{ng} / \mathrm{mL}$ after thyroid hormone withdrawal (with goal TSH of $>30 \mathrm{mIU} / \mathrm{L}$ ), at the time of administration of $100-200 \mathrm{mCi}$ of ${ }^{131} \mathrm{I}$ (for remnant ablation or treatment), was associated with the following NPVs for biochemical or structural recurrence at 6-12 months: $98.4 \%$ for ATA low-risk patients, $94.1 \%$ for ATA intermediate-risk patients, and $50 \%$ in the ATA high-risk group. They further reported that the NPVs increased to $97.2 \%$, and $100 \%$ for ATA intermediate- and high-risk patients, respectively, when the stimulated Tg values were combined with negative neck US findings at baseline (with no change in low-risk patients). Similar significant decreases in the risk of recurrence were seen when ATA intermediate- and high-risk patients had a normal postoperative neck US (660).

[B35] Role of postoperative radioisotope diagnostic scanning in clinical decision-making

Iodine radioisotope diagnostic testing may include ${ }^{131} \mathrm{I}$ or ${ }^{123}$ I diagnostic imaging with or without SPECT-CT, and/or RAI uptake measurements. Postoperative RAI planar imaging $\left({ }^{123} \mathrm{I}\right.$ or ${ }^{131} \mathrm{I}$, with or without SPECT-CT) has been reported to yield information that could alter clinical management (such as altering disease status assessment) in $25 \%-53 \%$ of patients, as reported in single-center, retrospective studies $(643,661,662)$. However, in a multivariate analysis of retrospective data, $\mathrm{Hu}$ et al. (663) reported that the use of $5 \mathrm{mCi}$ of ${ }^{131}$ I between 4 and 11 days prior to remnant ablation was independently associated with an increased risk of remnant ablation failure. In contrast, in a smaller retrospective study, the administration of 3-5 $\mathrm{mCi}$ of ${ }^{131}$ I for scanning 2-5 days prior to ablation in 37 patients was not associated with any significant reduction in remnant ablation success, compared to no pretherapy scanning in 63 patients ( ${ }^{131}$ I therapeutic activity of $100-200 \mathrm{mCi}$ used in both groups) (664). A possible relationship between ${ }^{131}$ I diagnostic scan activity on remnant ablation success was suggested in another retrospective study, in which success was lower following the use of $3 \mathrm{mCi}$ as compared to $1 \mathrm{mCi}$ of ${ }^{131} \mathrm{I}, 9$ days before therapeutic administration of $100 \mathrm{mCi}$ (665). In two small RCTs, there was no significant impact of ${ }^{131}$ I scanning compared to ${ }^{123}$ I scanning on the rate of successful remnant ablation $(666,667)$. The timing of whole-body diagnostic scans following administration of radioisotopes in reviewed studies ranged from about 24 to 72 hours for ${ }^{131} \mathrm{I}(643,662$, $663,665-667)$ and was 24 hours for ${ }^{123} \mathrm{I}(661,662,666)$. The tailoring of RAI therapeutic activity according to RAI neck uptake (measured 24 hours after administration of 1 $\mathrm{mCi}$ of ${ }^{131} \mathrm{I}$ ) was associated with a lower rate of remnant ablation success than fixed dosing, in another single-center retrospective observational study (668). Furthermore, in a multivariate analysis of retrospective data (adjusted for relevant risk factors), Verburg et al. (669) reported that the use of $1 \mathrm{mCi}$ of ${ }^{131} \mathrm{I}$ for calculation of RAI neck uptake 2 days before remnant ablation was independently associated with an increased risk of remnant ablation failure, although Yap and Murby showed that $1.1 \mathrm{mCi}^{131}$ I diagnostic scans did not adversely affect the success of ablation or recurrence rate at 3 years $(670)$.

There continues to be discussion on the utility of postoperative iodine radioisotope diagnostic scanning (with or without SPECT/CT) in guiding RAI therapeutic decision-making. Valuable information on disease status, remnant uptake, and the presence of residual RAI-avid disease may be obtained by such testing, which could alter management and potentially 
benefit outcome. Questions regarding the potentially negative impact of such scans with ${ }^{131}$ I on RAI therapeutic efficacy for successful remnant ablation ("stunning") may be mitigated or avoided by the use of either low-activity ${ }^{131} \mathrm{I}(1-3 \mathrm{mCi})$ or alternative isotopes such as ${ }^{123} \mathrm{I}$.

\section{[B36] What is the role of RAI (including remnant ablation, adjuvant therapy, or therapy for persistent disease) after thyroidectomy in the primary management of DTC?}

\section{- RECOMMENDATION 51 (details in Table 14)}

(A) RAI remnant ablation is not routinely recommended after thyroidectomy for ATA low-risk DTC patients. Consideration of specific features of the individual patient that could modulate recurrence risk, disease follow-up implications, and patient preferences are relevant to RAI decision-making.

\section{(Weak recommendation, Low-quality evidence)}

(B) RAI remnant ablation is not routinely recommended after lobectomy or total thyroidectomy for patients with unifocal papillary microcarcinoma, in the absence of other adverse features.

(Strong recommendation, Moderate-quality evidence)

(C) RAI remnant ablation is not routinely recommended after thyroidectomy for patients with multifocal papillary microcarcinoma in absence of other adverse features. Consideration of specific features of the individual patient that could modulate recurrence risk, disease follow-up implications, and patient preferences are relevant to RAI decision-making.

\section{(Weak recommendation, Low-quality evidence)}

(D) RAI adjuvant therapy should be considered after total thyroidectomy in ATA intermediate-risk level DTC patients.

\section{(Weak recommendation, Low-quality evidence)}

(E) RAI adjuvant therapy is routinely recommended after total thyroidectomy for ATA high risk DTC patients

\section{(Strong recommendation, Moderate-quality evidence)}

Depending on the postoperative risk stratification of the individual patient, the primary goal of postoperative administration of RAI after total thyroidectomy may include (i) RAI remnant ablation (to facilitate detection of recurrent disease and initial staging by tests such as Tg measurements or whole-body RAI scans), (ii) RAI adjuvant therapy (intended to improve disease-free survival by theoretically destroying suspected, but unproven residual disease, especially in patients at increased risk of disease recurrence), or (iii) RAI therapy (intended to improve disease-specific and disease-free survival by treating persistent disease in higher risk patients). Additional considerations in RAI decision-making may include patient comorbidities (and the potential impact of therapeutic doses of RAI or preparation for the procedure), feasible or preferred disease surveillance procedures, patient preferences (the latter being particularly important when clear data on therapeutic efficacy are lacking), or others. It is important to note that in patients with low-risk DTC, disease surveillance may be accomplished without RAI ablation using neck US and Tg with $\mathrm{Tg}$ antibody measurements while on thyroid hormone therapy.

We categorized the results of our review according to the ATA Risk of Recurrence Risk stratification (outlined in a preceding section of these guidelines). However, given that the ATA risk classification is relatively new and the majority of studies examining therapeutic efficacy of postsurgical RAI remnant ablation or therapy (adjuvant or for persistent disease) have been performed with attention to traditional mortality risk stratification systems such as the AJCC/TNM system, MACIS, National Thyroid Cancer Treatment Cooperative Study Group (NTCTCSG), or others, it was necessary to extrapolate the results of many studies according to estimated ATA risk level. We have also categorized some of the results of our evidence review according to the $\mathrm{AJCC} /$ TNM risk of mortality stratification system because this system has been in use longer in our field (Table 14). Evaluation of postoperative disease status and recommendations for RAI remnant ablation and adjuvant therapy can be found in algorithms in Figs. 5-8.

ATA low risk. Studies examining the impact of RAI remnant ablation/adjuvant therapy on long-term thyroid cancer outcomes in ATA low-risk patients are subject to limitations due to their observational nature (and potential for bias), as well as limited statistical power to detect relatively uncommon events (such as disease-related mortality). By definition, the risk of disease-specific mortality is low, the risk of persistent/recurrent disease is low (around 3\%), and there is no evidence that delayed discovery and treatment of persistent disease may decrease the chance of cure in these patients. In a retrospective multicenter registry study, 1298 DTC patients categorized as being in the ATA low-risk level, were followed for a median of 10.3 years, and there was no significant effect of RAI adjuvant therapy on overall or disease-free survival, using respective multivariate and stratified propensity analysis techniques (544). Prospective data from the NTCTCSG suggest that overall disease-specific and disease-free survival are not improved by RAI treatment in NTCTCSG stage I and II patients (i.e., patients aged $<45$ years with no distant metastases or patients aged $\geq 45$ years with a primary tumor $<4 \mathrm{~cm}$ in diameter, no extrathyroidal extension, and no nodal metastases), also using multivariate analyses and propensity analyses $(671,672)$. In two systematic reviews examining results of multivariate adjusted analyses, with a focus on low-risk DTC using classic clinicpathologic staging systems such as TNM/AJCC, the majority of studies did not show a significant effect of RAI adjuvant therapy in reducing thyroid cancer-related death, and conflicting findings of studies relating to outcomes of disease recurrence $(673,674)$. A more recent systematic review of the literature supported the findings of the earlier systematic reviews (675). It is important to note that in the studies summarized in this section on ATA low-risk disease (or equivalent), patients with multifocal PTC were generally included (if no other adverse features meeting criteria for upstaging were noted). To date, there is little evidence to suggest that RAI may improve disease-specific mortality in low-risk DTC patients, and there is some conflicting evidence 
Table 14. Characteristics According to the American Thyroid Association Risk Stratification System and AJCC/TNM Staging System That May Impact Postoperative Radioiodine Decision-Making

\begin{tabular}{|c|c|c|c|c|}
\hline $\begin{array}{l}\text { ATA risk } \\
\text { Staging (TNM) }\end{array}$ & Description & $\begin{array}{l}\text { Body of evidence } \\
\text { suggests RAI im- } \\
\text { proves disease- } \\
\text { specific survival? }\end{array}$ & $\begin{array}{l}\text { Body of evidence } \\
\text { suggests RAI im- } \\
\text { proves disease- } \\
\text { free survival? }\end{array}$ & Postsurgical RAI indicated? \\
\hline $\begin{array}{l}\text { ATA low risk } \\
\text { T1a } \\
\text { N0,Nx } \\
\text { M0,Mx }\end{array}$ & $\begin{array}{l}\text { Tumor size } \leq 1 \mathrm{~cm} \\
\text { (uni-or multi- } \\
\text { focal) }\end{array}$ & No & No & No \\
\hline $\begin{array}{l}\text { ATA low risk } \\
\text { T1b,T2 } \\
\text { N0, Nx } \\
\text { M0,Mx }\end{array}$ & $\begin{array}{c}\text { Tumor size } \\
>1-4 \mathrm{~cm}\end{array}$ & No & $\begin{array}{l}\text { Conflicting } \\
\text { observational } \\
\text { data }\end{array}$ & $\begin{array}{l}\text { Not routine }{ }^{\mathrm{b}} \text {-May be considered for } \\
\text { patients with aggressive histology or } \\
\text { vascular invasion (ATA intermedi- } \\
\text { ate risk). }\end{array}$ \\
\hline $\begin{array}{l}\text { ATA low to in- } \\
\text { termediate risk } \\
\text { T3 } \\
\text { N0,Nx } \\
\text { M0,Mx }\end{array}$ & Tumor size $>4 \mathrm{~cm}$ & Conflicting data & $\begin{array}{l}\text { Conflicting } \\
\text { observational } \\
\text { data }\end{array}$ & $\begin{array}{l}\text { Consider }{ }^{\mathrm{b}}-\text { Need to consider presence } \\
\text { of other adverse features. Advancing } \\
\text { age may favor RAI use in some } \\
\text { cases, but specific age and tumor } \\
\text { size cutoffs subject to some } \\
\text { uncertainty. }\end{array}$ \\
\hline $\begin{array}{l}\text { ATA low to in- } \\
\text { termediate risk } \\
\text { T3 } \\
\text { N0,Nx } \\
\text { M0,Mx }\end{array}$ & $\begin{array}{l}\text { Microscopic } \\
\text { ETE, any } \\
\text { tumor size }\end{array}$ & No & $\begin{array}{l}\text { Conflicting } \\
\text { observational } \\
\text { data }\end{array}$ & $\begin{array}{l}\text { Consider }- \text { Generally favored based } \\
\text { on risk of recurrent disease. Smaller } \\
\text { tumors with microscopic ETE may } \\
\text { not require RAI. }\end{array}$ \\
\hline $\begin{array}{l}\text { ATA low to in- } \\
\text { termediate risk } \\
\text { T1-3 } \\
\text { N1a } \\
\text { M0,Mx }\end{array}$ & $\begin{array}{l}\text { Central compart- } \\
\text { ment neck } \\
\text { lymph node } \\
\text { metastases }\end{array}$ & $\begin{array}{l}\text { No, except possi- } \\
\text { bly in subgroup } \\
\text { of patients } \geq 45 \\
\text { years of age } \\
\text { (NTCTCSG } \\
\text { Stage III) }\end{array}$ & $\begin{array}{l}\text { Conflicting } \\
\text { observational } \\
\text { data }\end{array}$ & $\begin{array}{l}\text { Consider }{ }^{\mathrm{b}} \text {-Generally favored, due to } \\
\text { somewhat higher risk of persistent } \\
\text { or recurrent disease, especially with } \\
\text { increasing number of large } \\
(>2-3 \mathrm{~cm}) \text { or clinically evident } \\
\text { lymph nodes or presence of extra- } \\
\text { nodal extension. Advancing age may } \\
\text { also favor RAI use. }{ }^{\text {a However, there }} \\
\text { is insufficient data to mandate RAI } \\
\text { use in patients with few }(<5) \\
\text { microscopic nodal metastases in } \\
\text { central compartment in absence of } \\
\text { other adverse features. }\end{array}$ \\
\hline $\begin{array}{l}\text { ATA low to in- } \\
\text { termediate risk } \\
\text { T1-3 } \\
\text { N1b } \\
\text { M0,Mx }\end{array}$ & $\begin{array}{l}\text { Lateral neck or } \\
\text { mediastinal } \\
\text { lymph node } \\
\text { metastases }\end{array}$ & $\begin{array}{l}\text { No, except possi- } \\
\text { bly in subgroup } \\
\text { of patients } \geq 45 \\
\text { years of age }\end{array}$ & $\begin{array}{l}\text { Conflicting } \\
\text { observational } \\
\text { data }\end{array}$ & $\begin{array}{l}\text { Consider }{ }^{\mathrm{b}} \text {-Generally favored, due to } \\
\text { higher risk of persistent or recurrent } \\
\text { disease, especially with increasing } \\
\text { number of macroscopic or clinically } \\
\text { evident lymph nodes or presence of } \\
\text { extranodal extension. Advancing } \\
\text { age may also favor RAI use. }\end{array}$ \\
\hline $\begin{array}{l}\text { ATA high risk } \\
\text { T4 } \\
\text { Any } \mathrm{N} \\
\text { Any } \mathrm{M}\end{array}$ & $\begin{array}{l}\text { Any size, } \\
\text { gross ETE }\end{array}$ & $\begin{array}{l}\text { Yes, } \\
\text { observational } \\
\text { data }\end{array}$ & $\begin{array}{l}\text { Yes, } \\
\text { observational } \\
\text { data }\end{array}$ & Yes \\
\hline $\begin{array}{l}\text { ATA high risk } \\
\text { M1 } \\
\text { Any T } \\
\text { Any N }\end{array}$ & Distant metastases & $\begin{array}{l}\text { Yes, } \\
\text { observational } \\
\text { data }\end{array}$ & $\begin{array}{l}\text { Yes, } \\
\text { observational } \\
\text { data }\end{array}$ & Yes \\
\hline
\end{tabular}

${ }^{a}$ Recent data from the NTCTCSG (National Thyroid Cancer Treatment Cooperative Study Group) have suggested that a more appropriate prognostic age cutoff for their and other classification systems could be 55 years, rather than 45 years, particularly for women.

${ }^{\mathrm{b}}$ In addition to standard clinicopathologic features, local factors such as the quality of preoperative and postoperative US evaluations, availability and quality of Tg measurements, experience of the operating surgeon, and clinical concerns of the local disease management team may also be considerations in postoperative RAI decision-making. 
on effect on recurrence, with newer data using the ATA risk system suggesting the lack of a significant effect. Furthermore, the majority of the best available observational evidence suggests that RAI adjuvant therapy is unlikely to improve disease-specific or disease-free survival in papillary microcarcinoma ( $<1 \mathrm{~cm}$, uni- or multifocal), in the absence of other higher risk features $(146,676-680)$. In a recent retrospective analysis of 704 papillary microcarcinoma patients whose initial risk level was ATA low or intermediate who were followed for a median of 64 months, there was no significant reduction in recurrence rates in patients treated with RAI compared to those not treated with RAI using a propensity score analysis (677). With respect to microcarcinomas of follicular cancer and Hürthle cell cancer, a recent SEER registry secondary data analysis suggested no disease-specific survival benefit in patients treated with RAI in a multivariate analysis adjusted for age, histology, disease extent, type of surgery, and external beam radiation therapy (the total number of patients in this study was 564) (681). A limitation of interpreting these data on follicular and Hürthle cell microcarcinomas is that some of the patients in the study had some adverse features and were not all considered low risk; however, the authors adjusted for relevant variables in their multivariate analysis (681). The role of RAI adjuvant therapy in ATA low-risk DTC should be clarified in the future, following completion of RCTs, such as the Iodine or Not (IoN) trial for low- and intermediate-risk patients (682) and ESTIMABL2 for low-risk patients.

ATA intermediate risk. Multivariate adjusted analyses from SEER suggest that postsurgical RAI treatment is associated with improved overall survival for aggressive PTC histologies such as tall cell, diffuse sclerosing, and insular variants $(683,684)$. Furthermore, multivariate adjusted analyses from SEER suggest that RAI treatment is associated with improved overall survival in node-positive adult patients with PTC or pT3 node-negative PTC, in which the primary tumor is $>4 \mathrm{~cm}$ or there is evidence of microscopic extrathyroidal extension (685). It is important to note, however, that in this SEER study the overall survival rate was very high in node-positive or pT3-node negative PTC patients aged $<45$ years, such that $99 \%$ and $98 \%$ of such individuals were alive after a median follow-up period of 6.8 years, with or without RAI treatment, respectively. The clinical significance of this approximately $1 \%$ absolute risk difference could be questioned. In contrast, in the same study, for individuals aged $\geq 65$ years, assuming the same median study follow-up period of 6.8 years, $73 \%$ of T3-node negative or node-positive PTC patients treated with RAI and $69 \%$ of those not treated with RAI were alive (685); in this older subgroup, the absolute risk difference would be estimated to be about $4 \%$.

There is some supportive evidence from multivariate and propensity analyses that there may be a benefit of adjuvant RAI treatment in improving overall and disease-specific survival as well as disease-free survival in patients with nodal metastases aged $\geq 45$ years, as such patients would be included in the NTCTCSG stage III category (671). Furthermore, in a single-center retrospective study from Hong Kong examining data from a subgroup of 421 patients with nodepositive PTC, lymph node failure-free survival was improved with postsurgical RAI treatment, with the greatest treatment benefits observed in patients with N1b disease, as well as with lymph nodes $>1 \mathrm{~cm}$ in diameter (686). RAI therapeutic efficacy in patients $<45$ years of age with nodal metastases are unclear because such patients are categorized as stage I by the NTCTCSG system and no significant benefit of RAI treatment was observed for the stage I group in that study, with no specific subgroup analysis reported according to node positivity (671). A single-center retrospective study from the Mayo Clinic examining 20-year cause-specific mortality and recurrence rate, suggested no significant benefit in PTC patients with a MACIS score of $<6$ who had positive lymph nodes, using respective univariate analyses (278). Given that age is incorporated in the MACIS score, it is possible that age was a contributing factor in the results of that analysis. In a subgroup of 352 patients with microscopic extrathyroidal extension from a single-center retrospective study, postsurgical RAI treatment was associated with a reduction in rate of local relapse (686). However, in the NTCTCSG study, microscopic extraglandular invasion would be classified as NTCTCSG stage I for patients $<45$ years of age and II for those $\geq 45$ years of age, and those stages were associated with lack of clear benefit of RAI. In a recent systematic review, Lamartina et al. (675) reported conflicting results on the impact of RAI treatment on disease recurrence, specifically indicating that 11 nonrandomized studies suggested a benefit, whereas 13 studies did not show a significant benefit. For patients with ATA intermediate-risk DTC, limited risk-group specific data examining RAI efficacy is available, but existing data suggest that the greatest potential benefit may be observed with adverse thyroid cancer histologies, increasing volume of nodal disease, lymph node disease outside the central neck, and advancing patient age. Benefits regarding survival or recurrence can be expected primarily in patients with higher risk of recurrent or persistent disease that is iodine avid. More studies are needed, including RCTs, to characterize RAI treatment efficacy in ATA intermediate-risk patients. The adjuvant therapeutic efficacy of RAI treatment in improving long-term thyroid cancer outcomes in the situation of isolated microscopic central neck nodal disease in the absence of other adverse features is unknown, so the relatively good overall prognosis of this group (as outlined in the preceding section of these guidelines) as well as the uncertain RAI therapeutic efficacy for this subgroup, are important considerations in RAI decision-making. Clearly more research is needed to understand the therapeutic efficacy in various subgroups of patients in the ATA intermediate-risk category.

ATA high risk. A prospective multicenter study reported a significant improvement in overall and disease-specific mortality, as well as disease-free survival in NTCTCSG stage III and IV patients, after statistical adjustment using multivariate and propensity stratified analyses (671). Furthermore, prospectively collected data from the SEER cancer registry suggest that postsurgical RAI therapy is associated with improved overall survival in patients with PTC with distant metastases (when distant metastases were combined with age $>45$ years, tumor size $>2 \mathrm{~cm}$, and positive lymph nodes at primary diagnosis) (687). Data from SEER also suggest that overall survival in patients with FTC with distant metastases more than doubled in patients receiving postsurgical RAI treatment (687). Thus, routine postsurgical RAI treatment is recommended in patients with ATA high-risk DTC. 
[B37] What is the role of molecular marker status in therapeutic RAl decision-making?

\section{- RECOMMENDATION 52}

The role of molecular testing in guiding postoperative RAI use has yet to be established; therefore, no molecular testing to guide postoperative RAI use can be recommended at this time.

\section{(No recommendation, Insufficient evidence)}

Preclinical studies show that the presence of the $B R A F^{V 600 E}$ mutation significantly reduces sodium-iodide symporter expression and RAI uptake (688). There are currently insufficient clinical data, however, to know whether the presence or absence of the $B R A F^{V 600 E}$ mutation or other genetic alterations in PTC may impact the success of adjuvant therapy or remnant ablation in PTC, or if adjustments in administered activity are warranted for any planned treatments. In a recent subgroup analysis of 134 PTC patients with T1aN0M0 disease, the rate of macroscopic structural disease recurrence was $0 \%$ in the no RAI group (24\% of whom were $B R A F$ positive), $2.6 \%$ in $39 B R A F$-positive patients who received RAI, and $1.7 \%$ in $B R A F$-negative patients who received RAI (mean follow-up of 5.3 years for the entire study) (565). Of the 97 T1aN0M0 patients who received postoperative RAI, the rate of biochemical persistence of disease (defined by a stimulated Tg of $>1 \mathrm{ng} / \mathrm{mL}$ ), was $13 \%$ in the $39 B R A F$-positive patients and $1.7 \%$ in the $B R A F$-negative patients; the standard activity for remnant ablation in this study was $30 \mathrm{mCi}$ in most cases (565). The relatively small number of patients who did not receive postoperative RAI and the relatively small number of structural disease recurrences in the T1aN0M0 subgroup of PTC patients and lack of randomization in this study may preclude meaningful analysis of RAI therapeutic efficacy. The ESTIMABL2 study will analyze the relevance of $B R A F$ status on outcome (registration number NCT01837745). There are no studies examining therapeutic efficacy of RAI in ATA high-risk patients, but the presence or absence of a $B R A F^{V 600 E}$ mutation in that situation would be unlikely to alter RAI decision-making at present. Thus, the role of molecular testing in guiding postoperative RAI use has yet to be established, and more research in this area is clearly needed. Moreover, in general, RCTs examining RAI therapeutic efficacy are needed, and ideally these should be appropriately stratified for ATA recurrence risk level and other important prognostic variables.

\section{[B38] How long does thyroid hormone need to be withdrawn in preparation for RAI remnant ablation/ treatment or diagnostic scanning?}

\section{- RECOMMENDATION 53}

(A) If thyroid hormone withdrawal is planned prior to RAI therapy or diagnostic testing, $\mathrm{LT}_{4}$ should be withdrawn for 3-4 weeks. Liothyronine ( $\left.\mathrm{LT}_{3}\right)$ may be substituted for $\mathrm{LT}_{4}$ in the initial weeks if $\mathrm{LT}_{4}$ is withdrawn for 4 or more weeks, and in such circumstances, $\mathrm{LT}_{3}$ should be withdrawn for at least 2 weeks. Serum TSH should be measured prior to radioisotope administration to evaluate the degree of TSH elevation.
(Strong recommendation, Moderate-quality evidence)

(B) A goal TSH of $>30 \mathrm{mIU} / \mathrm{L}$ has been generally adopted in preparation for RAI therapy or diagnostic testing, but there is uncertainty relating to the optimum TSH level associated with improvement in long-term outcomes.

\section{(Weak recommendation, Low-quality evidence)}

Thyrotropin stimulation before RAI remnant ablation/ therapy or scanning has been a long-established standard of care because early observational research suggested that a TSH >30 mIU/L was required for incompletely resected thyroid tumors to significantly concentrate ${ }^{131} \mathrm{I}$ (689). There have been two RCTs comparing various thyroid hormone withdrawal protocols prior to therapeutic or diagnostic iodine radioisotope administration (690,691). Lee et al. (691) reported on an open-label, single-center study, in which 291 patients with well-differentiated thyroid cancer (TNM stage T1-T3, N0/N1a,M0) were randomized to either (a) withdrawal $\mathrm{LT}_{4}$ for 4 weeks $(n=89)$, (b) withdrawal of $\mathrm{LT}_{4}$ for 4 weeks with substitution of $\mathrm{LT}_{3}$ for the first 2 weeks $(n=133)$, or (c) recombinant human TSH (rhTSH; with withdrawal of $\mathrm{LT}_{4}$ for a few days from the time of the first rhTSH injection to radioisotope administration) $(n=69)$ (691). In this trial, all patients received $30 \mathrm{mCi}$ of ${ }^{131} \mathrm{I}$ for remnant ablation and were prescribed a 2-week low-iodine diet (LID) pre-ablation. Although the randomization method was unclear, the baseline characteristics (including pre-ablation urinary iodine measurements) were well balanced among groups. Furthermore, the pre-ablation TSH was $>30$ in all patients in all groups in this trial, with no significant difference in mean preablation TSH levels. Moreover, the primary outcome, which was the rate of successful remnant ablation at 12 months, was not significantly different among groups (range 91.0\%$91.7 \%$ among groups). Upon administration of questionnaires in a double-blind fashion, there was no significant difference in quality of life during preparation for RAI ablation, between the $\mathrm{LT}_{4}$ withdrawal group and the $\mathrm{LT}_{4}$ withdrawal with $\mathrm{LT}_{3}$ substitution group; however, quality of life in both withdrawal groups prior to remnant ablation was significantly worse than after rhTSH preparation (691). Long-term outcome data from this trial were not reported. In a single-center trial, Leboeuf et al. (690) randomized 20 individuals with well-differentiated thyroid cancer awaiting RAI remnant ablation or diagnostic scanning to $\mathrm{LT}_{4}$ withdrawal and either (a) substitution of $\mathrm{LT}_{3}(50 \mu \mathrm{g} / \mathrm{d}$, divided as two capsules) for 21 days, followed by 2 weeks off $\mathrm{LT}_{3}$, or (b) identical-appearing placebo for $\mathrm{LT}_{3}$ (two pills per day) for 21 days. In both groups, either the $\mathrm{LT}_{3}$ or placebo was withdrawn for another 2 weeks, and weekly measurements were performed for serum TSH, free thyroxine, and free triiodothyronine (690). The primary outcome was the hypothyroidism symptom score (Billewicz scale), which was ascertained in a double-blind fashion at time of $\mathrm{LT}_{4}$ withdrawal and every 2 weeks until the end of the study. The randomization method was a computer-generated number sequence; the $\mathrm{LT}_{3}$ group was significantly older than the placebo group (mean age 64 compared to 46), suggesting imbalance in the randomization (690). Disease stage of participants was not reported. Approximately $15 \%$ of participants withdrew from this trial (two in the placebo group and one in the $\mathrm{LT}_{3}$ group). Leboeuf et al. (690) reported no significant differences between the two thyroid hormone 
withdrawal protocol groups for hypothyroid symptom scores at any time point in the trial in a protocol-based analysis. At the time of ablation or whole-body scanning, the mean TSH was not significantly different between groups. In summary, available evidence from recent RCTs suggests that either direct $\mathrm{LT}_{4}$ withdrawal or $\mathrm{LT}_{4}$ withdrawal with substitution of $\mathrm{LT}_{3}$ in initial weeks is associated with similar short-term quality of life and hypothyroidism symptom scores; moreover, the remnant ablation success rate appears comparable.

There is some conflicting observational evidence on whether any specific pre-RAI administration TSH level is associated with success of remnant ablation (692-696). For example, in a secondary analysis of a RAI remnant ablation activity RCT, Fallahi et al. (692) reported that a pre-RAI TSH of $>25$ following $\left(\mathrm{LT}_{4}\right.$ and $\left.\mathrm{LT}_{3}\right)$ thyroid hormone withdrawal was significantly associated with increased likelihood of successful remnant ablation (odds ratio 2.36, [95\% CI 1.28-4.35], $p=0.006$ ), after adjustment for RAI activity, baseline serum $\mathrm{Tg}$, on- $\mathrm{LT}_{4} \mathrm{TSH}$ level, sex, age, histology, baseline RAI uptake, and extent of surgery). In two retrospective studies, each including several hundred DTC patients who underwent thyroid hormone withdrawal, no significant association was observed between pre-RAI TSH and rate of successful remnant ablation, in respective multivariate analyses adjusted for relevant variables such as disease extent, ${ }^{131}$ I activity, and sex $(695,696)$. However, results of these two studies may not necessarily be extrapolated to TSH levels below $30 \mathrm{mU} / \mathrm{L}$, given that patients with such TSH thresholds were not generally considered eligible for RAI ablation in these studies. Pre-RAI ablation TSH was not a significant predictor of becoming disease free without further treatment in a secondary subgroup analysis of 50 patients who underwent thyroid hormone withdrawal, but the small number of patients in this subgroup may have limited the statistical power for a multivariate analysis (694). In summary, there is some uncertainty on the optimal level pre-RAI treatment TSH following thyroid hormone withdrawal in considering long-term outcome effects.

\section{[B39] Can rhTSH (Thyrogen) be used as an alternative to thyroxine withdrawal for remnant ablation or adjuvant therapy in patients who have undergone near-total or total thyroidectomy?}

\section{- RECOMMENDATION 54}

(A) In patients with ATA low-risk and ATA intermediaterisk DTC without extensive lymph node involvement (i.e., T1-T3, N0/Nx/N1a, M0), in whom RAI remnant ablation or adjuvant therapy is planned, preparation with rhTSH stimulation is an acceptable alternative to thyroid hormone withdrawal for achieving remnant ablation, based on evidence of superior short-term quality of life, noninferiority of remnant ablation efficacy, and multiple consistent observations suggesting no significant difference in long-term outcomes.

(Strong recommendation, Moderate-quality evidence)

(B) In patients with ATA intermediate-risk DTC who have extensive lymph node disease (multiple clinically involved $\mathrm{LN}$ ) in the absence of distant metastases, preparation with rhTSH stimulation may be considered as an alternative to thyroid hormone withdrawal prior to adjuvant RAI treatment.

\section{(Weak recommendation, Low-quality evidence)}

(C) In patients with ATA high-risk DTC with attendant higher risks of disease-related mortality and morbidity, more controlled data from long-term outcome studies are needed before rhTSH preparation for RAI adjuvant treatment can be recommended.

\section{(No recommendation, Insufficient evidence)}

(D) In patients with DTC of any risk level with significant comorbidity that may preclude thyroid hormone withdrawal prior to iodine RAI administration, rhTSH preparation should be considered. Significant comorbidity may include (a) a significant medical or psychiatric condition that could be acutely exacerbated with hypothyroidism, leading to a serious adverse event, or (b) inability to mount an adequate endogenous TSH response with thyroid hormone withdrawal.

\section{(Strong recommendation, Low-quality evidence)}

Recombinant human thyrotropin (trade name Thyrogen) is currently approved by many international authorities including the United States Food and Drug Administration (FDA) and Health Canada for use in preparation for RAI remnant ablation in patients who have undergone a near-total or total thyroidectomy for well-differentiated thyroid cancer and who do not have evidence of distant metastases (FDA, www.accessdata. fda.gov; Health Canada, http://webprod.hc-sc.gc.ca). Data from a compassionate use observational study suggest that rhTSH raises serum TSH measurements in patients who are unable to mount an endogenous TSH rise and appears to reduce the risk of hypothyroid-related complications in patients with significant medical or psychiatric comorbidity (697). Some of the complications that were reported to be avoided by use of rhTSH included worsening of psychiatric illness, respiratory compromise, central nervous system (CNS) compromise, aggravation of congestive heart failure, and aggravation of coronary artery disease (697).

Multiple RCTs have focused on short-term remnant ablation outcomes in low and intermediate risk DTC with lower risk features, using rhTSH compared to thyroid hormone withdrawal. In six RCTs of patients with well-differentiated thyroid cancer without distant metastases undergoing RAI remnant ablation (T1-T3, N1 or $\mathrm{N} 0$, all $\mathrm{M} 0)$, the rate of successful remnant ablation, was not significantly different after rhTSH preparation compared to thyroid hormone withdrawal, using ${ }^{131} \mathrm{I}$ dose activities ranging from 30 to 100 mCi (691,698-702). Patients with resected cervical lymph node metastases were included in five of these trials (691,699-702), and these may be assumed to be confined to the central neck, given the extent of primary surgery described in these studies. In one trial, a further inclusion restriction was fewer than five positive nodes at the time of the primary surgery (702). Some potential limitations of the existing RCTs in this area include lack of blinding of patients and treating physicians (because it was not feasible). In five of the RCTs that examined health-related quality of life around the time of remnant ablation, this outcome was significantly worse in patients who underwent thyroid hormone withdrawal compared to rhTSH preparation, and this was attributed to hypothyroid symptoms $(691,699-702)$. However, in three of these RCTs, which examined longer term 
quality of life, there was no significant difference in measurements between patients who had received rhTSH compared to those who had thyroid hormone withdrawal preparation, 3 or more months after RAI remnant ablation $(699,701,702)$. A smaller RCT including a total of 25 individuals who had incidentally discovered PTC in the course of thyroidectomy for multinodular goiter, showed that mean $\mathrm{Tg}$ measurements were similar at various time points out to about 20 months in 13 individuals who were prepared for remnant ablation using rhTSH within a week after thyroidectomy, compared to those prepared by $\mathrm{LT}_{4}$ withdrawal for 4-6 weeks postoperatively (703). A meta-analysis pooling data from 1535 patients in all seven trials described herein, suggested that the rates of remnant ablation success were not significantly different using rhTSH compared to thyroid hormone withdrawal (risk ratio 0.97 [95\% CI 0.94-1.01]) (704). Furthermore, a pooled analysis suggested that qualityof-life measures were superior on the day of remnant ablation in the rhTSH group, with no significant difference between groups 3 months later (704). Another meta-analysis including six of the previously mentioned RCTs, also suggested that the success of remnant ablation was not significantly different between patients prepared with rhTSH or thyroid hormone withdrawal, and this result was robust using a variety of definitions of remnant ablation success (705). In summary, the use of rhTSH for preparation for remnant ablation is associated with superior short-term quality of life and similar rates of successful remnant ablation compared to traditional thyroid hormone withdrawal.

There are some limited long-term outcome data following rhTSH preparation for RAI treatment compared to thyroid hormone withdrawal. In one aforementioned RCT in ATA low- and intermediate-risk patients (700), follow-up data were reported at a median of 3.7 years later for 51 of the original 63 patients, and rates of reoperation for cervical neck recurrence were essentially identical between groups (4\% of patients), with no deaths (706). In the same study, repeat treatment with ${ }^{131} \mathrm{I}$ for detectable $\mathrm{Tg}$ or imaging evidence of disease was performed in 4 of the 28 patients in the rhTSH group and 5 of the 23 patients in the hypothyroid group in this study (706). The low number of thyroid cancer-related deaths and recurrences in this small trial limit the ability to make meaningful statistical comparisons of long-term outcomes. In another RCT including 44 mixed risk-level DTC patients, who were subjected to either rhTSH preparation $(n=24)$ for RAI treatment within a week after surgery or 4-6 weeks of $\mathrm{LT}_{4}$ withdrawal $(n=20)$, after a mean follow-up period of about 52 months, only one individual in the rhTSH group was histologically proven to have recurrent disease (lymph nodes and bone) (707). However, this study was likely underpowered to detect meaningful differences in this outcome, and the final data analysis would be limited by lack of data available at the final follow-up (i.e., in progress) for $18 \%$ of the trial participants (707). In one prospective (694) and two retrospective $(708,709)$ observational studies including largely ATA low- and intermediate-risk DTC patients, no significant difference was observed in the long-term presence of clinically significant disease, regardless of whether rhTSH or thyroid hormone withdrawal was used in preparing for therapeutic RAI for DTC patients). In a subgroup analysis of 183 DTC patients with level N1b nodal disease from one of the aforementioned retrospective studies, the rate of NED at last follow-up was not significantly different in patients prepared with thyroid hormone withdrawal (26.8\%) compared to those prepared with rhTSH $(33.7 \%)$ (708). In a smaller retrospective, multicenter study, Pitoia et al. (710) reported that in 45 consecutive $\mathrm{Tg}$ antibodynegative patients with T3-T4 /N1-Nx/M0 disease, the absence of persistence or recurrence of disease after a mean follow-up of about 3 years was $72 \%$ in patients pretreated with rhTSH and $59 \%$ in those pretreated with thyroid hormone withdrawal $(p=0.03)$. In a multicenter retrospective analysis of patients with T4 disease with or without nodal or distant metastases (T4, N0/N1, M0/M1), the rate of stimulated $\mathrm{Tg}<2 \mathrm{ng} / \mathrm{mL} \mathrm{Tg}$ among antibody-negative patients was $67.7 \%$ (42 of 62) in patients prepared for RAI treatment with rhTSH, compared to $57.8 \%$ (37 of 64) in those prepared with thyroid hormone withdrawal (6-month followup) (711). In a retrospective analysis of 175 patients with RAI-avid metastatic disease to lungs and/or bone, the authors observed no significant difference in overall survival after a mean follow-up period of 5.5 years, among patients prepared prior to RAI treatment with rhTSH alone for all RAI treatments, thyroid hormone withdrawal for all RAI treatments, or thyroid hormone withdrawal for initial treatments followed by rhTSH for subsequent treatment(s) (712). In this study, whole-body and blood dosimetry studies were performed in all patients; therefore, the results may not be extrapolated to RAI fixed dosing. Some important differences among groups in this study that could have impacted the findings included differences in cumulative RAI activities received and longer follow-up in groups who had thyroid hormone withdrawal (712). Although the authors performed a multivariable analysis examining for predictors of overall survival, and method of TSH stimulation was not significant, this model did not adjust for these variables. In a two-center retrospective analysis comparing responses to treatment using RECIST 1.1 criteria in 56 patients with distant metastatic disease pretreated with either rhTSH or thyroid hormone withdrawal prior to RAI, there were no differences in outcomes between groups after a mean follow-up period of about 6 years (713). Also in this study, there were important baseline differences among groups, such as rates of use of dosimetry and mean cumulative RAI activity. Rates of xerostomia, leukopenia, or thrombocytopenia were not significantly different between treatment groups in this study. The overall mortality rate was $20 \%$ in the rhTSH group (3 of 15) and $7.3 \%$ in the thyroid hormone withdrawal group (3 of 41) $(p=0.188)(713)$, although the study was likely not sufficiently large to examine differences in this important outcome. The findings of the latter study cannot be readily extrapolated to fixed-dosing RAI treatment regimens because $80 \%$ of the individuals in the rhTSH group and $46 \%$ in the thyroid hormone withdrawal group had dosimetry-based RAI treatment. RCTs comparing rhTSH to thyroid hormone withdrawal preparation pre-RAI treatment, are clearly needed to guide clinical care in higher risk DTC patients.

\section{[B40] What activity of ${ }^{131}$ I should be used for remnant ablation or adjuvant therapy?}

\section{- RECOMMENDATION 55}

(A) If RAI remnant ablation is performed after total thyroidectomy for ATA low-risk thyroid cancer or intermediate-risk disease with lower risk features (i.e., low- 
volume central neck nodal metastases with no other known gross residual disease or any other adverse features), a low administered activity of approximately of $30 \mathrm{mCi}$ is generally favored over higher administered activities.

\section{(Strong recommendation, High-quality evidence)}

(B) Higher administered activities may need to be considered for patients receiving less than a total or near-total thyroidectomy in which a larger remnant is suspected or in which adjuvant therapy is intended.

\section{(Weak recommendation, Low-quality evidence)}

\section{- RECOMMENDATION 56}

When RAI is intended for initial adjuvant therapy to treat suspected microscopic residual disease, administered activities above those used for remnant ablation up to $150 \mathrm{mCi}$ are generally recommended (in absence of known distant metastases). It is uncertain whether routine use of higher administered activities $(>150 \mathrm{mCi})$ in this setting will reduce structural disease recurrence for T3 and N1 disease.

\section{(Weak recommendation, Low-quality evidence)}

Successful remnant ablation can be defined by an undetectable stimulated serum $\mathrm{Tg}$, in the absence of interfering $\mathrm{Tg}$ antibodies, with or without confirmatory nuclear or other imaging studies. An alternative definition in cases in which Tg antibodies are present is the absence of visible RAI uptake on a subsequent diagnostic RAI scan. In this section, we only included published original RCTs or systematic reviews/meta-analyses of such studies examining the impact of various ${ }^{131} \mathrm{I}$ dose activities on the rate of successful remnant ablation or thyroid cancer outcomes (including thyroid cancer-related deaths or recurrences) in adult patients with well-differentiated thyroid carcinoma who had been treated with total or near-total thyroidectomy and who were not known to have any gross residual disease following surgery. Our search yielded six RCTs $(692,699,701,714-716)$, the majority of which had had no blinding of patients and health care providers $(699,701,714-717)$.

In the trial by Fallahi et al. (692), the randomization program was prepared and executed by a technologist in the laboratory where the ${ }^{131}$ I activities were prepared for dispensing in coded vials, without revealing the administered activity to participants and health care providers. The pathologic stage of disease of patients included was TNM stage pT1 to pT3 in four trials $(699,714,715,717)$, whereas only pT1 or pT2 patients were eligible in one trial (701), and primary tumor size or tumor size staging was not reported in two trials $(692,716)$. Some patients with known smallvolume lymph node disease were included in five of the trials $(699,701,714,715,717)$, but patients with known lymph node disease were excluded from one trial (692), and lymph node staging was not reported in another trial (716). Although the specific levels and size of lymph node metastases at baseline were not clearly reported, data reported on surgical extent suggested that these were consistent with relatively nonbulky central neck nodal metastases resected in the course of thyroidectomy with or without central neck dissection $(699,701$, 714,715,717).

The ${ }^{131} \mathrm{I}$ activities compared were as follows: $30 \mathrm{mCi}$ compared to $100 \mathrm{mCi}$ in four trials $(692,699,701,714), 50$ $\mathrm{mCi}$ compared to $100 \mathrm{mCi}$ in two trials $(715,716)$, or $30 \mathrm{mCi}$ compared to $60 \mathrm{mCi}$ or $100 \mathrm{mCi}$ in one study comprising pooled long-term outcome data from two staged smaller trials from the same group (717).

The rate of successful remnant ablation was reported to be not inferior using an administered activity of $30 \mathrm{mCi}$ as compared to $100 \mathrm{mCi}$ in three trials after preparation with thyroid hormone withdrawal $(699,701,714)$ and in two trials after preparation with rhTSH $(699,701)$, including data from the two large factorial design trials in both comparisons $(699,701)$. Pilli et al. (715) reported that an administered activity of $50 \mathrm{mCi}$ was not inferior to $100 \mathrm{mCi}$ in achieving successful remnant ablation, following preparation with rhTSH. In contrast, Zaman et al. (716) suggested that 100 $\mathrm{mCi}$ may be superior to $50 \mathrm{mCi}$ following thyroid hormone withdrawal, but the small size (40 patients) and lack of reporting of statistical comparisons are important limitations of this study. The third largest trial (341 patients randomized) by Fallahi et al. (692) reported that an administered activity of $100 \mathrm{mCi}$ was superior to $30 \mathrm{mCi}$ in achieving successful remnant ablation after thyroid hormone withdrawal. The rate of initial successful remnant ablation (as defined by the primary authors) was highly variable among trials, and the following rates were reported following initial administration of $100 \mathrm{mCi}$ of ${ }^{131} \mathrm{I}$ : $64 \%$ in the trial from Fallahi et al. (692), 56\% in the trial from Maenpaa et al. (714), $89 \%$ in the trial from Mallick et al. (699), $67 \%$ in the trial from Pilli et al. (715), 94\% in the trial from Schlumberger et al. (701), and 60\% in the trial from Zaman et al. (716). The reasons explaining variability in the rates of successful RAI remnant ablation among trials are not completely understood but could potentially be due to differences in study populations, completeness of surgery (including size of the remaining remnant), or sensitivity of techniques used to evaluate outcomes (such as Tg assays or diagnostic imaging studies).

Short-term side effects in the weeks following remnant ablation have been reported to be more frequent in patients treated with $100 \mathrm{mCi}$ as compared to $30 \mathrm{mCi}$ activities by Mallick et al. (699), and a similar trend was reported by Maenpaa et al. (714). Repeat treatment with additional ${ }^{131} \mathrm{I}$ has been reported to be more frequent in patients treated with $30 \mathrm{mCi}$ as compared to higher activities in three trials $(692,699,717)$, but not in one trial (714). Long-term outcome data from randomized trials in this area are limited by relatively low event rates, potentially underpowering statistical analyses. Kukulska et al. (717) followed 390 patients that had been randomized to either 30,60 , or $100 \mathrm{mCi}$ administered activities for remnant ablation, and reported the following event rates after a median period of 10 years following treatment: local relapse in $2 \%$ following an initial $30 \mathrm{mCi}$ activity compared to $3 \%$ for initial administered activities of 60 or $100 \mathrm{mCi}$ (reported to be not significantly different), and distant metastatic recurrence in $0 \%$ of the patients in all of the treatment groups. Maenpaa et al. (714) followed 160 patients who were randomized to either 30 or $100 \mathrm{mCi}$ for a median of 51 months, and they reported the following outcomes: reoperation for resection of thyroid cancer in lymph nodes in $7 \%$ and $8 \%$ of patients who were treated with activities of 30 and $100 \mathrm{mCi}$, respectively; distant metastatic recurrence in $0 \%$ and $4 \%$ of patients treated with activities of 30 and $100 \mathrm{mCi}$, respectively; and no thyroid cancer-related deaths in any of the treatment groups. 
Overall, the rate of successful remnant ablation in patients who have undergone total or near-total thyroidectomy appears to be not inferior in patients treated with $30 \mathrm{mCi}$ compared to $100 \mathrm{mCi}$ in the majority of studies comparing these activities and particularly in studies achieving the highest successful ablation rates. Rates of short-term adverse effects may be higher after administration of $100 \mathrm{mCi}{ }^{131} \mathrm{I}$ compared to $30 \mathrm{mCi}$, in a small number of trials examining these outcomes. Limited long-term RCT data on the impact of various activities for remnant ablation or adjuvant therapy are available, but thyroid cancer-related recurrences or deaths do not appear to be higher with the use of lower initial activities for remnant ablation, compared to higher administered activities. Four recent systematic reviews and metaanalyses reported results that are supportive of these conclusions (718-721), although some of the predefined study inclusion criteria in these reviews were generally not as strict as defined in our review (particularly for variables such as the extent of primary surgery or the stringency of $\mathrm{Tg}$ threshold in the definition of success of remnant ablation); it is also important to note that in some of these meta-analyses, statistically significant heterogeneity (variability) of treatment effect was noted for the pooled analyses on successful remnant ablation $(718,720,721)$. A recent retrospective database analysis by Verburg et al. (722) with longer follow-up than most earlier studies adds a note of caution for the use of lower administered activities in older low-risk patients. They followed 698 patients with low-risk DTC (pT1 or pT2 and no involved LN) for at least 5 years. There were no long-term (10-15 year) overall survival or disease-specific survival differences in younger patients $(<45$ years old $)$ who received lower administered activities of ${ }^{131} \mathrm{I}(\leq 54 \mathrm{mCi})$ compared with those receiving higher administered activities. The older patients ( $\geq 45$ years old), however, who received lower administered activities of ${ }^{131} \mathrm{I}(\leq 54 \mathrm{mCi})$ did have a lower disease-specific survival compared with those receiving higher administered activities. Disease was defined as abnormal structural or functional imaging or a detectable serum $\mathrm{Tg}$ after TSH stimulation. The absolute disease-specific survival remained high even in the patients receiving lower administered activities of ${ }^{131} \mathrm{I}$, and there were no differences in overall survival in these older patients.

In 2009, the ATA guidelines task force recommended a fixed administered activity of between 100 and $200 \mathrm{mCi}$ for adjuvant RAI treatment if residual microscopic disease is suspected) or if an aggressive histologic variant of DTC was present (25). Since that time, at least five retrospective, single institution studies have compared clinical outcomes following various adjuvant RAI fixed activities in ATA intermediate-risk and ATA higher risk patients, without distant metastases (723-726). In comparing rates of disease persistence or recurrence in 225 ATA intermediate-risk DTC patients treated with 30 to $50 \mathrm{mCi}$ compared to $\geq 100 \mathrm{mCi}$ of adjuvant RAI, Castagna et al. (723) reported no significant difference in rates of successful remnant ablation or in longterm disease persistence/recurrence between groups. However, there were some statistically significant differences between the treatment groups that may have influenced these results, including higher numbers of men and individuals with lateral neck nodal disease and a longer follow-up period (which may increase the rate of detection) in the higher administered activity group of this study (723). In another study including 176 DTC patients with a primary tumor size $\leq 2 \mathrm{~cm}$ in diameter and microscopic extrathyroidal extension, no significant differences were found in a comparison of rates of successful remnant ablation and long-term recurrences in patients treated with $30 \mathrm{mCi}{ }^{131} \mathrm{I}$ compared with $149 \mathrm{mCi}$ (724). In this study, no recurrences were noted in either group after a median follow-up of 7.2 years. Although the mean primary tumor size was higher in the group treated with higher activities compared with the lower activity group in this study $(p<0.001)$, the difference in mean tumor diameter was only $2 \mathrm{~mm}$, so it may be of questionable clinical significance (724). Kruijff et al. (725) reported the results of multiple secondary subgroup analyses on data from 341 patients with T3 PTC, in which a postsurgical ${ }^{131}$ I administered activity of $\leq 75 \mathrm{mCi}$ was compared to $>75 \mathrm{mCi}$. In this study, the respective rates of disease recurrence, mortality, and stimulated $\mathrm{Tg}>2 \mathrm{ng} / \mathrm{mL}$ were not significantly different in the lower administered activity group (i.e., 7\%, 3\%, 72\%), compared to the higher activity group $(12 \%, 1.7 \%, 64 \%)$ (respective $p$-values of $0.55,0.43,0.40)$. Furthermore, in this study, a multivariate analysis using data from 1171 mixedrisk DTC patients without distant metastases suggested that there was no significant difference in risk of disease recurrence with the use of $>75 \mathrm{mCi}$ of ${ }^{131} \mathrm{I}$ postoperatively compared to $\leq 75 \mathrm{mCi}$ (higher administered activity hazard ratio 1.57 ([95\% CI 0.61-3.98], $p=0.341$ ), after adjustment for age, sex, primary tumor size, presence of vascular invasion, multifocality, and lymph node positivity, with a mean followup period of 60 months) (725). In another study comparing rates of disease structural recurrence/persistence in $181 \mathrm{pa}-$ tients with positive N1b lymph nodes, Sabra et al. (726) reported no statistically significant difference between the following approximate fixed administered activity categories: $75-139 \mathrm{mCi}$ with a median of $102 \mathrm{mCi}(31 \%), 140-$ $169 \mathrm{mCi}$ with a median of $150 \mathrm{mCi}(32 \%)$, and $170-468 \mathrm{mCi}$ with a median of $202 \mathrm{mCi}(23 \%)(p=0.17)$. Consistent with this finding, no significant correlation between RAI activity and best clinical response was observed in this study. In respective secondary subgroup analyses, a dose response was apparent in individuals $\geq 45$ years of age, but not younger individuals, and the authors cautioned about the use of fixed RAI activities exceeding $150 \mathrm{mCi}$ because of concerns about potential toxicity in the context of renal impairment (726). In the three studies utilizing either thyroid hormone withdrawal or rhTSH for RAI treatment preparation $(723,725,726)$, insufficient data were reported to make any meaningful interpretation on any relationship between administered activity in the context of preparation method. In one study, RAI adjuvant treatment was performed postoperatively, presumably without initiation of thyroid hormone because rhTSH was not reported to be used (724). None of the aforementioned studies (723-726) reported on RAI toxicity or quality of life outcomes; furthermore, T4 disease was not included in these studies. Overall, there is little evidence to suggest that increasing administered activities of adjuvant RAI is necessarily associated with improvement of clinical outcomes for patients with ATA intermediate- and high-risk disease without evidence of persistent disease. There is an important unmet need for RCTs examining thyroid cancer-related outcomes, quality of life, and toxicities in patients with ATA intermediate or higher level thyroid cancer, in the absence of gross residual disease or distant metastases. 


\section{[B41] Is a low-iodine diet necessary before remnant ablation?}

\section{- RECOMMENDATION 57}

A low iodine diet (LID) for approximately 1-2 weeks should be considered for patients undergoing RAI remnant ablation or treatment.

\section{(Weak recommendation, Low-quality evidence)}

It is important for health care providers to inquire about a history of possible high-dose iodine exposure (e.g., IV contrast, amiodarone, or others) in considering timing of scheduling RAI therapy or imaging. There are no studies examining whether the use of a LID in preparation for RAI remnant ablation or treatment impacts long-term diseaserelated recurrence or mortality rates. In a recent systematic review of observational studies in this area, the most commonly studied LIDs allowed for $\leq 50 \mu \mathrm{g} / \mathrm{d}$ of iodine for $1-2$ weeks and that the use of LIDs appeared to be associated with reduction in urinary iodine excretion as well as increase in ${ }^{131}$ I uptake, compared to no LID (727). There is conflicting evidence on the impact of a LID on the outcome of remnant ablation success (727), with the best available evidence largely restricted to retrospective analyses, using historical controls $(728,729)$. In a study including a total of 120 patients, the use of a 4-day LID (with seafood restriction for 1 week) was associated with a higher rate of remnant ablation success (defined by absent neck activity and stimulated $\mathrm{Tg}$ $<2 \mathrm{ng} / \mathrm{mL}$ ) compared to no LID (728). In a study including a total of 94 patients, comparing a more stringent LID to a less stringent diet of restricted salt/vitamins/seafood, each for 10 to 14 days, there was no significant difference in rate of successful remnant ablation, using a visually negative ${ }^{131} \mathrm{I}$ scan to define that outcome (729). The optimal stringency and duration of LID (if any) prior to therapeutic RAI is not known. In a RCT including 46 patients, the increase in ${ }^{131} \mathrm{I}$ uptake and reduction in urinary iodine excretion was not significantly different between patients who followed a LID for 2 weeks compared to 3 weeks prior to RAI scanning (730), suggesting that there may be little reason to extend the LID past 2 weeks. However, a lack of association between urinary iodine excretion and rate of successful thyroid ablation has been reported in patients not specifically prescribed a LID (731); the absence of a specific LID comparison group in this study may limit the generalizability of the findings to situations in which a specific LID is prescribed. Such findings may suggest, however, that the routine measurement of urinary iodine excretion, outside of possibly a research setting or suspected iodine contamination, may not be necessary. Although LIDs may be cumbersome or unpalatable, serious side effects are relatively infrequent (727), with case reports of potentially life-threatening hyponatremia occurring largely in elderly patients who were subject to thyroid hormone withdrawal, often in the presence of metastatic disease, sometimes concurrently treated with thiazide diuretics, and with a LID duration of longer than a week in the majority of cases (732). It is important to avoid restriction of noniodized salt during the LID, since this may be associated with hyponatremia, especially in patients undergoing thyroid hormone withdrawal. Some examples of LID descriptions for patients, may be found at the following websites: ATA (www.thyroid.org/faq-low-iodine-diet/), ThyCa: Thyroid Cancer Survivors' Association, Inc. (http:// thyca.org/rai.htm\#diet), Light of Life Foundation (www .checkyourneck.com/About-Thyroid-Cancer/Low-IodineCookbook), and Thyroid Cancer Canada (www.thyroid cancercanada.org/userfiles/files/LID_English_Aug_2013_ final.pdf).

\section{[B42] Should a posttherapy scan be performed following remnant ablation or adjuvant therapy?}

\section{- RECOMMENDATION 58}

A posttherapy WBS (with or without SPECT/CT) is recommended after RAI remnant ablation or treatment, to inform disease staging and document the RAI avidity of any structural disease.

\section{(Strong recommendation, Low-quality evidence)}

The literature on the utility of posttherapy RAI scans is largely based on single-center retrospective studies (643,733-736), many of which included a relatively large proportion of ATA intermediate- and high-risk DTC patients $(643,733,734)$. In a comparison of the results of pretherapy ${ }^{131}$ I scans to posttherapy scans, the rate of newly discovered lesions on posttherapy scans was reported to be between $6 \%$ and $13 \%$. In a study examining post-remnant ablation scans in 60 DTC patients, the disease stage was altered in $8.3 \%$ of individuals (735). In older literature, it had been reported that posttherapy scanning demonstrated new findings in $31 \%$ of 39 cases studied, but the detection of thyroid foci was included in that outcome, whereas almost a third of the patients (12 of 39) had a sizeable portion of their thyroid remaining following primary surgery (736). In the recent posttherapy scan literature, the ${ }^{131} \mathrm{I}$ activities ranged from 30 to $300 \mathrm{mCi}$ (733-735), and the timing of scans was between 2 and 12 days following therapeutic RAI $(643,733-735,737,738)$, with some studies prescribing a preparatory LID $(643,735,738)$ and others not prescribing such a diet $(733,734)$. In one study, posttherapy scan images were compared on the third and seventh day following ablative or therapeutic RAI administration for mixed-risk DTC (following thyroid hormone withdrawal) (738). The authors of this study reported that the concordance of lesions detected on both scans was $80.5 \%$ (108 of 135 patients), with $7.5 \%$ of early scans providing more information than late scans, and $12 \%$ of late scans providing more information than early scans (738). A limitation in interpreting the posttherapy scan literature is that all of the lesions identified on posttherapy scans were not always confirmed to represent structural disease (i.e., using crosssectional imaging, histopathology, or long-term outcome data), and readers of posttherapy scans were generally not specifically blinded to the results of other investigations, such as pretherapy RAI scans.

The potential utility of the combination of RAI posttherapy scanning in conjunction with SPECT/CT has been examined in multiple prospective $(737,739,740)$ and retrospective studies (741-743). The majority of these studies (737,739-742) have independently confirmed the presence of disease by means such as alternative imaging studies, histopathology, or clinical follow-up. In a single-center 
study of 170 patients with mixed risk level welldifferentiated thyroid cancer, the combination RAI posttherapy scanning and neck/thorax SPECT/CT, was estimated to have a sensitivity of $78 \%$ [95\% CI 60\%-90\%], with a specificity of $100 \%$ (negative or indeterminate scans were grouped as negative), for the outcome of persistent/ recurrent disease (median study follow-up period of 29 months) (737). Furthermore, in a multivariate analysis reported in this study, posttherapy RAI scanning with SPECT/ CT significantly independently predicted an increased risk of future disease persistence/recurrence (HR 65.2 [95\% CI 26.0-163.4) (737). In a single-center study of 81 DTC patients who underwent ${ }^{131} \mathrm{I}$ posttherapy scanning in conjunction with SPECT-spiral CT of the neck, $1.6 \%$ of the 61 patients with negative cervical scintigraphy-SPECT/CT had evidence of abnormal cervical scintigraphy 5 months later, whereas 3 of the 20 patients $(15 \%)$ with positive or indeterminate cervical posttherapy scintigraphy-SPECT/CT, had abnormal cervical scintigraphy 5 months later (741). The incremental value of SPECT/CT in impacting therapeutic planning appears to be greatest in studies in which its use was reserved for situations of posttherapy scan diagnostic uncertainty (impacted therapy in $24.4 \%$ [ 8 of 33] of cases, all of which went on to surgery) (739), or when disease was advanced and WBS was inconclusive (impacted management in 35\% [8 of 23] of such patients in another study) (740). The routine addition of neck/chest SPECT/CT to all posttherapy scans was estimated to alter postsurgical ATA recurrence risk estimate in $6.4 \%$ (7 of 109) of patients (743), impact therapeutic planning in about $2 \%$ of cases (742), and reduce the need for additional cross-sectional imaging in $20 \%$ of cases (29 of 148). In one study examining the use of routine cervical/ thoracic SPECT/CT in conjunction with posttherapy scanning, the SPECT/CT portion identified non-iodine avid lesions in $22 \%$ of patients (32 of 148), although the underlying pathologic diagnosis or long-term clinical significance of these lesions was not clearly reported (i.e., "tiny" lung nodules in 19 patients, mediastinal lymph nodes $<5 \mathrm{~mm}$ in 10 patients, and osteolytic bone metastases in three patients) (743). In situations in which SPECT/CT may not be feasible to perform in conjunction with a posttherapy RAI scan, clinical judgment needs to prevail on the utility of alternative cross-sectional imaging studies, considering factors such as clinical-pathologic stage, the completeness of surgery, inappropriate thyroglobulinemia, and, if performed, the posttherapy RAI scan result.

\section{[B43] Early management of DTC after initial therapy}

\section{[B44] What is the appropriate degree of initial TSH suppression?}

\section{- RECOMMENDATION 59}

(A) For high-risk thyroid cancer patients, initial TSH suppression to below $0.1 \mathrm{mU} / \mathrm{L}$ is recommended.

\section{(Strong recommendation, Moderate-quality evidence)}

(B) For intermediate-risk thyroid cancer patients, initial TSH suppression to $0.1-0.5 \mathrm{mU} / \mathrm{L}$ is recommended.

\section{(Weak recommendation, Low-quality evidence)}

(C) For low-risk patients who have undergone remnant ablation and have undetectable serum Tg levels, TSH may be maintained at the lower end of the reference range $(0.5-$ $2 \mathrm{mU} / \mathrm{L}$ ) while continuing surveillance for recurrence. Similar recommendations hold for low-risk patients who have not undergone remnant ablation and have undetectable serum $\mathrm{Tg}$ levels.

\section{(Weak recommendation, Low-quality evidence)}

(D) For low-risk patients who have undergone remnant ablation and have low-level serum Tg levels, TSH may be maintained at or slightly below the lower limit of normal $(0.1-0.5 \mathrm{mU} / \mathrm{L})$ while surveillance for recurrence is continued. Similar recommendations hold for low-risk patients who have not undergone remnant ablation, although serum Tg levels may be measurably higher and continued surveillance for recurrence applies.

\section{(Weak recommendation, Low-quality evidence)}

(E) For low-risk patients who have undergone lobectomy, $\mathrm{TSH}$ may be maintained in the mid to lower reference range $(0.5-2 \mathrm{mU} / \mathrm{L})$ while surveillance for recurrence is continued. Thyroid hormone therapy may not be needed if patients can maintain their serum TSH in this target range.

\section{(Weak recommendation, Low-quality evidence)}

DTC expresses the TSH receptor on the cell membrane and responds to TSH stimulation by increasing the expression of several thyroid specific proteins ( $\mathrm{Tg}$, sodium-iodide symporter) and by increasing the rates of cell growth (744). Suppression of $\mathrm{TSH}$, using supraphysiologic doses of $\mathrm{LT}_{4}$, is used commonly to treat patients with thyroid cancer in an effort to decrease the risk of recurrence $(275,671,745-747)$. A meta-analysis supported the efficacy of TSH suppression therapy in preventing major adverse clinical events $(\mathrm{RR}=0.73[\mathrm{CI}=0.60-0.88], p<0.05)$ (745). A large RCT from Japan (748) showed that disease-free survival was equivalent in patients with normal TSH (0.4$5 \mathrm{mU} / \mathrm{L}$ ) compared with those on $\mathrm{LT}_{4}$ suppression therapy (TSH $<0.01 \mathrm{mU} / \mathrm{L}$ ). Extent of residual disease is uncertain in these patients in that most did not undergo total thyroidectomy or RAI ablation and $\mathrm{Tg}$ levels were not monitored or reported, making direct comparisons to a North American approach difficult.

Retrospective and prospective studies have demonstrated that TSH suppression to below $0.1 \mathrm{mU} / \mathrm{L}$ may improve outcomes in high-risk thyroid cancer patients $(275,749)$, though no such evidence of benefit has been documented in low-risk patients. Higher degrees of suppression to $<0.03 \mathrm{mU} / \mathrm{L}$ may offer no additional benefit (746). A prospective, nonrandomized cohort study (671) of 2936 patients found that overall survival improved significantly when the TSH was suppressed to undetectable levels in patients with NTCTCSG stage III or IV disease and suppressed to the subnormal to undetectable range in patients with NTCTCSG stage II disease; however, in the latter group there was no incremental benefit from suppressing TSH to undetectable levels. Patients in the NTCTCSG stage II classification are somewhat different from AJCC/UICC stage II patients. Suppression of TSH was not beneficial in patients with NTCTCSG stage I disease. In another study, there was a positive association between serum TSH levels and the risk for recurrent 
disease and cancer-related mortality (750). Adverse effects of TSH suppression may include the known consequences of subclinical thyrotoxicosis, including exacerbation of angina in patients with ischemic heart disease, increased risk for atrial fibrillation in older patients (751), and increased risk of osteoporosis in postmenopausal women $(748,752-754)$. Therefore, optimal TSH goals for individual patients must balance the potential benefit of TSH suppression with the possible harm from subclinical thyrotoxicosis especially in patients with medical conditions that can be exacerbated with aggressive TSH suppression.

There is little evidence to guide TSH targets or the use of thyroid hormone in ATA low-risk patients who have undergone lobectomy. Most of the studies evaluating lobectomy for these patients do not discuss TSH targets or the use of thyroid hormone therapy or note that these data were unavailable in the databases studied (318,323-327). Vaisman et al. (328) noted that "levothyroxine was often not given after lobectomy if the patient maintained thyroid function tests within the reference range," while Matsuzu et al. (322) noted that "TSH suppression therapy was performed in most of the cases postoperatively, but the patients' TSH levels were not analyzed in this study." A recent study by Ebina et al. (755) retrospectively analyzed lowrisk patients who had undergone lobectomy and had not received thyroid hormone therapy. After a mean follow-up of 8.3 years, only $13 \%$ of the 674 patients undergoing lobectomy became overtly hypothyroid. The 10-year cause-specific and disease-specific survivals were not different between the patients who underwent thyroidectomy versus a lesser operation, although it was common for the patients undergoing lobectomy to also receive an ipsilateral central neck dissection. More research is needed in this area to help guide management of those patients undergoing lobectomy for low-risk DTC.

\section{[B45] Is there a role for adjunctive external beam radiation therapy or chemotherapy?}

\section{[B46] External beam radiation therapy}

\section{- RECOMMENDATION 60}

There is no role for routine adjuvant EBRT to the neck in patients with DTC after initial complete surgical removal of the tumor.

\section{(Strong recommendation, Low-quality evidence)}

The application of adjuvant neck/thyroid bed/loco-regional radiation therapy in DTC patients remains controversial. In particular, the use of radiation therapy within the context of initial/primary surgery/thyroidectomy has no meaningful literature support. There are reports of responses among patients with locally advanced disease $(756,757)$ and improved relapsefree and cause-specific survival in patients over age 60 with extrathyroidal extension but no gross residual disease (758), and selective use can be considered in these patients. It remains unknown whether external beam radiation therapy might reduce the risk for recurrence in the neck following adequate primary surgery and/or RAI treatment in patients with aggressive histologic subtypes (759). However, in the context of certain individual patients undergoing multiple and frequent serial neck re-operations for palliation of loco-regionally recurrent disease, adjuvant EBRT may be considered. In such contexts, the risks of anticipated additional serial re-operations versus the risks of EBRT must be carefully weighed to arrive at optimal decisions for individual patients. The approach to patients with gross incomplete surgical resection of disease is addressed in another section (Recommendation 72).

\section{[B47] Systemic adjuvant therapy}

\section{- RECOMMENDATION 61}

There is no role for routine systemic adjuvant therapy in patients with DTC (beyond RAI and/or TSH suppressive therapy using $\mathrm{LT}_{4}$ ).

\section{(Strong recommendation, Low-quality evidence)}

There are no clinical trial data to indicate that any adjuvant therapy beyond RAI and/or TSH suppressive therapy using $\mathrm{LT}_{4}$ has a net beneficial role in DTC patients. Furthermore, as the prognosis of DTC patients in complete remission and without any indication of active systemic disease is very good-and as toxicities, and even the risk of death, from use of kinase inhibitor therapies are appreciable - toxicities/risks have strong potential to exceed expected therapeutic benefit in the adjuvant context in most patients with DTC.

Whether populations of DTC patients might be identifiable who have sufficiently great future risks from recurrent disease to justify the corresponding risks attendant to the application of adjuvant systemic therapy (beyond RAI and/or TSH suppressive therapy using $\mathrm{LT}_{4}$ ) remains uncertain. Doxorubicin may act as a radiation sensitizer in some tumors of thyroid origin (760) and could be considered for patients with locally advanced disease undergoing external beam radiation therapy. It is unproven whether patients with rising $\mathrm{Tg}$ in the setting of no identifiable progression of anatomical disease have sufficiently high future risks from disease to justify the application of adjuvant systemic therapy beyond RAI and/or TSH suppressive therapy using $\mathrm{LT}_{4}$.

\section{[C1] DTC: LONG-TERM MANAGEMENT AND ADVANCED CANCER MANAGEMENT GUIDELINES}

\section{[C2] What are the appropriate features of long-term management?}

Accurate surveillance for possible recurrence in patients thought to be free of disease is a major goal of long-term follow-up. Tests with high specificity allow identification of patients unlikely to experience disease recurrence, so that less aggressive management strategies can be used that may be more cost effective and safe. Similarly, patients with a higher risk of recurrence are monitored more aggressively because it is believed that early detection of recurrent disease offers the best opportunity for effective treatment. A large study (761) found that the residual life span in disease-free patients treated with total or near-total thyroidectomy, ${ }^{131}$ I for remnant ablation, and in some cases high-dose ${ }^{131} \mathrm{I}$ for residual disease was similar to that in the general Dutch population. In contrast, the life expectancy for patients with persistent disease was reduced to $60 \%$ of that in the general population but varied widely depending upon tumor features. Age was not a factor in disease-specific mortality in a comparison of patients with age-matched individuals in the Dutch population. Treatment thus appears safe and does not shorten life expectancy. Although an increased incidence of second tumors in thyroid 
cancer patients has been recognized after the administration of high cumulative activities of ${ }^{131} \mathrm{I}(762,763)$, this elevated risk was not found to be associated with the use of ${ }^{131} \mathrm{I}$ in another study (764). RAI therapy in low-risk patients did not affect median overall survival in one study (765), but did increase second primary malignancies in another study (766). This risk of second primary malignancies after RAI therapy is discussed in more detail in section C33. Patients with persistent or recurrent disease are offered treatment to cure or to delay future morbidity or mortality. In the absence of such options, therapies to palliate by substantially reducing tumor burden or preventing tumor growth are utilized, with special attention paid to tumors threatening critical structures.

A second goal of long-term follow-up is to monitor thyroxine suppression or replacement therapy to avoid underreplacement or overly aggressive therapy (767).

\section{[C3] What are the criteria for absence of persistent tumor (excellent response)?}

In patients who have undergone total or near-total thyroidectomy and RAI treatment (remnant ablation, adjuvant therapy or therapy), disease-free status comprises all of the following (summarized in Table 13):

1. No clinical evidence of tumor

2. No imaging evidence of tumor by RAI imaging (no uptake outside the thyroid bed on the initial posttreatment WBS if performed, or if uptake outside the thyroid bed had been present, no imaging evidence of tumor on a recent diagnostic or posttherapy WBS) and/or neck US

3. Low serum $\mathrm{Tg}$ levels during $\mathrm{TSH}$ suppression ( $\mathrm{Tg}$ $<0.2 \mathrm{ng} / \mathrm{mL})$ or after stimulation $(\mathrm{Tg}<1 \mathrm{ng} / \mathrm{mL})$ in the absence of interfering antibodies

\section{[C4] What are the appropriate methods} for following patients after initial therapy?

\section{[C5] What is the role of serum Tg measurement in the follow-up of DTC?}

\section{- RECOMMENDATION 62}

(A) Serum Tg should be measured by an assay that is calibrated against the CRM457 standard. Thyroglobulin antibodies should be quantitatively assessed with every measurement of serum Tg. Ideally, serum $\mathrm{Tg}$ and anti- $\mathrm{Tg}$ antibodies should be assessed longitudinally in the same laboratory and using the same assay for a given patient.

\section{(Strong recommendation, High-quality evidence)}

(B) During initial follow-up, serum $\mathrm{Tg}$ on thyroxine therapy should be measured every 6-12 months. More frequent Tg measurements may be appropriate for ATA high-risk patients.

\section{(Strong recommendation, Moderate-quality evidence)}

(C) In ATA low- and intermediate-risk patients that achieve an excellent response to therapy, the utility of subsequent $\mathrm{Tg}$ testing is not established. The time interval between serum $\mathrm{Tg}$ measurements can be lengthened to at least 12-24 months.

\section{(Weak recommendation, Low-quality evidence)}

(D) Serum TSH should be measured at least every 12 months in all patients on thyroid hormone therapy.

\section{(Strong recommendation, Low-quality evidence)}

(E) ATA high-risk patients (regardless of response to therapy) and all patients with biochemical incomplete, structural incomplete, or indeterminate response should continue to have Tg measured at least every 6-12 months for several years.

\section{(Weak recommendation, Low-quality evidence)}

\section{- RECOMMENDATION 63}

(A) In ATA low-risk and intermediate-risk patients who have had remnant ablation or adjuvant therapy and negative cervical US, serum Tg should be measured at 6-18 months on thyroxine therapy with a sensitive $\mathrm{Tg}$ assay $(<0.2 \mathrm{ng} / \mathrm{mL})$ or after TSH stimulation to verify absence of disease (excellent response).

\section{(Strong recommendation, Moderate-quality evidence)}

(B) Repeat TSH-stimulated Tg testing is not recommended for low- and intermediate-risk patients with an excellent response to therapy.

\section{(Weak recommendation, Low-quality evidence)}

(C) Subsequent TSH-stimulated Tg testing may be considered in patients with an indeterminate, biochemical incomplete, or structural incomplete response following either additional therapies or a spontaneous decline in $\mathrm{Tg}$ values on thyroid hormone therapy over time in order to reassess response to therapy.

\section{(Weak recommendation, Low-quality evidence)}

Subsequent stimulated testing is rarely needed for those with NED, because there are rarely benefits seen in this patient population from repeated $\mathrm{TSH}$-stimulated $\mathrm{Tg}$ testing $(590,594,597,768)$. The use of sensitive methods for serum $\mathrm{Tg}$ may obviate the need for rhTSH stimulation in low-risk patients with a $\mathrm{Tg}$ on $\mathrm{LT}_{4}$ treatment below $0.1-0.2 \mathrm{ng} / \mathrm{mL}$ $(393,587,595,601,606,769)$.

\section{[C6] Serum Tg measurement and clinical utility}

Measurement of serum Tg levels is an important modality to monitor patients for residual or recurrent disease. Most laboratories currently use immunometric assays to measure serum $\mathrm{Tg}$, and it is important that these assays are calibrated against the CRM-457 international standard. Despite improvements in standardization of $\mathrm{Tg}$ assays, there is still a two-fold difference between some assays (316,770), leading to the recommendation that measurements in individual patients be performed with the same assay over time. Immunometric assays are prone to interference from anti-Tg autoantibodies, which commonly cause falsely low serum $\mathrm{Tg}$ measurements. Moreover, variability in Tg autoantibody assays may result in falsely negative antibody levels associated with a misleadingly undetectable serum Tg due to the antibodies that are present but not detected (771). Assays for antiTg autoantibodies suffer from a similar variance and lack of concordance as do Tg assays $(608,772)$, and both $\mathrm{Tg}$ and $\mathrm{Tg}$ autoantibody assays may be affected by heterophilic antibodies $(773,774)$. The presence of $\mathrm{Tg}$ autoantibodies should be suspected when the surgical pathology indicates the presence of background Hashimoto thyroiditis (775). While there 
is no method that reliably eliminates $\mathrm{Tg}$ antibody interference, radioimmunoassays for $\mathrm{Tg}$ may be less prone to antibody interference, which can occasionally result in falsely elevated Tg levels (776-778). However, radioimmunoassays for Tg are not as widely available, may be less sensitive than immunometric assays in detecting small amounts of residual tumor, and their role in the clinical care of patients is uncertain. In the absence of antibody interference, serum $\mathrm{Tg}$ has a high degree of sensitivity and specificity to detect thyroid cancer, especially after total thyroidectomy and remnant ablation. In patients with low risk for recurrence, serum $\mathrm{Tg}$ measurement at the time of remnant ablation/adjuvant therapy may be useful for prediction of subsequent disease-free status (605).

Most data come from studies using methods with a functional sensitivity of $1 \mathrm{ng} / \mathrm{mL}$. Functional sensitivity of many contemporary assays is $\leq 0.1 \mathrm{ng} / \mathrm{mL}$, which may lead to greater reliance of $\mathrm{Tg}$ on thyroid hormone therapy instead of performing $\mathrm{Tg}$ determination following $\mathrm{TSH}$ stimulation. Because TSH stimulation generally increases basal serum $\mathrm{Tg}$ by 5 - to 10 -fold, significant serum Tg levels $(>1-2 \mathrm{ng} / \mathrm{mL})$ found after TSH stimulation when using an assay with a functional sensitivity of $0.5-1 \mathrm{ng} / \mathrm{mL}$ may already be predicted in patients on LT4 treatment without TSH stimulation by a highly sensitive $\mathrm{Tg}$ assay when $\mathrm{Tg}$ levels are above $0.2 \mathrm{ng} / \mathrm{mL}$.

The highest degrees of sensitivity for serum $\mathrm{Tg}$ are noted following thyroid hormone withdrawal or stimulation using rhTSH (779). Serum Tg measurements obtained during thyroid hormone suppression of TSH and less commonly following $\mathrm{TSH}$ stimulation may fail to identify patients with relatively small amounts of residual tumor $(583,649$, 780,781). These minimal amounts of residual disease are often located in the neck, and performing neck US in these patients offers the best opportunity to recognize or exclude neoplastic disease even when serum $\mathrm{Tg}$ is undetectable (297,782,783). Conversely, even TSH-stimulated Tg measurement may fail to identify patients with clinically significant tumor because of anti-Tg antibodies or less commonly because of defective or absent production and secretion of immunoreactive $\mathrm{Tg}$ by tumor cells $(649,780)$. Tg levels should be interpreted in light of the pretest probability of clinically significant residual tumor. An aggressive or poorly differentiated tumor may be present despite low basal or stimulated $\mathrm{Tg}$; in contrast, a minimally elevated stimulated $\mathrm{Tg}$ may occur in patients at low risk for clinically significant morbidity (784). Nevertheless, a single rhTSH-stimulated serum $\mathrm{Tg}<0.5-1.0 \mathrm{ng} / \mathrm{mL}$ in the absence of anti- $\mathrm{Tg}$ antibody has an approximately $98 \%-99.5 \%$ likelihood of identifying patients completely free of tumor on follow-up (590,591,593,597,768). Repeating rhTSH-stimulated Tg measurements may not be necessary in most cases when surveillance includes an undetectable basal serum $\mathrm{Tg}$ and negative ultrasonography $(604,617)$. However, $0.5 \%-3 \%$ of patients may manifest clinical or biochemical recurrence in spite of an initial rhTSH-stimulated Tg of $<0.5 \mathrm{ng} / \mathrm{mL}(592)$.

Initial follow-up for low-risk patients (about $85 \%$ of postoperative patients) who have undergone total or neartotal thyroidectomy and ${ }^{131}$ I remnant ablation should be based mainly on TSH-suppressed Tg and cervical US, followed by TSH-stimulated serum $\mathrm{Tg}$ measurements if the TSH-suppressed Tg testing is undetectable $(583,785)$. How- ever, a Tg assay with a functional sensitivity of $0.1-0.2 \mathrm{ng} /$ $\mathrm{mL}$ may reduce the need to perform $\mathrm{TSH}$-stimulated $\mathrm{Tg}$ measurements during the initial and long-term follow-up of some patients. In one study using such an assay, a $\mathrm{T}_{4}$ suppressed serum $\mathrm{Tg}<0.1 \mathrm{ng} / \mathrm{mL}$ was only rarely $(2 \%)$ associated with an rhTSH-stimulated $\mathrm{Tg}>2 \mathrm{ng} / \mathrm{mL}$; however, $42 \%$ of the patients had baseline rhTSH-stimulated Tg elevation $>0.1 \mathrm{ng} / \mathrm{mL}$, but only one patient was found to have residual tumor (606). In another study using the same assay (787), a TSH-suppressed serum Tg level was $>0.1 \mathrm{ng} / \mathrm{mL}$ in $14 \%$ of patients, but the false-positive rate was $35 \%$ using an rhTSH-stimulated Tg cutoff of $>2 \mathrm{ng} / \mathrm{mL}$, raising the possibility of unnecessary testing and treatment. In low-risk patients not undergoing ablation, an ultrasensitive $\mathrm{Tg}$ was $<1 \mathrm{ng} / \mathrm{mL}$ in $91 \%$ and $<2 \mathrm{ng} / \mathrm{mL}$ in $96 \%$ of patients at 9 months after thyroidectomy (644). In a second-generation assay, a cutoff of $0.15 \mathrm{ng} / \mathrm{mL}$ was shown to have a NPV of $98.6 \%$ and $91 \%$ specificity for residual disease or potential recurrence (587). The only prospective study also documented increased sensitivity of detection of disease at the expense of reduced specificity (770), and receiver operating curves have shown that a Tg level on thyroid hormone therapy around $0.2-0.3 \mathrm{ng} / \mathrm{mL}$ portends the best sensitivity and specificity for detecting persistent disease. With the use of these sensitive Tg assays, it was concluded that an annual serum $\mathrm{Tg}$ on $\mathrm{LT}_{4}$ treatment with periodic neck US is adequate for detection of recurrence without need for rhTSH stimulation testing in those patients with a serum $\mathrm{Tg}<0.2-0.3 \mathrm{ng}$ / $\mathrm{mL}$ on thyroid hormone therapy (606). In patients at low to intermediate risk of recurrence, the utility of an undetectable postoperative nonstimulated $\mathrm{Tg}$ level is uncertain and may depend upon the functional sensitivity of the Tg assay, with some studies $(632,646)$ observing RAI-avid metastatic foci (usually in neck lymph nodes) in $8.5 \%-12 \%$ of such patients, while another study (630) noted negative scans in 63 of 63 patients when the baseline $\mathrm{Tg}$ was $<0.2 \mathrm{ng} / \mathrm{mL}$. The different results likely relate to both the degree of intermediate versus higher risk patients in the respective cohorts, the amount of residual thyroid tissue, the elapsed time since surgery, the cutoff for functional sensitivity of the Tg assays, as well as the sensitivity of the post-RAI imaging techniques.

Approximately $20 \%$ of patients who are clinically free of disease with serum Tg levels $<1 \mathrm{ng} / \mathrm{mL}$ during thyroid hormone suppression of TSH (785) will have a serum Tg level $>2 \mathrm{ng} / \mathrm{mL}$ after rhTSH or thyroid hormone withdrawal at 12 months after initial therapy with surgery and RAI. In this patient population, one-third will have identification of persistent or recurrent disease and of increasing $\mathrm{Tg}$ levels, and the other two-thirds will remain free of clinical disease and will have stable or decreasing stimulated serum Tg levels over time $(618,624)$. However, there may be a low likelihood of a rise in serum $\mathrm{Tg}$ to $>2 \mathrm{ng} / \mathrm{mL}$ when the basal serum $\mathrm{Tg}$ is $<0.1 \mathrm{ng} / \mathrm{mL}$ if a second-generation $\mathrm{Tg}$ immunochemiluminometric assay (ICMA) with a functional sensitivity of $0.05 \mathrm{ng} / \mathrm{mL}$ is employed (788). There is good evidence that a $\mathrm{Tg}$ cutoff level above $2 \mathrm{ng} / \mathrm{mL}$ following rhTSH stimulation is highly sensitive in identifying patients with persistent tumor (785,789-794). However, the results of serum Tg measurements made on the same serum specimen differ among assay methods (316). Therefore, the Tg cutoff may differ significantly among medical centers and laboratories. Further, the clinical significance of minimally 
detectable Tg levels is unclear, especially if only detected following TSH stimulation. However, receiver operating curves have shown that a $\mathrm{Tg}$ level on thyroid hormone around $0.2-0.3 \mathrm{ng} / \mathrm{mL}$ portends the best sensitivity and specificity for detecting persistent disease. In these patients, the trend in serum Tg over time will typically identify patients with clinically significant residual disease. A rising unstimulated or stimulated serum $\mathrm{Tg}$ indicates disease that is likely to become clinically apparent $(618,795)$. Thyroglobulin doubling time may have utility as a predictor of recurrence, analogous to the use of calcitonin doubling time for MTC $(622,796)$.

\section{[C7] Anti-Tg antibodies}

The presence of anti-Tg antibodies, which occur in approximately $25 \%$ of thyroid cancer patients (797) and $10 \%$ of the general population (798), will falsely lower serum $\mathrm{Tg}$ determinations in immunometric assays (799). The use of recovery assays in this setting to detect significant interference is controversial $(799,800)$. Serum anti-Tg antibody should be measured in conjunction with serum Tg assay by an immunometric method. Although assay standardization against the International Reference Preparation 65/93 has been recommended (608), wide-ranging variability in assay results and analytical sensitivity of the assay remains $(801,802)$. Use of recovery methods for anti-Tg antibody may suffer variable interferences (608). Anti-Tg antibody may rise transiently postoperatively as an apparent immune reaction to the surgery itself and may also rise after ablation therapy (611). Anti-Tg antibodies should be measured in a different assay if the routine anti-Tg antibody assay is negative in a patient with surgically proven Hashimoto thyroiditis (775). It may be useful to measure anti-Tg antibodies shortly after thyroidectomy and prior to ablation because high levels may herald the likelihood of recurrence in patients without Hashimoto thyroiditis (801). Similarly, recurrent or progressive disease is suggested in those patients initially positive for anti-Tg antibodies who then become negative but subsequently have rising levels of anti-Tg antibodies. Falling levels of anti-Tg antibodies may indicate successful therapy $(614,801)$. Thus, serial serum anti-Tg antibody quantification using the same methodology may serve as an imprecise surrogate marker of residual normal thyroid tissue, Hashimoto thyroiditis, or tumor $(608,609,615)$. Following total thyroidectomy and RAI remnant ablation, anti-Tg antibodies usually disappear over a median of about 3 years in patients without evidence of persistent disease $(611,615,616)$. Several studies demonstrate an increased risk of recurrence/persistent disease associated either with a new appearance of anti-Tg antibodies or rising titers (609-614). From a clinical perspective, anti-Tg antibody levels that are declining over time are considered a good prognostic sign, while rising antibody levels, in the absence of an acute injury to the thyroid (release of antigen by surgery or RAI treatment), significantly increases the risk that the patient will subsequently be diagnosed with persistent or recurrent thyroid cancer.

The recent development of liquid chromatography-tandem mass spectrometry assay of $\mathrm{Tg}$ holds promise for accurate $\mathrm{Tg}$ measurement in the presence of $\mathrm{Tg}$ autoantibodies (803$805)$, but further studies will be required to validate the assays in terms of functional sensitivity, correlations with immunoassay results, and patient outcomes, reflecting either excellent response or persistent disease (806).

\section{[C8] What is the role of serum Tg measurement in patients who have not undergone RAI remnant ablation?}

\section{- RECOMMENDATION 64}

Periodic serum $\mathrm{Tg}$ measurements on thyroid hormone therapy should be considered during follow-up of patients with DTC who have undergone less than total thyroidectomy and in patients who have had a total thyroidectomy but not RAI ablation. While specific cutoff levels of $\mathrm{Tg}$ that optimally distinguish normal residual thyroid tissue from persistent thyroid cancer are unknown, rising $\mathrm{Tg}$ values over time are suspicious for growing thyroid tissue or cancer.

\section{(Strong recommendation, Low-quality evidence)}

In low- and intermediate-risk patients who underwent a total thyroidectomy without remnant ablation or adjuvant therapy, the same strategy of follow-up is used, based on serum Tg determination on $\mathrm{LT}_{4}$ treatment and on neck US at 9-12 months. In most of these patients, neck US does not reveal any suspicious findings and the serum $\mathrm{Tg}$ is $<1 \mathrm{ng} / \mathrm{mL}$ on $\mathrm{LT}_{4}$ treatment, is low $(<2 \mathrm{ng} / \mathrm{mL})$ and will remain at a low level, or will decrease without any additional therapy over time (545). There is no need for rhTSH stimulation because $\mathrm{Tg}$ will increase to a value above $1 \mathrm{ng} / \mathrm{mL}$ in $50 \%$ of the cases, even in individuals without residual cancer, with the magnitude of increase being related to the size of normal thyroid remnants (783). These patients are followed on an annual basis with serum TSH and Tg determination.

In the few patients with a serum $\mathrm{Tg}$ that remains elevated over time, especially for those with a rising Tg, remnant ablation or adjuvant therapy with ${ }^{131}$ I may be considered with a posttherapy WBS if neck US is negative. There is no evidence in these low-risk patients that a delayed treatment over the postoperative treatment may adversely affect the outcome.

A cohort of 80 consecutive patients with very low-risk PTMC who had undergone near-total thyroidectomy without postoperative RAI treatment were studied over 5 years (783). The rhTSH-stimulated serum Tg levels were $\leq 1 \mathrm{ng} / \mathrm{mL}$ in 45 patients $(56 \%)$ and $>1 \mathrm{ng} / \mathrm{mL}$ in $35(44 \%)$ patients in whom rhTSH-stimulated Tg levels were as high as $25 \mathrm{ng} / \mathrm{mL}$. The diagnostic WBS revealed uptake in the thyroid bed but showed no pathological uptake in any patient, and thyroid bed uptake correlated with the rhTSH-stimulated serum Tg levels $(p<0.0001)$. Neck ultrasonography identified lymph node metastases in both Tg-positive and Tg-negative patients. The authors concluded that for follow-up of this group of patients: (i) diagnostic WBS was ineffective at detecting metastases; (ii) neck ultrasonography as the main surveillance tool was highly sensitive in detecting lymph node metastases; and (iii) detectable rhTSH-stimulated serum Tg levels mainly depended upon the size of thyroid remnants, which suggests that serum Tg determination should be performed primarily on thyroid hormone therapy when using a sensitive Tg assay (functional sensitivity $\leq 0.2 \mathrm{ng} / \mathrm{mL}$ ). In a series of 290 low-risk patients who had not undergone remnant ablation (545), serum Tg levels on $\mathrm{LT}_{4}$ became undetectable $(<1 \mathrm{ng} / \mathrm{mL})$ within $5-7$ years in $95 \%$ of the cohort and was $<0.1 \mathrm{ng} / \mathrm{mL}$ in $80 \%$ of a subset of these patients, using a sensitive assay to confirm the utility of $\mathrm{Tg}$ measurements on thyroid hormone treatment 
for routine follow-up. The frequency of follow-up is uncertain in patients who have not received RAI ablation and have sufficient residual thyroid tissue to produce measurable levels of serum $\mathrm{Tg}$, the magnitude of which will depend upon the mass of residual tissue and the degree of TSH suppression. It appears reasonable to consider periodic measurements of $\mathrm{Tg}$ as surveillance for a trend in rising values.

\section{[C9] What is the role of US and other imaging techniques (RAI SPECT/CT, CT, MRI, PET-CT) during follow-up?}

\section{[C10] Cervical ultrasonography}

\section{- RECOMMENDATION 65}

(A) Following surgery, cervical US to evaluate the thyroid bed and central and lateral cervical nodal compartments should be performed at 6-12 months and then periodically, depending on the patient's risk for recurrent disease and Tg status.

(Strong recommendation, Moderate-quality evidence)

(B) If a positive result would change management, ultrasonographically suspicious lymph nodes $\geq 8-10 \mathrm{~mm}$ (see Recommendation 71) in the smallest diameter should be biopsied for cytology with $\mathrm{Tg}$ measurement in the needle washout fluid.

\section{(Strong recommendation, Low-quality evidence)}

(C) Suspicious lymph nodes less than 8-10 mm in smallest diameter may be followed without biopsy with consideration for FNA or intervention if there is growth or if the node threatens vital structures.

\section{(Weak recommendation, Low-quality evidence)}

(D) Low-risk patients who have had remnant ablation, negative cervical US, and a low serum Tg on thyroid hormone therapy in a sensitive assay $(<0.2 \mathrm{ng} / \mathrm{mL})$ or after TSH stimulation $(\mathrm{Tg}<1 \mathrm{ng} / \mathrm{mL})$ can be followed primarily with clinical examination and $\mathrm{Tg}$ measurements on thyroid hormone replacement.

\section{(Weak recommendation, Low-quality evidence)}

Cervical ultrasonography is performed with a highfrequency probe $(\geq 10 \mathrm{MHz})$ and is highly sensitive in the detection of cervical metastases in patients with DTC (290,783,807). These studies primarily evaluate patients with PTC, and the utility of neck US for monitoring patients with low-risk FTC is not well-established. Neck US should interrogate all lymph node compartments and the thyroid bed. Frequently, US does not distinguish thyroid bed recurrences from benign nodules $(629,808)$. When an abnormality is found during the year after surgery in patients without any other suspicious findings, including low serum Tg on thyroid hormone therapy, follow-up may be performed with neck US.

A correlation performed between US findings and pathology at surgery (292) has shown for lymph nodes $>7 \mathrm{~mm}$ in the smallest diameter that a cystic appearance or hyperechoic punctuations in a context of thyroid cancer should be considered as malignant; lymph nodes with a hyperechoic hilum are reassuring; the type of vascularization (central: reassuring; peripheral: concerning) has a high sensitivity/specificity; a round shape, a hypoechoic appearance or the loss of the hyperechoic hilum by themselves does not justify a FNA biopsy (FNAB).

Interpretation of neck US should take into account all other clinical and biological data. In fact, the risk of recurrence is closely related to the initial lymph node status: most lymph node recurrences occur in already involved compartments; the risk increases with a higher number of $\mathrm{N} 1$ and a higher number of N1 with extracapsular extension (338) and with macroscopic rather than microscopic lymph node metastases $(335,809)$.

In low- and intermediate-risk patients, the risk of lymph node recurrence is low $(<2 \%)$ in patients with undetectable serum $\mathrm{Tg}$ and is much higher in those with detectable/elevated serum $\mathrm{Tg}$. In fact, $1 \mathrm{~g}$ of neoplastic thyroid tissue will increase the serum Tg by $\sim 1 \mathrm{ng} / \mathrm{mL}$ during $\mathrm{LT}_{4}$ treatment and by approximately $2-10 \mathrm{ng} / \mathrm{mL}$ following TSH stimulation $(788,800)$. Neck US can detect $\mathrm{N} 1$ as small as $2-3 \mathrm{~mm}$ in diameter (in patients in whom serum $\mathrm{Tg}$ may be low or undetectable), but benefits of their early discovery $(<8-10 \mathrm{~mm})$ is not demonstrated.

FNAB for cytology and Tg measurement in the aspirate fluid is performed for suspicious lymph nodes $\geq 8-10 \mathrm{~mm}$ in their smallest diameter. US guidance may improve the results of FNAB, in particular for small lymph nodes and those located deep in the neck. However, FNAB cytology misses thyroid cancer in a significant proportion (up to $20 \%$ ) of patients. The combination of cytology and serum $\mathrm{Tg}$ determination in the aspirate fluid increases sensitivity $(303,810,811)$. In cases of lymph node metastases, the $\mathrm{Tg}$ concentration in the aspirate fluid is often elevated $(>10 \mathrm{ng} / \mathrm{mL})$, and concentrations above this level are highly suspicious $(296,298,301)$. A Tg concentration in the aspirate fluid between 1 and $10 \mathrm{ng} / \mathrm{mL}$ is moderately suspicious for malignancy, and comparison of the Tg measurement in the aspirate fluid and the serum should be considered in these patients. Also, up to half of the FNAB performed for suspicious US findings are benign, demonstrating that selection of patients for FNAB needs to be improved $(296,298,812)$. Nonsuspicious and small nodes $(<8-10 \mathrm{~mm}$ in the smallest diameter) can be monitored with neck US.

\section{[C11] Diagnostic whole-body RAl scans}

\section{- RECOMMENDATION 66}

After the first posttreatment WBS performed following RAI remnant ablation or adjuvant therapy, low-risk and intermediate-risk patients (lower risk features) with an undetectable $\mathrm{Tg}$ on thyroid hormone with negative antiTg antibodies and a negative US (excellent response to therapy) do not require routine diagnostic WBS during follow-up.

(Strong recommendation, Moderate-quality evidence)

\section{- RECOMMENDATION 67}

(A) Diagnostic WBS, either following thyroid hormone withdrawal or rhTSH, 6-12 months after adjuvant RAI therapy can be useful in the follow-up of patients with high or intermediate risk (higher risk features) of persistent disease (see risk stratification system, section [B19]) and should be done with ${ }^{123} \mathrm{I}$ or low activity ${ }^{131} \mathrm{I}$.

\section{(Strong recommendation, Low-quality evidence)}

B) SPECT/CT RAI imaging is preferred over planar imaging in patients with uptake on planar imaging to better 
anatomically localize the RAI uptake and distinguish between likely tumors and nonspecific uptake

\section{(Weak recommendation, Moderate-quality evidence)}

Following RAI ablation or adjuvant therapy, when the posttherapy scan does not reveal uptake outside the thyroid bed, subsequent diagnostic WBSs have low sensitivity and are usually not necessary in low-risk patients who are clinically free of residual tumor and have an undetectable serum $\mathrm{Tg}$ level on thyroid hormone and negative cervical US $(583,785,813,814)$.

A diagnostic WBS may be indicated in three primary clinical settings: (i) patients with abnormal uptake outside the thyroid bed on posttherapy WBS, (ii) patients with poorly informative postablation WBS because of large thyroid remnants with high uptake of ${ }^{131} \mathrm{I}$ ( $>2 \%$ of the administered activity at the time of WBS) that may hamper the visualization of lower uptake in neck lymph nodes, and (iii) patients with $\mathrm{Tg}$ antibodies, at risk of false-negative $\mathrm{Tg}$ measurement, even when neck US does not show any suspicious findings. Iodine 123 is preferred over ${ }^{131} \mathrm{I}$ in these rare indications for diagnostic WBS, because it delivers lower radiation doses to the body and provides better quality images.

Iodine 131 or ${ }^{123}$ I whole-body scintigraphy includes planar images or images using a dual-head SPECT gamma camera of the whole body and spot images of the neck, mediastinum, and on any abnormal focus of RAI uptake. It may be performed after the administration of either a diagnostic (usually $2-5 \mathrm{mCi}$ ) or a therapeutic activity $(30-150 \mathrm{mCi})$ of RAI. Because of the lack of anatomical landmarks on planar images, it is often difficult to differentiate uptake in normal thyroid remnants from lymph node metastases (especially when thyroid remnants are large), uptake in lung metastases from rib lesions, or accumulation of RAI in intestine or bladder from a pelvic bone lesion. Hybrid cameras combine a dual-head SPECT gamma camera with a CT scanner in one gantry. This allows direct superimposition of functional and anatomical imaging. The radiation dose delivered to the patient by the low-dose CT scan is $2-5 \mathrm{mSv}$, a dose that is much lower than the dose delivered by the administration of $100 \mathrm{mCi}$ of ${ }^{131} \mathrm{I}$ (around $50 \mathrm{mSv}$ ).

Whole-body SPECT/CT performed after the administration of a diagnostic or a therapeutic activity $(30 \mathrm{mCi}$ or more $)$ of RAI is associated with (i) an increased number of patients with a diagnosis of metastatic lymph node and (ii) a decreased frequency of equivocal findings $(739,743,815-818)$. Furthermore, the CT portion of the SPECT/CT provides additional information on non-iodine-avid lesions; SPECT-CT changed tumor risk classifications in $25 \%$ of the patients according to the International Union Against Cancer classification and in $6 \%$ of the patients according to the ATA risk of recurrence classification; the SPECT-CT changed treatment management in 24 to $35 \%$ of patients, by decreasing the rate of equivocal findings. Finally, SPECT-CT avoids the need for further cross-sectional imaging studies such as contrast CT or MRI. Neoplastic lesions with low uptake of RAI or without any uptake may be a cause of false negative SPECT-CT.

Iodine 124 emits positrons, allowing PET/CT imaging in DTC patients. It is used as a dosimetric and also as a diagnostic tool to localize disease. In fact, for each neoplastic focus ${ }^{124}$ I PET/CT permits an accurate measurement of its volume as well as the uptake and half-life of ${ }^{124} \mathrm{I}$ in it, therefore allowing a reliable individual dosimetric assessment for each neoplastic focus.
The sensitivity of ${ }^{124}$ I-PET for the detection of residual thyroid tissue and/or metastatic DTC was reported to be higher than that of a diagnostic ${ }^{131}$ I planar WBS $(99 \%$ vs. 66\%) (819-821). Iodine 124 PET/CT has not yet been compared with ${ }^{131}$ I SPECT/CT in a large series of patients with DTC. Furthermore, ${ }^{124} \mathrm{I}$ is not yet widely available for clinical use and is primarily a research tool at this time.

\section{[C12] ${ }^{18}$ FDG-PET scanning}

\section{- RECOMMENDATION 68}

(A) ${ }^{18}$ FDG-PET scanning should be considered in highrisk DTC patients with elevated serum Tg (generally $>10 \mathrm{ng} / \mathrm{mL}$ ) with negative RAI imaging

\section{(Strong recommendation, Moderate-quality evidence)}

(B) ${ }^{18}$ FDG-PET scanning may also be considered as (i) a part of initial staging in poorly differentiated thyroid cancers and invasive Hürthle cell carcinomas, especially those with other evidence of disease on imaging or because of elevated serum Tg levels, (ii) a prognostic tool in patients with metastatic disease to identify lesions and patients at highest risk for rapid disease progression and disease-specific mortality, and (iii) an evaluation of posttreatment response following systemic or local therapy of metastatic or locally invasive disease.

\section{(Weak recommendation, Low-quality evidence)}

${ }^{18}$ FDG-PET/CT is primarily considered in high-risk DTC patients with elevated serum $\mathrm{Tg}$ (generally $>10 \mathrm{ng} / \mathrm{mL}$ ) with negative RAI imaging. In a meta-analysis of 25 studies that included 789 patients, the sensitivity of ${ }^{18}$ FDG-PET/CT was $83 \%$ (ranging from $50 \%$ to $100 \%$ ) and the specificity was $84 \%$ (ranging from $42 \%$ to $100 \%$ ) in non- ${ }^{131}$ I-avid DTC (822). Factors influencing ${ }^{18}$ FDG-PET/CT sensitivity included tumor dedifferentiation, larger tumor burden, and to a lesser extent, TSH stimulation.

${ }^{18}$ FDG-PET is more sensitive in patients with an aggressive histological subtype, including poorly differentiated, tall cell, and Hürthle cell thyroid cancer. ${ }^{18}$ FDG uptake on PET in metastatic DTC patients is a major negative predictive factor for response to RAI treatment and an independent prognostic factor for survival $(823,824)$. It can also identify lesions with high ${ }^{18}$ FDG uptake (standardized uptake value) that may be more aggressive and should be targeted for therapy or close monitoring. It is complementary to ${ }^{131} \mathrm{I}$ WBS, even in the presence of detectable ${ }^{131}$ I uptake in metastases, because ${ }^{18}$ FDG uptake may be present in neoplastic foci with no ${ }^{131} \mathrm{I}$ uptake.

In patients with a TSH-stimulated $\mathrm{Tg} \leq 10 \mathrm{ng} / \mathrm{mL}$, the sensitivity of ${ }^{18} \mathrm{FDG}$ is low, ranging from $<10 \%$ to $30 \%$. It is therefore recommended to consider ${ }^{18}$ FDG-PET only in DTC patients with a stimulated $\mathrm{Tg}$ level $\geq 10 \mathrm{ng} / \mathrm{mL}$. Of course, this level needs to be adapted and lowered in case of aggressive pathological variant of thyroid cancer that may produce low amounts of serum Tg. Furthermore, in patients with undetectable Tg levels but with persistent Tg antibodies the level of serum Tg cannot be reliably assessed and ${ }^{18}$ FDG-PET may localize disease in some of these patients.

The sensitivity of ${ }^{18}$ FDG-PET scanning may be slightly increased with TSH stimulation. A multicentric prospective study on 63 patients showed an increase in the number of lesions 
detected on the ${ }^{18}$ FDG-PET/CT performed after rhTSH stimulation compared to the ${ }^{18} \mathrm{FDG}-\mathrm{PET} / \mathrm{CT}$ performed on thyroid hormone treatment and without TSH stimulation (825). However, the sensitivity for detecting patients with at least one tumor site was not improved by the rhTSH stimulation. In this study, the lesions found only by rhTSH-PET contributed adequately to an altered therapeutic plan in four patients $(6 \%)$, and the clinical benefit of identifying these additional small foci remains to be proven. Its clinical benefit might be higher in patients with normal neck and chest CT scan and normal neck ultrasonography. A metaanalysis on seven studies including the previous study and comprising 168 patients confirmed these results and showed that ${ }^{18}$ FDG-PET/CT performed following TSH stimulation altered clinical management in only $9 \%$ of patients. Furthermore, false positives can be seen with ${ }^{18}$ FDG-PET imaging with or without TSH stimulation (825).

Results of ${ }^{18}$ FDG-PET/CT might alter the indications for ${ }^{131}$ I treatment or the decision for surgical removal of small tumor foci with ${ }^{18}$ FDG uptake. The frequency of falsepositive lesions varies among series from $0 \%$ to $39 \%$, and this high number justifies a FNAB with cytology and Tg measurement in the aspirate fluid in cases in which surgery is planned, based on ${ }^{18}$ FDG-PET results. The higher sensitivity of neck ultrasonography for the detection of small metastatic lymph nodes should be noted, with ${ }^{18}$ FDG-PET being more sensitive for some locations such as the retropharyngeal or the retro-clavicular regions (825).

To date, there is no evidence that TSH stimulation improves the prognostic value of ${ }^{18}$ FDG-PET imaging.

\section{[C13] CT and MRI}

\section{- RECOMMENDATION 69}

(A) Cross-sectional imaging of the neck and upper chest (CT, MRI) with IV contrast should be considered (i) in the setting of bulky and widely distributed recurrent nodal disease where US may not completely delineate disease, (ii) in the assessment of possible invasive recurrent disease where potential aerodigestive tract invasion requires complete assessment, or (iii) when neck US is felt to be inadequately visualizing possible neck nodal disease (high Tg, negative neck US).

(Strong recommendation, Moderate-quality evidence)

(B) CT imaging of the chest without IV contrast (imaging pulmonary parenchyma) or with IV contrast (to include the mediastinum) should be considered in high risk DTC patients with elevated serum Tg (generally $>10 \mathrm{ng} /$ $\mathrm{mL}$ ) or rising $\mathrm{Tg}$ antibodies with or without negative RAI imaging.

\section{(Strong recommendation, Moderate-quality evidence)}

(C) Imaging of other organs including MRI brain, MR skeletal survey, and/or CT or MRI of the abdomen should be considered in high-risk DTC patients with elevated serum $\mathrm{Tg}$ (generally $>10 \mathrm{ng} / \mathrm{mL}$ ) and negative neck and chest imaging who have symptoms referable to those organs or who are being prepared for TSH-stimulated RAI therapy (withdrawal or rhTSH) and may be at risk for complications of tumor swelling.

(Strong recommendation, Low-quality evidence)
In patients with elevated or rising $\mathrm{Tg}$ or anti-Tg antibodies and NED on neck US or RAI imaging (if performed), CT imaging of the neck and chest should be considered. The frequency of positive anatomic imaging increases with higher serum Tg levels above $10 \mathrm{ng} / \mathrm{mL}$. CT is the most frequently recommended first-line technique to search for lymph node metastases in patients with squamous cell carcinoma of the head and neck, and an injection of contrast medium is mandatory for the analysis of the neck and mediastinum (826). Radioiodine can be administered 4-8 weeks following the injection of contrast medium, because at that time a majority of the iodine contamination has disappeared in most patients (315). If there is a concern, a random urine iodine (and creatinine) prior to initiation of a LID and RAI testing or treatment can be measured to make sure the urine iodine is not high. Diagnostic CT scan may complement neck US for the detection of macrometastases in the central compartment, in the mediastinum, and behind the trachea (307-309), and it is the most sensitive tool for the detection of micrometastases in the lungs. Before revision surgery is contemplated, presumptive recurrent neck targets must be defined by highresolution radiographic anatomic studies such as US or spiral axial CT scan to complement ${ }^{18}$ FDG-PET/CT or RAI imaging and must be carefully defined to allow for adequate preoperative mapping and definitive surgical localization. In addition to nodal assessment axial scanning, including CT scan with contrast has utility in the evaluation of locally recurrent invasive disease and relationships with vessels. Such patients may present with hoarseness, vocal cord paralysis on laryngeal exam, progressive dysphagia or mass fixation to surrounding structures, respiratory symptoms including stridor or hemoptysis, and lesions with rapid progression/enlargement. Such lesions are incompletely evaluated with US alone, and axial CT scanning with contrast medium is indicated.

The use of MRI has also been advocated for imaging the neck and the mediastinum. It is performed with and without injection of gadolinium chelate as a contrast medium and does not require any injection of iodine contrast medium. The performance of MRI for imaging the neck and mediastinum has not been directly compared with CT on large numbers of thyroid cancer patients (827-829). Compared to CT scan, it may better delineate any involvement of the aerodigestive tract $(830,831)$. It is often used as second-line imaging technique in patients with demonstrated or suspicious lesions on CT scan in order to better delineate these lesions. In the lower part of the neck, movements of the aerodigestive axis during the procedure that may last several minutes will decrease the quality of images (414). Endoscopy of the trachea and or esophagus, with or without ultrasonography, looking for evidence of intraluminal extension can also be helpful in cases of suspected aerodigestive tract invasion. MRI is less sensitive than CT scan for the detection of lung micronodules.

Finally, whether these imaging techniques (CT and MRI) should be performed for diagnostic purposes or whether an ${ }^{18}$ FDG-PET/CT scan should be performed as the first-line imaging procedure for diagnosis is still a matter of debate. In the past, CT scan with injection of contrast medium was more sensitive for the detection of lymph node metastases (832), but with modern PET/CT equipment, the CT scan of the PET/ $\mathrm{CT}$ is as reliable as a CT scan used for radiology, and many lesions can be found on ${ }^{18}$ FDG-PET/CT scanning, even if no 
injection of contrast medium has been performed $(833,834)$. Delineation between lymph node metastases or local recurrence and vessels or the aerodigestive axis is often not well visualized on ${ }^{18}$ FDG-PET/CT in the absence of contrast injection, and if necessary other imaging techniques (CT and MRI with contrast medium) may be performed especially for a preoperative work-up. As a result, most patients with extensive disease should be considered for ${ }^{18} \mathrm{FDG}-\mathrm{PET} / \mathrm{CT}$ and CT imaging with contrast, and some patients will also be considered for MRI.

This imaging strategy is applied in patients with elevated serum $\mathrm{Tg}(>5-10 \mathrm{ng} / \mathrm{mL})$ and no other evidence of disease (neck and chest imaging), starting with a ${ }^{18}$ FDG-PET/CT $(822,833)$. In the past an empiric treatment was used in such patients, but recent studies have shown that ${ }^{18}$ FDG-PET/CT imaging is more sensitive and should be performed as the first-line approach, with empiric RAI treatment being considered only for those patients with no detectable ${ }^{18} \mathrm{FDG}$ uptake (833).

[C14] Using ongoing risk stratification (response to therapy) to guide disease long-term surveillance and therapeutic management decisions

Ongoing risk stratification allows the clinician to continue to provide individualized management recommendations as the risk estimates evolve over time. While the specific details of how surveillance and therapeutic strategies should be modified over time as a function of response to therapy reclassification within each ATA risk category remains to be defined, we do endorse the following concepts (more details in Table 13).

Excellent response: An excellent response to therapy should lead to a decrease in the intensity and frequency of follow-up and the degree of TSH suppression (this change in management will be most apparent in ATA intermediate- and high-risk patients).

Biochemical incomplete response: If associated with stable or declining serum $\mathrm{Tg}$ values, a biochemical incomplete response should lead to continued observation with ongoing TSH suppression in most patients. Rising Tg or anti$\mathrm{Tg}$ antibody values should prompt additional imaging and potentially additional therapies.

Structural incomplete response: A structural incomplete response may lead to additional treatments or ongoing observation depending on multiple clinico-pathologic factors including the size, location, rate of growth, RAI avidity, ${ }^{18}$ FDG avidity, and specific pathology of the structural lesions.

Indeterminate response: An indeterminate response should lead to continued observation with appropriate serial imaging of the nonspecific lesions and serum Tg monitoring. Nonspecific findings that become suspicious over time or rising Tg or anti-Tg antibody levels can be further evaluated with additional imaging or biopsy.

\section{[C15] What is the role of TSH suppression during thyroid hormone therapy in the long-term follow-up of DTC?}

\section{- RECOMMENDATION 70}

(A) In patients with a structural incomplete response to therapy, the serum TSH should be maintained below
$0.1 \mathrm{mU} / \mathrm{L}$ indefinitely in the absence of specific contraindications.

\section{(Strong recommendation, Moderate-quality evidence)}

(B) In patients with a biochemical incomplete response to therapy, the serum TSH should be maintained between 0.1 and $0.5 \mathrm{mU} / \mathrm{L}$, taking into account the initial ATA risk classification, $\mathrm{Tg}$ level, $\mathrm{Tg}$ trend over time, and risk of TSH suppression.

\section{(Weak recommendation, Low-quality evidence)}

(C) In patients who presented with high-risk disease but have an excellent (clinically and biochemically free of disease) or indeterminate response to therapy, consideration should be given to maintaining thyroid hormone therapy to achieve serum TSH levels of $0.1-0.5 \mathrm{mU} / \mathrm{L}$ for up to 5 years after which the degree of TSH suppression can by reduced with continued surveillance for recurrence.

\section{(Weak recommendation, Low-quality evidence)}

(D) In patients with an excellent (clinically and biochemically free of disease) or indeterminate response to therapy, especially those at low risk for recurrence, the serum TSH may be kept within the low reference range (0.5-2 mU/L).

\section{(Strong recommendation, Moderate-quality evidence)}

(E) In patients who have not undergone remnant ablation or adjuvant therapy who demonstrate an excellent or indeterminate response to therapy with a normal neck US, and low or undetectable suppressed serum $\mathrm{Tg}$, and $\mathrm{Tg}$ or anti-Tg antibodies that are not rising, the serum TSH can be allowed to rise to the low reference range (0.5-2 mU/L).

\section{(Weak recommendation, Low-quality evidence)}

A meta-analysis has suggested an association between thyroid hormone suppression therapy and reduction of major adverse clinical events (745). The appropriate degree of TSH suppression by thyroid hormone therapy is still unknown, especially in high-risk patients rendered free of disease. A constantly suppressed TSH $(0.05 \mathrm{mU} / \mathrm{L})$ was found in one study to be associated with a longer relapse-free survival than when serum TSH levels were always $1 \mathrm{mU} / \mathrm{L}$ or greater, and the degree of TSH suppression was an independent predictor of recurrence in multivariate analysis (749). Conversely, another large study found that disease stage, patient age, and ${ }^{131} \mathrm{I}$ therapy independently predicted disease progression, but the degree of TSH suppression did not (275). A third study showed that during $\mathrm{LT}_{4}$ therapy the mean $\mathrm{Tg}$ levels were significantly higher when TSH levels were normal than when TSH levels were suppressed $(<0.5 \mathrm{mU} / \mathrm{L})$ but only in patients with local or distant recurrence (835). A fourth study of 2936 patients found that overall survival improved significantly when the TSH was suppressed to $<0.1 \mathrm{mU} / \mathrm{L}$ in patients with NTCTCSG stage III or IV disease and to a range of $0.1 \mathrm{mU} / \mathrm{L}$ to about $0.5 \mathrm{mU} / \mathrm{L}$ in patients with NTCTCSG stage II disease; however, there was no incremental benefit from suppressing TSH to undetectable levels in stage II patients, and suppression of TSH was of no benefit in patients with stage I disease (671), and higher degrees of suppression to TSH of $<0.03 \mathrm{mU} / \mathrm{L}$ provided no additional benefit (746). Another study found that a serum TSH 
threshold of $2 \mathrm{mU} / \mathrm{L}$ differentiated best between patients free of disease and those with relapse or cancer-related mortality, which remained significant when age and tumor stage were included in a multivariate analysis (750). A prospective study showed that disease-free survival for low-risk patients without TSH suppression was not inferior to patients with TSH suppression (836). No prospective studies have been performed examining the risk of recurrence and death from thyroid cancer associated with varying serum TSH levels, based on the criteria outlined above in [C14] for the absence of tumor at 6-12 months post surgery and RAI ablation.

A recent observational study demonstrated increased risk of all-cause and cardiovascular mortality in DTC patients compared to a control population (837). The authors also showed that survival in the DTC patients was lower when the serum TSH was $<0.02 \mathrm{mU} / \mathrm{L}$, which is particularly relevant in patients with an excellent response to therapy in whom overtreatment should be avoided. An approach to balancing the risks of thyroxine suppression against the risks of tumor recurrence or progression has been presented in a recent review (747). This review helped to define patients at low, intermediate, and high risk of complications from TSH suppression therapy. Table 15 provides recommendations for TSH ranges based on response to thyroid cancer therapy weighed against risks of $\mathrm{LT}_{4}$ therapy, which is adapted from the review by Biondi and Cooper (747). In patients at high risk of adverse effects on heart and bone by TSH suppression therapy, the benefits of TSH suppression should be weighed against the potential risks. In peri- and postmenopausal women at risk for bone loss, adjunctive therapy with calcium supplements, vitamin $\mathrm{D}$, and other boneenhancing agents (bisphosphonates, denosumab, etc.) should be considered. $\beta$-Adrenergic blocking drugs may be considered in older patients to obviate increases in left ventricular mass and tachycardia $(838,839)$.

There are inadequate data to make a strong recommendation regarding the intensity and duration of TSH suppression in the biochemical incomplete response to therapy category. This category encompasses a variety of patients with low serum $\mathrm{Tg}$ levels (median nonstimulated $\mathrm{Tg} 3.6 \mathrm{ng} / \mathrm{mL}$ ) having been initially classified as ATA low risk (16\%-24\%), ATA intermediate risk $(47 \%-64 \%)$, or ATA high risk (18\%-21\%) $(539,607)$. Furthermore, the risk of development of structurally identifiable disease within this cohort is not uniform but rather is related to the ongoing behavior of residual disease as reflected by both the magnitude of the Tg elevation and to the rate of rise of the serum $\mathrm{Tg}$ or anti-Tg antibodies. Based on weak data and expert opinion, we recommend a goal TSH of 0.1-0.5 mIU/L for the majority of patients with a biochemical

Table 15. Thyrotropin Targets for Long-Term Thyroid Hormone Therapy

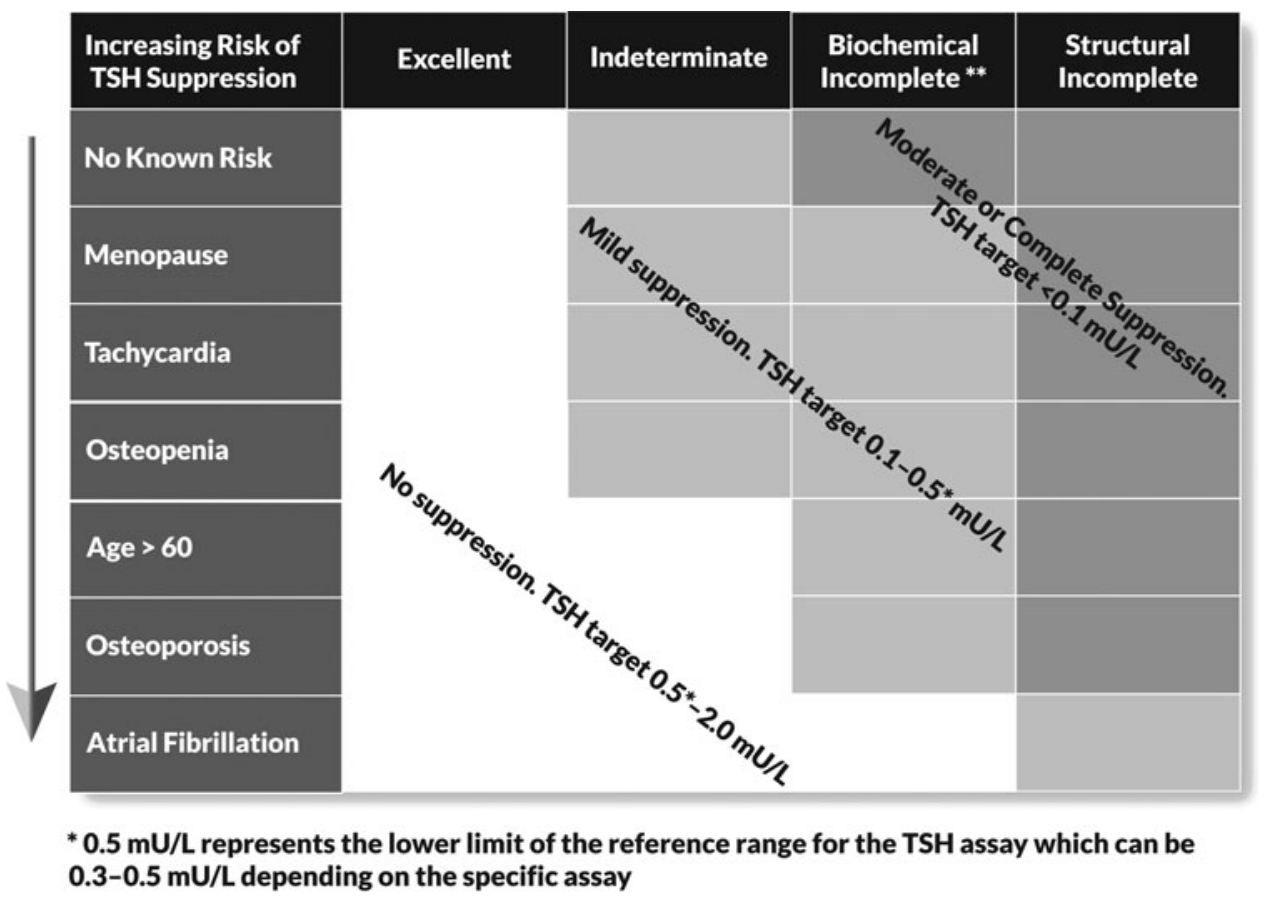
${ }^{* *}$ TSH target for patients with a biochemical incomplete response can be quite different based
on original ATA risk, Tg level, Tg trend over time and risk of TSH suppression

No suppression. TSH target $0.5^{*}-2.0 \mathrm{mU} / \mathrm{L}$

Mild suppression. TSH target $0.1-0.5^{*} \mathrm{mU} / \mathrm{L}$

Moderate or Complete suppression. TSH target $<0.1 \mathrm{mU} / \mathrm{L}$ 
incomplete response, recognizing that less intense TSH suppression $(0.5-2.0 \mathrm{mIU} / \mathrm{L})$ may be appropriate for ATA lowrisk patients with stable nonstimulated $\mathrm{Tg}$ values near the threshold for excellent response (e.g., nonstimulated $\mathrm{Tg}$ values in the $1-2 \mathrm{ng} / \mathrm{mL}$ range), while more intense TSH suppression $(<0.1 \mathrm{mIU} / \mathrm{L})$ may be desired in the setting of more elevated or rapidly rising $\mathrm{Tg}$ values.

\section{[C16] What is the most appropriate management of DTC patients with metastatic disease?}

Metastases may be discovered at the time of initial disease staging or may be identified during longitudinal follow-up. If metastases are found following initial therapy, some patients may subsequently experience a reduction in tumor burden with additional treatments that may offer a survival or palliative benefit (840-844). The preferred hierarchy of treatment for metastatic disease (in order) is surgical excision of locoregional disease in potentially curable patients, ${ }^{131}$ I therapy for RAI-responsive disease, external beam radiation therapy or other directed treatment modalities such as thermal ablation, TSH-suppressive thyroid hormone therapy for patients with stable or slowly progressive asymptomatic disease, and systemic therapy with kinase inhibitors (preferably by use of FDA-approved drugs or participation in clinical trials), especially for patients with significantly progressive macroscopic refractory disease. Clinical trials or kinase inhibitor therapy may be tried before external beam radiation therapy in special circumstances, in part because of the morbidity of external beam radiation and its relative lack of efficacy. However, localized treatments with thermal (radiofrequency or cryo-) ablation (845), ethanol ablation (846), or chemo-embolization (847) may be beneficial in patients with a single or a few metastases and in those with metastases at high risk of local complications; the treatments should be performed in such patients before the initiation of any systemic treatment. These modalities may control treated metastases, may avoid local complications, and may delay initiation of systemic treatment. Additionally, surgical therapy in selected incurable patients is important to prevent complications in targeted areas, such as the CNS and central neck compartment. Conversely, conservative intervention with TSH-suppressive thyroid hormone therapy may be appropriate for selected patients with stable asymptomatic local metastatic disease and most patients with stable asymptomatic non-CNS distant metastatic disease.

\section{[C17] What is the optimal directed approach to patients with suspected structural neck recurrence?}

\section{- RECOMMENDATION 71}

Therapeutic compartmental central and/or lateral neck dissection in a previously operated compartment, sparing uninvolved vital structures, should be performed for patients with biopsy-proven persistent or recurrent disease for central neck nodes $\geq 8 \mathrm{~mm}$ and lateral neck nodes $\geq 10 \mathrm{~mm}$ in the smallest dimension that can be localized on anatomic imaging.

\section{(Strong recommendation, Moderate-quality evidence)}

Persistent or recurrent nodal disease may result in local invasion and is the source of considerable patient and phy- sician anxiety (848). However, several observational studies suggest that low-volume recurrent nodal disease can be indolent and can be managed through active surveillance, although not all lesions in these series are documented as malignant $(629,849)$. Bulky or invasive recurrent disease is best treated surgically $(319,850-853)$.

The judgment to offer surgery for recurrent nodal disease in the neck is made with equipoise in two opposing decision elements: (i) the risks of revision surgery (which are typically higher than primary surgery due to scarring from previous surgery (854) balanced with (ii) the fact that surgical resection generally represents the optimal treatment of macroscopic gross nodal disease over other treatment options. An important element in this decision-making process is the availability of surgical expertise specifically in the performance of revision thyroid cancer nodal surgery, which is a discrete surgical skill set. The decision to treat cervical nodal recurrence surgically should be made with an appreciation of distant disease presence and progression but may be undertaken even in the setting of known distant metastasis for palliation of symptoms and prevention of aerodigestive tract obstruction. The decision for treatment and surgery specifically is best derived through collaborative team approach involving surgery, endocrinology, and importantly the patient and family (855). Therefore, cytologic confirmation of disease can be deferred if the findings of the FNA will not lead to additional evaluation or treatment. While we generally recommend cytologic confirmation of abnormal radiographic findings prior to surgical resection, we recognize that this may not be necessary (or possible) in every case (e.g., radiographic findings with a very high likelihood of malignancy, or the specific location of the lymph node makes it difficult/impossible to biopsy).

\section{[C18] Nodal size threshold}

Surgery is considered with the recognition of clinically apparent, macroscopic nodal disease through radiographic analysis including US (Table 7) and/or axial (CT) scanning rather than through isolated $\mathrm{Tg}$ elevation $(309,335,856)$. Given the risks of revision nodal surgery, a clearly defined preoperative radiographic target is mandatory. The risks of surgery relate in part to the exact location of the target node(s) and whether the compartment in question has been previously dissected such as recurrent central neck nodes after primary thyroidectomy. This target must be defined by high-resolution radiographic anatomic studies such as US or spiral CT scan with contrast, as a complement to ${ }^{18}$ FDG-PET/ CT or RAI-SPECT/CT when performed, to allow for adequate preoperative mapping and definitive surgical localization $(309,856)$. Ultrasound-guided FNA for cytology with Tg measurement in the aspiration sample can be performed in the setting of radiographically suspicious nodal recurrence keeping in mind that $\mathrm{Tg}$ rinsing may be positive with thyroid bed persistent benign thyroid remnant tissue if the patient has not been treated with RAI. Charcoal tattooing under US guidance may help the surgeon to localize the lymph node to be removed during surgery (344).

Malignant central neck nodes $\geq 8 \mathrm{~mm}$ and lateral neck nodes $\geq 10 \mathrm{~mm}$ in the smallest dimension that have undergone FNAB and can be localized on anatomic imaging (US with or without axial CT) can be considered surgical targets 
(309,857-859). Short-axis nodal diameter measurement is optimally employed in surgical decision-making for nodal malignancy. Smaller lesions are probably best managed with active surveillance (observation) with serial cross-sectional imaging, reserving FNA and subsequent intervention for documented structural disease progression. However, multiple factors in addition to size should be taken into account when considering surgical options, including proximity of given malignant nodes to adjacent vital structures and the functional status of the vocal cords. Patient comorbidities, motivation, and emotional concerns should also be taken into account along with primary tumor factors (high-grade histology, Tg doubling time, RAI avidity, ${ }^{18}$ FDG-PET avidity, and presence of molecular markers associated with aggressive behavior). Through thorough patient and multidisciplinary collaborative discussions, metastatic nodes $>8-10 \mathrm{~mm}$ can be carefully observed in properly selected patients with serial clinical and radiographic follow-up, with surgery being offered if they progress during follow-up and conservative follow-up being maintained if they are stable over time.

\section{[C19] Extent of nodal surgery}

Because of the increased risk of recurrence with focal "berry-picking" techniques, compartmental surgery is recommended (860,861). Planned compartmental dissection should be adjusted and be more limited depending on the surgeon's judgment of procedural safety as it relates to scarring/distortion of anatomy (from prior surgery and/or past radiation therapy) and the perception of impending complications. Typical revision lateral neck dissection involves levels II, III, and IV, while revision central neck dissection includes at least one paratracheal region with prelaryngeal and pretracheal subcompartments. Bilateral central neck dissection is offered only when dictated by disease distribution because of the risks of bilateral nerve injury and permanent hypoparathyroidism.

Basal $\mathrm{Tg}$ decreases by $60 \%-90 \%$ after compartmental dissection for recurrent nodal disease in modern series, but only $30 \%-50 \%$ of patients have unmeasurable basal $\mathrm{Tg}$ after such surgery, and it is difficult to predict who will respond to surgery with $\mathrm{Tg}$ reduction $(627,628,634,858,862-876)$. However, most series suggest surgery results in a high clearance rate of structural disease in over $80 \%$ of patients $(859,875)$.

\section{[C20] Ethanol injection}

Percutaneous ethanol injection for patients with metastatic lymph nodes is gaining interest as a nonsurgical directed therapy for patients with recurrent DTC. Most of the studies limited PEI to patients who had undergone previous neck dissections and RAI treatment, those who had FNA-proven DTC in the lymph node, and those with no known distant metastases.

One of the first studies examining the effectiveness of local metastatic lymph node control by PEI treated 14 patients with 29 lymph nodes (846). Twelve of the 14 patients had good loco-regional control in this study with short-term follow-up (mean 18 months). The largest study to date treated 63 patients with 109 metastatic lymph nodes between the years 2004 and 2009 (878). Ninety-two lymph nodes $(84 \%)$ were successfully ablated in this retrospective study with a mean follow-up of 38 months, and most re- quired one to three treatment sessions. Minor complications included brief discomfort at the PEI site, and there were no major complications.

A recent study retrospectively reviewed 25 patients who had 37 lymph nodes ablated between the years 1994 and 2012, with a relatively long follow-up of a mean of 65 months (879). All lymph nodes were successfully ablated in one to five treatment sessions by lack of flow on US. Most of the lymph nodes decreased in size and $46 \%$ completely disappeared. Serum Tg levels were reduced in most patients and brought into an acceptable range $(<2.4 \mathrm{ng} / \mathrm{mL})$ in $82 \%$ of patients with negative anti-Tg antibodies. There were no serious or long-term complications. Another recent study also demonstrated safety and efficacy of PEI in 21 patients with 41 metastatic lymph nodes (880). These investigators treated patients with only one session, and $24 \%$ of patients had a recurrence at the site of the injection.

Limitations of many of the studies included small numbers of patients, relatively short-term follow-up, and many patients with small lymph nodes $(<5-8 \mathrm{~mm})$.

A general consensus from studies and reviews is that PEI should be considered in patients who are poor surgical candidates. Many patients will likely need more than one treatment session and lymph nodes $>2 \mathrm{~cm}$ may be difficult to treat with PEI. Focal PEI treatment does represent a nonsurgical form of berry picking. Formal neck compartmental dissection is still the first-line therapy in DTC patients with clinically apparent or progressive lymph node metastases. When deciding for the optimal strategy of care for a patient's lymph node metastases, previous treatment modalities should also be taken into consideration.

\section{[C21] Radiofrequency or laser ablation}

The use of radiofrequency ablation (RFA) with local anesthesia in the treatment of recurrent thyroid cancer has been associated with a mean volume reduction that ranges between approximately $55 \%$ and $95 \%(881,882)$ and complete disappearance of the metastatic foci in $40 \%-60 \%$ of the cases $(845,882,883)$. As with alcohol ablation, multiple treatment sessions are often required. Complications include discomfort, pain, skin burn, and changes in the voice (884). Similar to alcohol ablation techniques, it appears that RFA may be most useful in high-risk surgical patients or in patients refusing additional surgery, rather than as a standard alternative to surgical resection of metastatic disease (883-885). More recently, preliminary findings using US-guided laser ablation for treatment of cervical lymph node metastases have been reported (886).

\section{[C22] Other therapeutic options}

Empiric RAI therapy for structurally identifiable disease that is not RAI avid by diagnostic scanning is very unlikely to have a significant tumoricidal effect and is therefore not generally recommended (887). Stereotactic radiotherapy (SBRT) can be successfully used to treat isolated metastatic disease foci, but it has no role in most patients with resectable lymph node metastases. EBRT using modern techniques such as intensity modulated radiotherapy and sterotactic radiation, is considered for loco-regional recurrence that is not surgically resectable or with extranodal extension or involvement of soft tissues, particularly in patients with no evidence of 
distant disease. Efficacy has been suggested only in retrospective studies on limited numbers of patients $(888,889)$. Likewise, systemic therapies (such as cytotoxic chemotherapy or kinase inhibitors) for loco-regional disease are considered only after all surgical and radiation therapy options have been exhausted.

\section{[C23] What is the surgical management of aerodigestive invasion?}

\section{- RECOMMENDATION 72}

When technically feasible, surgery for aerodigestive invasive disease is recommended in combination with RAI and/or EBRT.

(Strong Recommendation, Moderate-quality evidence)

For tumors that invade the upper aerodigestive tract, surgery combined with additional therapy such as ${ }^{131} \mathrm{I}$ and/or external beam radiation therapy is generally advised $(890,891)$. Patient outcome is related to complete resection of all gross disease with the preservation of function, with techniques ranging from shaving a tumor off the trachea or esophagus for superficial invasion, to more aggressive techniques when the trachea is more deeply invaded (e.g., direct intraluminal invasion), including tracheal resection and anastomosis or laryngopharyngoesophagectomy (892-894). Surgical decision-making can be complex and must balance oncologic surgical completeness with preservation of upper aerodigestive track head and neck function. In some circumstances such surgery represents a possible attempt for cure, and in other circumstances it offers significant regional neck palliation in patients with distant metastasis with impending asphyxiation or significant hemoptysis $(414,891)$.

\section{[C24] How should RAI therapy be considered for loco-regional or distant metastatic disease?}

For regional nodal metastases discovered on diagnostic WBS, RAI may be employed in patients with low-volume disease or in combination with surgery, although surgery is typically preferred in the presence of bulky disease or disease amenable to surgery. Radioiodine is also used adjunctively following surgery for regional nodal disease or aerodigestive invasion if residual RAI-avid disease is present or suspected. Significant variation exists nationally in the United States in regard to RAI use, irrespective of the degree of disease or risk of recurrence $(895,896)$. However, there are no randomized, controlled clinical trials demonstrating better patient outcomes after RAI therapy. One retrospective analysis indicated that a delay in RAI therapy of 6 months or more was associated with disease progression and reduced survival (897). In one study of 45 patients with persistent serum $\mathrm{Tg}$ elevation after reoperation for loco-regional recurrence, adjuvant RAI therapy demonstrated no benefit (898).

[C25] Administered activity of ${ }^{131} \mathrm{I}$ for loco-regional or metastatic disease

\section{- RECOMMENDATION 73}

(A) Although there are theoretical advantages to dosimetric approaches to the treatment of loco-regional or metastatic disease, no recommendation can be made about the superiority of one method of RAI administration over another (empiric high activity versus blood and/or body dosimetry versus lesional dosimetry).

\section{(No recommendation, Insufficient evidence)}

(B) Empirically administered amounts of ${ }^{131}$ I exceeding 150 $\mathrm{mCi}$ that often potentially exceed the maximum tolerable tissue dose should be avoided in patients over age 70 years.

\section{(Strong recommendation, Moderate-quality evidence)}

Despite the apparent effectiveness of ${ }^{131}$ I therapy in many patients, the optimal therapeutic activity remains uncertain and controversial $(895,899)$. There are three approaches to ${ }^{131}$ I therapy: empiric fixed amounts, therapy determined by the upper limit of blood and body dosimetry (900-907), and quantitative tumor or lesional dosimetry $(908,909)$. Dosimetric methods are often reserved for patients with distant metastases or unusual situations such as renal insufficiency (910,911), children $(912,913)$, the elderly, and those with extensive pulmonary metastases (914). Comparison of outcomes among these methods from published series is difficult $(895,897,900)$. No prospective randomized trial to address the optimal therapeutic approach has been published. One retrospective study concluded that patients with locoregional disease were more likely to respond after dosimetric therapy than after empiric treatment (915). Another study demonstrated improved efficacy of administration of dosimetric maximal activity after failure of empiric dosage (916). Arguments in favor of higher activities cite a positive relationship between the total ${ }^{131}$ I uptake per tumor mass and outcome (908), while others have not confirmed this relationship (917). In the future, the use of ${ }^{123} \mathrm{I}$ or ${ }^{131} \mathrm{I}$ with modern SPECT/CT or ${ }^{124}$ I PET-based dosimetry may facilitate whole-body and lesional dosimetry (918-920). In certain settings (e.g., in the patients with radiation-induced thyroid cancer seen after the Chernobyl accident), RAI treatment may be associated with good outcomes even in high-risk patients with metastatic disease (921).

The efficacy of RAI therapy is related to the mean radiation dose delivered to neoplastic foci and also to the radiosensitivity of tumor tissue (922). The radiosensitivity is higher in patients who are younger, with small metastases from welldifferentiated papillary or follicular carcinoma and with uptake of RAI but no or low ${ }^{18}$ FDG uptake.

The maximum tolerated radiation absorbed dose (MTRD), commonly defined as 200 rads (cGy) to the blood, is potentially exceeded in a significant number of patients undergoing empiric treatment with various amounts of ${ }^{131} \mathrm{I}$. In one study (923) $1 \%-22 \%$ of patients treated with ${ }^{131} \mathrm{I}$ according to dosimetry calculations would have theoretically exceeded the MTRD had they been empirically treated with 100-300 $\mathrm{mCi}$ of ${ }^{131} \mathrm{I}$. Another study (924) found that an empirically administered ${ }^{131} \mathrm{I}$ activity of $200 \mathrm{mCi}$ would exceed the MTRD in $8 \%-15 \%$ of patients younger than age 70 and $22 \%-$ $38 \%$ of patients aged 70 years and older. Administering $250 \mathrm{mCi}$ empirically would have exceeded the MTRD in $22 \%$ of patients younger than 70 and $50 \%$ of patients 70 and older. These estimates imply the need for caution in administering empiric activities higher than $100-150 \mathrm{mCi}$ in certain populations such as elderly patients and patients with renal insufficiency. 
[C26] Use of rhTSH (Thyrogen) to prepare patients for ${ }^{131}$ I therapy for loco-regional or metastatic disease

\section{- RECOMMENDATION 74}

There are currently insufficient outcome data to recommend rhTSH-mediated therapy for all patients with distant metastatic disease being treated with ${ }^{131} \mathrm{I}$.

\section{(No Recommendation, Insufficient evidence)}

\section{- RECOMMENDATION 75}

Recombinant human TSH-mediated therapy may be indicated in selected patients with underlying comorbidities making iatrogenic hypothyroidism potentially risky, in patients with pituitary disease whose serum TSH cannot be raised, or in patients in whom a delay in therapy might be deleterious. Such patients should be given the same or higher activity that would have been given had they been prepared with hypothyroidism or a dosimetrically determined activity.

\section{(Strong Recommendation, Low-quality evidence)}

No randomized trial comparing thyroid hormone withdrawal therapy to rhTSH-mediated therapy for treatment of distant metastatic disease has been reported, but there is a growing body of nonrandomized studies exploring the use of rhTSH to prepare patients for therapy of metastatic disease (713,925-934). One small comparative study showed that the radiation dose to metastatic foci is lower with rhTSH than that following withdrawal (935). Many of these case reports and series report disease stabilization or improvement in some patients following rhTSH-mediated ${ }^{131} \mathrm{I}$ therapy, but whether the efficacy of this preparation is comparable to thyroid hormone withdrawal is unknown. Extreme or prolonged elevations of TSH from either thyroid hormone withdrawal or rhTSH may acutely stimulate tumor growth and mass of metastases (930,936-938). With metastatic deposits in the brain or in close relation to the spinal cord or the superior vena cava, such swelling may severely compromise neurologic function or produce a superior vena cava syndrome, respectively. When distant metastases are evident, MRI of the brain and spine is recommended to detect the presence of critical metastases prior to treatment (see sections [C28] and [C39]). When metastases are detected, institution of temporary high-dose corticosteroid therapy is recommended for trying to limit the risk of acute tumor swelling and compromised function. Dexamethasone has been employed in doses of 2-4 mg every 8 hours starting 6-12 hours prior to rhTSH and RAI dosing or after 10-12 days of thyroid hormone withdrawal, with the steroids continued in a tapering dosage schedule for 1 week post therapy, for 48-72 hours after rhTSH administration, or for 72 hours after re-institution of thyroxine therapy when thyroid hormone withdrawal was employed (927). In patients with these critical metastases, consideration should be given to preparation with either a reduced dose of rhTSH or to attenuating the degree and duration of endogenous TSH elevation after thyroid hormone withdrawal while monitoring serum TSH levels. This can be achieved by the temporary addition of $\mathrm{LT}_{3}$ therapy to thyroxine replacement. Satisfactory RAI treatment with either empiric or dosimetric activities should be feasible after achieving TSH levels of 30-50 mU/L. When thyroid hormone withdrawal has been employed, $\mathrm{LT}_{4}$ therapy should recommence once the dose of RAI is administered in order to reduce the duration of TSH elevation.

\section{[C27] Use of lithium in ${ }^{131}$ I therapy}

\section{- RECOMMENDATION 76}

Since there are no outcome data that demonstrate a better outcome of patients treated with lithium as an adjunct to

${ }^{131}$ I therapy, the data are insufficient to recommend lithium therapy.

\section{(No recommendation, Insufficient evidence)}

Lithium inhibits iodine release from the thyroid without impairing iodine uptake, thus enhancing ${ }^{131} \mathrm{I}$ retention in normal thyroid and tumor cells (939). One study (940) found that lithium increased the estimated ${ }^{131}$ I radiation dose in metastatic tumors on average by more than 2 -fold, but primarily in those tumors that rapidly cleared iodine. On the other hand, a different study was unable to document any clinical advantage of lithium therapy on outcome in patients with metastatic disease, despite an increase in RAI uptake in tumor deposits (941). Lithium use may be associated with adverse events and needs to be precisely managed.

\section{[C28] How should distant metastatic disease to various organs be treated?}

The overall approach to treatment of distant metastatic thyroid cancer is based upon the following observations and oncologic principles:

1. Morbidity and mortality are increased in patients with distant metastases, but individual prognosis depends upon factors including histology of the primary tumor, distribution and number of sites of metastasis (e.g., brain, bone, lung), tumor burden, age at diagnosis of metastases, and ${ }^{18}$ FDG and RAI avidity $(842,933,942-948)$.

2. Improved survival is associated with responsiveness to directed therapy (surgery, EBRT, thermal ablation, etc.) and/or RAI (842,933,942-948).

3 . In the absence of demonstrated survival benefit, certain interventions can provide significant palliation or reduce morbidity $(847,949-951)$.

4. Treatment of a specific metastatic area must be considered in light of the patient's performance status and other sites of disease; for example, $5 \%-20 \%$ of patients with distant metastases die from progressive cervical disease $(948,952)$.

5. Longitudinal re-evaluation of patient status and continuing reassessment of potential benefit and risk of intervention are required.

6. In the setting of overall poor anticipated outcome of patients with radiographically evident or symptomatic metastases that do not respond to RAI, the complexity of multidisciplinary treatment considerations and the availability of prospective clinical trials should encourage the clinician to refer such patients to tertiary centers with particular expertise.

7. Mutation profiling of metastatic tumor (to detect abnormalities in genes such as BRAF, TERT, RAS, or PAX8/ 
$P P A R \gamma$ ) has not yet definitively proven to be of value for estimating patient prognosis or for predicting response to treatments such as anti-angiogenic kinase inhibitors, although the presence of certain mutations such as $B R A F^{V 600 E}$ or $P A X 8 / P P A R \gamma$ are required for some clinical trials. Thus, routine mutation profiling cannot be recommended at this time outside of research settings.

There is little if any benefit derived from the treatment of RAI-refractory DTC with RAI (953). Although RAIrefractory tumors often may harbor $B R A F^{V 600 E}$ mutations and RAI-avid tumors are overrepresented with $R A S$ mutations, RAI therapy has not been shown to be more effective in patients with $R A S$ mutations (954).

\section{[C29] Treatment of pulmonary metastases}

\section{- RECOMMENDATION 77}

(A) Pulmonary micrometastases should be treated with RAI therapy and RAI therapy should be repeated every 6 12 months as long as disease continues to concentrate RAI and respond clinically because the highest rates of complete remission are reported in these subgroups.

\section{(Strong recommendation, Moderate-quality evidence)}

(B) The selection of RAI activity to administer for pulmonary micrometastases can be empiric (100-200 $\mathrm{mCi}$, or $100-150 \mathrm{mCi}$ for patients $\geq 70$ years old) or estimated by dosimetry to limit whole-body retention to $80 \mathrm{mCi}$ at 48 hours and $200 \mathrm{cGy}$ to the bone marrow.

(Strong recommendation, Moderate-quality evidence)

\section{- RECOMMENDATION 78}

Radioiodine-avid macronodular metastases may be treated with RAI and treatment may be repeated when objective benefit is demonstrated (decrease in the size of the lesions, decreasing Tg), but complete remission is not common and survival remains poor. The selection of RAI activity to administer can be made empirically (100-200 mCi) or by lesional dosimetry or whole-body dosimetry if available in order to limit whole-body retention to $80 \mathrm{mCi}$ at 48 hours and $200 \mathrm{cGy}$ to the bone marrow.

\section{(Weak recommendation, Low-quality evidence)}

In the management of the patient with pulmonary metastases, key criteria for therapeutic decisions include (i) size of metastatic lesions (macronodular typically detected by chest radiography, micronodular typically detected by CT, lesions beneath the resolution of CT); (ii) avidity for RAI and, if applicable, response to prior RAI therapy; and (iii) stability (or lack thereof) of metastatic lesions. Pulmonary pneumonitis and fibrosis are rare complications of high-dose RAI treatment. Dosimetric approaches to therapy with a limit of $80 \mathrm{mCi}$ whole-body retention at 48 hours and 200 cGy to the bone marrow should be considered in patients with diffuse ${ }^{131}$ I pulmonary uptake (955). If pulmonary fibrosis is suspected, then appropriate periodic pulmonary function testing and consultation should be obtained. The presence of pulmonary fibrosis may limit the ability to further treat metastatic disease with RAI (956).

Patients with pulmonary micrometastases $(<2 \mathrm{~mm}$, generally not seen on anatomic imaging) that are RAI avid have the highest rates of complete remission after treatment with RAI $(942,947,957,958)$. These patients should be treated with RAI therapy repeatedly every $6-12$ months as long as disease continues to concentrate RAI and respond clinically.

A precise definition of "responding clinically" is not feasible given the wide variation in disease presentation and response to therapy. A meaningful response to RAI treatment is generally associated with a significant reduction in serum $\mathrm{Tg}$ and/or in the size or rate of growth of metastases or structurally apparent disease. In contrast, a reduction in serum $\mathrm{Tg}$ and in RAI uptake with no concomitant decrease or with an increase in tumor size suggests refractoriness to RAI therapy. In the presence of widespread metastases, especially when in bone, additional RAI may temporarily stabilize progression, but it is unlikely to result in cure. The risks of bone marrow suppression or pulmonary fibrosis should generate caution when repeated doses of RAI are being considered. Absolute neutrophil count and platelet counts are the usual markers of bone marrow suppression, and pulmonary function testing including diffusing capacity of the lungs for carbon monoxide can be markers of pulmonary toxicity. Other approaches (see sections [C36-C41]) should be considered once maximal cumulative tolerable radiation doses have been administered.

Macronodular pulmonary metastases may also be treated with RAI if demonstrated to be iodine avid. How many doses of RAI to give and how often to give RAI is a decision that must be individualized based on the disease response to treatment, age of the patient, and the presence or absence of other metastatic lesions $(942,947)$. The presence of significant side effects including bone marrow suppression and salivary gland damage may temper enthusiasm for additional RAI therapy (959), and risk of second malignancies after RAI treatment remains controversial $(766,960,961)$. Patients with solitary pulmonary DTC metastases may be considered for surgical resection, although the potential benefit weighed against the risk of surgery is unclear.

The likelihood of significant long-term benefit of ${ }^{131} \mathrm{I}$ treatment in patients with elevated $\mathrm{Tg}$ and negative diagnostic RAI scans is very low. (962,963). While some reduction in serum Tg may be observed after such empiric therapy, one analysis concluded that there was no good evidence either for or against such treatment (964). In one small retrospective series of patients with structural disease but negative diagnostic ${ }^{131}$ I WBSs, additional RAI therapy was associated with stability of disease in $44 \%$, but progression of structural disease occurred in $56 \%$ of the patients (887).

\section{[C30] RAI treatment of bone metastases}

\section{- RECOMMENDATION 79}

(A) RAI therapy of iodine-avid bone metastases has been associated with improved survival and should be employed, although RAI is rarely curative.

\section{(Strong recommendation, Moderate-quality evidence)}

(B) The RAI activity administered can be given empirically (100-200 mCi) or determined by dosimetry.

\section{(Weak recommendation, Low-quality evidence)}

RAI therapy for patients with bone metastases is rarely curative, but some patients with RAI-avid bone metastases may benefit from this therapy $(842,947)$. A dosimetrically 
determined administered dose of RAI may be beneficial for patients with bone metastases (908), although this is not proven in controlled studies. Patients undergoing RAI therapy for bone metastases should also be considered for directed therapy of bone metastases that are visible on anatomical imaging (section [C38]). This may include surgery, external beam radiation therapy, and other focal treatment modalities. These patients should also be considered for systemic therapy with bone-directed agents (section [C47]).

\section{[C31] When should empiric RAI therapy be considered for Tg-positive, RAI diagnostic scan-negative patients?}

\section{- RECOMMENDATION 80}

In the absence of structurally evident disease, patients with stimulated serum $\mathrm{Tg}<10 \mathrm{ng} / \mathrm{mL}$ with thyroid hormone withdrawal or $<5 \mathrm{ng} / \mathrm{mL}$ with $\mathrm{rhTSH}$ (indeterminate response) can be followed without empiric RAI therapy on continued thyroid hormone therapy alone, reserving additional therapies for those with rising serum $\mathrm{Tg}$ levels over time or other evidence of structural disease progression.

(Weak recommendation, Low-quality evidence)

\section{- RECOMMENDATION 81}

Empiric (100-200 mCi) or dosimetrically determined RAI therapy may be considered in patients with more significantly elevated serum Tg levels (see Recommendation 80), rapidly rising serum $\mathrm{Tg}$ levels, or rising ant-Tg antibody levels, in whom imaging (anatomic neck/chest imaging and/ or ${ }^{18}$ FDG-PET/CT) has failed to reveal a tumor source that is amenable to directed therapy. The risk of high cumulative administered activities of RAI must be balanced against uncertain long-term benefits. If empiric RAI therapy is given and the posttherapy scan is negative, the patient should be considered to have RAI-refractory disease and no further RAI therapy should be administered.

(Weak recommendation, Low-quality evidence)

\section{- RECOMMENDATION 82}

If persistent nonresectable disease is localized after an empiric dose of RAI, and there is objective evidence of significant tumor reduction, then consideration can be made for RAI therapy to be repeated until the tumor has been eradicated or the tumor no longer responds to treatment. The risk of repeated therapeutic doses of RAI must be balanced against uncertain long-term benefits.

\section{(Weak recommendation, Low-quality evidence)}

Factors to consider when selecting patients for empiric RAI therapy include the level of serum Tg elevation and the results of ${ }^{18}$ FDG-PET scanning, if performed. Since tumors that are ${ }^{18}$ FDG-PET positive generally do not concentrate RAI (965), RAI therapy is much less likely to be efficacious $(965,966)$ and it is unlikely to alter the poorer outcome in such patients (823). Because of this, it is reasonable to perform ${ }^{18}$ FDG-PET/CT scanning prior to consideration of empiric RAI therapy (967).

The cutoff value of serum $\mathrm{Tg}$ above which a patient should be treated with an empiric dose of RAI is not clear. Most studies have reported primarily on patients with Tg levels after $T_{4}$ withdrawal of $10 \mathrm{ng} / \mathrm{mL}$ or higher; it has been suggested that a corresponding level after rhTSH stimulation would be $5 \mathrm{ng} / \mathrm{mL}$ $(321,782,962,968,969)$. Patients with a suppressed (624) or stimulated (970) serum $\mathrm{Tg}$ of $5 \mathrm{ng} / \mathrm{mL}$ or higher are unlikely to demonstrate a decline without therapy, and they have higher rates of subsequent structural recurrence than those with lower serum Tg levels (970). In addition, a rising serum $\mathrm{Tg}$ indicates disease that is likely to become clinically apparent, particularly if it is rapidly rising $(622,971,972)$.

If serum Tg levels suggest residual or recurrent disease, but diagnostic RAI WBS imaging is negative and structural imaging does not reveal disease that is amenable to directed therapy (surgical, thermal ablation, EBRT, alcohol ablation, see section [C17 and C38]), then empiric therapy with RAI (100-200 mCi) or dosimetrically determined RAI activities can be considered for two purposes: (i) to aid in disease localization, and/or (ii) as therapy for nonsurgical disease. This approach may identify the location of persistent disease in approximately $50 \%$ of patients $(968,973,974)$, although the reported range of success is wide. From a therapeutic perspective, over half of patients experience a fall in serum Tg after empiric RAI therapy in patients with negative diagnostic WBS $(963,969,975,976)$; however, there is no evidence for improved survival with empiric therapy in this setting $(782,962,968)$. Further, there is evidence that $\mathrm{Tg}$ levels may decline without specific therapy in a significant proportion of patients with Tg levels $<10 \mathrm{ng} / \mathrm{mL}(539,618-$ $620,624,782,970-972,975,977-979)$. The most compelling evidence for benefit from empiric RAI therapy is for pulmonary metastases, which are not amenable to surgical management or EBRT $(782,844,980)$.

\section{[C32] What is the management of complications of RAI therapy?}

\section{- RECOMMENDATION 83}

The evidence is insufficient to recommend for or against the routine use of measures to prevent salivary gland damage after RAI therapy.

\section{(No recommendation, Low-quality evidence)}

\section{- RECOMMENDATION 84}

Patients with xerostomia are at increased risk of dental caries and should discuss preventive strategies with their dental/oral health professional.

\section{(Weak recommendation, Low-quality evidence)}

\section{- RECOMMENDATION 85}

Surgical correction should be considered for nasolacrimal outflow obstruction, which often presents as excessive tearing (epiphora) but also predisposes to infection.

(Strong recommendation, Low-quality evidence)

While RAI appears to be a reasonably safe therapy, it is associated with a cumulative dose-related low risk of early- and late-onset complications such as salivary gland damage, dental caries (981), nasolacrimal duct obstruction (982), and secondary malignancies $(762,763,961,983,984)$, and it may likely contribute to long-term dysphagia (985). Therefore, it is important to ensure that the benefits of RAI therapy, especially repeated courses, outweigh the potential risks. There is probably no dose 
of RAI that is completely safe, nor is there any maximum cumulative dose that could not be used in selected situations. However, with higher individual and cumulative doses there are increased risks of side effects as discussed previously.

For acute transient loss of taste or change in taste and sialadentitis, recommended measures to prevent damage to the salivary glands have included hydration, sour candies, amifostine, and cholinergic agents (986), but evidence is insufficient to recommend for or against these modalities. One study suggested sour candy may actually increase salivary gland damage when given within 1 hour of RAI therapy, as compared to its use until 24 hours post therapy (987). Another study showed that the use of lemon slices within 20 min of ${ }^{124} \mathrm{I}$ administration resulted in increased radiation absorbed dose to the salivary glands (988). A different study suggested that early use and multiple administered doses of lemon juice transiently decreased radiation exposure to the parotid glands (989), so the exact role and details of use of sialagogues to prevent salivary gland damage remains uncertain. Patients with painful sialadenitis may receive pain relief from local application of ice. For chronic salivary gland complications, such as dry mouth and dental caries, cholinergic agents may increase salivary flow (986). Interventional sialendoscopy has been shown in a number of small studies to be an effective treatment in patients with RAI-induced sialadenitis that is unresponsive to medical therapy (990-992).

\section{[C33] How should patients who have received RAI therapy be monitored for risk of secondary malignancies?}

\section{- RECOMMENDATION 86}

Although patients should be counseled on the risks of second primary malignancy with RAI treatment for DTC, the absolute increase in risk of developing a second primary malignancy attributable to RAI treatment is considered small and does not warrant specific screening to any extent greater than age-appropriate general population health screening.

\section{(Weak recommendation, Low-quality evidence)}

Most long-term follow-up studies variably report a very low risk of secondary malignancies (bone and soft tissue malignancies, including breast, colorectal, kidney, and salivary cancers, and leukemia) in long-term survivors (762,763). A meta-analysis of two large multicenter studies showed that the $\mathrm{RR}$ of second malignancies was significantly increased at 1.19 ([95\% CI 1.04-1.36], $p<0.010$ ), relative to thyroid cancer survivors not treated with RAI, although the absolute increase in second primary malignancy risk attributable to RAI is considered to be small (961). The risk of leukemia was also significantly increased in thyroid cancer survivors treated with RAI, with a RR of 2.5 ([95\% CI 1.13-5.53], $p<0.024$ ) (961). Studies on T1N0 PTC from the SEER registry suggested that the excess risk of leukemia after RAI treatment was greater in young individuals compared with older individuals. The excess risk of leukemia was significantly greater in patients aged $<45$ years (standardized incidence rate of 5.32 [95\% CI 2.75-9.30] for those aged $<45$ years versus 2.26 [95\% CI 1.43-3.39] in older individuals) (766). The risk of secondary malignancies is dose related (763), with an excess absolute risk of 14.4 solid cancers and of 0.8 leukemias per gigabecquerel $(1 \mathrm{GBq}=27$
$\mathrm{mCi}$ ) of ${ }^{131} \mathrm{I}$ at 10,000 person-years of follow-up. Cumulative ${ }^{131}$ I activities above $500-600 \mathrm{mCi}$ are associated with a significant increase in risk. In theory, the risk of second primary malignancies increases with higher administered activities. There is no direct evidence of increased risk of secondary malignancies after a single administration of $30-100 \mathrm{mCi}$ in comparison to the observed risk of second primary cancer in thyroid cancer patients who have not been treated with ${ }^{131} \mathrm{I}$. The risk is clearly increased in patients who have been treated with a large cumulative activity that is higher than $600 \mathrm{mCi}$ (763), which suggests a dose-effect relationship. This is an argument for using the minimal activity necessary to treat each patient. There appears to be an increased risk of breast cancer in women with thyroid cancer $(762,983,993)$. It is unclear whether this is due to screening bias, RAI therapy, or other factors. An elevated risk of breast cancer with ${ }^{131}$ I was not observed in another study (764). The use of laxatives may decrease radiation exposure of the bowel, particularly in patients treated after prolonged withdrawal of thyroid hormone, and vigorous oral hydration will reduce exposure of the bladder and gonads (22).

\section{[C34] What other testing should patients receiving RAI therapy undergo?}

\section{- RECOMMENDATION 87}

Patients receiving therapeutic doses of RAI should have baseline complete blood count and assessment of renal function.

\section{(Weak recommendation, Low-quality evidence)}

Published data indicate that when administered activities are selected to remain below $200 \mathrm{cGy}$ to the bone marrow, minimal transient effects are noted in white blood cell and platelet counts (955). However, persistent mild decrements in white blood cell count and/or platelets are seen in some patients who have received multiple RAI therapies. Further, radiation to the bone marrow is impacted by several factors, including renal function. The kidneys are a major means of iodine excretion from the body, and physiologic radioisotope study research in nonthyroidectomized individuals has shown that renal impairment significantly reduces RAI excretion (994).

\section{[C35] How should patients be counseled about RAl therapy and pregnancy, breastfeeding, and gonadal function?}

\section{- RECOMMENDATION 88}

Women of childbearing age receiving RAI therapy should have a negative screening evaluation for pregnancy prior to RAI administration and avoid pregnancy for 6-12 months after receiving RAI.

\section{(Strong recommendation, Low-quality evidence)}

\section{- RECOMMENDATION 89}

Radioactive iodine should not be given to nursing women. Depending on the clinical situation, RAI therapy could be deferred until lactating women have stopped breastfeeding or pumping for at least 3 months. A diagnostic ${ }^{123} \mathrm{I}$ or low-dose ${ }^{131}$ I scan should be considered in recently lactating women to detect breast uptake that may warrant deferral of therapy. 
(Strong recommendation, Moderate-quality evidence)

\section{- RECOMMENDATION 90}

Men receiving cumulative RAI activities $\geq 400 \mathrm{mCi}$ should be counseled on potential risks of infertility.

\section{(Weak recommendation, Low-quality evidence)}

Women about to receive RAI therapy should first undergo pregnancy testing. Gonadal tissue is exposed to radiation from RAI in the blood, urine, and feces. Temporary amenorrhea/ oligomenorrhea lasting 4-10 months occurs in 20\%-27\% of menstruating women after ${ }^{131}$ I therapy for thyroid cancer. Although the numbers of patients studied are small, long-term rates of infertility, miscarriage, and fetal malformation do not appear to be elevated in women after RAI therapy (995-997). One recent large retrospective cohort study showed that use of RAI was associated with delayed childbearing and decreased birthrate in later years, although it is unclear if this is due to reproductive choice or reproductive health (998). Another large retrospective study suggested that pregnancy should be postponed for 1 year after therapy because of an increase in miscarriage rate (999), although this was not confirmed in a subsequent study (1000). Ovarian damage from RAI therapy may result in menopause occurring approximately 1 year earlier than in the general population, but this result was not associated with cumulative dose administered or the age at which the therapy was given (1001). Radioiodine is also significantly concentrated in lactating breast tissue (1002). Therefore, RAI should not be given to women who are breastfeeding (1003). A diagnostic ${ }^{123}$ I or low-dose ${ }^{131}$ I scan can be employed in recently lactating women to detect breast uptake that may warrant deferral of therapy (662). Dopaminergic agents might be useful in decreasing breast exposure in recently lactating women, although caution should be exercised given the risk of serious, albeit rare, side effects associated with their routine use to suppress postpartum lactation $(1003,1004)$.

In men, RAI therapy may be associated with a temporary reduction in sperm counts and elevated serum folliclestimulating hormone levels $(1005,1006)$. Higher cumulative activities (500-800 $\mathrm{mCi}$ ) in men are associated with an increased risk of persistent elevation of serum folliclestimulating hormone levels, but fertility and risks of miscarriage or congenital abnormalities in subsequent pregnancies are not changed with moderate RAI activities $(\sim 200$ $\mathrm{mCi})(1007,1008)$. Permanent male infertility is unlikely with a single ablative activity of RAI, but theoretically there could be cumulative damage with multiple treatments. It has been suggested that sperm banking be considered in men who may receive cumulative RAI activities $\geq 400 \mathrm{mCi}$ (1006). Gonadal radiation exposure is reduced with good hydration, frequent micturition to empty the bladder, and avoidance of constipation (1009). Some specialists recommend that men wait 3 months (or one full sperm cycle) to avoid the potential for transient chromosomal abnormalities.

\section{[C36] How is RAI-refractory DTC classified?}

\section{- RECOMMENDATION 91}

Radioiodine-refractory structurally evident DTC is classified in patients with appropriate TSH stimulation and iodine preparation in four basic ways: (i) the malignant/ metastatic tissue does not ever concentrate RAI (no uptake outside the thyroid bed at the first therapeutic WBS), (ii) the tumor tissue loses the ability to concentrate RAI after previous evidence of RAI-avid disease (in the absence of stable iodine contamination), (iii) RAI is concentrated in some lesions but not in others; and (iv) metastatic disease progresses despite significant concentration of RAI.

When a patient with DTC is classified as refractory to RAI, there is no indication for further RAI treatment.

\section{(Weak recommendation, Low-quality evidence)}

The prognosis of patients with DTC is usually favorable, even when metastatic RAI-avid disease is present. For this reason, ${ }^{131} \mathrm{I}$ is considered the gold standard in the treatment of metastatic disease. However, many DTC patients with advanced disease do not respond or become refractory to ${ }^{131} \mathrm{I}$, with some of these patients dying within 3-5 years, but there are also long-term survivors with very slowly progressive disease.

Iodine 131 refractory DTC includes four categories of patients (1010). In the first category, the malignant/metastatic tissue does not concentrate ${ }^{131}$ I outside the thyroid bed at the first therapeutic WBS. There is no evidence in these patients that further treatment with RAI may be of any benefit. Furthermore, patients with measurable disease with an absence of ${ }^{131}$ I uptake on subsequent diagnostic WBS may also be considered refractory because even when uptake is seen on posttherapy scan, it will likely have limited benefit. In the second category, the tumor tissue loses the ability to concentrate ${ }^{131}$ I (in the absence of stable iodine contamination) after previous evidence of uptake. This often occurs in patients with large and multiple metastases and is due to the eradication by ${ }^{131} \mathrm{I}$ treatment of differentiated cells able to concentrate RAI but not of poorly differentiated cells that do not concentrate ${ }^{131} \mathrm{I}$ and that generally will progress. In the third category, ${ }^{131}$ I uptake is retained in some lesions but not in others; this pattern is frequent in patients with multiple large metastases as shown by ${ }^{124}$ I studies on PET scan (918) and by comparing results of ${ }^{18}$ FDG-PET scan with ${ }^{131}$ I WBS. In these patients, progression is likely to occur in metastases without uptake (in particular when ${ }^{18}$ FDG uptake is present), and RAI treatment will not be beneficial on the overall outcome of the disease. Some patients may have predominantly RAI-avid disease with only a few lesions that do not concentrate RAI. While these patients technically meet the definition of RAI-refractory disease, they may benefit from a combination of RAI therapy for the majority of the lesions and directed therapy (section [C38]; Recommendation 93) for those few lesions that do not concentrate RAI. In the fourth category, metastatic disease progresses despite significant uptake of ${ }^{131}$ I (new lesions, progressive growth of lesions, continual rise in serum $\mathrm{Tg}$ within months of RAI therapy). The definition of any of these possibilities depends on imaging modalities, including a posttherapy ${ }^{131} \mathrm{I}$ WBS combined with other imaging modalities, such as CT scan, MRI, or ${ }^{18}$ FDG PET/CT. The criteria for RAI-refractory disease remain somewhat controversial. Future studies will hopefully help to refine this definition.

Predictive factors for tumor response to RAI treatment are indeed the presence of ${ }^{131}$ I uptake by tumor, and among those patients with RAI uptake, younger age, well-differentiated histotype, small metastases, and low ${ }^{18}$ FDG uptake. Indeed these 
parameters are closely interrelated $(823,824,844)$ and may permit predicting the outcome of RAI treatment at the time of the discovery of the metastases. About two-thirds of patients with metastases demonstrate ${ }^{131}$ I uptake in their metastases, and only half of them will be cured with repeated courses of ${ }^{131}$ I treatment.

Less clear is the case of patients with visible RAI uptake in all the known lesions, who are not cured despite several treatment courses but whose disease remains stable and does not progress according to RECIST criteria. It is controversial whether these patients should be considered ${ }^{131}$ I refractory and whether RAI treatment should be abandoned in favor of other treatment modalities. The probability to obtain a cure with further treatments is low and side effects may increase, including the risk of secondary cancers and leukemias $(762,763,844,961)$. Several parameters should then be taken into consideration for the decision to continue treatment with RAI, including response to previous treatment courses, high or significant ${ }^{131}$ I uptake after previous treatment courses, low ${ }^{18} \mathrm{FDG}$ uptake in tumor foci, and limited side effects from the RAI therapy.

\section{[C37] Which patients with metastatic thyroid cancer can be followed without additional therapy?}

\section{- RECOMMENDATION 92}

(A) Patients with ${ }^{131}$ I-refractory metastatic DTC that is asymptomatic, stable, or minimally progressive who are not likely to develop rapidly progressive, clinically significant complications and do not have indications for directed therapy can be monitored on TSH-suppressive thyroid hormone therapy with serial radiographic imaging every 3-12 months.

\section{(Weak recommendation, Low-quality evidence)}

(B) BRAF or other mutational testing is not routinely recommended for prognostic purposes in patients with RAIrefractory, progressive, locally advanced, or metastatic DTC.

\section{(Weak recommendation, Moderate-quality evidence)}

Patients with ${ }^{131}$ I-refractory metastatic DTC often have an indolent clinical course, with no apparent symptoms or adverse impact from their disease burden for many years. In the absence of tolerable therapies with significant likelihood of inducing durable complete remission or improving overall survival, treatment should be limited to interventions that prevent morbidity or palliate symptoms. Thus, once ${ }^{131} \mathrm{I}$ refractory metastatic disease is identified, attention should be directed toward (i) determining the extent of metastatic disease by staging imaging studies such as CT, ${ }^{18}$ FDG PET/CT, or MRI as described in sections [C9-C13]; (ii) assessing degree of current or potential symptoms from the disease; (iii) understanding the comorbidities that might influence the choice of therapies for the metastatic disease; and (iv) determining the rate of progression of radiographically evident lesions. Serial assessment of the size and development of metastatic lesions can be enhanced by applying criteria similar to RECIST, as commonly used to assess tumor response in clinical trials (1011). Representative soft tissue metastatic lesions, typically $>1 \mathrm{~cm}$, are identified as "targets" on cross-sectional imaging, with the longest diameter of each lesion measured. Disease extent can be considered to be stable or minimally progressive if the sum of the longest diameters of the target lesions increases $<20 \%$ in the absence of new metastatic foci on sequential imaging over 12-15 months of follow-up. No study has identified an appropriate frequency for repeat imaging, but it is reasonable to repeat the staging imaging studies within 3-12 months based on the disease burden and locations of lesions $(953,1012)$. More frequent assessment could be considered once metastatic disease is identified and/or in response to intercurrent patient symptoms, and less frequent imaging performed once a pattern of stability is identified. The development of progressive disease, either by RECIST assessment identifying at least $20 \%$ increase in sum of longest diameters of target lesions, the appearance of significant new metastatic lesions, or development of disease-related symptoms should warrant consideration of appropriate systemic therapies (section [C41]) beyond TSH-suppressive thyroid hormone and/or directed therapies (sections [C15] and [C38]). Although serum Tg levels should be measured as biomarkers of the disease extent, patients should not be identified as having progressive disease and requiring more aggressive therapy solely on the basis of rising levels of $\mathrm{Tg}$; accelerating increases in $\mathrm{Tg}$ levels should, however, lead to consideration of more frequent and comprehensive imaging in efforts to identify previously occult structural correlates.

In each of two recent international, randomized phase III trials of kinase inhibitors for therapy of progressive, RAIrefractory, metastatic DTC, patients whose tumors contained the $B R A F^{V 600 E}$ mutation who were randomized to the placebo treatment arms experienced similar progression-free survival compared with those whose tumors did not harbor a $B R A F$ mutation $(1013,1014)$. Thus, $B R A F$ mutation status does not appear to be of prognostic value once patients reach this degree of advanced disease that would have qualified them for these clinical trials, that is, RAI-refractory, locally advanced, or metastatic DTC that has progressed by RECIST within 13 or 14 months.

\section{[C38] What is the role for directed therapy in advanced thyroid cancer? \\ - RECOMMENDATION 93}

(A) Both stereotactic radiation and thermal ablation (RFA and cryoablation) show a high efficacy in treating individual distant metastases with relatively few side effects and may be considered as valid alternatives to surgery.

(Weak recommendation, Moderate-quality evidence)

(B) Stereotactic radiation or thermal ablation should be considered prior to initiation of systemic treatment when the individual distant metastases are symptomatic or at high risk of local complications.

(Strong recommendation, Moderate-quality evidence)

Several local treatment modalities other than surgery may be used to treat brain, lung, liver, and bone lesions from thyroid carcinoma. These local treatment modalities should be considered prior to initiation of systemic treatment when the individual distant metastases are symptomatic or at high risk of local complications. They may also be helpful in case 
of progression in a single lesion in patients with otherwise controlled disease during systemic treatment. In these patients, benefits can be achieved in preventing local complications, in improving symptoms such as pain, in delaying the initiation of systemic treatments, and even in improving survival. These techniques can be a less aggressive alternative to surgery and may be indicated in cases of lung metastases associated with insufficient respiratory reserve, poor patient clinical status, or after multiple previous surgical resections, local recurrence at the site of previous surgery, or refusal of additional surgery.

In selected patients, the techniques may be an alternative to surgery as first-line treatment, and they may induce local tumor control with a similar efficacy to surgical resection. Of interest, long-term benefits in terms of disease control have been reported in patients with a single or few metastases and in whom the disease is slowly progressive.

Interventional radiology (thermal ablation and cement injections), SBRT, or intensity modulated radiotherapy are the most frequently used techniques. The main principle of these techniques is to selectively treat the lesion, be minimally invasive, and be well tolerated with relatively few side effects. The indications and the feasibility of each technique depend on the location and the size of the lesion to be treated. Experience with metastases from thyroid cancer is scarce, and most available data have been obtained in patients with metastases from nonthyroid cancers.

SBRT allows for delivering high radiation doses in few fractions to the target tumoral lesion with a high degree of precision, minimizing the radiation of normal surrounding tissue. It has been used in several trials to treat brain, liver, lung, and bone metastases.

For patients with few (one to three) brain metastases, SBRT is as effective as surgery and can be repeated in case of appearance of new brain lesions. It is usually well tolerated, and brain necrosis that occurred in less than $10 \%$ of cases is usually limited and had no clinical consequences. The patient outcome depends mostly on the progression rate of extracerebral lesions (1015).

Data on lung and liver metastases are available only in retrospective studies on low numbers of patients and with a median follow-up of less than 1 year in most cases and in one prospective study (1016). This study included patients with many different primary tumors, including $10 \%$ of the patients having thyroid cancer. They showed a local control rate ranging from $63 \%$ to $98 \%$ in lung lesions and from $57 \%$ to $100 \%$ in liver lesions, with a cumulative dose delivered ranging from 20 to $75 \mathrm{~Gy}$ in $5-15$ fractions. The local tumor control seems to be long lasting with complete response ranging from $70 \%$ to $90 \%$ at 2-3 years. Furthermore, rare $(<3 \%)$ grade $3-4$ toxicities (pneumonitis, pleural effusion, intestinal complications) were reported (1017). These toxicities are much less common that those associated with percutaneous treatment modalities. The local control rate seems to be dose-related, but the optimal protocol for SBRT is still not established.

Concerning bone lesions, radiotherapy plays an important role because it can complement surgery in case of incomplete resection or be used alone for pain relief or palliation. In many cases, usual radiotherapy can be given. However, the major limitation of radiotherapy in spine lesions is the cumulative dose to the spinal cord. SBRT compared to standard radiotherapy demonstrated a higher efficacy on tumor control and for limiting radiation to the spinal cord, especially in patients who need to be re-irradiated. A local tumor control rate of bone lesions for SBRT ranging from $88 \%$ to $100 \%$ was reported especially for lesions that were previously surgically resected, with a pain relief rate of $30 \%-83 \%$. SBRT protocols differed among studies, with a maximum of 30 Gy administrated in one to five fractions but a single dose of 12.5-15 Gy seems to achieve similar results (1018). Spinal myelopathy or vertebral fractures are the most important side effects, especially in case of large-volume lesions.

Percutaneous thermal ablation is aimed at destroying tumor foci by increasing (RFA) or decreasing (cryoablation) temperatures sufficiently to induce irreversible cellular damages.

RFA is performed for liver, lung, and bone tumor foci. Clinical trials showed high efficacy of RFA for liver lesions, especially from colorectal cancer with long-term local disease control ranging from $40 \%$ to $80 \%$, depending on lesion size, and a prolonged overall survival in treated patients $(1019,1020)$.

In a total of 100 lung lesions including primary lung tumors and metastases, RFA was both efficient and well tolerated, with a complete tumor control rate of $93 \%$ at 18 months (1021). A multicenter prospective trial on 183 lung metastases from cancer other than colorectal showed a complete response rate of $88 \%$ at 1 year and an overall survival of $92 \%$ and $64 \%$ at 1 year and at 2 years, respectively (1022). Recurrence occurs more frequently in lesions $>3 \mathrm{~cm}$ and can be detected early after ablation (within 3 months) by ${ }^{18}$ FDG PET/CT $(824,1016)$. Cases of delayed recurrence have also been reported, and long-term follow-up is needed. RFA may be considered for lung lesions $<3 \mathrm{~cm}$ of diameter, without soft tissue or mediastinum invasion and without contact with large vessels. Furthermore, repeated treatments can be performed on the same lesion and multiple lesions can be treated in the same patient.

RFA or cryoablation of bone lesions showed promising results with rapid (1-7 days) and long-lasting pain control $(1023,1024)$. Cryoablation is a safe technique to treat or to stabilize bone lesions, is frequently associated with cementoplasty to consolidate the bone and avoid subsequent complications, and can treat larger lesions than RFA.

Local disease control was achieved in the few reported cases of lung and bone metastases from thyroid cancer treated by thermal ablation $(883,1016,1025)$. The association of cryoablation and cementoplasty seems promising in purely lytic bone metastases from thyroid cancer. Furthermore, in three patients with liver metastases from thyroid cancer (two MTC and one FTC) RFA was feasible and reduced local symptoms (1026). Multidisciplinary treatment for metastases from thyroid cancer especially for bone metastasesincluding, for example, thermal ablation (RFA or cryoablation) and/or cementoplasty associated with systemic treatment (RAI, bone-directed agents, or chemotherapy) — can improve the patient's quality of life by reducing pain and prolonging time to skeletal events, delaying initiation of systemic treatment, and even improving patient survival (1027).

The toxicity of thermal ablation is generally low, but pneumothorax or pleural effusion was observed in up to $50 \%$ of RFA procedures for lung lesions, but it rarely required further treatments. Local pain or transient neurological deficit or vertebral fracture can occur in case of ablation of bone 
lesions $(5 \%-6 \%)$, and intestinal perforation, abdominal pain, or intraperitoneal bleeding was observed following ablation of liver lesions $(1021,1028)$.

Published experience using thermal ablation and stereotactic radiation in thyroid cancer patients is limited, and recommendations are currently based on more robust evidence in other solid tumors. Randomized prospective studies comparing the efficacy and tolerability of these different techniques are lacking, and their choice in clinical practice is based on local experience, lesion location as well as patient status and preference.

\section{[C39] Treatment of brain metastases}

\section{- RECOMMENDATION 94}

While surgical resection and stereotactic EBRT are the mainstays of therapy for CNS metastases, RAI can be considered if CNS metastases concentrate RAI. If RAI is being considered, stereotactic EBRT and concomitant glucocorticoid therapy are recommended prior to RAI therapy to minimize the effects of a potential TSH-induced increase in tumor size and RAI-induced inflammatory response.

\section{(Weak recommendation, Low-quality evidence)}

Brain metastases typically occur in older patients with more advanced disease and are associated with a poor prognosis (933). Surgical resection and stereotactic EBRT are the mainstays of therapy $(933,1029,1030)$. There are few data showing efficacy of RAI. Stereotactic radiation therapy is preferred to whole-brain radiation because life expectancy in patients with brain metastases may be prolonged, and stereotactic radiation induces less short- and long-term toxicity compared with whole-brain radiation (fatigue, headache, cognitive decline, and behavioral changes), and it may be effective even in patients with multiple brain lesions.

\section{[C40] Who should be considered for clinical trials?}

\section{- RECOMMENDATION 95}

Patients should be considered for referral to participate in prospective therapeutic clinical trials based upon specific eligibility requirements for given studies and the likelihood that the patient may or may not benefit from study participation. Clinicians considering referral of patients for trials should review available treatment options and eligibility criteria, preferably through discussions with trial center personnel and review of trial materials at the website www.clinicaltrials.org.

(Strong recommendation, Moderate-quality evidence)

A therapeutic clinical trial is a systematic investigation of the effectiveness and safety of a potential new or modified treatment or combination of treatments, potentially including medications, surgery, radiation therapy, and/or other novel or revised approaches. A broad variety of such trials may exist at any given time, which can generally be identified through online databases such as www.clinicaltrials.org, best supplemented with direct contact with the institutions conducting trials of particular interest so as to ensure trial availability and patient eligibility. There is limited evidence that enrollment into clinical trials is associated with lower overall cancerspecific mortality for patients with common cancers, even within contexts in which approved and "standard of care" therapies already exist (1031). The reasons for this association are unclear, but there is no evidence to suggest that trial participation is deleterious to patient outcomes, and it may be beneficial. Participation in a clinical trial should be considered in any situation wherein there exists no effective or proven standard of care, or when a standard of care is being compared with a promising new or investigational therapy. Adjuvant therapy trials may be appropriate for patients at high risk for disease recurrence following primary treatment who wish to pursue aggressive therapy. For patients with RAI-refractory carcinoma that is locally advanced or metastatic, clinical trials may be appropriate in the setting of disease that is considered progressive by RECIST criteria, especially if progression occurred after use of an approved kinase inhibitor such as lenvatinib or sorafenib, and/or if approved therapies are otherwise unaffordable to a specific patient. Patients demonstrated to have specific therapeutically targetable tumor alterations, such as $B R A F$ mutations or $P A X 8 / P P A R \gamma$ or $A L K$ rearrangements, can also be considered for trials testing therapies specifically targeting these alterations. However, given the indolent nature of metastatic disease in most patients, therapeutic clinical trial participation should not be considered for patients with stable, asymptomatic metastatic disease unless agents with significant likelihood of complete remission, prolongation of survival, or biologic impact such as redifferentiation that could sensitize to definitive therapy are available (see section [C37]).

\section{[C41] What is the role of systemic therapy (kinase inhibitors, other selective therapies, conventional chemotherapy, bisphosphonates, denosumab) in treating metastatic DTC?}

Systemic therapeutics of several types in selected clinical contexts appear to provide clinical benefit in treating metastatic DTC (1032). Benefit has been demonstrated in the form of improved progression-free survival (delay in time to disease progression or death) in three randomized, doubleblinded, placebo-controlled clinical trials: vandetanib (1033), sorafenib (1013), and lenvatinib (1034). Benefit has also been demonstrated in the form of induced durable tumor regression (1035-1037).

However, randomized clinical trial data are not yet available to address many additional critical questions, including effects of systemic therapies of various types on survival and quality of life, or to address critical issues of optimal patient selection/inclusion/exclusion criteria for therapy and duration of treatment.

To date, no clinical trial has demonstrated an overall survival advantage or improved quality of life from use of any therapy in RAI-refractory DTC $(1013,1034)$. Consequently, therapeutic decisions are presently based upon the convergence of expert opinion and patient preference/philosophy, thus emphasizing the critical need to address the above questions definitively through clinical trials. It is therefore important to involve highly skilled clinicians familiar with RAI-refractory disease and these systemic therapies in decision-making until definitive guidelines can be developed based upon more rigorous data. As a guide, evidence-based recommendations with expert consensus have been recently published (953).

It should also be highlighted that, broadly construed, systemic therapy encompasses not only more recently emerging 
"targeted" approaches, but also historical "mainstay" therapies including TSH suppression and RAI. Although more "novel" approaches have attracted attention recently, it is important to optimally apply fundamental approaches. In this regard, therapeutic RAI should also be used to optimal effect prior to the initiation of more recent/novel therapies. To accomplish this requires attention to detail, including ensuring adequate TSH stimulation, patient adherence to low-iodine pre-RAI therapy dietary restrictions, and avoidance of proximal preceding iodine contamination from IV contrast agents, with verification by urinary iodine concentration measurements in selected cases. In this context, occasional patients previously declared "RAI-refractory" can instead be found to have RAI-responsive disease when such details are attended to if previously neglected.

Also important is the consideration of alternatives to the use of systemic therapy, such as the application of surgery or other localized approaches (including radiation therapy or thermal ablation approaches). In patients in whom the threats imposed by cancer are more localized, directed approaches may have greater potential to control localized disease and symptoms compared to systemic therapies, especially given the absence of data to indicate survival benefit or improved quality of life from the application of systemic therapies in advanced RAI-refractory DTC.

It is also critically important to ensure that the disease prompting therapy represents metastatic thyroid cancer. In particular, because pulmonary nodules attributable to benign causes are common, the presence of pulmonary nodules does not in and of itself justify the application of systemic therapy. Thus, in cases of diagnostic uncertainty in which the result would have definitive therapeutic implications, biopsy is required, especially when Tg levels are low/unhelpful (such as in the presence of anti-Tg antibodies). Conversely, stable, asymptomatic pulmonary nodules of a few millimeters in size likely do not justify invasive assessment or systemic therapy.

The introduction of systemic therapy requires that both the clinician and the patient agree that clinical benefits are expected to exceed risks for that individual patient. The problem in this determination, however, is that it is often very difficult to precisely define such risks and benefits because they vary greatly depending upon patient context and they are often poorly articulated in the literature. It is also critical to weigh not just risks of death and injury, but also risks of systemic therapies on quality of life, especially as viewed by a particular patient considering treatment. Hence, the decision is not based solely on benefits and risks of therapy, but also on patient value judgments. Issues of risks and benefits are reviewed in this context in conjunction with each therapeutic modality below. Finally, it is important that the involved care team (physicians, physician assistants, nurse practitioners, nurses) be experienced in the use and management of toxicities associated with these therapies.

\section{[C42] Kinase inhibitors}

\section{- RECOMMENDATION 96}

(A) Kinase inhibitor therapy should be considered in RAIrefractory DTC patients with metastatic, rapidly progressive, symptomatic, and/or imminently threatening disease not otherwise amenable to local control using other approaches.
Kinase inhibitors that are FDA approved for differentiated thyroid carcinoma or other available kinase inhibitors (preferably within the context of therapeutic clinical trials) can be considered since the impact of these agents on overall survival and quality of life remains to be defined.

\section{(Weak recommendation, Moderate-quality evidence)}

(B) Patients who are candidates for kinase inhibitor therapy should be thoroughly counseled on the potential risks and benefits of this therapy as well as alternative therapeutic approaches including best supportive care. Appropriate informed consent should be obtained and documented in the medical record prior to initiation of any therapy, regardless of whether the patient is being treated in the context of a clinical trial.

\section{(Strong recommendation, Low-quality evidence)}

Cytotoxic chemotherapy has historically produced disappointing results in patients with DTC (1038). Kinase inhibitors, many of which share the common target of the VEGF receptor (VEGFR) (e.g., sorafenib, pazopanib, sunitinib, lenvatinib, axitinib, cabozantinib, and vandetanib), have recently emerged as highly promising therapies for metastatic RAI-refractory DTC (1032). Kinase inhibitors, however, are associated with numerous adverse effects including diarrhea, fatigue, induced hypertension (requiring initiation of antihypertensive therapy in about half of all previously normotensive individuals), hepatotoxicity, skin changes, nausea, increased $\mathrm{LT}_{4}$ dosage requirement, changes in taste, and weight loss. These potential side effects have high probability of negatively impacting quality of life and/or necessitating dosage reductions in many patients and treatment discontinuation in up to $20 \%$ of patients. Furthermore, these agents are also associated with more serious and potentially fatal risks including of thrombosis, bleeding, heart failure, hepatotoxicity, gastrointestinal tract fistula formation, and intestinal perforation (1039). Overall, the risk of therapyrelated death in cancer patients treated with oral kinase inhibitors is about $1.5 \%-2 \%(\mathrm{RR}=2.23, p<0.023$, compared with randomized placebo treated control cancer patients) based upon meta-analysis of results from 10 recently published randomized trials conducted in several cancers (1039). In the setting of thyroid cancer, lenvatinib (now approved in the United States for use in RAI-refractory DTC) was associated with severe toxicities in $75 \%$ of patients and therapy-attributed mortality in $2.3 \%$ of patients (1034). While risk of drug-related death is relatively low, the knowledge of potentially fatal therapeutic outcomes should prompt considerable restraint in the use of kinase inhibitors, especially in patients who are asymptomatic and/ or with stable or slowly progressive disease and in patients who can otherwise be effectively treated using directed therapies.

Three randomized placebo-controlled clinical trials (phase 2 , vandetanib; phase 3 , sorafenib and lenvatinib) had been published by the time of the writing of these guidelines, each demonstrating delayed time to disease progression among kinase inhibitor-treated patients relative to those treated with a placebo $(1013,1033,1034)$. On this basis, sorafenib and lenvatinib were approved for use in the United States and the European Union for patients with advanced RAI-refractory 
DTC. Sorafenib or vandetanib treatment were each associated with progression-free survival prolonged by 5 months, with $<15 \%$ objective response rates, but with no improvement on overall survival demonstrated to date. Assessments comparing sorafenib to placebo outcomes demonstrated overall lower quality of life among sorafenib-treated patients relative to those treated with placebo, despite improved progression-free survival. Lenvatinib therapy was associated with longer prolonged median progression-free survival by 14.7 months compared with placebo, with a RECIST response rate of $65 \%$, with some complete responses also reported. Despite these very encouraging results and regulatory approval, however, no statistically significant impact of lenvatinib (or any other kinase inhibitor) therapy on overall survival has yet been observed (1034). However, survival data in this context, where all three randomized and placebocontrolled DTC trials allowed crossover of placebo-treated patients to kinase inhibitor, must be interpreted with great caution. The crossover trial designs effectively "contaminated" overall survival data, with many placebo-treated patients later crossing over to receive open-label kinase inhibitor. Hence, the absence of a proven effect of kinase inhibitors on overall survival in these trials can best be interpreted as indicating that, among study populations, a delay in initiation of kinase inhibitor therapy until observation of RECIST disease progression among initially placebo-treated patients did not adversely affect overall survival.

Additional VEGFR-directed kinase inhibitors including axitinib, pazopanib, cabozantinib, and sunitinib also have activity in metastatic DTC based upon phase 2 therapeutic trials (1035-1037). No multi-arm comparison "superiority", phase 3 trial data in DTC are available to inform decisionmaking with regard to selection among available and mostly similarly targeted kinase inhibitors. Recent regulatory approval in DTC and more abundant outcome data in response to lenvatinib and sorafenib therapy in DTC $(1013,1034$,
1040-1043) prompt prominent consideration of either lenvatinib or sorafenib as a first-line therapy.

Despite encouraging trial results, the extent to which any kinase inhibitor may prolong overall survival and in which contexts remains undefined. In some situations, however, the decision to initiate kinase inhibitor therapy is straightforward. For example, patients with oxygen dependence attributable to DTC lung metastases are not only adversely affected by their cancers, but also imminently threatened. Such patients have potential to gain symptomatic benefit even in the absence of definite extension of life. At the other extreme, many patients with metastatic DTC are asymptomatic from their cancers and are not expected to be threatened by their cancers in the foreseeable future. These patients should remain on TSH-suppressive treatment as their primary therapeutic intervention rather than be exposed to kinase inhibitor therapy. For patients with disease between these two extremes, it is critical that risks and benefits of therapy, anticipated side effects, goals, patient context, and the therapeutic philosophy of each particular patient be thoroughly vetted so as to best individualize therapy. This decision is ideally made within the context of specialized centers with comprehensive knowledge of the natural history of the disease and of the effects of available therapies.

As a general "expert consensus" guideline, structurally progressive, symptomatic, and/or imminently threatening DTC (wherein disease progression is expected to require intervention and/or to produce morbidity or mortality in $<6$ months) that is RAI refractory and not amenable to satisfactory control using directed approaches (e.g., surgery, radiation therapy, thermal ablation) should prompt consideration of kinase inhibitor therapy. Specific characteristics impacting decision-making for starting such therapy are outlined in Table 16.

Patients who are candidates for kinase inhibitor therapy should be thoroughly counseled with regard not only to

Table 16. Factors to Review When Considering Kinase Inhibitor Therapy ${ }^{\mathrm{a}}$

Factors favoring kinase inhibitor therapy

Imminently threatening disease progression expected to require intervention and/or to produce morbidity or mortality in $<6$ months (e.g., pulmonary lesions or lymphadenopathy likely to rapidly invade airways, produce dyspnea, or cause bronchial obstruction).

Symptomatic disease (e.g., exertional dyspnea, painful unresectable adenopathy), not adequately addressable using directed therapy.

Diffuse disease progression as opposed to focal progression (e.g., in multiple lung metastases, as opposed to a few growing lesions)
Factors discouraging kinase inhibitor therapy

Comorbidity including

- Active or recent intestinal disease (e.g., diverticulitis, inflammatory bowel disease, recent bowel resection)

- Liver disease

- Recent bleeding (e.g., ulcer/GI bleed) or coagulopathy

- Recent cardiovascular event(s) (e.g., CVA, MI)

- Recent tracheal radiation therapy (this is associated with increased risks of aerodigestive fistula with kinase inhibitor therapy)

- Cachexia/low weight/poor nutrition

- Poorly controlled hypertension

- Prolonged QTc interval/history of significant arrhythmia (includes ventricular and bradyarrhythmias)

- Untreated brain metastases (controversial)

- Recent suicidal ideation (suicide has been reported in depressed patients receiving TKIs)

Life expectancy based upon other comorbidities estimated to be too brief to justify systemic therapy

\footnotetext{
${ }^{\mathrm{a} B o n e}$ metastases are often poorly responsive to kinase inhibitor therapy (see Bone-Directed Agents in section [C47]).
} GI, gastrointestinal; CVA, cerebrovascular accident; MI, myocardial infarction; TKI, tyrosine kinase inhibitor. 
potential benefits, but also about potential side effects and risks of therapy and alternative therapeutic approaches including best supportive care, as should be the case with any medical therapeutic decision-making (1044). Such extensive and comprehensive discussions are particularly important to undertake in the context of kinase inhibitor therapy because of the high probability of side effects of these agents and because of their presently uncertain effects on patient overall survival and quality of life (1045).

Another critical and complex question often faced in treating patients with kinase inhibitor therapy centers on when treatment should be discontinued once initiated. In general, therapy should be continued so long as net benefit exceeds net detriment. In case of slow RECIST disease progression after significant tumor response, treatment may be maintained so long as overall disease control is maintained providing that toxicities are manageable. Progression rate should be taken into account, and when global progression is rapid, therapy should be discontinued. However, there are times when focal/oligometastatic disease progression amenable to directed therapies is noted. In such instances, superimposed loco-regional therapies in the setting of maintained systemic therapy can sometimes be in the best interest of a particular patient. For instance, in the setting of regressed lung metastases and yet progression at a solitary bone site, maintained systemic therapy with superimposed directed radiation therapy may be reasonable.

\section{fails}

[C43] Patients for whom first-line kinase inhibitor therapy

\section{- RECOMMENDATION 97}

Patients who have disease progression while on initial kinase inhibitor therapy without prohibitive adverse effects should be considered for second-line kinase inhibitor therapy. Ideally, such therapy should be undertaken within the context of therapeutic clinical trials.

\section{(Weak recommendation, Low-quality evidence)}

DTC patients who progress through first-line line kinase inhibitor therapy commonly respond to a second similarly targeted agent, and thus they should be considered candidates for second-line kinase inhibitor therapy $(1014,1046)$. Hence, the selection of an agent for initial therapy may be less critical in some senses because many patient will go on to receive several kinase inhibitors over their disease courses. In the near future, other treatment modalities, such as RAI resensitization therapy, immunotherapy, or drugs directed to other targets, may also offer additional therapeutic options.

[C44] Management of toxicities from kinase inhibitor therapy

\section{- RECOMMENDATION 98}

Proactive monitoring and timely intervention in response to emergent toxicities are critical components of management in patients receiving kinase inhibitor therapy.

\section{(Strong recommendation, Low-quality evidence)}

Patients treated with kinase inhibitors are subject to many potential toxicities that can negatively impact quality of life or even be fatal; as a consequence, great care must be taken not only in selecting appropriate patients for therapy, but also in monitoring patients once they are receiving kinase inhibitor therapy (1044). Some toxicities (e.g., fatigue, diarrhea, gastrointestinal symptoms, cutaneous effects) will be symptomatic and can be more easily addressed upon close communication with patients. However, there are also more serious potential toxicities that might not be expected to immediately lead to patient symptoms (e.g., hepatotoxicity, prolonged QTc) that require proactive screening (Table 17).

Hypertension and hepatotoxicity are especially important to serially monitor and rapidly address. Cardiotoxicity is also important to be aware of and to monitor according to patient risk factors, agent used, and induced symptoms/signs. In particular, sunitinib induces a decreased cardiac ejection fraction in about $20 \%$ of treated patients (1047). Furthermore, kinase inhibitors can induce QTc prolongation that should prompt careful consideration of coadministered drugs and periodic electrocardiography. Also important is that kinase inhibitor half-life is long; consequently, severe toxicities should prompt complete cessation of kinase inhibitor therapy for an adequate amount of time to allow drug levels to decline before resumption at a lower dosage.

Serum TSH level frequently increases during kinase inhibitor therapy; this should lead to frequent TSH assessment in conjunction with therapy initiation and upon therapy cessation, with responsive modifications thyroid hormone therapy where indicated.

\section{[C45] Other novel agents}

\section{- RECOMMENDATION 99}

Agents without established efficacy in DTC should be used primarily within the context of therapeutic clinical trials.

\section{(Strong recommendation, Low-quality evidence)}

A variety of novel agents have been and/or are being tested as candidate therapeutics in progressive metastatic RAIrefractory DTC $(1032,1048)$. At the time of the writing of these guidelines, however, only kinase inhibitors have shown sufficient promise to consider use other than within the context of therapeutic clinical trials. Agents of particular interest for further testing include BRAF kinase inhibitors because PTC frequently harbors the constitutively activating $B R A F^{V 600 E}$ mutation and the inhibitors have already shown efficacy and been approved for use in BRAF mutant melanoma (10491051). Inhibitors of MEK kinase and other signaling pathways are also of considerable investigational interest. In addition, promising initial results in response to use of kinase inhibition to "resensitize" RAI-refractory tumors to RAI have been reported (1052). Data developed to date, however, do not yet favor the use of these novel approaches over use of VEGFRdirected kinase inhibitors unless within the context of therapeutic clinical trials or alternatively when used as "salvage" therapies after disease progression has occurred despite prior VEGFR-directed kinase inhibitor therapy.

\section{[C46] Cytotoxic chemotherapy}

\section{- RECOMMENDATION 100}

Cytotoxic chemotherapy can be considered in RAIrefractory DTC patients with metastatic, rapidly progressive, 
Table 17. Potential Toxicities and Recommended Screening or Monitoring Approaches in Patients Started on Kinase Inhibitor Therapy

\begin{tabular}{|c|c|}
\hline Toxicity & Recommended screening/monitoring \\
\hline Hypertension & $\begin{array}{l}\text { Frequent blood pressure monitoring, most critical during the first } 8 \text { weeks of } \\
\text { therapy; if hypertension is induced, therapy should be individualized to patient } \\
\text { response } \\
\text { - Note: effective and expeditious management of hypertension is critical - and } \\
\text { may reduce potential for cardiotoxicity. If antihypertensive therapy is } \\
\text { needed, calcium channel blockers (e.g., amlodipine) may be most effective. }\end{array}$ \\
\hline Cutaneous/mucocutaneous toxicities & $\begin{array}{l}\text { Careful patient reporting of rash/mouth sores, patient awareness and education } \\
\text { related to increased potential for photosentization/sunburn. }\end{array}$ \\
\hline Hepatotoxicity & $\begin{array}{l}\text { Serial assessment of alanine serum transferase (AST), alkaline phosphatase and } \\
\text { bilirubin - most critical during the first } 8 \text { weeks of therapy } \\
\text { - Note: dose reduction of kinase inhibitor therapy is commonly required due to } \\
\text { hepatic toxicity }\end{array}$ \\
\hline Cardiotoxicity & $\begin{array}{l}\text { ECG pretherapy and frequently during therapy } \\
\text { - Hold (or do not initiate) kinase inhibitor therapy if QTc }>480 \mathrm{~ms} \\
\text { Echocardiogram: elective, but recommended in any patient with cardiac } \\
\text { history and especially important in patient with hypertension, symptoms } \\
\text { consistent with congestive heart failure or coronary artery disease and in } \\
\text { patients receiving sunitinib }\end{array}$ \\
\hline Hypothyroidism & $\begin{array}{l}\text { TSH should be assessed frequently, with levothyroxine dosage altered in response } \\
\text { to rising TSH if observed }\end{array}$ \\
\hline Nephrotoxicity & Serial serum creatinine, urine analysis with protein determination, \\
\hline Hematological toxicities & Serial CBC/diff \\
\hline Pancreatitis & Serial amylase \\
\hline Teratogenicity & $\begin{array}{l}\text { Pretherapy pregnancy testing and effective contraception in women and men of } \\
\text { childbearing potential }\end{array}$ \\
\hline
\end{tabular}

CBC, complete blood count; ECG, electrocardiography.

symptomatic, and/or imminently threatening disease not otherwise amenable to control through other approaches, including kinase inhibitors. Too few data exist to recommend specific cytotoxic regimens, and use within the context of a therapeutic clinical trial is preferred.

\section{(Weak recommendation, Low-quality evidence)}

Although doxorubicin was approved for use in thyroid cancer by the US FDA in 1974 and has some utility in anaplastic thyroid cancer, cytotoxic chemotherapy has historically produced disappointing results when used to treat RAIrefractory DTC (1038). Cytotoxic chemotherapy, however, may have selective benefit in patients unresponsive to kinase inhibitors and perhaps also in some patients with poorly differentiated thyroid cancer (1053). Data are limited and primarily anecdotal.

\section{[C47] Bone-directed agents}

\section{- RECOMMENDATION 101}

Bisphosphonate or denosumab therapy should be considered in patients with diffuse and/or symptomatic bone metastases from RAI-refractory DTC, either alone or concomitantly with other systemic therapies. Adequate renal function (bisphosphonates) and calcium level (bisphosphonates and denosumab) should be documented prior to each dose, and dental evaluation should take place before initial use.

\section{(Strong recommendation, Moderate-quality evidence)}

Metastatic bone disease represents a particularly challenging clinical problem in patients with RAI-refractory DTC, especially given the high rate of multiple skeletalrelated events in patients following detection of an initial bone lesion (1054). Patients with a small number of threatening and/or symptomatic bone lesions are generally best treated with focal approaches such as radiation therapy and/ or surgery and/or thermoablation. Many patients, however, suffer from diffuse progression of bone metastases that are not amenable to effective control using focal therapies alone. In such patients, focal therapy to symptomatic lesions or lesions at high risk of complications may be beneficial and should be performed before initiation of systemic treatment.

Unfortunately, kinase inhibitors appear to be less effective in controlling bone metastatic disease in comparison to disease at other soft tissue sites such as lungs and lymph nodes. Progression of bone metastases while on kinase inhibitor therapy commonly occurs despite maintained benefit with respect to disease at other metastatic sites. Hence, kinase inhibitor therapy cannot be relied upon to control diffuse bone metastases in many patients with RAI-refractory DTC.

In other solid tumors, bone-directed therapeutics including bisphosphonates (especially zoledronic acid) and the RANK ligand-directed agent denosumab have been shown to delay time to occurrence of subsequent skeletal-related adverse events (fracture, pain, neurologic complications) and to improve symptoms, and these agents may provide benefits for patients 
with diffuse bone metastases $(1055,1056)$. The determination of benefits across several tumor types suggests that they may be broadly generalizable, prompting FDA approval for their general use in patients with solid tumor bone metastases. Two small studies have suggested benefit from bisphosphonates specifically within the context of DTC bone metastases $(951,1057)$.

Risks of bisphosphonates and RANK ligand-directed agents include hypocalcemia, which can be severe, prompting the recommended concomitant use of supplemental calcium and vitamin $\mathrm{D}$ therapy. Moreover, these agents also moderately increase the risk of nonhealing oral lesions and jaw osteonecrosis; thus, candidates for this therapy should undergo dental/oral surgical evaluation prior to their initiation so as to minimize these risks (1055).

Recent studies have overall shown equivalence or superiority of denosumab to zoledronic acid in delaying bonerelated adverse events in several solid tumors, with similar risk of jaw osteonecrosis, greater incidence of hypocalcemia, and less nephrotoxicity (1058). However, no thyroid-specific data related to the efficacy of denosumab have yet been published. If the patient's renal function is impaired, there is sometimes justification for beginning with RANK liganddirected/denosumab therapy because this agent seems to produce fewer adverse renal effects, albeit the oral/jaw effects are similar to bisphosphonates in magnitude.

Expert consensus is that bone-directed therapy should be strongly considered in patients with multiple progressing and/or symptomatic bone metastases, likely best beginning with bisphosphonate/zoledronic acid (assuming calcium and renal function permit). Candidates for such therapy should be cleared by their dentist/oral surgeon prior to therapy initiation; in addition, calcium and vitamin $\mathrm{D}$ therapy should be ongoing in conjunction with any intended bone-directed therapeutic. In general, there is consensus that the administration of zoledronic acid therapy every 3 months (rather than every month) is a reasonable initial approach in terms of dosing interval, but randomized trial data are unavailable to definitively clarify this issue. Expert consensus is that bone-directed therapy should be used in the setting of diffuse bone metastases even if kinase inhibitor therapy is intended or ongoing.

\section{[D1] DIRECTIONS FOR FUTURE RESEARCH}

[D2] Optimizing molecular markers for diagnosis, prognosis, and therapeutic targets

Significant progress has been made over the last several years in understanding the genetic mechanisms of thyroid cancer and creating molecular tests for cancer diagnosis in thyroid nodules. This process is currently going through an accelerated phase, which is expected to continue into the future. The Cancer Genome Atlas (TCGA) and work from multiple research laboratories led to the identification of mutations and other driver genetic alterations in more than $90 \%$ of thyroid cancers, making it one of the best characterized human cancers from a genetic standpoint (1059). The TCGA focused only on PTC. Moreover, next-generation sequencing technologies may allow detection of most of these alterations in a limited cell sample obtained by FNA. Progress in identifying mutational, other genetic (gene expression, miRNA), and epigenetic markers of thyroid cancer is expected to result in a significantly improved accuracy of cancer detection in thyroid nodules as compared to the currently available clinical tests. If such progress continues, it is expected that future molecular tests will be able to predict the risk of cancer in thyroid nodules with high accuracy, dramatically reducing the uncertainty of indeterminate FNA cytology. Furthermore, as the cost of next-generation sequencing of human DNA continues to decrease and the analytical tools become more efficient, it should be expected that the cost of molecular testing will decrease, enabling costefficient utilization of testing.

Molecular markers are expected to have a significant impact on cancer prognostication. While the BRAF status can be considered as a relatively sensitive prognostic marker for papillary cancer, it cannot be used in isolation for tumor prognostication. However, recent results obtained by broad tumor genotyping show that several specific molecular signatures (such as presence of several driver mutations, TP53 mutation, TERT mutation in isolation or in combination with $B R A F)$ are found in a small fraction of well-differentiated papillary and follicular cancers and appear to be associated with more aggressive tumor behavior. It is expected that these molecular signatures will be confirmed and perhaps further improved in additional studies and will offer a more specific detection of well-differentiated thyroid cancers that have high risks of tumor recurrence and cancer-related mortality. Furthermore, research may identify how such data may inform therapeutic decision-making (e.g., surgical extent [if any], treatment with multi-kinase and specific kinase inhibitors or their combination). Discoveries of new gene mutations/rearrangements involved in the pathogenesis of thyroid cancer, such as those of $A L K$ and NTRK3, are expected to offer new effective therapeutic targets. Finally, new therapeutic approaches to target genes commonly mutated in thyroid cancer, such as the RAS genes, are in development and expected to enter clinical trials in the future. Prospective long-term outcome studies, ideally in the form of RCTs, will be needed to define the optimal surgical and postsurgical management of patients based on these molecular signatures. Such research may enable personalized, evidence-based care of patients with thyroid cancer across the disease trajectory.

\section{[D3] Active surveillance of DTC primary tumors}

Our Japanese colleagues have provided compelling data that an active surveillance management approach to papillary microcarcinoma is a safe and effective alternative to immediate surgical resection in properly selected patients $(143,149)$.

Unfortunately, no clinical features or molecular abnormality in isolation can reliably differentiate the relatively small number of PTMC patients destined to develop clinically significant progression from the larger population of people that harbor indolent PTMCs that will not cause significant disease. Therefore, additional studies are needed to identify specific risk factors that would favor surgical resection over active surveillance.

Furthermore, additional studies are needed to define important management issues that arise during an active surveillance follow-up approach. These issues include the frequency of US evaluations required during follow-up, optimal TSH goals, the potential role of serum Tg in follow-up, and specific indications for surgical intervention (e.g., what measurements should be used to define a clinically significant 
increase in the size of the primary tumor, what constitutes clinically significant lymph node metastases).

Finally, studies that examine decision-making and acceptability of an active surveillance approach to thyroid cancer in patients, family members, and clinicians are required to better understand how to implement this novel management approach outside of Japan. These studies should ideally be performed in the context of an Institutional Review Board-approved clinical trial.

\section{[D4] Improved risk stratification}

While the AJCC/UICC TNM staging provides valuable information with regard to disease-specific mortality, studies are needed to determine if inclusion of additional prognostic variables into the AJCC staging system could improve its predictive ability. Potential variables for consideration include the specific histology (well-differentiated thyroid cancer versus poorly differentiated thyroid cancer), molecular profile, size and location of distant metastases (pulmonary metastases versus bone metastases versus brain metastases), functional status of the metastases (RAI avid versus ${ }^{18}$ FDGPET avid), and effectiveness of initial therapy (completeness of resection; effectiveness of RAI, external beam radiation, or other systemic therapies).

Furthermore, additional studies will be required to determine if there is any significant incremental benefit of adding these specific prognostic variables to the 2009 Initial Risk Stratification system.

Since the response to therapy dynamic (ongoing) risk stratification systems were primarily optimized and validated on DTC patients that had total thyroidectomy and RAI remnant ablation or adjuvant therapy, additional studies are needed to refine the definitions of excellent, biochemical incomplete, structural incomplete, and indeterminate responses in patients treated with total thyroidectomy without RAI ablation and in patients treated with less than total thyroidectomy (602). Furthermore, additional studies are needed to define what types of cross-sectional/functional imaging are required to rule out structural disease in order to classify a patient as having a biochemical incomplete response to therapy (based on initial risk, serum Tg, signs/symptoms, or other imaging results).

Another area of significant research interest centers on identifying specific clinical situations in which the molecular findings provide clinically meaningful information that goes beyond what is predicted by standard clinico-pathological staging. Molecular findings that provide these types of prognostic information or guide optimal initial/ongoing treatment decisions have the potential to significantly alter clinical management.

[D5] Improving our understanding of the risks and benefits of DTC treatments and optimal implementation/utilization

In achieving a better understanding of the risks and benefits of DTC treatments (such as extent of primary surgery or secondary surgery for recurrence, RAI ablation/treatment, and thyroid hormone suppressive therapy), more prospective long-term outcome research is needed, and in particular, RCTs. In the case of relatively uncommon adverse effects of treatments, prospective surveillance research is also needed. Vaisman and colleagues (633) are conducting a prospective, nonrandomized study to determine which patients with lowto intermediate-risk DTC should receive RAI remnant ablation based on the postoperative stimulated serum $\mathrm{Tg}$ level.
Longer-term follow-up and randomized multicenter studies are needed to determine how this commonly used biomarker can be applied to decision-making in a majority of patients with low- to intermediate-risk DTC. Prospective collection of data on quality of life and related outcomes (when relevant) is also needed in DTC trials. Using an evidence-based approach to knowledge synthesis (systematic reviews, meta-analyses, and clinical practice guidelines) of data can also enable evidence-informed clinical practice. Barriers to dissemination and implementation of clinical practice guideline recommendations need to be overcome. Evidence-based guidelines may need to be formally adapted to various practice settings to enable their implementation. Important gaps in patientdirected knowledge translation have been recently identified by thyroid cancer patients and survivors $(1060,1061)$. The development of plain language educational materials, including decision aids or other decision support tools would be helpful for use as adjuncts in physician counseling of patients about diagnostic and treatment options. Decision aids and other decision support have been associated with improvement in patients' medical knowledge and reducing decisional conflict, in general oncology $(1062,1063)$, with a recent RCT demonstrating benefits of a decision aid in thyroid cancer survivors considering RAI remnant ablation (1064).

\section{[D6] Issues with measurement of $\mathrm{Tg}$ and anti-Tg anti- bodies}

Current methodologies for both $\mathrm{Tg}$ and anti-Tg antibodies remain problematic in many ways that hopefully will be overcome in the future. For Tg assays, these include interference with $\mathrm{Tg}$ measurement by the presence of anti-Tg and heterophile antibodies and the use of a host of different methods with varying results in terms of sensitivity or detection limits. Assay calibration or standardization may be successful only if the same certified reference standard is employed, which currently is CRM-457. While more "ultrasensitive" Tg assays have been developed, we need to determine the true clinical significance or utility of measurable levels below $0.2 \mathrm{ng} / \mathrm{mL}$ (either on suppression or after TSH stimulation) as indicating evidence of residual disease or outcome. Recently developed mass spectrometry-based assays have offered some promise, but are yet to be validated (806). Competitive immunoassays have not provided an alternative in view of their unpredictability (771). In regard to anti-Tg antibodies, we need to better characterize the various epitopes of interfering antibodies to better understand their effect in different sera in order to interpret the associated spectrum of results obtained for measurable Tg. We will need to do better than approximating Tg levels by the ratio of ICMA Tg to Tg measured by competitive immunoassay (1065). Authoritative bodies such as the National Academy of Clinical Biochemistry should consider mandating specific methodology rather than recommending general guidance. It remains to be seen whether the future standard might entail adoption of a modified ICMA, radioimmunoassay, or mass spectrometry methodology.

\section{[D7] Management of metastatic cervical adenopathy de-} tected on US

With the advent of improved technology, increased utilization, and specialized operator experience, US imaging can identify small-volume metastatic cervical lymph nodes. From the surgical pathology literature analyzing specimens 
from prophylactic lateral and central neck dissections with normal preoperative cervical lymph node sonography, up to $90 \%$ of patients with papillary cancers $<1 \mathrm{~cm}$ have metastatic level VI lymph nodes and up to $40 \%$ have metastatic lateral neck lymph nodes $(359,1066,1067)$. Yet, in the absence of these dissections, this is not the observed clinical locoregional recurrence rate for these patients. It should not be surprising then, that during extended surveillance, US will be able to detect small-volume metastatic disease that may represent a stable reservoir of residual cancer. On the other hand, grossly involved metastatic lymph nodes were at one time minimally invaded by metastatic thyroid cancer. The challenge is to differentiate between low-volume metastatic disease that progresses with potential clinical consequences, and that which remains stable. To date, only one study has addressed this question, and no sonographic, pathologic, demographic, or molecular feature predicted outcome (849). In addition, it is unclear if growth itself is a harbinger of decreased survival. Therefore, observational studies using standardized US scanning protocols are first required to define the magnitude of this scenario and define predictors of disease progression. Subsequently, randomized controlled interventional trials could be designed to address change in outcome, such as development of additional loco-regional disease, appearance of distant metastases, or disease-specific survival.

[D8] Novel therapies for systemic RAI-refractory disease

Over the years, a wide variety of agents have been used in preclinical (valproic acid, trichostatin, depsipeptide, 5azacytidine, arsenic tri-oxide) and clinical (retinoids, thiazolidinediones) models to "redifferentiate" thyroid cancer cells in order to restore RAI avidity. While these agents showed limited clinical effectiveness, the observation that oncogenic activation of the MAP kinase pathway was associated with downregulation of the genes involved in iodine metabolism suggested an alternative, targeted approach to redifferentiation therapy. Recently, two proof-of-principle pilot clinical trials have confirmed that targeted blockade of the MAP kinase pathway can result in clinically relevant restoration of RAI avidity in a substantial percentage of RAI-refractory thyroid cancer patients $(1052,1068)$. In the first trial, 1 month of the MEK inhibitor selumetinib increased RAI uptake in 12 of 20 iodine-refractory thyroid cancer patients, with structural tumor shrinkage seen in five of the eight patients that achieved lesional dosimetry high enough to warrant RAI therapy (1052). In the second trial, 1 month of the BRAF inhibitor dabrabenib restored RAI avidity on diagnostic WBS in 6 of 10 RAI-refractory patients, resulting in structural responses in two patients and a decrease in serum $\mathrm{Tg}$ in four patients (1068). While the primary focus of current redifferentiation trials has been in the setting of RAI-refractory distant metastases, future studies are needed to define the role of redifferentiation therapy in the high-risk adjuvant therapy setting and in the RAI-responsive metastatic disease setting in an effort to enhance the tumoricidal effect of RAI before the tumors become RAI refractory.

Efforts to develop additional and further improved systemic therapeutic approaches to RAI-refractory metastatic DTC presently involve a wide a variety of approaches. First, the question arises as to whether kinase inhibitors with already demonstrated clinical activity in DTC can be made more effective as single agents. For example, based upon data indicating strong correlation between achieved pazopanib levels and extent of clinical response in DTC, indicating absence of response to pazopanib therapy in the lowest quintile of pazopanib drug levels, one clinical trial is presently examining the feasibility and potential benefits of individualization of pazopanib therapy with the goal of achieving target drug levels in the highest achievable fraction of patients, in hopes of improving the fraction of patients benefiting (NCT01552356). Another study is examining the differential impacts of continuous versus intermittent pazopanib dosing in thyroid cancer (NCT01813136).

Generally predicated upon identification of synergistic interactions in preclinical models, several studies are examining the question of whether therapy combining several agents may improve outcomes in thyroid cancer. Although the majority of combinatorial studies assess effects of multiple coadministered small molecule therapeutics, several are using kinase inhibitors in combination with RAI in efforts to enhance RAI avidity and clinical efficacy in the context of RAI-refractory disease (e.g., NCT00970359). Still other trials are focusing on novel nontraditional therapies, including engineered candidate virotherapeutics (e.g., NCT01229865).

Alternatively, another active area of investigation involves efforts to therapeutically target specific alterations (mutations, translocation) found in thyroid cancers to individualize therapy and thereby potentially improve outcomes in the process; one such recent example is the use of the $\mathrm{BRAF}^{\mathrm{V} 600 \mathrm{E}}$ inhibitor vemurafenib in $B R A F^{V 600 E}$ PTC (NCT01286753), with many additional mutation-specific therapeutic trials under development or underway.

Finally, immunotherapy including checkpoint inhibitors (e.g., PD-1/PD-L1) has shown promise in other cancers (1069-1071) and is being investigated in advanced RAIrefractory thyroid cancer.

\section{[D9] Survivorship care}

The American Cancer Society estimates that there will be 62,450 new cases of thyroid cancer diagnosed in 2015 , but only 1950 deaths from thyroid cancer (www.cancer.org/cancer/ thyroidcancer/detailedguide/thyroid-cancer-key-statistics). According to SEER database statistics, there are more than 600,000 people living with thyroid cancer in the United States alone (seer.cancer.gov/statfacts/html/thyro.html). Despite these large numbers of patients living with thyroid cancer, there is only a modest amount of peer-reviewed literature studying thyroid cancer survivors.

The majority of the literature involving thyroid cancer survivors relates directly to the short- and long-term effects of thyroid cancer therapies: surgery, RAI, and lifetime thyroid hormone therapy. There is very little information regarding the impact of the diagnosis itself or the effect of living with persistent disease such as Tg-positive, scan-negative thyroid cancer. A thoughtful and comprehensive analysis of thyroid cancer survivors will likely require both qualitative studies with in-depth interviews of survivors that will represent as many demographics of thyroid cancer patients as possible. Additionally, or as a next step, the development and/or utilization of a validated survey type instrument needs to be developed. This instrument would be designed to assess the quality of life of thyroid cancer survivors in a more quantitative manner, allowing for rigorous statistical analyses, and will help identifying areas to target that may improve the lives of thyroid cancer survivors (1072). 
Further research also needs to be performed in addressing patient and survivor care needs throughout the active treatment and survivorship trajectory, including issues such as identification and management of treatment-related side effects $(1060,1061)$, psychosocial distress $(1060,1061)$, persistent fatigue $(1073,1074)$, financial impact (12), and cancer-related worry (1075).

\section{ACKNOWLEDGMENTS}

The task force wishes to thank Ms. Bobbi Smith, Executive Director, ATA, and Ms. Sharleene Cano, Assistant to the Taskforce, for their constant help and support, as well as Ms. Vicki Wright (Division of Endocrinology, University of Colorado School of Medicine) for her assistance in manuscript preparation. We thank Dr. Joshua Klopper (Division of Endocrinology, University of Colorado School of Medicine) on behalf of the CAC Quality Of Life Task Force for the contribution of the Survivorship Care section [D9]. We also thank Dr. Irwin Klein (North Shore University Hospital, Manhasset, New York) for his input on TSH targets for long-term thyroid hormone therapy (Table 15). We would like to thank the ATA members who responded to our survey in preparation for this iteration of the guidelines as well as manuscript review prior to journal submission, and the leadership of ThyCa: Thyroid Cancer Survivors' Association, Inc. and Thyroid Cancer Canada who provided written feedback on our survey. A.M.S. holds a Cancer Care Ontario Health Services Research Chair, which enabled protected time for research and contribution to these guidelines. These guidelines were funded by the ATA without support from any commercial sources. The patient organization, ThyCa: Thyroid Cancer Survivors' Association, Inc., contributed an unrestricted educational grant toward the development of the thyroid nodules and differentiated thyroid cancer guidelines.

The following groups reviewed and endorsed the final document: American Association of Clinical Endocrinologists; American Association of Endocrine Surgeons; American Head and Neck Society; Asia Oceania Thyroid Association (AOTA); British Society of Nuclear Medicine; Canadian Association of Otolaryngology Head and Neck Surgery; The Endocrine Society; Endocrine Society of Australia; European Thyroid Association; International Association of Endocrine Surgeons; International Federation of Head and Neck Oncologic Societie; Japanese Thyroid Association; Latin American Thyroid Society; Society of Surgical Oncology; Ukrainian Association of Endocrine Surgeons.

\section{DISCLAIMER}

It is our goal in formulating these guidelines, and the ATA's goal in providing support for the development of these guidelines, that they assist in the clinical care of patients and share what we believe is current, rational, and optimal medical practice. In some circumstances, it may be apparent that the level of care recommended may be best provided in limited centers with specific expertise. Finally, it is not the intent of these guidelines to replace individual decision-making, the wishes of the patient or family, or clinical judgment.

\section{AUTHOR DISCLOSURE STATEMENT}

These guidelines were funded by the ATA without support from any commercial sources.
K.C.B., G.M.D., F.P., G.A.R., A.M.S., and K.S. have no significant financial or competing interests to disclose.

B.R.H. has received grant/research support from Veracyte and Genzyme, as well as a one-time speaker honorarium from Genzyme. E.K.A. has received research support from Asuragen, Veracyte, and Novo Nordisk. He has been a consultant for NPS Pharmaceuticals, Genzyme, and Veracyte as well as on the Scientific Advisory Board for Asuragen. S.J.M. has received grant/research support from Veracyte and Asuragen. She has been on the scientific advisory committee for Asuragen and has been a CME speaker for Genzyme. Y.N. has been a consultant for Quest Diagnostics. His institution, UPMC, has a service agreement with CBLPath to provide molecular testing for various tumors. M.S. has received grant/research support from Genzyme, Bayer, AstraZeneca, Exelixis, and Eisai. He has been a consultant for Genzyme, Bayer, AstraZeneca, Exelixis, and Eisai. S.I.S. has received grant/research support from Genzyme and the National Cancer Institute. He is a consultant for Veracyte, Exelixis, Bayer, AstraZeneca, Eisai, Genzyme, Novo Nordisk, and Eli Lilly. He has received honoraria from Onyx, and he has fiduciary responsibility as Chairman of International Thyroid Oncology Group. J.A.S. has received one-time speaker honorarium from Exelixis and is a member/ATA representative on the Data Monitoring Committee for the Medullary Thyroid Cancer Registry called for by the FDA and funded by NovoNordisk, Astra Zeneca, GlaxoSmithKline, and Eli Lilly. D.L.S. has received grant/research support from Astra-Zeneca. R.M.T. is a consultant for Genzyme, Novo Nordisk, AstraZeneca, Bayer/Onyx, and Veracyte. L.W. has been a consultant for Asuragen, Interpace Diagnostics, Eisei, and IBSA. He has received speaker honoraria from Genzyme.

E.K.A. and R.M.T. received consulting payments through stock options from Veracyte. This has been reviewed and discussed with the Ethics Committee and reviewed by the ATA Board. E.K.A. and R.M.T. were recused from review and approval of the molecular markers sections. They divested these stock options prior to submission of this document for journal review.

\section{REFERENCES}

1. Vander JB, Gaston EA, Dawber TR 1968 The significance of nontoxic thyroid nodules. Final report of a 15year study of the incidence of thyroid malignancy. Ann Intern Med 69:537-540.

2. Tunbridge WM, Evered DC, Hall R, Appleton D, Brewis M, Clark F, Evans JG, Young E, Bird T, Smith PA 1977 The spectrum of thyroid disease in a community: the Whickham survey. Clin Endocrinol (Oxf) 7:481-493.

3. Tan GH, Gharib H 1997 Thyroid incidentalomas: management approaches to nonpalpable nodules discovered incidentally on thyroid imaging. Ann Intern Med 126: 226-231.

4. Guth S, Theune U, Aberle J, Galach A, Bamberger CM 2009 Very high prevalence of thyroid nodules detected by high frequency $(13 \mathrm{MHz})$ ultrasound examination. Eur J Clin Invest 39:699-706.

5. Hegedus L 2004 Clinical practice. The thyroid nodule. N Engl J Med 351:1764-1771.

6. Mandel SJ 2004 A 64-year-old woman with a thyroid nodule. JAMA 292:2632-2642.

7. Sherman SI 2003 Thyroid carcinoma. Lancet 361:501-511. 
8. Siegel R, Ma J, Zou Z, Jemal A 2014 Cancer statistics, 2014. CA Cancer J Clin 64:9-29.

9. Davies L, Welch HG 2014 Current thyroid cancer trends in the United States. JAMA Otolaryngol Head Neck Surg 140:317-322.

10. Leenhardt L, Bernier MO, Boin-Pineau MH, Conte DB, Maréchaud R, Niccoli-Sire P, Nocaudie M, Orgiazzi J, Schlumberger M, Wémeau JL, Cherie-Challine L, De Vathaire F 2004 Advances in diagnostic practices affect thyroid cancer incidence in France. Eur J Endocrinol 150:133-139.

11. Brito JP, Al Nofal A, Montori V, Hay ID, Morris JC III 2015 The impact of subclinical disease and mechanism of detection on the rise in thyroid cancer incidence: a population-based study in Olmsted County, Minnesota during 1935 through 2012. Thyroid 25:999-1007.

12. Aschebrook-Kilfoy B, Schechter RB, Shih YC, Kaplan EL, Chiu BC, Angelos P, Grogan RH 2013 The clinical and economic burden of a sustained increase in thyroid cancer incidence. Cancer Epidemiol Biomarkers Prev 22:1252-1259.

13. Singer PA, Cooper DS, Daniels GH, Ladenson PW, Greenspan FS, Levy EG, Braverman LE, Clark OH, McDougall IR, Ain KV, Dorfman SG 1996 Treatment guidelines for patients with thyroid nodules and welldifferentiated thyroid cancer. American Thyroid Association. Arch Intern Med 156:2165-2172.

14. Francis GL, Waguespack SG, Bauer AJ, Angelos P, Benvenga S, Cerutti JM, Dinauer CA, Hamilton J, Hay ID, Luster M, Parisi MT, Rachmiel M, Thompson GB, Yamashita S 2015 Management guidelines for children with thyroid nodules and differentiated thyroid cancer. Thyroid 25:716-759.

15. Lewis MH, Gohagan JK, Merenstein DJ 2007 The locality rule and the physician's dilemma: local medical practices vs the national standard of care. JAMA 297:2633-2637.

16. Harrison MB, Graham ID, van den Hoek J, Dogherty EJ, Carley ME, Angus V 2013 Guideline adaptation and implementation planning: a prospective observational study. Implement Sci 8:49-63.

17. Carlson RW, Larsen JK, McClure J, Fitzgerald CL, Venook AP, Benson AB III, Anderson BO 2014 International adaptations of NCCN Clinical Practice Guidelines in Oncology. J Natl Compr Canc Netw 12:643-648.

18. Gharib H, Papini E, Paschke R, Duick DS, Valcavi R, Hegedus L, Vitti P 2010 American Association of Clinical Endocrinologists, Associazione Medici Endocrinologi, and European Thyroid Association Medical guidelines for clinical practice for the diagnosis and management of thyroid nodules: executive summary of recommendations. Endocr Pract 16:468-475.

19. Perros P, Boelaert K, Colley S, Evans C, Evans RM, Gerrard BG, Gilbert J, Harrison B, Johnson SJ, Giles TE, Moss L, Lewington V, Newbold K, Taylor J, Thakker RV, Watkinson J, Williams GR 2014 Guidelines for the management of thyroid cancer. Clin Endocrinol (Oxf) 81 Suppl 1:1-122.

20. Leenhardt L, Erdogan MF, Hegedus L, Mandel SJ, Paschke R, Rago T, Russ G 20132013 European Thyroid Association guidelines for cervical ultrasound scan and ultrasound-guided techniques in the postoperative management of patients with thyroid cancer. Eur Thyroid J 2:147-159.
21. Silberstein EB, Alavi A, Balon HR, Clarke SE, Divgi C, Gelfand MJ, Goldsmith SJ, Jadvar H, Marcus CS, Martin WH, Parker JA, Royal HD, Sarkar SD, Stabin M, Waxman AD 2012 The SNMMI practice guideline for therapy of thyroid disease with 131I 3.0. J Nucl Med 53:1633-1651.

22. Luster M, Clarke SE, Dietlein M, Lassmann M, Lind P, Oyen WJ, Tennvall J, Bombardieri E 2008 Guidelines for radioiodine therapy of differentiated thyroid cancer. Eur J Nucl Med Mol Imaging 35:1941-1959.

23. Takami H, Ito Y, Okamoto T, Onoda N, Noguchi H, Yoshida A 2014 Revisiting the guidelines issued by the Japanese Society of Thyroid Surgeons and Japan Association of Endocrine Surgeons: a gradual move towards consensus between Japanese and Western practice in the management of thyroid carcinoma. World J Surg 38:2002-2010.

24. Cooper DS, Doherty GM, Haugen BR, Kloos RT, Lee SL, Mandel SJ, Mazzaferri EL, McIver B, Sherman SI, Tuttle RM 2006 Management guidelines for patients with thyroid nodules and differentiated thyroid cancer. Thyroid 16:109-142.

25. Cooper DS, Doherty GM, Haugen BR, Kloos RT, Lee SL, Mandel SJ, Mazzaferri EL, McIver B, Pacini F, Schlumberger M, Sherman SI, Steward DL, Tuttle RM 2009 Revised American Thyroid Association management guidelines for patients with thyroid nodules and differentiated thyroid cancer. Thyroid 19:11671214.

26. Qaseem A, Snow V, Owens DK, Shekelle P 2010 The development of clinical practice guidelines and guidance statements of the American College of Physicians: summary of methods. Ann Intern Med 153:194-199.

27. Schunemann HJ, Oxman AD, Brozek J, Glasziou P, Jaeschke R, Vist GE, Williams JW Jr, Kunz R, Craig J, Montori VM, Bossuyt P, Guyatt GH 2008 Grading quality of evidence and strength of recommendations for diagnostic tests and strategies. BMJ 336:1106-1110.

28. Brozek JL, Akl EA, Jaeschke R, Lang DM, Bossuyt P, Glasziou P, Helfand M, Ueffing E, Alonso-Coello P, Meerpohl J, Phillips B, Horvath AR, Bousquet J, Guyatt GH, Schunemann HJ 2009 Grading quality of evidence and strength of recommendations in clinical practice guidelines: part 2 of 3 . The GRADE approach to grading quality of evidence about diagnostic tests and strategies. Allergy 64:1109-1116.

29. Bossuyt PM, Reitsma JB, Bruns DE, Gatsonis CA, Glasziou PP, Irwig LM, Lijmer JG, Moher D, Rennie D, de Vet HC 2003 Towards complete and accurate reporting of studies of diagnostic accuracy: the STARD Initiative. Ann Intern Med 138:40-44.

30. Leung AN, Bull TM, Jaeschke R, Lockwood CJ, Boiselle PM, Hurwitz LM, James AH, McCullough LB, Menda Y, Paidas MJ, Royal HD, Tapson VF, Winer-Muram HT, Chervenak FA, Cody DD, McNitt-Gray MF, Stave CD, Tuttle BD 2011 An official American Thoracic Society/Society of Thoracic Radiology clinical practice guideline: evaluation of suspected pulmonary embolism in pregnancy. Am J Respir Crit Care Med 184: 1200-1208.

31. Whiting PF, Rutjes AW, Westwood ME, Mallett S, Deeks JJ, Reitsma JB, Leeflang MM, Sterne JA, Bossuyt PM 2011 QUADAS-2: a revised tool for the quality assessment of diagnostic accuracy studies. Ann Intern Med 155:529-536. 
32. Marqusee E, Benson CB, Frates MC, Doubilet PM, Larsen PR, Cibas ES, Mandel SJ 2000 Usefulness of ultrasonography in the management of nodular thyroid disease. Ann Intern Med 133:696-700.

33. Hagag P, Strauss S, Weiss M 1998 Role of ultrasoundguided fine-needle aspiration biopsy in evaluation of nonpalpable thyroid nodules. Thyroid 8:989-995.

34. Charkes ND 2006 On the prevalence of familial nonmedullary thyroid cancer in multiply affected kindreds. Thyroid 16:181-186.

35. Capezzone M, Marchisotta S, Cantara S, Busonero G, Brilli L, Pazaitou-Panayiotou K, Carli AF, Caruso G, Toti P, Capitani S, Pammolli A, Pacini F 2008 Familial non-medullary thyroid carcinoma displays the features of clinical anticipation suggestive of a distinct biological entity. Endocr Relat Cancer 15:1075-1081.

36. Moses W, Weng J, Kebebew E 2011 Prevalence, clinicopathologic features, and somatic genetic mutation profile in familial versus sporadic nonmedullary thyroid cancer. Thyroid 21:367-371.

37. Ito Y, Kakudo K, Hirokawa M, Fukushima M, Yabuta T, Tomoda C, Inoue H, Kihara M, Higashiyama T, Uruno T, Takamura Y, Miya A, Kobayashi K, Matsuzuka F, Miyauchi A 2009 Biological behavior and prognosis of familial papillary thyroid carcinoma. Surgery 145:100-105.

38. Park YJ, Ahn HY, Choi HS, Kim KW, Park do J, Cho BY 2012 The long-term outcomes of the second generation of familial nonmedullary thyroid carcinoma are more aggressive than sporadic cases. Thyroid 22:356-362.

39. Mazeh H, Benavidez J, Poehls JL, Youngwirth L, Chen H, Sippel RS 2012 In patients with thyroid cancer of follicular cell origin, a family history of nonmedullary thyroid cancer in one first-degree relative is associated with more aggressive disease. Thyroid 22:3-8.

40. Robenshtok E, Tzvetov G, Grozinsky-Glasberg S, ShragaSlutzky I, Weinstein R, Lazar L, Serov S, Singer J, Hirsch D, Shimon I, Benbassat C 2011 Clinical characteristics and outcome of familial nonmedullary thyroid cancer: a retrospective controlled study. Thyroid 21:43-48.

41. Richards ML 2010 Familial syndromes associated with thyroid cancer in the era of personalized medicine. Thyroid 20:707-713.

42. Curtis RE, Rowlings PA, Deeg HJ, Shriner DA, Socie G, Travis LB, Horowitz MM, Witherspoon RP, Hoover RN, Sobocinski KA, Fraumeni JF Jr, Boice JD Jr 1997 Solid cancers after bone marrow transplantation. N Engl J Med 336:897-904.

43. Pacini F, Vorontsova T, Demidchik EP, Molinaro E, Agate L, Romei C, Shavrova E, Cherstvoy ED, Ivashkevitch Y, Kuchinskaya E, Schlumberger M, Ronga G, Filesi M, Pinchera A 1997 Post-Chernobyl thyroid carcinoma in Belarus children and adolescents: comparison with naturally occurring thyroid carcinoma in Italy and France. J Clin Endocrinol Metab 82:3563-3569.

44. Gharib H, Papini E 2007 Thyroid nodules: clinical importance, assessment, and treatment. Endocrinol Metab Clin North Am 36:707-35, vi.

45. Boelaert K, Horacek J, Holder RL, Watkinson JC, Sheppard MC, Franklyn JA 2006 Serum thyrotropin concentration as a novel predictor of malignancy in thyroid nodules investigated by fine-needle aspiration. $\mathbf{J}$ Clin Endocrinol Metab 91:4295-4301.

46. Haymart MR, Repplinger DJ, Leverson GE, Elson DF, Sippel RS, Jaume JC, Chen H 2008 Higher serum thyroid stimulating hormone level in thyroid nodule patients is associated with greater risks of differentiated thyroid cancer and advanced tumor stage. J Clin Endocrinol Metab 93:809-814.

47. Repplinger D, Bargren A, Zhang YW, Adler JT, Haymart M, Chen H 2008 Is Hashimoto's thyroiditis a risk factor for papillary thyroid cancer? J Surg Res 150:49-52.

48. Suh I, Vriens MR, Guerrero MA, Griffin A, Shen WT, Duh QY, Clark OH, Kebebew E 2010 Serum thyroglobulin is a poor diagnostic biomarker of malignancy in follicular and Hürthle-cell neoplasms of the thyroid. Am J Surg 200:41-46.

49. Lee EK, Chung KW, Min HS, Kim TS, Kim TH, Ryu JS, Jung YS, Kim SK, Lee YJ 2012 Preoperative serum thyroglobulin as a useful predictive marker to differentiate follicular thyroid cancer from benign nodules in indeterminate nodules. J Korean Med Sci 27:1014-1018.

50. Elisei R, Bottici V, Luchetti F, Di Coscio G, Romei C, Grasso L, Miccoli P, Iacconi P, Basolo F, Pinchera A, Pacini F 2004 Impact of routine measurement of serum calcitonin on the diagnosis and outcome of medullary thyroid cancer: experience in 10,864 patients with nodular thyroid disorders. J Clin Endocrinol Metab 89:163-168.

51. Hahm JR, Lee MS, Min YK, Lee MK, Kim KW, Nam SJ, Yang JH, Chung JH 2001 Routine measurement of serum calcitonin is useful for early detection of medullary thyroid carcinoma in patients with nodular thyroid diseases. Thyroid 11:73-80.

52. Niccoli P, Wion-Barbot N, Caron P, Henry JF, de Micco C, Saint Andre JP, Bigorgne JC, Modigliani E, ConteDevolx B 1997 Interest of routine measurement of serum calcitonin: study in a large series of thyroidectomized patients. The French Medullary Study Group. J Clin Endocrinol Metab 82:338-341.

53. Costante G, Meringolo D, Durante C, Bianchi D, Nocera M, Tumino S, Crocetti U, Attard M, Maranghi M, Torlontano M, Filetti S 2007 Predictive value of serum calcitonin levels for preoperative diagnosis of medullary thyroid carcinoma in a cohort of 5817 consecutive patients with thyroid nodules. J Clin Endocrinol Metab 92:450-455.

54. Chambon G, Alovisetti C, Idoux-Louche C, Reynaud C, Rodier M, Guedj AM, Chapuis H, Lallemant JG, Lallemant B 2011 The use of preoperative routine measurement of basal serum thyrocalcitonin in candidates for thyroidectomy due to nodular thyroid disorders: results from 2733 consecutive patients. J Clin Endocrinol Metab 96:75-81.

55. Colombo C, Verga U, Mian C, Ferrero S, Perrino M, Vicentini L, Dazzi D, Opocher G, Pelizzo MR, BeckPeccoz P, Fugazzola L 2012 Comparison of calcium and pentagastrin tests for the diagnosis and follow-up of medullary thyroid cancer. J Clin Endocrinol Metab 97: 905-913.

56. Karga H, Giagourta I, Papaioannou G, Doumouchtsis K, Polymeris A, Thanou S, Papamichael K, Zerva C 2011 Changes in risk factors and Tumor Node Metastasis stage of sporadic medullary thyroid carcinoma over 41 years, before and after the routine measurements of serum calcitonin. Metabolism 60:604-608.

57. Cheung K, Roman SA, Wang TS, Walker HD, Sosa JA 2008 Calcitonin measurement in the evaluation of thyroid nodules in the United States: a cost-effectiveness and decision analysis. J Clin Endocrinol Metab 93: 2173-2180. 
58. Gagel RF, Hoff AO, Cote GE 2005 Medullary thyroid carcinoma. In: Braverman L, Utiger R (eds) Werner and Ingbar's The Thyroid. Lippincott Williams and Wilkins, Philadelphia, PA, pp 967-988.

59. Diazzi C, Madeo B, Taliani E, Zirilli L, Romano S, Granata AR, De Santis MC, Simoni M, Cioni K, Carani C, Rochira V 2013 The diagnostic value of calcitonin measurement in wash-out fluid from fine-needle aspiration of thyroid nodules in the diagnosis of medullary thyroid cancer. Endocr Pract 19:769-779.

60. Soelberg KK, Bonnema SJ, Brix TH, Hegedus L 2012 Risk of malignancy in thyroid incidentalomas detected by $18 \mathrm{~F}$-fluorodeoxyglucose positron emission tomography: a systematic review. Thyroid 22:918-925.

61. Chen W, Parsons M, Torigian DA, Zhuang H, Alavi A 2009 Evaluation of thyroid FDG uptake incidentally identified on FDG-PET/CT imaging. Nucl Med Commun 30:240-244.

62. Nishimori H, Tabah R, Hickeson M, How J 2011 Incidental thyroid "PETomas": clinical significance and novel description of the self-resolving variant of focal FDG-PET thyroid uptake. Can J Surg 54:83-88.

63. Hall TL, Layfield LJ, Philippe A, Rosenthal DL 1989 Sources of diagnostic error in fine needle aspiration of the thyroid. Cancer 63:718-725.

64. Alexander EK, Heering JP, Benson CB, Frates MC, Doubilet PM, Cibas ES, Marqusee E 2002 Assessment of nondiagnostic ultrasound-guided fine needle aspirations of thyroid nodules. J Clin Endocrinol Metab 87: 4924-4927.

65. Smith-Bindman R, Lebda P, Feldstein VA, Sellami D, Goldstein RB, Brasic N, Jin C, Kornak J 2013 Risk of thyroid cancer based on thyroid ultrasound imaging characteristics: results of a population-based study. JAMA Intern Med 173:1788-1796.

66. Brito JP, Gionfriddo MR, Al NA, Boehmer KR, Leppin AL, Reading C, Callstrom M, Elraiyah TA, Prokop LJ, Stan MN, Murad MH, Morris JC, Montori VM 2014 The accuracy of thyroid nodule ultrasound to predict thyroid cancer: systematic review and meta-analysis. J Clin Endocrinol Metab 99:1253-1263.

67. Langer JE, Agarwal R, Zhuang H, Huang SS, Mandel SJ 2011 Correlation of findings from iodine 123 scan and ultrasonography in the recommendation for thyroid fineneedle aspiration biopsy. Endocr Pract 17:699-706.

68. Danese D, Sciacchitano S, Farsetti A, Andreoli M, Pontecorvi A 1998 Diagnostic accuracy of conventional versus sonography-guided fine-needle aspiration biopsy of thyroid nodules. Thyroid 8:15-21.

69. Carmeci C, Jeffrey RB, McDougall IR, Nowels KW, Weigel RJ 1998 Ultrasound-guided fine-needle aspiration biopsy of thyroid masses. Thyroid 8:283-289.

70. Kwak JY, Han KH, Yoon JH, Moon HJ, Son EJ, Park SH, Jung HK, Choi JS, Kim BM, Kim EK 2011 Thyroid imaging reporting and data system for US features of nodules: a step in establishing better stratification of cancer risk. Radiology 260:892-899.

71. Moon WJ, Jung SL, Lee JH, Na DG, Baek JH, Lee YH, Kim J, Kim HS, Byun JS, Lee DH 2008 Benign and malignant thyroid nodules: US differentiation-multicenter retrospective study. Radiology 247:762-770.

72. Moon HJ, Kwak JY, Kim MJ, Son EJ, Kim EK 2010 Can vascularity at power Doppler US help predict thyroid malignancy? Radiology 255:260-269.
73. Salmaslioglu A, Erbil Y, Dural C, Issever H, Kapran Y, Ozarmagan S, Tezelman S 2008 Predictive value of sonographic features in preoperative evaluation of malignant thyroid nodules in a multinodular goiter. World $\mathbf{J}$ Surg 32:1948-1954.

74. Papini E, Guglielmi R, Bianchini A, Crescenzi A, Taccogna S, Nardi F, Panunzi C, Rinaldi R, Toscano V, Pacella CM 2002 Risk of malignancy in nonpalpable thyroid nodules: predictive value of ultrasound and color-Doppler features. J Clin Endocrinol Metab 87:1941-1946.

75. Gul K, Ersoy R, Dirikoc A, Korukluoglu B, Ersoy PE, Aydin R, Ugras SN, Belenli OK, Cakir B 2009 Ultrasonographic evaluation of thyroid nodules: comparison of ultrasonographic, cytological, and histopathological findings. Endocrine 36:464-472.

76. Cappelli C, Pirola I, Cumetti D, Micheletti L, Tironi A, Gandossi E, Martino E, Cherubini L, Agosti B, Castellano M, Mattanza C, Rosei EA 2005 Is the anteroposterior and transverse diameter ratio of nonpalpable thyroid nodules a sonographic criteria for recommending fine-needle aspiration cytology? Clin Endocrinol (Oxf) 63:689-693.

77. Frates MC, Benson CB, Doubilet PM, Kunreuther E, Contreras M, Cibas ES, Orcutt J, Moore FD Jr, Larsen PR, Marqusee E, Alexander EK 2006 Prevalence and distribution of carcinoma in patients with solitary and multiple thyroid nodules on sonography. J Clin Endocrinol Metab 91:3411-3417.

78. Moon HJ, Sung JM, Kim EK, Yoon JH, Youk JH, Kwak JY 2012 Diagnostic performance of gray-scale US and elastography in solid thyroid nodules. Radiology 262: 1002-1013.

79. Kim DS, Kim JH, Na DG, Park SH, Kim E, Chang KH, Sohn CH, Choi YH 2009 Sonographic features of follicular variant papillary thyroid carcinomas in comparison with conventional papillary thyroid carcinomas. J Ultrasound Med 28:1685-1692.

80. Park YJ, Kim JA, Son EJ, Youk JH, Kim EK, Kwak JY, Park CS 2014 Thyroid nodules with macrocalcification: sonographic findings predictive of malignancy. Yonsei Med J 55:339-344.

81. Cappelli C, Castellano M, Pirola I, Cumetti D, Agosti B, Gandossi E, Agabiti Rosei E 2007 The predictive value of ultrasound findings in the management of thyroid nodules. QJM 100:29-35.

82. Jeh SK, Jung SL, Kim BS, Lee YS 2007 Evaluating the degree of conformity of papillary carcinoma and follicular carcinoma to the reported ultrasonographic findings of malignant thyroid tumor. Korean J Radiol 8:192-197.

83. Machens A, Holzhausen HJ, Dralle H 2005 The prognostic value of primary tumor size in papillary and follicular thyroid carcinoma. Cancer 103:2269-2273.

84. Nam-Goong IS, Kim HY, Gong G, Lee HK, Hong SJ, Kim WB, Shong YK 2004 Ultrasonography-guided fineneedle aspiration of thyroid incidentaloma: correlation with pathological findings. Clin Endocrinol (Oxf) 60: 21-28.

85. Henrichsen TL, Reading CC, Charboneau JW, Donovan DJ, Sebo TJ, Hay ID 2010 Cystic change in thyroid carcinoma: prevalence and estimated volume in 360 carcinomas. J Clin Ultrasound 38:361-366.

86. Lee MJ, Kim EK, Kwak JY, Kim MJ 2009 Partially cystic thyroid nodules on ultrasound: probability of malignancy and sonographic differentiation. Thyroid 19: 341-346. 
87. Kim DW, Lee EJ, In HS, Kim SJ 2010 Sonographic differentiation of partially cystic thyroid nodules: a prospective study. AJNR Am J Neuroradiol 31:1961-1966.

88. Bonavita JA, Mayo J, Babb J, Bennett G, Oweity T, Macari M, Yee J 2009 Pattern recognition of benign nodules at ultrasound of the thyroid: which nodules can be left alone? AJR Am J Roentgenol 193:207-213.

89. Horvath E, Majlis S, Rossi R, Franco C, Niedmann JP, Castro A, Dominguez M 2009 An ultrasonogram reporting system for thyroid nodules stratifying cancer risk for clinical management. J Clin Endocrinol Metab 94: 1748-1751.

90. Tae HJ, Lim DJ, Baek KH, Park WC, Lee YS, Choi JE, Lee JM, Kang MI, Cha BY, Son HY, Lee KW, Kang SK 2007 Diagnostic value of ultrasonography to distinguish between benign and malignant lesions in the management of thyroid nodules. Thyroid 17:461-466.

91. Shimura H, Haraguchi K, Hiejima Y, Fukunari N, Fujimoto Y, Katagiri M, Koyanagi N, Kurita T, Miyakawa M, Miyamoto Y, Suzuki N, Suzuki S, Kanbe M, Kato Y, Murakami T, Tohno E, Tsunoda-Shimizu H, Yamada K, Ueno E, Kobayashi K, Kobayashi T, Yokozawa T, Kitaoka M 2005 Distinct diagnostic criteria for ultrasonographic examination of papillary thyroid carcinoma: a multicenter study. Thyroid 15:251-258.

92. Russ G, Royer B, Bigorgne C, Rouxel A, BienvenuPerrard M, Leenhardt L 2013 Prospective evaluation of thyroid imaging reporting and data system on 4550 nodules with and without elastography. Eur J Endocrinol 168:649-655.

93. Cheng SP, Lee JJ, Lin JL, Chuang SM, Chien MN, Liu CL 2013 Characterization of thyroid nodules using the proposed thyroid imaging reporting and data system (TIRADS). Head Neck 35:541-547.

94. Ito Y, Amino N, Yokozawa T, Ota H, Ohshita M, Murata N, Morita S, Kobayashi K, Miyauchi A 2007 Ultrasonographic evaluation of thyroid nodules in 900 patients: comparison among ultrasonographic, cytological, and histological findings. Thyroid 17:1269-1276.

95. Ito $\mathrm{Y}$, Miyauchi A, Kihara M, Higashiyama T, Kobayashi K, Miya A 2014 Patient age is significantly related to the progression of papillary microcarcinoma of the thyroid under observation. Thyroid 24:27-34.

96. Hamming JF, Goslings BM, van Steenis GJ, van Ravenswaay $\mathrm{CH}$, Hermans J, van de Velde CJ 1990 The value of fine-needle aspiration biopsy in patients with nodular thyroid disease divided into groups of suspicion of malignant neoplasms on clinical grounds. Arch Intern Med 150:113-116.

97. Rago T, Santini F, Scutari M, Pinchera A, Vitti P 2007 Elastography: new developments in ultrasound for predicting malignancy in thyroid nodules. J Clin Endocrinol Metab 92:2917-2922.

98. Azizi G, Keller J, Lewis M, Puett D, Rivenbark K, Malchoff C 2013 Performance of elastography for the evaluation of thyroid nodules: a prospective study. Thyroid 23:734-740.

99. Baloch ZW, LiVolsi VA, Asa SL, Rosai J, Merino MJ, Randolph G, Vielh P, DeMay RM, Sidawy MK, Frable WJ 2008 Diagnostic terminology and morphologic criteria for cytologic diagnosis of thyroid lesions: a synopsis of the National Cancer Institute Thyroid FineNeedle Aspiration State of the Science Conference. Diagn Cytopathol 36:425-437.
100. Crippa S, Mazzucchelli L, Cibas ES, Ali SZ 2010 The Bethesda System for reporting thyroid fine-needle aspiration specimens. Am J Clin Pathol 134:343-344.

101. Theoharis CG, Schofield KM, Hammers L, Udelsman R, Chhieng DC 2009 The Bethesda thyroid fine-needle aspiration classification system: year 1 at an academic institution. Thyroid 19:1215-1223.

102. Luu MH, Fischer AH, Pisharodi L, Owens CL 2011 Improved preoperative definitive diagnosis of papillary thyroid carcinoma in FNAs prepared with both ThinPrep and conventional smears compared with FNAs prepared with ThinPrep alone. Cancer Cytopathol 119:68-73.

103. Bongiovanni M, Spitale A, Faquin WC, Mazzucchelli L, Baloch ZW 2012 The Bethesda System for Reporting Thyroid Cytopathology: a meta-analysis. Acta Cytol 56: 333-339.

104. Nayar R, Ivanovic M 2009 The indeterminate thyroid fine-needle aspiration: experience from an academic center using terminology similar to that proposed in the 2007 National Cancer Institute Thyroid Fine Needle Aspiration State of the Science Conference. Cancer 117: 195-202.

105. Ohori NP, Schoedel KE 2011 Variability in the atypia of undetermined significance/follicular lesion of undetermined significance diagnosis in the Bethesda System for Reporting Thyroid Cytopathology: sources and recommendations. Acta Cytol 55:492-498.

106. Cibas ES, Baloch ZW, Fellegara G, LiVolsi VA, Raab SS, Rosai J, Diggans J, Friedman L, Kennedy GC, Kloos RT, Lanman RB, Mandel SJ, Sindy N, Steward DL, Zeiger MA, Haugen BR, Alexander EK 2013 A prospective assessment defining the limitations of thyroid nodule pathologic evaluation. Ann Intern Med 159: 325-332.

107. Nishino M, Wang HH 2014 Should the thyroid AUS/ FLUS category be further stratified by malignancy risk? Cancer Cytopathol 122:481-483.

108. Crothers BA, Henry MR, Firat P 2010 Nondiagnostic/ unsatisfactory. In: Ali SZ, Cibas ES (eds) The Bethesda System for Reporting Thyroid Cytopathology. Springer, pp 5-14.

109. Baloch ZW, Tam D, Langer J, Mandel S, LiVolsi VA, Gupta PK 2000 Ultrasound-guided fine-needle aspiration biopsy of the thyroid: role of on-site assessment and multiple cytologic preparations. Diagn Cytopathol 23: 425-429.

110. Braga M, Cavalcanti TC, Collaco LM, Graf H 2001 Efficacy of ultrasound-guided fine-needle aspiration biopsy in the diagnosis of complex thyroid nodules. J Clin Endocrinol Metab 86:4089-4091.

111. Redman R, Zalaznick H, Mazzaferri EL, Massoll NA 2006 The impact of assessing specimen adequacy and number of needle passes for fine-needle aspiration biopsy of thyroid nodules. Thyroid 16:55-60.

112. Orija IB, Pineyro M, Biscotti C, Reddy SS, Hamrahian AH 2007 Value of repeating a nondiagnostic thyroid fine-needle aspiration biopsy. Endocr Pract 13:735-742.

113. Wu HH, Rose C, Elsheikh TM 2012 The Bethesda system for reporting thyroid cytopathology: an experience of 1,382 cases in a community practice setting with the implication for risk of neoplasm and risk of malignancy. Diagn Cytopathol 40:399-403.

114. Layfield LJ, Abrams J, Cochand-Priollet B, Evans D, Gharib H, Greenspan F, Henry M, LiVolsi V, Merino M, 
Michael CW, Wang H, Wells SA 2008 Post-thyroid FNA testing and treatment options: a synopsis of the National Cancer Institute Thyroid Fine Needle Aspiration State of the Science Conference. Diagn Cytopathol 36:442-448.

115. Singh RS, Wang HH 2011 Timing of repeat thyroid fineneedle aspiration in the management of thyroid nodules. Acta Cytol 55:544-548.

116. Lubitz CC, Nagarkatti SS, Faquin WC, Samir AE, Hassan MC, Barbesino G, Ross DS, Randolph GW, Gaz RD, Stephen AE, Hodin RA, Daniels GH, Parangi S 2012 Diagnostic yield of nondiagnostic thyroid nodules is not altered by timing of repeat biopsy. Thyroid 22: 590-594.

117. Choi YS, Hong SW, Kwak JY, Moon HJ, Kim EK 2012 Clinical and ultrasonographic findings affecting nondiagnostic results upon the second fine needle aspiration for thyroid nodules. Ann Surg Oncol 19: 2304-2309.

118. Moon HJ, Kwak JY, Choi YS, Kim EK 2012 How to manage thyroid nodules with two consecutive nondiagnostic results on ultrasonography-guided fine-needle aspiration. World J Surg 36:586-592.

119. Na DG, Kim JH, Sung JY, Baek JH, Jung KC, Lee H, Yoo H 2012 Core-needle biopsy is more useful than repeat fine-needle aspiration in thyroid nodules read as nondiagnostic or atypia of undetermined significance by the Bethesda system for reporting thyroid cytopathology. Thyroid 22:468-475.

120. Nam SY, Han BK, Ko EY, Kang SS, Hahn SY, Hwang JY, Nam MY, Kim JW, Chung JH, Oh YL, Shin JH 2010 $B R A F^{V 600 E}$ mutation analysis of thyroid nodules needle aspirates in relation to their ultrasongraphic classification: a potential guide for selection of samples for molecular analysis. Thyroid 20:273-279.

121. Yip L, Nikiforova MN, Carty SE, Yim JH, Stang MT, Tublin MJ, LeBeau SO, Hodak SP, Ogilvie JB, Nikiforov YE 2009 Optimizing surgical treatment of papillary thyroid carcinoma associated with BRAF mutation. Surgery 146:1215-1223.

122. Cantara S, Capezzone M, Marchisotta S, Capuano S, Busonero G, Toti P, Di SA, Caruso G, Carli AF, Brilli L, Montanaro A, Pacini F 2010 Impact of proto-oncogene mutation detection in cytological specimens from thyroid nodules improves the diagnostic accuracy of cytology. $\mathbf{J}$ Clin Endocrinol Metab 95:1365-1369.

123. Renshaw AA, Pinnar N 2007 Comparison of thyroid fine-needle aspiration and core needle biopsy. Am J Clin Pathol 128:370-374.

124. Yeon JS, Baek JH, Lim HK, Ha EJ, Kim JK, Song DE, Kim TY, Lee JH 2013 Thyroid nodules with initially nondiagnostic cytologic results: the role of core-needle biopsy. Radiology 268:274-280.

125. Deleted.

126. Hatada T, Okada K, Ishii H, Ichii S, Utsunomiya J 1998 Evaluation of ultrasound-guided fine-needle aspiration biopsy for thyroid nodules. Am J Surg 175:133-136.

127. Deleted.

128. Cesur M, Corapcioglu D, Bulut S, Gursoy A, Yilmaz AE, Erdogan N, Kamel N 2006 Comparison of palpationguided fine-needle aspiration biopsy to ultrasoundguided fine-needle aspiration biopsy in the evaluation of thyroid nodules. Thyroid 16:555-561.

129. Chehade JM, Silverberg AB, Kim J, Case C, Mooradian AD 2001 Role of repeated fine-needle aspiration of thyroid nodules with benign cytologic features. Endocr Pract 7:237-243.

130. Orlandi A, Puscar A, Capriata E, Fideleff H 2005 Repeated fine-needle aspiration of the thyroid in benign nodular thyroid disease: critical evaluation of long-term follow-up. Thyroid 15:274-278.

131. Oertel YC, Miyahara-Felipe L, Mendoza MG, Yu K 2007 Value of repeated fine needle aspirations of the thyroid: an analysis of over ten thousand FNAs. Thyroid 17:1061-1066.

132. Erdogan MF, Kamel N, Aras D, Akdogan A, Baskal N, Erdogan G 1998 Value of re-aspirations in benign nodular thyroid disease. Thyroid 8:1087-1090.

133. Illouz F, Rodien P, Saint-Andre JP, Triau S, LaboureauSoares S, Dubois S, Vielle B, Hamy A, Rohmer V 2007 Usefulness of repeated fine-needle cytology in the follow-up of non-operated thyroid nodules. Eur J Endocrinol 156:303-308.

134. Tee YY, Lowe AJ, Brand CA, Judson RT 2007 Fineneedle aspiration may miss a third of all malignancy in palpable thyroid nodules: a comprehensive literature review. Ann Surg 246:714-720.

135. Pinchot SN, Al-Wagih H, Schaefer S, Sippel R, Chen H 2009 Accuracy of fine-needle aspiration biopsy for predicting neoplasm or carcinoma in thyroid nodules $4 \mathrm{~cm}$ or larger. Arch Surg 144:649-655.

136. Kuru B, Gulcelik NE, Gulcelik MA, Dincer H 2010 The false-negative rate of fine-needle aspiration cytology for diagnosing thyroid carcinoma in thyroid nodules. Langenbecks Arch Surg 395:127-132.

137. Wharry LI, McCoy KL, Stang MT, Armstrong MJ, LeBeau SO, Tublin ME, Sholosh B, Silbermann A, Ohori NP, Nikiforov YE, Hodak SP, Carty SE, Yip L 2014 Thyroid nodules $(>/=4 \mathrm{~cm})$ : can ultrasound and cytology reliably exclude cancer? World J Surg 38:614-621.

138. Yoon JH, Kwak JY, Moon HJ, Kim MJ, Kim EK 2011 The diagnostic accuracy of ultrasound-guided fineneedle aspiration biopsy and the sonographic differences between benign and malignant thyroid nodules $3 \mathrm{~cm}$ or larger. Thyroid 21:993-1000.

139. Porterfield JR Jr, Grant CS, Dean DS, Thompson GB, Farley DR, Richards ML, Reading CC, Charboneau JW, Vollrath BK, Sebo TJ 2008 Reliability of benign fine needle aspiration cytology of large thyroid nodules. Surgery 144:963-968.

140. Nou E, Kwong N, Alexander LK, Cibas ES, Marqusee E, Alexander EK 2014 Determination of the optimal time interval for repeat evaluation after a benign thyroid nodule aspiration. J Clin Endocrinol Metab 99:510-516.

141. Mazzaferri EL 2007 Management of low-risk differentiated thyroid cancer. Endocr Pract 13:498-512.

142. Hay ID 2007 Management of patients with low-risk papillary thyroid carcinoma. Endocr Pract 13:521-533.

143. Sugitani I, Toda K, Yamada K, Yamamoto N, Ikenaga M, Fujimoto Y 2010 Three distinctly different kinds of papillary thyroid microcarcinoma should be recognized: our treatment strategies and outcomes. World J Surg 34: 1222-1231.

144. Yu XM, Wan Y, Sippel RS, Chen H 2011 Should all papillary thyroid microcarcinomas be aggressively treated? An analysis of 18,445 cases. Ann Surg 254:653660.

145. Chow SM, Law SC, Chan JK, Au SK, Yau S, Lau WH 2003 Papillary microcarcinoma of the thyroid-prog- 
nostic significance of lymph node metastasis and multifocality. Cancer 98:31-40.

146. Hay ID, Hutchinson ME, Gonzalez-Losada T, McIver B, Reinalda ME, Grant CS, Thompson GB, Sebo TJ, Goellner JR 2008 Papillary thyroid microcarcinoma: a study of 900 cases observed in a 60-year period. Surgery 144:980-987.

147. Roti E, degli Uberti EC, Bondanelli M, Braverman LE 2008 Thyroid papillary microcarcinoma: a descriptive and meta-analysis study. Eur J Endocrinol 159: 659-673.

148. Giordano D, Gradoni P, Oretti G, Molina E, Ferri T 2010 Treatment and prognostic factors of papillary thyroid microcarcinoma. Clin Otolaryngol 35:118-124.

149. Ito $\mathrm{Y}$, Miyauchi A, Inoue H, Fukushima M, Kihara M, Higashiyama T, Tomoda C, Takamura Y, Kobayashi K, Miya A 2010 An observational trial for papillary thyroid microcarcinoma in Japanese patients. World J Surg 34: 28-35.

150. Niemeier LA, Kuffner AH, Song C, Carty SE, Hodak SP, Yip L, Ferris RL, Tseng GC, Seethala RR, LeBeau SO, Stang MT, Coyne C, Johnson JT, Stewart AF, Nikiforov YE 2012 A combined molecular-pathologic score improves risk stratification of thyroid papillary microcarcinoma. Cancer 118:2069-2077.

151. Roh JL, Kim JM, Park CI 2008 Central cervical nodal metastasis from papillary thyroid microcarcinoma: pattern and factors predictive of nodal metastasis. Ann Surg Oncol 15:2482-2486.

152. Lin KL, Wang OC, Zhang XH, Dai XX, Hu XQ, Qu JM 2010 The BRAF mutation is predictive of aggressive clinicopathological characteristics in papillary thyroid microcarcinoma. Ann Surg Oncol 17:3294-3300.

153. Zheng X, Wei S, Han Y, Li Y, Yu Y, Yun X, Ren X, Gao M 2013 Papillary microcarcinoma of the thyroid: clinical characteristics and BRAF(V600E) mutational status of 977 cases. Ann Surg Oncol 20:2266-2273.

154. Melo M, da Rocha AG, Vinagre J, Batista R, Peixoto J, Tavares C, Celestino R, Almeida A, Salgado C, Eloy C, Castro P, Prazeres H, Lima J, Amaro T, Lobo C, Martins MJ, Moura M, Cavaco B, Leite V, Cameselle-Teijeiro JM, Carrilho F, Carvalheiro M, Maximo V, Sobrinho-Simoes M, Soares P 2014 TERT promoter mutations are a major indicator of poor outcome in differentiated thyroid carcinomas. J Clin Endocrinol Metab 99:E754-E765.

155. Xing M, Liu R, Liu X, Murugan AK, Zhu G, Zeiger MA, Pai S, Bishop J $2014 B R A F^{V 600 E}$ and TERT promoter mutations cooperatively identify the most aggressive papillary thyroid cancer with highest recurrence. J Clin Oncol 32:2718-2726.

156. Nikiforova MN, Kimura ET, Gandhi M, Biddinger PW, Knauf JA, Basolo F, Zhu Z, Giannini R, Salvatore G, Fusco A, Santoro M, Fagin JA, Nikiforov YE 2003 BRAF mutations in thyroid tumors are restricted to papillary carcinomas and anaplastic or poorly differentiated carcinomas arising from papillary carcinomas. J Clin Endocrinol Metab 88:5399-5404.

157. Ricarte-Filho JC, Ryder M, Chitale DA, Rivera M, Heguy A, Ladanyi M, Janakiraman M, Solit D, Knauf JA, Tuttle RM, Ghossein RA, Fagin JA 2009 Mutational profile of advanced primary and metastatic radioactive iodine-refractory thyroid cancers reveals distinct pathogenetic roles for BRAF, PIK3CA, and AKT1. Cancer Res 69:4885-4893.
158. Febbo PG, Ladanyi M, Aldape KD, De Marzo AM, Hammond ME, Hayes DF, Iafrate AJ, Kelley RK, Marcucci G, Ogino S, Pao W, Sgroi DC, Birkeland ML 2011 NCCN Task Force report: Evaluating the clinical utility of tumor markers in oncology. J Natl Compr Canc Netw 9(Suppl 5):S1-S32.

159. Bernet V, Hupart KH, Parangi S, Woeber KA 2014 AACE/ACE disease state commentary: molecular diagnostic testing of thyroid nodules with indeterminate cytopathology. Endocr Pract 20:360-363.

160. Ferris RL, Baloch Z, Bernet V, Chen A, Fahey TJ III, Ganly I, Hodak SP, Kebebew E, Patel KN, Shaha A, Steward DL, Tufano RP, Wiseman SM, Carty SE; American Thyroid Association Surgical Affairs Committee 2015 American Thyroid Association Statement on Surgical Application of Molecular Profiling for Thyroid Nodules: Current Impact on Perioperative Decision Making. Thyroid 25:760-768.

161. Xing M, Haugen BR, Schlumberger M 2013 Progress in molecular-based management of differentiated thyroid cancer. Lancet 381:1058-1069.

162. Nikiforov YE, Ohori NP, Hodak SP, Carty SE, LeBeau SO, Ferris RL, Yip L, Seethala RR, Tublin ME, Stang MT, Coyne C, Johnson JT, Stewart AF, Nikiforova MN 2011 Impact of mutational testing on the diagnosis and management of patients with cytologically indeterminate thyroid nodules: a prospective analysis of 1056 FNA samples. J Clin Endocrinol Metab 96: 3390-3397.

163. Alexander EK, Kennedy GC, Baloch ZW, Cibas ES, Chudova D, Diggans J, Friedman L, Kloos RT, LiVolsi VA, Mandel SJ, Raab SS, Rosai J, Steward DL, Walsh PS, Wilde JI, Zeiger MA, Lanman RB, Haugen BR 2012 Preoperative diagnosis of benign thyroid nodules with indeterminate cytology. N Engl J Med 367:705-715.

164. Bartolazzi A, Orlandi F, Saggiorato E, Volante M, Arecco F, Rossetto R, Palestini N, Ghigo E, Papotti M, Bussolati G, Martegani MP, Pantellini F, Carpi A, Giovagnoli MR, Monti S, Toscano V, Sciacchitano S, Pennelli GM, Mian C, Pelizzo MR, Rugge M, Troncone G, Palombini L, Chiappetta G, Botti G, Vecchione A, Bellocco R 2008 Galectin-3-expression analysis in the surgical selection of follicular thyroid nodules with indeterminate fine-needle aspiration cytology: a prospective multicentre study. Lancet Oncol 9:543-549.

165. Nikiforov YE, Steward DL, Robinson-Smith TM, Haugen BR, Klopper JP, Zhu Z, Fagin JA, Falciglia M, Weber K, Nikiforova MN 2009 Molecular testing for mutations in improving the fine-needle aspiration diagnosis of thyroid nodules. J Clin Endocrinol Metab 94: 2092-2098.

166. Moses W, Weng J, Sansano I, Peng M, Khanafshar E, Ljung BM, Duh QY, Clark OH, Kebebew E 2010 Molecular testing for somatic mutations improves the accuracy of thyroid fine-needle aspiration biopsy. World J Surg 34:2589-2594.

167. Beaudenon-Huibregtse S, Alexander EK, Guttler RB, Hershman JM, Babu V, Blevins TC, Moore P, Andruss B, Labourier E 2014 Centralized molecular testing for oncogenic gene mutations complements the local cytopathologic diagnosis of thyroid nodules. Thyroid 24: 1479-1487.

168. Liu S, Gao A, Zhang B, Zhang Z, Zhao Y, Chen P, Ji M, Hou P, Shi B 2014 Assessment of molecular testing in 
fine-needle aspiration biopsy samples: an experience in a Chinese population. Exp Mol Pathol 97:292-297.

169. Nikiforov YE, Nikiforova MN 2011 Molecular genetics and diagnosis of thyroid cancer. Nat Rev Endocrinol 7: 569-580.

170. Nikiforov YE, Carty SE, Chiosea SI, Coyne C, Duvvuri U, Ferris RL, Gooding WE, Hodak SP, LeBeau SO, Ohori NP, Seethala RR, Tublin ME, Yip L, Nikiforova MN 2014 Highly accurate diagnosis of cancer in thyroid nodules with follicular neoplasm/suspicious for a follicular neoplasm cytology by ThyroSeq v2 next-generation sequencing assay. Cancer 120:3627-3634.

171. Alexander EK, Schorr M, Klopper J, Kim C, Sipos J, Nabhan F, Parker C, Steward DL, Mandel SJ, Haugen BR 2014 Multicenter clinical experience with the Afirma gene expression classifier. J Clin Endocrinol Metab 99: $119-125$.

172. Harrell RM, Bimston DN 2014 Surgical utility of Afirma: effects of high cancer prevalence and oncocytic cell types in patients with indeterminate thyroid cytology. Endocr Pract 20:364-369.

173. McIver B, Castro MR, Morris JC, Bernet V, Smallridge R, Henry M, Kosok L, Reddi H 2014 An independent study of a gene expression classifier (Afirma) in the evaluation of cytologically indeterminate thyroid nodules. J Clin Endocrinol Metab 99:4069-4077.

174. Marti JL, Avadhani V, Donatelli LA, Niyogi S, Wang B, Wong RJ, Shaha AR, Ghossein RA, Lin O, Morris LG, Ho AS 2015 Wide inter-institutional variation in performance of a molecular classifier for indeterminate thyroid nodules. Ann Surg Oncol 22:3996-4001.

175. Franco C, Martinez V, Allamand JP, Medina F, Glasinovic A, Osorio M, Schachter D 2009 Molecular markers in thyroid fine-needle aspiration biopsy: a prospective study. Appl Immunohistochem Mol Morphol 17:211215.

176. Fadda G, Rossi ED, Raffaelli M, Pontecorvi A, Sioletic S, Morassi F, Lombardi CP, Zannoni GF, Rindi G 2011 Follicular thyroid neoplasms can be classified as lowand high-risk according to HBME-1 and Galectin-3 expression on liquid-based fine-needle cytology. Eur J Endocrinol 165:447-453.

177. Lubitz CC, Fahey TJ III 2005 The differentiation of benign and malignant thyroid nodules. Adv Surg 39: 355-377.

178. Prasad NB, Somervell H, Tufano RP, Dackiw AP, Marohn MR, Califano JA, Wang Y, Westra WH, Clark DP, Umbricht CB, Libutti SK, Zeiger MA 2008 Identification of genes differentially expressed in benign versus malignant thyroid tumors. Clin Cancer Res 14:33273337.

179. Lappinga PJ, Kip NS, Jin L, Lloyd RV, Henry MR, Zhang J, Nassar A 2010 HMGA2 gene expression analysis performed on cytologic smears to distinguish benign from malignant thyroid nodules. Cancer Cytopathol 118:287-297.

180. Chen YT, Kitabayashi N, Zhou XK, Fahey TJ III, Scognamiglio T 2008 MicroRNA analysis as a potential diagnostic tool for papillary thyroid carcinoma. Mod Pathol 21:1139-1146.

181. Nikiforova MN, Tseng GC, Steward D, Diorio D, Nikiforov YE 2008 MicroRNA expression profiling of thyroid tumors: biological significance and diagnostic utility. J Clin Endocrinol Metab 93:1600-1608.
182. Mazeh H, Mizrahi I, Halle D, Ilyayev N, Stojadinovic A, Trink B, Mitrani-Rosenbaum S, Roistacher M, Ariel I, Eid A, Freund HR, Nissan A 2011 Development of a microRNA-based molecular assay for the detection of papillary thyroid carcinoma in aspiration biopsy samples. Thyroid 21:111-118.

183. Shen R, Liyanarachchi S, Li W, Wakely PE Jr, Saji M, Huang J, Nagy R, Farrell T, Ringel MD, de la Chapelle A, Kloos RT, He H 2012 MicroRNA signature in thyroid fine needle aspiration cytology applied to "atypia of undetermined significance" cases. Thyroid 22:9-16.

184. Keutgen XM, Filicori F, Crowley MJ, Wang Y, Scognamiglio T, Hoda R, Buitrago D, Cooper D, Zeiger MA, Zarnegar R, Elemento O, Fahey TJ III $2012 \mathrm{~A}$ panel of four miRNAs accurately differentiates malignant from benign indeterminate thyroid lesions on fine needle aspiration. Clin Cancer Res 18:2032-2038.

185. Agretti P, Ferrarini E, Rago T, Candelieri A, De Marco G, Dimida A, Niccolai F, Molinaro A, Di Coscio G, Pinchera A, Vitti P, Tonacchera M 2012 MicroRNA expression profile helps to distinguish benign nodules from papillary thyroid carcinomas starting from cells of fine-needle aspiration. Eur $\mathbf{J}$ Endocrinol 167:393-400.

186. Labourier E, Shifrin A, Busseniers AE, Lupo MA, Manganelli ML, Andruss B, Wylie D, Beaudenon-Huibregtse S 2015 Molecular testing for miRNA, mRNA, and DNA on fine-needle aspiration improves the preoperative diagnosis of thyroid nodules with indeterminate cytology. $\mathrm{J}$ Clin Endocrinol Metab 100:2743-2750.

187. Milas M, Shin J, Gupta M, Novosel T, Nasr C, Brainard J, Mitchell J, Berber E, Siperstein A 2010 Circulating thyrotropin receptor mRNA as a novel marker of thyroid cancer: clinical applications learned from 1758 samples. Ann Surg 252:643-651.

188. McGovern MM, Benach MO, Wallenstein S, Desnick RJ, Keenlyside R 1999 Quality assurance in molecular genetic testing laboratories. JAMA 281:835-840.

189. McGovern MM, Elles R, Beretta I, Somerville MJ, Hoefler G, Keinanen M, Barton D, Carson N, Dequeker E, Brdicka R, Blazkova A, Ayme S, Schnieders B, Muller CR, Dalen V, Martinez AA, Kristoffersson U, Ozguc M, Mueller H, Boone J, Lubin IM, Sequeiros J, Taruscio D, Williamson B, Mainland L, Yoshikura H, Ronchi E 2007 Report of an international survey of molecular genetic testing laboratories. Community Genet 10:123-131.

190. Krane JF, Nayar R, Renshaw AA 2010 Atypia of undetermined significance/follicular lesion of undetermined significance. In: Ali SZ, Cibas ES (eds) The Bethesda System for Reporting Thyroid Cytopathology. Springer, pp 37-49.

191. Bongiovanni M, Crippa S, Baloch Z, Piana S, Spitale A, Pagni F, Mazzucchelli L, Di BC, Faquin W 2012 Comparison of 5-tiered and 6-tiered diagnostic systems for the reporting of thyroid cytopathology: a multiinstitutional study. Cancer Cytopathol 120:117-125.

192. Davidov T, Trooskin SZ, Shanker BA, Yip D, Eng O, Crystal J, Hu J, Chernyavsky VS, Deen MF, May M, Artymyshyn RL 2010 Routine second-opinion cytopathology review of thyroid fine needle aspiration biopsies reduces diagnostic thyroidectomy. Surgery 148: 1294-1299. 
193. Baloch Z, LiVolsi VA, Jain P, Jain R, Aljada I, Mandel S, Langer JE, Gupta PK 2003 Role of repeat fine-needle aspiration biopsy (FNAB) in the management of thyroid nodules. Diagn Cytopathol 29:203-206.

194. Yassa L, Cibas ES, Benson CB, Frates MC, Doubilet PM, Gawande AA, Moore FD Jr, Kim BW, Nose V, Marqusee E, Larsen PR, Alexander EK 2007 Long-term assessment of a multidisciplinary approach to thyroid nodule diagnostic evaluation. Cancer 111:508-516.

195. Yang J, Schnadig V, Logrono R, Wasserman PG 2007 Fine-needle aspiration of thyroid nodules: a study of 4703 patients with histologic and clinical correlations. Cancer 111:306-315.

196. Vanderlaan PA, Marqusee E, Krane JF 2011 Clinical outcome for atypia of undetermined significance in thyroid fine-needle aspirations: should repeated fna be the preferred initial approach? Am J Clin Pathol 135:770-775.

197. Nasrollah N, Trimboli P, Rossi F, Amendola S, Guidobaldi L, Ventura C, Maglio R, Nigri G, Romanelli F, Valabrega S, Crescenzi A 2014 Patient's comfort with and tolerability of thyroid core needle biopsy. Endocrine 45:79-83.

198. Kim SK, Hwang TS, Yoo YB, Han HS, Kim DL, Song KH, Lim SD, Kim WS, Paik NS 2011 Surgical results of thyroid nodules according to a management guideline based on the BRAF(V600E) mutation status. J Clin Endocrinol Metab 96:658-664.

199. Adeniran AJ, Hui P, Chhieng DC, Prasad ML, Schofield $\mathrm{K}$, Theoharis C 2011 BRAF mutation testing of thyroid fine-needle aspiration specimens enhances the predictability of malignancy in thyroid follicular lesions of undetermined significance. Acta Cytol 55:570-575.

200. Lastra RR, Pramick MR, Crammer CJ, LiVolsi VA, Baloch ZW 2014 Implications of a suspicious afirma test result in thyroid fine-needle aspiration cytology: an institutional experience. Cancer Cytopathol 122:737-744.

201. Jeong SH, Hong HS, Lee EH, Cha JG, Park JS, Kwak JJ 2013 Outcome of thyroid nodules characterized as atypia of undetermined significance or follicular lesion of undetermined significance and correlation with ultrasound features and BRAF(V600E) mutation analysis. AJR Am J Roentgenol 201:W854-W860.

202. Yoo WS, Choi HS, Cho SW, Moon JH, Kim KW, Park HJ, Park SY, Choi SI, Choi SH, Lim S, Yi KH, Park do J, Jang HC, Park YJ 2014 The role of ultrasound findings in the management of thyroid nodules with atypia or follicular lesions of undetermined significance. Clin Endocrinol (Oxf) 80:735-742.

203. Gweon HM, Son EJ, Youk JH, Kim JA 2013 Thyroid nodules with Bethesda system III cytology: can ultrasonography guide the next step? Ann Surg Oncol 20:30833088.

204. Kim DW, Lee EJ, Jung SJ, Ryu JH, Kim YM 2011 Role of sonographic diagnosis in managing Bethesda class III nodules. AJNR Am J Neuroradiol 32:2136-2141.

205. Rosario PW 2014 Thyroid nodules with atypia or follicular lesions of undetermined significance (Bethesda Category III): importance of ultrasonography and cytological subcategory. Thyroid 24:1115-1120.

206. Henry MR, DeMay RM, Berezowski K 2010 Follicular neoplasm/suspicious for a follicular neoplasm. In: Ali SZ, Cibas ES (eds) The Bethesda System for Reporting Thyroid Cytopathology. Springer, pp 51-58.

207. Faquin WC, Michael CW, Renshaw AA 2010 Follicular neoplasm, Hürthle cell type/suspicious for a follicular neoplasm, Hürthle cell type. In: Ali SZ, Cibas ES (eds) The Bethesda System for Reporting Thyroid Cytopathology. Springer, pp 59-73.

208. Gupta N, Dasyam AK, Carty SE, Nikiforova MN, Ohori NP, Armstrong M, Yip L, LeBeau SO, McCoy KL, Coyne C, Stang MT, Johnson J, Ferris RL, Seethala R, Nikiforov YE, Hodak SP 2013 RAS mutations in thyroid FNA specimens are highly predictive of predominantly low-risk follicular-pattern cancers. J Clin Endocrinol Metab 98:E914-E922.

209. Wang HH, Filie AC, Clark DP 2010 Suspicious for malignancy. In: Ali SZ, Cibas ES (eds) The Bethesda System for Reporting Thyroid Cytopathology. Springer, pp 75-89.

210. Moon HJ, Kwak JY, Kim EK, Choi JR, Hong SW, Kim MJ, Son EJ 2009 The role of BRAFV600E mutation and ultrasonography for the surgical management of a thyroid nodule suspicious for papillary thyroid carcinoma on cytology. Ann Surg Oncol 16:3125-3131.

211. Adeniran AJ, Theoharis C, Hui P, Prasad ML, Hammers L, Carling T, Udelsman R, Chhieng DC 2011 Reflex BRAF testing in thyroid fine-needle aspiration biopsy with equivocal and positive interpretation: a prospective study. Thyroid 21:717-723.

212. Jara SM, Bhatnagar R, Guan H, Gocke CD, Ali SZ, Tufano RP 2014 Utility of BRAF mutation detection in fine-needle aspiration biopsy samples read as "suspicious for papillary thyroid carcinoma". Head Neck doi: 10.1002/hed.23829. [Epub ahead of print].

213. de Geus-Oei LF, Pieters GF, Bonenkamp JJ, Mudde AH, Bleeker-Rovers CP, Corstens FH, Oyen WJ 2006 18FFDG PET reduces unnecessary hemithyroidectomies for thyroid nodules with inconclusive cytologic results. J Nucl Med 47:770-775.

214. Kresnik E, Gallowitsch HJ, Mikosch P, Stettner H, Igerc I, Gomez I, Kumnig G, Lind P 2003 Fluorine-18fluorodeoxyglucose positron emission tomography in the preoperative assessment of thyroid nodules in an endemic goiter area. Surgery 133:294-299.

215. Kim JM, Ryu JS, Kim TY, Kim WB, Kwon GY, Gong G, Moon DH, Kim SC, Hong SJ, Shong YK 2007 18Ffluorodeoxyglucose positron emission tomography does not predict malignancy in thyroid nodules cytologically diagnosed as follicular neoplasm. J Clin Endocrinol Metab 92:1630-1634.

216. Sebastianes FM, Cerci JJ, Zanoni PH, Soares J Jr, Chibana LK, Tomimori EK, de Camargo RY, Izaki M, Giorgi MC, Eluf-Neto J, Meneghetti JC, Pereira MA 2007 Role of 18F-fluorodeoxyglucose positron emission tomography in preoperative assessment of cytologically indeterminate thyroid nodules. J Clin Endocrinol Metab 92:4485-4488.

217. Hales NW, Krempl GA, Medina JE 2008 Is there a role for fluorodeoxyglucose positron emission tomography/ computed tomography in cytologically indeterminate thyroid nodules? Am J Otolaryngol 29:113-118.

218. Traugott AL, Dehdashti F, Trinkaus K, Cohen M, Fialkowski E, Quayle F, Hussain H, Davila R, Ylagan L, Moley JF 2010 Exclusion of malignancy in thyroid nodules with indeterminate fine-needle aspiration cytology after negative 18 F-fluorodeoxyglucose positron emission tomography: interim analysis. World J Surg 34:1247-1253.

219. Smith RB, Robinson RA, Hoffman HT, Graham MM 2008 Preoperative FDG-PET imaging to assess the ma- 
lignant potential of follicular neoplasms of the thyroid. Otolaryngol Head Neck Surg 138:101-106.

220. Deandreis D, Al Ghuzlan A, Auperin A, Vielh P, Caillou B, Chami L, Lumbroso J, Travagli JP, Hartl D, Baudin E, Schlumberger M, Leboulleux S 2012 Is (18)Ffluorodeoxyglucose-PET/CT useful for the presurgical characterization of thyroid nodules with indeterminate fine needle aspiration cytology? Thyroid 22:165-172.

221. Vriens D, de Wilt JH, van der Wilt GJ, Netea-Maier RT, Oyen WJ, de Geus-Oei LF 2011 The role of [18F]-2fluoro-2-deoxy-d-glucose-positron emission tomography in thyroid nodules with indeterminate fine-needle aspiration biopsy: systematic review and meta-analysis of the literature. Cancer 117:4582-4594.

222. Wang N, Zhai H, Lu Y 2013 Is fluorine-18 fluorodeoxyglucose positron emission tomography useful for the thyroid nodules with indeterminate fine needle aspiration biopsy? A meta-analysis of the literature. J Otolaryngol Head Neck Surg 42:38-45.

223. Vriens D, Adang EM, Netea-Maier RT, Smit JW, de Wilt JH, Oyen WJ, de Geus-Oei LF 2014 Cost-effectiveness of FDG-PET/CT for cytologically indeterminate thyroid nodules: a decision analytic approach. J Clin Endocrinol Metab 99:3263-3274.

224. Duren M, Yavuz N, Bukey Y, Ozyegin MA, Gundogdu S, Acbay O, Hatemi H, Uslu I, Onsel C, Aksoy F, Oz F, Unal G, Duren E 2000 Impact of initial surgical treatment on survival of patients with differentiated thyroid cancer: experience of an endocrine surgery center in an iodine-deficient region. World J Surg 24:1290-1294.

225. Stojadinovic A, Peoples GE, Libutti SK, Henry LR, Eberhardt J, Howard RS, Gur D, Elster EA, Nissan A 2009 Development of a clinical decision model for thyroid nodules. BMC Surg 9:12.

226. Tuttle RM, Lemar H, Burch HB 1998 Clinical features associated with an increased risk of thyroid malignancy in patients with follicular neoplasia by fine-needle aspiration. Thyroid 8:377-383.

227. Goldstein RE, Netterville JL, Burkey B, Johnson JE 2002 Implications of follicular neoplasms, atypia, and lesions suspicious for malignancy diagnosed by fine-needle aspiration of thyroid nodules. Ann Surg 235:656-662.

228. Schlinkert RT, van Heerden JA, Goellner JR, Gharib H, Smith SL, Rosales RF, Weaver AL 1997 Factors that predict malignant thyroid lesions when fine-needle aspiration is "suspicious for follicular neoplasm". Mayo Clin Proc 72:913-916.

229. Mehta RS, Carty SE, Ohori NP, Hodak SP, Coyne C, LeBeau SO, Tublin ME, Stang MT, Johnson JT, McCoy KL, Nikiforova MN, Nikiforov YE, Yip L 2013 Nodule size is an independent predictor of malignancy in mutation-negative nodules with follicular lesion of undetermined significance cytology. Surgery 154:730-736.

230. McCoy KL, Carty SE, Armstrong MJ, Seethala RR, Ohori NP, Kabaker AS, Stang MT, Hodak SP, Nikiforov YE, Yip L 2012 Intraoperative pathologic examination in the era of molecular testing for differentiated thyroid cancer. J Am Coll Surg 215:546-554.

231. Kandil E, Krishnan B, Noureldine SI, Yao L, Tufano RP 2013 Hemithyroidectomy: a meta-analysis of postoperative need for hormone replacement and complications. ORL J Otorhinolaryngol Relat Spec 75:6-17.

232. Sosa JA, Bowman HM, Tielsch JM, Powe NR, Gordon TA, Udelsman R 1998 The importance of surgeon ex- perience for clinical and economic outcomes from thyroidectomy. Ann Surg 228:320-330.

233. Loyo M, Tufano RP, Gourin CG 2013 National trends in thyroid surgery and the effect of volume on short-term outcomes. Laryngoscope 123:2056-2063.

234. Verloop H, Louwerens M, Schoones JW, Kievit J, Smit JW, Dekkers OM 2012 Risk of hypothyroidism following hemithyroidectomy: systematic review and metaanalysis of prognostic studies. J Clin Endocrinol Metab 97:2243-2255.

235. Brito JP, Yarur AJ, Prokop LJ, McIver B, Murad MH, Montori VM 2013 Prevalence of thyroid cancer in multinodular goiter versus single nodule: a systematic review and meta-analysis. Thyroid 23:449-455.

236. Kwak JY, Koo H, Youk JH, Kim MJ, Moon HJ, Son EJ, Kim EK 2010 Value of US correlation of a thyroid nodule with initially benign cytologic results. Radiology 254:292-300.

237. Brauer VF, Eder P, Miehle K, Wiesner TD, Hasenclever $\mathrm{H}$, Paschke R 2005 Interobserver variation for ultrasound determination of thyroid nodule volumes. Thyroid 15: 1169-1175.

238. Alexander EK, Hurwitz S, Heering JP, Benson CB, Frates MC, Doubilet PM, Cibas ES, Larsen PR, Marqusee E 2003 Natural history of benign solid and cystic thyroid nodules. Ann Intern Med 138:315-318.

239. Papini E, Petrucci L, Guglielmi R, Panunzi C, Rinaldi R, Bacci V, Crescenzi A, Nardi F, Fabbrini R, Pacella CM 1998 Long-term changes in nodular goiter: a 5-year prospective randomized trial of levothyroxine suppressive therapy for benign cold thyroid nodules. J Clin Endocrinol Metab 83:780-783.

240. Erdogan MF, Gursoy A, Erdogan G 2006 Natural course of benign thyroid nodules in a moderately iodinedeficient area. Clin Endocrinol (Oxf) 65:767-771.

241. Durante C, Costante G, Lucisano G, Bruno R, Meringolo D, Paciaroni A, Puxeddu E, Torlontano M, Tumino S, Attard M, Lamartina L, Nicolucci A, Filetti S 2015 The natural history of benign thyroid nodules. JAMA 313: 926-935.

242. Rosario PW, Purisch S 2010 Ultrasonographic characteristics as a criterion for repeat cytology in benign thyroid nodules. Arq Bras Endocrinol Metabol 54: 52-55.

243. Zelmanovitz F, Genro S, Gross JL 1998 Suppressive therapy with levothyroxine for solitary thyroid nodules: a double-blind controlled clinical study and cumulative meta-analyses. J Clin Endocrinol Metab 83:3881-3885.

244. Wemeau JL, Caron P, Schvartz C, Schlienger JL, Orgiazzi J, Cousty C, Vlaeminck-Guillem V 2002 Effects of thyroid-stimulating hormone suppression with levothyroxine in reducing the volume of solitary thyroid nodules and improving extranodular nonpalpable changes: a randomized, double-blind, placebo-controlled trial by the French Thyroid Research Group. J Clin Endocrinol Metab 87:4928-4934.

245. Castro MR, Caraballo PJ, Morris JC 2002 Effectiveness of thyroid hormone suppressive therapy in benign solitary thyroid nodules: a meta-analysis. J Clin Endocrinol Metab 87:4154-4159.

246. Yousef A, Clark J, Doi SA 2010 Thyroxine suppression therapy for benign, non-functioning solitary thyroid nodules: a quality-effects meta-analysis. Clin Med Res 8:150-158. 
247. Sdano MT, Falciglia M, Welge JA, Steward DL 2005 Efficacy of thyroid hormone suppression for benign thyroid nodules: meta-analysis of randomized trials. Otolaryngol Head Neck Surg 133:391-396.

248. Grussendorf M, Reiners C, Paschke R, Wegscheider K 2011 Reduction of thyroid nodule volume by levothyroxine and iodine alone and in combination: a randomized, placebo-controlled trial. J Clin Endocrinol Metab 96:2786-2795.

249. Puzziello A, Carrano M, Angrisani E, Marotta V, Faggiano A, Zeppa P, Vitale M 2014 Evolution of benign thyroid nodules under levothyroxine non-suppressive therapy. J Endocrinol Invest 37:1181-1186.

250. Bennedbaek FN, Hegedus L 2003 Treatment of recurrent thyroid cysts with ethanol: a randomized double-blind controlled trial. J Clin Endocrinol Metab 88:5773-5777.

251. Valcavi R, Frasoldati A 2004 Ultrasound-guided percutaneous ethanol injection therapy in thyroid cystic nodules. Endocr Pract 10:269-275.

252. Antonelli A, Campatelli A, Di VA, Alberti B, Baldi V, Salvioni G, Fallahi P, Baschieri L 1994 Comparison between ethanol sclerotherapy and emptying with injection of saline in treatment of thyroid cysts. Clin Investig 72:971-974.

253. Verde G, Papini E, Pacella CM, Gallotti C, Delpiano S, Strada S, Fabbrini R, Bizzarri G, Rinaldi R, Panunzi C 1994 Ultrasound guided percutaneous ethanol injection in the treatment of cystic thyroid nodules. Clin Endocrinol (Oxf) 41:719-724.

254. Chen AY, Bernet VJ, Carty SE, Davies TF, Ganly I, Inabnet WB III, Shaha AR 2014 American Thyroid Association statement on optimal surgical management of goiter. Thyroid 24:181-189.

255. Shin JJ, Caragacianu D, Randolph GW 2015 Impact of thyroid nodule size on prevalence and post-test probability of malignancy: a systematic review. Laryngoscope 125:263-272.

256. Marley EF, Oertel YC 1997 Fine-needle aspiration of thyroid lesions in 57 pregnant and postpartum women. Diagn Cytopathol 16:122-125.

257. Kung AW, Chau MT, Lao TT, Tam SC, Low LC 2002 The effect of pregnancy on thyroid nodule formation. $\mathrm{J}$ Clin Endocrinol Metab 87:1010-1014.

258. Moosa M, Mazzaferri EL 1997 Outcome of differentiated thyroid cancer diagnosed in pregnant women. J Clin Endocrinol Metab 82:2862-2866.

259. Rosen IB, Korman M, Walfish PG 1997 Thyroid nodular disease in pregnancy: current diagnosis and management. Clin Obstet Gynecol 40:81-89.

260. McLeod DS, Watters KF, Carpenter AD, Ladenson PW, Cooper DS, Ding EL 2012 Thyrotropin and thyroid cancer diagnosis: a systematic review and doseresponse meta-analysis. J Clin Endocrinol Metab 97: 2682-2692.

261. Karger S, Schotz S, Stumvoll M, Berger F, Fuhrer D 2010 Impact of pregnancy on prevalence of goitre and nodular thyroid disease in women living in a region of borderline sufficient iodine supply. Horm Metab Res 42: 137-142.

262. Messuti I, Corvisieri S, Bardesono F, Rapa I, Giorcelli J, Pellerito R, Volante M, Orlandi F 2014 Impact of pregnancy on prognosis of differentiated thyroid cancer: clinical and molecular features. Eur J Endocrinol 170: 659-666.
263. Vannucchi G, Perrino M, Rossi S, Colombo C, Vicentini L, Dazzi D, Beck-Peccoz P, Fugazzola L 2010 Clinical and molecular features of differentiated thyroid cancer diagnosed during pregnancy. Eur J Endocrinol 162:145-151.

264. Mestman JH, Goodwin TM, Montoro MM 1995 Thyroid disorders of pregnancy. Endocrinol Metab Clin North Am 24:41-71.

265. Herzon FS, Morris DM, Segal MN, Rauch G, Parnell T 1994 Coexistent thyroid cancer and pregnancy. Arch Otolaryngol Head Neck Surg 120:1191-1193.

266. Mazzaferri EL, Jhiang SM 1994 Long-term impact of initial surgical and medical therapy on papillary and follicular thyroid cancer. Am J Med 97:418-428.

267. Kuy S, Roman SA, Desai R, Sosa JA 2009 Outcomes following thyroid and parathyroid surgery in pregnant women. Arch Surg 144:399-406.

268. Aschebrook-Kilfoy B, Ward MH, Sabra MM, Devesa SS 2011 Thyroid cancer incidence patterns in the United States by histologic type, 1992-2006. Thyroid 21:125-134.

269. Hundahl SA, Fleming ID, Fremgen AM, Menck HR 1998 A National Cancer Data Base report on 53,856 cases of thyroid carcinoma treated in the U.S., 19851995 [see commetns]. Cancer 83:2638-2648.

270. Hay ID, Bergstralh EJ, Goellner JR, Ebersold JR, Grant CS 1993 Predicting outcome in papillary thyroid carcinoma: development of a reliable prognostic scoring system in a cohort of 1779 patients surgically treated at one institution during 1940 through 1989. Surgery 114: 1050-1057.

271. Shah MD, Hall FT, Eski SJ, Witterick IJ, Walfish PG, Freeman JL 2003 Clinical course of thyroid carcinoma after neck dissection. Laryngoscope 113:2102-2107.

272. Wang TS, Dubner S, Sznyter LA, Heller KS 2004 Incidence of metastatic well-differentiated thyroid cancer in cervical lymph nodes. Arch Otolaryngol Head Neck Surg 130:110-113.

273. Mazzaferri EL 1999 An overview of the management of papillary and follicular thyroid carcinoma. Thyroid 9:421-427.

274. Mazzaferri EL 2000 Long-term outcome of patients with differentiated thyroid carcinoma: effect of therapy. Endocr Pract 6:469-476.

275. Cooper DS, Specker B, Ho M, Sperling M, Ladenson PW, Ross DS, Ain KB, Bigos ST, Brierley JD, Haugen BR, Klein I, Robbins J, Sherman SI, Taylor T, Maxon HR III 1998 Thyrotropin suppression and disease progression in patients with differentiated thyroid cancer: results from the National Thyroid Cancer Treatment Cooperative Registry. Thyroid 8:737-744.

276. Lin JD, Chao TC, Huang MJ, Weng HF, Tzen KY 1998 Use of radioactive iodine for thyroid remnant ablation in well-differentiated thyroid carcinoma to replace thyroid reoperation. Am J Clin Oncol 21:77-81.

277. Brierley JD, Panzarella T, Tsang RW, Gospodarowicz MK, O'Sullivan B 1997 A comparison of different staging systems predictability of patient outcome. Thyroid carcinoma as an example. Cancer 79:2414-2423.

278. Hay ID, Thompson GB, Grant CS, Bergstralh EJ, Dvorak CE, Gorman CA, Maurer MS, McIver B, Mullan BP, Oberg AL, Powell CC, van Heerden JA, Goellner JR 2002 Papillary thyroid carcinoma managed at the Mayo Clinic during six decades (1940-1999): temporal trends in initial therapy and long-term outcome in 2444 consecutively treated patients. World J Surg 26:879-885. 
279. Gourin CG, Tufano RP, Forastiere AA, Koch WM, Pawlik TM, Bristow RE 2010 Volume-based trends in thyroid surgery. Arch Otolaryngol Head Neck Surg 136:1191-1198.

280. Stavrakis AI, Ituarte PH, Ko CY, Yeh MW 2007 Surgeon volume as a predictor of outcomes in inpatient and outpatient endocrine surgery. Surgery 142:887-899.

281. Grebe SK, Hay ID 1996 Thyroid cancer nodal metastases: biologic significance and therapeutic considerations. Surg Oncol Clin N Am 5:43-63.

282. Scheumann GF, Gimm O, Wegener G, Hundeshagen H, Dralle H 1994 Prognostic significance and surgical management of locoregional lymph node metastases in papillary thyroid cancer. World J Surg 18:559-567.

283. Ito Y, Uruno T, Nakano K, Takamura Y, Miya A, Kobayashi K, Yokozawa T, Matsuzuka F, Kuma S, Kuma K, Miyauchi A 2003 An observation trial without surgical treatment in patients with papillary microcarcinoma of the thyroid. Thyroid 13:381-387.

284. Hay ID, Grant CS, van Heerden JA, Goellner JR, Ebersold JR, Bergstralh EJ 1992 Papillary thyroid microcarcinoma: a study of 535 cases observed in a 50-year period. Surgery 112:1139-1146.

285. Qubain SW, Nakano S, Baba M, Takao S, Aikou T 2002 Distribution of lymph node micrometastasis in pN0 well-differentiated thyroid carcinoma. Surgery 131:249256.

286. Arturi F, Russo D, Giuffrida D, Ippolito A, Perrotti N, Vigneri R, Filetti S 1997 Early diagnosis by genetic analysis of differentiated thyroid cancer metastases in small lymph nodes. J Clin Endocrinol Metab 82:1638-1641.

287. Solorzano CC, Carneiro DM, Ramirez M, Lee TM, Irvin GL, III 2004 Surgeon-performed ultrasound in the management of thyroid malignancy. Am Surg 70:576-580.

288. Shimamoto K, Satake H, Sawaki A, Ishigaki T, Funahashi H, Imai T 1998 Preoperative staging of thyroid papillary carcinoma with ultrasonography. Eur J Radiol 29:4-10.

289. Stulak JM, Grant CS, Farley DR, Thompson GB, van Heerden JA, Hay ID, Reading CC, Charboneau JW 2006 Value of preoperative ultrasonography in the surgical management of initial and reoperative papillary thyroid cancer. Arch Surg 141:489-494.

290. Kouvaraki MA, Shapiro SE, Fornage BD, EdeikenMonro BS, Sherman SI, Vassilopoulou-Sellin R, Lee JE, Evans DB 2003 Role of preoperative ultrasonography in the surgical management of patients with thyroid cancer. Surgery 134:946-954.

291. O'Connell K, Yen TW, Quiroz F, Evans DB, Wang TS 2013 The utility of routine preoperative cervical ultrasonography in patients undergoing thyroidectomy for differentiated thyroid cancer. Surgery 154:697-701.

292. Leboulleux S, Girard E, Rose M, Travagli JP, Sabbah N, Caillou B, Hartl DM, Lassau N, Baudin E, Schlumberger M 2007 Ultrasound criteria of malignancy for cervical lymph nodes in patients followed up for differentiated thyroid cancer. J Clin Endocrinol Metab 92:3590-3594.

293. Frasoldati A, Valcavi R 2004 Challenges in neck ultrasonography: lymphadenopathy and parathyroid glands. Endocr Pract 10:261-268.

294. Kuna SK, Bracic I, Tesic V, Kuna K, Herceg GH, Dodig D 2006 Ultrasonographic differentiation of benign from malignant neck lymphadenopathy in thyroid cancer. J Ultrasound Med 25:1531-1537.
295. Park JH, Lee YS, Kim BW, Chang HS, Park CS 2012 Skip lateral neck node metastases in papillary thyroid carcinoma. World J Surg 36:743-747.

296. Snozek CL, Chambers EP, Reading CC, Sebo TJ, Sistrunk JW, Singh RJ, Grebe SK 2007 Serum thyroglobulin, high-resolution ultrasound, and lymph node thyroglobulin in diagnosis of differentiated thyroid carcinoma nodal metastases. J Clin Endocrinol Metab 92: 4278-4281.

297. Frasoldati A, Pesenti M, Gallo M, Caroggio A, Salvo D, Valcavi R 2003 Diagnosis of neck recurrences in patients with differentiated thyroid carcinoma. Cancer 97:90-96.

298. Boi F, Baghino G, Atzeni F, Lai ML, Faa G, Mariotti S 2006 The diagnostic value for differentiated thyroid carcinoma metastases of thyroglobulin $(\mathrm{Tg})$ measurement in washout fluid from fine-needle aspiration biopsy of neck lymph nodes is maintained in the presence of circulating anti-Tg antibodies. J Clin Endocrinol Metab 91:1364-1369.

299. Jeon MJ, Park JW, Han JM, Yim JH, Song DE, Gong G, Kim TY, Baek JH, Lee JH, Shong YK, Kim WB 2013 Serum antithyroglobulin antibodies interfere with thyroglobulin detection in fine-needle aspirates of metastatic neck nodes in papillary thyroid carcinoma. J Clin Endocrinol Metab 98:153-160.

300. Chung J, Kim EK, Lim H, Son EJ, Yoon JH, Youk JH, Kim JA, Moon HJ, Kwak JY 2014 Optimal indication of thyroglobulin measurement in fine-needle aspiration for detecting lateral metastatic lymph nodes in patients with papillary thyroid carcinoma. Head Neck 36:795-801.

301. Grani G, Fumarola A 2014 Thyroglobulin in lymph node fine-needle aspiration wash-out: a systematic review and meta-analysis of diagnostic accuracy. J Clin Endocrinol Metab 99:1970-1982.

302. Pak K, Suh S, Hong H, Cheon GJ, Hahn SK, Kang KW, Kim EE, Lee DS, Chung JK 2015 Diagnostic values of thyroglobulin measurement in fine-needle aspiration of lymph nodes in patients with thyroid cancer. Endocrine 49:70-77.

303. Pacini F, Fugazzola L, Lippi F, Ceccarelli C, Centoni R, Miccoli P, Elisei R, Pinchera A 1992 Detection of thyroglobulin in fine needle aspirates of nonthyroidal neck masses: a clue to the diagnosis of metastatic differentiated thyroid cancer. J Clin Endocrinol Metab 74:1401-1404.

304. Moon JH, Kim YI, Lim JA, Choi HS, Cho SW, Kim KW, Park HJ, Paeng JC, Park YJ, Yi KH, Park do J, Kim SE, Chung JK 2013 Thyroglobulin in washout fluid from lymph node fine-needle aspiration biopsy in papillary thyroid cancer: large-scale validation of the cutoff value to determine malignancy and evaluation of discrepant results. J Clin Endocrinol Metab 98:1061-1068.

305. Giovanella L, Bongiovanni M, Trimboli P 2013 Diagnostic value of thyroglobulin assay in cervical lymph node fine-needle aspirations for metastatic differentiated thyroid cancer. Curr Opin Oncol 25:6-13.

306. Stephenson BM, Wheeler MH, Clark OH 1994 The role of total thyroidectomy in the management of differentiated thyroid cancer. Curr Opin Gen Surg 2:53-59.

307. Ahn JE, Lee JH, Yi JS, Shong YK, Hong SJ, Lee DH, Choi CG, Kim SJ 2008 Diagnostic accuracy of CT and ultrasonography for evaluating metastatic cervical lymph nodes in patients with thyroid cancer. World J Surg 32: $1552-1558$. 
308. Choi JS, Kim J, Kwak JY, Kim MJ, Chang HS, Kim EK 2009 Preoperative staging of papillary thyroid carcinoma: comparison of ultrasound imaging and CT. AJR Am J Roentgenol 193:871-878.

309. Lesnik D, Cunnane ME, Zurakowski D, Acar GO, Ecevit C, Mace A, Kamani D, Randolph GW 2014 Papillary thyroid carcinoma nodal surgery directed by a preoperative radiographic map utilizing $\mathrm{CT}$ scan and ultrasound in all primary and reoperative patients. Head Neck 36:191-202.

310. Jeong HS, Baek CH, Son YI, Choi JY, Kim HJ, Ko YH, Chung JH, Baek HJ 2006 Integrated 18F-FDG PET/CT for the initial evaluation of cervical node level of patients with papillary thyroid carcinoma: comparison with ultrasound and contrast-enhanced CT. Clin Endocrinol (Oxf) 65:402-407.

311. Kaplan SL, Mandel SJ, Muller R, Baloch ZW, Thaler ER, Loevner LA 2009 The role of MR imaging in detecting nodal disease in thyroidectomy patients with rising thyroglobulin levels. AJNR Am J Neuroradiol 30:608-612.

312. Andersen PE, Kinsella J, Loree TR, Shaha AR, Shah JP 1995 Differentiated carcinoma of the thyroid with extrathyroidal extension. Am J Surg 170:467-470.

313. Kim E, Park JS, Son KR, Kim JH, Jeon SJ, Na DG 2008 Preoperative diagnosis of cervical metastatic lymph nodes in papillary thyroid carcinoma: comparison of ultrasound, computed tomography, and combined ultrasound with computed tomography. Thyroid 18:411-418.

314. Yeh MW, Bauer AJ, Bernet VA, Ferris RL, Loevner LA, Mandel SJ, Orloff LA, Randolph GW, Steward DL 2015 American Thyroid Association statement on preoperative imaging for thyroid cancer surgery. Thyroid 25:3-14.

315. Padovani RP, Kasamatsu TS, Nakabashi CC, Camacho CP, Andreoni DM, Malouf EZ, Marone MM, Maciel RM, Biscolla RP 2012 One month is sufficient for urinary iodine to return to its baseline value after the use of water-soluble iodinated contrast agents in postthyroidectomy patients requiring radioiodine therapy. Thyroid 22:926-930.

316. Spencer CA, Bergoglio LM, Kazarosyan M, Fatemi S, LoPresti JS 2005 Clinical impact of thyroglobulin (Tg) and $\mathrm{Tg}$ autoantibody method differences on the management of patients with differentiated thyroid carcinomas. J Clin Endocrinol Metab 90:5566-5575.

317. McLeod DS, Cooper DS, Ladenson PW, Ain KB, Brierley JD, Fein HG, Haugen BR, Jonklaas J, Magner J, Ross DS, Skarulis MC, Steward DL, Maxon HR, Sherman SI; for the National Thyroid Cancer Treatment Cooperative Study Group 2014 Prognosis of differentiated thyroid cancer in relation to serum thyrotropin and thyroglobulin antibody status at time of diagnosis. Thyroid 24:35-42.

318. Bilimoria KY, Bentrem DJ, Ko CY, Stewart AK, Winchester DP, Talamonti MS, Sturgeon C 2007 Extent of surgery affects survival for papillary thyroid cancer. Ann Surg 246:375-381.

319. Grant CS, Hay ID, Gough IR, Bergstralh EJ, Goellner JR, McConahey WM 1988 Local recurrence in papillary thyroid carcinoma: is extent of surgical resection important? Surgery 104:954-962.

320. Hay ID, Grant CS, Bergstralh EJ, Thompson GB, van Heerden JA, Goellner JR 1998 Unilateral total lobectomy: is it sufficient surgical treatment for patients with AMES low-risk papillary thyroid carcinoma? Surgery 124:958-964.
321. Mazzaferri EL, Kloos RT 2001 Clinical review 128: Current approaches to primary therapy for papillary and follicular thyroid cancer. J Clin Endocrinol Metab 86: 1447-1463.

322. Matsuzu K, Sugino K, Masudo K, Nagahama M, Kitagawa W, Shibuya H, Ohkuwa K, Uruno T, Suzuki A, Magoshi S, Akaishi J, Masaki C, Kawano M, Suganuma N, Rino Y, Masuda M, Kameyama K, Takami H, Ito K 2014 Thyroid lobectomy for papillary thyroid cancer: long-term follow-up study of 1,088 cases. World J Surg 38:68-79.

323. Barney BM, Hitchcock YJ, Sharma P, Shrieve DC, Tward JD 2011 Overall and cause-specific survival for patients undergoing lobectomy, near-total, or total thyroidectomy for differentiated thyroid cancer. Head Neck 33:645-649.

324. Mendelsohn AH, Elashoff DA, Abemayor E, St John MA 2010 Surgery for papillary thyroid carcinoma: is lobectomy enough? Arch Otolaryngol Head Neck Surg 136: $1055-1061$.

325. Haigh PI, Urbach DR, Rotstein LE 2005 Extent of thyroidectomy is not a major determinant of survival in lowor high-risk papillary thyroid cancer. Ann Surg Oncol 12:81-89.

326. Nixon IJ, Ganly I, Patel SG, Palmer FL, Whitcher MM, Tuttle RM, Shaha A, Shah JP 2012 Thyroid lobectomy for treatment of well differentiated intrathyroid malignancy. Surgery 151:571-579.

327. Adam MA, Pura J, Gu L, Dinan MA, Tyler DS, Reed SD, Scheri R, Roman SA, Sosa JA 2014 Extent of surgery for papillary thyroid cancer is not associated with survival: an analysis of 61,775 patients. Ann Surg 260: 601-605.

328. Vaisman F, Shaha A, Fish S, Michael TR 2011 Initial therapy with either thyroid lobectomy or total thyroidectomy without radioactive iodine remnant ablation is associated with very low rates of structural disease recurrence in properly selected patients with differentiated thyroid cancer. Clin Endocrinol (Oxf) 75:112-119.

329. Tuggle CT, Roman S, Udelsman R, Sosa JA 2011 Sameday thyroidectomy: a review of practice patterns and outcomes for 1,168 procedures in New York State. Ann Surg Oncol 18:1035-1040.

330. Tuggle CT, Roman SA, Wang TS, Boudourakis L, Thomas DC, Udelsman R, Ann SJ 2008 Pediatric endocrine surgery: who is operating on our children? Surgery 144:869-877.

331. Kandil E, Noureldine SI, Abbas A, Tufano RP 2013 The impact of surgical volume on patient outcomes following thyroid surgery. Surgery 154:1346-1352.

332. Duclos A, Peix JL, Colin C, Kraimps JL, Menegaux F, Pattou F, Sebag F, Touzet S, Bourdy S, Voirin N, Lifante JC 2012 Influence of experience on performance of individual surgeons in thyroid surgery: prospective cross sectional multicentre study. BMJ 344:d8041.

333. Hauch A, Al-Qurayshi Z, Randolph G, Kandil E 2014 Total thyroidectomy is associated with increased risk of complications for low- and high-volume surgeons. Ann Surg Oncol 21:3844-3852.

334. Hughes DT, White ML, Miller BS, Gauger PG, Burney RE, Doherty GM 2010 Influence of prophylactic central lymph node dissection on postoperative thyroglobulin levels and radioiodine treatment in papillary thyroid cancer. Surgery 148:1100-1106. 
335. Randolph GW, Duh QY, Heller KS, LiVolsi VA, Mandel SJ, Steward DL, Tufano RP, Tuttle RM 2012 The prognostic significance of nodal metastases from papillary thyroid carcinoma can be stratified based on the size and number of metastatic lymph nodes, as well as the presence of extranodal extension. Thyroid 22:11441152.

336. Podnos YD, Smith D, Wagman LD, Ellenhorn JD 2005 The implication of lymph node metastasis on survival in patients with well-differentiated thyroid cancer. Am Surg 71:731-734.

337. Zaydfudim V, Feurer ID, Griffin MR, Phay JE 2008 The impact of lymph node involvement on survival in patients with papillary and follicular thyroid carcinoma. Surgery 144:1070-1077.

338. Leboulleux S, Rubino C, Baudin E, Caillou B, Hartl DM, Bidart JM, Travagli JP, Schlumberger M 2005 Prognostic factors for persistent or recurrent disease of papillary thyroid carcinoma with neck lymph node metastases and/or tumor extension beyond the thyroid capsule at initial diagnosis. J Clin Endocrinol Metab 90:5723-5729.

339. Sugitani I, Kasai N, Fujimoto Y, Yanagisawa A 2004 A novel classification system for patients with PTC: addition of the new variables of large $(3 \mathrm{~cm}$ or greater) nodal metastases and reclassification during the follow-up period. Surgery 135:139-148.

340. Adam MA, Pura J, Goffredo P, Dinan MA, Reed SD, Scheri RP, Hyslop T, Roman SA, Sosa JA 2015 Presence and number of lymph node metastases are associated with compromised survival for patients younger than age 45 years with papillary thyroid cancer. J Clin Oncol 33: 2370-2375.

341. Robbins KT, Shaha AR, Medina JE, Califano JA, Wolf GT, Ferlito A, Som PM, Day TA 2008 Consensus statement on the classification and terminology of neck dissection. Arch Otolaryngol Head Neck Surg 134: 536-538.

342. Hwang HS, Orloff LA 2011 Efficacy of preoperative neck ultrasound in the detection of cervical lymph node metastasis from thyroid cancer. Laryngoscope 121: 487-491.

343. Mulla M, Schulte KM 2012 Central cervical lymph node metastases in papillary thyroid cancer: a systematic review of imaging-guided and prophylactic removal of the central compartment. Clin Endocrinol (Oxf) 76:131-136.

344. Hartl DM, Leboulleux S, Al Ghuzlan A, Baudin E, Chami L, Schlumberger M, Travagli JP 2012 Optimization of staging of the neck with prophylactic central and lateral neck dissection for papillary thyroid carcinoma. Ann Surg 255:777-783.

345. Popadich A, Levin O, Lee JC, Smooke-Praw S, Ro K, Fazel M, Arora A, Tolley NS, Palazzo F, Learoyd DL, Sidhu S, Delbridge L, Sywak M, Yeh MW 2011 A multicenter cohort study of total thyroidectomy and routine central lymph node dissection for $\mathrm{cNO}$ papillary thyroid cancer. Surgery 150:1048-1057.

346. Lang BH, Wong KP, Wan KY, Lo CY 2012 Impact of routine unilateral central neck dissection on preablative and postablative stimulated thyroglobulin levels after total thyroidectomy in papillary thyroid carcinoma. Ann Surg Oncol 19:60-67.

347. Wang TS, Evans DB, Fareau GG, Carroll T, Yen TW 2012 Effect of prophylactic central compartment neck dissection on serum thyroglobulin and recommendations for adjuvant radioactive iodine in patients with differentiated thyroid cancer. Ann Surg Oncol 19:4217-4222.

348. Carty SE, Cooper DS, Doherty GM, Duh QY, Kloos RT, Mandel SJ, Randolph GW, Stack BC Jr, Steward DL, Terris DJ, Thompson GB, Tufano RP, Tuttle RM, Udelsman R 2009 Consensus statement on the terminology and classification of central neck dissection for thyroid cancer. Thyroid 19:1153-1158.

349. Chisholm EJ, Kulinskaya E, Tolley NS 2009 Systematic review and meta-analysis of the adverse effects of thyroidectomy combined with central neck dissection as compared with thyroidectomy alone. Laryngoscope 119:1135-1139.

350. Bonnet S, Hartl D, Leboulleux S, Baudin E, Lumbroso JD, Al Ghuzlan A, Chami L, Schlumberger M, Travagli JP 2009 Prophylactic lymph node dissection for papillary thyroid cancer less than $2 \mathrm{~cm}$ : implications for radioiodine treatment. J Clin Endocrinol Metab 94:11621167.

351. Sancho JJ, Lennard TW, Paunovic I, Triponez F, SitgesSerra A 2014 Prophylactic central neck disection in papillary thyroid cancer: a consensus report of the European Society of Endocrine Surgeons (ESES). Langenbecks Arch Surg 399:155-163.

352. Zetoune T, Keutgen X, Buitrago D, Aldailami H, Shao H, Mazumdar M, Fahey TJ III, Zarnegar R 2010 Prophylactic central neck dissection and local recurrence in papillary thyroid cancer: a meta-analysis. Ann Surg Oncol 17:3287-3293.

353. Barczynski M, Konturek A, Stopa M, Nowak W 2013 Prophylactic central neck dissection for papillary thyroid cancer. Br J Surg 100:410-418.

354. Hartl DM, Mamelle E, Borget I, Leboulleux S, Mirghani H, Schlumberger M 2013 Influence of prophylactic neck dissection on rate of retreatment for papillary thyroid carcinoma. World J Surg 37:1951-1958.

355. Sywak M, Cornford L, Roach P, Stalberg P, Sidhu S, Delbridge L 2006 Routine ipsilateral level VI lymphadenectomy reduces postoperative thyroglobulin levels in papillary thyroid cancer. Surgery 140:1000-1005.

356. Laird AM, Gauger PG, Miller BS, Doherty GM 2012 Evaluation of postoperative radioactive iodine scans in patients who underwent prophylactic central lymph node dissection. World J Surg 36:1268-1273.

357. Costa S, Giugliano G, Santoro L, Ywata De Carvalho A, Massaro MA, Gibelli B, De Fiori E, Grosso E, Ansarin M, Calabrese L 2009 Role of prophylactic central neck dissection in cN0 papillary thyroid cancer. Acta Otorhinolaryngol Ital 29:61-69.

358. Ryu IS, Song CI, Choi SH, Roh JL, Nam SY, Kim SY 2014 Lymph node ratio of the central compartment is a significant predictor for locoregional recurrence after prophylactic central neck dissection in patients with thyroid papillary carcinoma. Ann Surg Oncol 21:277-283.

359. Moreno MA, Edeiken-Monroe BS, Siegel ER, Sherman SI, Clayman GL 2012 In papillary thyroid cancer, preoperative central neck ultrasound detects only macroscopic surgical disease, but negative findings predict excellent long-term regional control and survival. Thyroid 22:347-355.

360. Yoo D, Ajmal S, Gowda S, Machan J, Monchik J, Mazzaglia P 2012 Level VI lymph node dissection does not decrease radioiodine uptake in patients undergoing 
radioiodine ablation for differentiated thyroid cancer. World J Surg 36:1255-1261.

361. Roh JL, Park JY, Park CI 2007 Total thyroidectomy plus neck dissection in differentiated papillary thyroid carcinoma patients: pattern of nodal metastasis, morbidity, recurrence, and postoperative levels of serum parathyroid hormone. Ann Surg 245:604-610.

362. Cavicchi O, Piccin O, Caliceti U, De Cataldis A, Pasquali R, Ceroni AR 2007 Transient hypoparathyroidism following thyroidectomy: a prospective study and multivariate analysis of 604 consecutive patients. Otolaryngol Head Neck Surg 137:654-658.

363. Raffaelli M, De Crea C, Sessa L, Giustacchini P, Revelli L, Bellantone C, Lombardi CP 2012 Prospective evaluation of total thyroidectomy versus ipsilateral versus bilateral central neck dissection in patients with clinically node-negative papillary thyroid carcinoma. Surgery 152:957-964.

364. Viola D, Materazzi G, Valerio L, Molinaro E, Agate L, Faviana P, Seccia V, Sensi E, Romei C, Piaggi P, Torregrossa L, Sellari-Franceschini S, Basolo F, Vitti P, Elisei R, Miccoli P 2015 Prophylactic central compartment lymph node dissection in papillary thyroid carcinoma: clinical implications derived from the first prospective randomized controlled single institution study. J Clin Endocrinol Metab 100:1316-1324.

365. Lang BH, Ng SH, Lau LL, Cowling BJ, Wong KP, Wan KY 2013 A systematic review and meta-analysis of prophylactic central neck dissection on short-term locoregional recurrence in papillary thyroid carcinoma after total thyroidectomy. Thyroid 23:1087-1098.

366. Wang TS, Cheung K, Farrokhyar F, Roman SA, Sosa JA 2013 A meta-analysis of the effect of prophylactic central compartment neck dissection on locoregional recurrence rates in patients with papillary thyroid cancer. Ann Surg Oncol 20:3477-3483.

367. Gyorki DE, Untch B, Tuttle RM, Shaha AR 2013 Prophylactic central neck dissection in differentiated thyroid cancer: an assessment of the evidence. Ann Surg Oncol 20:2285-2289.

368. Haymart MR, Banerjee M, Stewart AK, Koenig RJ, Birkmeyer JD, Griggs JJ 2011 Use of radioactive iodine for thyroid cancer. JAMA 306:721-728.

369. Howell GM, Nikiforova MN, Carty SE, Armstrong MJ, Hodak SP, Stang MT, McCoy KL, Nikiforov YE, Yip L $2013 B R A F^{V 600 E}$ mutation independently predicts central compartment lymph node metastasis in patients with papillary thyroid cancer. Ann Surg Oncol 20:47-52.

370. Xing M, Alzahrani AS, Carson KA, Viola D, Elisei R, Bendlova B, Yip L, Mian C, Vianello F, Tuttle RM, Robenshtok E, Fagin JA, Puxeddu E, Fugazzola L, Czarniecka A, Jarzab B, O’Neill CJ, Sywak MS, Lam AK, Riesco-Eizaguirre G, Santisteban P, Nakayama H, Tufano RP, Pai SI, Zeiger MA, Westra WH, Clark DP, Clifton-Bligh R, Sidransky D, Ladenson PW, Sykorova V 2013 Association between $B R A F^{V 600 E}$ mutation and mortality in patients with papillary thyroid cancer. JAMA 309:1493-1501.

371. Kim TH, Park YJ, Lim JA, Ahn HY, Lee EK, Lee YJ, Kim KW, Hahn SK, Youn YK, Kim KH, Cho BY, Park do J 2012 The association of the BRAF(V600E) mutation with prognostic factors and poor clinical outcome in papillary thyroid cancer: a meta-analysis. Cancer 118:1764-1773.

372. Ito Y, Yoshida H, Kihara M, Kobayashi K, Miya A, Miyauchi A 2014 BRAF(V600E) mutation analysis in papillary thyroid carcinoma: is it useful for all patients? World J Surg 38:679-687.

373. Gouveia C, Can NT, Bostrom A, Grenert JP, van Zante A, Orloff LA 2013 Lack of association of BRAF mutation with negative prognostic indicators in papillary thyroid carcinoma: the University of California, San Francisco, experience. JAMA Otolaryngol Head Neck Surg 139:1164-1170.

374. Dutenhefner SE, Marui S, Santos AB, de Lima EU, Inoue M, Neto JS, Shiang C, Fukushima JT, Cernea CR, Friguglietti CU 2013 BRAF: a tool in the decision to perform elective neck dissection? Thyroid 23:15411546.

375. Lee KC, Li C, Schneider EB, Wang Y, Somervell H, Krafft M, Umbricht CB, Zeiger MA 2012 Is BRAF mutation associated with lymph node metastasis in patients with papillary thyroid cancer? Surgery 152: 977-983.

376. Xing M 2010 Prognostic utility of BRAF mutation in papillary thyroid cancer. Mol Cell Endocrinol 321: 86-93.

377. Sugitani I, Fujimoto Y, Yamada K, Yamamoto N 2008 Prospective outcomes of selective lymph node dissection for papillary thyroid carcinoma based on preoperative ultrasonography. World J Surg 32:2494-2502.

378. Ito Y, Miyauchi A 2007 Lateral and mediastinal lymph node dissection in differentiated thyroid carcinoma: indications, benefits, and risks. World J Surg 31:905-915.

379. Gemsenjager E, Perren A, Seifert B, Schuler G, Schweizer I, Heitz PU 2003 Lymph node surgery in papillary thyroid carcinoma. J Am Coll Surg 197:182-190.

380. Kouvaraki MA, Lee JE, Shapiro SE, Sherman SI, Evans DB 2004 Preventable reoperations for persistent and recurrent papillary thyroid carcinoma. Surgery 136: 1183-1191.

381. Ito Y, Tomoda C, Uruno T, Takamura Y, Miya A, Kobayashi K, Matsuzuka F, Kuma K, Miyauchi A 2004 Preoperative ultrasonographic examination for lymph node metastasis: usefulness when designing lymph node dissection for papillary microcarcinoma of the thyroid. World J Surg 28:498-501.

382. Erdem E, Gulcelik MA, Kuru B, Alagol H 2003 Comparison of completion thyroidectomy and primary surgery for differentiated thyroid carcinoma. Eur J Surg Oncol 29:747-749.

383. Tan MP, Agarwal G, Reeve TS, Barraclough BH, Delbridge LW 2002 Impact of timing on completion thyroidectomy for thyroid cancer. Br J Surg 89:802-804.

384. Untch BR, Palmer FL, Ganly I, Patel SG, Michael TR, Shah JP, Shaha AA 2014 Oncologic outcomes after completion thyroidectomy for patients with welldifferentiated thyroid carcinoma. Ann Surg Oncol 21: 1374-1378.

385. Barbesino G, Goldfarb M, Parangi S, Yang J, Ross DS, Daniels GH 2012 Thyroid lobe ablation with radioactive iodine as an alternative to completion thyroidectomy after hemithyroidectomy in patients with follicular thyroid carcinoma: long-term follow-up. Thyroid 22:369-376.

386. Santra A, Bal S, Mahargan S, Bal C 2011 Long-term outcome of lobar ablation versus completion thyroidectomy in differentiated thyroid cancer. Nucl Med Commun 32:52-58.

387. Giovanella L, Piccardo A, Paone G, Foppiani L, Treglia G, Ceriani L 2013 Thyroid lobe ablation with iodine- ${ }^{131}$ I 
in patients with differentiated thyroid carcinoma: a randomized comparison between 1.1 and $3.7 \mathrm{GBq}$ activities. Nucl Med Commun 34:767-770.

388. O'Connor AM, Bennett CL, Stacey D, Barry M, Col NF, Eden KB, Entwistle VA, Fiset V, Holmes-Rovner M, Khangura S, Llewellyn-Thomas H, Rovner D 2009 Decision aids for people facing health treatment or screening decisions. Cochrane Database Syst Rev (3): CD001431.

389. Feldman-Stewart D, Capirci C, Brennenstuhl S, Tong C, Abacioglu U, Gawkowska-Suwinska M, van Gils F, Heyda A, Igdem S, Macias V, Grillo IM, Moynihan C, Pijls-Johannesma M, Parker C, Pimentel N, Wördehoff H 2011 Information for decision making by patients with early-stage prostate cancer: a comparison across 9 countries. Med Decis Making 31:754-766.

390. Haynes RB, McKibbon KA, Kanani R 1996 Systematic review of randomised trials of interventions to assist patients to follow prescriptions for medications. Lancet 348:383-386.

391. Weiss SM, Wengert PA Jr, Martinez EM, Sewall W, Kopp E 1996 Patient satisfaction with decisionmaking for breast cancer therapy. Ann Surg Oncol 3:285-289.

392. Street RL Jr, Voigt B 1997 Patient participation in deciding breast cancer treatment and subsequent quality of life. Med Decis Making 17:298-306.

393. Abdul-Sater L, Henry M, Majdan A, Mijovic T, Franklin JH, Brandt MG, Black MJ, Hier MP, Payne RJ 2011 What are thyroidectomy patients really concerned about? Otolaryngol Head Neck Surg 144:685-690.

394. Husson O, Haak HR, Oranje WA, Mols F, Reemst PH, van de Poll-Franse LV 2011 Health-related quality of life among thyroid cancer survivors: a systematic review. Clin Endocrinol (Oxf) 75:544-554.

395. Stojadinovic A, Shaha AR, Orlikoff RF, Nissan A, Kornak MF, Singh B, Boyle JO, Shah JP, Brennan MF, Kraus DH 2002 Prospective functional voice assessment in patients undergoing thyroid surgery. Ann Surg 236: 823-832.

396. Soylu L, Ozbas S, Uslu HY, Kocak S 2007 The evaluation of the causes of subjective voice disturbances after thyroid surgery. Am J Surg 194:317-322.

397. Wilson JA, Deary IJ, Millar A, Mackenzie K 2002 The quality of life impact of dysphonia. Clin Otolaryngol Allied Sci 27:179-182.

398. Jones SM, Carding PN, Drinnan MJ 2006 Exploring the relationship between severity of dysphonia and voicerelated quality of life. Clin Otolaryngol 31:411-417.

399. Munch S, deKryger L 2001 A piece of my mind. Moral wounds: complicated complications. JAMA 285:11311132.

400. Cohen SM, Kim J, Roy N, Asche C, Courey M 2012 Prevalence and causes of dysphonia in a large treatmentseeking population. Laryngoscope 122:343-348.

401. Singer MC, Iverson KC, Terris DJ 2012 Thyroidectomyrelated malpractice claims. Otolaryngol Head Neck Surg 146:358-361.

402. Chandrasekhar SS, Randolph GW, Seidman MD, Rosenfeld RM, Angelos P, Barkmeier-Kraemer J, Benninger MS, Blumin JH, Dennis G, Hanks J, Haymart MR, Kloos RT, Seals B, Schreibstein JM, Thomas MA, Waddington C, Warren B, Robertson PJ 2013 Clinical practice guideline: improving voice outcomes after thyroid surgery. Otolaryngol Head Neck Surg 148: S1-37.

403. Farrag TY, Samlan RA, Lin FR, Tufano RP 2006 The utility of evaluating true vocal fold motion before thyroid surgery. Laryngoscope 116:235-238.

404. Roh JL, Yoon YH, Park CI 2009 Recurrent laryngeal nerve paralysis in patients with papillary thyroid carcinomas: evaluation and management of resulting vocal dysfunction. Am J Surg 197:459-465.

405. Randolph GW, Kamani D 2006 The importance of preoperative laryngoscopy in patients undergoing thyroidectomy: voice, vocal cord function, and the preoperative detection of invasive thyroid malignancy. Surgery 139: 357-362.

406. Eadie TL, Kapsner M, Rosenzweig J, Waugh P, Hillel A, Merati A 2010 The role of experience on judgments of dysphonia. J Voice 24:564-573.

407. Rowe-Jones JM, Rosswick RP, Leighton SE 1993 Benign thyroid disease and vocal cord palsy. Ann R Coll Surg Engl 75:241-244.

408. Shin JJ, Grillo HC, Mathisen D, Katlic MR, Zurakowski D, Kamani D, Randolph GW 2011 The surgical management of goiter: Part I. Preoperative evaluation. Laryngoscope 121:60-67.

409. Bergenfelz A, Jansson S, Kristoffersson A, Martensson H, Reihner E, Wallin G, Lausen I 2008 Complications to thyroid surgery: results as reported in a database from a multicenter audit comprising 3,660 patients. Langenbecks Arch Surg 393:667-673.

410. Green KM, de Carpentier JP 1999 Are pre-operative vocal fold checks necessary? J Laryngol Otol 113:642644.

411. Cheng SP, Lee JJ, Liu TP, Lee KS, Liu CL 2012 Preoperative ultrasonography assessment of vocal cord movement during thyroid and parathyroid surgery. World J Surg 36:2509-2515.

412. Terris DJ, Snyder S, Carneiro-Pla D, Inabnet WB III, Kandil E, Orloff L, Shindo M, Tufano RP, Tuttle RM, Urken M, Yeh MW 2013 American Thyroid Association statement on outpatient thyroidectomy. Thyroid 23: 1193-1202.

413. Dralle H, Sekulla C, Haerting J, Timmermann W, Neumann HJ, Kruse E, Grond S, Muhlig HP, Richter C, Voss J, Thomusch O, Lippert H, Gastinger I, Brauckhoff M, Gimm O 2004 Risk factors of paralysis and functional outcome after recurrent laryngeal nerve monitoring in thyroid surgery. Surgery 136:1310-1322.

414. Shindo ML, Caruana S, Kandil E, McCaffrey JC, Orloff L, Porterfield JR, Randolph G, Shaha A, Shin J, Terris D 2014 Management of invasive well-differentiated thyroid cancer an American Head and Neck Society consensus statement. Head Neck 36:1379-1390.

415. Falk SA, McCaffrey TV 1995 Management of the recurrent laryngeal nerve in suspected and proven thyroid cancer. Otolaryngol Head Neck Surg 113:42-48.

416. Jatzko GR, Lisborg PH, Muller MG, Wette VM 1994 Recurrent nerve palsy after thyroid operations_-principal nerve identification and a literature review. Surgery 115:139-144.

417. Lo CY, Kwok KF, Yuen PW 2000 A prospective evaluation of recurrent laryngeal nerve paralysis during thyroidectomy. Arch Surg 135:204-207.

418. Curran AJ, Smyth D, Sheehan SJ, Joyce W, Hayes DB, Walsh MA 1997 Recurrent laryngeal nerve dysfunction 
following carotid endarterectomy. J R Coll Surg Edinb 42:168-170.

419. Rosenthal LH, Benninger MS, Deeb RH 2007 Vocal fold immobility: a longitudinal analysis of etiology over 20 years. Laryngoscope 117:1864-1870.

420. Kriskovich MD, Apfelbaum RI, Haller JR 2000 Vocal fold paralysis after anterior cervical spine surgery: incidence, mechanism, and prevention of injury. Laryngoscope 110:1467-1473.

421. Benninger MS, Crumley RL, Ford CN, Gould WJ, Hanson DG, Ossoff RH, Sataloff RT 1994 Evaluation and treatment of the unilateral paralyzed vocal fold. Otolaryngol Head Neck Surg 111:497-508.

422. Grundfast KM, Harley E 1989 Vocal cord paralysis. Otolaryngol Clin North Am 22:569-597.

423. Hermann M, Alk G, Roka R, Glaser K, Freissmuth M 2002 Laryngeal recurrent nerve injury in surgery for benign thyroid diseases: effect of nerve dissection and impact of individual surgeon in more than 27,000 nerves at risk. Ann Surg 235:261-268.

424. Randolph GW, Dralle H, Abdullah H, Barczynski M, Bellantone R, Brauckhoff M, Carnaille B, Cherenko S, Chiang FY, Dionigi G, Finck C, Hartl D, Kamani D, Lorenz K, Miccolli P, Mihai R, Miyauchi A, Orloff L, Perrier N, Poveda MD, Romanchishen A, Serpell J, Sitges-Serra A, Sloan T, Van SS, Snyder S, Takami H, Volpi E, Woodson G 2011 Electrophysiologic recurrent laryngeal nerve monitoring during thyroid and parathyroid surgery: international standards guideline statement. Laryngoscope 121(Suppl 1):S1S16.

425. Pisanu A, Porceddu G, Podda M, Cois A, Uccheddu A 2014 Systematic review with meta-analysis of studies comparing intraoperative neuromonitoring of recurrent laryngeal nerves versus visualization alone during thyroidectomy. J Surg Res 188:152-161.

426. Barczynski M, Konturek A, Stopa M, Honowska A, Nowak W 2012 Randomized controlled trial of visualization versus neuromonitoring of the external branch of the superior laryngeal nerve during thyroidectomy. World J Surg 36:1340-1347.

427. Chan WF, Lang BH, Lo CY 2006 The role of intraoperative neuromonitoring of recurrent laryngeal nerve during thyroidectomy: a comparative study on 1000 nerves at risk. Surgery 140:866-872.

428. Musholt TJ, Clerici T, Dralle H, Frilling A, Goretzki PE, Hermann MM, Kussmann J, Lorenz K, Nies C, Schabram J, Schabram P, Scheuba C, Simon D, Steinmuller T, Trupka AW, Wahl RA, Zielke A, Bockisch A, Karges W, Luster M, Schmid KW 2011 German Association of Endocrine Surgeons practice guidelines for the surgical treatment of benign thyroid disease. Langenbecks Arch Surg 396:639-649.

429. Sturgeon C, Sturgeon T, Angelos P 2009 Neuromonitoring in thyroid surgery: attitudes, usage patterns, and predictors of use among endocrine surgeons. World J Surg 33:417-425.

430. Horne SK, Gal TJ, Brennan JA 2007 Prevalence and patterns of intraoperative nerve monitoring for thyroidectomy. Otolaryngol Head Neck Surg 136:952-956.

431. Sadowski SM, Soardo P, Leuchter I, Robert JH, Triponez F 2013 Systematic use of recurrent laryngeal nerve neuromonitoring changes the operative strategy in planned bilateral thyroidectomy. Thyroid 23:329-333.
432. Goretzki PE, Schwarz K, Brinkmann J, Wirowski D, Lammers BJ 2010 The impact of intraoperative neuromonitoring (IONM) on surgical strategy in bilateral thyroid diseases: is it worth the effort? World J Surg 34:1274-1284.

433. Melin M, Schwarz K, Lammers BJ, Goretzki PE 2013 IONM-guided goiter surgery leading to two-stage thyroidectomy-indication and results. Langenbecks Arch Surg 398:411-418.

434. Barczynski M, Randolph GW, Cernea CR, Dralle H, Dionigi G, Alesina PF, Mihai R, Finck C, Lombardi D, Hartl DM, Miyauchi A, Serpell J, Snyder S, Volpi E, Woodson G, Kraimps JL, Hisham AN 2013 External branch of the superior laryngeal nerve monitoring during thyroid and parathyroid surgery: International Neural Monitoring Study Group standards guideline statement. Laryngoscope 123(Suppl 4):S1-S14.

435. Randolph GW, Clark OH 2013 Principles in thyroid surgery. In: Randolph GW (ed) Surgery of the Thyroid and Parathyroid Glands. 2nd edition. Elsevier, Philadelphia, PA, pp 273-293.

436. Lorente-Poch L, Sancho JJ, Ruiz S, Sitges-Serra A 2015 Importance of in situ preservation of parathyroid glands during total thyroidectomy. Br J Surg 102:359-367.

437. Friedman AD, Burns JA, Heaton JT, Zeitels SM 2010 Early versus late injection medialization for unilateral vocal cord paralysis. Laryngoscope 120:2042-2046.

438. Arviso LC, Johns MM III, Mathison CC, Klein AM 2010 Long-term outcomes of injection laryngoplasty in patients with potentially recoverable vocal fold paralysis. Laryngoscope 120:2237-2240.

439. Yung KC, Likhterov I, Courey MS 2011 Effect of temporary vocal fold injection medialization on the rate of permanent medialization laryngoplasty in unilateral vocal fold paralysis patients. Laryngoscope 121:2191-2194.

440. Carty SE, Doherty GM, Inabnet WB III, Pasieka JL, Randolph GW, Shaha AR, Terris DJ, Tufano RP, Tuttle RM 2012 American Thyroid Association statement on the essential elements of interdisciplinary communication of perioperative information for patients undergoing thyroid cancer surgery. Thyroid 22:395-399.

441. DeLellis RA, Lloyd RV, Heitz PU, Eng C 2004 World Health Organization Classification of Tumours. Pathology and Genetics of Tumours of Endocrine Organs. IARC Press, Lyon.

442. Haigh PI, Urbach DR 2005 The treatment and prognosis of Hürthle cell follicular thyroid carcinoma compared with its non-Hürthle cell counterpart. Surgery 138:11521157.

443. Shaha AR, Loree TR, Shah JP 1995 Prognostic factors and risk group analysis in follicular carcinoma of the thyroid. Surgery 118:1131-1136.

444. Ganly I, Ricarte FJ, Eng S, Ghossein R, Morris LG, Liang Y, Socci N, Kannan K, Mo Q, Fagin JA, Chan TA 2013 Genomic dissection of Hürthle cell carcinoma reveals a unique class of thyroid malignancy. J Clin Endocrinol Metab 98:E962-E972.

445. Baloch ZW, LiVolsi VA 2002 Follicular-patterned lesions of the thyroid: the bane of the pathologist. Am J Clin Pathol 117:143-150.

446. D’Avanzo A, Treseler P, Ituarte PH, Wong M, Streja L, Greenspan FS, Siperstein AE, Duh QY, Clark OH 2004 Follicular thyroid carcinoma: histology and prognosis. Cancer 100:1123-1129. 
447. Rosai J 2005 Handling of thyroid follicular patterned lesions. Endocr Pathol 16:279-283.

448. Nikiforov YE, Ohori NP 2012 Follicular carcinoma. In: Nikiforov YE, Biddinger PW, Thompson LDR (eds) Diagnostic Pathology and Molecular Genetics of the Thyroid. 1st edition. Lippincott, Philadelphia, PA, pp 152-182.

449. Yamashita H, Noguchi S, Murakami N, Kawamoto H, Watanabe S 1997 Extracapsular invasion of lymph node metastasis is an indicator of distant metastasis and poor prognosis in patients with thyroid papillary carcinoma. Cancer 80:2268-2272.

450. Lango M, Flieder D, Arrangoiz R, Veloski C, Yu JQ, Li T, Burtness B, Mehra R, Galloway T, Ridge JA 2013 Extranodal extension of metastatic papillary thyroid carcinoma: correlation with biochemical endpoints, nodal persistence, and systemic disease progression. Thyroid 23:1099-1105.

451. Falvo L, Catania A, D’Andrea V, Marzullo A, Giustiniani MC, De Antoni E 2005 Prognostic importance of histologic vascular invasion in papillary thyroid carcinoma. Ann Surg 241:640-646.

452. Gardner RE, Tuttle RM, Burman KD, Haddady S, Truman C, Sparling YH, Wartofsky L, Sessions RB, Ringel MD 2000 Prognostic importance of vascular invasion in papillary thyroid carcinoma. Arch Otolaryngol Head Neck Surg 126:309-312.

453. Mete O, Asa SL 2011 Pathological definition and clinical significance of vascular invasion in thyroid carcinomas of follicular epithelial derivation. Mod Pathol 24:15451552.

454. Brennan MD, Bergstralh EJ, van Heerden JA, McConahey WM 1991 Follicular thyroid cancer treated at the Mayo Clinic, 1946 through 1970: initial manifestations, pathologic findings, therapy, and outcome. Mayo Clin Proc 66:11-22.

455. Collini P, Sampietro G, Pilotti S 2004 Extensive vascular invasion is a marker of risk of relapse in encapsulated non-Hürthle cell follicular carcinoma of the thyroid gland: a clinicopathological study of 18 consecutive cases from a single institution with a 11-year median follow-up. Histopathology 44:35-39.

456. Lang W, Choritz H, Hundeshagen H 1986 Risk factors in follicular thyroid carcinomas. A retrospective follow-up study covering a 14-year period with emphasis on morphological findings. Am J Surg Pathol 10:246-255.

457. Nikiforov YE, Ohori NP 2012 Papillary carcinoma. In: Nikiforov YE, Biddinger PW, Thompson LDR (eds) Diagnostic Pathology and Molecular Genetics of the Thyroid. 1st edition. Lippincott, Philadelphia, PA, pp 183-262.

458. Hawk WA, Hazard JB 1976 The many appearances of papillary carcinoma of the thyroid. Cleve Clin Q 43: 207-215.

459. Leung AK, Chow SM, Law SC 2008 Clinical features and outcome of the tall cell variant of papillary thyroid carcinoma. Laryngoscope 118:32-38.

460. Moreno EA, Rodriguez Gonzalez JM, Sola PJ, Soria CT, Parrilla PP 1993 Prognostic value of the tall cell variety of papillary cancer of the thyroid. Eur J Surg Oncol 19: 517-521.

461. Ostrowski ML, Merino MJ 1996 Tall cell variant of papillary thyroid carcinoma: a reassessment and immunohistochemical study with comparison to the usual type of papillary carcinoma of the thyroid. Am J Surg Pathol 20:964-974.
462. Johnson TL, Lloyd RV, Thompson NW, Beierwaltes WH, Sisson JC 1988 Prognostic implications of the tall cell variant of papillary thyroid carcinoma. Am J Surg Pathol 12:22-27.

463. Michels JJ, Jacques M, Henry-Amar M, Bardet S 2007 Prevalence and prognostic significance of tall cell variant of papillary thyroid carcinoma. Hum Pathol 38:212-219.

464. Ghossein RA, Leboeuf R, Patel KN, Rivera M, Katabi N, Carlson DL, Tallini G, Shaha A, Singh B, Tuttle RM 2007 Tall cell variant of papillary thyroid carcinoma without extrathyroid extension: biologic behavior and clinical implications. Thyroid 17:655-661.

465. Morris LG, Shaha AR, Tuttle RM, Sikora AG, Ganly I 2010 Tall-cell variant of papillary thyroid carcinoma: a matched-pair analysis of survival. Thyroid 20:153-158.

466. Xing M 2005 BRAF mutation in thyroid cancer. Endocr Relat Cancer 12:245-262.

467. Chen JH, Faquin WC, Lloyd RV, Nose V 2011 Clinicopathological and molecular characterization of nine cases of columnar cell variant of papillary thyroid carcinoma. Mod Pathol 24:739-749.

468. Evans HL 1986 Columnar-cell carcinoma of the thyroid. A report of two cases of an aggressive variant of thyroid carcinoma. Am J Clin Pathol 85:77-80.

469. Evans HL 1996 Encapsulated columnar-cell neoplasms of the thyroid. A report of four cases suggesting a favorable prognosis. Am J Surg Pathol 20:1205-1211.

470. Wenig BM, Thompson LD, Adair CF, Shmookler B, Heffess CS 1998 Thyroid papillary carcinoma of columnar cell type: a clinicopathologic study of 16 cases. Cancer 82:740-753.

471. Asioli S, Erickson LA, Sebo TJ, Zhang J, Jin L, Thompson GB, Lloyd RV 2010 Papillary thyroid carcinoma with prominent hobnail features: a new aggressive variant of moderately differentiated papillary carcinoma. A clinicopathologic, immunohistochemical, and molecular study of eight cases. Am J Surg Pathol 34:44-52.

472. Motosugi U, Murata S, Nagata K, Yasuda M, Shimizu M 2009 Thyroid papillary carcinoma with micropapillary and hobnail growth pattern: a histological variant with intermediate malignancy? Thyroid 19:535-537.

473. Lubitz CC, Economopoulos KP, Pawlak AC, Lynch K, Dias-Santagata D, Faquin WC, Sadow PM 2014 Hobnail variant of papillary thyroid carcinoma: an institutional case series and molecular profile. Thyroid 24:958-965.

474. Mizukami Y, Noguchi M, Michigishi T, Nonomura A, Hashimoto T, Otakes S, Nakamura S, Matsubara F 1992 Papillary thyroid carcinoma in Kanazawa, Japan: prognostic significance of histological subtypes. Histopathology 20:243-250.

475. Nikiforov YE, Erickson LA, Nikiforova MN, Caudill CM, Lloyd RV 2001 Solid variant of papillary thyroid carcinoma: incidence, clinical-pathologic characteristics, molecular analysis, and biologic behavior. Am J Surg Pathol 25:1478-1484.

476. Cardis E, Howe G, Ron E, Bebeshko V, Bogdanova T, Bouville A, Carr Z, Chumak V, Davis S, Demidchik Y, Drozdovitch V, Gentner N, Gudzenko N, Hatch M, Ivanov V, Jacob P, Kapitonova E, Kenigsberg Y, Kesminiene A, Kopecky KJ, Kryuchkov V, Loos A, Pinchera A, Reiners C, Repacholi M, Shibata Y, Shore RE, Thomas G, Tirmarche M, Yamashita S, Zvonova I 2006 Cancer consequences of the Chernobyl accident: 20 years on. J Radiol Prot 26:127-140. 
477. Nikiforov YE 2006 Radiation-induced thyroid cancer: what we have learned from Chernobyl. Endocr Pathol 17:307-317.

478. Volante M, Collini P, Nikiforov YE, Sakamoto A, Kakudo K, Katoh R, Lloyd RV, LiVolsi VA, Papotti M, Sobrinho-Simoes M, Bussolati G, Rosai J 2007 Poorly differentiated thyroid carcinoma: the Turin proposal for the use of uniform diagnostic criteria and an algorithmic diagnostic approach. Am J Surg Pathol 31:1256-1264.

479. Asioli S, Erickson LA, Righi A, Jin L, Volante M, Jenkins S, Papotti M, Bussolati G, Lloyd RV 2010 Poorly differentiated carcinoma of the thyroid: validation of the Turin proposal and analysis of IMP3 expression. Mod Pathol 23:1269-1278.

480. Fukushima M, Ito Y, Hirokawa M, Akasu H, Shimizu K, Miyauchi A 2009 Clinicopathologic characteristics and prognosis of diffuse sclerosing variant of papillary thyroid carcinoma in Japan: an 18-year experience at a single institution. World J Surg 33:958-962.

481. Koo JS, Hong S, Park CS 2009 Diffuse sclerosing variant is a major subtype of papillary thyroid carcinoma in the young. Thyroid 19:1225-1231.

482. Regalbuto C, Malandrino P, Tumminia A, Le Moli R, Vigneri R, Pezzino V 2011 A diffuse sclerosing variant of papillary thyroid carcinoma: clinical and pathologic features and outcomes of 34 consecutive cases. Thyroid 21:383-389.

483. Lam AK, Lo CY 2006 Diffuse sclerosing variant of papillary carcinoma of the thyroid: a 35-year comparative study at a single institution. Ann Surg Oncol 13: 176-181.

484. Rivera M, Ricarte-Filho J, Knauf J, Shaha A, Tuttle M, Fagin JA, Ghossein RA 2010 Molecular genotyping of papillary thyroid carcinoma follicular variant according to its histological subtypes (encapsulated vs infiltrative) reveals distinct BRAF and RAS mutation patterns. Mod Pathol 23:1191-1200.

485. Howitt BE, Jia Y, Sholl LM, Barletta JA 2013 Molecular alterations in partially-encapsulated or well-circumscribed follicular variant of papillary thyroid carcinoma. Thyroid 23:1256-1262.

486. Liu J, Singh B, Tallini G, Carlson DL, Katabi N, Shaha A, Tuttle RM, Ghossein RA 2006 Follicular variant of papillary thyroid carcinoma: a clinicopathologic study of a problematic entity. Cancer 107:1255-1264.

487. Piana S, Frasoldati A, Di Felice E, Gardini G, Tallini G, Rosai J 2010 Encapsulated well-differentiated follicularpatterned thyroid carcinomas do not play a significant role in the fatality rates from thyroid carcinoma. Am J Surg Pathol 34:868-872.

488. Proietti A, Giannini R, Ugolini C, Miccoli M, Fontanini G, Di Coscio G, Romani R, Berti P, Miccoli P, Basolo F 2010 BRAF status of follicular variant of papillary thyroid carcinoma and its relationship to its clinical and cytological features. Thyroid 20:1263-1270.

489. Chan J 2002 Strict criteria should be applied in the diagnosis of encapsulated follicular variant of papillary thyroid carcinoma. Am J Clin Pathol 117:16-18.

490. Vivero M, Kraft S, Barletta JA 2013 Risk stratification of follicular variant of papillary thyroid carcinoma. Thyroid 23:273-279.

491. Baloch ZW, LiVolsi VA 2000 Encapsulated follicular variant of papillary thyroid carcinoma with bone metastases. Mod Pathol 13:861-865.
492. Hunt JL, Dacic S, Barnes EL, Bures JC 2002 Encapsulated follicular variant of papillary thyroid carcinoma. Am J Clin Pathol 118:602-603.

493. Rivera M, Ricarte-Filho J, Patel S, Tuttle M, Shaha A, Shah JP, Fagin JA, Ghossein RA 2010 Encapsulated thyroid tumors of follicular cell origin with high grade features (high mitotic rate/tumor necrosis): a clinicopathologic and molecular study. Hum Pathol 41:172-180.

494. O’Neill CJ, Vaughan L, Learoyd DL, Sidhu SB, Delbridge LW, Sywak MS 2011 Management of follicular thyroid carcinoma should be individualised based on degree of capsular and vascular invasion. Eur J Surg Oncol 37:181-185.

495. van Heerden JA, Hay ID, Goellner JR, Salomao D, Ebersold JR, Bergstralh EJ, Grant CS 1992 Follicular thyroid carcinoma with capsular invasion alone: a nonthreatening malignancy. Surgery 112:1130-1136.

496. Goldstein NS, Czako P, Neill JS 2000 Metastatic minimally invasive (encapsulated) follicular and Hürthle cell thyroid carcinoma: a study of 34 patients. Mod Pathol 13:123-130.

497. Sugino K, Ito K, Nagahama M, Kitagawa W, Shibuya H, Ohkuwa K, Yano Y, Uruno T, Akaishi J, Kameyama K, Ito $\mathrm{K} 2011$ Prognosis and prognostic factors for distant metastases and tumor mortality in follicular thyroid carcinoma. Thyroid 21:751-757.

498. Sugino K, Kameyama K, Ito K, Nagahama M, Kitagawa W, Shibuya H, Ohkuwa K, Yano Y, Uruno T, Akaishi J, Suzuki A, Masaki C, Ito K 2012 Outcomes and prognostic factors of 251 patients with minimally invasive follicular thyroid carcinoma. Thyroid 22:798-804.

499. Ghossein RA, Hiltzik DH, Carlson DL, Patel S, Shaha A, Shah JP, Tuttle RM, Singh B 2006 Prognostic factors of recurrence in encapsulated Hürthle cell carcinoma of the thyroid gland: a clinicopathologic study of 50 cases. Cancer 106:1669-1676.

500. Huang CC, Hsueh C, Liu FH, Chao TC, Lin JD 2011 Diagnostic and therapeutic strategies for minimally and widely invasive follicular thyroid carcinomas. Surg Oncol 20:1-6.

501. Lo CY, Chan WF, Lam KY, Wan KY 2005 Follicular thyroid carcinoma: the role of histology and staging systems in predicting survival. Ann Surg 242:708-715.

502. Slade I, Bacchelli C, Davies H, Murray A, Abbaszadeh F, Hanks S, Barfoot R, Burke A, Chisholm J, Hewitt M, Jenkinson H, King D, Morland B, Pizer B, Prescott K, Saggar A, Side L, Traunecker H, Vaidya S, Ward P, Futreal PA, Vujanic G, Nicholson AG, Sebire N, Turnbull C, Priest JR, Pritchard-Jones K, Houlston R, Stiller C, Stratton MR, Douglas J, Rahman N 2011 DICER1 syndrome: clarifying the diagnosis, clinical features and management implications of a pleiotropic tumour predisposition syndrome. J Med Genet 48:273-278.

503. Cetta F, Montalto G, Gori M, Curia MC, Cama A, Olschwang S 2000 Germline mutations of the APC gene in patients with familial adenomatous polyposisassociated thyroid carcinoma: results from a European cooperative study. J Clin Endocrinol Metab 85:286-292.

504. Harach HR, Williams GT, Williams ED 1994 Familial adenomatous polyposis associated thyroid carcinoma: a distinct type of follicular cell neoplasm. Histopathology 25:549-561.

505. Hirokawa M, Maekawa M, Kuma S, Miyauchi A 2010 Cribriform-morular variant of papillary thyroid carcino- 
ma-cytological and immunocytochemical findings of 18 cases. Diagn Cytopathol 38:890-896.

506. Jung CK, Choi YJ, Lee KY, Bae JS, Kim HJ, Yoon SK, Son YI, Chung JH, Oh YL 2009 The cytological, clinical, and pathological features of the cribriform-morular variant of papillary thyroid carcinoma and mutation analysis of CTNNB1 and BRAF genes. Thyroid 19:905-913.

507. Koo JS, Jung W, Hong SW 2011 Cytologic characteristics and beta-catenin immunocytochemistry on smear slide of cribriform-morular variant of papillary thyroid carcinoma. Acta Cytol 55:13-18.

508. Ito Y, Miyauchi A, Ishikawa H, Hirokawa M, Kudo T, Tomoda C, Miya A 2011 Our experience of treatment of cribriform morular variant of papillary thyroid carcinoma; difference in clinicopathological features of FAP-associated and sporadic patients. Endocr J 58: 685-689.

509. Hollander MC, Blumenthal GM, Dennis PA 2011 PTEN loss in the continuum of common cancers, rare syndromes and mouse models. Nat Rev Cancer 11:289-301.

510. Laury AR, Bongiovanni M, Tille JC, Kozakewich H, Nose V 2011 Thyroid pathology in PTEN-hamartoma tumor syndrome: characteristic findings of a distinct entity. Thyroid 21:135-144.

511. Nose V 2011 Familial thyroid cancer: a review. Mod Pathol 24(Suppl 2):S19-S33.

512. Harach HR, Soubeyran I, Brown A, Bonneau D, Longy M 1999 Thyroid pathologic findings in patients with Cowden disease. Ann Diagn Pathol 3:331-340.

513. Hemmings CT 2003 Thyroid pathology in four patients with Cowden's disease. Pathology 35:311-314.

514. Volante M, Landolfi S, Chiusa L, Palestini N, Motta M, Codegone A, Torchio B, Papotti MG 2004 Poorly differentiated carcinomas of the thyroid with trabecular, insular, and solid patterns: a clinicopathologic study of 183 patients. Cancer 100:950-957.

515. Sakamoto A, Kasai N, Sugano H 1983 Poorly differentiated carcinoma of the thyroid. A clinicopathologic entity for a high-risk group of papillary and follicular carcinomas. Cancer 52:1849-1855.

516. Carcangiu ML, Zampi G, Rosai J 1984 Poorly differentiated ("insular") thyroid carcinoma. A reinterpretation of Langhans" "wuchernde Struma". Am J Surg Pathol 8:655-668.

517. Pulcrano M, Boukheris H, Talbot M, Caillou B, Dupuy C, Virion A, De Vathaire F, Schlumberger M 2007 Poorly differentiated follicular thyroid carcinoma: prognostic factors and relevance of histological classification. Thyroid 17:639-646.

518. Papotti M, Botto MF, Favero A, Palestini N, Bussolati G 1993 Poorly differentiated thyroid carcinomas with primordial cell component. A group of aggressive lesions sharing insular, trabecular, and solid patterns. Am J Surg Pathol 17:291-301.

519. Decaussin M, Bernard MH, Adeleine P, Treilleux I, Peix JL, Pugeat M, Tourniaire J, Berger N 2002 Thyroid carcinomas with distant metastases: a review of 111 cases with emphasis on the prognostic significance of an insular component. Am J Surg Pathol 26:1007-1015.

520. Hiltzik D, Carlson DL, Tuttle RM, Chuai S, Ishill N, Shaha A, Shah JP, Singh B, Ghossein RA 2006 Poorly differentiated thyroid carcinomas defined on the basis of mitosis and necrosis: a clinicopathologic study of 58 patients. Cancer 106:1286-1295.
521. Gnemmi V, Renaud F, Do Cao C, Salleron J, Lion G, Wemeau JL, Copin MC, Carnaille B, Leteurtre E, Pattou F, Aubert S 2014 Poorly differentiated thyroid carcinomas: application of the Turin proposal provides prognostic results similar to those from the assessment of high-grade features. Histopathology 64:263-273.

522. Sherman SI 1999 Toward a standard clinicopathologic staging approach for differentiated thyroid carcinoma. Semin Surg Oncol 16:12-15.

523. Yang L, Shen W, Sakamoto N 2013 Population-based study evaluating and predicting the probability of death resulting from thyroid cancer and other causes among patients with thyroid cancer. J Clin Oncol 31: 468-474.

524. Onitilo AA, Engel JM, Lundgren CI, Hall P, Thalib L, Doi SA 2009 Simplifying the TNM system for clinical use in differentiated thyroid cancer. J Clin Oncol 27:1872-1878.

525. Mankarios D, Baade P, Youl P, Mortimer RH, Onitilo AA, Russell A, Doi SA 2013 Validation of the QTNM staging system for cancer-specific survival in patients with differentiated thyroid cancer. Endocrine 46:300-308.

526. Sherman SI, Brierley JD, Sperling M, Ain KB, Bigos ST, Cooper DS, Haugen BR, Ho M, Klein I, Ladenson PW, Robbins J, Ross DS, Specker B, Taylor T, Maxon HR III 1998 Prospective multicenter study of thyroiscarcinoma treatment: initial analysis of staging and outcome. National Thyroid Cancer Treatment Cooperative Study Registry Group. Cancer 83:1012-1021.

527. Lang BH, Chow SM, Lo CY, Law SC, Lam KY 2007 Staging systems for papillary thyroid carcinoma: a study of 2 tertiary referral centers. Ann Surg 246:114-121.

528. Lang BH, Lo CY, Chan WF, Lam KY, Wan KY 2007 Staging systems for papillary thyroid carcinoma: a review and comparison. Ann Surg 245:366-378.

529. Voutilainen PE, Siironen P, Franssila KO, Sivula A, Haapiainen RK, Haglund CH 2003 AMES, MACIS and TNM prognostic classifications in papillary thyroid carcinoma. Anticancer Res 23:4283-4288.

530. Yildirim E 2005 A model for predicting outcomes in patients with differentiated thyroid cancer and model performance in comparison with other classification systems. J Am Coll Surg 200:378-392.

531. Jonklaas J, Nogueras-Gonzalez G, Munsell M, Litofsky D, Ain KB, Bigos ST, Brierley JD, Cooper DS, Haugen BR, Ladenson PW, Magner J, Robbins J, Ross DS, Skarulis MC, Steward DL, Maxon HR, Sherman SI 2012 The impact of age and gender on papillary thyroid cancer survival. J Clin Endocrinol Metab 97:E878-E887.

532. Bischoff LA, Curry J, Ahmed I, Pribitkin E, Miller JL 2013 Is above age 45 appropriate for upstaging welldifferentiated papillary thyroid cancer? Endocr Pract 19: 995-997.

533. Ganly I, Nixon IJ, Wang LY, Palmer FL, Migliacci JC, Aniss A, Sywak M, Eskander A, Freeman JL, Campbell MJ, Shen WT, Vaisman F, Momesso D, Corbo R, Vaisman M, Shaha AM, Tuttle RM, Shah JP, Patel SG 2015 Survival from differentiated thyroid cancer: what has age got to do with it? Thyroid 25:1106-1114.

534. D'Avanzo A, Ituarte P, Treseler P, Kebebew E, Wu J, Wong M, Duh QY, Siperstein AE, Clark OH 2004 Prognostic scoring systems in patients with follicular thyroid cancer: a comparison of different staging systems in predicting the patient outcome. Thyroid 14:453-458. 
535. Lang BH, Lo CY, Chan WF, Lam KY, Wan KY 2007 Staging systems for follicular thyroid carcinoma: application to 171 consecutive patients treated in a tertiary referral centre. Endocr Relat Cancer 14:29-42.

536. Orlov S, Orlov D, Shaytzag M, Dowar M, Tabatabaie V, Dwek P, Yip J, Hu C, Freeman JL, Walfish PG 2009 Influence of age and primary tumor size on the risk for residual/recurrent well-differentiated thyroid carcinoma. Head Neck 31:782-788.

537. Baek SK, Jung KY, Kang SM, Kwon SY, Woo JS, Cho SH, Chung EJ 2010 Clinical risk factors associated with cervical lymph node recurrence in papillary thyroid carcinoma. Thyroid 20:147-152.

538. Tuttle RM, Tala H, Shah J, Leboeuf R, Ghossein R, Gonen M, Brokhin M, Omry G, Fagin JA, Shaha A 2010 Estimating risk of recurrence in differentiated thyroid cancer after total thyroidectomy and radioactive iodine remnant ablation: using response to therapy variables to modify the initial risk estimates predicted by the new American Thyroid Association staging system. Thyroid 20:1341-1349.

539. Vaisman F, Momesso D, Bulzico DA, Pessoa CH, Dias F, Corbo R, Vaisman M, Tuttle RM 2012 Spontaneous remission in thyroid cancer patients after biochemical incomplete response to initial therapy. Clin Endocrinol (Oxf) 77:132-138.

540. Pacini F, Schlumberger M, Dralle H, Elisei R, Smit JW, Wiersinga W 2006 European consensus for the management of patients with differentiated thyroid carcinoma of the follicular epithelium. Eur J Endocrinol 154:787-803.

541. Camargo R, Corigliano S, Friguglietti C, Gauna A, Harach R, Munizaga F, Niepomniszcze H, Pitoia F, Pretell E, Vaisman M, Ward LS, Wohllk N, Tomimori E 2009 Latin American Thyroid Society recommendations for the management of thyroid nodules. Arq Bras Endocrinol Metabol 53:1167-1175.

542. Castagna MG, Maino F, Cipri C, Belardini V, Theodoropoulou A, Cevenini G, Pacini F 2011 Delayed risk stratification, to include the response to initial treatment (surgery and radioiodine ablation), has better outcome predictivity in differentiated thyroid cancer patients. Eur J Endocrinol 165:441-446.

543. Pitoia F, Bueno F, Urciuoli C, Abelleira E, Cross G, Tuttle RM 2013 Outcomes of patients with differentiated thyroid cancer risk-stratified according to the American Thyroid Association and Latin American Thyroid Society risk of recurrence classification systems. Thyroid 23:1401-1407.

544. Schvartz C, Bonnetain F, Dabakuyo S, Gauthier M, Cueff A, Fieffe S, Pochart JM, Cochet I, Crevisy E, Dalac A, Papathanassiou D, Toubeau M 2012 Impact on overall survival of radioactive iodine in low-risk differentiated thyroid cancer patients. J Clin Endocrinol Metab 97:1526-1535.

545. Durante C, Montesano T, Attard M, Torlontano M, Monzani F, Costante G, Meringolo D, Ferdeghini M, Tumino S, Lamartina L, Paciaroni A, Massa M, Giacomelli L, Ronga G, Filetti S 2012 Long-term surveillance of papillary thyroid cancer patients who do not undergo postoperative radioiodine remnant ablation: is there a role for serum thyroglobulin measurement? J Clin Endocrinol Metab 97:2748-2753.

546. Kim KM, Park JB, Bae KS, Kim CB, Kang DR, Kang SJ 2013 Clinical prognostic index for recurrence of papil- lary thyroid carcinoma including intraoperative findings. Endocr J 60:291-297.

547. Ito Y, Kudo T, Kihara M, Takamura Y, Kobayashi K, Miya A, Miyauchi A 2012 Prognosis of low-risk papillary thyroid carcinoma patients: its relationship with the size of primary tumors. Endocr J 59:119-125.

548. Lee J, Song Y, Soh EY 2014 Prognostic significance of the number of metastatic lymph nodes to stratify the risk of recurrence. World J Surg 38:858-862.

549. Clain JB, Scherl S, Dos RL, Turk A, Wenig BM, Mehra S, Karle WE, Urken ML 2014 Extrathyroidal extension predicts extranodal extension in patients with positive lymph nodes: an important association that may affect clinical management. Thyroid 24:951-957.

550. Riemann B, Kramer JA, Schmid KW, Dralle H, Dietlein M, Schicha H, Sauerland C, Frankewitsch T, Schober O 2010 Risk stratification of patients with locally aggressive differentiated thyroid cancer. Results of the MSDS trial. Nuklearmedizin 49:79-84.

551. Nixon IJ, Ganly I, Patel S, Palmer FL, Whitcher MM, Tuttle RM, Shaha AR, Shah JP 2011 The impact of microscopic extrathyroid extension on outcome in patients with clinical T1 and T2 well-differentiated thyroid cancer. Surgery 150:1242-1249.

552. Jukkola A, Bloigu R, Ebeling T, Salmela P, Blanco G 2004 Prognostic factors in differentiated thyroid carcinomas and their implications for current staging classifications. Endocr Relat Cancer 11:571-579.

553. Ito $\mathrm{Y}$, Tomoda C, Uruno T, Takamura Y, Miya A, Kobayashi K, Matsuzuka F, Kuma K, Miyauchi A 2006 Prognostic significance of extrathyroid extension of papillary thyroid carcinoma: massive but not minimal extension affects the relapse-free survival. World J Surg 30:780-786.

554. Radowsky JS, Howard RS, Burch HB, Stojadinovic A 2014 Impact of degree of extrathyroidal extension of disease on papillary thyroid cancer outcome. Thyroid 24: 241-244.

555. Fukushima M, Ito Y, Hirokawa M, Miya A, Shimizu K, Miyauchi A 2010 Prognostic impact of extrathyroid extension and clinical lymph node metastasis in papillary thyroid carcinoma depend on carcinoma size. World J Surg 34:3007-3014.

556. Baloch ZW, Shafique K, Flannagan M, LiVolsi VA 2010 Encapsulated classic and follicular variants of papillary thyroid carcinoma: comparative clinicopathologic study. Endocr Pract 16:952-959.

557. Deleted.

558. Nishida T, Katayama S, Tsujimoto M 2002 The clinicopathological significance of histologic vascular invasion in differentiated thyroid carcinoma. Am J Surg 183:80-86.

559. Akslen LA, Myking AO, Salvesen H, Varhaug JE 1992 Prognostic importance of various clinicopathological features in papillary thyroid carcinoma. Eur J Cancer 29A:44-51.

560. Simpson WJ, McKinney SE, Carruthers JS, Gospodarowicz MK, Sutcliffe SB, Panzarella T 1987 Papillary and follicular thyroid cancer. Prognostic factors in 1,578 patients. Am J Med 83:479-488.

561. Mai KT, Khanna P, Yazdi HM, Perkins DG, Veinot JP, Thomas J, Lamba M, Nair BD 2002 Differentiated thyroid carcinomas with vascular invasion: a comparative study of follicular, Hurthle cell and papillary thyroid carcinoma. Pathology 34:239-244. 
562. Furlan JC, Bedard YC, Rosen IB 2004 Clinicopathologic significance of histologic vascular invasion in papillary and follicular thyroid carcinomas. J Am Coll Surg 198: 341-348.

563. Tufano RP, Teixeira GV, Bishop J, Carson KA, Xing M 2012 BRAF mutation in papillary thyroid cancer and its value in tailoring initial treatment: a systematic review and meta-analysis. Medicine (Baltimore) 91:274-286.

564. Xing M, Westra WH, Tufano RP, Cohen Y, Rosenbaum E, Rhoden KJ, Carson KA, Vasko V, Larin A, Tallini G, Tolaney S, Holt EH, Hui P, Umbricht CB, Basaria S, Ewertz M, Tufaro AP, Califano JA, Ringel MD, Zeiger MA, Sidransky D, Ladenson PW 2005 BRAF mutation predicts a poorer clinical prognosis for papillary thyroid cancer. J Clin Endocrinol Metab 90:6373-6379.

565. Elisei R, Viola D, Torregrossa L, Giannini R, Romei C, Ugolini C, Molinaro E, Agate L, Biagini A, Lupi C, Valerio L, Materazzi G, Miccoli P, Piaggi P, Pinchera A, Vitti P, Basolo F 2012 The BRAF(V600E) mutation is an independent, poor prognostic factor for the outcome of patients with low-risk intrathyroid papillary thyroid carcinoma: single-institution results from a large cohort study. J Clin Endocrinol Metab 97:4390-4398.

566. Kim TY, Kim WB, Rhee YS, Song JY, Kim JM, Gong G, Lee S, Kim SY, Kim SC, Hong SJ, Shong YK 2006 The BRAF mutation is useful for prediction of clinical recurrence in low-risk patients with conventional papillary thyroid carcinoma. Clin Endocrinol (Oxf) 65:364-368.

567. Li C, Lee KC, Schneider EB, Zeiger MA 2012 $B R A F^{V 600 E}$ mutation and its association with clinicopathological features of papillary thyroid cancer: a metaanalysis. J Clin Endocrinol Metab 97:4559-4570.

568. Fernandez IJ, Piccin O, Sciascia S, Cavicchi O, Repaci A, Vicennati V, Fiorentino M 2013 Clinical significance of BRAF mutation in thyroid papillary cancer. Otolaryngol Head Neck Surg 148:919-925.

569. Prescott JD, Sadow PM, Hodin RA, Le LP, Gaz RD, Randolph GW, Stephen AE, Parangi S, Daniels GH, Lubitz CC $2012 B R A F^{V 600 E}$ status adds incremental value to current risk classification systems in predicting papillary thyroid carcinoma recurrence. Surgery 152: 984-990.

570. Sedliarou I, Saenko V, Lantsov D, Rogounovitch T, Namba H, Abrosimov A, Lushnikov E, Kumagai A, Nakashima M, Meirmanov S, Mine M, Hayashi T, Yamashita S 2004 The BRAFT1796A transversion is a prevalent mutational event in human thyroid microcarcinoma. Int J Oncol 25:1729-1735.

571. Xing M 2007 BRAF mutation in papillary thyroid cancer: pathogenic role, molecular bases, and clinical implications. Endocr Rev 28:742-762.

572. Park YJ, Kim YA, Lee YJ, Kim SH, Park SY, Kim KW, Chung JK, Youn YK, Kim KH, Park do J, Cho BY 2010 Papillary microcarcinoma in comparison with larger papillary thyroid carcinoma in BRAF(V600E) mutation, clinicopathological features, and immunohistochemical findings. Head Neck 32:38-45.

573. Namba H, Nakashima M, Hayashi T, Hayashida N, Maeda S, Rogounovitch TI, Ohtsuru A, Saenko VA, Kanematsu T, Yamashita S 2003 Clinical implication of hot spot BRAF mutation, V599E, in papillary thyroid cancers. J Clin Endocrinol Metab 88:4393-4397.

574. Kwak JY, Kim EK, Chung WY, Moon HJ, Kim MJ, Choi JR 2009 Association of BRAFV600E mutation with poor clinical prognostic factors and US features in Korean patients with papillary thyroid microcarcinoma. Radiology 253:854-860.

575. Rodolico V, Cabibi D, Pizzolanti G, Richiusa P, Gebbia N, Martorana A, Russo A, Amato MC, Galluzzo A, Giordano C 2007 BRAF ${ }^{\text {VGOOE }}$ mutation and p27 kip1 expression in papillary carcinomas of the thyroid $<$ or $=$ $1 \mathrm{~cm}$ and their paired lymph node metastases. Cancer 110:1218-1226.

576. Kimura ET, Nikiforova MN, Zhu Z, Knauf JA, Nikiforov YE, Fagin JA 2003 High prevalence of BRAF mutations in thyroid cancer: genetic evidence for constitutive activation of the RET/PTC-RAS-BRAF signaling pathway in papillary thyroid carcinoma. Cancer Res 63:14541457.

577. Henderson YC, Shellenberger TD, Williams MD, ElNaggar AK, Fredrick MJ, Cieply KM, Clayman GL 2009 High rate of BRAF and RET/PTC dual mutations associated with recurrent papillary thyroid carcinoma. Clin Cancer Res 15:485-491.

578. Nikiforova MN, Wald AI, Roy S, Durso MB, Nikiforov YE 2013 Targeted next-generation sequencing panel (ThyroSeq) for detection of mutations in thyroid cancer. J Clin Endocrinol Metab 98:E1852-E1860.

579. Liu X, Bishop J, Shan Y, Pai S, Liu D, Murugan AK, Sun H, El-Naggar AK, Xing M 2013 Highly prevalent TERT promoter mutations in aggressive thyroid cancers. Endocr Relat Cancer 20:603-610.

580. Landa I, Ganly I, Chan TA, Mitsutake N, Matsuse M, Ibrahimpasic T, Ghossein RA, Fagin JA 2013 Frequent somatic TERT promoter mutations in thyroid cancer: higher prevalence in advanced forms of the disease. $\mathrm{J}$ Clin Endocrinol Metab 98:E1562-E1566.

581. Liu X, Qu S, Liu R, Sheng C, Shi X, Zhu G, Murugan AK, Guan H, Yu H, Wang Y, Sun H, Shan Z, Teng W, Xing M 2014 TERT promoter mutations and their association with $B R A F^{V 600 E}$ mutation and aggressive clinicopathological characteristics of thyroid cancer. J Clin Endocrinol Metab 99:E1130-E1136.

582. Tuttle RM, Leboeuf R 2008 Follow up approaches in thyroid cancer: a risk adapted paradigm. Endocrinol Metab Clin North Am 37:419-435, ix-x.

583. Schlumberger M, Berg G, Cohen O, Duntas L, Jamar F, Jarzab B, Limbert E, Lind P, Pacini F, Reiners C, Sanchez FF, Toft A, Wiersinga WM 2004 Follow-up of low-risk patients with differentiated thyroid carcinoma: a European perspective. Eur J Endocrinol 150:105-112.

584. Tuttle RM 2008 Risk-adapted management of thyroid cancer. Endocr Pract 14:764-774.

585. Deleted.

586. Berger F, Friedrich U, Knesewitsch P, Hahn K 2011 Diagnostic 131I whole-body scintigraphy 1 year after thyroablative therapy in patients with differentiated thyroid cancer: correlation of results to the individual risk profile and long-term follow-up. Eur J Nucl Med Mol Imaging 38:451-458.

587. Malandrino P, Latina A, Marescalco S, Spadaro A, Regalbuto C, Fulco RA, Scollo C, Vigneri R, Pellegriti G 2011 Risk-adapted management of differentiated thyroid cancer assessed by a sensitive measurement of basal serum thyroglobulin. J Clin Endocrinol Metab 96:17031709.

588. Soyluk O, Boztepe H, Aral F, Alagol F, Ozbey NC 2011 Papillary thyroid carcinoma patients assessed to be at 
low or intermediary risk after primary treatment are at greater risk of long term recurrence if they are thyroglobulin antibody positive or do not have distinctly low thyroglobulin at initial assessment. Thyroid 21:13011308.

589. Piccardo A, Arecco F, Morbelli S, Bianchi P, Barbera F, Finessi M, Corvisieri S, Pestarino E, Foppiani L, Villavecchia G, Cabria M, Orlandi F 2010 Low thyroglobulin concentrations after thyroidectomy increase the prognostic value of undetectable thyroglobulin levels on levo-thyroxine suppressive treatment in low-risk differentiated thyroid cancer. J Endocrinol Invest 33:83-87.

590. Castagna MG, Brilli L, Pilli T, Montanaro A, Cipri C, Fioravanti C, Sestini F, Capezzone M, Pacini F 2008 Limited value of repeat recombinant human thyrotropin (rhTSH)-stimulated thyroglobulin testing in differentiated thyroid carcinoma patients with previous negative rhTSHstimulated thyroglobulin and undetectable basal serum thyroglobulin levels. J Clin Endocrinol Metab 93:76-81.

591. Kloos RT, Mazzaferri EL 2005 A single recombinant human thyrotropin-stimulated serum thyroglobulin measurement predicts differentiated thyroid carcinoma metastases three to five years later. J Clin Endocrinol Metab 90:5047-5057.

592. Kloos RT 2010 Thyroid cancer recurrence in patients clinically free of disease with undetectable or very low serum thyroglobulin values. J Clin Endocrinol Metab 95:5241-5248.

593. Han JM, Kim WB, Yim JH, Kim WG, Kim TY, Ryu JS, Gong G, Sung TY, Yoon JH, Hong SJ, Kim EY, Shong YK 2012 Long-term clinical outcome of differentiated thyroid cancer patients with undetectable stimulated thyroglobulin level one year after initial treatment. Thyroid 22:784-790.

594. Rosario PW, Furtado MS, Mineiro Filho AF, Lacerda RX, Calsolari MR 2012 Value of repeat stimulated thyroglobulin testing in patients with differentiated thyroid carcinoma considered to be free of disease in the first year after ablation. Thyroid 22:482-486.

595. Brassard M, Borget I, Edet-Sanson A, Giraudet AL, Mundler O, Toubeau M, Bonichon F, Borson-Chazot F, Leenhardt L, Schvartz C, Dejax C, Brenot-Rossi I, Toubert ME, Torlontano M, Benhamou E, Schlumberger M 2011 Long-term follow-up of patients with papillary and follicular thyroid cancer: a prospective study on 715 patients. J Clin Endocrinol Metab 96:1352-1359.

596. Pelttari H, Valimaki MJ, Loyttyniemi E, Schalin-Jantti C 2010 Post-ablative serum thyroglobulin is an independent predictor of recurrence in low-risk differentiated thyroid carcinoma: a 16-year follow-up study. Eur J Endocrinol 163:757-763.

597. Klubo-Gwiezdzinska J, Burman KD, Van Nostrand D, Wartofsky L 2011 Does an undetectable rhTSH-stimulated Tg level 12 months after initial treatment of thyroid cancer indicate remission? Clin Endocrinol (Oxf) 74:111117.

598. Crocetti U, Durante C, Attard M, Maniglia A, Tumino S, Bruno R, Bonfitto N, Dicembrino F, Varraso A, Meringolo D, Filetti S, Trischitta V, Torlontano M 2008 Predictive value of recombinant human TSH stimulation and neck ultrasonography in differentiated thyroid cancer patients. Thyroid 18:1049-1053.

599. Torlontano M, Attard M, Crocetti U, Tumino S, Bruno R, Costante G, D’Azzo G, Meringolo D, Ferretti E,
Sacco R, Arturi F, Filetti S 2004 Follow-up of low risk patients with papillary thyroid cancer: role of neck ultrasonography in detecting lymph node metastases. J Clin Endocrinol Metab 89:3402-3407.

600. Verburg FA, Stokkel MP, Duren C, Verkooijen RB, Mader U, van Isselt JW, Marlowe RJ, Smit JW, Reiners C, Luster M 2010 No survival difference after successful (131)I ablation between patients with initially low-risk and high-risk differentiated thyroid cancer. Eur J Nucl Med Mol Imaging 37:276-283.

601. Giovanella L, Maffioli M, Ceriani L, De PD, Spriano G 2009 Unstimulated high sensitive thyroglobulin measurement predicts outcome of differentiated thyroid carcinoma. Clin Chem Lab Med 47:1001-1004.

602. Momesso DP, Tuttle RM 2014 Update on differentiated thyroid cancer staging. Endocrinol Metab Clin North Am 43:401-421.

603. Lemb J, Hufner M, Meller B, Homayounfar K, Sahlmann C, Meller J 2013 How reliable is secondary risk stratification with stimulated thyroglobulin in patients with differentiated thyroid carcinoma? Results from a retrospective study. Nuklearmedizin 52:88-96.

604. Nascimento C, Borget I, Al Ghuzlan A, Deandreis D, Chami L, Travagli JP, Hartl D, Lumbroso J, Chougnet C, Lacroix L, Baudin E, Schlumberger M, Leboulleux S 2011 Persistent disease and recurrence in differentiated thyroid cancer patients with undetectable postoperative stimulated thyroglobulin level. Endocr Relat Cancer 18:R29-R40.

605. Webb RC, Howard RS, Stojadinovic A, Gaitonde DY, Wallace MK, Ahmed J, Burch HB 2012 The utility of serum thyroglobulin measurement at the time of remnant ablation for predicting disease-free status in patients with differentiated thyroid cancer: a metaanalysis involving 3947 patients. J Clin Endocrinol Metab 97:2754-2763.

606. Chindris AM, Diehl NN, Crook JE, Fatourechi V, Smallridge RC 2012 Undetectable sensitive serum thyroglobulin $(<0.1 \mathrm{ng} / \mathrm{mL})$ in 163 patients with follicular cell-derived thyroid cancer: results of rhTSH stimulation and neck ultrasonography and long-term biochemical and clinical follow-up. J Clin Endocrinol Metab 97:2714-2723.

607. Vaisman F, Tala H, Grewal R, Tuttle RM 2011 In differentiated thyroid cancer, an incomplete structural response to therapy is associated with significantly worse clinical outcomes than only an incomplete thyroglobulin response. Thyroid 21:1317-1322.

608. Verburg FA, Luster M, Cupini C, Chiovato L, Duntas L, Elisei R, Feldt-Rasmussen U, Rimmele H, Seregni E, Smit JW, Theimer C, Giovanella L 2013 Implications of thyroglobulin antibody positivity in patients with differentiated thyroid cancer: a clinical position statement. Thyroid 23:1211-1225.

609. Spencer CA, Takeuchi M, Kazarosyan M, Wang CC, Guttler RB, Singer PA, Fatemi S, LoPresti JS, Nicoloff JT 1998 Serum thyroglobulin autoantibodies: prevalence, influence on serum thyroglobulin measurement, and prognostic significance in patients with differentiated thyroid carcinoma. J Clin Endocrinol Metab 83:1121-1127.

610. Chung JK, Park YJ, Kim TY, So Y, Kim SK, Park DJ, Lee DS, Lee MC, Cho BY 2002 Clinical significance of elevated level of serum antithyroglobulin antibody in patients with differentiated thyroid cancer after thyroid ablation. Clin Endocrinol (Oxf) 57:215-221. 
611. Gorges R, Maniecki M, Jentzen W, Sheu SN, Mann K, Bockisch A, Janssen OE 2005 Development and clinical impact of thyroglobulin antibodies in patients with differentiated thyroid carcinoma during the first 3 years after thyroidectomy. Eur J Endocrinol 153:49-55.

612. Seo JH, Lee SW, Ahn BC, Lee J 2010 Recurrence detection in differentiated thyroid cancer patients with elevated serum level of antithyroglobulin antibody: special emphasis on using (18)F-FDG PET/CT. Clin Endocrinol (Oxf) 72:558-563.

613. Adil A, Jafri RA, Waqar A, Abbasi SA, Matiul H, Asghar AH, Jilani A, Naz I 2003 Frequency and clinical importance of anti-Tg auto-antibodies (ATG). J Coll Physicians Surg Pak 13:504-506.

614. Kim WG, Yoon JH, Kim WB, Kim TY, Kim EY, Kim JM, Ryu JS, Gong G, Hong SJ, Shong YK 2008 Change of serum antithyroglobulin antibody levels is useful for prediction of clinical recurrence in thyroglobulinnegative patients with differentiated thyroid carcinoma. $\mathbf{J}$ Clin Endocrinol Metab 93:4683-4689.

615. Chiovato L, Latrofa F, Braverman LE, Pacini F, Capezzone M, Masserini L, Grasso L, Pinchera A 2003 Disappearance of humoral thyroid autoimmunity after complete removal of thyroid antigens. Ann Intern Med 139:346-351.

616. Thomas D, Liakos V, Vassiliou E, Hatzimarkou F, Tsatsoulis A, Kaldrimides P 2007 Possible reasons for different pattern disappearance of thyroglobulin and thyroid peroxidase autoantibodies in patients with differentiated thyroid carcinoma following total thyroidectomy and iodine-131 ablation. J Endocrinol Invest 30: 173-180.

617. Castagna MG, Tala Jury HP, Cipri C, Belardini V, Fioravanti C, Pasqui L, Sestini F, Theodoropoulou A, Pacini F 2011 The use of ultrasensitive thyroglobulin assays reduces but does not abolish the need for TSH stimulation in patients with differentiated thyroid carcinoma. J Endocrinol Invest 34:e219-e223.

618. Baudin E, Do Cao C, Cailleux AF, Leboulleux S, Travagli JP, Schlumberger M 2003 Positive predictive value of serum thyroglobulin levels, measured during the first year of follow-up after thyroid hormone withdrawal, in thyroid cancer patients. J Clin Endocrinol Metab 88: 1107-1111.

619. Pineda JD, Lee T, Ain K, Reynolds JC, Robbins J 1995 Iodine-131 therapy for thyroid cancer patients with elevated thyroglobulin and negative diagnostic scan. J Clin Endocrinol Metab 80:1488-1492.

620. Alzahrani AS, Mohamed G, Al Shammary A, Aldasouqi S, Abdal Salam S, Shoukri M 2005 Long-term course and predictive factors of elevated serum thyroglobulin and negative diagnostic radioiodine whole body scan in differentiated thyroid cancer. $\mathrm{J}$ Endocrinol Invest 28:540-546.

621. Valadao MM, Rosario PW, Borges MA, Costa GB, Rezende LL, Padrao EL, Barroso AL, Purisch S 2006 Positive predictive value of detectable stimulated tg during the first year after therapy of thyroid cancer and the value of comparison with $\mathrm{Tg}$-ablation and $\mathrm{Tg}$ measured after 24 months. Thyroid 16:1145-1149.

622. Miyauchi A, Kudo T, Miya A, Kobayashi K, Ito Y, Takamura Y, Higashiyama T, Fukushima M, Kihara M, Inoue H, Tomoda C, Yabuta T, Masuoka H 2011 Prognostic impact of serum thyroglobulin doubling-time un- der thyrotropin suppression in patients with papillary thyroid carcinoma who underwent total thyroidectomy. Thyroid 21:707-716.

623. Wong H, Wong KP, Yau T, Tang V, Leung R, Chiu J, Lang BH 2012 Is there a role for unstimulated thyroglobulin velocity in predicting recurrence in papillary thyroid carcinoma patients with detectable thyroglobulin after radioiodine ablation? Ann Surg Oncol 19:3479-3485.

624. Padovani RP, Robenshtok E, Brokhin M, Tuttle RM 2012 Even without additional therapy, serum thyroglobulin concentrations often decline for years after total thyroidectomy and radioactive remnant ablation in patients with differentiated thyroid cancer. Thyroid 22: 778-783.

625. Hsieh CJ, Wang PW 2014 Sequential changes of serum antithyroglobulin antibody levels are a good predictor of disease activity in thyroglobulin-negative patients with papillary thyroid carcinoma. Thyroid 24:488-493.

626. Schuff KG, Weber SM, Givi B, Samuels MH, Andersen PE, Cohen JI 2008 Efficacy of nodal dissection for treatment of persistent/recurrent papillary thyroid cancer. Laryngoscope 118:768-775.

627. Al-Saif O, Farrar WB, Bloomston M, Porter K, Ringel MD, Kloos RT 2010 Long-term efficacy of lymph node reoperation for persistent papillary thyroid cancer. J Clin Endocrinol Metab 95:2187-2194.

628. Yim JH, Kim WB, Kim EY, Kim WG, Kim TY, Ryu JS, Gong G, Hong SJ, Shong YK 2011 The outcomes of first reoperation for locoregionally recurrent/persistent papillary thyroid carcinoma in patients who initially underwent total thyroidectomy and remnant ablation. J Clin Endocrinol Metab 96:2049-2056.

629. Rondeau G, Fish S, Hann LE, Fagin JA, Tuttle RM 2011 Ultrasonographically detected small thyroid bed nodules identified after total thyroidectomy for differentiated thyroid cancer seldom show clinically significant structural progression. Thyroid 21:845-853.

630. Giovanella L, Ceriani L, Suriano S, Ghelfo A, Maffioli M 2008 Thyroglobulin measurement before rhTSHaided 131I ablation in detecting metastases from differentiated thyroid carcinoma. Clin Endocrinol (Oxf) 69:659-663.

631. Giovanella L, Ceriani L, Ghelfo A, Keller F 2005 Thyroglobulin assay 4 weeks after thyroidectomy predicts outcome in low-risk papillary thyroid carcinoma. Clin Chem Lab Med 43:843-847.

632. Phan HT, Jager PL, van der Wal JE, Sluiter WJ, Plukker JT, Dierckx RA, Wolffenbuttel BH, Links TP 2008 The follow-up of patients with differentiated thyroid cancer and undetectable thyroglobulin ( $\mathrm{Tg}$ ) and $\mathrm{Tg}$ antibodies during ablation. Eur J Endocrinol 158:77-83.

633. Vaisman A, Orlov S, Yip J, Hu C, Lim T, Dowar M, Freeman JL, Walfish PG 2010 Application of postsurgical stimulated thyroglobulin for radioiodine remnant ablation selection in low-risk papillary thyroid carcinoma. Head Neck 32:689-698.

634. Kim TY, Kim WB, Kim ES, Ryu JS, Yeo JS, Kim SC, Hong SJ, Shong YK 2005 Serum thyroglobulin levels at the time of 131I remnant ablation just after thyroidectomy are useful for early prediction of clinical recurrence in low-risk patients with differentiated thyroid carcinoma. J Clin Endocrinol Metab 90:1440-1445.

635. Toubeau M, Touzery C, Arveux P, Chaplain G, Vaillant $\mathrm{G}$, Berriolo A, Riedinger JM, Boichot C, Cochet A, 
Brunotte F 2004 Predictive value for disease progression of serum thyroglobulin levels measured in the postoperative period and after (131)I ablation therapy in patients with differentiated thyroid cancer. J Nucl Med 45:988-994.

636. Piccardo A, Arecco F, Puntoni M, Foppiani L, Cabria M, Corvisieri S, Arlandini A, Altrinetti V, Bandelloni R, Orlandi F 2013 Focus on high-risk DTC patients: high postoperative serum thyroglobulin level is a strong predictor of disease persistence and is associated to progression-free survival and overall survival. Clin Nucl Med 38:18-24.

637. Polachek A, Hirsch D, Tzvetov G, Grozinsky-Glasberg S, Slutski I, Singer J, Weinstein R, Shimon I, Benbassat CA 2011 Prognostic value of post-thyroidectomy thyroglobulin levels in patients with differentiated thyroid cancer. J Endocrinol Invest 34:855-860.

638. Hall FT, Beasley NJ, Eski SJ, Witterick IJ, Walfish PG, Freeman JL 2003 Predictive value of serum thyroglobulin after surgery for thyroid carcinoma. Laryngoscope 113:77-81.

639. Heemstra KA, Liu YY, Stokkel M, Kievit J, Corssmit E, Pereira AM, Romijn JA, Smit JW 2007 Serum thyroglobulin concentrations predict disease-free remission and death in differentiated thyroid carcinoma. Clin Endocrinol (Oxf) 66:58-64.

640. Ronga G, Filesi M, Ventroni G, Vestri AR, Signore A 1999 Value of the first serum thyroglobulin level after total thyroidectomy for the diagnosis of metastases from differentiated thyroid carcinoma. Eur $\mathbf{J}$ Nucl Med 26: $1448-1452$.

641. Lin JD, Huang MJ, Hsu BR, Chao TC, Hsueh C, Liu FH, Liou MJ, Weng HF 2002 Significance of postoperative serum thyroglobulin levels in patients with papillary and follicular thyroid carcinomas. J Surg Oncol 80:45-51.

642. Ibrahimpasic T, Nixon IJ, Palmer FL, Whitcher MM, Tuttle RM, Shaha A, Patel SG, Shah JP, Ganly I 2012 Undetectable thyroglobulin after total thyroidectomy in patients with low- and intermediate-risk papillary thyroid cancer-is there a need for radioactive iodine therapy? Surgery 152:1096-1105.

643. Avram AM, Fig LM, Frey KA, Gross MD, Wong KK 2013 Preablation 131-I scans with SPECT/CT in postoperative thyroid cancer patients: what is the impact on staging? J Clin Endocrinol Metab 98:1163-1171.

644. Nascimento C, Borget I, Troalen F, Al Ghuzlan A, Deandreis D, Hartl D, Lumbroso J, Chougnet CN, Baudin E, Schlumberger M, Leboulleux S 2013 Ultrasensitive serum thyroglobulin measurement is useful for the follow-up of patients treated with total thyroidectomy without radioactive iodine ablation. Eur $\mathbf{J}$ Endocrinol 169:689-693.

645. Rosario PW, Xavier AC, Calsolari MR 2011 Value of postoperative thyroglobulin and ultrasonography for the indication of ablation and 131I activity in patients with thyroid cancer and low risk of recurrence. Thyroid 21:49-53.

646. Robenshtok E, Grewal RK, Fish S, Sabra M, Tuttle RM 2013 A low postoperative nonstimulated serum thyroglobulin level does not exclude the presence of radioactive iodine avid metastatic foci in intermediate-risk differentiated thyroid cancer patients. Thyroid 23:436-442.

647. de Rosario PW, Guimaraes VC, Maia FF, Fagundes TA, Purisch S, Padrao EL, Rezende LL, Barroso AL 2005
Thyroglobulin before ablation and correlation with posttreatment scanning. Laryngoscope 115:264-267.

648. Park EK, Chung JK, Lim IH, Park do J, Lee DS, Lee MC, Cho BY 2009 Recurrent/metastatic thyroid carcinomas false negative for serum thyroglobulin but positive by posttherapy I-131 whole body scans. Eur J Nucl Med Mol Imaging 36:172-179.

649. Giovanella L, Suriano S, Ceriani L, Verburg FA 2011 Undetectable thyroglobulin in patients with differentiated thyroid carcinoma and residual radioiodine uptake on a postablation whole-body scan. Clin Nucl Med 36:109-112.

650. Oyen WJ, Verhagen C, Saris E, van den Broek WJ, Pieters GF, Corsten FH 2000 Follow-up regimen of differentiated thyroid carcinoma in thyroidectomized patients after thyroid hormone withdrawal. J Nucl Med 41:643-646.

651. Tamilia M, Al-Kahtani N, Rochon L, Hier MP, Payne RJ, Holcroft CA, Black MJ 2011 Serum thyroglobulin predicts thyroid remnant ablation failure with $30 \mathrm{mCi}$ iodine-131 treatment in patients with papillary thyroid carcinoma. Nucl Med Commun 32:212-220.

652. Bernier MO, Morel O, Rodien P, Muratet JP, Giraud P, Rohmer V, Jeanguillaume C, Bigorgne JC, Jallet P 2005 Prognostic value of an increase in the serum thyroglobulin level at the time of the first ablative radioiodine treatment in patients with differentiated thyroid cancer. Eur J Nucl Med Mol Imaging 32:1418-1421.

653. Feldt-Rasmussen U, Petersen PH, Date J, Madsen CM 1982 Serum thyroglobulin in patients undergoing subtotal thyroidectomy for toxic and nontoxic goiter. J Endocrinol Invest 5:161-164.

654. Feldt-Rasmussen U, Petersen PH, Nielsen H, Date J, Madsen CM 1978 Thyroglobulin of varying molecular sizes with different disappearance rates in plasma following subtotal thyroidectomy. Clin Endocrinol (Oxf) 9:205-214.

655. Izumi M, Kubo I, Taura M, Yamashita S, Morimoto I, Ohtakara S, Okamoto S, Kumagai LF, Nagataki S 1986 Kinetic study of immunoreactive human thyroglobulin. J Clin Endocrinol Metab 62:410-412.

656. Hocevar M, Auersperg M, Stanovnik L 1997 The dynamics of serum thyroglobulin elimination from the body after thyroid surgery. Eur J Surg Oncol 23:208-210.

657. Gerfo PL, Colacchio T, Colacchio D, Feind C 1978 Serum clearance rates of immunologically reactive thyroglobulin. Cancer 42:164-166.

658. Giovanella L, Ceriani L, Maffioli M 2010 Postsurgery serum thyroglobulin disappearance kinetic in patients with differentiated thyroid carcinoma. Head Neck 32: 568-571.

659. Lee JI, Chung YJ, Cho BY, Chong S, Seok JW, Park SJ 2013 Postoperative-stimulated serum thyroglobulin measured at the time of 131I ablation is useful for the prediction of disease status in patients with differentiated thyroid carcinoma. Surgery 153:828-835.

660. Lepoutre-Lussey C, Maddah D, Golmard JL, Russ G, Tissier F, Tresallet C, Menegaux F, Aurengo A, Leenhardt L 2014 Post-operative neck ultrasound and risk stratification in differentiated thyroid cancer patients with initial lymph node involvement. Eur J Endocrinol 170:837-846.

661. Chen MK, Yasrebi M, Samii J, Staib LH, Doddamane I, Cheng DW 2012 The utility of I-123 pretherapy scan in 
I-131 radioiodine therapy for thyroid cancer. Thyroid 22:304-309.

662. Van Nostrand D, Aiken M, Atkins F, Moreau S, Garcia C, Acio E, Burman K, Wartofsky L 2009 The utility of radioiodine scans prior to iodine 131 ablation in patients with well-differentiated thyroid cancer. Thyroid 19:849855.

663. Hu YH, Wang PW, Wang ST, Lee CH, Chen HY, Chou FF, Huang YE, Huang HH 2004 Influence of 131I diagnostic dose on subsequent ablation in patients with differentiated thyroid carcinoma: discrepancy between the presence of visually apparent stunning and the impairment of successful ablation. Nucl Med Commun 25:793-797.

664. Morris LF, Waxman AD, Braunstein GD 2001 The nonimpact of thyroid stunning: remnant ablation rates in 131I-scanned and nonscanned individuals. J Clin Endocrinol Metab 86:3507-3511.

665. Muratet JP, Daver A, Minier JF, Larra F 1998 Influence of scanning doses of iodine-131 on subsequent first ablative treatment outcome in patients operated on for differentiated thyroid carcinoma. J Nucl Med 39:1546-1550.

666. Silberstein EB 2007 Comparison of outcomes after (123)I versus (131)I pre-ablation imaging before radioiodine ablation in differentiated thyroid carcinoma. $\mathrm{J}$ Nucl Med 48:1043-1046.

667. Leger FA, Izembart M, Dagousset F, Barritault L, Baillet G, Chevalier A, Clerc J 1998 Decreased uptake of therapeutic doses of iodine-131 after $185-\mathrm{MBq}$ iodine131 diagnostic imaging for thyroid remnants in differentiated thyroid carcinoma. Eur J Nucl Med 25:242-246.

668. Verkooijen RB, Verburg FA, van Isselt JW, Lips CJ, Smit JW, Stokkel MP 2008 The success rate of I-131 ablation in differentiated thyroid cancer: comparison of uptake-related and fixed-dose strategies. Eur J Endocrinol 159:301-307.

669. Verburg FA, Verkooijen RB, Stokkel MP, van Isselt JW 2009 The success of 131I ablation in thyroid cancer patients is significantly reduced after a diagnostic activity of $40 \mathrm{MBq} 131 \mathrm{I}$. Nuklearmedizin 48:138-142.

670. Yap BK, Murby B 2014 No adverse affect in clinical outcome using low preablation diagnostic (131)I activity in differentiated thyroid cancer: refuting thyroidstunning effect. J Clin Endocrinol Metab 99:2433-2440.

671. Jonklaas J, Sarlis NJ, Litofsky D, Ain KB, Bigos ST, Brierley JD, Cooper DS, Haugen BR, Ladenson PW, Magner J, Robbins J, Ross DS, Skarulis M, Maxon HR, Sherman SI 2006 Outcomes of patients with differentiated thyroid carcinoma following initial therapy. Thyroid 16:1229-1242.

672. Jonklaas J, Cooper DS, Ain KB, Bigos T, Brierley JD, Haugen BR, Ladenson PW, Magner J, Ross DS, Skarulis MC, Steward DL, Maxon HR, Sherman SI 2010 Radioiodine therapy in patients with stage I differentiated thyroid cancer. Thyroid 20:1423-1424.

673. Sacks W, Fung CH, Chang JT, Waxman A, Braunstein GD 2010 The effectiveness of radioactive iodine for treatment of low-risk thyroid cancer: a systematic analysis of the peer-reviewed literature from 1966 to April 2008. Thyroid 20:1235-1245.

674. Sawka AM, Brierley JD, Tsang RW, Thabane L, Rotstein L, Gafni A, Straus S, Goldstein DP 2008 An updated systematic review and commentary examining the effectiveness of radioactive iodine remnant ablation in well-differentiated thyroid cancer. Endocrinol Metab Clin North Am 37:457-480.

675. Lamartina L, Durante C, Filetti S, Cooper DS 2015 Lowrisk differentiated thyroid cancer and radioiodine remnant ablation: a systematic review of the literature. J Clin Endocrinol Metab 100:1748-1761.

676. Ross DS, Litofsky D, Ain KB, Bigos T, Brierley JD, Cooper DS, Haugen BR, Jonklaas J, Ladenson PW, Magner J, Robbins J, Skarulis MC, Steward DL, Maxon HR, Sherman SI 2009 Recurrence after treatment of micropapillary thyroid cancer. Thyroid 19:1043-1048.

677. Kim HJ, Kim NK, Choi JH, Kim SW, Jin SM, Suh S, Bae JC, Min YK, Chung JH, Kim SW 2013 Radioactive iodine ablation does not prevent recurrences in patients with papillary thyroid microcarcinoma. Clin Endocrinol (Oxf) 78:614-620.

678. Baudin E, Travagli JP, Ropers J, Mancusi F, BrunoBossio G, Caillou B, Cailleux AF, Lumbroso JD, Parmentier C, Schlumberger M 1998 Microcarcinoma of the thyroid gland: the Gustave-Roussy Institute experience. Cancer 83:553-559.

679. Creach KM, Siegel BA, Nussenbaum B, Grigsby PW 2012 Radioactive iodine therapy decreases recurrence in thyroid papillary microcarcinoma. ISRN Endocrinol 2012:816386.

680. Lin HW, Bhattacharyya N 2009 Survival impact of treatment options for papillary microcarcinoma of the thyroid. Laryngoscope 119:1983-1987.

681. Kuo EJ, Roman SA, Sosa JA 2013 Patients with follicular and Hurthle cell microcarcinomas have compromised survival: a population level study of 22,738 patients. Surgery 154:1246-1253.

682. Mallick U, Harmer C, Hackshaw A, Moss L 2012 Iodine or Not (IoN) for low-risk differentiated thyroid cancer: the next UK National Cancer Research Network randomised trial following HiLo. Clin Oncol (R Coll Radiol ) 24:159-161.

683. Kazaure HS, Roman SA, Sosa JA 2012 Aggressive variants of papillary thyroid cancer: incidence, characteristics and predictors of survival among 43,738 patients. Ann Surg Oncol 19:1874-1880.

684. Kazaure HS, Roman SA, Sosa JA 2012 Insular thyroid cancer: a population-level analysis of patient characteristics and predictors of survival. Cancer 118:3260-3267.

685. Ruel E, Thomas S, Dinan M, Perkins JM, Roman SA, Sosa JA 2015 Adjuvant radioactive iodine therapy is associated with improved survival for patients with intermediate-risk papillary thyroid cancer. J Clin Endocrinol Metab 100:1529-1536.

686. Chow SM, Yau S, Kwan CK, Poon PC, Law SC 2006 Local and regional control in patients with papillary thyroid carcinoma: specific indications of external radiotherapy and radioactive iodine according to $\mathrm{T}$ and $\mathrm{N}$ categories in AJCC 6th edition. Endocr Relat Cancer 13:1159-1172.

687. Podnos YD, Smith DD, Wagman LD, Ellenhorn JD 2007 Survival in patients with papillary thyroid cancer is not affected by the use of radioactive isotope. J Surg Oncol 96:3-7.

688. Chakravarty D, Santos E, Ryder M, Knauf JA, Liao XH, West BL, Bollag G, Kolesnick R, Thin TH, Rosen N, Zanzonico P, Larson SM, Refetoff S, Ghossein R, Fagin JA 2011 Small-molecule MAPK inhibitors restore radioiodine incorporation in mouse thyroid cancers with conditional BRAF activation. J Clin Invest 121:4700-4711.

689. Edmonds CJ, Hayes S, Kermode JC, Thompson BD 1977 Measurement of serum TSH and thyroid hormones in the 
management of treatment of thyroid carcinoma with radioiodine. Br J Radiol 50:799-807.

690. Leboeuf R, Perron P, Carpentier AC, Verreault J, Langlois MF 2007 L-T3 preparation for whole-body scintigraphy: a randomized-controlled trial. Clin Endocrinol (Oxf) 67:839-844.

691. Lee J, Yun MJ, Nam KH, Chung WY, Soh EY, Park CS 2010 Quality of life and effectiveness comparisons of thyroxine withdrawal, triiodothyronine withdrawal, and recombinant thyroid-stimulating hormone administration for low-dose radioiodine remnant ablation of differentiated thyroid carcinoma. Thyroid 20:173-179.

692. Fallahi B, Beiki D, Takavar A, Fard-Esfahani A, Gilani KA, Saghari M, Eftekhari M 2012 Low versus high radioiodine dose in postoperative ablation of residual thyroid tissue in patients with differentiated thyroid carcinoma: a large randomized clinical trial. Nucl Med Commun 33:275-282.

693. Goropoulos A, Karamoshos K, Christodoulou A, Ntitsias T, Paulou K, Samaras A, Xirou P, Efstratiou I 2004 Value of the cervical compartments in the surgical treatment of papillary thyroid carcinoma. World J Surg 28:1275-1281.

694. Molinaro E, Giani C, Agate L, Biagini A, Pieruzzi L, Bianchi F, Brozzi F, Ceccarelli C, Viola D, Piaggi P, Vitti P, Pacini F, Elisei R 2013 Patients with differentiated thyroid cancer who underwent radioiodine thyroid remnant ablation with low-activity ${ }^{131}$ I after either recombinant human TSH or thyroid hormone therapy withdrawal showed the same outcome after a 10-year follow-up. J Clin Endocrinol Metab 98:2693-2700.

695. Prpic M, Dabelic N, Stanicic J, Jukic T, Milosevic M, Kusic Z 2012 Adjuvant thyroid remnant ablation in patients with differentiated thyroid carcinoma confined to the thyroid: a comparison of ablation success with different activities of radioiodine (I-131). Ann Nucl Med 26:744-751.

696. Karam M, Gianoukakis A, Feustel PJ, Cheema A, Postal ES, Cooper JA 2003 Influence of diagnostic and therapeutic doses on thyroid remnant ablation rates. Nucl Med Commun 24:489-495.

697. Robbins RJ, Driedger A, Magner J 2006 Recombinant human thyrotropin-assisted radioiodine therapy for patients with metastatic thyroid cancer who could not elevate endogenous thyrotropin or be withdrawn from thyroxine. Thyroid 16:1121-1130.

698. Chianelli M, Todino V, Graziano FM, Panunzi C, Pace D, Guglielmi R, Signore A, Papini E 2009 Low-activity (2.0 GBq; $54 \mathrm{mCi}$ ) radioiodine post-surgical remnant ablation in thyroid cancer: comparison between hormone withdrawal and use of rhTSH in low-risk patients. Eur J Endocrinol 160:431-436.

699. Mallick U, Harmer C, Yap B, Wadsley J, Clarke S, Moss L, Nicol A, Clark PM, Farnell K, McCready R, Smellie J, Franklyn JA, John R, Nutting CM, Newbold K, Lemon C, Gerrard G, Abdel-Hamid A, Hardman J, Macias E, Roques T, Whitaker S, Vijayan R, Alvarez P, Beare S, Forsyth S, Kadalayil L, Hackshaw A 2012 Ablation with low-dose radioiodine and thyrotropin alfa in thyroid cancer. N Engl J Med 366:1674-1685.

700. Pacini F, Ladenson PW, Schlumberger M, Driedger A, Luster M, Kloos RT, Sherman S, Haugen B, Corone C, Molinaro E, Elisei R, Ceccarelli C, Pinchera A, Wahl RL, Leboulleux S, Ricard M, Yoo J, Busaidy NL, Del- passand E, Hanscheid H, Felbinger R, Lassmann M, Reiners C 2006 Radioiodine ablation of thyroid remnants after preparation with recombinant human thyrotropin in differentiated thyroid carcinoma: results of an international, randomized, controlled study. J Clin Endocrinol Metab 91:926-932.

701. Schlumberger M, Catargi B, Borget I, Deandreis D, Zerdoud S, Bridji B, Bardet S, Leenhardt L, Bastie D, Schvartz C, Vera P, Morel O, Benisvy D, Bournaud C, Bonichon F, Dejax C, Toubert ME, Leboulleux S, Ricard M, Benhamou E 2012 Strategies of radioiodine ablation in patients with low-risk thyroid cancer. N Engl J Med 366: $1663-1673$.

702. Taieb D, Sebag F, Cherenko M, Baumstarck-Barrau K, Fortanier C, Farman-Ara B, de Micco C, Vaillant J, Thomas S, Conte-Devolx B, Loundou A, Auquier P, Henry JF, Mundler O 2009 Quality of life changes and clinical outcomes in thyroid cancer patients undergoing radioiodine remnant ablation (RRA) with recombinant human TSH (rhTSH): a randomized controlled study. Clin Endocrinol (Oxf) 71:115-123.

703. Emmanouilidis N, Muller JA, Jager MD, Kaaden S, Helfritz FA, Guner Z, Kespohl H, Knitsch W, Knapp WH, Klempnauer J, Scheumann GF 2009 Surgery and radioablation therapy combined: introducing a 1-weekcondensed procedure bonding total thyroidectomy and radioablation therapy with recombinant human TSH. Eur J Endocrinol 161:763-769.

704. Tu J, Wang S, Huo Z, Lin Y, Li X, Wang S 2014 Recombinant human thyrotropin-aided versus thyroid hormone withdrawal-aided radioiodine treatment for differentiated thyroid cancer after total thyroidectomy: a meta-analysis. Radiother Oncol 110:25-30.

705. Pak K, Cheon GJ, Kang KW, Kim SJ, Kim IJ, Kim EE, Lee DS, Chung JK 2014 The effectiveness of recombinant human thyroid-stimulating hormone versus thyroid hormone withdrawal prior to radioiodine remnant ablation in thyroid cancer: a meta-analysis of randomized controlled trials. J Korean Med Sci 29:811-817.

706. Elisei R, Schlumberger M, Driedger A, Reiners C, Kloos RT, Sherman SI, Haugen B, Corone C, Molinaro E, Grasso L, Leboulleux S, Rachinsky I, Luster M, Lassmann M, Busaidy NL, Wahl RL, Pacini F, Cho SY, Magner J, Pinchera A, Ladenson PW 2009 Follow-up of low-risk differentiated thyroid cancer patients who underwent radioiodine ablation of postsurgical thyroid remnants after either recombinant human thyrotropin or thyroid hormone withdrawal. J Clin Endocrinol Metab 94:4171-4179.

707. Emmanouilidis N, Schrem H, Winkler M, Klempnauer J, Scheumann GF 2013 Long-term results after treatment of very low-, low-, and high-risk thyroid cancers in a combined setting of thyroidectomy and radio ablation therapy in euthyroidism. Int J Endocrinol 2013:769473.

708. Hugo J, Robenshtok E, Grewal R, Larson S, Tuttle RM 2012 Recombinant human thyroid stimulating hormoneassisted radioactive iodine remnant ablation in thyroid cancer patients at intermediate to high risk of recurrence. Thyroid 22:1007-1015.

709. Rosario PW, Mineiro Filho AF, Lacerda RX, Calsolari MR 2012 Long-term follow-up of at least five years after recombinant human thyrotropin compared to levothyroxine withdrawal for thyroid remnant ablation with radioactive iodine. Thyroid 22:332-333. 
710. Pitoia F, Marlowe RJ, Abelleira E, Faure EN, Bueno F, Schwarzstein D, Lutfi RJ, Niepomniszcze H 2012 Radioiodine thyroid remnant ablation after recombinant human thyrotropin or thyroid hormone withdrawal in patients with high-risk differentiated thyroid cancer. J Thyroid Res 2012:481568.

711. Bartenstein P, Calabuig EC, Maini CL, Mazzarotto R, Muros de Fuentes MA, Petrich T, Rodrigues FJ, Vallejo Casas JA, Vianello F, Basso M, Balaguer MG, Haug A, Monari F, Vano RS, Sciuto R, Magner J 2014 High-risk patients with differentiated thyroid cancer T4 primary tumors achieve remnant ablation equally well using rhTSH or thyroid hormone withdrawal. Thyroid 24:480487.

712. Tala H, Robbins R, Fagin JA, Larson SM, Tuttle RM 2011 Five-year survival is similar in thyroid cancer patients with distant metastases prepared for radioactive iodine therapy with either thyroid hormone withdrawal or recombinant human TSH. J Clin Endocrinol Metab 96:2105-2111.

713. Klubo-Gwiezdzinska J, Burman KD, Van Nostrand D, Mete M, Jonklaas J, Wartofsky L 2012 Radioiodine treatment of metastatic thyroid cancer: relative efficacy and side effect profile of preparation by thyroid hormone withdrawal versus recombinant human thyrotropin. Thyroid 22:310-317.

714. Maenpaa HO, Heikkonen J, Vaalavirta L, Tenhunen M, Joensuu H 2008 Low vs. high radioiodine activity to ablate the thyroid after thyroidectomy for cancer: a randomized study. PLoS One 3:e1885.

715. Pilli T, Brianzoni E, Capoccetti F, Castagna MG, Fattori S, Poggiu A, Rossi G, Ferretti F, Guarino E, Burroni L, Vattimo A, Cipri C, Pacini F 2007 A comparison of 1850 $(50 \mathrm{mCi})$ and $3700 \mathrm{MBq}(100 \mathrm{mCi})$ 131-iodine administered doses for recombinant thyrotropin-stimulated postoperative thyroid remnant ablation in differentiated thyroid cancer. J Clin Endocrinol Metab 92:3542-3546.

716. Zaman M, Toor R, Kamal S, Maqbool M, Habib S, Niaz K 2006 A randomized clinical trial comparing $50 \mathrm{mCi}$ and $100 \mathrm{mCi}$ of iodine-131 for ablation of differentiated thyroid cancers. J Pak Med Assoc 56:353-356.

717. Kukulska A, Krajewska J, Gawkowska-Suwinska M, Puch Z, Paliczka-Cieslik E, Roskosz J, HandkiewiczJunak D, Jarzab M, Gubala E, Jarzab B 2010 Radioiodine thyroid remnant ablation in patients with differentiated thyroid carcinoma (DTC): prospective comparison of long-term outcomes of treatment with 30,60 and 100 mCi. Thyroid Res 3:9.

718. Fang Y, Ding Y, Guo Q, Xing J, Long Y, Zong Z 2013 Radioiodine therapy for patients with differentiated thyroid cancer after thyroidectomy: direct comparison and network meta-analyses. J Endocrinol Invest 36:896902.

719. Ma C, Tang L, Fu H, Li J, Wang H 2013 rhTSH-aided low-activity versus high-activity regimens of radioiodine in residual ablation for differentiated thyroid cancer: a meta-analysis. Nucl Med Commun 34:1150-1156.

720. Cheng W, Ma C, Fu H, Li J, Chen S, Wu S, Wang H 2013 Low- or high-dose radioiodine remnant ablation for differentiated thyroid carcinoma: a meta-analysis. J Clin Endocrinol Metab 98:1353-1360.

721. Valachis A, Nearchou A 2013 High versus low radioiodine activity in patients with differentiated thyroid cancer: a meta-analysis. Acta Oncol 52:1055-1061.
722. Verburg FA, Mader U, Reiners C, Hanscheid H 2014 Long term survival in DTC is worse after low-activity initial post-surgical I-131 therapy in both high and low risk patients. J Clin Endocrinol Metab 99:4487-4496.

723. Castagna MG, Cevenini G, Theodoropoulou A, Maino F, Memmo S, Claudia C, Belardini V, Brianzoni E, Pacini F 2013 Post-surgical thyroid ablation with low or high radioiodine activities results in similar outcomes in intermediate risk differentiated thyroid cancer patients. Eur J Endocrinol 169:23-29.

724. Han JM, Kim WG, Kim TY, Jeon MJ, Ryu JS, Song DE, Hong SJ, Shong YK, Kim WB 2014 Effects of low-dose and high-dose postoperative radioiodine therapy on the clinical outcome in patients with small differentiated thyroid cancer having microscopic extrathyroidal extension. Thyroid 24:820-825.

725. Kruijff S, Aniss AM, Chen P, Sidhu SB, Delbridge LW, Robinson B, Clifton-Bligh RJ, Roach P, Gill AJ, Learoyd D, Sywak MS 2013 Decreasing the dose of radioiodine for remnant ablation does not increase structural recurrence rates in papillary thyroid carcinoma. Surgery 154: 1337-1344.

726. Sabra M, Grewal R, Ghossein RM, Tuttle RM 2014 Higher administered activities of radioactive iodine are associated with less structural persistent response in older, but not younger, papillary thyroid cancer patients with lateral neck lymph node metastases. Thyroid 24:1088-1095.

727. Sawka AM, Ibrahim-Zada I, Galacgac P, Tsang RW, Brierley JD, Ezzat S, Goldstein DP 2010 Dietary iodine restriction in preparation for radioactive iodine treatment or scanning in well-differentiated thyroid cancer: a systematic review. Thyroid 20:1129-1138.

728. Pluijmen MJ, Eustatia-Rutten C, Goslings BM, Stokkel MP, Arias AM, Diamant M, Romijn JA, Smit JW 2003 Effects of low-iodide diet on postsurgical radioiodide ablation therapy in patients with differentiated thyroid carcinoma. Clin Endocrinol (Oxf) 58:428-435.

729. Morris LF, Wilder MS, Waxman AD, Braunstein GD 2001 Reevaluation of the impact of a stringent lowiodine diet on ablation rates in radioiodine treatment of thyroid carcinoma. Thyroid 11:749-755.

730. Morsch EP, Vanacor R, Furlanetto TW, Schmid H 2011 Two weeks of a low-iodine diet are equivalent to 3 weeks for lowering urinary iodine and increasing thyroid radioactive iodine uptake. Thyroid 21:61-67.

731. Tala Jury HP, Castagna MG, Fioravanti C, Cipri C, Brianzoni E, Pacini F 2010 Lack of association between urinary iodine excretion and successful thyroid ablation in thyroid cancer patients. J Clin Endocrinol Metab 95:230-237.

732. Al Nozha OM, Vautour L, How J 2011 Life-threatening hyponatremia following a low-iodine diet: a case report and review of all reported cases. Endocr Pract 17:e113-e117.

733. Sherman SI, Tielens ET, Sostre S, Wharam MD Jr, Ladenson PW 1994 Clinical utility of posttreatment radioiodine scans in the management of patients with thyroid carcinoma. J Clin Endocrinol Metab 78:629-634.

734. Fatourechi V, Hay ID, Mullan BP, Wiseman GA, Eghbali-Fatourechi GZ, Thorson LM, Gorman CA 2000 Are posttherapy radioiodine scans informative and do they influence subsequent therapy of patients with differentiated thyroid cancer? Thyroid 10:573-577.

735. Souza Rosario PW, Barroso AL, Rezende LL, Padrao EL, Fagundes TA, Penna GC, Purisch S 2004 Post I-131 
therapy scanning in patients with thyroid carcinoma metastases: an unnecessary cost or a relevant contribution? Clin Nucl Med 29:795-798.

736. Spies WG, Wojtowicz CH, Spies SM, Shah AY, Zimmer AM 1989 Value of post-therapy whole-body I-131 imaging in the evaluation of patients with thyroid carcinoma having undergone high-dose I-131 therapy. Clin Nucl Med 14:793-800.

737. Ciappuccini R, Heutte N, Trzepla G, Rame JP, Vaur D, Aide N, Bardet S 2011 Postablation (131)I scintigraphy with neck and thorax SPECT-CT and stimulated serum thyroglobulin level predict the outcome of patients with differentiated thyroid cancer. Eur J Endocrinol 164:961969.

738. Salvatori M, Perotti G, Villani MF, Mazza R, Maussier ML, Indovina L, Sigismondi A, Dottorini ME, Giordano A 2013 Determining the appropriate time of execution of an I-131 post-therapy whole-body scan: comparison between early and late imaging. Nucl Med Commun 34:900-908.

739. Kohlfuerst S, Igerc I, Lobnig $M$, Gallowitsch HJ, Gomez-Segovia I, Matschnig S, Mayr J, Mikosch P, Beheshti M, Lind P 2009 Posttherapeutic (131)I SPECTCT offers high diagnostic accuracy when the findings on conventional planar imaging are inconclusive and allows a tailored patient treatment regimen. Eur J Nucl Med Mol Imaging 36:886-893.

740. Chen L, Luo Q, Shen Y, Yu Y, Yuan Z, Lu H, Zhu R 2008 Incremental value of 131I SPECT/CT in the management of patients with differentiated thyroid carcinoma. J Nucl Med 49:1952-1957.

741. Schmidt D, Linke R, Uder M, Kuwert T 2010 Five months' follow-up of patients with and without iodinepositive lymph node metastases of thyroid carcinoma as disclosed by (131)I-SPECT/CT at the first radioablation. Eur J Nucl Med Mol Imaging 37:699-705.

742. Maruoka Y, Abe K, Baba S, Isoda T, Sawamoto H, Tanabe Y, Sasaki M, Honda H 2012 Incremental diagnostic value of SPECT/CT with 131I scintigraphy after radioiodine therapy in patients with well-differentiated thyroid carcinoma. Radiology 265:902-909.

743. Grewal RK, Tuttle RM, Fox J, Borkar S, Chou JF, Gonen M, Strauss HW, Larson SM, Schoder H 2010 The effect of posttherapy 131I SPECT/CT on risk classification and management of patients with differentiated thyroid cancer. J Nucl Med 51:1361-1367.

744. Brabant G 2008 Thyrotropin suppressive therapy in thyroid carcinoma: what are the targets? J Clin Endocrinol Metab 93:1167-1169.

745. McGriff NJ, Csako G, Gourgiotis L, Lori CG, Pucino F, Sarlis NJ 2002 Effects of thyroid hormone suppression therapy on adverse clinical outcomes in thyroid cancer. Ann Med 34:554-564.

746. Diessl S, Holzberger B, Mader U, Grelle I, Smit JW, Buck AK, Reiners C, Verburg FA 2012 Impact of moderate vs stringent TSH suppression on survival in advanced differentiated thyroid carcinoma. Clin Endocrinol (Oxf) 76:586-592.

747. Biondi B, Cooper DS 2010 Benefits of thyrotropin suppression versus the risks of adverse effects in differentiated thyroid cancer. Thyroid 20:135-146.

748. Sugitani I, Fujimoto Y 2011 Effect of postoperative thyrotropin suppressive therapy on bone mineral density in patients with papillary thyroid carcinoma: a prospective controlled study. Surgery 150:1250-1257.
749. Pujol P, Daures JP, Nsakala N, Baldet L, Bringer J, Jaffiol C 1996 Degree of thyrotropin suppression as a prognostic determinant in differentiated thyroid cancer. J Clin Endocrinol Metab 81:4318-4323.

750. Hovens GC, Stokkel MP, Kievit J, Corssmit EP, Pereira AM, Romijn JA, Smit JW 2007 Associations of serum thyrotropin concentrations with recurrence and death in differentiated thyroid cancer. J Clin Endocrinol Metab 92:2610-2615.

751. Sawin CT, Geller A, Wolf PA, Belanger AJ, Baker E, Bacharach P, Wilson PW, Benjamin EJ, D'Agostino RB 1994 Low serum thyrotropin concentrations as a risk factor for atrial fibrillation in older persons. N Engl J Med 331:1249-1252.

752. Toft AD 2001 Clinical practice. Subclinical hyperthyroidism. N Engl J Med 345:512-516.

753. Chen CH, Chen JF, Yang BY, Liu RT, Tung SC, Chien WY, Lu YC, Kuo MC, Hsieh CJ, Wang PW 2004 Bone mineral density in women receiving thyroxine suppressive therapy for differentiated thyroid carcinoma. J Formos Med Assoc 103:442-447.

754. Panico A, Lupoli GA, Fonderico F, Marciello F, Martinelli A, Assante R, Lupoli G 2009 Osteoporosis and thyrotropin-suppressive therapy: reduced effectiveness of alendronate. Thyroid 19:437-442.

755. Ebina A, Sugitani I, Fujimoto Y, Yamada K 2014 Riskadapted management of papillary thyroid carcinoma according to our own risk group classification system: is thyroid lobectomy the treatment of choice for low-risk patients? Surgery 156: 1579-1588.

756. Ford D, Giridharan S, McConkey C, Hartley A, Brammer C, Watkinson JC, Glaholm J 2003 External beam radiotherapy in the management of differentiated thyroid cancer. Clin Oncol (R Coll Radiol ) 15:337-341.

757. Terezakis SA, Lee KS, Ghossein RA, Rivera M, Tuttle RM, Wolden SL, Zelefsky MJ, Wong RJ, Patel SG, Pfister DG, Shaha AR, Lee NY 2009 Role of external beam radiotherapy in patients with advanced or recurrent nonanaplastic thyroid cancer: Memorial Sloan-Kettering Cancer Center experience. Int J Radiat Oncol Biol Phys 73:795-801.

758. Brierley J, Tsang R, Panzarella T, Bana N 2005 Prognostic factors and the effect of treatment with radioactive iodine and external beam radiation on patients with differentiated thyroid cancer seen at a single institution over 40 years. Clin Endocrinol (Oxf) 63:418-427.

759. Sanders EM Jr, LiVolsi VA, Brierley J, Shin J, Randolph GW 2007 An evidence-based review of poorly differentiated thyroid cancer. World J Surg 31:934-945.

760. Kim JH, Leeper RD 1987 Treatment of locally advanced thyroid carcinoma with combination doxorubicin and radiation therapy. Cancer 60:2372-2375.

761. Links TP, van Tol KM, Jager PL, Plukker JT, Piers DA, Boezen HM, Dullaart RP, de Vries EG, Sluiter WJ 2005 Life expectancy in differentiated thyroid cancer: a novel approach to survival analysis. Endocr Relat Cancer 12: 273-280.

762. Brown AP, Chen J, Hitchcock YJ, Szabo A, Shrieve DC, Tward JD 2008 The risk of second primary malignancies up to three decades after the treatment of differentiated thyroid cancer. J Clin Endocrinol Metab 93:504-515.

763. Rubino C, de Vathaire F, Dottorini ME, Hall P, Schvartz C, Couette JE, Dondon MG, Abbas MT, Langlois C, 
Schlumberger M 2003 Second primary malignancies in thyroid cancer patients. Br J Cancer 89:1638-1644.

764. Berthe E, Henry-Amar M, Michels JJ, Rame JP, Berthet P, Babin E, Icard P, Samama G, Galateau-Salle F, Mahoudeau J, Bardet S 2004 Risk of second primary cancer following differentiated thyroid cancer. Eur J Nucl Med Mol Imaging 31:685-691.

765. Kim S, Wei JP, Braveman JM, Brams DM 2004 Predicting outcome and directing therapy for papillary thyroid carcinoma. Arch Surg 139:390-394.

766. Iyer NG, Morris LG, Tuttle RM, Shaha AR, Ganly I 2011 Rising incidence of second cancers in patients with low-risk (T1N0) thyroid cancer who receive radioactive iodine therapy. Cancer 117:4439-4446.

767. Biondi B, Filetti S, Schlumberger M 2005 Thyroidhormone therapy and thyroid cancer: a reassessment. Nat Clin Pract Endocrinol Metab 1:32-40.

768. Diaz-Soto G, Puig-Domingo M, Martinez-Pino I, Martinez de Osaba MJ, Mora M, Rivera-Fillat F, Halperin I 2011 Do thyroid cancer patients with basal undetectable $\mathrm{Tg}$ measured by current immunoassays require rhTSH testing? Exp Clin Endocrinol Diabetes 119:348-352.

769. Smallridge RC, Meek SE, Morgan MA, Gates GS, Fox TP, Grebe S, Fatourechi V 2007 Monitoring thyroglobulin in a sensitive immunoassay has comparable sensitivity to recombinant human TSH-stimulated thyroglobulin in follow-up of thyroid cancer patients. J Clin Endocrinol Metab 92:82-87.

770. Schlumberger M, Hitzel A, Toubert ME, Corone C, Troalen F, Schlageter MH, Claustrat F, Koscielny S, Taieb D, Toubeau M, Bonichon F, Borson-Chazot F, Leenhardt L, Schvartz C, Dejax C, Brenot-Rossi I, Torlontano M, Tenenbaum F, Bardet S, Bussiere F, Girard JJ, Morel O, Schneegans O, Schlienger JL, Prost A, So D, Archambeaud F, Ricard M, Benhamou E 2007 Comparison of seven serum thyroglobulin assays in the follow-up of papillary and follicular thyroid cancer patients. J Clin Endocrinol Metab 92:2487-2495.

771. Spencer CA 2011 Clinical review: Clinical utility of thyroglobulin antibody ( $\mathrm{TgAb}$ ) measurements for patients with differentiated thyroid cancers (DTC). J Clin Endocrinol Metab 96:3615-3627.

772. Taylor KP, Parkington D, Bradbury S, Simpson HL, Jefferies SJ, Halsall DJ 2011 Concordance between thyroglobulin antibody assays. Ann Clin Biochem 48: 367-369.

773. Giovanella L, Keller F, Ceriani L, Tozzoli R 2009 Heterophile antibodies may falsely increase or decrease thyroglobulin measurement in patients with differentiated thyroid carcinoma. Clin Chem Lab Med 47:952-954.

774. Verburg FA, Waschle K, Reiners C, Giovanella L, Lentjes EG 2010 Heterophile antibodies rarely influence the measurement of thyroglobulin and thyroglobulin antibodies in differentiated thyroid cancer patients. Horm Metab Res 42:736-739.

775. Latrofa F, Ricci D, Montanelli L, Rocchi R, Piaggi P, Sisti E, Grasso L, Basolo F, Ugolini C, Pinchera A, Vitti P 2012 Lymphocytic thyroiditis on histology correlates with serum thyroglobulin autoantibodies in patients with papillary thyroid carcinoma: impact on detection of serum thyroglobulin. J Clin Endocrinol Metab 97:23802387.

776. Giovanella L, Ceriani L 2011 Comparison of thyroglobulin antibody interference in first- and second- generation thyroglobulin immunoassays. Clin Chem Lab Med 49:1025-1027.

777. Stanojevic M, Savin S, Cvejic D, Djukic A, Jeremic M, Zivancevic SS 2009 Comparison of the influence of thyroglobulin antibodies on serum thyroglobulin values from two different immunoassays in post surgical differentiated thyroid carcinoma patients. J Clin Lab Anal 23:341-346.

778. Stanojevic M, Savin S, Cvejic D, Dukic A, Zivancevic SS 2009 Correlation of thyroglobulin concentrations measured by radioimmunoassay and immunoradiometric assay and the influence of thyroglobulin antibody. $\mathbf{J}$ Immunoassay Immunochem 30:197-207.

779. Eustatia-Rutten CF, Smit JW, Romijn JA, van der KleijCorssmit EP, Pereira AM, Stokkel MP, Kievit J 2004 Diagnostic value of serum thyroglobulin measurements in the follow-up of differentiated thyroid carcinoma, a structured meta-analysis. Clin Endocrinol (Oxf) 61: 61-74.

780. Bachelot A, Leboulleux S, Baudin E, Hartl DM, Caillou B, Travagli JP, Schlumberger M 2005 Neck recurrence from thyroid carcinoma: serum thyroglobulin and highdose total body scan are not reliable criteria for cure after radioiodine treatment. Clin Endocrinol (Oxf) 62:376379.

781. Cherk MH, Francis P, Topliss DJ, Bailey M, Kalff V 2012 Incidence and implications of negative serum thyroglobulin but positive I-131 whole-body scans in patients with well-differentiated thyroid cancer prepared with rhTSH or thyroid hormone withdrawal. Clin Endocrinol (Oxf) 76:734-740.

782. Pacini F, Agate L, Elisei R, Capezzone M, Ceccarelli C, Lippi F, Molinaro E, Pinchera A 2001 Outcome of differentiated thyroid cancer with detectable serum $\mathrm{Tg}$ and negative diagnostic (131)I whole body scan: comparison of patients treated with high (131)I activities versus untreated patients. J Clin Endocrinol Metab 86:4092-4097.

783. Torlontano M, Crocetti U, Augello G, D'Aloiso L, Bonfitto N, Varraso A, Dicembrino F, Modoni S, Frusciante V, Di GA, Bruno R, Filetti S, Trischitta V 2006 Comparative evaluation of recombinant human thyrotropin-stimulated thyroglobulin levels, 131I wholebody scintigraphy, and neck ultrasonography in the follow-up of patients with papillary thyroid microcarcinoma who have not undergone radioiodine therapy. J Clin Endocrinol Metab 91:60-63.

784. Robbins RJ, Srivastava S, Shaha A, Ghossein R, Larson SM, Fleisher M, Tuttle RM 2004 Factors influencing the basal and recombinant human thyrotropin-stimulated serum thyroglobulin in patients with metastatic thyroid carcinoma. J Clin Endocrinol Metab 89:6010-6016.

785. Mazzaferri EL, Robbins RJ, Spencer CA, Braverman LE, Pacini F, Wartofsky L, Haugen BR, Sherman SI, Cooper DS, Braunstein GD, Lee S, Davies TF, Arafah BM, Ladenson PW, Pinchera A 2003 A consensus report of the role of serum thyroglobulin as a monitoring method for low-risk patients with papillary thyroid carcinoma. J Clin Endocrinol Metab 88:1433-1441.

786. Deleted.

787. Iervasi A, Iervasi G, Ferdeghini M, Solimeo C, Bottoni A, Rossi L, Colato C, Zucchelli GC 2007 Clinical relevance of highly sensitive Tg assay in monitoring patients treated for differentiated thyroid cancer. Clin Endocrinol (Oxf) 67:434-441. 
788. Spencer C, Fatemi S, Singer P, Nicoloff J, Lopresti J 2010 Serum basal thyroglobulin measured by a secondgeneration assay correlates with the recombinant human thyrotropin-stimulated thyroglobulin response in patients treated for differentiated thyroid cancer. Thyroid 20: 587-595.

789. Haugen BR, Pacini F, Reiners C, Schlumberger M, Ladenson PW, Sherman SI, Cooper DS, Graham KE, Braverman LE, Skarulis MC, Davies TF, DeGroot LJ, Mazzaferri EL, Daniels GH, Ross DS, Luster M, Samuels MH, Becker DV, Maxon HR III, Cavalieri RR, Spencer CA, McEllin K, Weintraub BD, Ridgway EC 1999 A comparison of recombinant human thyrotropin and thyroid hormone withdrawal for the detection of thyroid remnant or cancer. J Clin Endocrinol Metab 84: 3877-3885.

790. David A, Blotta A, Bondanelli M, Rossi R, Roti E, Braverman LE, Busutti L, degli Uberti EC 2001 Serum thyroglobulin concentrations and (131)I whole-body scan results in patients with differentiated thyroid carcinoma after administration of recombinant human thyroidstimulating hormone. J Nucl Med 42:1470-1475.

791. Mazzaferri EL, Kloos RT 2002 Is diagnostic iodine-131 scanning with recombinant human TSH useful in the follow-up of differentiated thyroid cancer after thyroid ablation? J Clin Endocrinol Metab 87:1490-1498.

792. Haugen BR, Ridgway EC, McLaughlin BA, McDermott MT 2002 Clinical comparison of whole-body radioiodine scan and serum thyroglobulin after stimulation with recombinant human thyrotropin. Thyroid 12:37-43.

793. Lima N, Cavaliere H, Tomimori E, Knobel M, MedeirosNeto G 2002 Prognostic value of serial serum thyroglobulin determinations after total thyroidectomy for differentiated thyroid cancer. J Endocrinol Invest 25: $110-115$.

794. Wartofsky L 2002 Management of low-risk welldifferentiated thyroid cancer based only on thyroglobulin measurement after recombinant human thyrotropin. Thyroid 12:583-590.

795. Schaap J, Eustatia-Rutten CF, Stokkel M, Links TP, Diamant M, van der Velde EA, Romijn JA, Smit JW 2002 Does radioiodine therapy have disadvantageous effects in non-iodine accumulating differentiated thyroid carcinoma? Clin Endocrinol (Oxf) 57:117-124.

796. Pacini F, Sabra MM, Tuttle RM 2011 Clinical relevance of thyroglobulin doubling time in the management of patients with differentiated thyroid cancer. Thyroid 21: 691-692.

797. Spencer CA, LoPresti JS, Fatemi S, Nicoloff JT 1999 Detection of residual and recurrent differentiated thyroid carcinoma by serum thyroglobulin measurement. Thyroid 9:435-441.

798. Hollowell JG, Staehling NW, Flanders WD, Hannon WH, Gunter EW, Spencer CA, Braverman LE 2002 Serum TSH, T(4), and thyroid antibodies in the United States population (1988 to 1994): National Health and Nutrition Examination Survey (NHANES III). J Clin Endocrinol Metab 87:489-499.

799. Spencer CA 2004 Challenges of serum thyroglobulin ( Tg) measurement in the presence of $\mathrm{Tg}$ autoantibodies. $\mathrm{J}$ Clin Endocrinol Metab 89:3702-3704.

800. Bachelot A, Cailleux AF, Klain M, Baudin E, Ricard M, Bellon N, Caillou B, Travagli JP, Schlumberger M 2002 Relationship between tumor burden and serum thyro- globulin level in patients with papillary and follicular thyroid carcinoma. Thyroid 12:707-711.

801. Spencer C, Fatemi S 2013 Thyroglobulin antibody ( $\mathrm{TgAb}$ ) methods - strengths, pitfalls and clinical utility for monitoring $\mathrm{TgAb}$-positive patients with differentiated thyroid cancer. Best Pract Res Clin Endocrinol Metab 27:701-712.

802. Nygaard B, Bentzen J, Laurberg P, Pedersen SM, Bastholt L, Handberg A, Rytter C, Godballe C, Faber J 2012 Large discrepancy in the results of sensitive measurements of thyroglobulin antibodies in the follow-up on thyroid cancer: a diagnostic dilemma. Eur Thyroid J 1: 193-197.

803. Hoofnagle AN, Becker JO, Wener MH, Heinecke JW 2008 Quantification of thyroglobulin, a low-abundance serum protein, by immunoaffinity peptide enrichment and tandem mass spectrometry. Clin Chem 54:17961804.

804. Clarke NJ, Zhang Y, Reitz RE 2012 A novel mass spectrometry-based assay for the accurate measurement of thyroglobulin from patient samples containing antithyroglobulin autoantibodies. J Investig Med 60:11571163.

805. Kushnir MM, Rockwood AL, Roberts WL, Abraham D, Hoofnagle AN, Meikle AW 2013 Measurement of thyroglobulin by liquid chromatography-tandem mass spectrometry in serum and plasma in the presence of antithyroglobulin autoantibodies. Clin Chem 59:982-990.

806. Hoofnagle AN, Roth MY 2013 Clinical review: improving the measurement of serum thyroglobulin with mass spectrometry. J Clin Endocrinol Metab 98:13431352.

807. Pacini F, Molinaro E, Castagna MG, Agate L, Elisei R, Ceccarelli C, Lippi F, Taddei D, Grasso L, Pinchera A 2003 Recombinant human thyrotropin-stimulated serum thyroglobulin combined with neck ultrasonography has the highest sensitivity in monitoring differentiated thyroid carcinoma. J Clin Endocrinol Metab 88:3668-3673.

808. Shin JH, Han BK, Ko EY, Kang SS 2007 Sonographic findings in the surgical bed after thyroidectomy: comparison of recurrent tumors and nonrecurrent lesions. $\mathbf{J}$ Ultrasound Med 26:1359-1366.

809. Bardet S, Malville E, Rame JP, Babin E, Samama G, De RD, Michels JJ, Reznik Y, Henry-Amar M 2008 Macroscopic lymph-node involvement and neck dissection predict lymph-node recurrence in papillary thyroid carcinoma. Eur J Endocrinol 158:551-560.

810. Torres MR, Nobrega Neto SH, Rosas RJ, Martins AL, Ramos AL, da Cruz TR 2014 Thyroglobulin in the washout fluid of lymph-node biopsy: what is its role in the follow-up of differentiated thyroid carcinoma? Thyroid 24:7-18.

811. Frasoldati A, Toschi E, Zini M, Flora M, Caroggio A, Dotti C, Valcavi R 1999 Role of thyroglobulin measurement in fine-needle aspiration biopsies of cervical lymph nodes in patients with differentiated thyroid cancer. Thyroid 9:105-111.

812. Baloch ZW, Barroeta JE, Walsh J, Gupta PK, LiVolsi VA, Langer JE, Mandel SJ 2008 Utility of thyroglobulin measurement in fine-needle aspiration biopsy specimens of lymph nodes in the diagnosis of recurrent thyroid carcinoma. Cytojournal 5:1-8.

813. Pacini F, Capezzone M, Elisei R, Ceccarelli C, Taddei D, Pinchera A 2002 Diagnostic 131-iodine whole-body scan 
may be avoided in thyroid cancer patients who have undetectable stimulated serum $\mathrm{Tg}$ levels after initial treatment. J Clin Endocrinol Metab 87:1499-1501.

814. Torlontano M, Crocetti U, D'Aloiso L, Bonfitto N, Di GA, Modoni S, Valle G, Frusciante V, Bisceglia M, Filetti S, Schlumberger M, Trischitta V 2003 Serum thyroglobulin and 131I whole body scan after recombinant human TSH stimulation in the follow-up of low-risk patients with differentiated thyroid cancer. Eur J Endocrinol 148:19-24.

815. Aide N, Heutte N, Rame JP, Rousseau E, Loiseau C, Henry-Amar M, Bardet S 2009 Clinical relevance of single-photon emission computed tomography/computed tomography of the neck and thorax in postablation (131)I scintigraphy for thyroid cancer. J Clin Endocrinol Metab 94:2075-2084.

816. Schmidt D, Szikszai A, Linke R, Bautz W, Kuwert T 2009 Impact of 131I SPECT/spiral CT on nodal staging of differentiated thyroid carcinoma at the first radioablation. J Nucl Med 50:18-23.

817. Jeong SY, Lee SW, Kim HW, Song BI, Ahn BC, Lee J 2014 Clinical applications of SPECT/CT after first I-131 ablation in patients with differentiated thyroid cancer. Clin Endocrinol (Oxf) 81:445-451.

818. Tharp K, Israel O, Hausmann J, Bettman L, Martin WH, Daitzchman M, Sandler MP, Delbeke D 2004 Impact of 131I-SPECT/CT images obtained with an integrated system in the follow-up of patients with thyroid carcinoma. Eur J Nucl Med Mol Imaging 31:1435-1442.

819. Freudenberg LS, Jentzen W, Stahl A, Bockisch A, Rosenbaum-Krumme SJ 2011 Clinical applications of 124I-PET/CT in patients with differentiated thyroid cancer. Eur J Nucl Med Mol Imaging 38(Suppl 1):S48S56.

820. Van Nostrand D, Moreau S, Bandaru VV, Atkins F, Chennupati S, Mete M, Burman K, Wartofsky L 2010 (124)I positron emission tomography versus (131)I planar imaging in the identification of residual thyroid tissue and/or metastasis in patients who have welldifferentiated thyroid cancer. Thyroid 20:879-883.

821. Phan HT, Jager PL, Paans AM, Plukker JT, Sturkenboom MG, Sluiter WJ, Wolffenbuttel BH, Dierckx RA, Links TP 2008 The diagnostic value of 124I-PET in patients with differentiated thyroid cancer. Eur J Nucl Med Mol Imaging 35:958-965.

822. Leboulleux S, Schroeder PR, Schlumberger M, Ladenson PW 2007 The role of PET in follow-up of patients treated for differentiated epithelial thyroid cancers. Nat Clin Pract Endocrinol Metab 3:112-121.

823. Robbins RJ, Wan Q, Grewal RK, Reibke R, Gonen M, Strauss HW, Tuttle RM, Drucker W, Larson SM 2006 Real-time prognosis for metastatic thyroid carcinoma based on 2-[18F]fluoro-2-deoxy-D-glucose-positron emission tomography scanning. J Clin Endocrinol Metab 91:498-505.

824. Deandreis D, Al Ghuzlan A, Leboulleux S, Lacroix L, Garsi JP, Talbot M, Lumbroso J, Baudin E, Caillou B, Bidart JM, Schlumberger M 2011 Do histological, immunohistochemical, and metabolic (radioiodine and fluorodeoxyglucose uptakes) patterns of metastatic thyroid cancer correlate with patient outcome? Endocr Relat Cancer 18:159-169.

825. Leboulleux S, Schroeder PR, Busaidy NL, Auperin A, Corone C, Jacene HA, Ewertz ME, Bournaud C, Wahl
RL, Sherman SI, Ladenson PW, Schlumberger M 2009 Assessment of the incremental value of recombinant thyrotropin stimulation before 2-[18F]-fluoro-2-deoxyD-glucose positron emission tomography/computed tomography imaging to localize residual differentiated thyroid cancer. J Clin Endocrinol Metab 94:13101316.

826. Yoon DY, Hwang HS, Chang SK, Rho YS, Ahn HY, Kim JH, Lee IJ 2009 CT, MR, US,18F-FDG PET/CT, and their combined use for the assessment of cervical lymph node metastases in squamous cell carcinoma of the head and neck. Eur Radiol 19:634-642.

827. Takashima S, Sone S, Takayama F, Wang Q, Kobayashi T, Horii A, Yoshida JI 1998 Papillary thyroid carcinoma: MR diagnosis of lymph node metastasis. AJNR Am J Neuroradiol 19:509-513.

828. Gross ND, Weissman JL, Talbot JM, Andersen PE, Wax MK, Cohen JI 2001 MRI detection of cervical metastasis from differentiated thyroid carcinoma. Laryngoscope 111:1905-1909.

829. Toubert ME, Cyna-Gorse F, Zagdanski AM, NoelWekstein S, Cattan P, Billotey C, Sarfati E, Rain JD 1999 Cervicomediastinal magnetic resonance imaging in persistent or recurrent papillary thyroid carcinoma: clinical use and limits. Thyroid 9:591-597.

830. Wang JC, Takashima S, Takayama F, Kawakami S, Saito A, Matsushita T, Matsuba H, Kobayashi S 2001 Tracheal invasion by thyroid carcinoma: prediction using MR imaging. AJR Am J Roentgenol 177:929-936.

831. Wang J, Takashima S, Matsushita T, Takayama F, Kobayashi T, Kadoya M 2003 Esophageal invasion by thyroid carcinomas: prediction using magnetic resonance imaging. J Comput Assist Tomogr 27:18-25.

832. Lee DH, Kang WJ, Seo HS, Kim E, Kim JH, Son KR, Na DG 2009 Detection of metastatic cervical lymph nodes in recurrent papillary thyroid carcinoma: computed tomography versus positron emission tomography-computed tomography. J Comput Assist Tomogr 33:805-810.

833. Rosario PW, Mourao GF, dos Santos JB, Calsolari MR 2014 Is empirical radioactive iodine therapy still a valid approach to patients with thyroid cancer and elevated thyroglobulin? Thyroid 24:533-536.

834. Leboulleux S, El Bez I, I, Borget I, Elleuch M, Déandreis D, Al Ghuzlan A, Chougnet C, Bidault F, Mirghani H, Lumbroso J, Hartl D, Baudin E, Schlumberger M 2012 Postradioiodine treatment whole-body scan in the era of 18-fluorodeoxyglucose positron emission tomography for differentiated thyroid carcinoma with elevated serum thyroglobulin levels. Thyroid 22:832-838.

835. Wang PW, Wang ST, Liu RT, Chien WY, Tung SC, Lu YC, Chen HY, Lee CH 1999 Levothyroxine suppression of thyroglobulin in patients with differentiated thyroid carcinoma. J Clin Endocrinol Metab 84:4549-4553.

836. Sugitani I, Fujimoto Y 2010 Does postoperative thyrotropin suppression therapy truly decrease recurrence in papillary thyroid carcinoma? A randomized controlled trial. J Clin Endocrinol Metab 95:4576-4583.

837. Klein Hesselink EN, Klein Hesselink MS, de Bock GH, Gansevoort RT, Bakker SJ, Vredeveld EJ, van der HorstSchrivers AN, van der Horst IC, Kamphuisen PW, Plukker JT, Links TP, Lefrandt JD 2013 Long-term cardiovascular mortality in patients with differentiated thyroid carcinoma: an observational study. J Clin Oncol 31:4046-4053. 
838. Shargorodsky M, Serov S, Gavish D, Leibovitz E, Harpaz D, Zimlichman R 2006 Long-term thyrotropinsuppressive therapy with levothyroxine impairs small and large artery elasticity and increases left ventricular mass in patients with thyroid carcinoma. Thyroid 16:381-386.

839. Taillard V, Sardinoux M, Oudot C, Fesler P, Rugale C, Raingeard I, Renard E, Ribstein J, du CG 2011 Early detection of isolated left ventricular diastolic dysfunction in high-risk differentiated thyroid carcinoma patients on TSH-suppressive therapy. Clin Endocrinol (Oxf) 75: 709-714.

840. Leeper RD 1973 The effect of 131 I therapy on survival of patients with metastatic papillary or follicular thyroid carcinoma. J Clin Endocrinol Metab 36:1143-1152.

841. Beierwaltes WH, Nishiyama RH, Thompson NW, Copp JE, Kubo A 1982 Survival time and "cure" in papillary and follicular thyroid carcinoma with distant metastases: statistics following University of Michigan therapy. J Nucl Med 23:561-568.

842. Bernier MO, Leenhardt L, Hoang C, Aurengo A, Mary JY, Menegaux F, Enkaoua E, Turpin G, Chiras J, Saillant G, Hejblum G 2001 Survival and therapeutic modalities in patients with bone metastases of differentiated thyroid carcinomas. J Clin Endocrinol Metab 86:1568-1573.

843. Sampson E, Brierley JD, Le LW, Rotstein L, Tsang RW 2007 Clinical management and outcome of papillary and follicular (differentiated) thyroid cancer presenting with distant metastasis at diagnosis. Cancer 110:1451-1456.

844. Durante C, Haddy N, Baudin E, Leboulleux S, Hartl D, Travagli JP, Caillou B, Ricard M, Lumbroso JD, De Vathaire F, Schlumberger M 2006 Long-term outcome of 444 patients with distant metastases from papillary and follicular thyroid carcinoma: benefits and limits of radioiodine therapy. J Clin Endocrinol Metab 91:28922899.

845. Dupuy DE, Monchik JM, Decrea C, Pisharodi L 2001 Radiofrequency ablation of regional recurrence from welldifferentiated thyroid malignancy. Surgery 130:971-977.

846. Lewis BD, Hay ID, Charboneau JW, McIver B, Reading CC, Goellner JR 2002 Percutaneous ethanol injection for treatment of cervical lymph node metastases in patients with papillary thyroid carcinoma. AJR Am J Roentgenol 178:699-704.

847. Eustatia-Rutten CF, Romijn JA, Guijt MJ, Vielvoye GJ, van den Berg R, Corssmit EP, Pereira AM, Smit JW 2003 Outcome of palliative embolization of bone metastases in differentiated thyroid carcinoma. J Clin Endocrinol Metab 88:3184-3189.

848. Misra S, Meiyappan S, Heus L, Freeman J, Rotstein L, Brierley JD, Tsang RW, Rodin G, Ezzat S, Goldstein DP, Sawka AM 2013 Patients' experiences following localregional recurrence of thyroid cancer: a qualitative study. J Surg Oncol 108:47-51.

849. Robenshtok E, Fish S, Bach A, Dominguez JM, Shaha A, Tuttle RM 2012 Suspicious cervical lymph nodes detected after thyroidectomy for papillary thyroid cancer usually remain stable over years in properly selected patients. J Clin Endocrinol Metab 97:2706-2713.

850. Ito Y, Higashiyama T, Takamura Y, Kobayashi K, Miya A, Miyauchi A 2011 Prognosis of patients with papillary thyroid carcinoma showing postoperative recurrence to the central neck. World J Surg 35:767-772.

851. Uchida H, Imai T, Kikumori T, Hayashi H, Sato S, Noda S, Idota A, Kiuchi T 2013 Long-term results of surgery for papillary thyroid carcinoma with local recurrence. Surg Today 43:848-853.

852. Newman KD, Black T, Heller G, Azizkhan RG, Holcomb GW III, Sklar C, Vlamis V, Haase GM, La Quaglia MP 1998 Differentiated thyroid cancer: determinants of disease progression in patients $<21$ years of age at diagnosis: a report from the Surgical Discipline Committee of the Children's Cancer Group. Ann Surg 227:533-541.

853. Robie DK, Dinauer CW, Tuttle RM, Ward DT, Parry R, McClellan D, Svec R, Adair C, Francis G 1998 The impact of initial surgical management on outcome in young patients with differentiated thyroid cancer. J Pediatr Surg 33:1134-1138.

854. Chadwick D, Kinsman R, Walton P 2012 The British Association of Endocrine and Thyroid Surgeons 2012. 4th edition. Dendrite Clinical Systems, Ltd, Oxfordshire, United Kingdom, pp 3-188.

855. Rosenthal MS, Angelos P, Cooper DS, Fassler C, Finder SG, Hays MT, Tendler B, Braunstein GD 2013 Clinical and professional ethics guidelines for the practice of thyroidology. Thyroid 23:1203-1210.

856. Yeh M, Bernet V, Ferris R, Loevner L, Mandel S, Orloff L, Randolph G, Steward D 2015 American Thyroid Association statement on preoperative imaging for thyroid cancer surgery. Thyroid 25:3-14.

857. Tufano RP, Clayman G, Heller KS, Inabnet WB, Kebebew E, Shaha A, Steward DL, Tuttle RM 2014 Management of recurrent/persistent nodal disease in patients with differentiated thyroid cancer: a critical review of the risks and benefits of surgical intervention versus active surveillance. Thyroid 25:15-27.

858. Phelan E, Kamani D, Shin J, Randolph GW 2013 Neural monitored revision thyroid cancer surgery: surgical safety and thyroglobulin response. Otolaryngol Head Neck Surg 149:47-52.

859. Urken ML, Milas M, Randolph GW, Tufano R, Bergman D, Bernet V, Brett EM, Brierley JD, Cobin R, Doherty G, Klopper J, Lee S, Machac J, Mechanick JI, Orloff LA, Ross D, Smallridge RC, Terris DJ, Clain JB, Tuttle M 2015 A review of the management of recurrent and persistent metastatic lymph nodes in well differentiated thyroid cancer: a multifactorial decision making guide created for the Thyroid Cancer Care Collaborative. Head Neck 37:605-614.

860. Merdad M, Eskander A, Kroeker T, Freeman JL 2012 Predictors of level II and Vb neck disease in metastatic papillary thyroid cancer. Arch Otolaryngol Head Neck Surg 138: 1030-1033.

861. Eskander A, Merdad M, Freeman JL, Witterick IJ 2013 Pattern of spread to the lateral neck in metastatic welldifferentiated thyroid cancer: a systematic review and meta-analysis. Thyroid 23:583-592.

862. Schuff KG 2011 Management of recurrent/persistent papillary thyroid carcinoma: efficacy of the surgical option. J Clin Endocrinol Metab 96:2038-2039.

863. McCoy KL, Yim JH, Tublin ME, Burmeister LA, Ogilvie JB, Carty SE 2007 Same-day ultrasound guidance in reoperation for locally recurrent papillary thyroid cancer. Surgery 142:965-972.

864. Hughes DT, Laird AM, Miller BS, Gauger PG, Doherty GM 2012 Reoperative lymph node dissection for recurrent papillary thyroid cancer and effect on serum thyroglobulin. Ann Surg Oncol 19:2951-2957. 
865. Roh JL, Kim JM, Park CI 2011 Central compartment reoperation for recurrent/persistent differentiated thyroid cancer: patterns of recurrence, morbidity, and prediction of postoperative hypocalcemia. Ann Surg Oncol 18:1312-1318.

866. Shaha AR 2012 Recurrent differentiated thyroid cancer. Endocr Pract 18:600-603.

867. Palme CE, Waseem Z, Raza SN, Eski S, Walfish P, Freeman JL 2004 Management and outcome of recurrent well-differentiated thyroid carcinoma. Arch Otolaryngol Head Neck Surg 130:819-824.

868. Clayman GL, Agarwal G, Edeiken BS, Waguespack SG, Roberts DB, Sherman SI 2011 Long-term outcome of comprehensive central compartment dissection in patients with recurrent/persistent papillary thyroid carcinoma. Thyroid 21:1309-1316.

869. Clayman GL, Shellenberger TD, Ginsberg LE, Edeiken BS, El-Naggar AK, Sellin RV, Waguespack SG, Roberts DB, Mishra A, Sherman SI 2009 Approach and safety of comprehensive central compartment dissection in patients with recurrent papillary thyroid carcinoma. Head Neck 31:1152-1163.

870. Chao TC, Jeng LB, Lin JD, Chen MF 1997 Reoperative thyroid surgery. World J Surg 21:644-647.

871. Erbil Y, Sari S, Agcaoglu O, Ersoz F, Bayraktar A, Salmaslioglu A, Gozkun O, Adalet I, Ozarmagan S 2010 Radio-guided excision of metastatic lymph nodes in thyroid carcinoma: a safe technique for previously operated neck compartments. World J Surg 34:25812588.

872. Alzahrani AS, Raef H, Sultan A, Al Sobhi S, Ingemansson S, Ahmed M, Al Mahfouz A 2002 Impact of cervical lymph node dissection on serum TG and the course of disease in TG-positive, radioactive iodine whole body scan-negative recurrent/persistent papillary thyroid cancer. J Endocrinol Invest 25:526-531.

873. Travagli JP, Cailleux AF, Ricard M, Baudin E, Caillou B, Parmentier C, Schlumberger M 1998 Combination of radioiodine (131I) and probe-guided surgery for persistent or recurrent thyroid carcinoma. J Clin Endocrinol Metab 83:2675-2680.

874. Lee L, Steward DL 2008 Sonographically-directed neck dissection for recurrent thyroid carcinoma. Laryngoscope 118:991-994.

875. Steward DL 2012 Update in utility of secondary node dissection for papillary thyroid cancer. J Clin Endocrinol Metab 97:3393-3398.

876. Rubello D, Salvatori M, Casara D, Piotto A, Toniato A, Gross MD, Al-Nahhas A, Muzzio PC, Pelizzo MR 2007 $99 \mathrm{mTc}$-sestamibi radio-guided surgery of loco-regional 131Iodine-negative recurrent thyroid cancer. Eur J Surg Oncol 33:902-906.

877. Deleted.

878. Heilo A, Sigstad E, Fagerlid KH, Haskjold OI, Groholt KK, Berner A, Bjoro T, Jorgensen LH 2011 Efficacy of ultrasound-guided percutaneous ethanol injection treatment in patients with a limited number of metastatic cervical lymph nodes from papillary thyroid carcinoma. J Clin Endocrinol Metab 96:2750-2755.

879. Hay ID, Lee RA, Davidge-Pitts C, Reading CC, Charboneau JW 2013 Long-term outcome of ultrasoundguided percutaneous ethanol ablation of selected "recurrent" neck nodal metastases in 25 patients with TNM stages III or IVA papillary thyroid carcinoma previously treated by surgery and 131I therapy. Surgery 154:14481454.

880. Guenette JP, Monchik JM, Dupuy DE 2013 Imageguided ablation of postsurgical locoregional recurrence of biopsy-proven well-differentiated thyroid carcinoma. J Vasc Interv Radiol 24:672-679.

881. Park KW, Shin JH, Han BK, Ko EY, Chung JH 2011 Inoperable symptomatic recurrent thyroid cancers: preliminary result of radiofrequency ablation. Ann Surg Oncol 18:2564-2568.

882. Baek JH, Kim YS, Sung JY, Choi H, Lee JH 2011 Locoregional control of metastatic well-differentiated thyroid cancer by ultrasound-guided radiofrequency ablation. AJR Am J Roentgenol 197:W331-W336.

883. Monchik JM, Donatini G, Iannuccilli J, Dupuy DE 2006 Radiofrequency ablation and percutaneous ethanol injection treatment for recurrent local and distant welldifferentiated thyroid carcinoma. Ann Surg 244:296-304.

884. Shin JE, Baek JH, Lee JH 2013 Radiofrequency and ethanol ablation for the treatment of recurrent thyroid cancers: current status and challenges. Curr Opin Oncol 25:14-19.

885. Na DG, Lee JH, Jung SL, Kim JH, Sung JY, Shin JH, Kim EK, Lee JH, Kim DW, Park JS, Kim KS, Baek SM, Lee Y, Chong S, Sim JS, Huh JY, Bae JI, Kim KT, Han SY, Bae MY, Kim YS, Baek JH 2012 Radiofrequency ablation of benign thyroid nodules and recurrent thyroid cancers: consensus statement and recommendations. Korean J Radiol 13:117-125.

886. Papini E, Bizzarri G, Bianchini A, Valle D, Misischi I, Guglielmi R, Salvatori M, Solbiati L, Crescenzi A, Pacella CM, Gharib H 2013 Percutaneous ultrasoundguided laser ablation is effective for treating selected nodal metastases in papillary thyroid cancer. J Clin Endocrinol Metab 98:E92-E97.

887. Sabra MM, Grewal RK, Tala H, Larson SM, Tuttle RM 2012 Clinical outcomes following empiric radioiodine therapy in patients with structurally identifiable metastatic follicular cell-derived thyroid carcinoma with negative diagnostic but positive post-therapy 131I whole-body scans. Thyroid 22:877-883.

888. Schwartz DL, Lobo MJ, Ang KK, Morrison WH, Rosenthal DI, Ahamad A, Evans DB, Clayman G, Sherman SI, Garden AS 2009 Postoperative external beam radiotherapy for differentiated thyroid cancer: outcomes and morbidity with conformal treatment. Int J Radiat Oncol Biol Phys 74:1083-1091.

889. Romesser PB, Sherman EJ, Shaha AR, Lian M, Wong RJ, Sabra M, Rao SS, Fagin JA, Tuttle RM, Lee NY 2014 External beam radiotherapy with or without concurrent chemotherapy in advanced or recurrent nonanaplastic non-medullary thyroid cancer. J Surg Oncol 110:375-382.

890. Ge JH, Zhao RL, Hu JL, Zhou WA 2004 Surgical treatment of advanced thyroid carcinoma with aerodigestive invasion. Zhonghua Er Bi Yan Hou Ke Za Zhi 39:237-240. (In Chinese.)

891. Avenia N, Ragusa M, Monacelli M, Calzolari F, Daddi N, Di CL, Semeraro A, Puma F 2004 Locally advanced thyroid cancer: therapeutic options. Chir Ital 56:501508.

892. McCaffrey JC 2000 Evaluation and treatment of aerodigestive tract invasion by well-differentiated thyroid carcinoma. Cancer Control 7:246-252. 
893. Musholt TJ, Musholt PB, Behrend M, Raab R, Scheumann GF, Klempnauer J 1999 Invasive differentiated thyroid carcinoma: tracheal resection and reconstruction procedures in the hands of the endocrine surgeon. Surgery 126:1078-1087.

894. Czaja JM, McCaffrey TV 1997 The surgical management of laryngotracheal invasion by well-differentiated papillary thyroid carcinoma. Arch Otolaryngol Head Neck Surg 123:484-490.

895. Haymart MR, Muenz DG, Stewart AK, Griggs JJ, Banerjee M 2013 Disease severity and radioactive iodine use for thyroid cancer. J Clin Endocrinol Metab 98:678686.

896. Van Nostrand D 2009 The benefits and risks of I-131 therapy in patients with well-differentiated thyroid cancer. Thyroid 19:1381-1391.

897. Higashi T, Nishii R, Yamada S, Nakamoto Y, Ishizu K, Kawase S, Togashi K, Itasaka S, Hiraoka M, Misaki T, Konishi J 2011 Delayed initial radioactive iodine therapy resulted in poor survival in patients with metastatic differentiated thyroid carcinoma: a retrospective statistical analysis of 198 cases. J Nucl Med 52:683-689.

898. Yim JH, Kim WB, Kim EY, Kim WG, Kim TY, Ryu JS, Moon DH, Sung TY, Yoon JH, Kim SC, Hong SJ, Shong YK 2011 Adjuvant radioactive therapy after reoperation for locoregionally recurrent papillary thyroid cancer in patients who initially underwent total thyroidectomy and high-dose remnant ablation. J Clin Endocrinol Metab 96:3695-3700.

899. Van Nostrand D, Wartofsky L 2007 Radioiodine in the treatment of thyroid cancer. Endocrinol Metab Clin North Am 36:807-822, vii-viii.

900. Van Nostrand D, Atkins F, Yeganeh F, Acio E, Bursaw R, Wartofsky L 2002 Dosimetrically determined doses of radioiodine for the treatment of metastatic thyroid carcinoma. Thyroid 12:121-134.

901. Chiesa C, Castellani MR, Vellani C, Orunesu E, Negri A, Azzeroni R, Botta F, Maccauro M, Aliberti G, Seregni E, Lassmann M, Bombardieri E 2009 Individualized dosimetry in the management of metastatic differentiated thyroid cancer. Q J Nucl Med Mol Imaging 53:546-561.

902. Lassmann M, Reiners C, Luster M 2010 Dosimetry and thyroid cancer: the individual dosage of radioiodine. Endocr Relat Cancer 17:R161-R172.

903. Verburg FA, Hanscheid H, Biko J, Hategan MC, Lassmann M, Kreissl MC, Reiners C, Luster M 2010 Dosimetry-guided high-activity (131)I therapy in patients with advanced differentiated thyroid carcinoma: initial experience. Eur J Nucl Med Mol Imaging 37:896-903.

904. Lassmann M, Hanscheid H, Chiesa C, Hindorf C, Flux G, Luster M 2008 EANM Dosimetry Committee series on standard operational procedures for pre-therapeutic dosimetry I: blood and bone marrow dosimetry in differentiated thyroid cancer therapy. Eur J Nucl Med Mol Imaging 35:1405-1412.

905. Robbins RJ, Schlumberger MJ 2005 The evolving role of (131)I for the treatment of differentiated thyroid carcinoma. J Nucl Med 46 Suppl 1:28S-37S.

906. Van Nostrand D, Atkins F, Moreau S, Aiken M, Kulkarni K, Wu JS, Burman KD, Wartofsky L 2009 Utility of the radioiodine whole-body retention at 48 hours for modifying empiric activity of 131-iodine for the treatment of metastatic well-differentiated thyroid carcinoma. Thyroid 19:1093-1098.
907. Dorn R, Kopp J, Vogt H, Heidenreich P, Carroll RG, Gulec SA 2003 Dosimetry-guided radioactive iodine treatment in patients with metastatic differentiated thyroid cancer: largest safe dose using a risk-adapted approach. J Nucl Med 44:451-456.

908. Maxon HR, Thomas SR, Hertzberg VS, Kereiakes JG, Chen IW, Sperling MI, Saenger EL 1983 Relation between effective radiation dose and outcome of radioiodine therapy for thyroid cancer. N Engl J Med 309: 937-941.

909. Thomas SR, Maxon HR, Kereiakes JG 1976 In vivo quantitation of lesion radioactivity using external counting methods. Med Phys 03:253-255.

910. Holst JP, Burman KD, Atkins F, Umans JG, Jonklaas J 2005 Radioiodine therapy for thyroid cancer and hyperthyroidism in patients with end-stage renal disease on hemodialysis. Thyroid 15:1321-1331.

911. Driedger AA, Quirk S, McDonald TJ, Ledger S, Gray D, Wall W, Yoo J 2006 A pragmatic protocol for I-131 rhTSH-stimulated ablation therapy in patients with renal failure. Clin Nucl Med 31:454-457.

912. Jarzab B, Handkiewicz-Junak D, Wloch J 2005 Juvenile differentiated thyroid carcinoma and the role of radioiodine in its treatment: a qualitative review. Endocr Relat Cancer 12:773-803.

913. Verburg FA, Biko J, Diessl S, Demidchik Y, Drozd V, Rivkees SA, Reiners C, Hanscheid H 2011 I-131 activities as high as safely administrable (AHASA) for the treatment of children and adolescents with advanced differentiated thyroid cancer. J Clin Endocrinol Metab 96:E1268-E1271.

914. Ma C, Xie J, Liu W, Wang G, Zuo S, Wang X, Wu F 2010 Recombinant human thyrotropin (rhTSH) aided radioiodine treatment for residual or metastatic differentiated thyroid cancer. Cochrane Database Syst Rev (11):CD008302.

915. Klubo-Gwiezdzinska J, Van Nostrand D, Atkins F, Burman K, Jonklaas J, Mete M, Wartofsky L 2011 Efficacy of dosimetric versus empiric prescribed activity of 131I for therapy of differentiated thyroid cancer. J Clin Endocrinol Metab 96:3217-3225.

916. Lee JJ, Chung JK, Kim SE, Kang WJ, Park do J, Lee DS, Cho BY, Lee MC 2008 Maximal safe dose of I-131 after failure of standard fixed dose therapy in patients with differentiated thyroid carcinoma. Ann Nucl Med 22: 727-734.

917. Samuel AM, Rajashekharrao B, Shah DH 1998 Pulmonary metastases in children and adolescents with welldifferentiated thyroid cancer. J Nucl Med 39:1531-1536.

918. Sgouros G, Kolbert KS, Sheikh A, Pentlow KS, Mun EF, Barth A, Robbins RJ, Larson SM 2004 Patient-specific dosimetry for 131I thyroid cancer therapy using 124I PET and 3-dimensional-internal dosimetry (3D-ID) software. J Nucl Med 45:1366-1372.

919. Jentzen W, Freudenberg L, Eising EG, Sonnenschein W, Knust J, Bockisch A 2008 Optimized 124I PET dosimetry protocol for radioiodine therapy of differentiated thyroid cancer. J Nucl Med 49:1017-1023.

920. Pettinato C, Monari F, Nanni C, Allegri V, Marcatili S, Civollani S, Cima S, Spezi E, Mazzarotto R, Fanti S 2012 Usefulness of 124I PET/CT imaging to predict absorbed doses in patients affected by metastatic thyroid cancer and treated with 131I. Q J Nucl Med Mol Imaging 56:509-514. 
921. Reiners C, Biko J, Haenscheid H, Hebestreit H, Kirinjuk S, Baranowski O, Marlowe RJ, Demidchik E, Drozd V, Demidchik Y 2013 Twenty-five years after Chernobyl: outcome of radioiodine treatment in children and adolescents with very high-risk radiation-induced differentiated thyroid carcinoma. J Clin Endocrinol Metab 98: 3039-3048.

922. Schlumberger M, Lacroix L, Russo D, Filetti S, Bidart JM 2007 Defects in iodide metabolism in thyroid cancer and implications for the follow-up and treatment of patients. Nat Clin Pract Endocrinol Metab 3:260-269.

923. Kulkarni K, Van Nostrand D, Atkins F, Aiken M, Burman K, Wartofsky L 2006 The relative frequency in which empiric dosages of radioiodine would potentially overtreat or undertreat patients who have metastatic well-differentiated thyroid cancer. Thyroid 16:10191023.

924. Tuttle RM, Leboeuf R, Robbins RJ, Qualey R, Pentlow K, Larson SM, Chan CY 2006 Empiric radioactive iodine dosing regimens frequently exceed maximum tolerated activity levels in elderly patients with thyroid cancer. J Nucl Med 47:1587-1591.

925. Rudavsky AZ, Freeman LM 1997 Treatment of scannegative, thyroglobulin-positive metastatic thyroid cancer using radioiodine 131I and recombinant human thyroid stimulating hormone. J Clin Endocrinol Metab 82: 11-14.

926. Ringel MD, Ladenson PW 1996 Diagnostic accuracy of 131I scanning with recombinant human thyrotropin versus thyroid hormone withdrawal in a patient with metastatic thyroid carcinoma and hypopituitarism. J Clin Endocrinol Metab 81:1724-1725.

927. Luster M, Lassmann M, Haenscheid H, Michalowski U, Incerti C, Reiners C 2000 Use of recombinant human thyrotropin before radioiodine therapy in patients with advanced differentiated thyroid carcinoma. J Clin Endocrinol Metab 85:3640-3645.

928. Mariani G, Ferdeghini M, Augeri C, Villa G, Taddei GZ, Scopinaro G, Boni G, Bodei L, Rabitti C, Molinari E, Bianchi R 2000 Clinical experience with recombinant human thyrotrophin (rhTSH) in the management of patients with differentiated thyroid cancer. Cancer Biother Radiopharm 15:211-217.

929. Perros P 1999 Recombinant human thyroid-stimulating hormone (rhTSH) in the radioablation of well-differentiated thyroid cancer: preliminary therapeutic experience. J Endocrinol Invest 22:30-34.

930. Lippi F, Capezzone M, Angelini F, Taddei D, Molinaro E, Pinchera A, Pacini F 2001 Radioiodine treatment of metastatic differentiated thyroid cancer in patients on Lthyroxine, using recombinant human TSH. Eur J Endocrinol 144:5-11.

931. Pellegriti G, Scollo C, Giuffrida D, Vigneri R, Squatrito S, Pezzino V 2001 Usefulness of recombinant human thyrotropin in the radiometabolic treatment of selected patients with thyroid cancer. Thyroid 11:1025-1030.

932. Adler ML, Macapinlac HA, Robbins RJ 1998 Radioiodine treatment of thyroid cancer with the aid of recombinant human thyrotropin. Endocr Pract 4:282-286.

933. Chiu AC, Delpassand ES, Sherman SI 1997 Prognosis and treatment of brain metastases in thyroid carcinoma. J Clin Endocrinol Metab 82:3637-3642.

934. Lau WF, Zacharin MR, Waters K, Wheeler G, Johnston V, Hicks RJ 2006 Management of paediatric thyroid carcinoma: recent experience with recombinant human thyroid stimulating hormone in preparation for radioiodine therapy. Intern Med J 36:564-570.

935. Potzi C, Moameni A, Karanikas G, Preitfellner J, Becherer A, Pirich C, Dudczak R 2006 Comparison of iodine uptake in tumour and nontumour tissue under thyroid hormone deprivation and with recombinant human thyrotropin in thyroid cancer patients. Clin Endocrinol (Oxf) 65:519-523.

936. Vargas GE, Uy H, Bazan C, Guise TA, Bruder JM 1999 Hemiplegia after thyrotropin alfa in a hypothyroid patient with thyroid carcinoma metastatic to the brain. $\mathrm{J}$ Clin Endocrinol Metab 84:3867-3871.

937. Robbins RJ, Voelker E, Wang W, Macapinlac HA, Larson SM 2000 Compassionate use of recombinant human thyrotropin to facilitate radioiodine therapy: case report and review of literature. Endocr Pract 6:460-464.

938. Braga M, Ringel MD, Cooper DS 2001 Sudden enlargement of local recurrent thyroid tumor after recombinant human TSH administration. J Clin Endocrinol Metab 86:5148-5151.

939. Pons F, Carrio I, Estorch M, Ginjaume M, Pons J, Milian R 1987 Lithium as an adjuvant of iodine-131 uptake when treating patients with well-differentiated thyroid carcinoma. Clin Nucl Med 12:644-647.

940. Koong SS, Reynolds JC, Movius EG, Keenan AM, Ain KB, Lakshmanan MC, Robbins J 1999 Lithium as a potential adjuvant to 131I therapy of metastatic, well differentiated thyroid carcinoma. J Clin Endocrinol Metab 84:912-916.

941. Liu YY, van der Pluijm G, Karperien M, Stokkel MP, Pereira AM, Morreau J, Kievit J, Romijn JA, Smit JW 2006 Lithium as adjuvant to radioiodine therapy in differentiated thyroid carcinoma: clinical and in vitro studies. Clin Endocrinol (Oxf) 64:617-624.

942. Ronga G, Filesi M, Montesano T, Di Nicola AD, Pace C, Travascio L, Ventroni G, Antonaci A, Vestri AR 2004 Lung metastases from differentiated thyroid carcinoma. A 40 years' experience. Q J Nucl Med Mol Imaging 48: 12-19.

943. Lin JD, Chao TC, Chou SC, Hsueh C 2004 Papillary thyroid carcinomas with lung metastases. Thyroid 14: 1091-1096.

944. Shoup M, Stojadinovic A, Nissan A, Ghossein RA, Freedman S, Brennan MF, Shah JP, Shaha AR 2003 Prognostic indicators of outcomes in patients with distant metastases from differentiated thyroid carcinoma. J Am Coll Surg 197:191-197.

945. Zettinig G, Fueger BJ, Passler C, Kaserer K, Pirich C, Dudczak R, Niederle B 2002 Long-term follow-up of patients with bone metastases from differentiated thyroid carcinoma-surgery or conventional therapy? Clin Endocrinol (Oxf) 56:377-382.

946. Pittas AG, Adler M, Fazzari M, Tickoo S, Rosai J, Larson SM, Robbins RJ 2000 Bone metastases from thyroid carcinoma: clinical characteristics and prognostic variables in one hundred forty-six patients. Thyroid 10: 261-268.

947. Schlumberger M, Challeton C, De Vathaire F, Travagli JP, Gardet P, Lumbroso JD, Francese C, Fontaine F, Ricard M, Parmentier C 1996 Radioactive iodine treatment and external radiotherapy for lung and bone metastases from thyroid carcinoma. J Nucl Med 37:598605. 
948. Dinneen SF, Valimaki MJ, Bergstralh EJ, Goellner JR, Gorman CA, Hay ID 1995 Distant metastases in papillary thyroid carcinoma: 100 cases observed at one institution during 5 decades. J Clin Endocrinol Metab 80: 2041-2045.

949. Foote RL, Brown PD, Garces YI, McIver B, Kasperbauer JL 2003 Is there a role for radiation therapy in the management of Hürthle cell carcinoma? Int $\mathrm{J}$ Radiat Oncol Biol Phys 56:1067-1072.

950. Pak H, Gourgiotis L, Chang WI, Guthrie LC, Skarulis MC, Reynolds JC, Merino MJ, Schrump DS, Libutti SK, Alexander HR Jr, Sarlis NJ 2003 Role of metastasectomy in the management of thyroid carcinoma: the NIH experience. J Surg Oncol 82:10-18.

951. Vitale G, Fonderico F, Martignetti A, Caraglia M, Ciccarelli A, Nuzzo V, Abbruzzese A, Lupoli G 2001 Pamidronate improves the quality of life and induces clinical remission of bone metastases in patients with thyroid cancer. Br J Cancer 84:1586-1590.

952. Kitamura Y, Shimizu K, Nagahama M, Sugino K, Ozaki O, Mimura T, Ito K, Ito K, Tanaka S 1999 Immediate causes of death in thyroid carcinoma: clinicopathological analysis of 161 fatal cases. J Clin Endocrinol Metab 84: 4043-4049.

953. Brose MS, Smit J, Capdevila J, Elisei R, Nutting C, Pitoia F, Robinson B, Schlumberger M, Shong YK, Takami H 2012 Regional approaches to the management of patients with advanced, radioactive iodine-refractory differentiated thyroid carcinoma. Expert Rev Anticancer Ther 12:1137-1147.

954. Sabra MM, Dominguez JM, Grewal RK, Larson SM, Ghossein RA, Tuttle RM, Fagin JA 2013 Clinical outcomes and molecular profile of differentiated thyroid cancers with radioiodine-avid distant metastases. J Clin Endocrinol Metab 98:E829-E836.

955. Benua RS, Cicale NR, Sonenberg M, Rawson RW 1962 The relation of radioiodine dosimetry to results and complications in the treatment of metastatic thyroid cancer. Am J Roentgenol Radium Ther Nucl Med 87:171-182.

956. Hebestreit H, Biko J, Drozd V, Demidchik Y, Burkhardt A, Trusen A, Beer M, Reiners C 2011 Pulmonary fibrosis in youth treated with radioiodine for juvenile thyroid cancer and lung metastases after Chernobyl. Eur J Nucl Med Mol Imaging 38:1683-1690.

957. Ilgan S, Karacalioglu AO, Pabuscu Y, Atac GK, Arslan N, Ozturk E, Gunalp B, Ozguven MA 2004 Iodine-131 treatment and high-resolution CT: results in patients with lung metastases from differentiated thyroid carcinoma. Eur J Nucl Med Mol Imaging 31:825-830.

958. Hod N, Hagag P, Baumer M, Sandbank J, Horne T 2005 Differentiated thyroid carcinoma in children and young adults: evaluation of response to treatment. Clin Nucl Med 30:387-390.

959. Van Nostrand D, Freitas J 2006 Side effects of 131I for ablation and treatment of well differentiated thyroid carcinoma. In: Wartofsky L, Van Nostrand D (eds) Thyroid Cancer: A Comprehensive Guide to Clinical Management. 2nd edition. Humana Press, Totowa, NJ, pp 459-485.

960. Lang BH, Wong IO, Wong KP, Cowling BJ, Wan KY 2012 Risk of second primary malignancy in differentiated thyroid carcinoma treated with radioactive iodine therapy. Surgery 151:844-850.

961. Sawka AM, Thabane L, Parlea L, Ibrahim-Zada I, Tsang RW, Brierley JD, Straus S, Ezzat S, Goldstein DP 2009
Second primary malignancy risk after radioactive iodine treatment for thyroid cancer: a systematic review and meta-analysis. Thyroid 19:451-457.

962. Fatourechi V, Hay ID, Javedan H, Wiseman GA, Mullan BP, Gorman CA 2002 Lack of impact of radioiodine therapy in tg-positive, diagnostic whole-body scannegative patients with follicular cell-derived thyroid cancer. J Clin Endocrinol Metab 87:1521-1526.

963. Koh JM, Kim ES, Ryu JS, Hong SJ, Kim WB, Shong YK 2003 Effects of therapeutic doses of 131I in thyroid papillary carcinoma patients with elevated thyroglobulin level and negative 131I whole-body scan: comparative study. Clin Endocrinol (Oxf) 58:421-427.

964. Ma C, Kuang A, Xie J 2009 Radioiodine therapy for differentiated thyroid carcinoma with thyroglobulin positive and radioactive iodine negative metastases. Cochrane Database Syst Rev1-38.

965. Wang W, Larson SM, Tuttle RM, Kalaigian H, Kolbert K, Sonenberg M, Robbins RJ 2001 Resistance of [18f]fluorodeoxyglucose-avid metastatic thyroid cancer lesions to treatment with high-dose radioactive iodine. Thyroid 11:1169-1175.

966. Salvatore B, Paone G, Klain M, Storto G, Nicolai E, D’Amico D, Della Morte AM, Pace L, Salvatore M 2008 Fluorodeoxyglucose PET/CT in patients with differentiated thyroid cancer and elevated thyroglobulin after total thyroidectomy and (131)I ablation. Q J Nucl Med Mol Imaging 52:2-8.

967. Kloos RT 2008 Approach to the patient with a positive serum thyroglobulin and a negative radioiodine scan after initial therapy for differentiated thyroid cancer. J Clin Endocrinol Metab 93:1519-1525.

968. van Tol KM, Jager PL, de Vries EG, Piers DA, Boezen HM, Sluiter WJ, Dullaart RP, Links TP 2003 Outcome in patients with differentiated thyroid cancer with negative diagnostic whole-body scanning and detectable stimulated thyroglobulin. Eur J Endocrinol 148:589-596.

969. Kabasakal L, Selcuk NA, Shafipour H, Ozmen O, Onsel C, Uslu I 2004 Treatment of iodine-negative thyroglobulinpositive thyroid cancer: differences in outcome in patients with macrometastases and patients with micrometastases. Eur J Nucl Med Mol Imaging 31:1500-1504.

970. Yim JH, Kim EY, Bae KW, Kim WG, Kim TY, Ryu JS, Gong G, Hong SJ, Yoon JH, Shong YK 2013 Long-term consequence of elevated thyroglobulin in differentiated thyroid cancer. Thyroid 23:58-63.

971. Black EG, Sheppard MC, Hoffenberg R 1987 Serial serum thyroglobulin measurements in the management of differentiated thyroid carcinoma. Clin Endocrinol (Oxf) 27:115-120.

972. Huang SH, Wang PW, Huang YE, Chou FF, Liu RT, Tung SC, Chen JF, Kuo MC, Hsieh JR, Hsieh HH 2006 Sequential follow-up of serum thyroglobulin and whole body scan in thyroid cancer patients without initial metastasis. Thyroid 16:1273-1278.

973. Schlumberger M, Mancusi F, Baudin E, Pacini F 1997 131I therapy for elevated thyroglobulin levels. Thyroid 7:273-276.

974. Ma C, Xie J, Kuang A 2005 Is empiric 131I therapy justified for patients with positive thyroglobulin and negative 131I whole-body scanning results? J Nucl Med 46:1164-1170.

975. Chao M 2010 Management of differentiated thyroid cancer with rising thyroglobulin and negative diagnostic 
radioiodine whole body scan. Clin Oncol (R Coll Radiol) 22:438-447.

976. Sinha P, Conrad GR, West HC 2011 Response of thyroglobulin to radioiodine therapy in thyroglobulin-elevated negative iodine scintigraphy (TENIS) syndrome. Anticancer Res 31:2109-2112.

977. Ozata M, Suzuki S, Miyamoto T, Liu RT, Fierro-Renoy F, DeGroot LJ 1994 Serum thyroglobulin in the followup of patients with treated differentiated thyroid cancer. $\mathbf{J}$ Clin Endocrinol Metab 79:98-105.

978. Kim WG, Ryu JS, Kim EY, Lee JH, Baek JH, Yoon JH, Hong SJ, Kim ES, Kim TY, Kim WB, Shong YK 2010 Empiric high-dose 131-iodine therapy lacks efficacy for treated papillary thyroid cancer patients with detectable serum thyroglobulin, but negative cervical sonography and 18F-fluorodeoxyglucose positron emission tomography scan. J Clin Endocrinol Metab 95:1169-1173.

979. Biko J, Reiners C, Kreissl MC, Verburg FA, Demidchik Y, Drozd V 2011 Favourable course of disease after incomplete remission on (131)I therapy in children with pulmonary metastases of papillary thyroid carcinoma: 10 years follow-up. Eur J Nucl Med Mol Imaging 38:651655.

980. Schlumberger M, Arcangioli O, Piekarski JD, Tubiana M, Parmentier C 1988 Detection and treatment of lung metastases of differentiated thyroid carcinoma in patients with normal chest X-rays. J Nucl Med 29:1790-1794.

981. Walter MA, Turtschi CP, Schindler C, Minnig P, MullerBrand J, Muller B 2007 The dental safety profile of highdose radioiodine therapy for thyroid cancer: long-term results of a longitudinal cohort study. J Nucl Med 48: $1620-1625$.

982. Kloos RT, Duvuuri V, Jhiang SM, Cahill KV, Foster JA, Burns JA 2002 Nasolacrimal drainage system obstruction from radioactive iodine therapy for thyroid carcinoma. J Clin Endocrinol Metab 87:5817-5820.

983. Sandeep TC, Strachan MW, Reynolds RM, Brewster DH, Scelo G, Pukkala E, Hemminki K, Anderson A, Tracey E, Friis S, McBride ML, Kee-Seng C, PompeKirn V, Kliewer EV, Tonita JM, Jonasson JG, Martos C, Boffetta P, Brennan P 2006 Second primary cancers in thyroid cancer patients: a multinational record linkage study. J Clin Endocrinol Metab 91:1819-1825.

984. Subramanian S, Goldstein DP, Parlea L, Thabane L, Ezzat S, Ibrahim-Zada I, Straus S, Brierley JD, Tsang RW, Gafni A, Rotstein L, Sawka AM 2007 Second primary malignancy risk in thyroid cancer survivors: a systematic review and meta-analysis. Thyroid 17:12771288.

985. Almeida JP, Sanabria AE, Lima EN, Kowalski LP 2011 Late side effects of radioactive iodine on salivary gland function in patients with thyroid cancer. Head Neck 33:686-690.

986. Mandel SJ, Mandel L 2003 Radioactive iodine and the salivary glands. Thyroid 13:265-271.

987. Nakada K, Ishibashi T, Takei T, Hirata K, Shinohara K, Katoh S, Zhao S, Tamaki N, Noguchi Y, Noguchi S 2005 Does lemon candy decrease salivary gland damage after radioiodine therapy for thyroid cancer? J Nucl Med 46:261-266.

988. Jentzen W, Balschuweit D, Schmitz J, Freudenberg L, Eising E, Hilbel T, Bockisch A, Stahl A 2010 The influence of saliva flow stimulation on the absorbed radiation dose to the salivary glands during radioiodine therapy of thyroid cancer using 124I PET(/CT) imaging. Eur J Nucl Med Mol Imaging 37:2298-2306.

989. Van Nostrand D, Bandaru V, Chennupati S, Wexler J, Kulkarni K, Atkins F, Mete M, Gadwale G 2010 Radiopharmacokinetics of radioiodine in the parotid glands after the administration of lemon juice. Thyroid 20:1113-1119.

990. Bomeli SR, Schaitkin B, Carrau RL, Walvekar RR 2009 Interventional sialendoscopy for treatment of radioiodineinduced sialadenitis. Laryngoscope 119:864-867.

991. Prendes BL, Orloff LA, Eisele DW 2012 Therapeutic sialendoscopy for the management of radioiodine sialadenitis. Arch Otolaryngol Head Neck Surg 138:15-19.

992. Bhayani MK, Acharya V, Kongkiatkamon S, Farah S, Roberts DB, Sterba J, Chambers MS, Lai SY 2015 Sialendoscopy for patients with radioiodine-induced sialadenitis and xerostomia. Thyroid 25:834-838.

993. Chen AY, Levy L, Goepfert H, Brown BW, Spitz MR, Vassilopoulou-Sellin R 2001 The development of breast carcinoma in women with thyroid carcinoma. Cancer 92:225-231.

994. Perry WF, Hughes JF 1952 The urinary excretion and thyroid uptake of iodine in renal disease. J Clin Invest 31:457-463.

995. Vini L, Hyer S, Al-Saadi A, Pratt B, Harmer C 2002 Prognosis for fertility and ovarian function after treatment with radioiodine for thyroid cancer. Postgrad Med J 78:92-93.

996. Dottorini ME, Lomuscio G, Mazzucchelli L, Vignati A, Colombo L 1995 Assessment of female fertility and carcinogenesis after iodine-131 therapy for differentiated thyroid carcinoma. J Nucl Med 36:21-27.

997. Sawka AM, Lakra DC, Lea J, Alshehri B, Tsang RW, Brierley JD, Straus S, Thabane L, Gafni A, Ezzat S, George SR, Goldstein DP 2008 A systematic review examining the effects of therapeutic radioactive iodine on ovarian function and future pregnancy in female thyroid cancer survivors. Clin Endocrinol (Oxf) 69:479490.

998. Wu JX, Young S, Ro K, Li N, Leung AM, Chiu HK, Harari A, Yeh MW 2015 Reproductive outcomes and nononcologic complications after radioactive iodine ablation for well-differentiated thyroid cancer. Thyroid 25:133-138.

999. Schlumberger M, De Vathaire F, Ceccarelli C, Delisle MJ, Francese C, Couette JE, Pinchera A, Parmentier C 1996 Exposure to radioactive iodine-131 for scintigraphy or therapy does not preclude pregnancy in thyroid cancer patients. J Nucl Med 37:606-612.

1000. Garsi JP, Schlumberger M, Rubino C, Ricard M, Labbe M, Ceccarelli C, Schvartz C, Henri-Amar M, Bardet S, De Vathaire F 2008 Therapeutic administration of 131I for differentiated thyroid cancer: radiation dose to ovaries and outcome of pregnancies. J Nucl Med 49:845852.

1001. Ceccarelli C, Bencivelli W, Morciano D, Pinchera A, Pacini F 2001 131I therapy for differentiated thyroid cancer leads to an earlier onset of menopause: results of a retrospective study. J Clin Endocrinol Metab 86:35123515.

1002. Bakheet SM, Powe J, Hammami MM 1998 Unilateral radioiodine breast uptake. Clin Nucl Med 23:170-171.

1003. Sisson JC, Freitas J, McDougall IR, Dauer LT, Hurley JR, Brierley JD, Edinboro CH, Rosenthal D, Thomas MJ, 
Wexler JA, Asamoah E, Avram AM, Milas M, Greenlee C 2011 Radiation safety in the treatment of patients with thyroid diseases by radioiodine 131I : practice recommendations of the American Thyroid Association. Thyroid 21:335-346.

1004. Bernard N, Jantzem H, Becker M, Pecriaux C, BenardLaribiere A, Montastruc JL, Descotes J, Vial T 2015 Severe adverse effects of bromocriptine in lactation inhibition: a pharmacovigilance survey. BJOG 122:1244-1251.

1005. Wichers M, Benz E, Palmedo H, Biersack HJ, Grunwald F, Klingmuller D 2000 Testicular function after radioiodine therapy for thyroid carcinoma. Eur J Nucl Med 27:503-507.

1006. Hyer S, Vini L, O’Connell M, Pratt B, Harmer C 2002 Testicular dose and fertility in men following I(131) therapy for thyroid cancer. Clin Endocrinol (Oxf) 56: $755-758$.

1007. Lushbaugh CC, Casarett GW 1976 The effects of gonadal irradiation in clinical radiation therapy: a review. Cancer 37:1111-1125.

1008. Sarkar SD, Beierwaltes WH, Gill SP, Cowley BJ 1976 Subsequent fertility and birth histories of children and adolescents treated with $131 \mathrm{I}$ for thyroid cancer. J Nucl Med 17:460-464.

1009. Mazzaferri EL 2002 Gonadal damage from 131I therapy for thyroid cancer. Clin Endocrinol (Oxf) 57:313-314.

1010. Schlumberger M, Brose M, Elisei R, Leboulleux S, Luster M, Pitoia F, Pacini F 2014 Definition and management of radioactive iodine-refractory differentiated thyroid cancer. Lancet Diabetes Endocrinol 2:356-358.

1011. Eisenhauer EA, Therasse P, Bogaerts J, Schwartz LH, Sargent D, Ford R, Dancey J, Arbuck S, Gwyther S, Mooney M, Rubinstein L, Shankar L, Dodd L, Kaplan R, Lacombe D, Verweij J 2009 New response evaluation criteria in solid tumours: revised RECIST guideline (version 1.1). Eur J Cancer 45:228-247.

1012. Schlumberger M, Sherman SI 2012 Approach to the patient with advanced differentiated thyroid cancer. Eur J Endocrinol 166:5-11.

1013. Brose MS, Nutting CM, Jarzab B, Elisei R, Siena S, Bastholt L, de la Fouchardiere C, Pacini F, Paschke R, Shong YK, Sherman SI, Smit JW, Chung J, Kappeler C, Pena C, Molnar I, Schlumberger MJ 2014 Sorafenib in radioactive iodine-refractory, locally advanced or metastatic differentiated thyroid cancer: a randomised, double-blind, phase 3 trial. Lancet 384:319-328.

1014. Schlumberger M, Tahara M, Wirth LJ, Robinson B, Brose MS, Elisei R, Dutcus CE, de las Heras B, Zhu J, Habra MA, Newbold K, Shah MH, Hoff AO, Gianoukakis AG, Kiyota N, Hiram M, Kim SB, Krzyzanowska MK, Sherman SI 2014 A phase 3, multicenter, doubleblind, placebo-controlled trial of lenvatinib (E7080) in patients with 131I-refractory differentiated thyroid cancer (SELECT). J Clin Oncol 32:5S. (Abstract.).

1015. Kocher M, Soffietti R, Abacioglu U, Villa S, Fauchon F, Baumert BG, Fariselli L, Tzuk-Shina T, Kortmann RD, Carrie C, Ben HM, Kouri M, Valeinis E, van den Berge D, Collette S, Collette L, Mueller RP 2011 Adjuvant wholebrain radiotherapy versus observation after radiosurgery or surgical resection of one to three cerebral metastases: results of the EORTC 22952-26001 study. J Clin Oncol 29:134-141.

1016. Bonichon F, Palussière J, Godbert Y, Pulido M, Descat E, Devillers A, Meunier C, Leboulleux S, de Baère T,
Galy-Lacour C, Lagoarde-Segot L, Cazeau AL 2013 Diagnostic accuracy of 18F-FDG PET/CT for assessing response to radiofrequency ablation treatment in lung metastases: a multicentre prospective study. Eur J Nucl Med Mol Imaging 40:1817-1827.

1017. Lo SS, Fakiris AJ, Chang EL, Mayr NA, Wang JZ, Papiez L, Teh BS, $\mu$ garry RC, Cardenes HR, Timmerman RD 2010 Stereotactic body radiation therapy: a novel treatment modality. Nat Rev Clin Oncol 7:44-54.

1018. Lo SS, Fakiris AJ, Teh BS, Cardenes HR, Henderson MA, Forquer JA, Papiez L, $\mu$ garry RC, Wang JZ, Li K, Mayr NA, Timmerman RD 2009 Stereotactic body radiation therapy for oligometastases. Expert Rev Anticancer Ther 9:621-635.

1019. de Baere T, Elias D, Dromain C, Din MG, Kuoch V, Ducreux M, Boige V, Lassau N, Marteau V, Lasser P, Roche A 2000 Radiofrequency ablation of 100 hepatic metastases with a mean follow-up of more than 1 year. AJR Am J Roentgenol 175:1619-1625.

1020. Solbiati L, Livraghi T, Goldberg SN, Ierace T, Meloni F, Dellanoce M, Cova L, Halpern EF, Gazelle GS 2001 Percutaneous radio-frequency ablation of hepatic metastases from colorectal cancer: long-term results in 117 patients. Radiology 221:159-166.

1021. de Baere T, Palussiere J, Auperin A, Hakime A, AbdelRehim M, Kind M, Dromain C, Ravaud A, Tebboune N, Boige V, Malka D, Lafont C, Ducreux M 2006 Midterm local efficacy and survival after radiofrequency ablation of lung tumors with minimum follow-up of 1 year: prospective evaluation. Radiology 240:587-596.

1022. Lencioni R, Crocetti L, Cioni R, Suh R, Glenn D, Regge D, Helmberger T, Gillams AR, Frilling A, Ambrogi M, Bartolozzi C, Mussi A 2008 Response to radiofrequency ablation of pulmonary tumours: a prospective, intentionto-treat, multicentre clinical trial (the RAPTURE study). Lancet Oncol 9:621-628.

1023. Goetz MP, Callstrom MR, Charboneau JW, Farrell MA, Maus TP, Welch TJ, Wong GY, Sloan JA, Novotny PJ, Petersen IA, Beres RA, Regge D, Capanna R, Saker MB, Gronemeyer DH, Gevargez A, Ahrar K, Choti MA, de Baere TJ, Rubin J 2004 Percutaneous image-guided radiofrequency ablation of painful metastases involving bone: a multicenter study. J Clin Oncol 22:300-306.

1024. Dupuy DE, Liu D, Hartfeil D, Hanna L, Blume JD, Ahrar K, Lopez R, Safran H, DiPetrillo T 2010 Percutaneous radiofrequency ablation of painful osseous metastases: a multicenter American College of Radiology Imaging Network trial. Cancer 116:989-997.

1025. Deandreis D, Leboulleux S, Dromain C, Auperin A, Coulot J, Lumbroso J, Deschamps F, Rao P, Schlumberger M, de Baere T 2011 Role of FDG PET/CT and chest $\mathrm{CT}$ in the follow-up of lung lesions treated with radiofrequency ablation. Radiology 258:270-276.

1026. Wertenbroek MW, Links TP, Prins TR, Plukker JT, van der Jagt EJ, de Jong KP 2008 Radiofrequency ablation of hepatic metastases from thyroid carcinoma. Thyroid 18:1105-1110.

1027. Quan GM, Pointillart V, Palussiere J, Bonichon F 2012 Multidisciplinary treatment and survival of patients with vertebral metastases from thyroid carcinoma. Thyroid 22:125-130.

1028. Kurup AN, Callstrom MR 2010 Ablation of skeletal metastases: current status. J Vasc Interv Radiol 21:S242S250. 
1029. McWilliams RR, Giannini C, Hay ID, Atkinson JL, Stafford SL, Buckner JC 2003 Management of brain metastases from thyroid carcinoma: a study of 16 pathologically confirmed cases over 25 years. Cancer 98: 356-362.

1030. Henriques de Figueiredo B, Godbert Y, Soubeyran I, Carrat X, Lagarde P, Cazeau AL, Italiano A, Sargos P, Kantor G, Loiseau H, Bonichon F 2014 Brain metastases from thyroid carcinoma: a retrospective study of 21 patients. Thyroid 24:270-276.

1031. Chow CJ, Habermann EB, Abraham A, Zhu Y, Vickers SM, Rothenberger DA, Al-Refaie WB 2013 Does enrollment in cancer trials improve survival? J Am Coll Surg 216:774-780.

1032. Anderson RT, Linnehan JE, Tongbram V, Keating K, Wirth LJ 2013 Clinical, safety, and economic evidence in radioactive iodine-refractory differentiated thyroid cancer: a systematic literature review. Thyroid 23:392407.

1033. Leboulleux S, Bastholt L, Krause T, de la Fouchardiere C, Tennvall J, Awada A, Gomez JM, Bonichon F, Leenhardt L, Soufflet C, Licour M, Schlumberger MJ 2012 Vandetanib in locally advanced or metastatic differentiated thyroid cancer: a randomised, double-blind, phase 2 trial. Lancet Oncol 13:897-905.

1034. Schlumberger M, Tahara M, Wirth LJ, Robinson B, Brose MS, Elisei R, Habra MA, Newbold K, Shah MH, Hoff AO, Gianoukakis AG, Kiyota N, Taylor MH, Kim SB, Krzyzanowska MK, Dutcus CE, de las Heras B, Zhu J, Sherman SI 2015 Lenvatinib versus placebo in radioiodine-refractory thyroid cancer. N Engl J Med 372:621-630.

1035. Cohen EE, Rosen LS, Vokes EE, Kies MS, Forastiere AA, Worden FP, Kane MA, Sherman E, Kim S, Bycott P, Tortorici M, Shalinsky DR, Liau KF, Cohen RB 2008 Axitinib is an active treatment for all histologic subtypes of advanced thyroid cancer: results from a phase II study. J Clin Oncol 26:4708-4713.

1036. Bible KC, Suman VJ, Molina JR, Smallridge RC, Maples WJ, Menefee ME, Rubin J, Sideras K, Morris JC III, McIver B, Burton JK, Webster KP, Bieber C, Traynor AM, Flynn PJ, Goh BC, Tang H, Ivy SP, Erlichman C 2010 Efficacy of pazopanib in progressive, radioiodinerefractory, metastatic differentiated thyroid cancers: results of a phase 2 consortium study. Lancet Oncol 11:962-972.

1037. Carr LL, Mankoff DA, Goulart BH, Eaton KD, Capell PT, Kell EM, Bauman JE, Martins RG 2010 Phase II study of daily sunitinib in FDG-PET-positive, iodinerefractory differentiated thyroid cancer and metastatic medullary carcinoma of the thyroid with functional imaging correlation. Clin Cancer Res 16:5260-5268.

1038. Droz JP, Schlumberger M, Rougier P, Ghosn M, Gardet P, Parmentier C 1990 Chemotherapy in metastatic nonanaplastic thyroid cancer: experience at the Institut Gustave-Roussy. Tumori 76:480-483.

1039. Schutz FA, Je Y, Richards CJ, Choueiri TK 2012 Metaanalysis of randomized controlled trials for the incidence and risk of treatment-related mortality in patients with cancer treated with vascular endothelial growth factor tyrosine kinase inhibitors. J Clin Oncol 30:871-877.

1040. Schneider TC, Abdulrahman RM, Corssmit EP, Morreau H, Smit JW, Kapiteijn E 2012 Long-term analysis of the efficacy and tolerability of sorafenib in advanced radio- iodine refractory differentiated thyroid carcinoma: final results of a phase II trial. Eur J Endocrinol 167:643-650.

1041. Ahmed M, Barbachano Y, Riddell A, Hickey J, Newbold KL, Viros A, Harrington KJ, Marais R, Nutting CM 2011 Analysis of the efficacy and toxicity of sorafenib in thyroid cancer: a phase II study in a UK based population. Eur J Endocrinol 165:315-322.

1042. Kloos RT, Ringel MD, Knopp MV, Hall NC, King M, Stevens R, Liang J, Wakely PE Jr, Vasko VV, Saji M, Rittenberry J, Wei L, Arbogast D, Collamore M, Wright JJ, Grever M, Shah MH 2009 Phase II trial of sorafenib in metastatic thyroid cancer. J Clin Oncol 27:1675-1684.

1043. Gupta-Abramson V, Troxel AB, Nellore A, Puttaswamy K, Redlinger M, Ransone K, Mandel SJ, Flaherty KT, Loevner LA, O’Dwyer PJ, Brose MS 2008 Phase II trial of sorafenib in advanced thyroid cancer. J Clin Oncol 26:4714-4719.

1044. Carhill AA, Cabanillas ME, Jimenez C, Waguespack SG, Habra MA, Hu M, Ying A, Vassilopoulou-Sellin R, Gagel RF, Sherman SI, Busaidy NL 2013 The noninvestigational use of tyrosine kinase inhibitors in thyroid cancer: establishing a standard for patient safety and monitoring. J Clin Endocrinol Metab 98:31-42.

1045. Bible KC, Ain KB, Rosenthal MS 2014 Protein kinase inhibitor therapy in advanced thyroid cancer: ethical challenges and potential solutions. Int $\mathrm{J}$ Endocr Oncol 1:145-151.

1046. Massicotte MH, Brassard M, Claude-Desroches M, Borget I, Bonichon F, Giraudet AL, Do Cao C, Chougnet CN, Leboulleux S, Baudin E, Schlumberger M, de la Fouchardiere C 2014 Tyrosine kinase inhibitor treatments in patients with metastatic thyroid carcinomas: a retrospective study of the TUTHYREF network. Eur J Endocrinol 170:575-582.

1047. Di Lorenzo G, Autorino R, Bruni G, Carteni G, Ricevuto E, Tudini M, Ficorella C, Romano C, Aieta M, Giordano A, Giuliano M, Gonnella A, De Nunzio C, Rizzo M, Montesarchio V, Ewer M, De Placido S 2009 Cardiovascular toxicity following sunitinib therapy in metastatic renal cell carcinoma: a multicenter analysis. Ann Oncol 20:1535-1542.

1048. Harris PJ, Bible KC 2011 Emerging therapeutics for advanced thyroid malignancies: rationale and targeted approaches. Expert Opin Investig Drugs 20: 1357-1375.

1049. Hauschild A, Grob JJ, Demidov LV, Jouary T, Gutzmer R, Millward M, Rutkowski P, Blank CU, Miller WH Jr, Kaempgen E, Martin-Algarra S, Karaszewska B, Mauch C, Chiarion-Sileni V, Martin AM, Swann S, Haney P, Mirakhur B, Guckert ME, Goodman V, Chapman PB 2012 Dabrafenib in BRAF-mutated metastatic melanoma: a multicentre, open-label, phase 3 randomised controlled trial. Lancet 380:358-365.

1050. Falchook GS, Long GV, Kurzrock R, Kim KB, Arkenau TH, Brown MP, Hamid O, Infante JR, Millward M, Pavlick AC, O'Day SJ, Blackman SC, Curtis CM, Lebowitz P, Ma B, Ouellet D, Kefford RF 2012 Dabrafenib in patients with melanoma, untreated brain metastases, and other solid tumours: a phase 1 dose-escalation trial. Lancet 379:1893-1901.

1051. Kudchadkar R, Paraiso KH, Smalley KS 2012 Targeting mutant BRAF in melanoma: current status and future development of combination therapy strategies. Cancer J 18:124-131. 
1052. Ho AL, Grewal RK, Leboeuf R, Sherman EJ, Pfister DG, Deandreis D, Pentlow KS, Zanzonico PB, Haque S, Gavane S, Ghossein RA, Ricarte-Filho JC, Dominguez JM, Shen R, Tuttle RM, Larson SM, Fagin JA 2013 Selumetinib-enhanced radioiodine uptake in advanced thyroid cancer. N Engl J Med 368:623-632.

1053. Spano JP, Vano Y, Vignot S, De La Motte RT, Hassani L, Mouawad R, Menegaux F, Khayat D, Leenhardt L 2012 GEMOX regimen in the treatment of metastatic differentiated refractory thyroid carcinoma. Med Oncol 29:1421-1428.

1054. Farooki A, Leung V, Tala H, Tuttle RM 2012 Skeletalrelated events due to bone metastases from differentiated thyroid cancer. J Clin Endocrinol Metab 97: 2433-2439.

1055. Coleman R, Woodward E, Brown J, Cameron D, Bell R, Dodwell D, Keane M, Gil M, Davies C, Burkinshaw R, Houston SJ, Grieve RJ, Barrett-Lee PJ, Thorpe H 2011 Safety of zoledronic acid and incidence of osteonecrosis of the jaw (ONJ) during adjuvant therapy in a randomised phase III trial (AZURE: BIG 01-04) for women with stage II/III breast cancer. Breast Cancer Res Treat 127:429-438.

1056. Wardley A, Davidson N, Barrett-Lee P, Hong A, Mansi J, Dodwell D, Murphy R, Mason T, Cameron D 2005 Zoledronic acid significantly improves pain scores and quality of life in breast cancer patients with bone metastases: a randomised, crossover study of community vs hospital bisphosphonate administration. $\mathrm{Br} \mathrm{J}$ Cancer 92:1869-1876.

1057. Orita Y, Sugitani I, Toda K, Manabe J, Fujimoto Y 2011 Zoledronic acid in the treatment of bone metastases from differentiated thyroid carcinoma. Thyroid 21:31-35.

1058. Lipton A, Fizazi K, Stopeck AT, Henry DH, Brown JE, Yardley DA, Richardson GE, Siena S, Maroto P, Clemens M, Bilynskyy B, Charu V, Beuzeboc P, Rader M, Viniegra M, Saad F, Ke C, Braun A, Jun S 2012 Superiority of denosumab to zoledronic acid for prevention of skeletal-related events: a combined analysis of 3 pivotal, randomised, phase 3 trials. Eur J Cancer 48:3082-3092.

1059. Cancer Genome Atlas Research Network 2014 Integrated genomic characterization of papillary thyroid carcinoma. Cell 159:676-690.

1060. Goldfarb M, Casillas J 2014 Unmet information and support needs in newly diagnosed thyroid cancer: comparison of adolescents/young adults (AYA) and older patients. J Cancer Surviv 8:394-401.

1061. Banach R, Bartes B, Farnell K, Rimmele H, Shey J, Singer S, Verburg FA, Luster M 2013 Results of the Thyroid Cancer Alliance international patient/survivor survey: Psychosocial/informational support needs, treatment side effects and international differences in care. Hormones (Athens) 12:428-438.

1062. O'Brien MA, Whelan TJ, Villasis-Keever M, Gafni A, Charles C, Roberts R, Schiff S, Cai W 2009 Are cancerrelated decision aids effective? A systematic review and meta-analysis. J Clin Oncol 27:974-985.

1063. Spiegle G, Al-Sukhni E, Schmocker S, Gagliardi AR, Victor JC, Baxter NN, Kennedy ED 2013 Patient decision aids for cancer treatment: are there any alternatives? Cancer 119:189-200.

1064. Sawka AM, Straus S, Rotstein L, Brierley JD, Tsang RW, Asa S, Segal P, Kelly C, Zahedi A, Freeman J, Solomon P, Anderson J, Thorpe KE, Gafni A, Rodin G,
Goldstein DP 2012 Randomized controlled trial of a computerized decision aid on adjuvant radioactive iodine treatment for patients with early-stage papillary thyroid cancer. J Clin Oncol 30:2906-2911.

1065. Spencer C, Petrovic I, Fatemi S 2011 Current thyroglobulin autoantibody ( $\mathrm{TgAb}$ ) assays often fail to detect interfering $\mathrm{TgAb}$ that can result in the reporting of falsely low/undetectable serum Tg IMA values for patients with differentiated thyroid cancer. J Clin Endocrinol Metab 96:1283-1291.

1066. Ito Y, Tomoda C, Uruno T, Takamura Y, Miya A, Kobayashi K, Matsuzuka F, Kuma K, Miyauchi A 2005 Ultrasonographically and anatomopathologically detectable node metastases in the lateral compartment as indicators of worse relapse-free survival in patients with papillary thyroid carcinoma. World J Surg 29:917-920.

1067. Ito Y, Tomoda C, Uruno T, Takamura Y, Miya A, Kobayashi K, Matsuzuka F, Kuma K, Miyauchi A 2006 Clinical significance of metastasis to the central compartment from papillary microcarcinoma of the thyroid. World J Surg 30:91-99.

1068. Rothenberg SM, McFadden DG, Palmer EL, Daniels GH, Wirth LJ 2015 Redifferentiation of iodine-refractory $B R A F^{V 600 E}$-mutant metastatic papillary thyroid cancer with dabrafenib. Clin Cancer Res 21:1028-1035.

1069. Topalian SL, Hodi FS, Brahmer JR, Gettinger SN, Smith DC, McDermott DF, Powderly JD, Carvajal RD, Sosman JA, Atkins MB, Leming PD, Spigel DR, Antonia SJ, Horn L, Drake CG, Pardoll DM, Chen L, Sharfman WH, Anders RA, Taube JM, McMiller TL, Xu H, Korman AJ, Jure-Kunkel M, Agrawal S, McDonald D, Kollia GD, Gupta A, Wigginton JM, Sznol M 2012 Safety, activity, and immune correlates of anti-PD-1 antibody in cancer. N Engl J Med 366:2443-2454.

1070. Brahmer JR, Tykodi SS, Chow LQ, Hwu WJ, Topalian SL, Hwu P, Drake CG, Camacho LH, Kauh J, Odunsi K, Pitot HC, Hamid O, Bhatia S, Martins R, Eaton K, Chen S, Salay TM, Alaparthy S, Grosso JF, Korman AJ, Parker SM, Agrawal S, Goldberg SM, Pardoll DM, Gupta A, Wigginton JM 2012 Safety and activity of anti-PD-L1 antibody in patients with advanced cancer. N Engl J Med 366:2455-2465.

1071. Hamid O, Robert C, Daud A, Hodi FS, Hwu WJ, Kefford R, Wolchok JD, Hersey P, Joseph RW, Weber JS, Dronca R, Gangadhar TC, Patnaik A, Zarour H, Joshua AM, Gergich K, Elassaiss-Schaap J, Algazi A, Mateus C, Boasberg P, Tumeh PC, Chmielowski B, Ebbinghaus SW, Li XN, Kang SP, Ribas A 2013 Safety and tumor responses with lambrolizumab (anti-PD-1) in melanoma. N Engl J Med 369:134-144.

1072. Husson O, Haak HR, Mols F, Nieuwenhuijzen GA, Nieuwlaat WA, Reemst PH, Huysmans DA, Toorians AW, van de Poll-Franse LV 2013 Development of a disease-specific health-related quality of life questionnaire (THYCA-QoL) for thyroid cancer survivors. Acta Oncol 52:447-454.

1073. Sawka AM, Naeem A, Jones J, Lowe J, Segal P, Goguen J, Gilbert J, Zahedi A, Kelly C, Ezzat S 2014 Persistent posttreatment fatigue in thyroid cancer survivors: a scoping review. Endocrinol Metab Clin North Am 43: 475-494.

1074. To J, Goldberg AS, Jones J, Zhang J, Lowe J, Ezzat S, Gilbert J, Zahedi A, Segal P, Sawka AM 2014 A systematic review of randomized controlled trials for man- 
agement of persistent post-treatment fatigue in thyroid cancer survivors. Thyroid 25:198-210.

1075. Bresner L, Banach R, Rodin G, Thabane L, Ezzat S, Sawka AM 2015 Cancer-related worry in Canadian thyroid cancer survivors. J Clin Endocrinol Metab 100:977-985.

1076. Cibas ES, Ali SZ 2009 The Bethesda System For Reporting Thyroid Cytopathology. Am J Clin Pathol 132: 658-665.

1077. Edge SB, Byrd DR, Compton CC, Fritz AG, Greene FL, Trotti A 2010 Thyroid cancer staging. In: Edge SB, Byrd DR, Compton CC, Fritz AG, Greene FL, Trotti A (eds) AJCC Cancer Staging Manual. 7th edition. SpringerVerlag, New York, pp 59-64.
1078. Rosario PW, Mineiro Filho AF, Prates BS, Silva LC, Calsolari MR 2012 Postoperative stimulated thyroglobulin of less than $1 \mathrm{ng} / \mathrm{mL}$ as a criterion to spare low-risk patients with papillary thyroid cancer from radioactive iodine ablation. Thyroid 22:1140-1143.

Address correspondence to: Bryan R. Haugen, MD University of Colorado School of Medicine Aurora, $\mathrm{CO}$

E-mail: bryan.haugen@ucdenver.edu 